Fall 1997

\title{
1997 Miracle Yearbook
}

\section{Cedarville College}

Follow this and additional works at: https://digitalcommons.cedarville.edu/yearbooks

Part of the Higher Education Commons, Organizational Communication Commons, and the Public Relations and Advertising Commons

\section{Recommended Citation}

Cedarville College, "1997 Miracle Yearbook" (1997). Yearbooks. 3.

https://digitalcommons.cedarville.edu/yearbooks/3

This Book is brought to you for free and open access by DigitalCommons@Cedarville, a service of the Centennial Library. It has been accepted for inclusion in Yearbooks by an authorized administrator of DigitalCommons@Cedarville. For more information, please contact digitalcommons@cedarville.edu. 


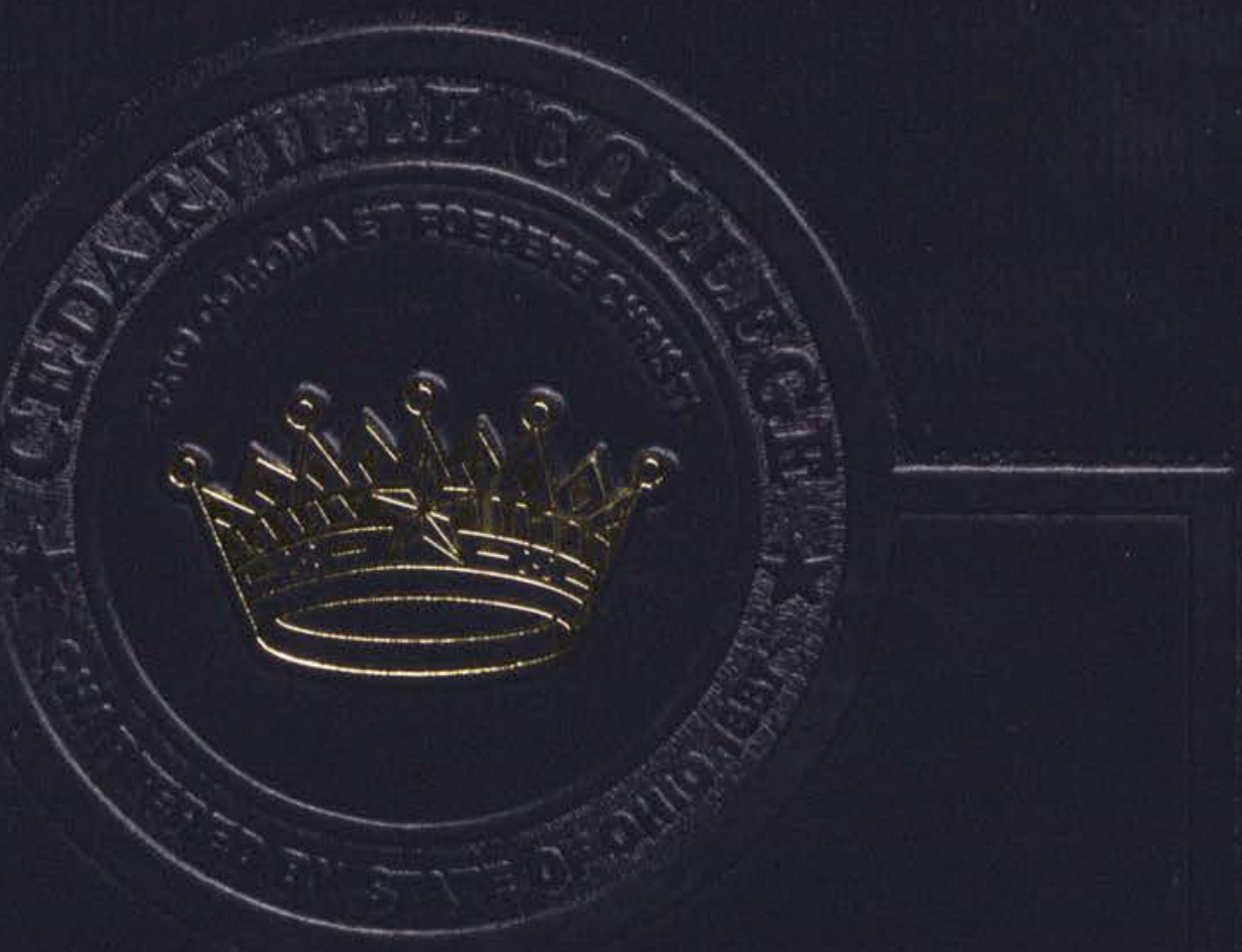

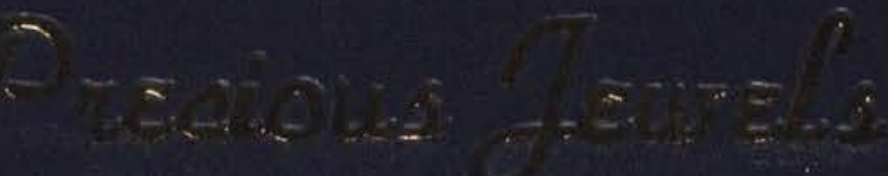

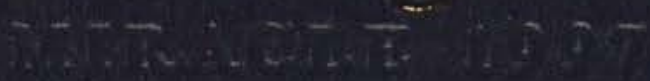




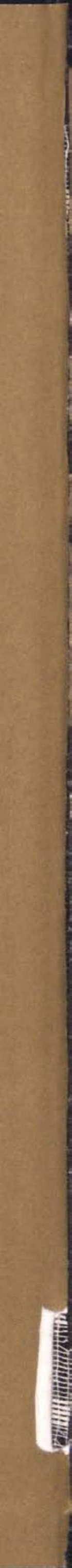




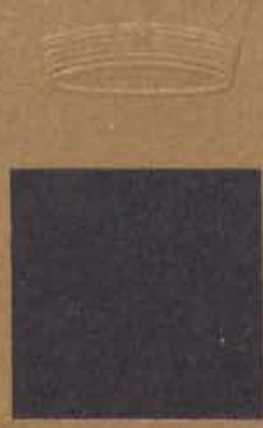

Student Life, Page 6

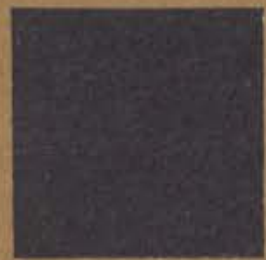

Acadrmic1, Page 58

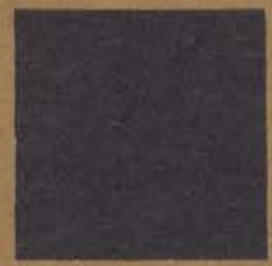

Christian Ninistries. Page 152

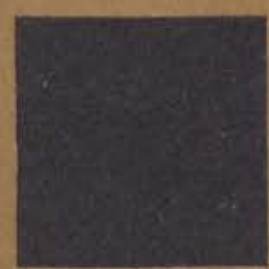

Organizations, Page 186

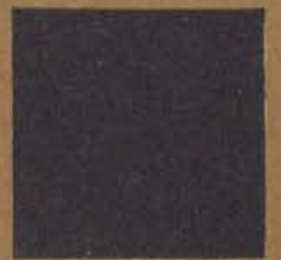

Athletics. Page 200

Contents 

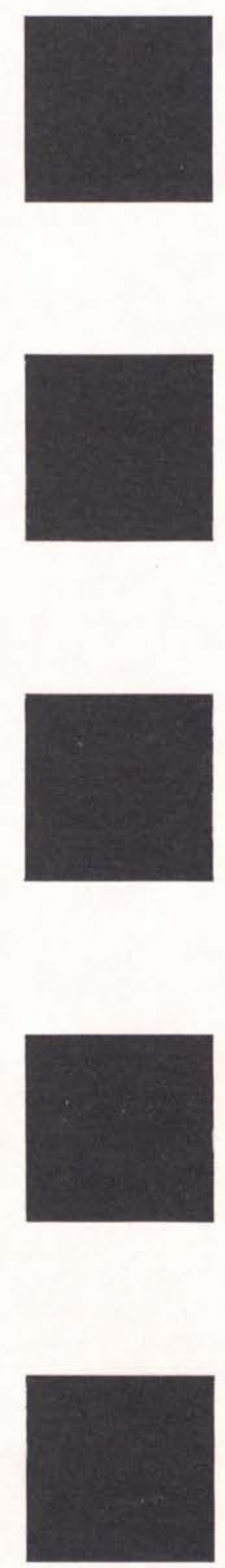


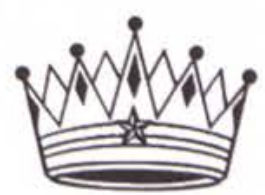

"Yhowing that you were not redermed with perishable things like siluer or gold, . . Gut with precious blood, as of a famb unblemished and spotless, the Glood of Christ.

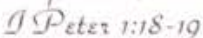

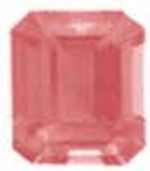

"There is gold and multitude of rubies, Gut the lips of knowledge are a precious jewel."

Proverbs 20:15

"Again, the kingdom of heauen is like a merchant seeking fine prazls, and upon finding one prarl of great value, he went and sold all that he had, and Gought it.

Natthew 13:45

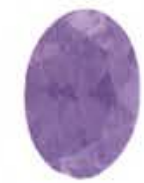

"Now if any man builds upon the foundation with gold, silver, precious stones, wood, hay, or straw, each man a work will become zuident, for the day will show it, because it is to $b_{\varepsilon}$ revealed with fire, and the fire itself will test the quality of each man's work.

$$
\text { ICozinthians } 3: 12
$$

"Do you not know that those who sun in a race all zun. Gut only one receives the prize? - Rus in such a way that you may win. Ig Timothy 2:5

\section{Cedarville College}

P.O. Box 601

Cedarville, $\mathrm{OH} 45314$ 
Another year is complete, and another Miracle marks its passing. This year has brought celebration with the opening of the new Ministry Center, and has also brought sorrow with the deaths of three students. We have witnessed another Presidential election, welcomed a new class of more than eight hundred freshmen, and bid farewell to another class of seniors. Change has been in the air as the college has prepared for new structure and growth by designing graduate programs, dividing into new departmental Schools, and exploring a distance-learning program. There are new faces on the faculty, and familiar faces are gone. Technology upgrades have altered the way many classes are taught, and ways that students do their work.

But for all of the changes and unique events, life at $\mathrm{Ce}$ darville remains much the same. Students still take the same classes and do the same things for fun. People still paint the rock and study outside whenever the sun shows itself. The changes quickly become part of our lives, the rough edges wear off, the feeling of strangeness fades.

The theme for the 1996-97 Miracle is "Precious Jewels."
In light of both change and stability, it is a phrase with different levels of meaning.

First, it is a reminder of our time here at Cedarville. We have learned together, both in classes and out of them, and built experiences and friendships that will last a lifetime. We have made many memories, laughed and cried, and each of those moments is a precious jewel to be prized.

Secondly, the theme reminds us that everything we do has lasting significance. Whether a freshman just beginning, or a senior preparing to graduate, we should keep in mind those things that are most important. "No other foundation can anyone lay than that which is laid, which is Jesus Christ. Now if anyone builds on this foundation with gold, silver, precious stones, wood, hay, straw, each one's work will become clear; for the Day will declare it, because it will be revealed by fire; and the fire will test each one's work, of what sort it is" (I Cor. 3:1113). We can make our actions unimportant and transitory, or we can choose to build with materials that will last an eternity.

The Miracle staff hopes that you have found this year filled with precious jewels of both kinds.

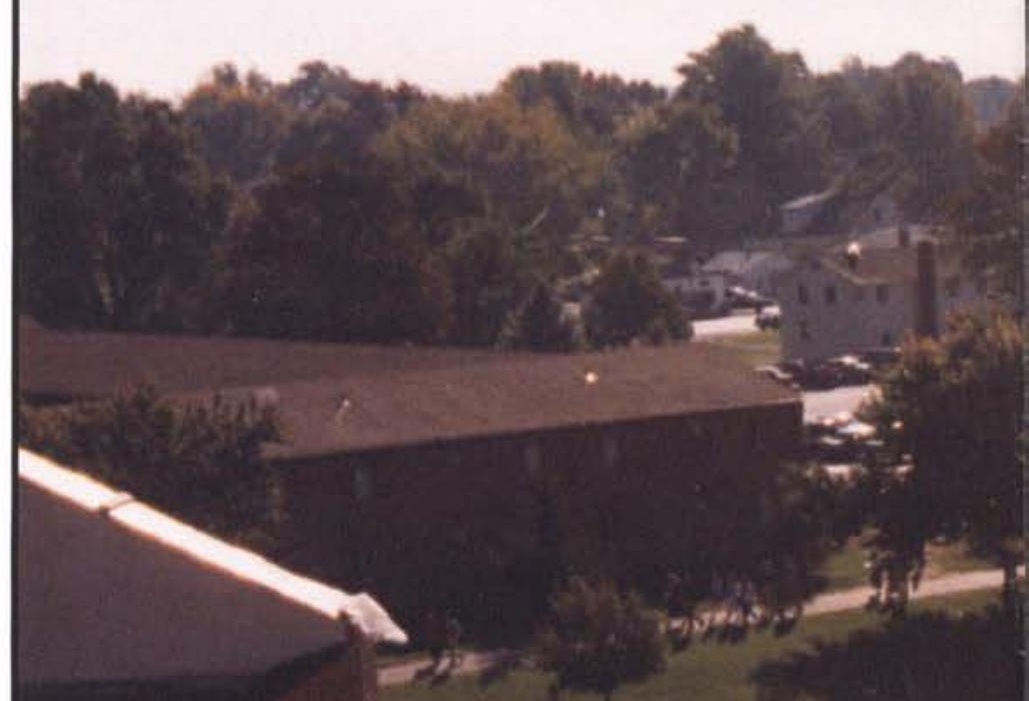



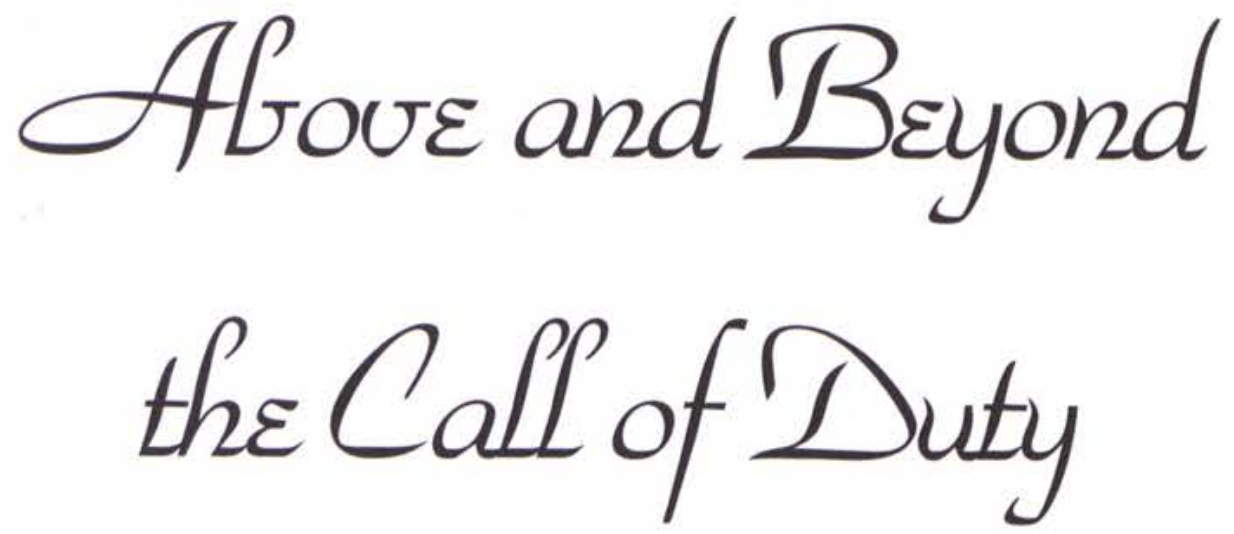

Gy Heather Fourman

Serving God and helping others to serve Him better is what Dr. Cheryl Fawcett is all about. Ever since coming to Cedarville in 1991 as an Assistant Professor of Christian Education, she has tried to use the abilities God has given her to serve Him to her greatest potential.

Dr. Fawcett earned a Bachelor of Religious Education degree from Baptist Bible College, a Master of Arts in Christian Ministries from Wheaton Graduate School, and a Doctor of Education from Trinity Evangelical Divinity School. In 1990, Dr. Fawcett was awarded the Professor A. L. Wedell Christian Education Award from Trinity Evangelical Divinity School, and in October of 1996, she was honored by BBC as Alumnus of the Year. Dr. Fawcett has served as Director of Christian Education for two churches - Tabernacle Baptist Church in Ithaca, New York, and Faith Baptist Church in Winfield, Illinois. Prior to coming to Cedarville, she was the Director of Student Activities at $\mathrm{BBC}$, where she also taught classes and served as the associate director of the Teen Leadership Conference, a position she held for twenty years. In addition to her responsibilities here at the college, Dr. Fawcett teaches in understand the Ten the AWANA ministry at Commandments. Shawnee Hills Baptist Church in Jamestown, and accepts

According to Dr. Jack Riggs, Chairman of the Bible

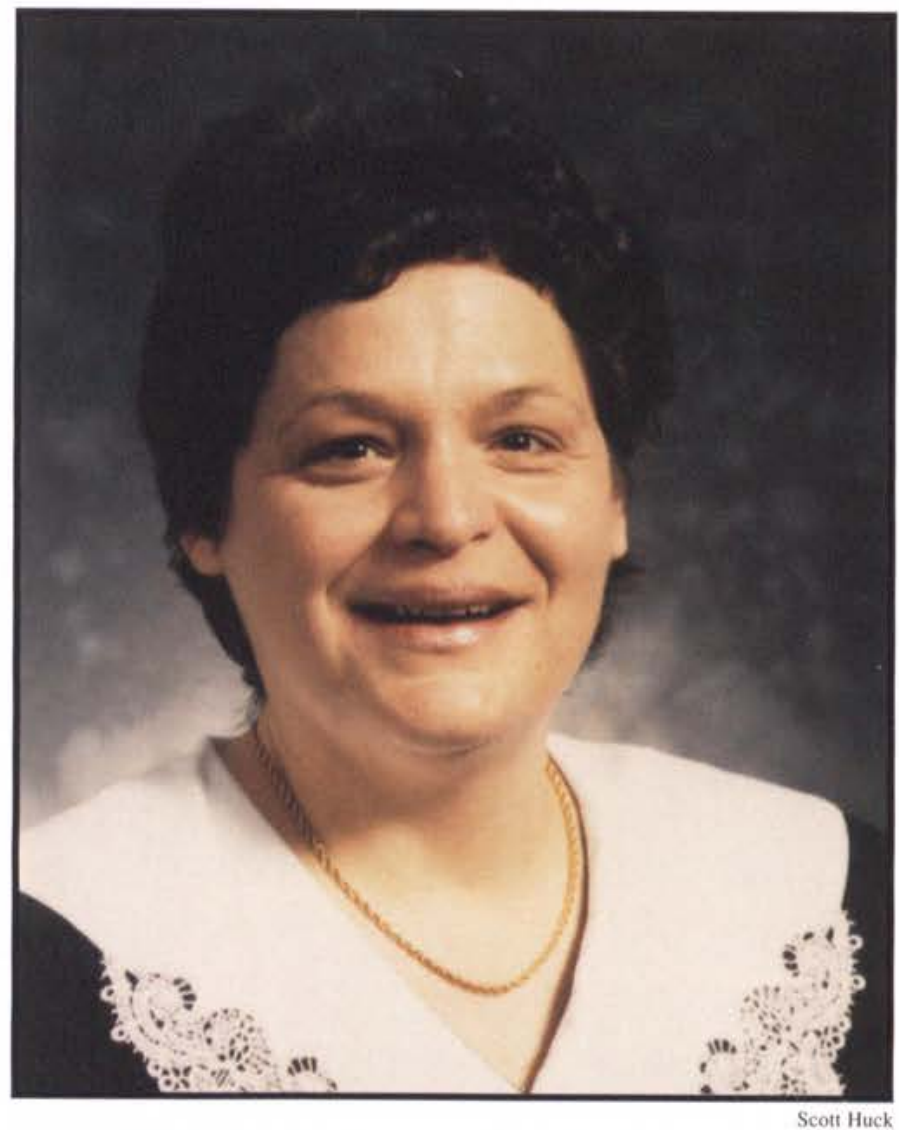

many invitations to speak at retreats and seminars. She has written a short series of children's books entitled Know and Grow, which was inspired by her experience with AWANA. She has co-authored another book called I Have a Question About God, which teaches children basic theological concepts, and is currently working on a third project meant to help children department, Dr. Fawcett has helped make Christian Education the fastest growing program in the Bible department; the number of students studying Christian Education has at least tripled under Dr. Fawcett's guidance. She has worked hard to develop new curricula for the program, adding new courses in small group ministry, discipleship, and women in ministry. She

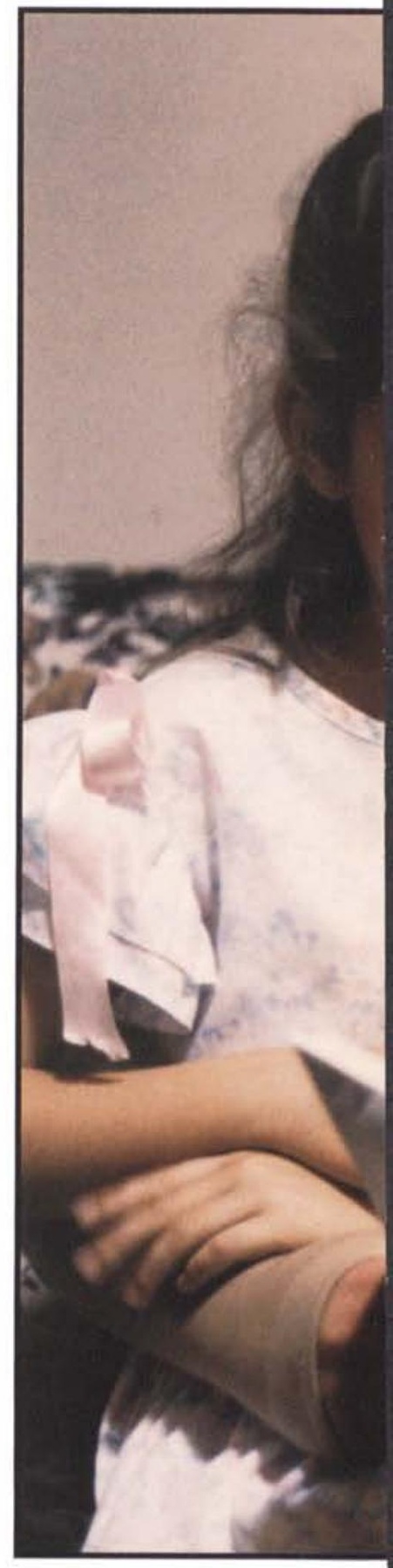

has also done a great deal to broaden the experiences of her students through internships.

Challenging her students is Dr. Fawcett's passion. When she first arrived at Cedarville, she shared with the student body that her desire was to be "up close and personal." Dr. Fawcett's approach means that she offers her friendship and prayer support to her students. She said, "For me, the highest compliment that a student can say to me is that I am their friend." Her desire to teach Bible classes reflects her longing to help bring about change in her students in a way that will influence them for eternity. She said, "I wanted to 

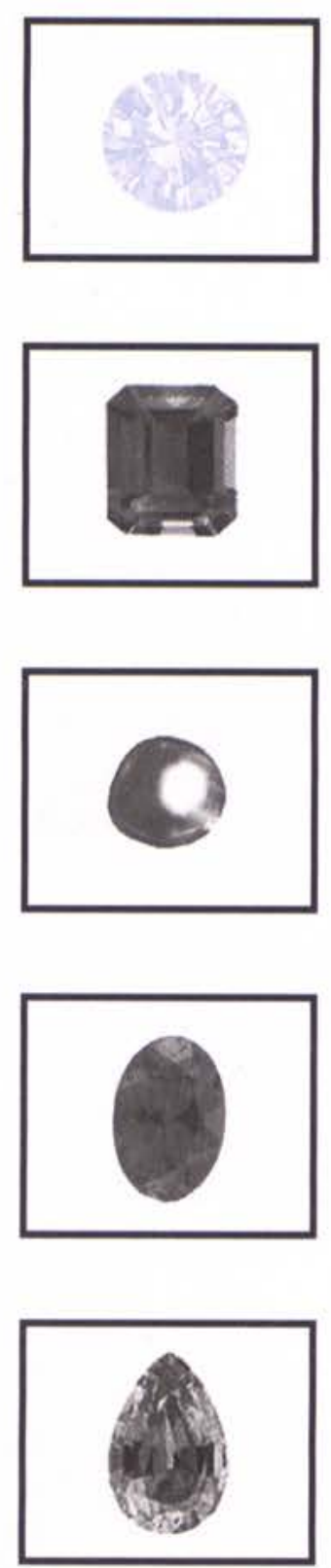

"'Knowing that you were not redermed with prrishafle things like siluer or gold,... Gut with precious flood, as of a Lamb unflemishrd and spotLess, the Glood of Christ."

$$
\text { I) Peter 1:18-19 }
$$

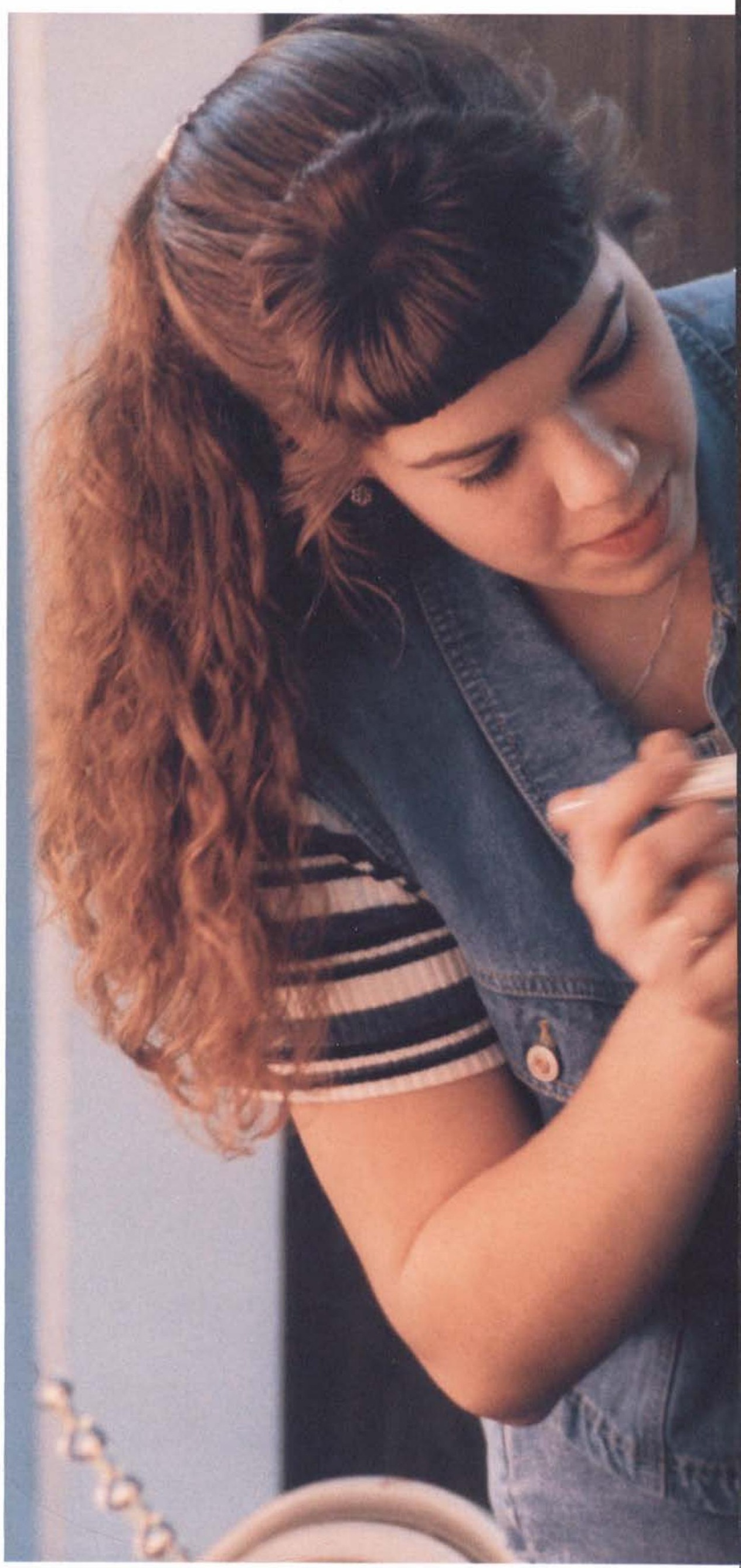




\section{Student Life}

Influential rococo painters, the Sapir-Whorf hypothesis, ligaments attached to the knee, and the semantic range of the word "walk." College is a constant barrage of facts and theories. But, as any student will tell you, there is a lot more to life at Cedarville than academics.

For the outdoor adventurer, John Bryan State Park and the historic Indian Mound are a short trek away. Several local malls have enough variety to satisfy the habitual shopper. Dayton, Cincinnati, and Columbus offer theaters and museums. There are dozens of concerts to choose from, both on campus and off. Paintballing, skiing, bowling, and rollerskating facilities can all be found nearby, and students keep several pizza places in business.

For those with a mind closer to home, the campus offers a number of ways to forget homework. Intramural sports, intercollegiate games, racquetball, and pick-up basketball are as close as the gym. The Gavelyte offers a place to hang out and play board games, ping pong, or pool.

Perhaps the most memorable times in college are those in the dorm, with its impromptu wrestling matches, late night talks, unit and hall meetings, and the dozens of close friendships that form over the years. Mixing with our fellow students remains the best part of college.

Keturah Stork 


\section{Discovering the "Ville \\ by CRochelle =Marcum and CRachel English}

he first few days of ollege life can be overwhelm-

the right frame of mind for the weekend.

On Friday, the RA's helped ing as new students face long registration lines, stacks of paperwork, moving in, and meeting new people. Getting Started is designed to make all those things as painless as possible.

More than 800 freshmen and transfers arrived on campus on the weekend of September 20, and the Campus Activities Office was ready for them, with the help of about 120 upperclassmen volunteers.

Getting Started '96 actually began a week before the official fall quarter, when the Resident Assistants arrived for their training sessions. Small-group leaders and support staff trickled in on Wednesday and Thursday for detailed instructions on what would be happening during the next few days, followed by a trip to Captain Bogey's to get everyone in

class picture, taken in the rain. That was followed by some time for everyone in the small groups to get to know each other. The rest of the evening included small group pictures, games, food, and fellowship.

The rain stopped long enough on Sunday that a number of people braved the mud for the annual Hike to the Gorge. After that was another small group meeting, this time to help new students figure out the busy schedule of meetings and activities that would make up the rest of the week.

Getting Started is the first thing that most students remember about their time at Cedarville. It is a tribute to the hard work of everyone involved that those memories are good ones.
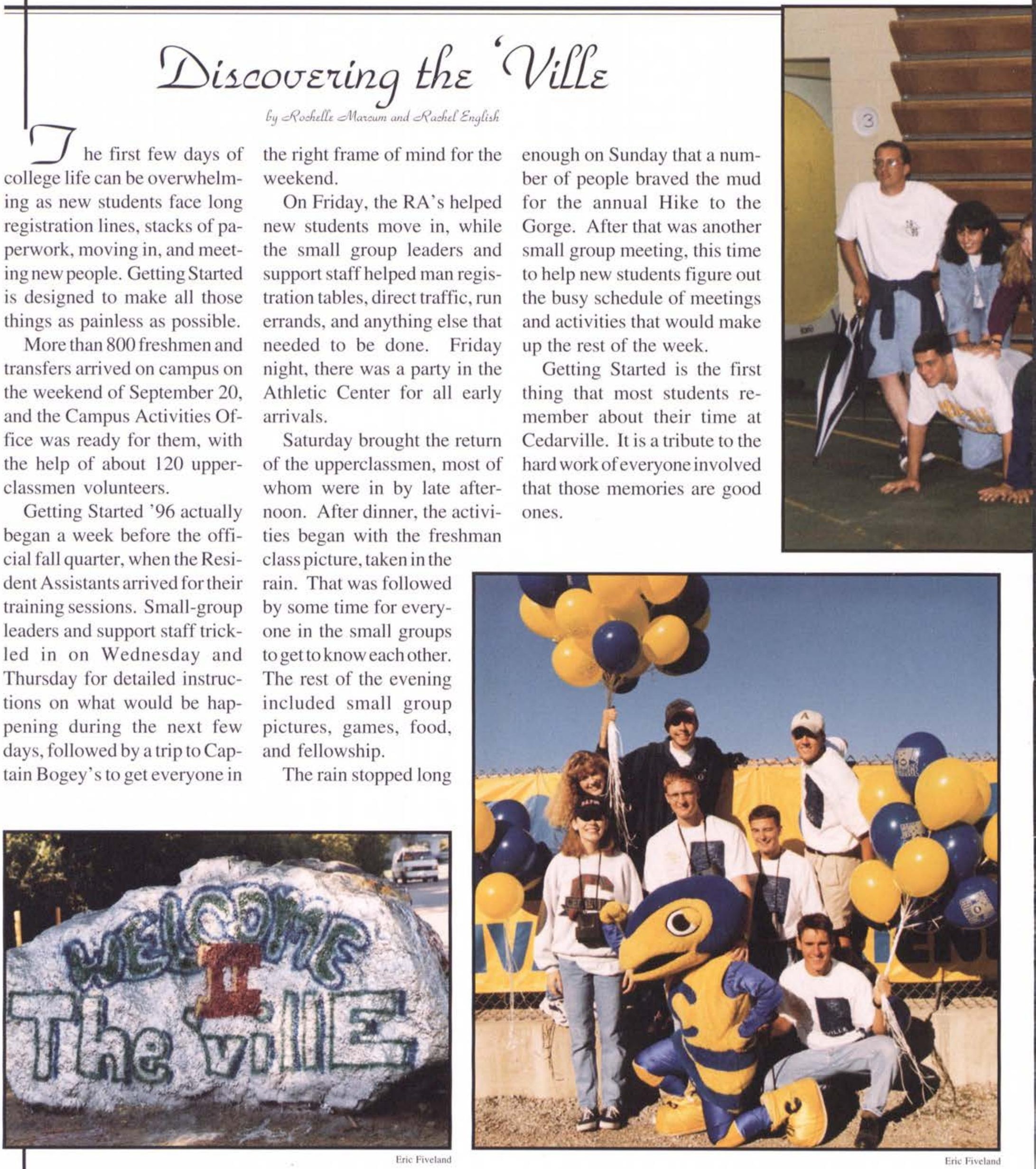


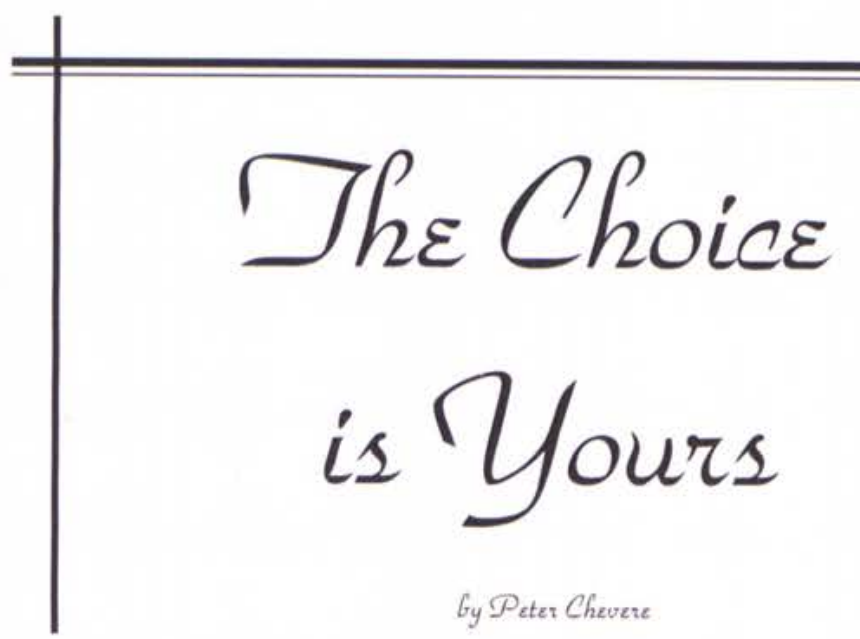

$(1)$ ctober 11 th brought the annual Organizational Fair, with hundreds of students filling the Athletic Center for food, games, and a chance to look over the numerous clubs, societies, and organizations. The fair allows the smaller organizations to interact with students on the same footing as the larger ones.

In order to attract attention, each organization uses games and activities of all kinds at their booths. Returning favorites included the Joust, the

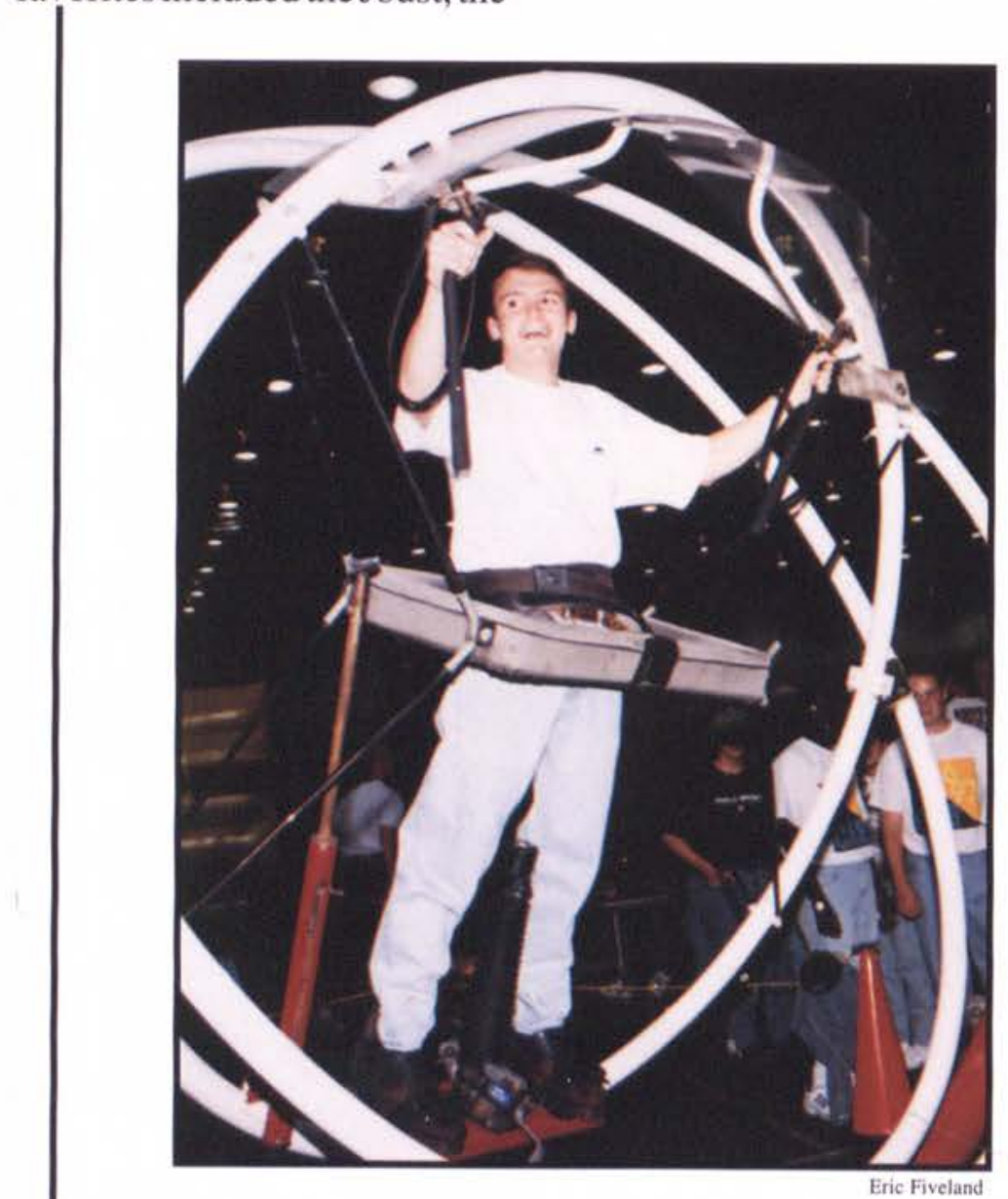

Gyro, and the Bungee Run. WSRN's karaoke table was a popular site as well. Many booths offered prizes, ranging from candy to T-shirts and gift certificates. The Student Government Association coordinated the evening and provided food and drinks for everyone.

As usual, the Fair was a success, both for students and for organizations. It was a fun time of interaction for all involved.
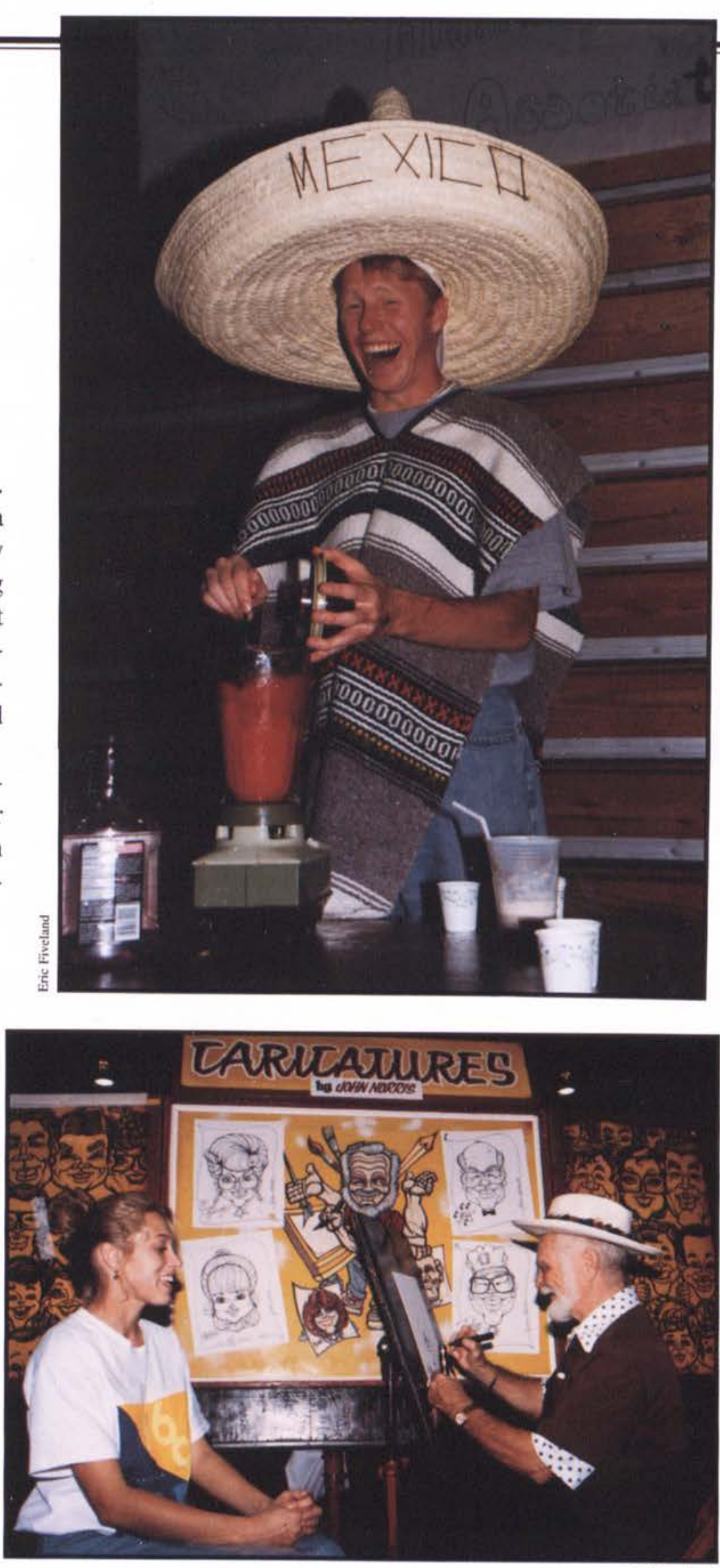

Eric Fiveland

Above: Reade Faulkner stirs up a little "south of the border trouble" at the UAA booth. Below left: Scott Thomson lives on the edge as he experiences weightlessness. Below right: Joy Beitler has her true nature sketched in a caricature. 


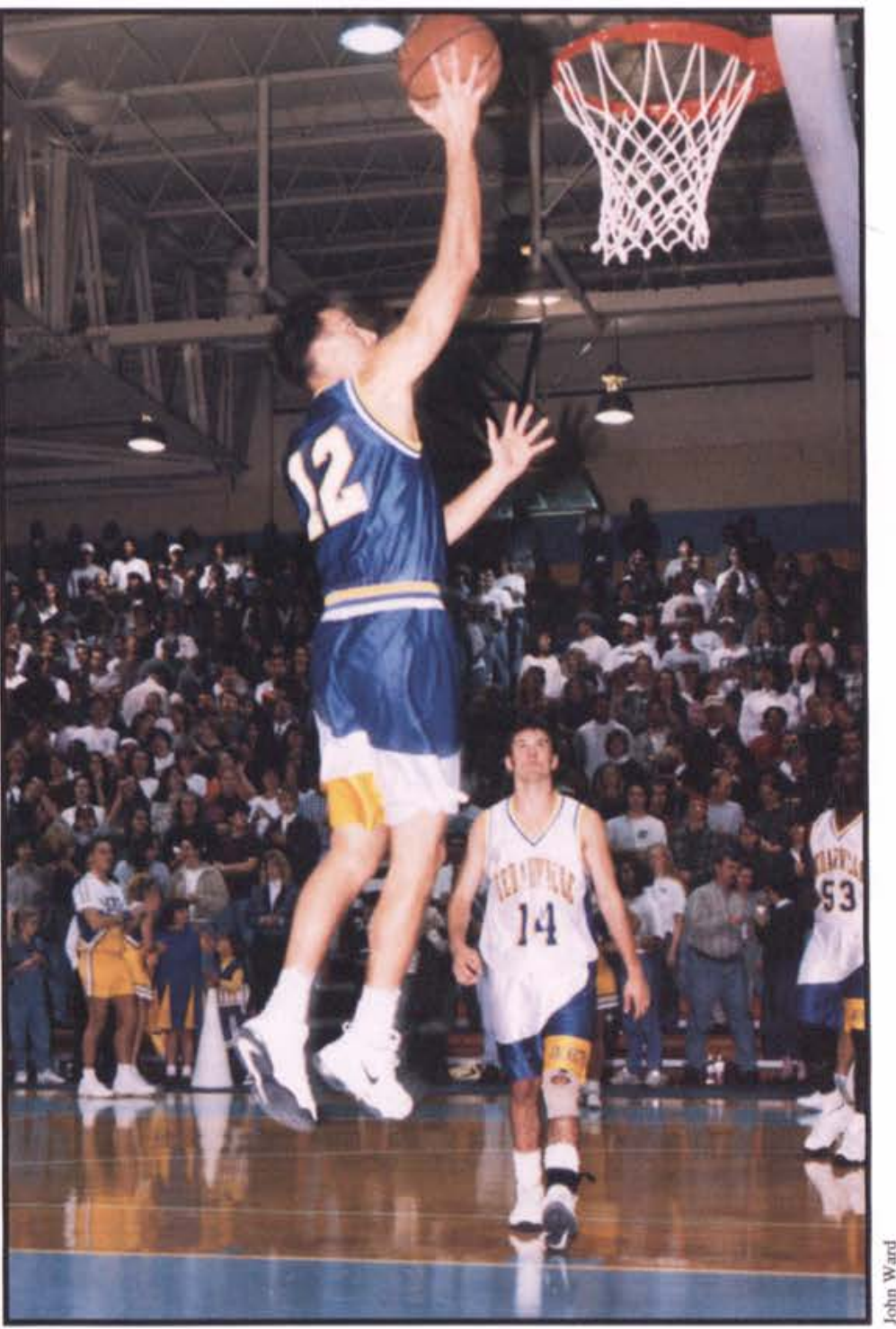

\section{Out to Win}

by Dim Hotehisis

$(\bigcap$ n Friday, October 18, hundreds of Cedarville students, alumni, family, and friends poured into the Athletic Center for Moonlight Madness, the annual kickoff of the new basketball season. While the crowds arrived, the Pep Band started the festivities with a number of favorite songs, including "Jackets Go," "Hang On Sloopy," and "You Can Call Me Al." Dr. Dixon officially opened the evening with prayer, followed by the Pledge of $\mathrm{Al}$ legiance. Then the lights went out, and, with a background of cheers and screams from the audience, Dr. Matson introduced the team. There were quite a few new faces on the Yellow Jackets this year, in addition to a few returning players. They played a scrimmage, demonstrating their skill and getting a feel for the home crowd. The evening ended with a slam dunk contest. Linton Ellis won by having a fellow team member sit in a chair, and jumping over both to jam the ball while the crowd cheered him on. It was an exciting way to close out the night and open a new season of Cedarville basketball.
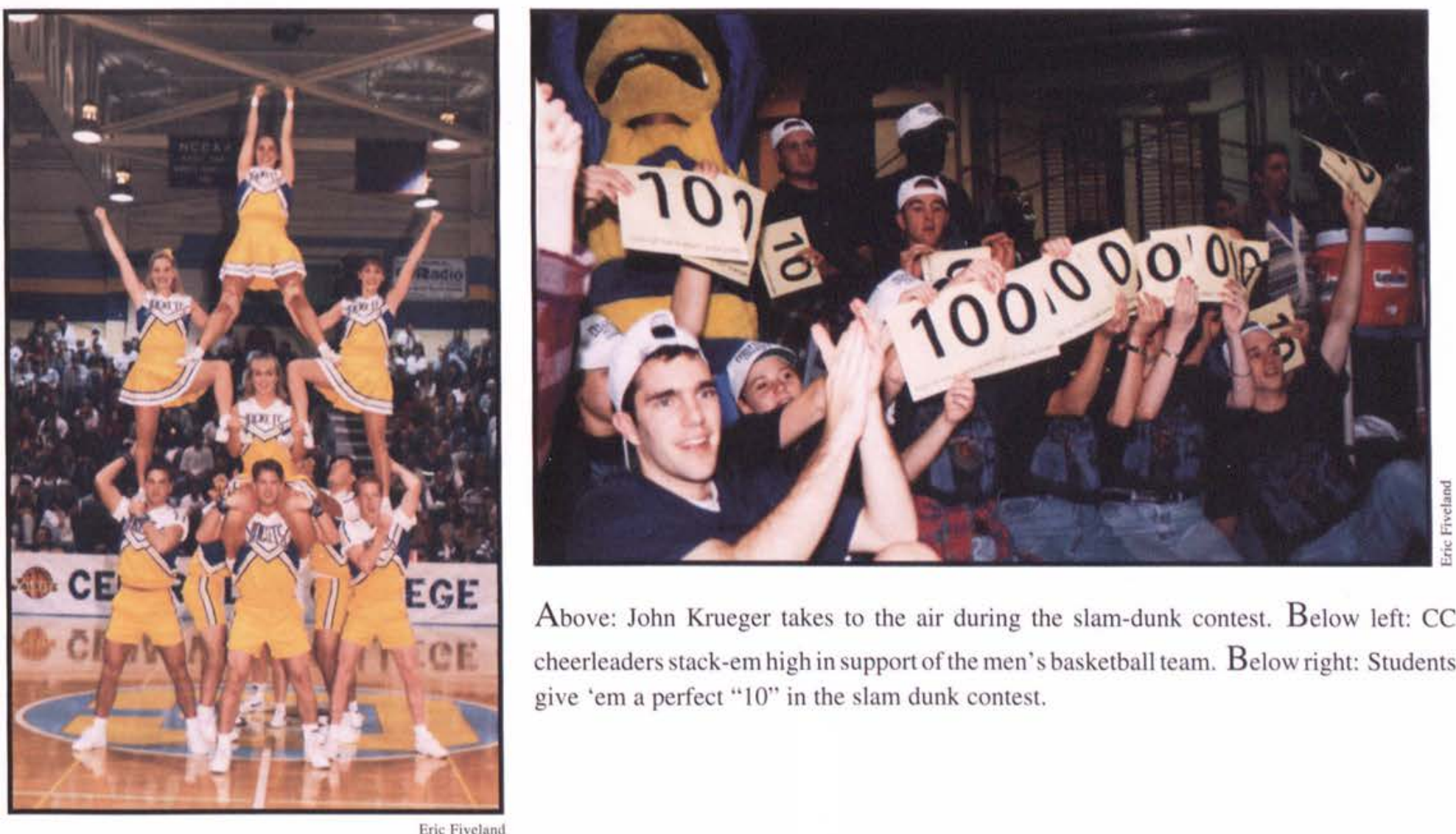

Above: John Krueger takes to the air during the slam-dunk contest. Below left: CC cheerleaders stack-em high in support of the men's basketball team. Below right: Students give "em a perfect " 10 " in the slam dunk contest. 
7 he theme of this year's Royalty Banquet was "A Table for Two." Chuck's was redecorated in black, burgundy, and gold with a lighted city skyline covering one wall, in order to resemble a dinner club from the 1940's. In keeping with the atmosphere, Jody Hovis and the Lab Band provided 30's and 40 's jazz music during the dinner. After the music, Stacy Saville and Dan Scott did a George Burns and Gracie Allen sketch that received a lot of applause from the assembled diners.

Brian White announced this year's Homecoming Grand Marshalls, Vice President and Mrs. Donald Rickard, and presented them with a silver bowl and a quilt embroidered with the history of Greene County, Ohio. In conclusion to the dinner portion of the evening, Josh Amos announced the identities of the Homecoming Attendents

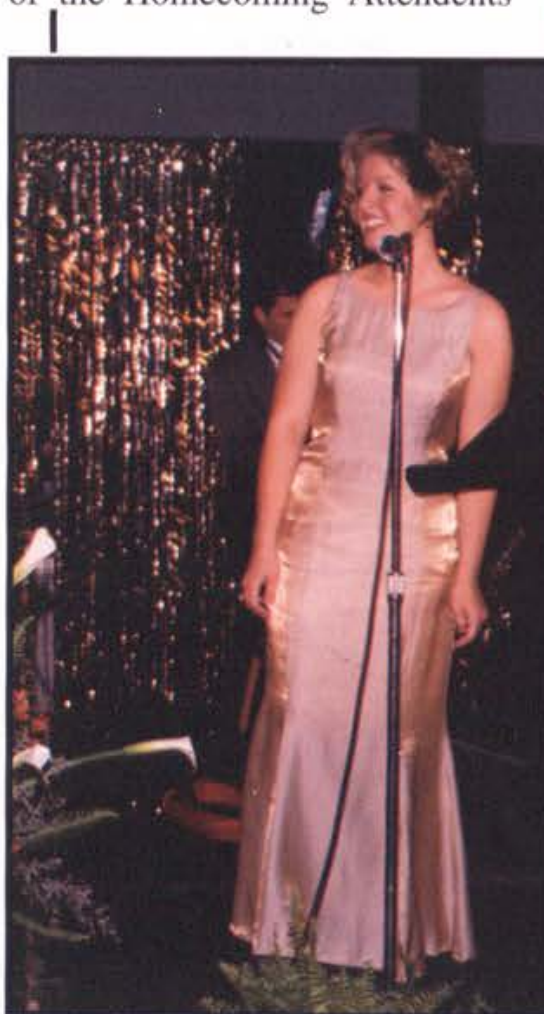

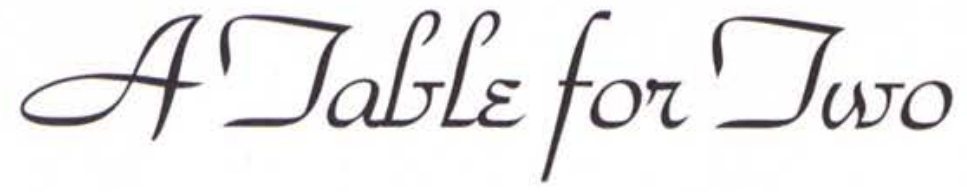

by Mraghan Lemke and theQueen, who was crowned by the Rickards.Immediately following the dinner was a concert by Out of the Grey, Christian musicians Scott and Christine Dente. They performed songs from their three albums, punctuated by up-beat dialogue and teasing banter. Their only musical accompaniment was Scott's skilled and intense-guitar playing. They quickly developed a rapport with the crowd, and played two encores before they finished. It was an evening filled with good music and fun for all who participated.
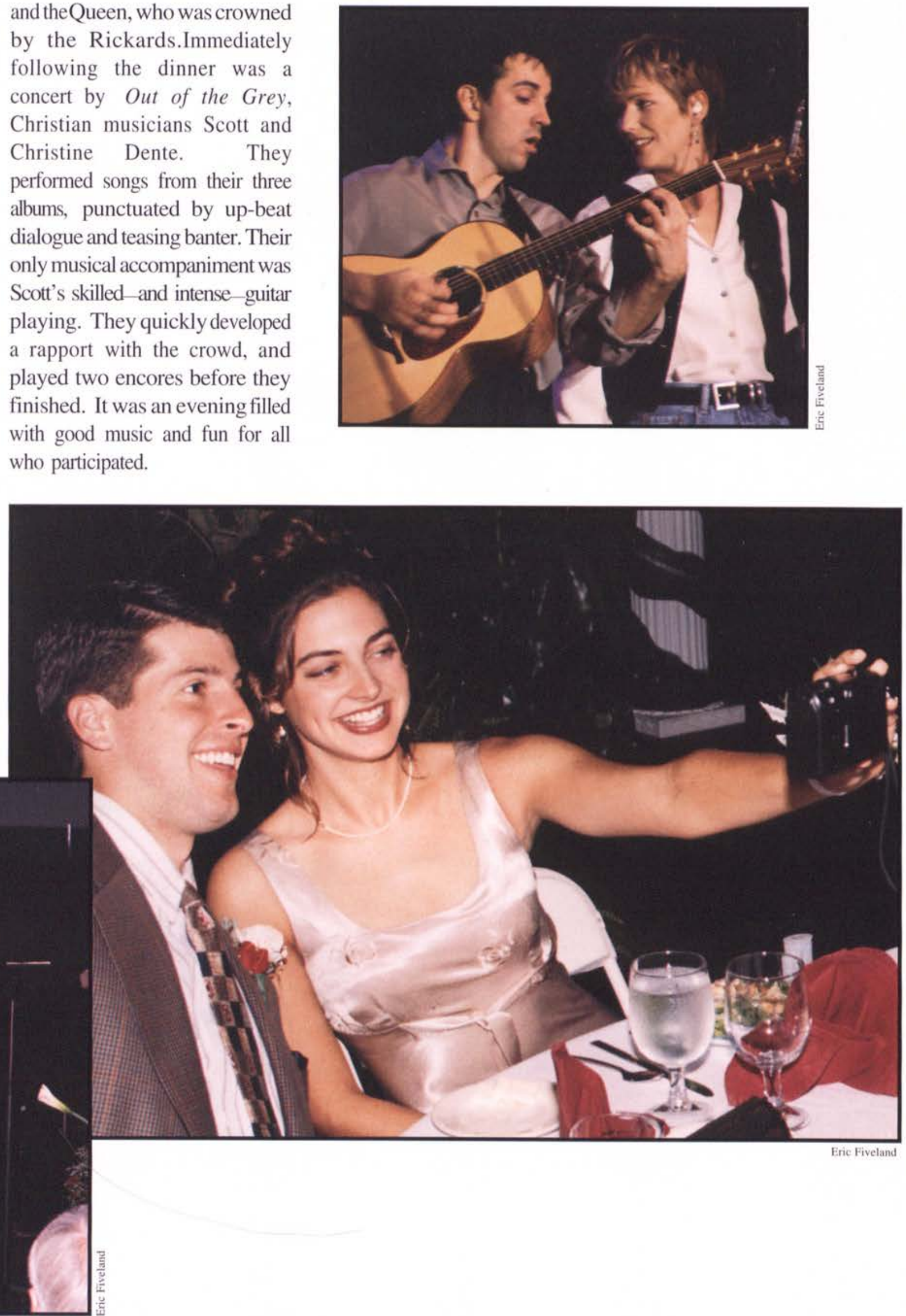

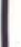




\section{Crowned with Glory}

Gy Keturah Stork

$\mathrm{Ca}_{\text {r a L y n }}$ Ludwig was crowned Homecoming Queen on Saturday, October 12 , at the 1996 Royalty Banquet. She is a senior nursing major, and while she describes her life as ordinary, it is far from boring.

She lives in Brooklyn, New York with her parents, three older brothers, and cat named Pokey. Although she's from the city, Sara enjoys the outdoors, and likes to play soccer and go for hikes.

Because her father is a Baptist pastor, Sara knew about Cedarville, but she chose to apply to several state schools first. When she visited the Cedar- ville campus, its slower pace and the friendly students caught her attention. The Christian atmosphere is what convinced her to attend.

This year, as she is preparing for graduation, Sara says that her spiritual growth and her close friends rank as the most important parts of college that she will take with her.

Sara tells an amusing story about her nomination as Homecoming Queen. As her father brought Sara to college her first year, he told her to work hard and to become class valedictorian and Homecoming Queen. Sara immediately dismissed this idea. She did not recall her father's words when, during her junior year, several friends nominated her for Queen. These friends raised enough support, and the following summer, Sara received a letter stating that she had been chosen as a candidate. Her first reaction was terror at the prospect of speaking in front of the entire student body.

Sara decided that God had something important for her to share with her fellow students. During her chapel speech, she told the college family about her internal conflict over her major. Dreaming of one day becoming a nurse, she had studied hard and looked forward to her clinical experience. She feared that her dream had ended when, beginning her clinical work, she discovered that she could not stomach the actual hands-on practice. She began to despair over what God wanted her to do if she could not use her nursing skills.

The turning point occurred on her MIS trip to Togo, West Africa. Sara suddenly felt confident to complete her nursing responsibilities and saw that
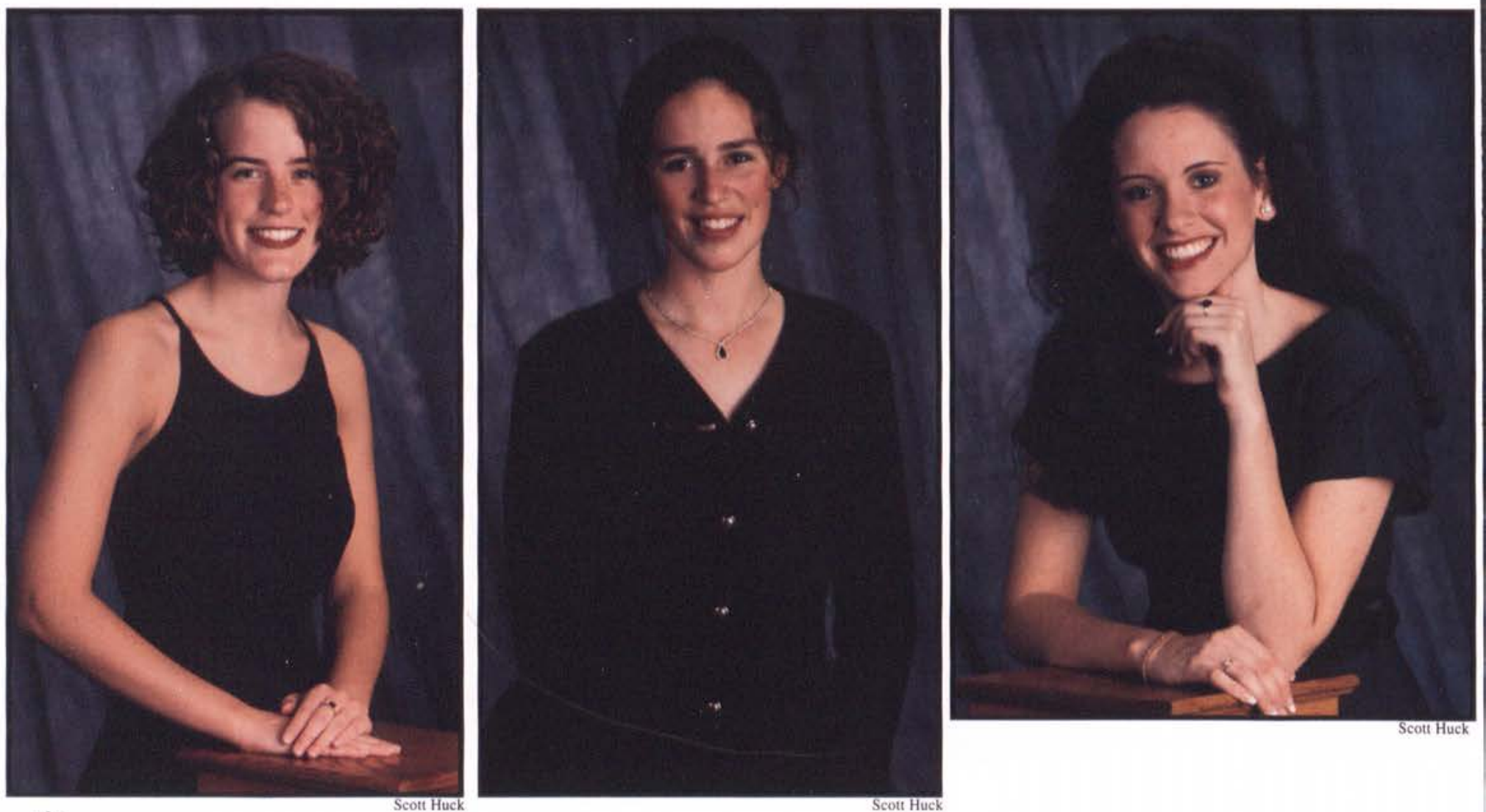
God could use her to minister to the patients there.

Being Homecoming Queen has filled this year with exciting memories, but Sara is also ready to begin "real life." She will miss the many friends she has made at college, but after four years, she knows that the time has come to apply all that she has gained here. She advises underclassmen to take one-hundred percent advantage of the academic and friendship opportunities at Cedarville, and to get involved with ministries. All of these things will have a positive influence and will follow students long after they leave college.

Right, queen: Sara Ludwig is a senior nursing major. Her life verse is Ps.28:7a, "The LORD is my strength and my shield; my heart trusts in him, and I am helped."
Bottom left: Cindy Parsons, from Charleston, West Virginia, is an organizational communications major. Cindy is interested in missions, and her life verse is Philippians 3:8. Bottom center: Krystal Bailey is an accounting major from Marshalltown, Iowa. Her ambition is to obtain her CPA and use her skills in the ministry. Bottom right: Paula Faris is from Jackson, MI, and majors in broadcasting with an emphasis in television production. She is interning at CNNSI this spring. (Opposite page) Upper left: Sarah Gross, junior attendant. Bottom left: Jennifer Tegtmeier, sophomore attendant. Bottom center: Geneva Roberts, freshman attendant. Bottom right: Alissa Whitten is an organizational communications major from Romeo, MI. She plans to pursue a career in law after working corporately for a few years. Psalm 145 is one of her favorite passages.
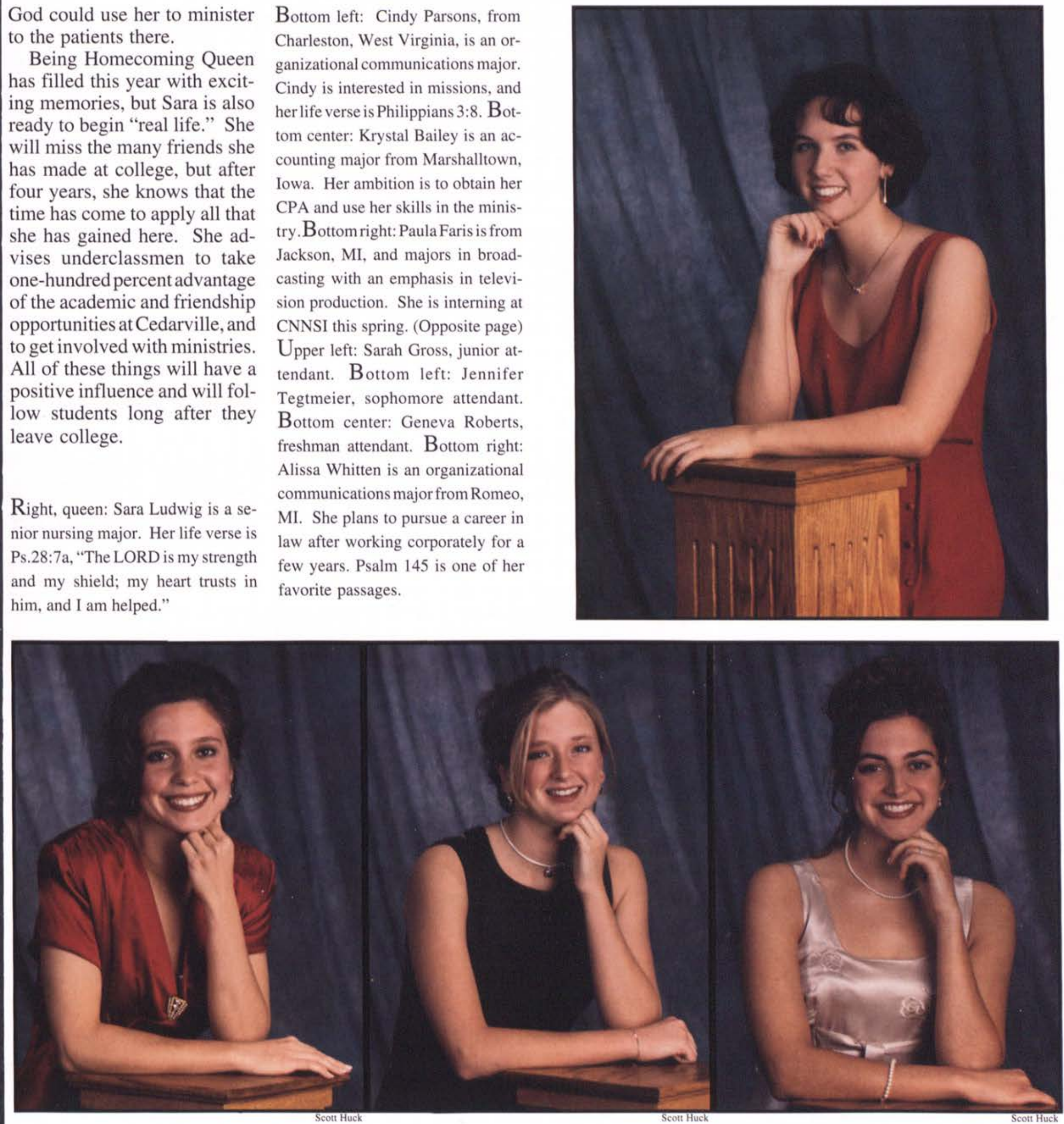

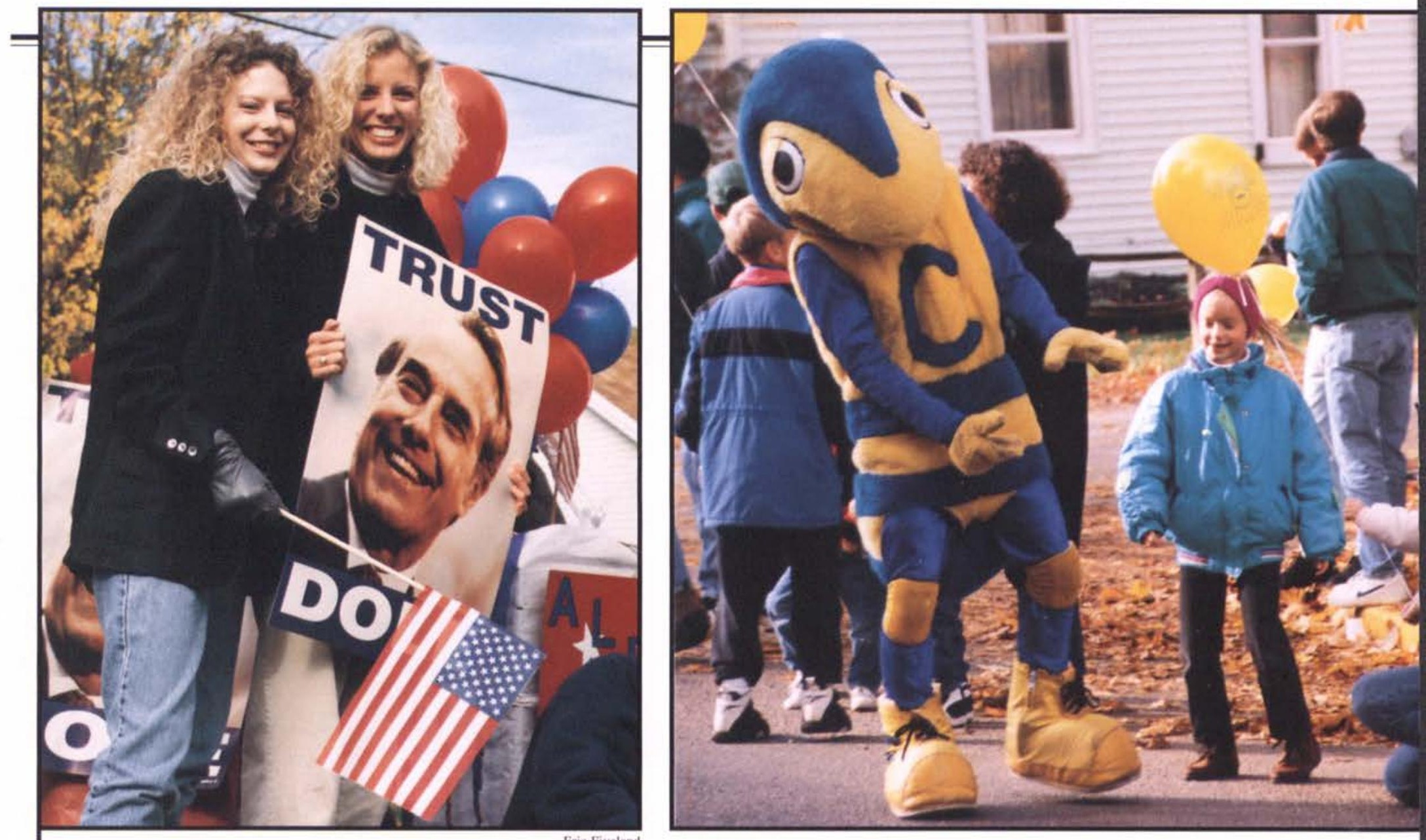

Eiric Fiveland

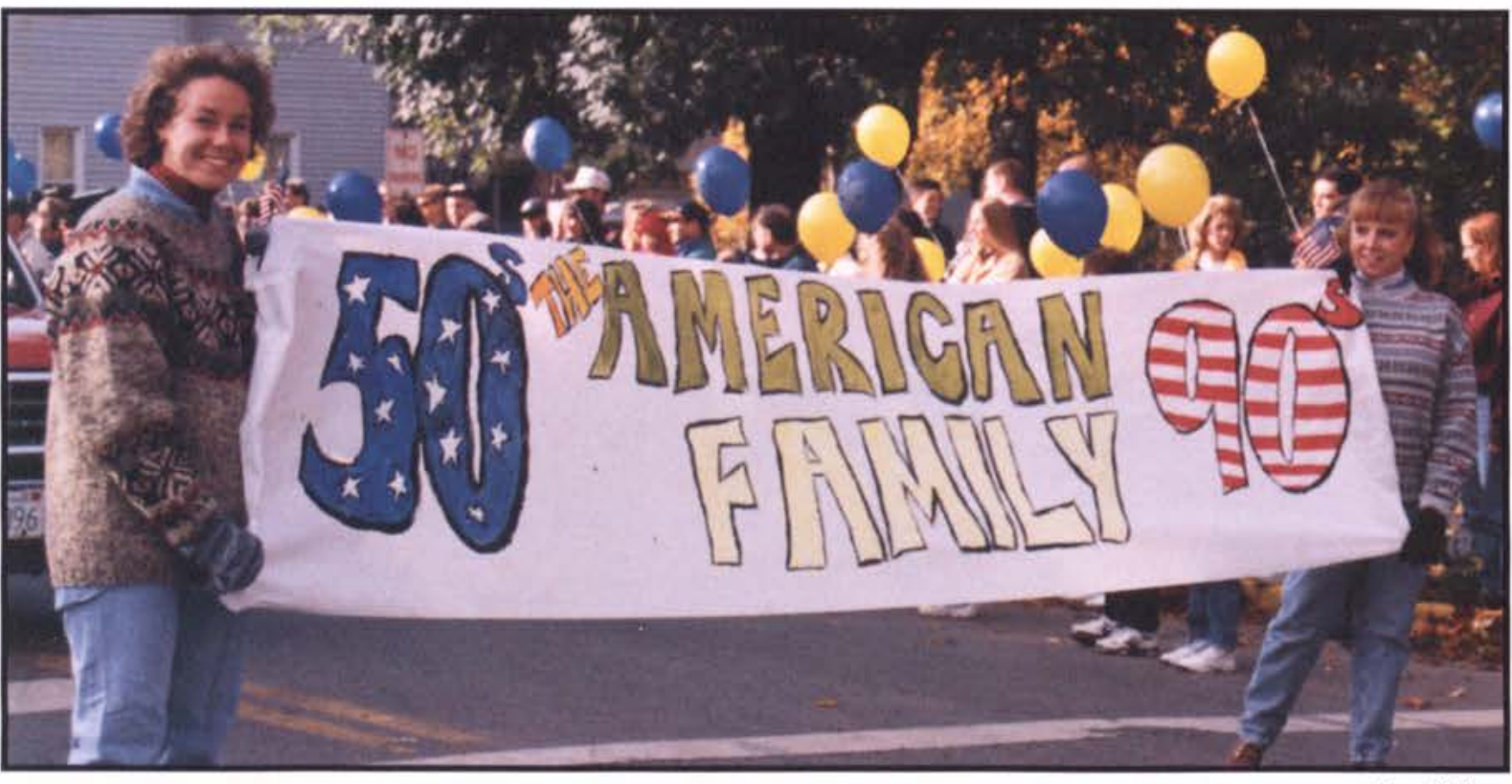

Laura Milligat

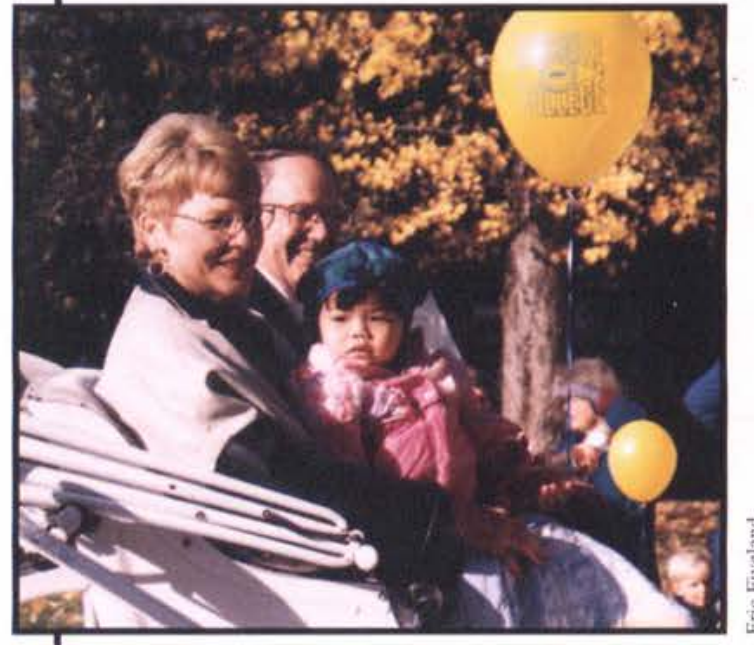

Above left: Jen Benson and Lisa Hunsberger show their support for presidential candidate Bob Dole. Above: The Bee greets an onlooker. Below left: Dr. and Mrs. Dixon ride with their youngest grandchild, Ellie. Above middle: Tiffany Zimmerman and Sheri Wilson walk with the Junior class float. 


\section{Patriotic Procession}

Gy Ronniz Schacfer

$\subseteq$ aturday morning of Homecoming Weekend brought with it rays of sunshine and a full schedule of activities.

The alumni soccer game led off the action. At 9:00, former Cedarville soccer players proved they could still kick a ball with some authority.

The big event that involved Cedarville College students, alumni, and residents of the town was the parade at 10:00. The sidewalks were lined with people as floats, carriages, and convert- ibles slowly made their way up Main Street, the occupants handing out candy to eager children. In addition, onlookers were treated to yellow and blue balloons, root beer floats, American flags, apple pies, smiles, and waves during the festive hourlong parade.

Following the parade was the highly-anticipated dedication of the Ministry Center. It was a rare opportunity for both current students and alumni to worship the Lord together in one of the most momentous chapel services of the year. During this ceremonial occasion, it was announced that the building would be named the Dixon Ministry Center after the college's president and his wife. The Christian Ministries wing of the building was named for Bob and Polly Reese, and the Department of Music wing was named for Bill and Nora Bolthouse. Lastly, thechapelitself wasnamed the Jeremiah Chapel in honor of James T. Jeremiah and his deceased wife Ruby.

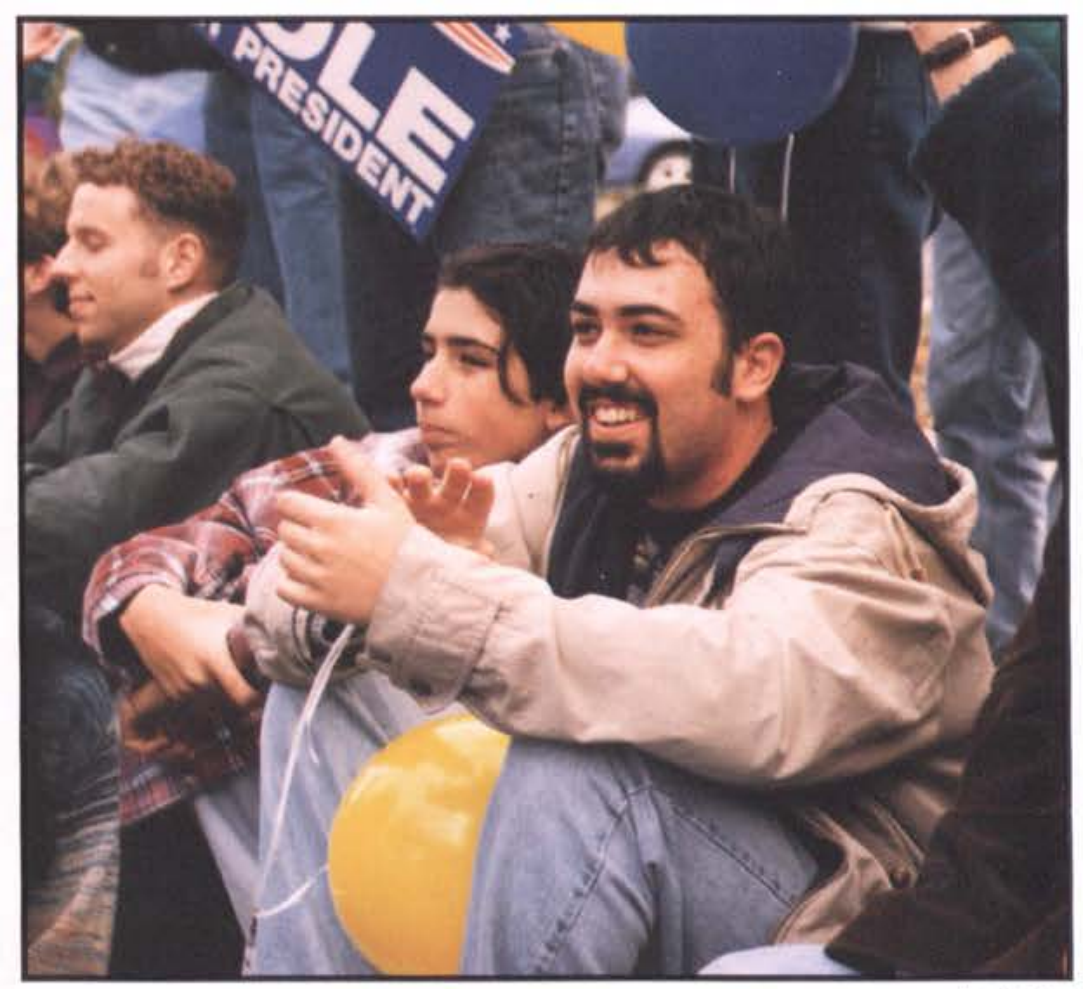

Above: Josh Kimmel enjoys the entertaining parade. Right: Barry Gorsuch poses as the Statue of Liberty.

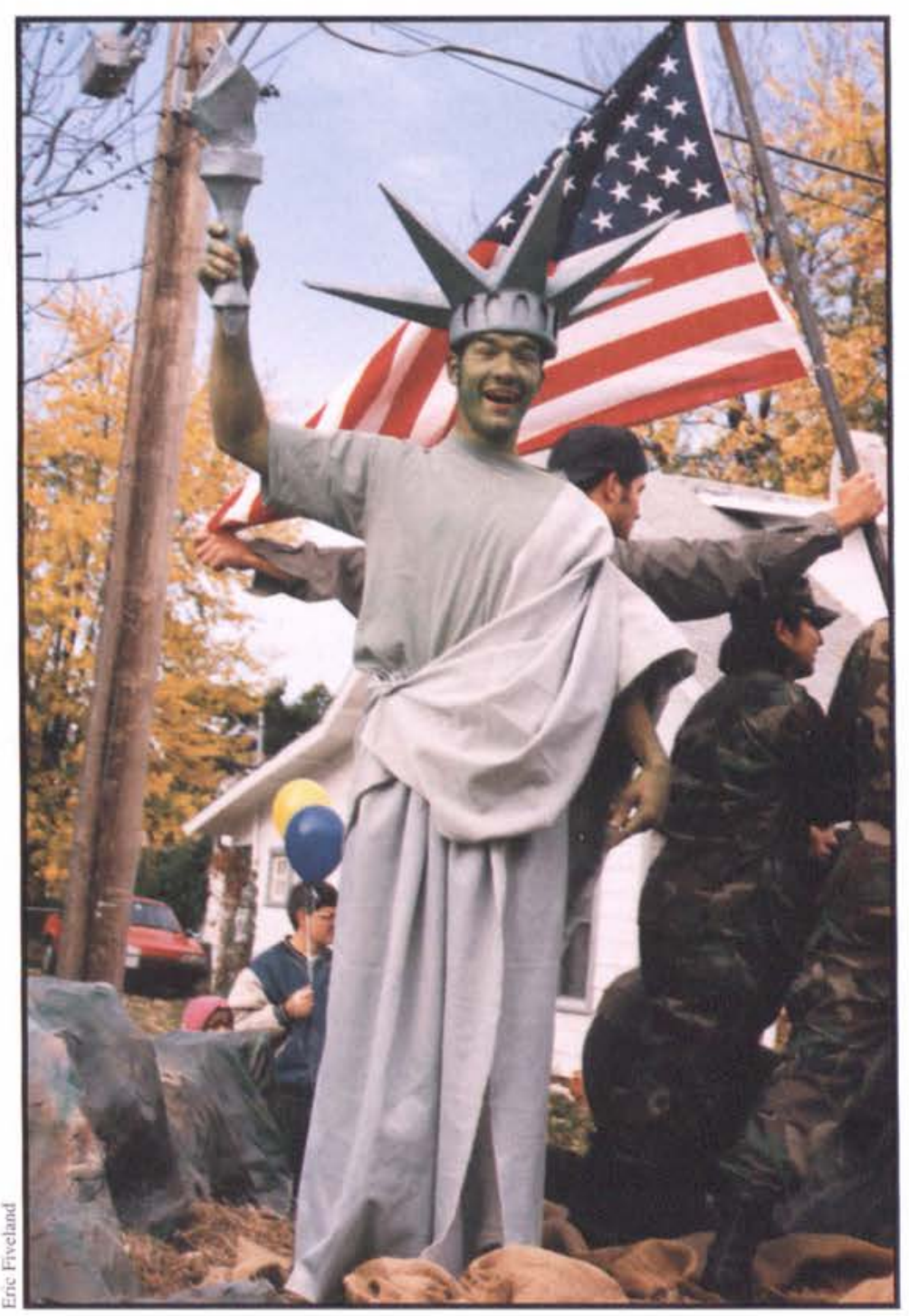




\section{Exploring the C Riches of God 1 Word}

Gy Iesse Deconto. Chris Asheraft, and CRachel'I. Stewart

\section{7} he speaker at the Fall Bible Conference was Dr. David Jeremiah, graduate of Cedarville and Dallas Theological Seminary, and senior pastor of Shadow Mountain Community Church in El Cajon, California. Dr. Jeremiah has ministries through many forms of media. He began his radio career as a disc jockey for WEEC out of Springfield, Ohio, and co-founded Cedarville's WCDR. Today, he ministers to millions through programs such as Turning Point, his syndicated radio broadcast. Dr. Jeremiah is also an accomplished author, but his heart remains in the pulpit. He said that if he had to give up every ministry except one, he would remain a pastor.

The theme of the fall conference was the Beatitudes. Dr. Jeremiah began with a series of challenges to Christians to discipline their lives, and showed how each of the Beatitudes helps the Christian become more like Christ.

Dr. Joseph M. Stowell III,

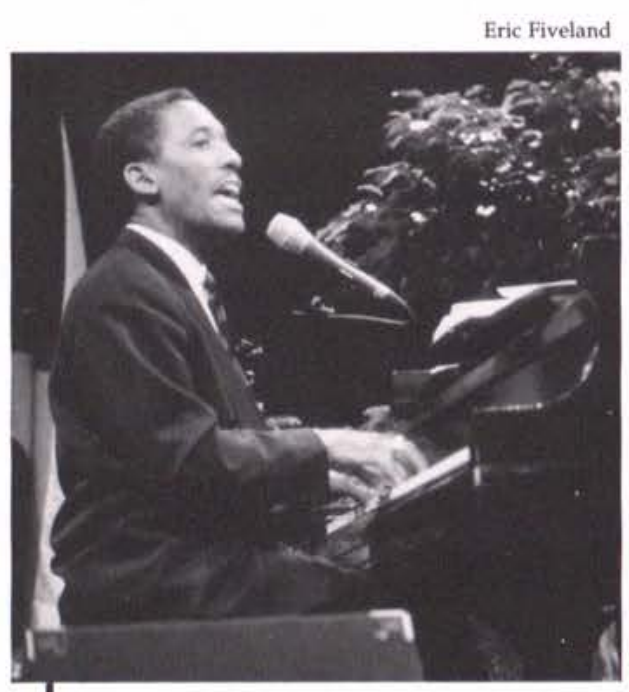

Above: Dr. Richard Farmer asks for a phrase from the balcony and five notes from the floor. the president of Moody Bible Institute (MBI), was the speaker at the Winter Enrichment Conference. Dr. Stowell graduated from Cedarville College and Dallas Theological Seminary and has pastored churches in Ohio, Indiana, and Michigan. He founded Southgate Baptist Church in Springfield, which grew in six years to almost 500 members. Dr. Stowell has written numerous books, including Perilous

Dr. Stowell has a special heart for pastoring, which he displayed throughout the Conference. He focused on the human need of a personal relationship with God. He said, Pursuits and Fan the Flame.
"We will never know intimacy with God until we know how desperately we need Him, for we dearly desire what we depend on."

The theme for the Spring Missions Conference was "Your Future: Built on a Foundation of Service." This year, missionaries representing 24 missions agencies attended. The Conference focused on service. Dr. Dennis Sullivan, BMM Medical Missionary to the Central African Republic and Dr. Viggo Olsen, ABWE Medical Missionary to Bangladesh, spoke about their medical ministry during chapel. Dr. Wendell Kempton, President of ABWE spoke in the

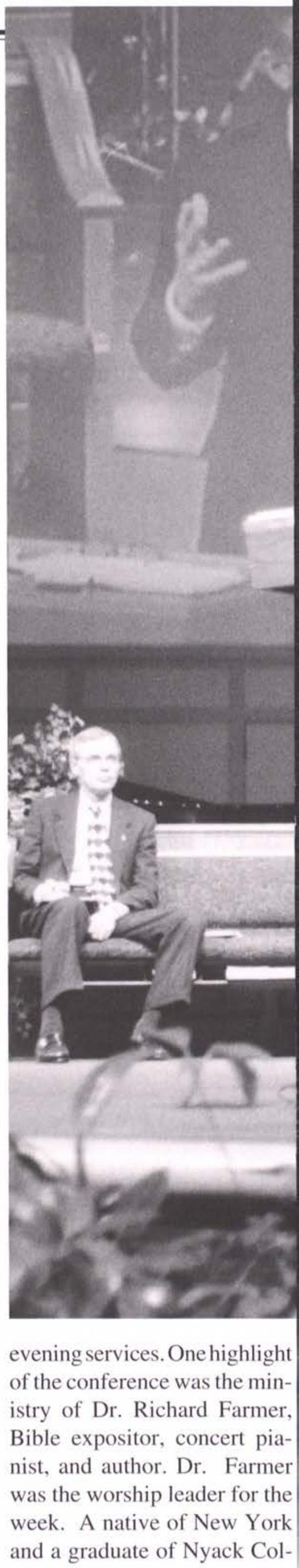

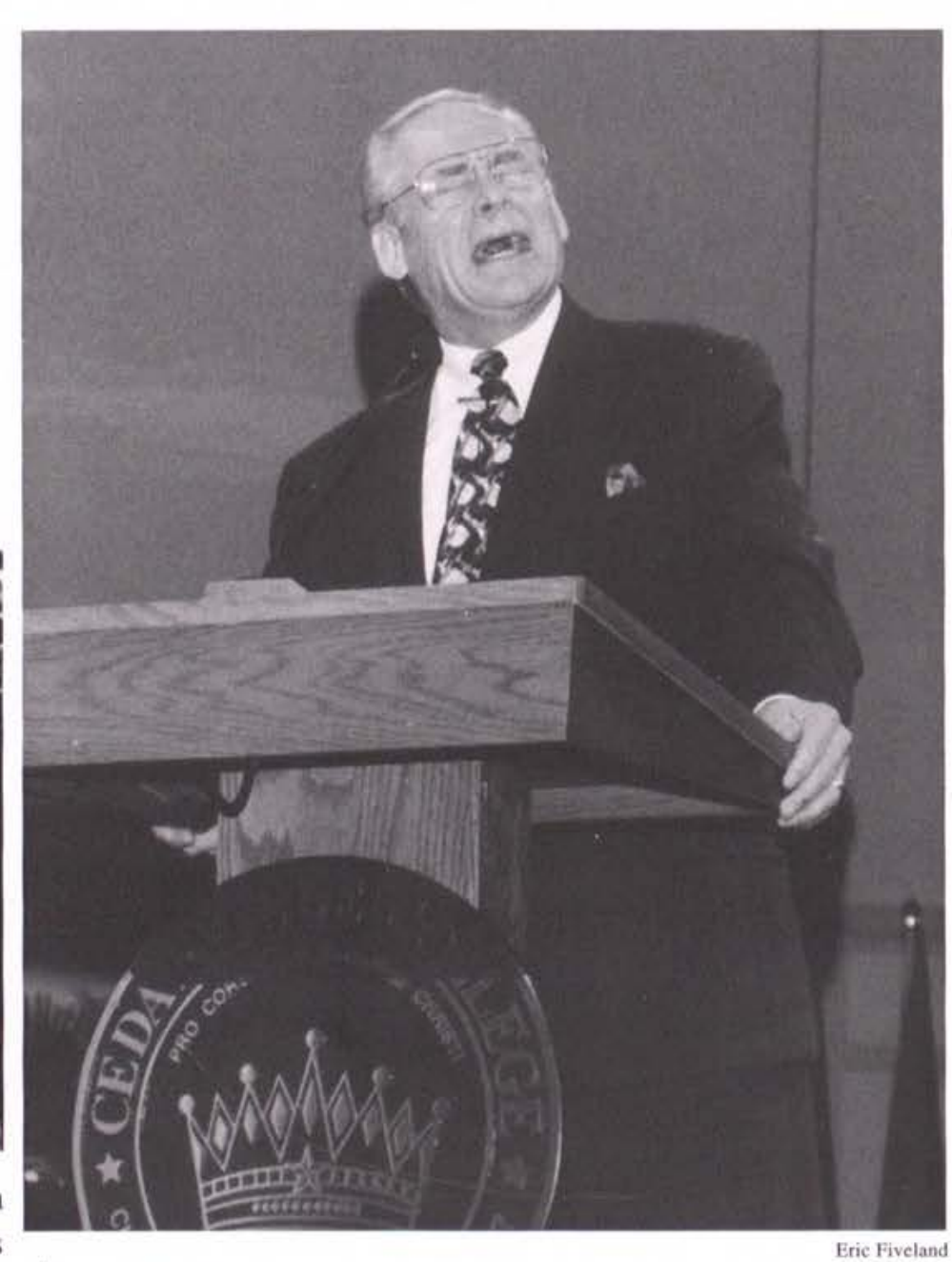

Above: Dr. Wendell Kempton passionately shares his convictions. 


\title{
The Crowning Jewel
}

\author{
Gy Heatier CMarsceau
}

C

hapel at Cedarville has been held in many places since its establishment. Founders Hall was the first home of the college's chapel. As the student body began to grow, chapel moved to what is now the bookstore, then to Alford Auditorium and on to the James T. Jeremiah Chapel in 1976.

For twenty years, the gold and blue auditorium has been chapel for Cedarville students. The day finally arrived to begin building a new place of worship for the college, and on October 7 the college family officially moved into the new Jeremiah Chapel. Nervous excitement and anticipation best describes the feeling among the students, faculty, staff, and visitors the morning the college moved to the new chapel. Everyone packed into the old building, many taking seats in the aisles and on the platform. Students waved wildly at Dr. Dixon as he videotaped the historic event. Pastor Rohm gave the announcements and prayer requests, and then instructed everyone to take a hymnal as they left for the new building. As everyone filed out, they joined in singing "What a Mighty God We Serve." Dr. and Mrs. Dixon led the student body across the bridge and into the nearly finished Ministry Center while being serenaded by a brass ensemble.

Mr. DiCuirci and the praise band welcomed the college family with various praise choruses. Dixon then called the construction and maintenance workers onto the stage, commending them for their hard

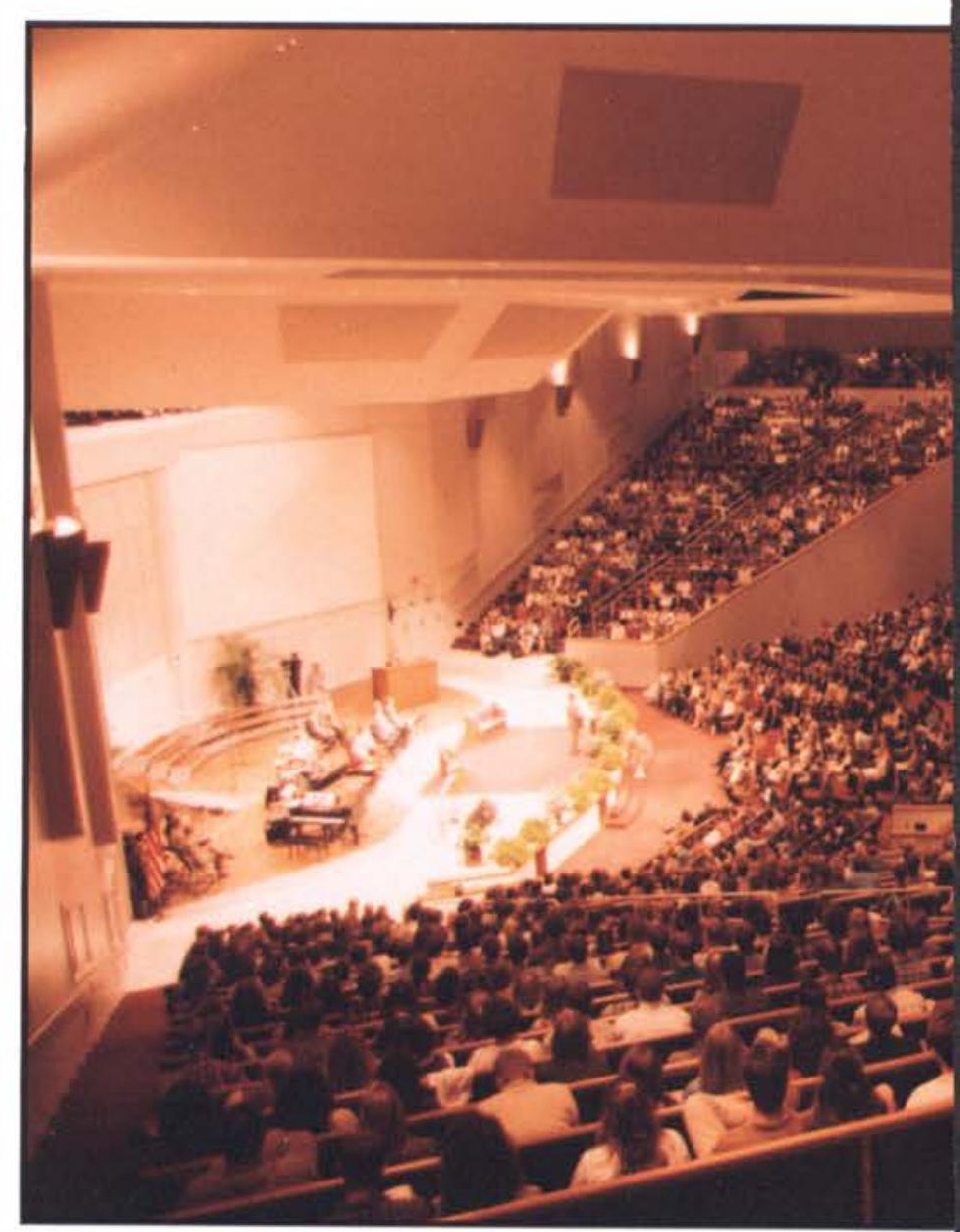

work. The college family showed their thanks with a standing ovation for each group of workers. Dixon also voiced his appreciation to the vice presidents of the college for their work and dedication. The Concert Chorale sang an a cappella arrangement of "A Mighty Fortress Is Our God," taking full advantage of the auditorium's acoustics.

Dixon spoke about the history of the chapel at Cedarville and the importance of this new building to the college. "Each part of this building is important to our mission," he said. 


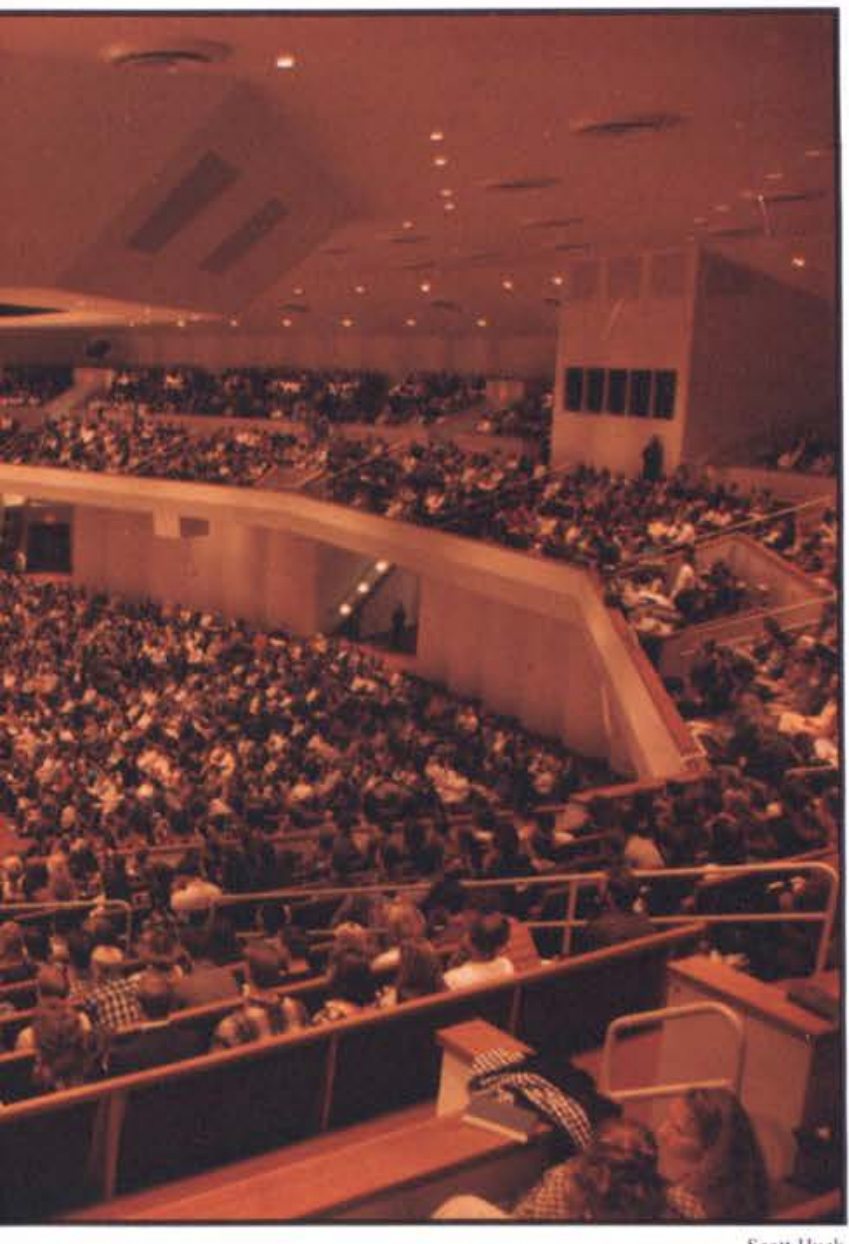

Scott Huck

He also pointed out that Cedarville has never lost its focus on the importance of chapel to the college family. "[In here] we will sing together [and] experience our God answering prayer. This building is about God," he said.

The administration has been planning the building for several years because they realized that the student body would soon outgrow the old chapel. After looking at various options, constructing a new building seemed like the best choice.

The Ministry Center was to be finished before the students came back this school year, but Cedarville weather was a major hindrance to the construction. Snow and rain made it impossible to complete the building according to the original schedule.

Leigh Hunt, Head of New Construction at Cedarville, said that construction workers numbered approximately 175 at various times. "Many College Building Services people stepped in and worked side by side with the contractors' employees in order to meet finishing schedules," Hunt said.
The result of all this hard work was a 120,000 square foot building containing a chapel auditorium which holds 293 pews and 3,540 people. Dr. Martin Clark, Vice President of Development, said that the layout of the chapel, with smaller ground floor seating and the wraparound balcony, gives the sense of closeness and the unity that the college desired.

While the emphasis has been on the grand opening of the auditorium, the Ministry Center has many functions beyond the daily chapel service. The
Christian Ministries Department, with its expanded offices and new practice rooms, and the Music Department, with extensive teaching studios, rehearsal and pedagogy facilities, and recital hall, are enjoying their new home in the Ministry Center.

The Ministry Center is a building meant for the students to enjoy. Clark said, "Hopefully everything about the Ministry Center will enhance the experience of the students at Cedarville." 


\section{College Unites in Prayer}

$$
\text { Gy Charis } \mathcal{P}_{\text {erez }}
$$

C

edarville College recognizes prayer as one of the essential components of the Christian walk. Every year, two mornings are set aside for special times of prayer.

October 30 brought students, faculty, and staff together on the national Day of Prayer. Students and their advisors met insmallgroupsat 8:30and 11:30; prayer was focused upon individual students' personal and family needs.

During chapel,musiciansfrom Grace Baptist Church led the collegefamily in worship, praising Godforthe freedom togather publicly in prayer. Dr. Dixon prayed for students' unsaved parents and grandparents, and Brian White, SGA President, prayed for the senior class.
Dr. Robert Gromacki conducted the chapel message. He asked the question "Are we willing to accept rebuke when we know we have sinned?" Dr. Gromacki emphasized the importance of distinguishing sorrow from repentance, and challenged the college family to examine their attitudes and relationships, to consider grudges held, and to become aware of their own shortcomings.

May 1 was the second observation of the Day of Prayer. The Campus Activities Board sponsored a prayer breakfast. Dr. David Drullinger spoke from I Tim. 2:1. His points focused on the Priority, People, Particulars, and Purposes of prayer. The basic pattern of prayer development is self, God, others, and the unsaved.

Chapel was a special blend of worship, prayer, and devotion. The call to worship was brought by the Jubilate choir with the songs "No Doubt About It" and "Jesus the Very Thought of Thee." Jim Cato led the worship time, which was interspersed with specialfocus prayers.

John Allen Hays presented achallengingdevotional onmature prayer. Before interceding for others, one must deal with the sin of his own life, for sin creates a separation between you and your God" (Is 59:2).
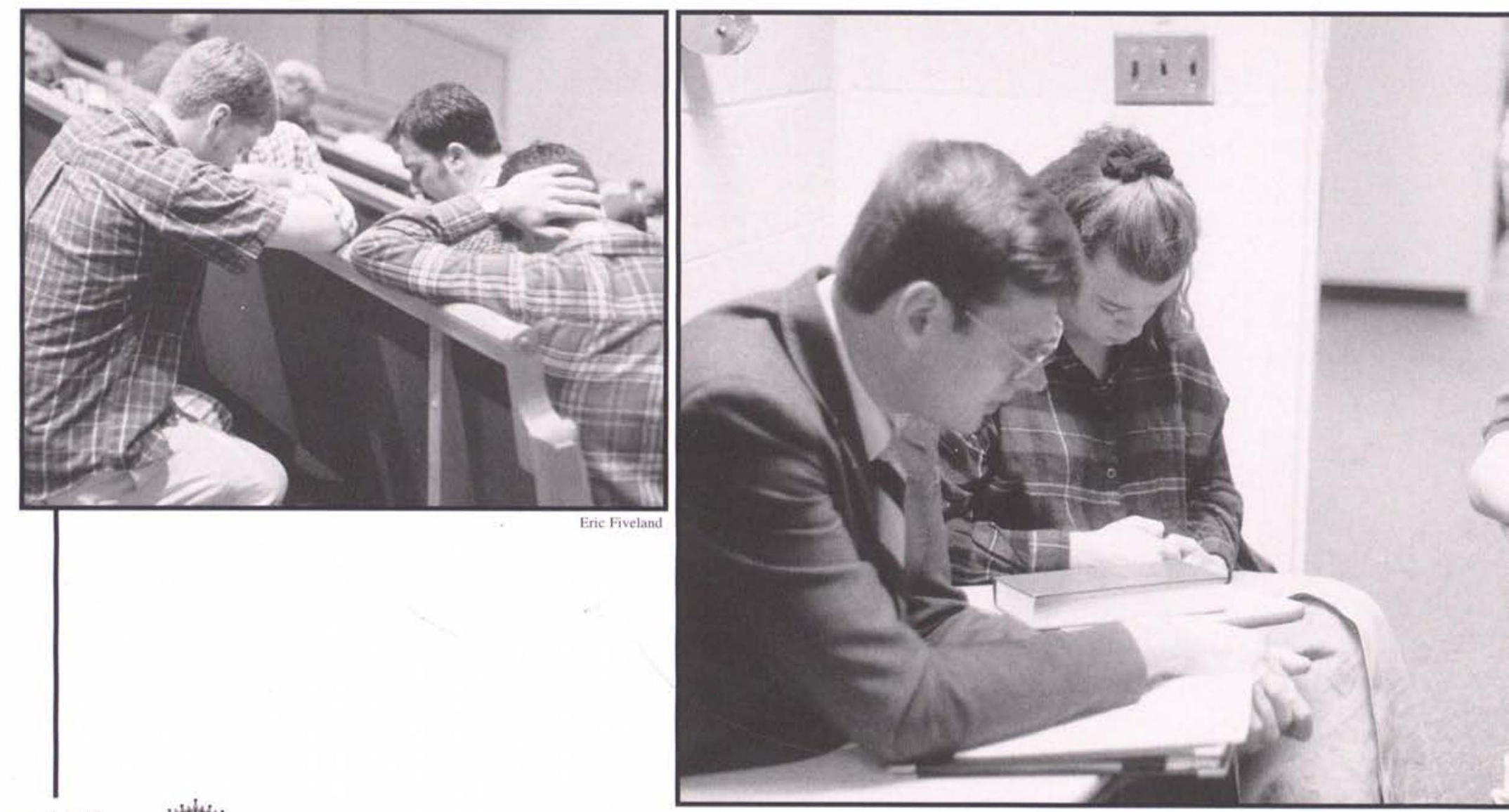


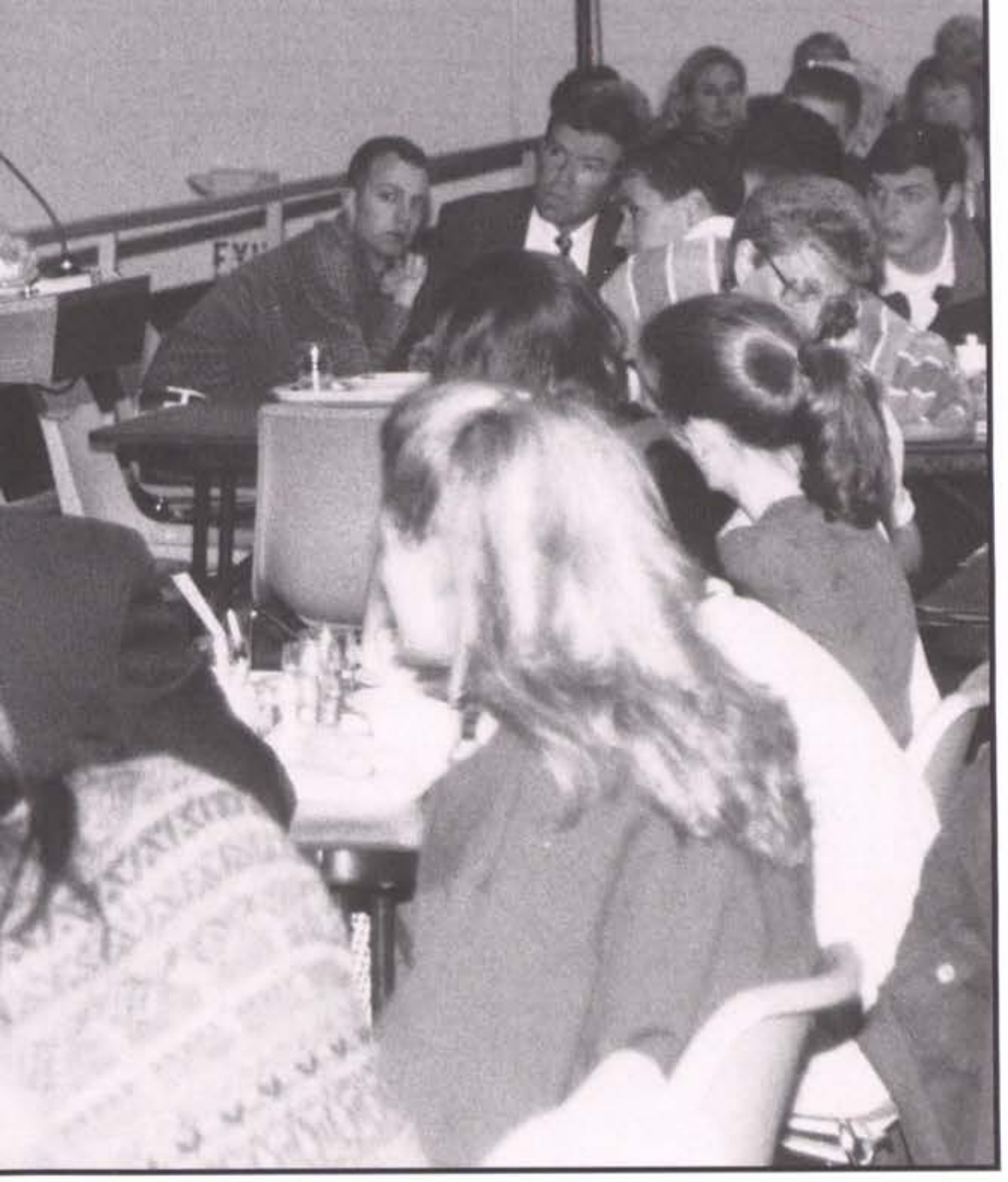

Above middle: Dr. Drullinger speaks at the Prayer Breakfast. Above right: Mr. Sweetser taking a moment by himself. Lower left: Ben Barnhart, Josh Kimmel, and Jay Manchesky kneel and pray together during the chapel service. Lower middle: Dr. Gollmer spends time with students in prayer. Lower right: Day of Prayer Chapel.
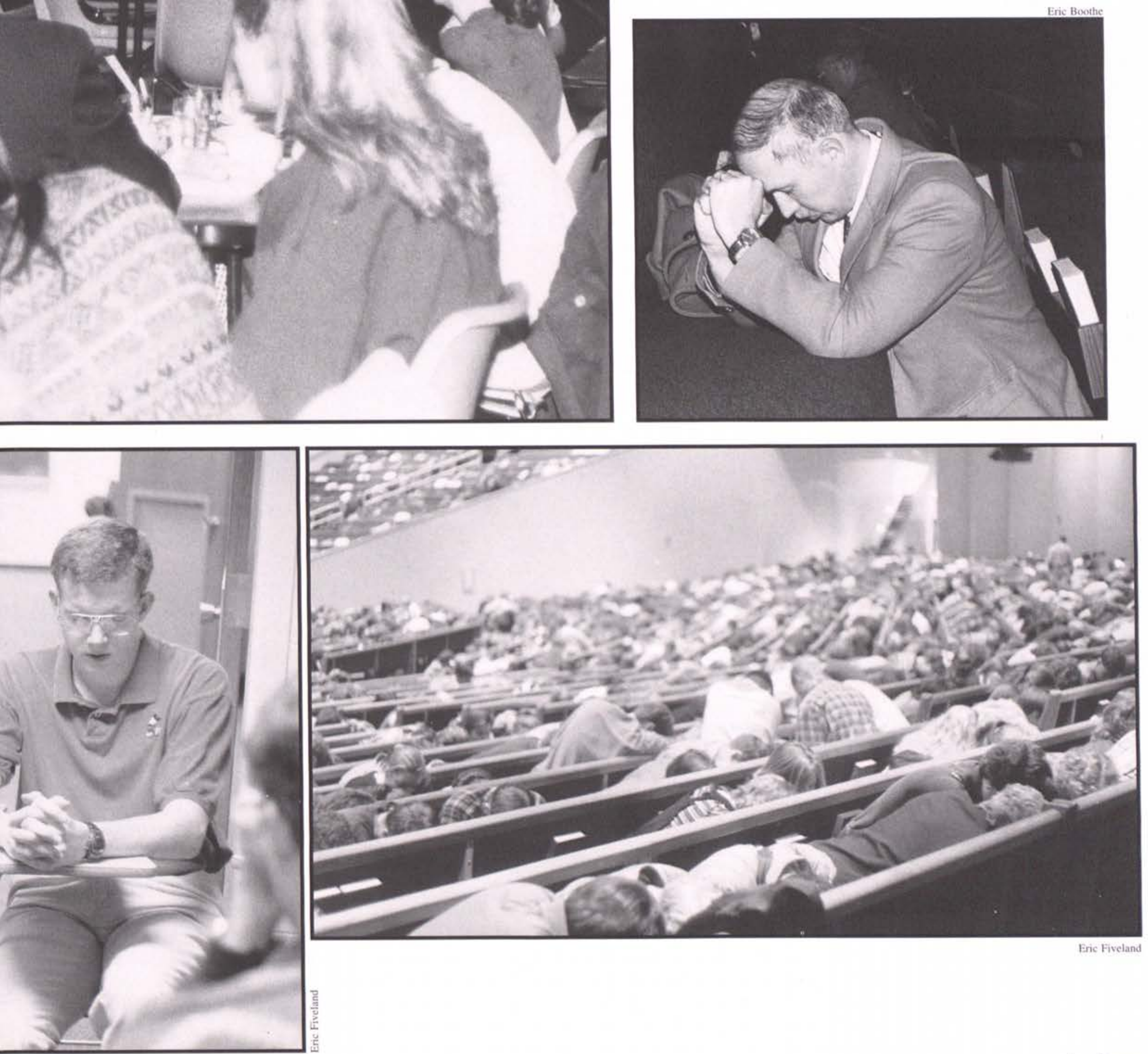

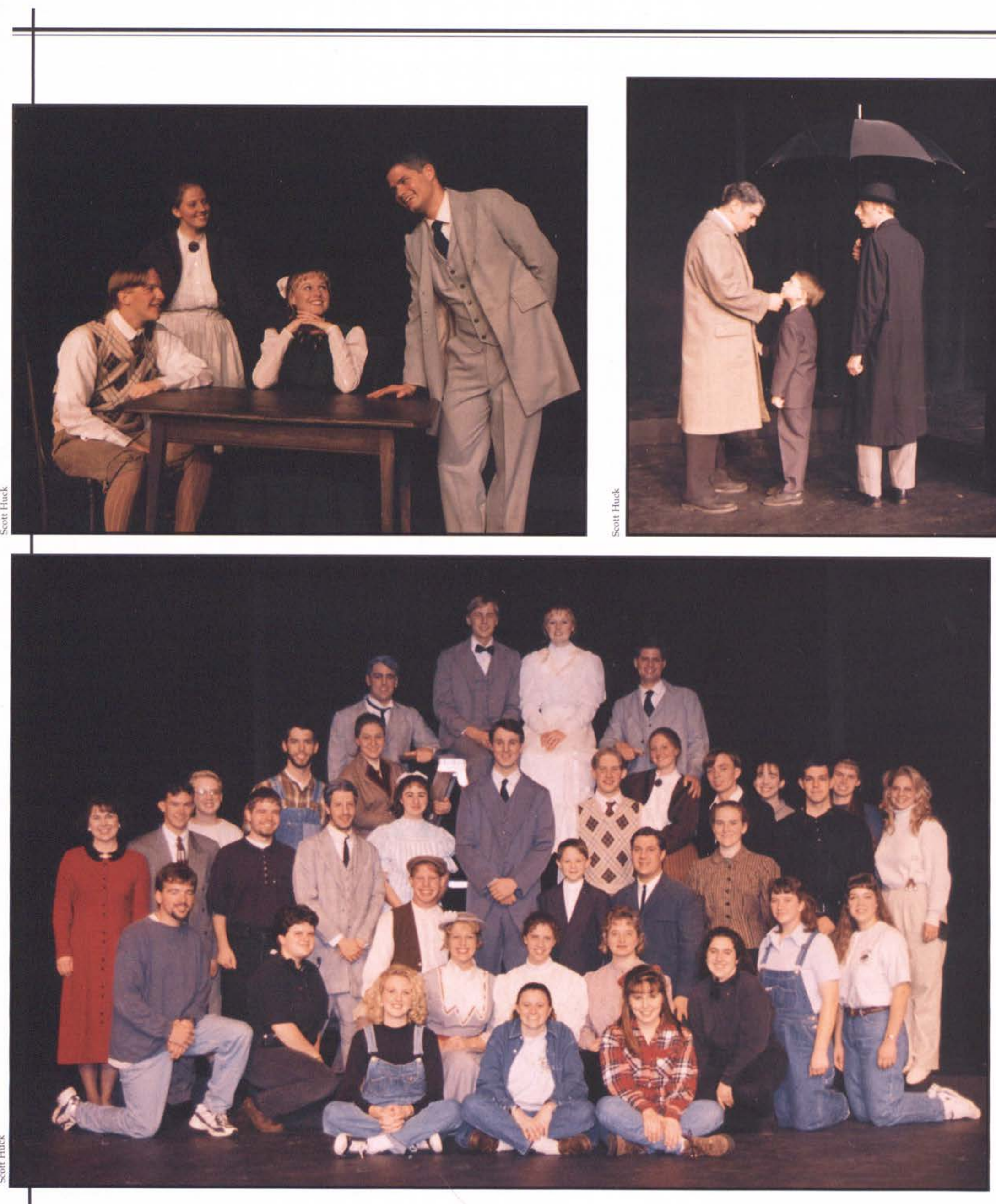

Above: row 1 sitting (1-r): Liz Tessmann, Christina Rising, Sara Romang. row 2 kneeling: Jeff Hyatt, Anne Miller, Jody Hovis, Amy Cartzendafner, Martha Failor, Ghena Marchetti, Kelly Cochrell, Summer Schafer. row 3 kneeling: Mike Mc Cubbin, Jay Leightenheimer, Chris Williams. row 4 standing: Mrs. Rebecca Baker, Brooke Taylor, Rebekah Sorensen, Dallas Hill, Todd Musser, Anna Lankhorst, Adam Kane, Josh Strychalski, Jennifer Myers. row 5 standing: Matt Jopson, Christina Jackson, Jean Estes, Aaron Berning, Charis Perez, Paul Best, Rebecca Kniowski, A'Linda Davis. row 6 standing: Chris Vitarelli, Colin Castelow, Trisha Johnson, Andrew Marshall.
24 student life
witity 


\section{Skits Encourage Students}

\section{by Joy Wickholm}

oward the end of Fall quarter, a few students rehearse for Thursdays Live, a collection of skits, narratives, and music used to communicate the importance of sexual purity. The title of this year's production was "98\% Pure and Mostly True"; referring to the fact that "mostly pure is really impure."

Associate Dean Carl Ruby suggested that there were three types of people present: those who were pure, those plagued with guilt, and those who were actively impure.

The drama examined sexual pressures from several different aspects. One humorous skit demonstrated the pressure during a first date. Julie Forstrum and Craig Hamer were stationed at "Look-Out Point." Craig wrestled with his desire to make "themove" with Julie, while Julie wrestled with not wanting to scar her reputation. After an intense psychological battle, Craigfinally blurted out "Doyou want to pray together?"

Krista Price performed a soliloquy. She reminisced abouther dating life, which had leftherinan off-campus kitchen surrounded by cookies she was saving for special friends. She thought about aformerboyfriendand theirphysical relationship. They, like many couples, started by giving small parts of their purity to one another, and then more. When brought back to the present, Krista finds that, during her reflection, she had eaten all of the cookies, leaving none for someone special.

The final skit of the evening was a celebration of Mandi Orme and Craig Hamer's anniversary at the park where they first held hands. As the evening progresses, they return to the car, and eventually engage in sex. The entire scenario is narrated by the characters to their respective roommates. Each reveal their excitement, horror, and guiltfor theirirresponsibility. Anson Hanbury concluded the skit with the song "All is Forgiven." The ultimate message of the evening was that sexual purity is a gift of God, but stepping over God's boundary can forfeit His protection.
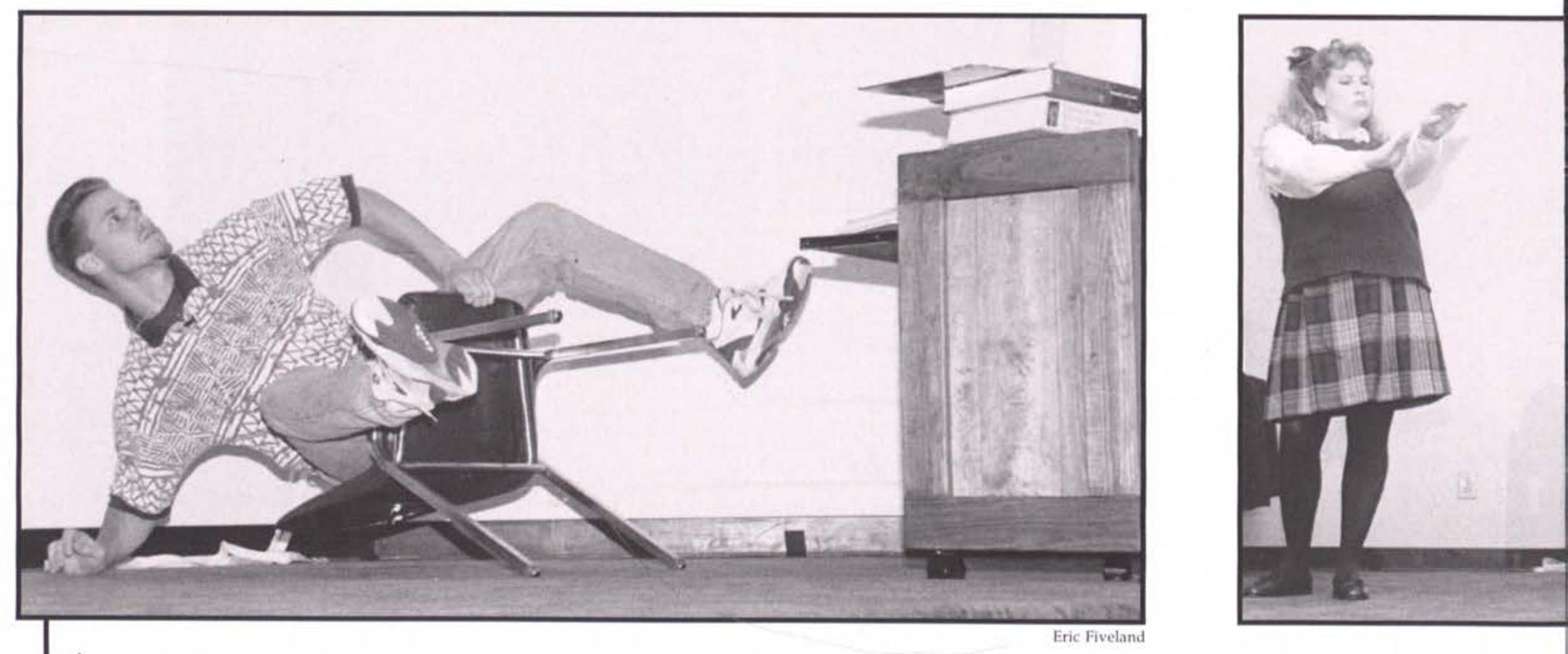

Above: Bill Workman falls backward as his computer speaks to him about the importance of a pure mind. 


\section{Indy,

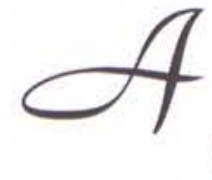 \\ Jungle, \\ and \oto}

(O)

n Wednesday, December 4, 1996, curfew was extended for half an hour for Christmas Open House, the one evening of the year when the dorms are open to all visitors. Once again, each hall and unit in every dorm scurried around on the night before, putting up decorations and getting costumes together. They were all competing for the first prize, dinner at any restaurant, for the top 4 winners. Some halls actually spent days preparing for the event, while others managed to transform their entire living quarters in a matter of hours.

The evening could be summed up as a campus-wide party. Every dorm was packed as hundreds of students, and a few faculty and staff members, toured from building to building, seeing friends' rooms, and simply enjoying the festive atmosphere. As usual, WSRN filled the airwaves with Christmas music.

Willets 3rd floor Central (Jodie Hager) won the women's hall award, and received a trip to the Olive Garden. Their theme was "A Jingle in the Jungle." As guests entered their hall, their first stop was the ABWE mission board, who sent them to the nurses' station for shots. They then proceeded into the jungle, complete with trees, dirt, darkness, and strange-looking creatures who jumped out at them.

McChesney's 1st floor back hall (Tim Simon) won for the guys' halls. They did an "Indiana Jones Christmas," complete with tour guides, leaves, twigs, and even poison ivy. There was an Ark of the Covenant room and a pit that everyone had to rope-swing over. A huge beachball volleyball, decorated like a boulder, chased each group out the door.

Maddox units 15 and 16 (Becky Ivey) won for the ladies' units with a "Cavegirl

Christmas." Their costumes were Flintstone outfits, and they created a dark cave atmosphere by lining the walls and ceiling with black garbage bags. A unit member with a club stood outside directing visitors into the "cave."

Lawlor's units 14 and 18
(Steve Keary) won the prize for their "Mission Impossible Training Course," which included stations for fingerprinting, interrogation, a training course, and firing range.

One hall or unit from each dorm was also awarded a pizza party for their efforts. Some of the best themes and decorations were "Christmas in the Nunnery" (the Hill), "Christmas in Dixonville" (Faith), the "Wizard of Oz" theme (Printy), and a unit who gift wrapped themselves (Lawlor).

Almost every hall and unit participated this year. It was a perfect break before exams and an opportunity for students to meet those who live around them.
Right: Marshall 5 and 6 built a castle on "The Hill". row 1 (1-r): Kevin Armstrong and Mike Gribbin. row 2 (l-r): John Gray, Dave Hassenzahl, Dr. Dixon, Chad Snow, Glenn Baker, Wayne Arnold, and Neal Stark.

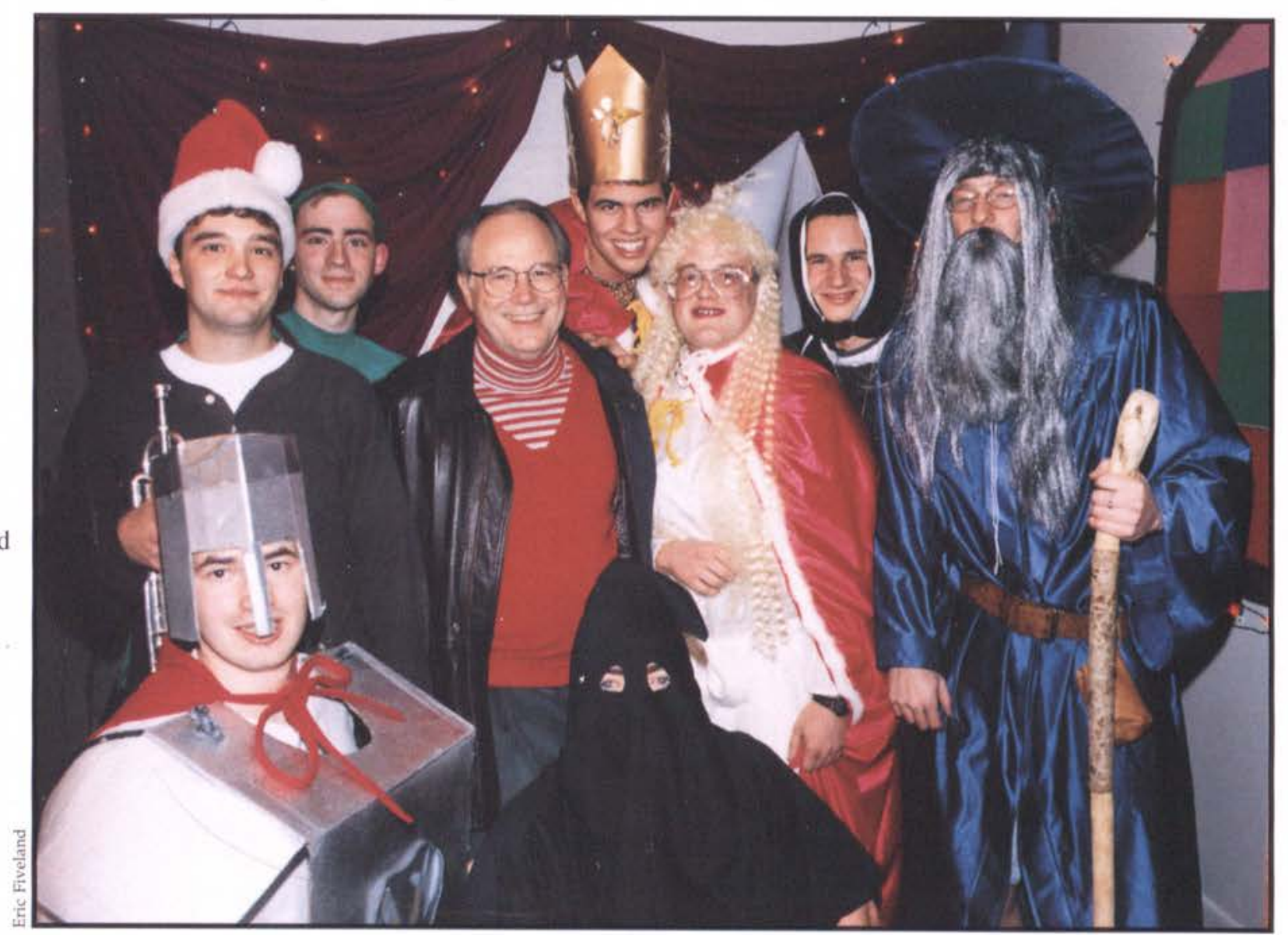




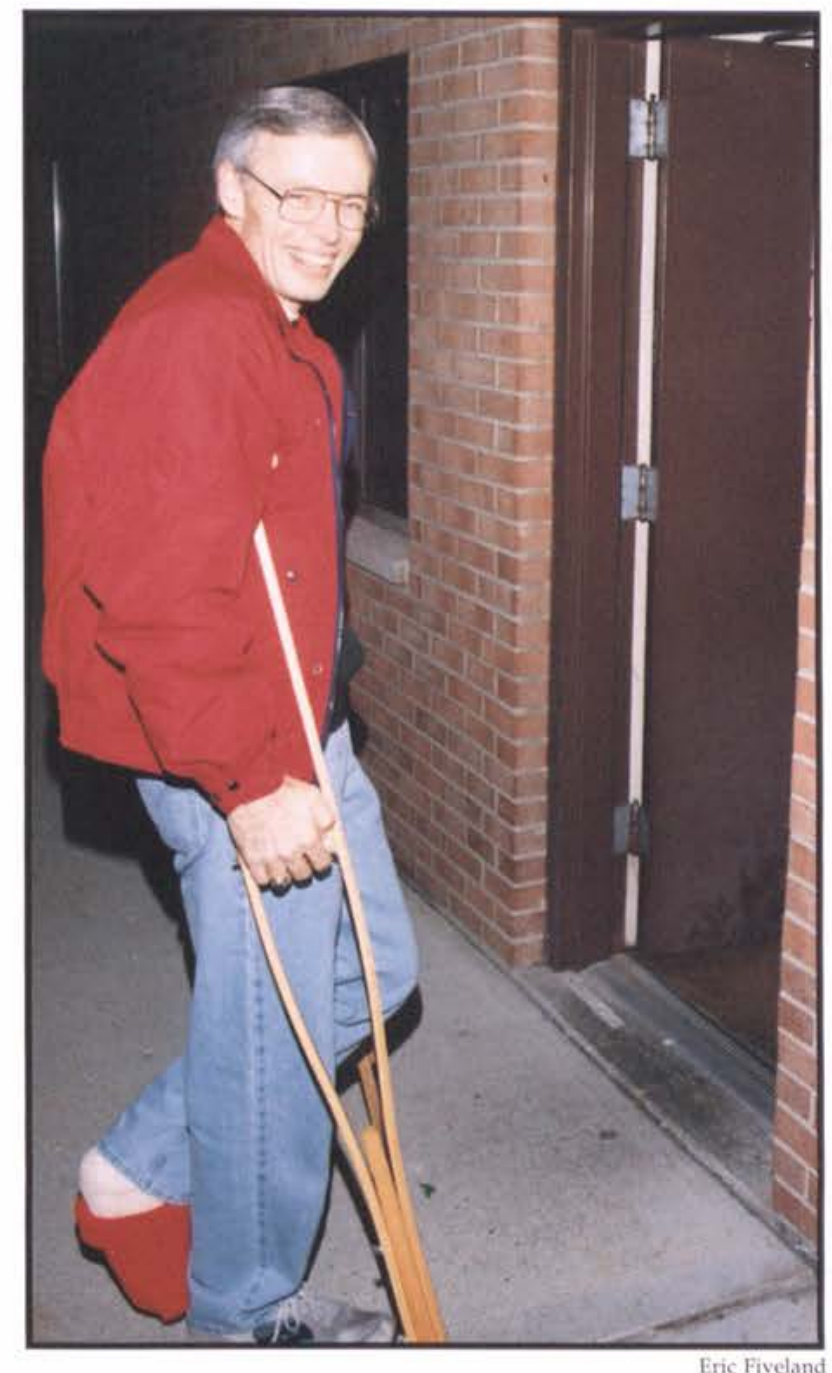

Left: Pastor Rohm hobbles from dorm to dorm. Below left: Kirk Le Blanc administers CPR to Todd Jones as Loren Pierson clears a path for the victim. Below right: Sometimes a picture is worth a thousand words. Below: Dr. Dixon is chillin' in the hood. Actually, isn't that Printy? (I-r): Doreen Fuhr, Kristi Gleason, Dr. Dixon, Daphne Diaz, Julie Forstrom, and Shannon Boynton.
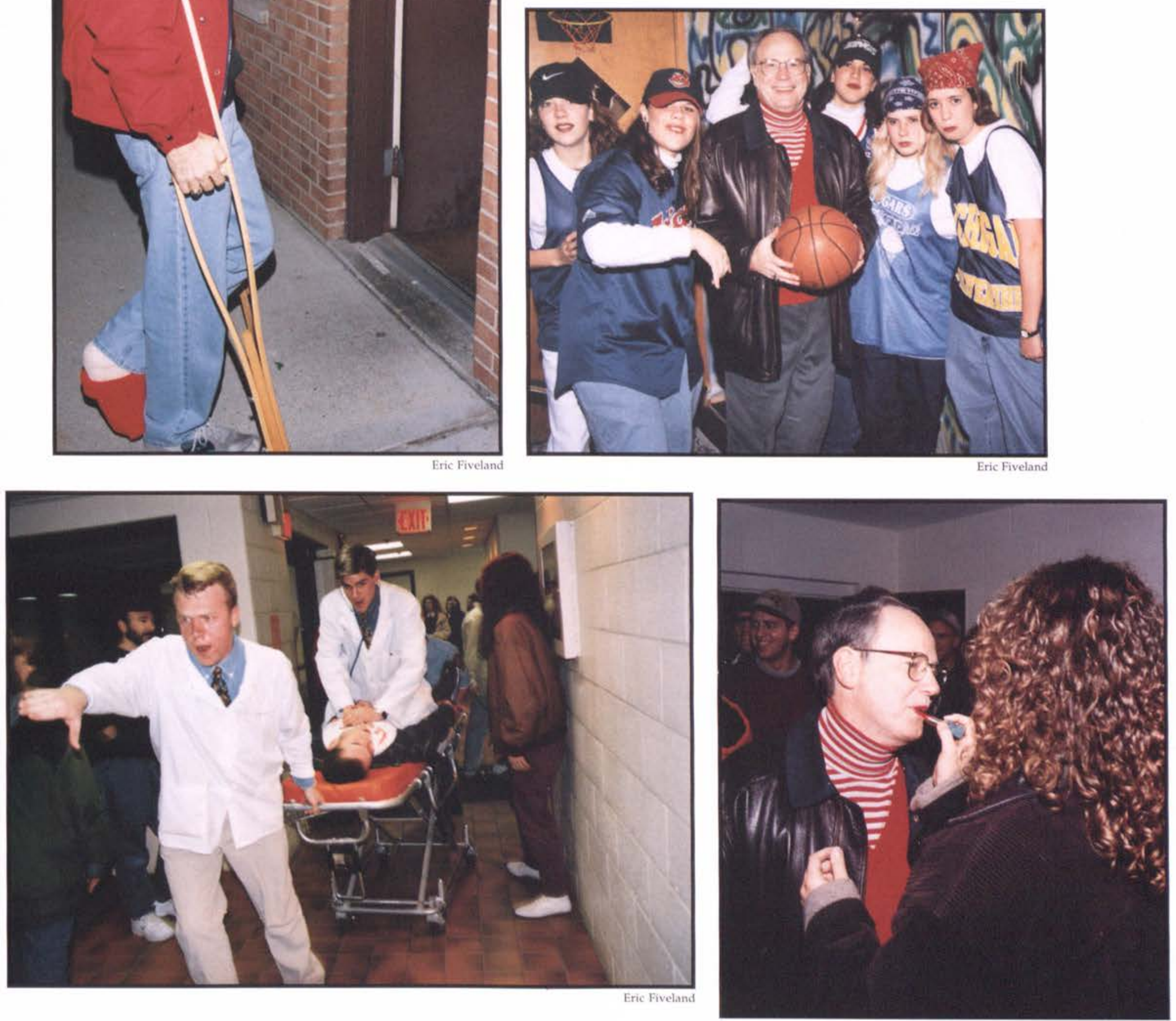
With the opening of the Dixon Ministry Center this year, the Campus Activities Board selected an unusually diverse line-up of performers for the annual Concert Series as a celebration of the new facility.

The first major concert came with the grand opening of the DMC on Homecoming Weekend; the college family and visitors were treated to a two-part concert by Stephen Nielson and Ovid Young, and the Cathedrals. Nielson and Young are a duo-piano ensemble who have seven recordings and three volumes of arrangements to their credit; they played a wide variety of classical and popular music. Following their portion of the concert, the Cathedrals switched musical styles, singing a lively array of southern gospel music. Glen Payne and George Younce, original members of the Cathedrals, and Ernie Haas, Scott Fowler, and Roger Bennett, who joined in 1979, gave an energetic performance and delighted the audience with their comic behavior and powerful testimony.

On January 14, the Columbus Symphony Orchestra, under conductor Peter Stafford Wilson, performed an evening of classical music and Broadway favorites. Wilson immediately captured the attention and imagination of the audience with his dry humor and his conversational explanations of the musical selections.

December 5 brought with it a Christmas concert by the Canadian Brass. Charles Daellenbach, Jens Lindemann, David Ohanian, Ronald Romm, and Eugene Watts played classical, Christmas, and popular music colored by their unique sound and wit. Among the most popular pieces of the evening was Peter Schikele's Hornsmoke, a brass "opera" in which each musician donned a western costume and acted out a part while playing.

The King's Singers performed on February 21. David Hurley, Nigel Short, Bob Chilcott, Philip Lawson, Gabriel Crouch, and Stephen Connolly form one of the most sought-after and highly acclaimed vocal ensembles in the world.

Steve Green concluded the Concert Series on May 3. He performed selections from his newest album, The Letter, and favorites from his previous recordings.
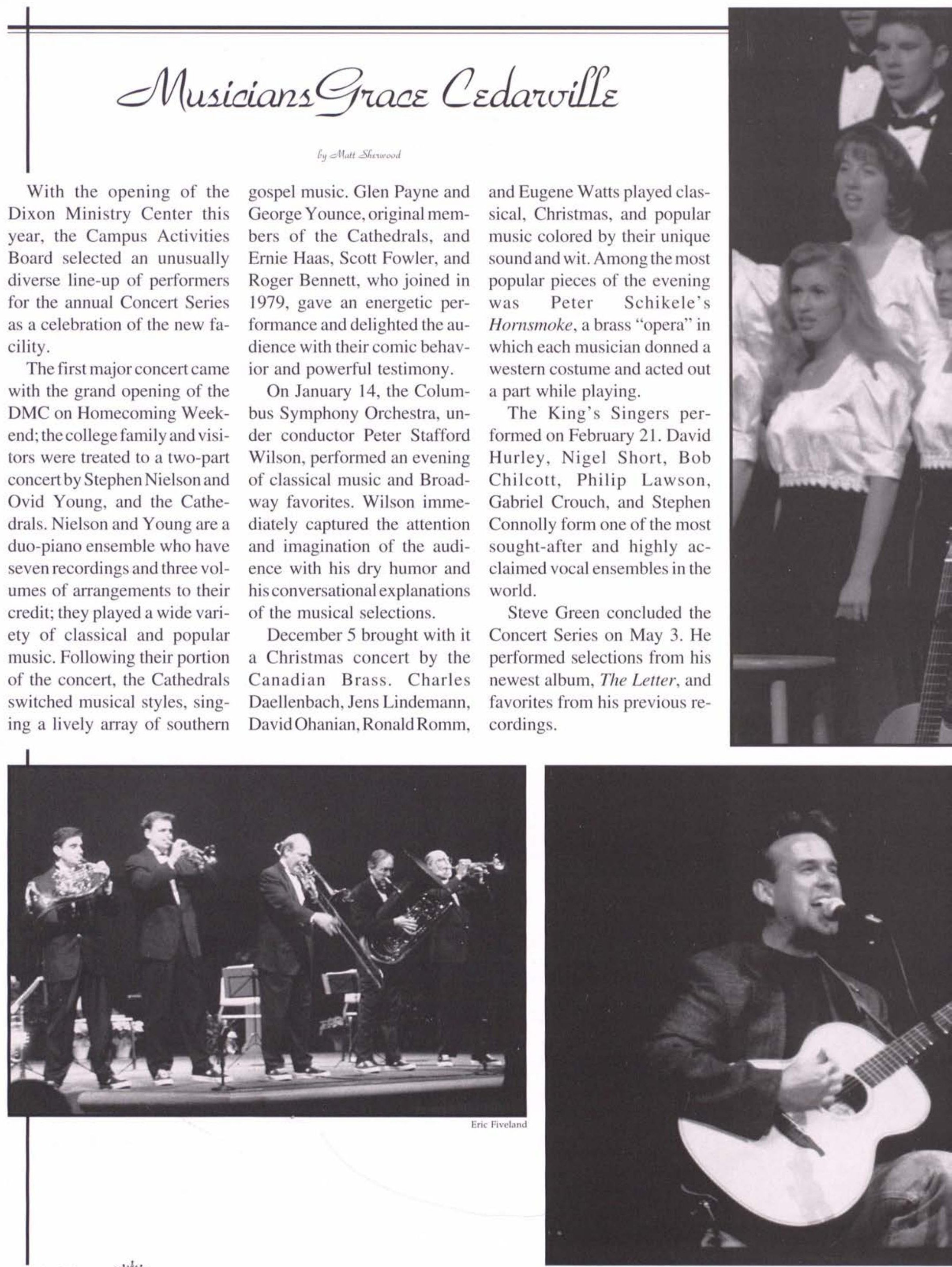


\section{Sharing \\ the Experience}

$\mathcal{L}$

i'l Sibs weekend is a time for students to host their brothers, sisters, nieces, nephews, cousins, and friends here at the 'Ville. This year, about 500 sibs were on campus February 7 and 8 . The Campus Activities Board, the Admissions Office, and a number of student organizations worked together to make the weekend a memorable one. On Friday night, siblings enjoyed the annual Winter Blast in the Athletic Center. Tonya Crevier, speaker, entertainer, and one of the most talented basketball handlers in the world presented her ball handling routine and gave her testimony. There were also games, food, caricatures, and "rides" like the Cosmic Orbiter and an air ball pen. For jazz enthusiasts, the Lab Band presented a free concert in the chapel. And to top out the night, Pi Sigma Nu organized a Late Skate in Columbus. Saturday, sibs enjoyed free time in the gym and a baseball card show. In the evening, the Yellow Jackets hosted Malone and Tiffin for exciting back-toback women's and men's basketball games. Afterwards came an annual treat - the ADO/DOE Cupid's Bash, which involved live music, skits, prizes, icebreakers, and games. The popu-

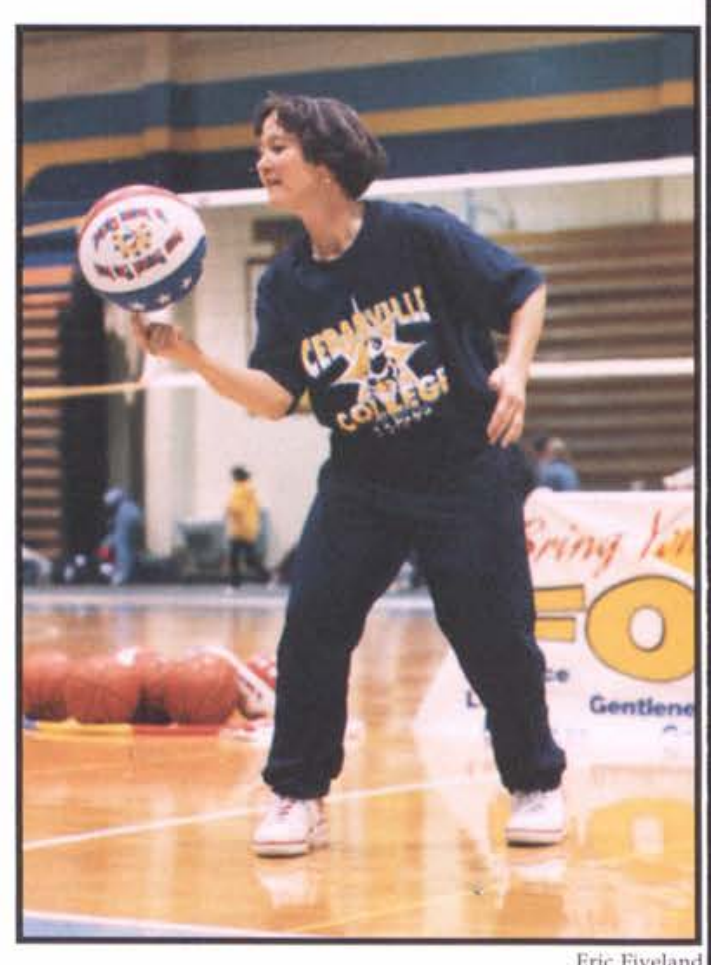

Eric Fiveland
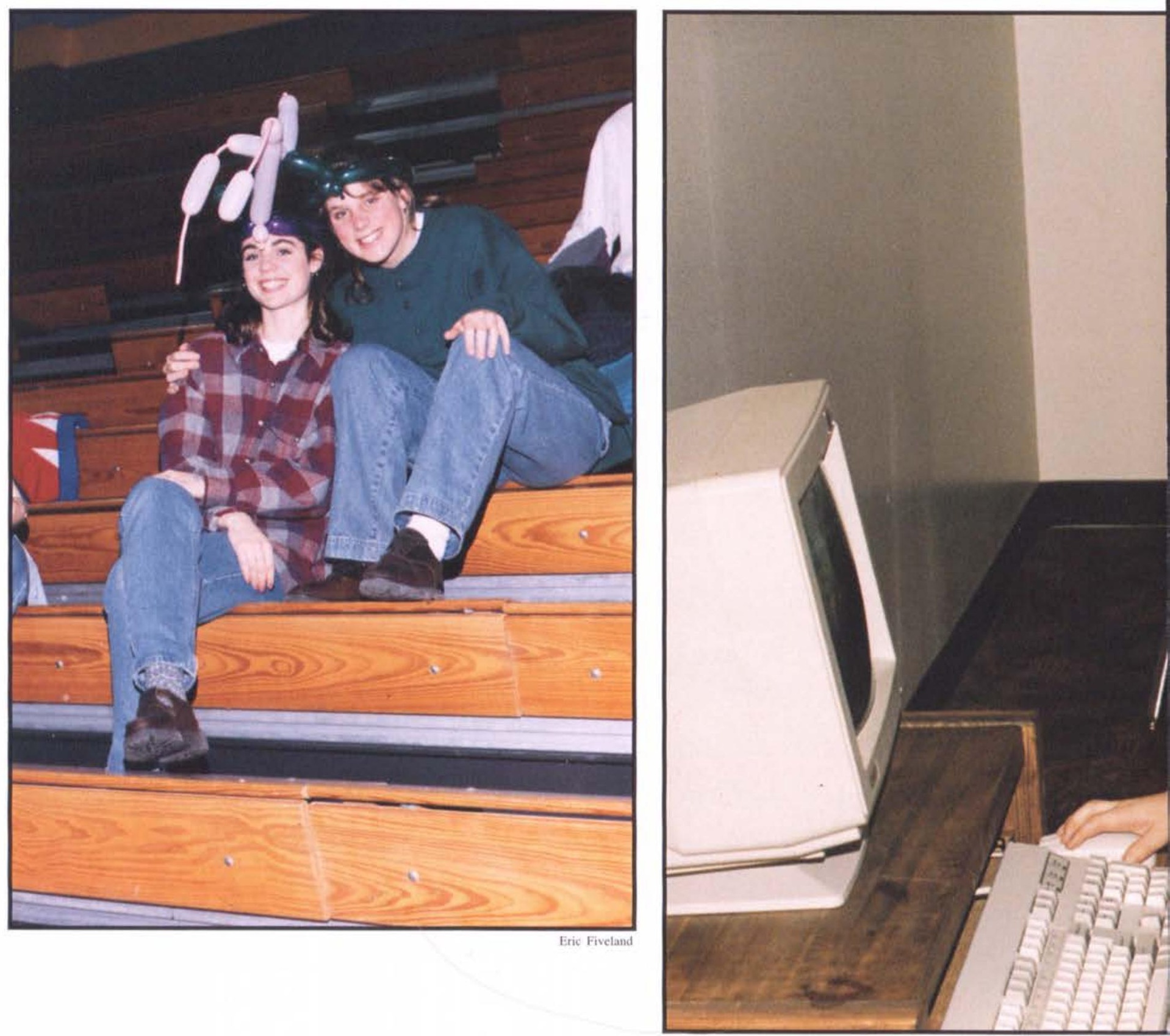
lar dating game joined a lucky couple up for the rest of the evening on a "mini-date" that was video taped and shown at the end of the party. Older brothers and sisters had the chance to show off - or look silly — by playing "How well do you know your Li'l Sib?". Li'l Sibs weekend is one more great memory for our Cedarville scrapbooks. Brothers and sisters were able to spend time together having fun, and future Cedarvillestudents had achance to get acquainted with college life.

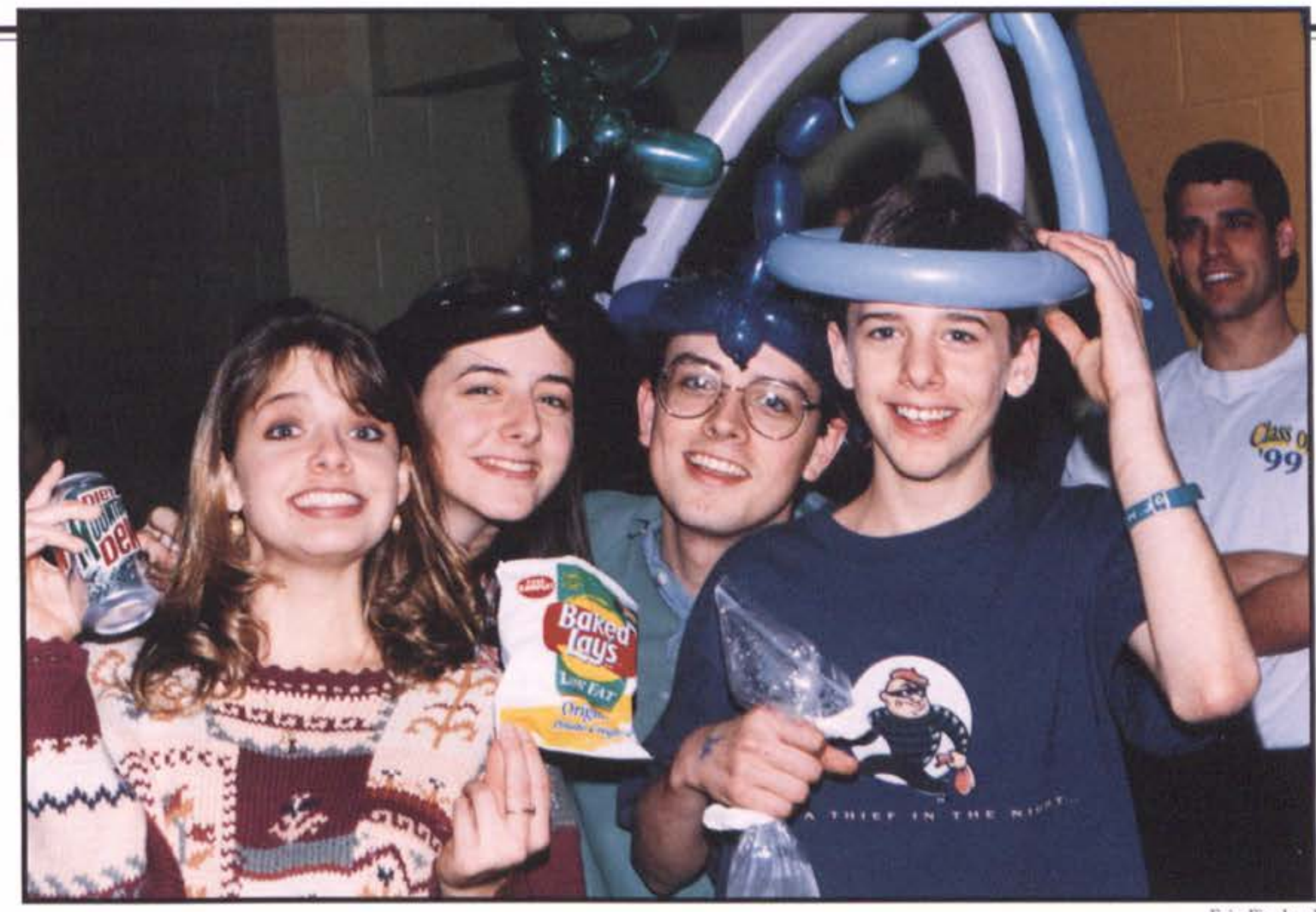

Eric Fiveland
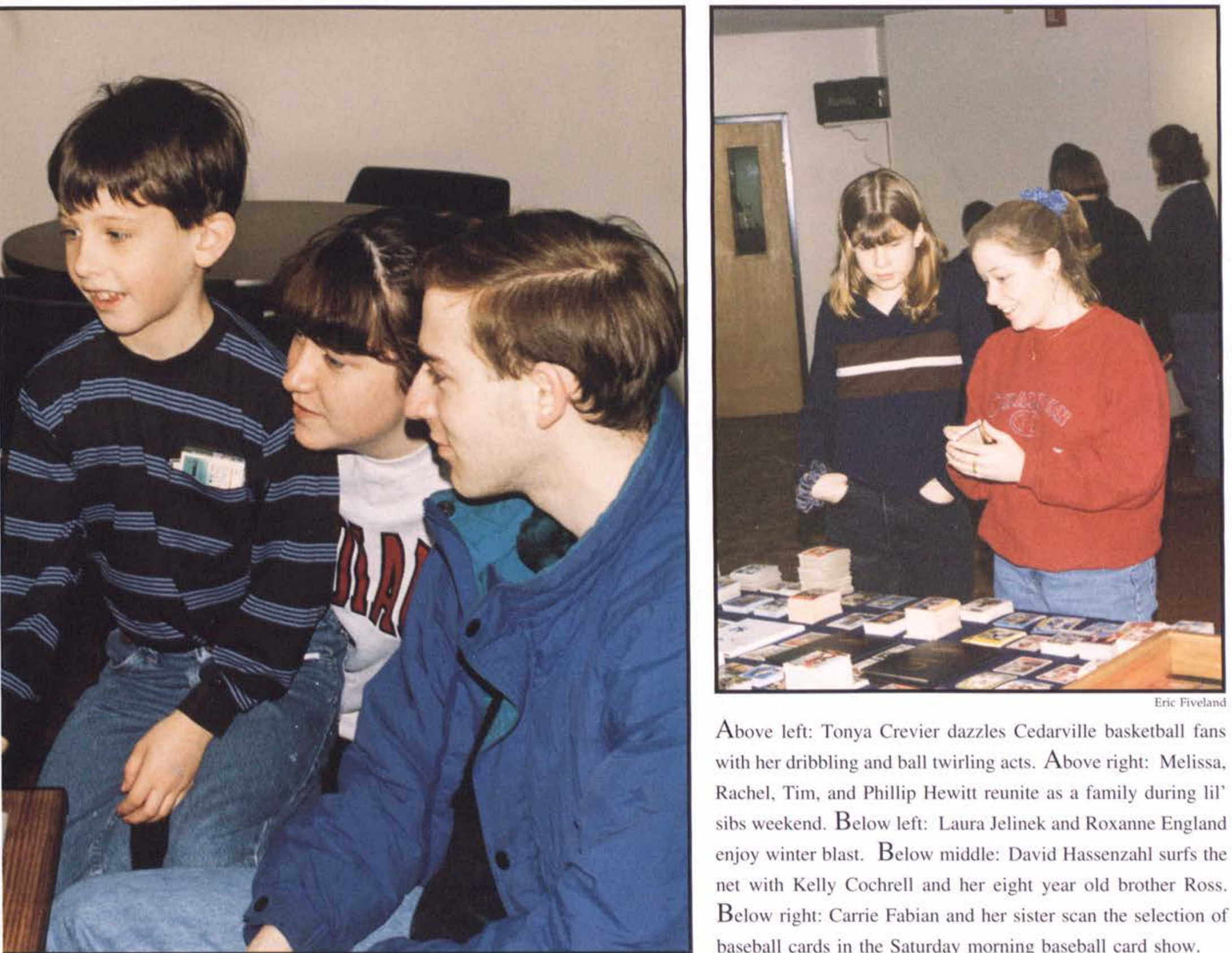

Above left: Tonya Crevier dazzles Cedarville basketball fans with her dribbling and ball twirling acts. Above right: Melissa, Rachel, Tim, and Phillip Hewitt reunite as a family during lil sibs weekend. Below left: Laura Jelinek and Roxanne England enjoy winter blast. Below middle: David Hassenzahl surfs the net with Kelly Cochrell and her eight year old brother Ross. Below right: Carrie Fabian and her sister scan the selection of baseball cards in the Saturday morning baseball card show. 

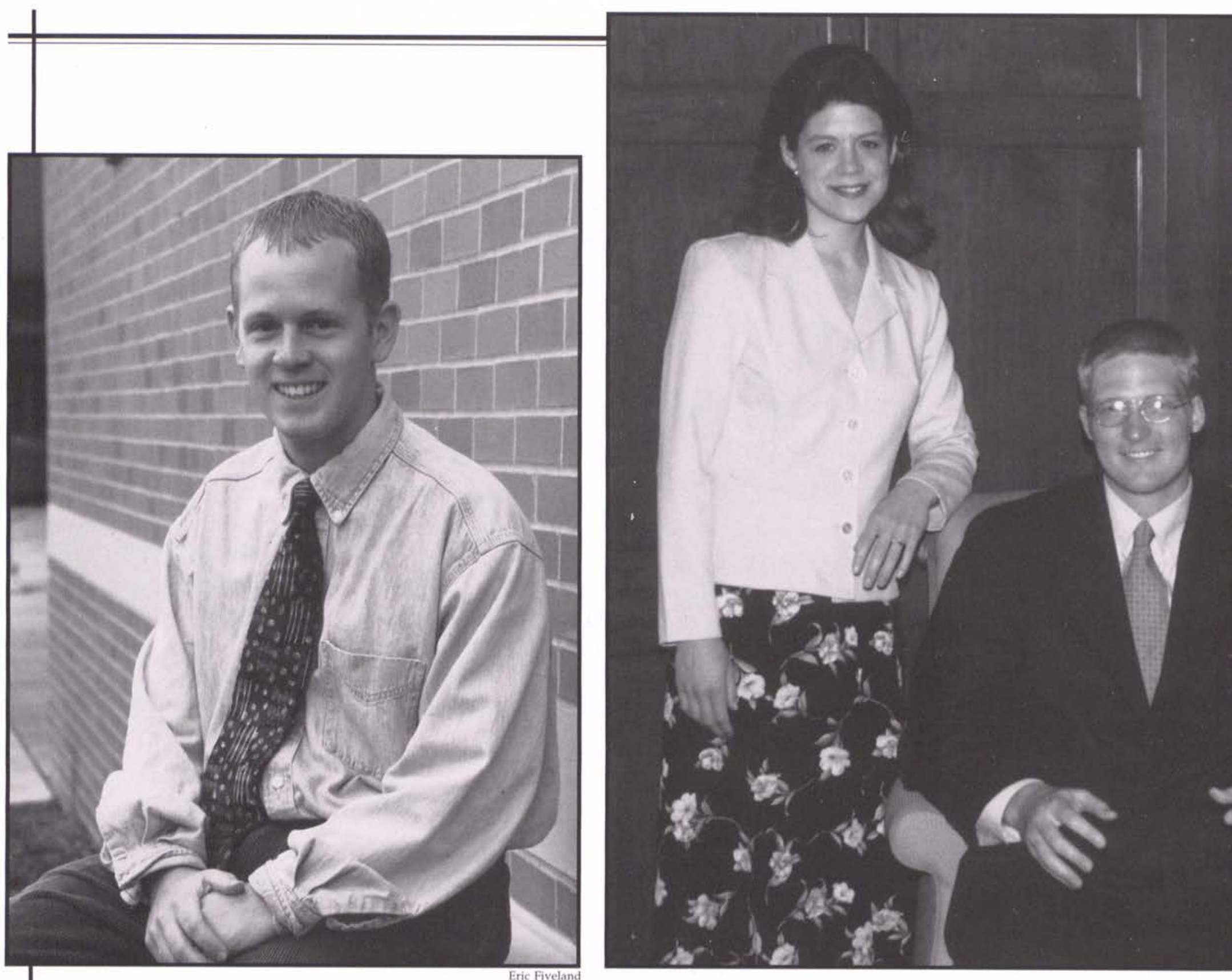

Above left: Bradford Illian performed his senior voice recital this year. Above right: Elizabeth Roseboom, Jamin Ferner and Christina Jackson all gave speech recitals. Below left: Josh Rupp prepares for his senior recital on May 24. Below middle: Jessica Shuring, Chet Jenkins, Chris Pagnard, Sara Clayton, and Kimberly Miller enjoy success at their music recitals. Below right: Sara Clayton poses with her flute.
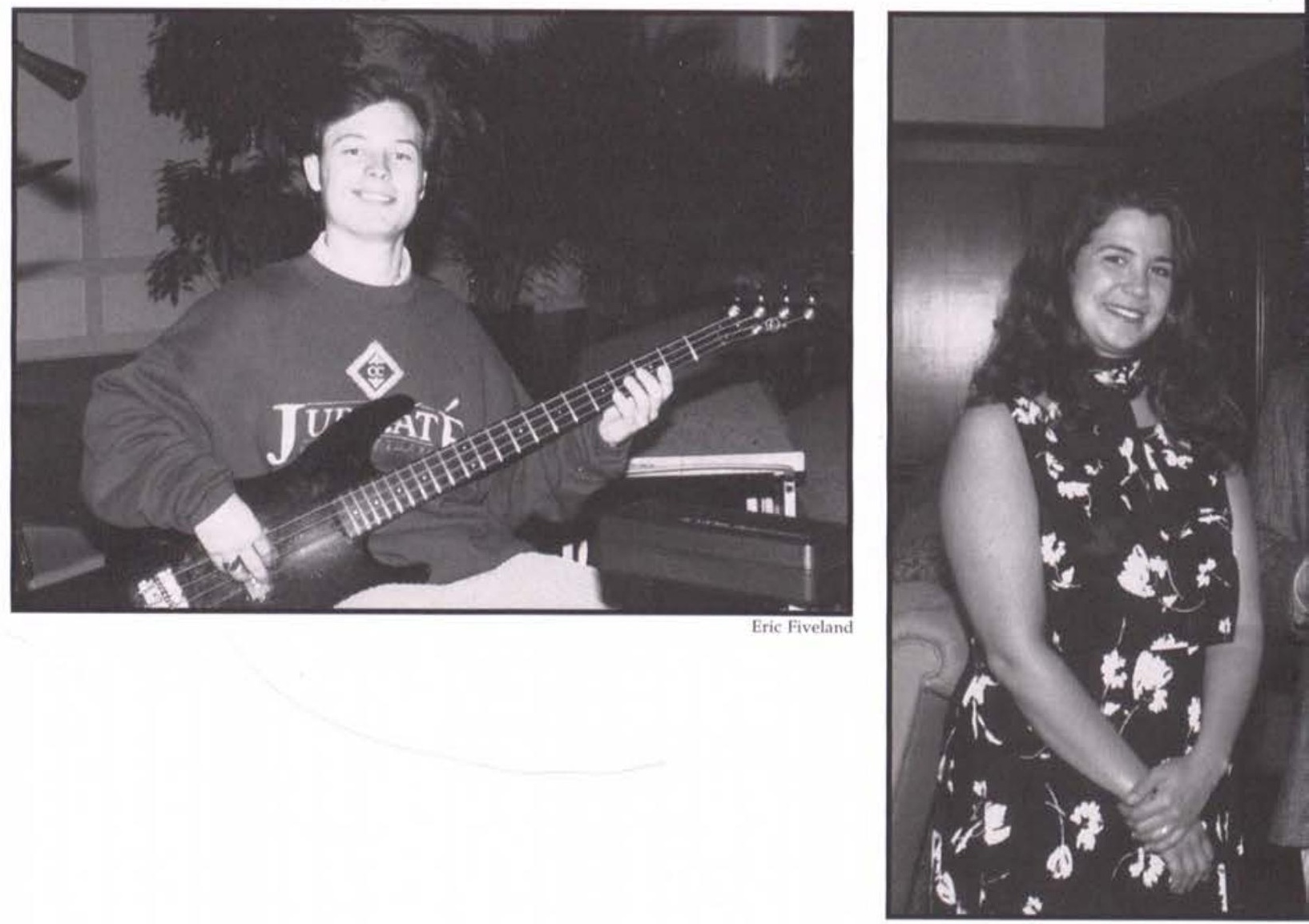


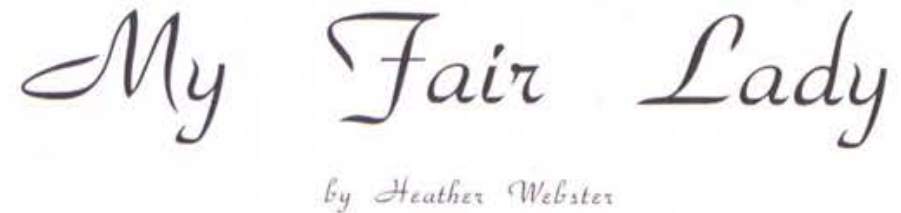

7 his year's spring production, My Fair Lady, was the most lavish play in Cedarville history, according to Professor of Communication Arts/Drama Dr. David Robey.

My Fair Lady is based on George Bernard Shaw's play Pygmalion and has been called one of the most popular Broadway musicals of all time. Its repertoire includes such songs as "The Rain in Spain," "I'm Getting Married in the Morning," "On the Street Where You Live," and "I Could Have Danced All Night."

Junior Jody Hovis played Eliza Doolittle, and was joined on stage by 25 students and one faculty member, Assistant Professor of Communication Arts Gary Barker.

In 1986, when Cedarville last
producedMy Fair Lady, Barker was the stage manager under Robey. In this year's performance, Barker brought to life the delightful Alfred P. Doolittle. Robey said that he especially appreciated his fine work and that it is an honor for Cedarville to have a licensed professional actor like Barker.

Augmenting the performance were Ed Supplee, who directed the 11-piece orchestra, and Sally Moore, who choreographed the show.

The play showed the differences between the upper class and the lower class. One would expect the upper class to be happier because of all they have, but really the poor are the happier people, Robey said. Eliza is the one who realizes the importance of hard work, achieving goals and furthering herself in life. One of the most poignant statements in the play comes when Eliza says to Higgins, "I sold flowers, I didn't sell myself. Now you've made a lady of me, I'm not fit to sell anything else."

Hovis said the most challenging thing about the part of Eliza was her shift in accent; she went from a strong cockney to a refined English.

"The thing I love about Eliza is her ability to fill a room with her presence. She's a bit of a charmer," Hovis said.
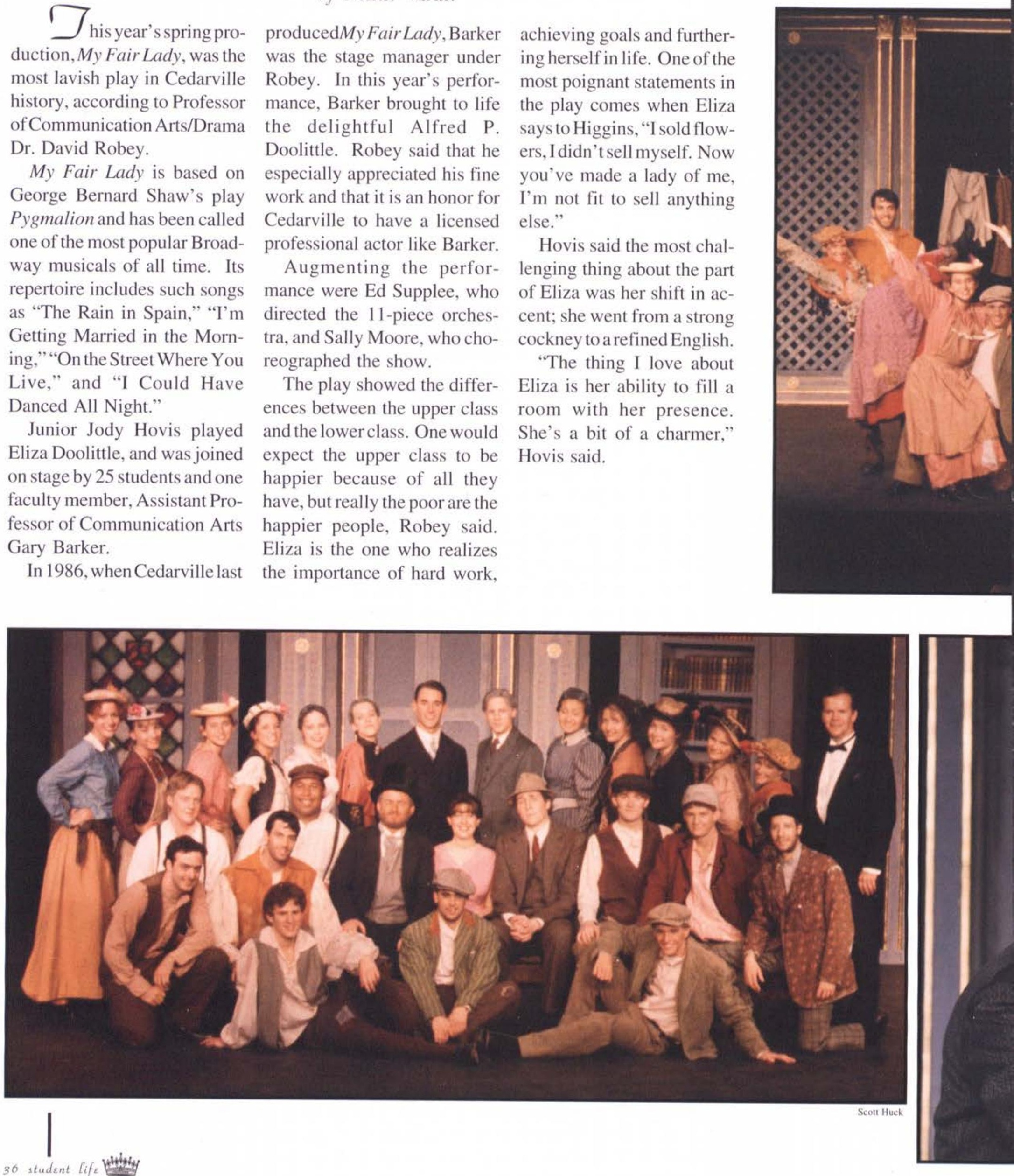


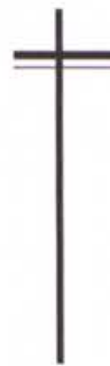

Right: With Heidi Dean and Shannah Campbell, Anson Hanbury and Mark Button, or Det. Robert E. Lee and Sergeant Kennedy, are once again perplexed. Below left: row 1(1-r): Daniel Scott, Rebekah Sorensen, Heidi Dean, Sara Romang, Amy Cartzendafner, Laura Milligan, Cheyenne Leslie, Holly Sorensen. row 2: Todd Musser, Joy Wickholm, Gary Barker, Rebekah Crosson, Shannah Campbell, Mark Button, Anson Hanbury, Stacy Saville, Jeremy Brown, Angela Wenzel, Daman Douridas. row 3: Brenon Christofer, Mark Schleith, Jamin Ferner, Colin Castelow, Heather Webster, Chris Pierre. Below middle: Colin Castelow directs Dan Scott and Chris Pierre as they perfect their performance. Below right: Mark Button accuses Todd Musser of the crime as Jamin Ferner looks on.

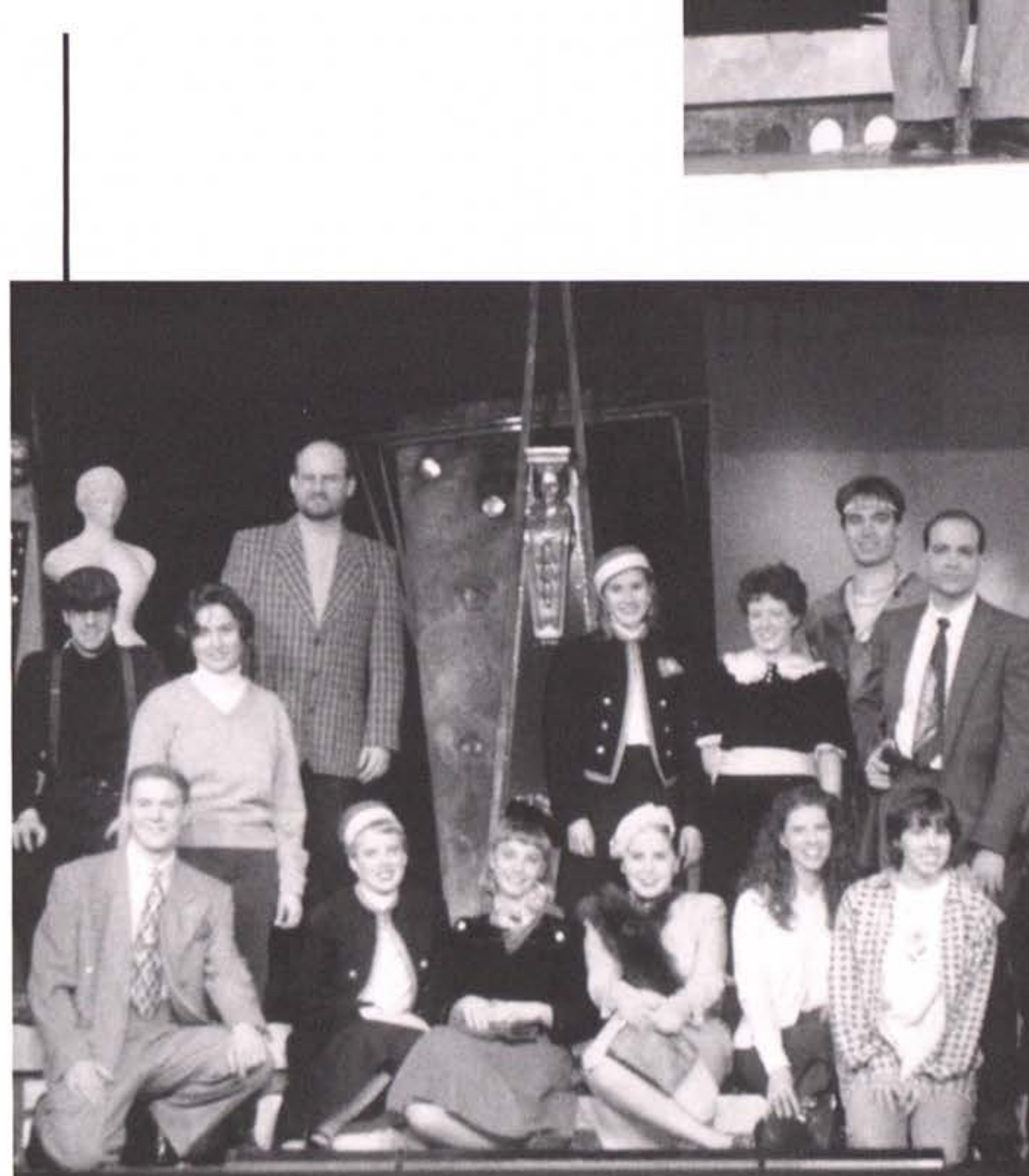

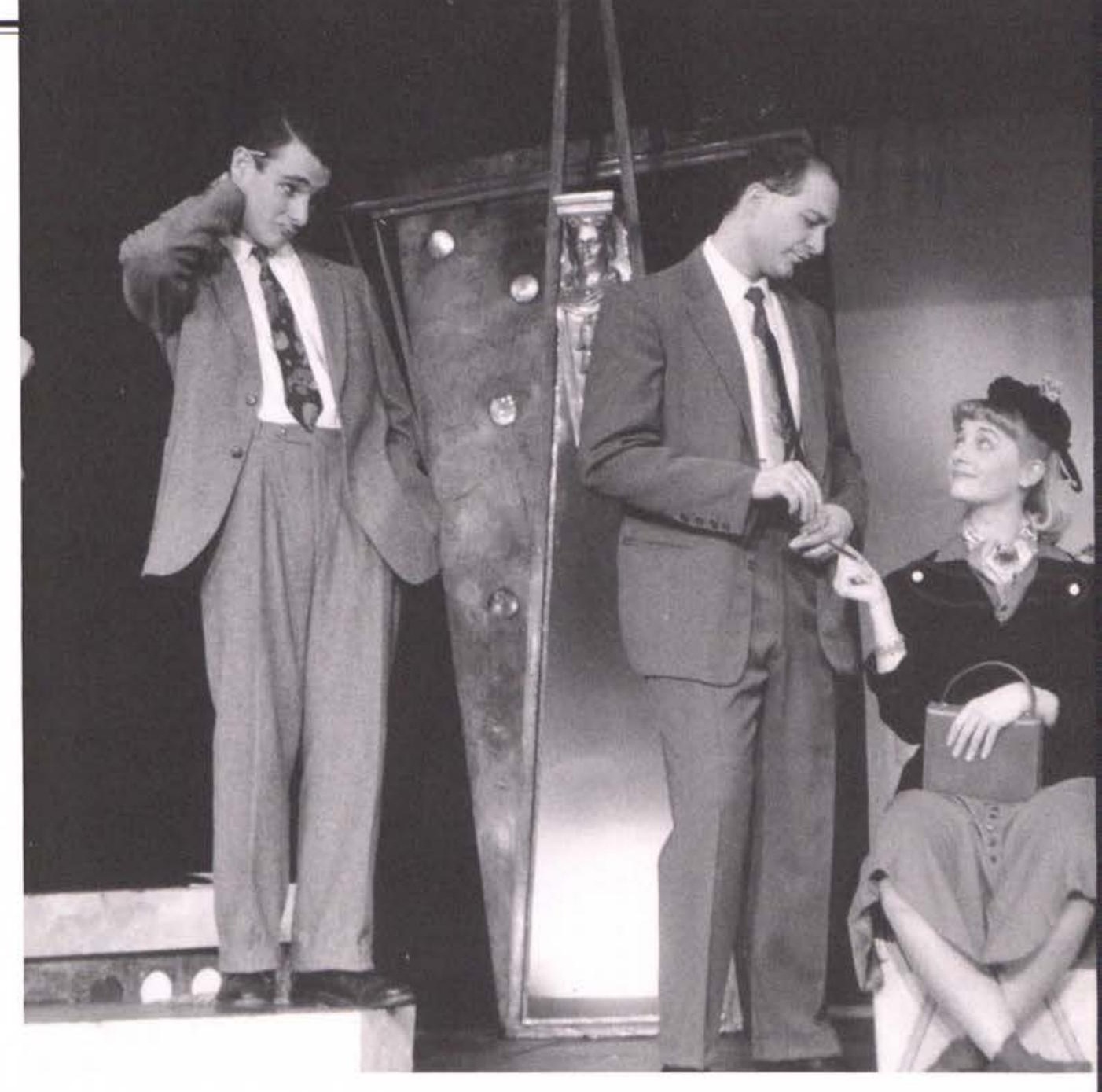




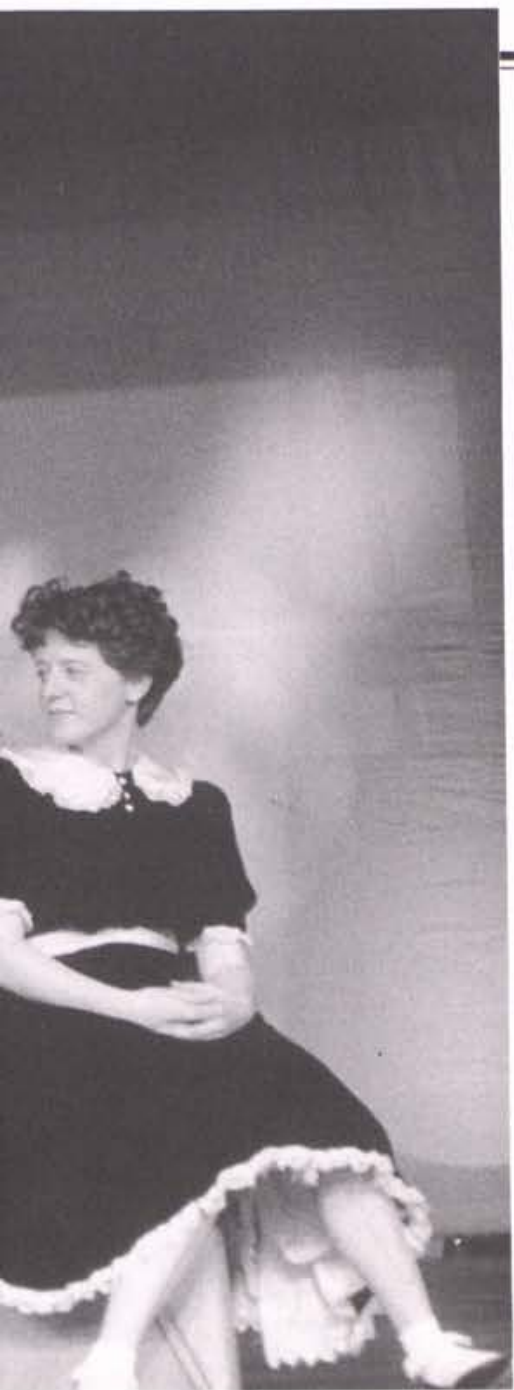

\section{Ceserve Juo for CMurder}

$$
\text { Gy Heathe: Mrfite: }
$$

I

$f$ you were in Alford Auditorium during the first few weeks of winter quarter, you probably wondered what was happening to the stage. What kind of play would need such an eccentric set? From the pink pillar with bust on top, to the leopard-skin covered chairs, the set of the winter play Reserve Two for Murder could definitely leave you confused.

The setting of the play is New York during the 1940's. The time is evening, and the place is a theater. The conflict is a murder in the first act. Witnesses are questioned in the second, and motive is revealed in the third.

One unusual feature of the play was the fact that it included audience participation. The usherettes passed out voting slips, and the audience was able to vote on whom they thought had committed the murder.

The play was a classic "whodunit." Everyone was a suspect, and the entire audience was "held" in the theater until the murderer was discovered.

The cast consisted of fifteen students portraying characters from all walks of life. The major roles included sophomore
Chris Pierre as a doctor, senior Mark Button as a police sergeant, and sophomore Sara Romang as a society dame. Juniors Stacy Saville and Angela Wenzel played actresses, and sophomore Dan Scott was the house manager. Freshmen Jeremy Brown and Brenon Christofer had key roles, and senior Jamin Ferner contributed a bit of comedy as a police officer. Junior Anson Hanbury played the detective who solved the case.
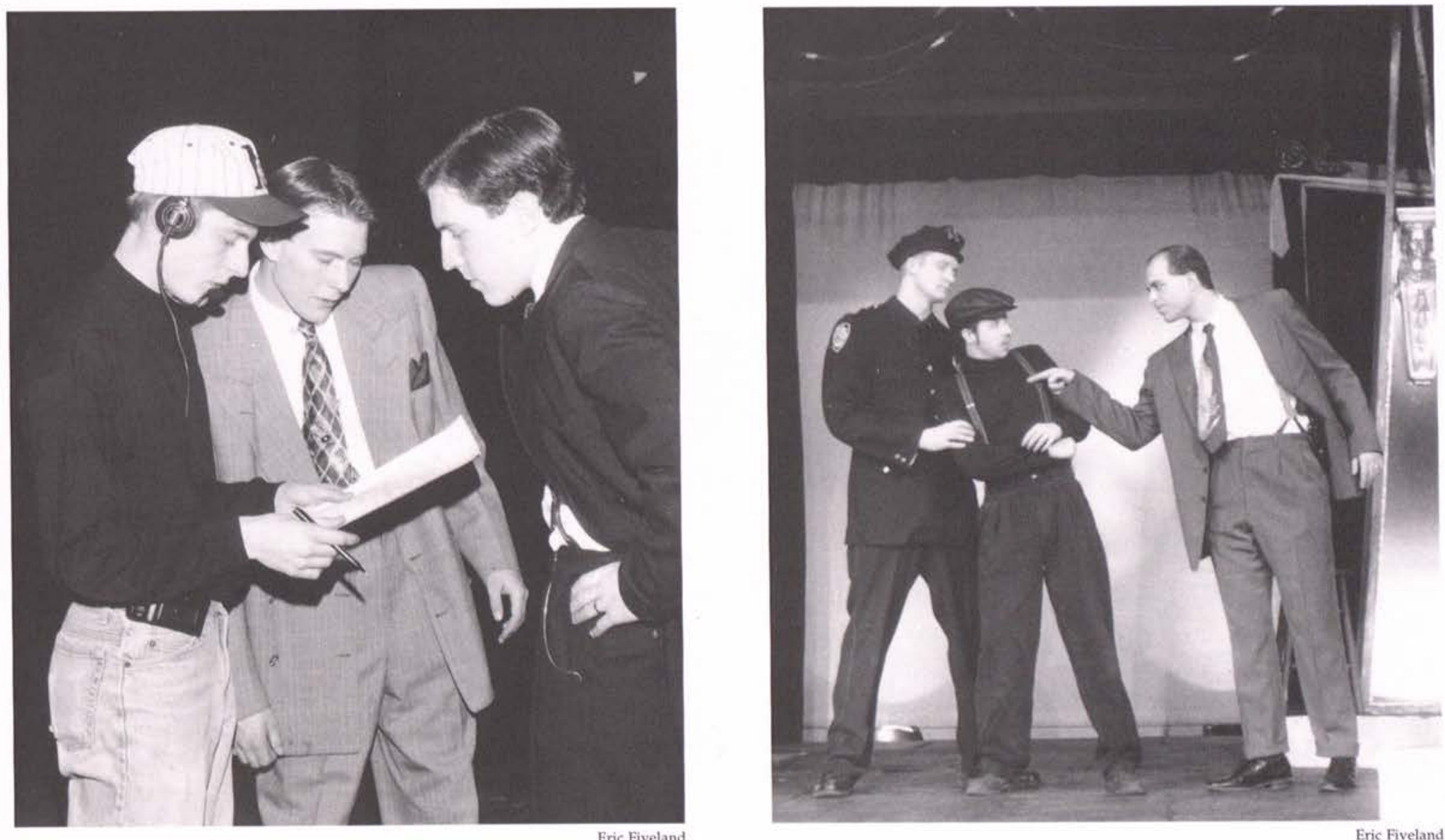

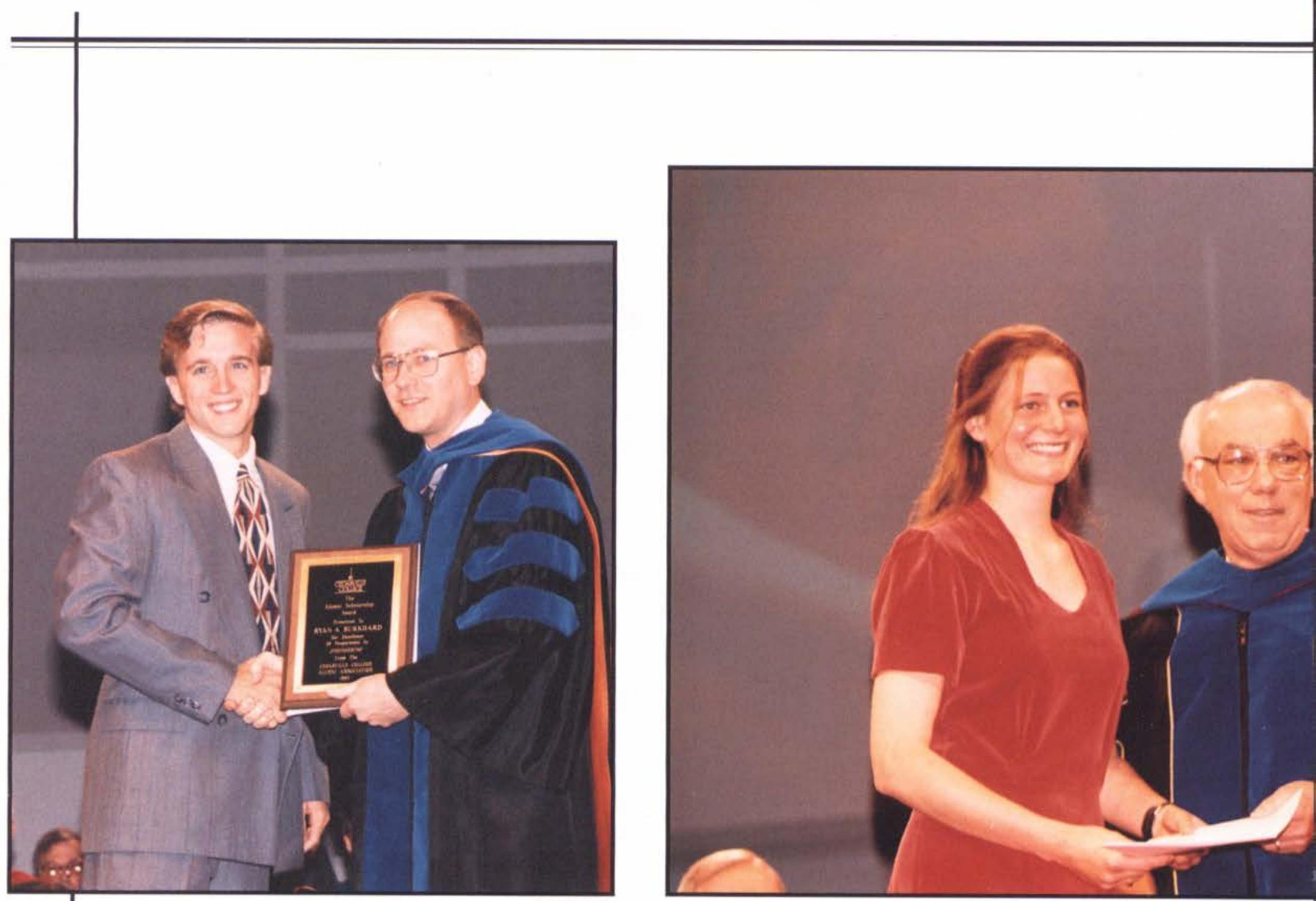

Eric Fiveland

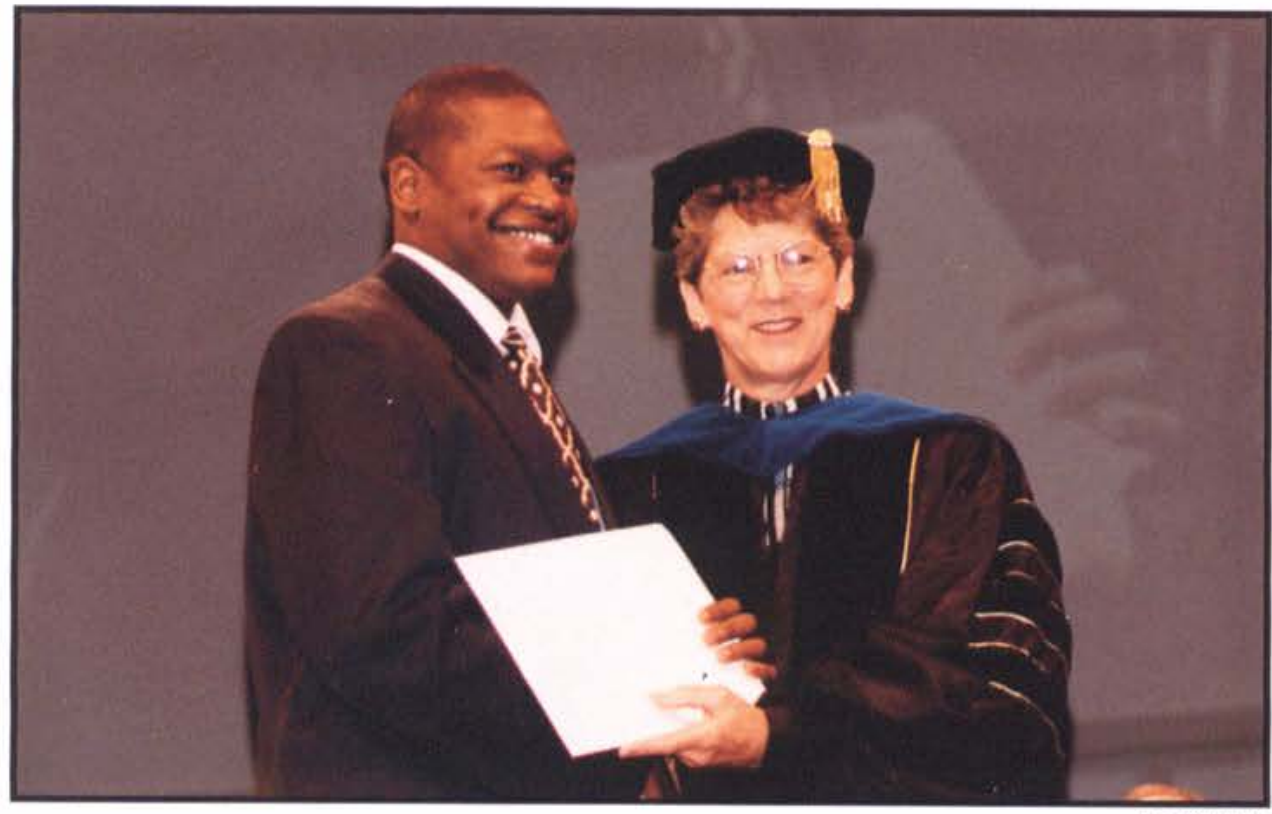

Eric Fiveland

Above left: Ryan Burkhard accepts his scholarship in Engeneering from Dr. Zavodney. Above right: Presian Smyers smiled proudly when she recieved her award from Dr. Wetzel. Below left: Nathan Payne recieves his nursing award from Dr. Alyn. Below middle: Staff member of the year, Murtha Kaercher gives her acceptence speech. Below right: One of the awards given out on Honors Day.

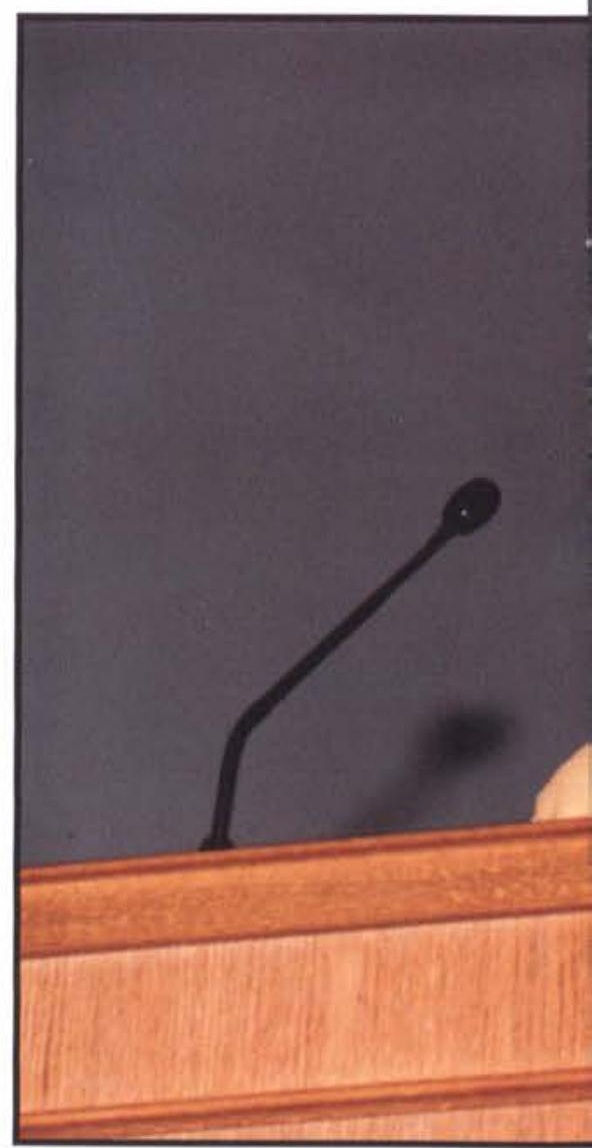




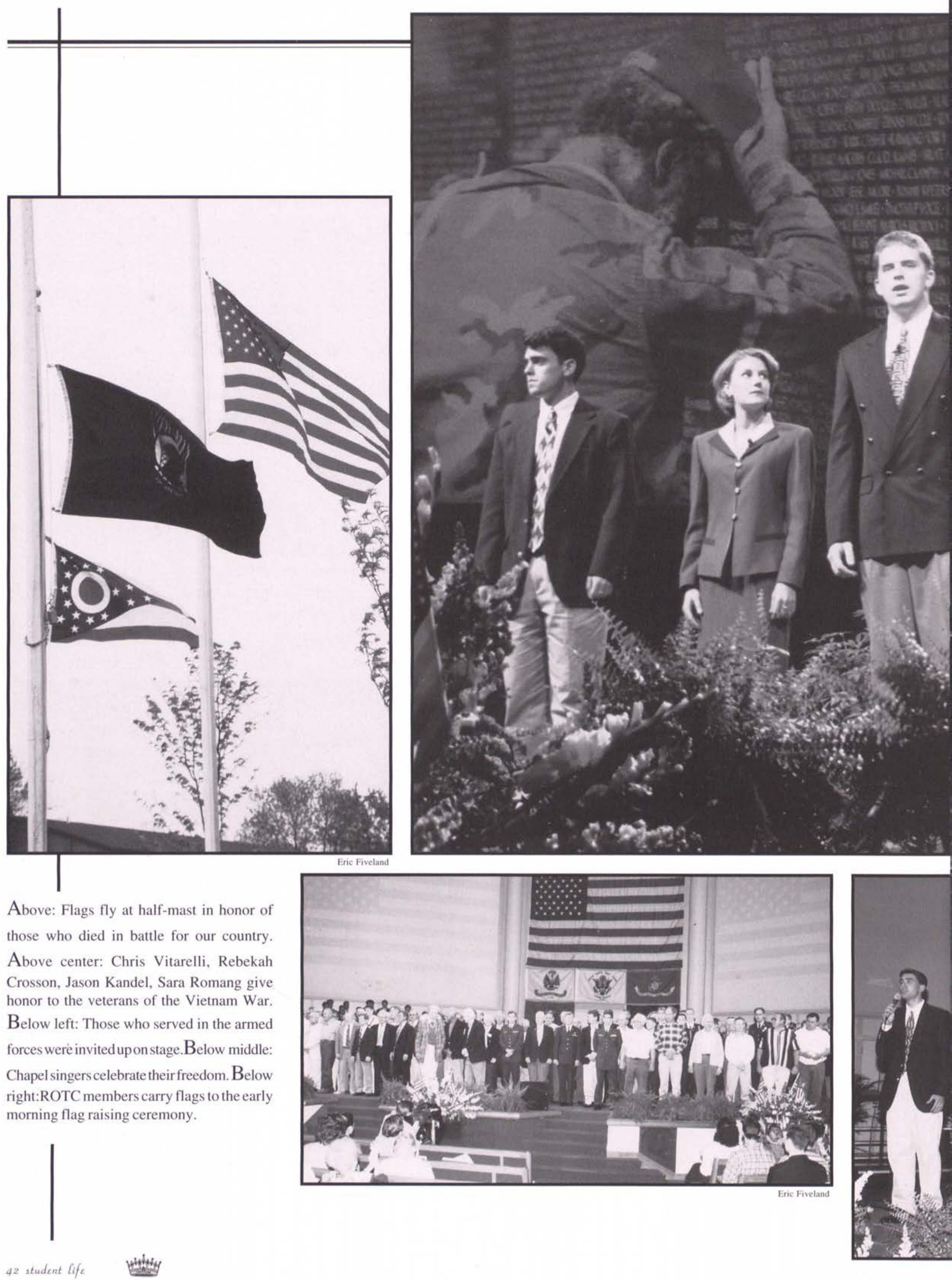




\section{Parents visit' 'Ville}

0 arents of many Cedarville students poured in from all across the nation to enjoy the concerts and events scheduled during this special weekend.

The Honor's chapel kicked things off on Friday morning. Thanks to the spaciousness of the Jeremiah Chapel, students and parents alike could comfortably enjoy the service, as awards for academic excellence were given.

The next two major events were the Lawn Fest and the Pops Concert. The Pops Concert, which was held later on Friday evening, provided the parents with a taste of the richness of Cedarville's music program. The Brass Choir, Men's Glee Club, Lab Band, The Concert Chorale, and Symphonic

by Bonnix Scharfes

Bandallperformedmagnificently. Well-knownnumbers suchas The Lion Sleeps Tonight, In the Mood, Hakuna Matata, and The Battle of Jericho were performed by these vocal and instrumental groups for the audience's listeningenjoyment.

Also during the weekend, Cedarville's track team hosted the NCCAA national track meet, which took place on both Friday and Saturday. Those who attended watched the men's and women'steams achieve success, as the women's team once again won the national NCCAA title and the men's team finished third in the national standings.

Another highlight of the weekend was the Steve Green concert heldon Saturday night. Throughout the concert, Green blended the music to meet the tastes of adults, college students, and children alike. His focus on the importance of the family was evident, as well as his ability to get the audience involved in worship.

Of course, the weekend would not have been complete without the play My Fair Lady. With more than one hundred cast and crew members, this production was one of the largest Cedarville has ever seen. As a result, Alford Auditorium was packed with delighted people who watched the characters comealive through the scenery, costumes, music, and superb acting of all involved.

Sunday marked the last day of parent's weekend. The churches were filled as students escorted their parents to morning services. Following the services, the families shared a final meal together and said farewell.
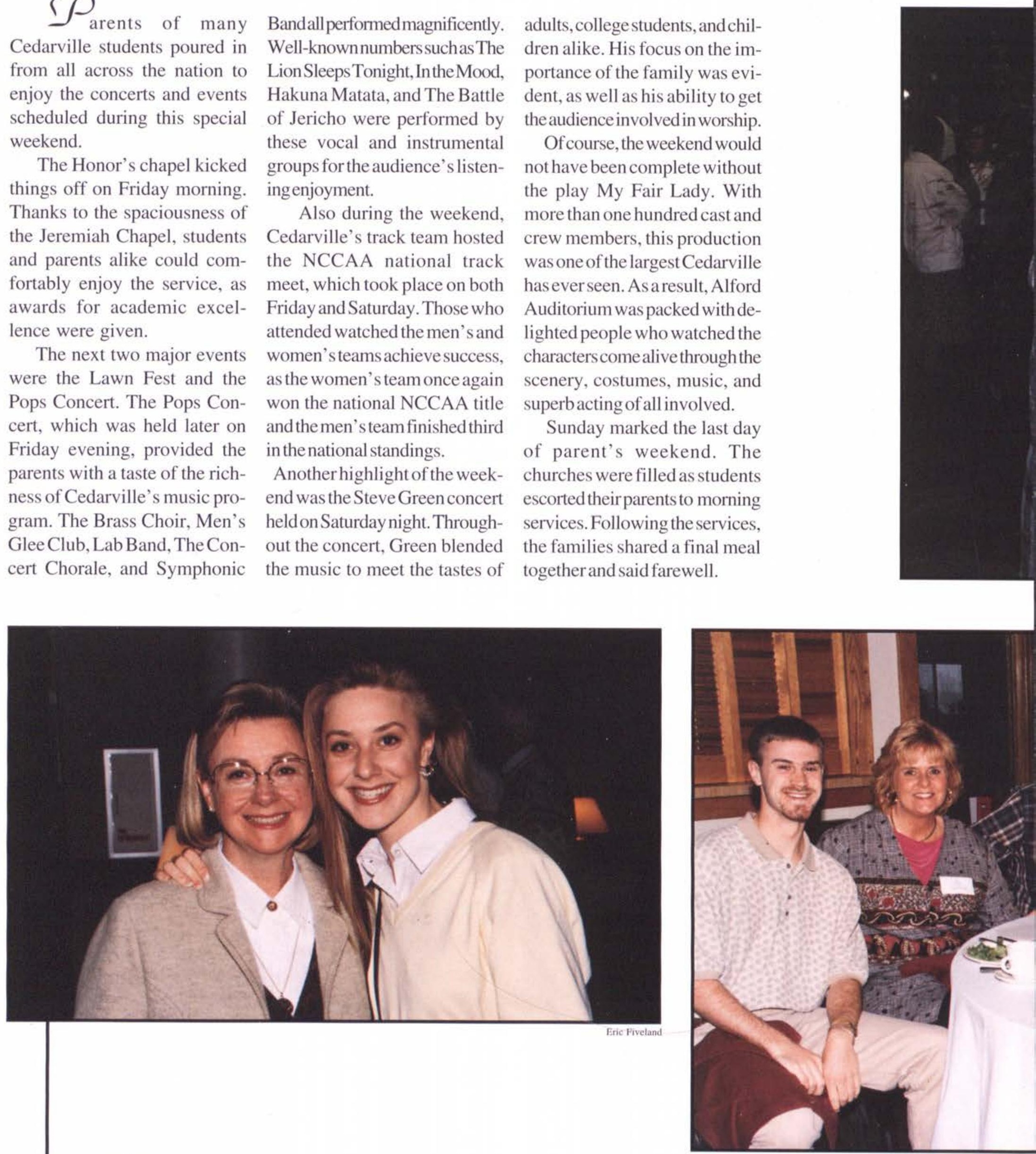
Left: Guests anticipate the Steve Green concert. Right: Brooke Frazier and her mom look through the CD's and music of Steve Green. Below left: Jennifer Hangosky and her mom enjoy their time together during Parent's Weekend. Below middle: Dale Pinkley, his parents, his sister and brother-in-law Janine and Scott Borling enjoy a time of fellowship. Below right: Parents get a chance to sample the food at Chuck's.

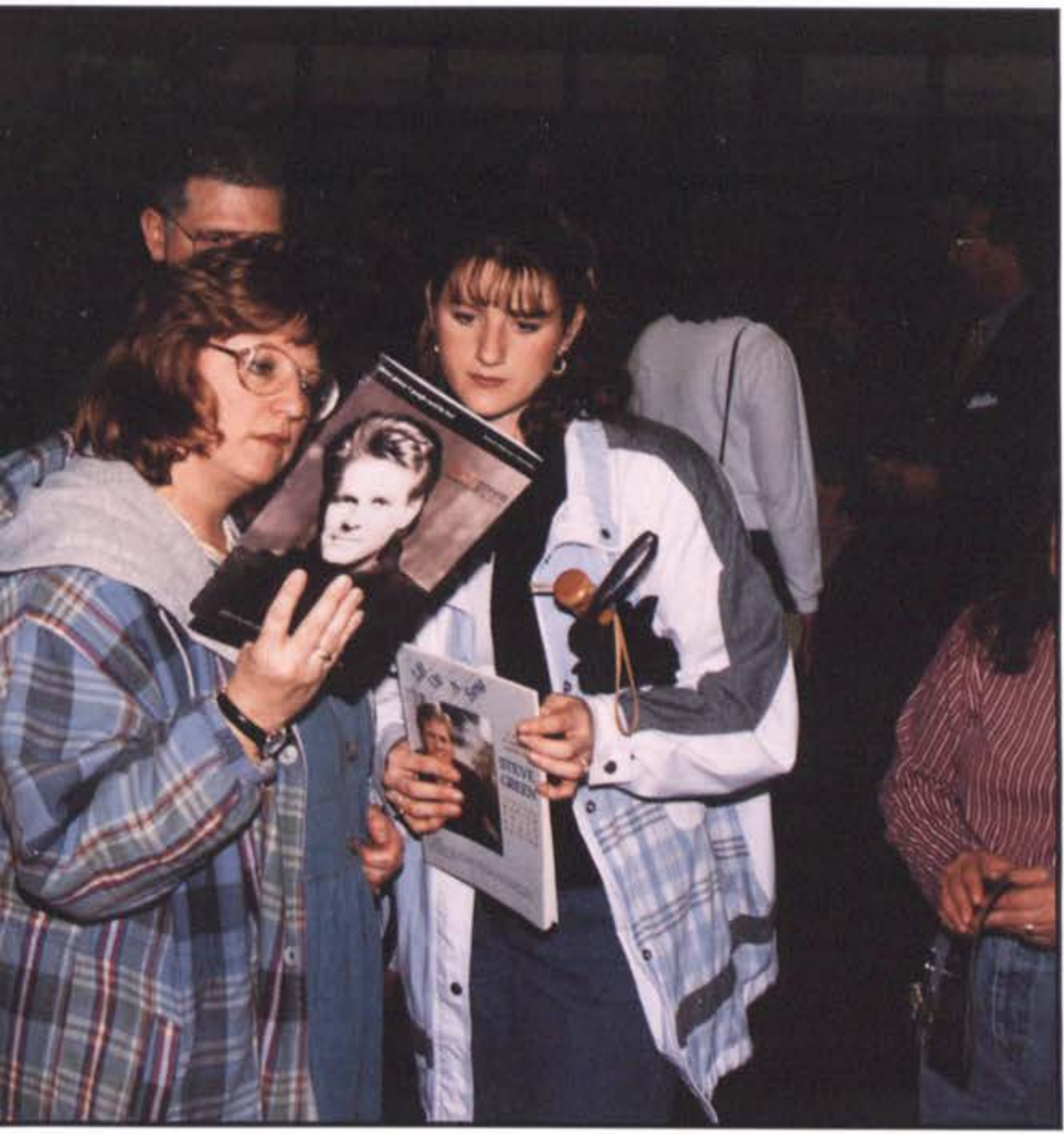

Eric Fiveland
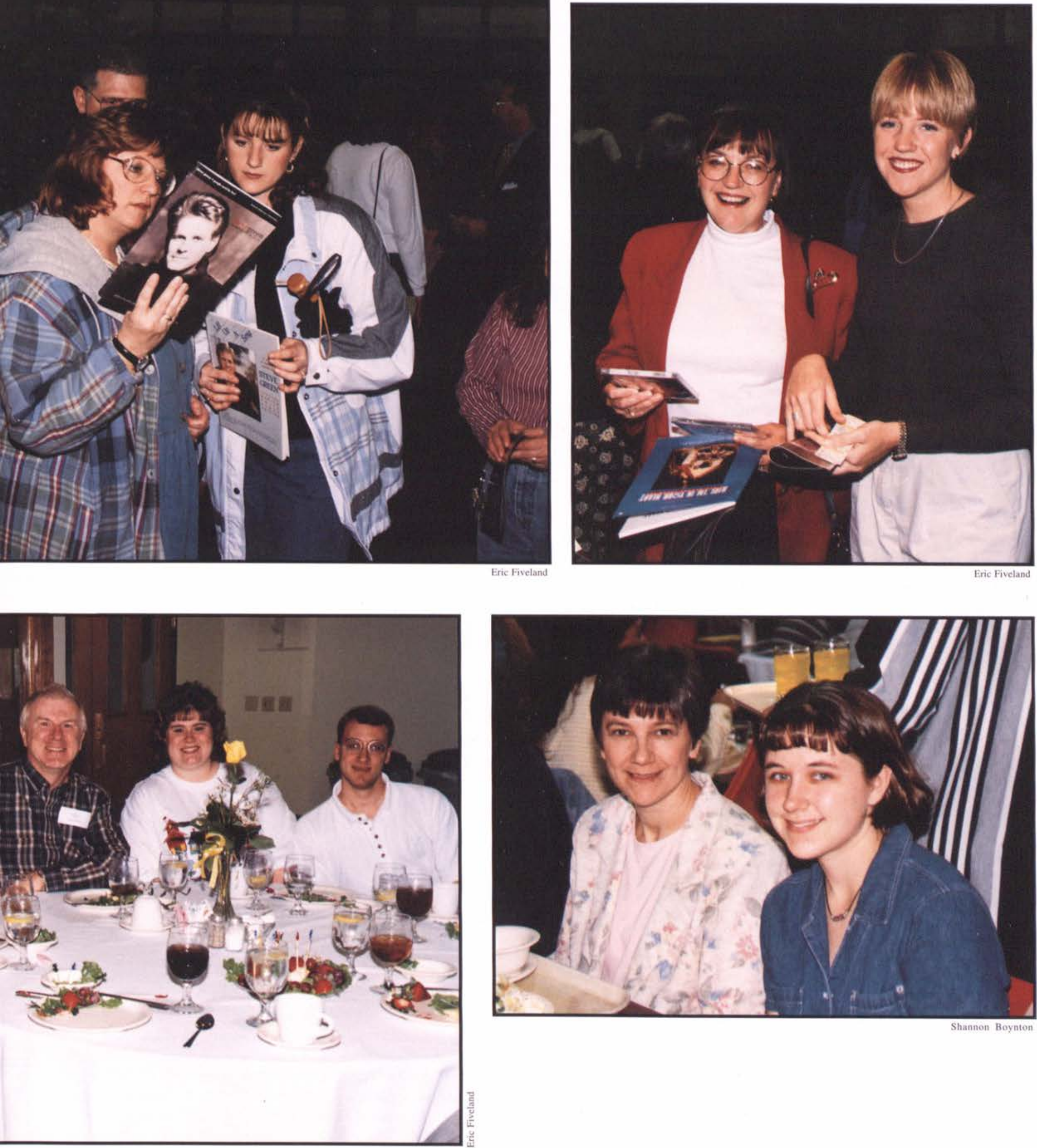


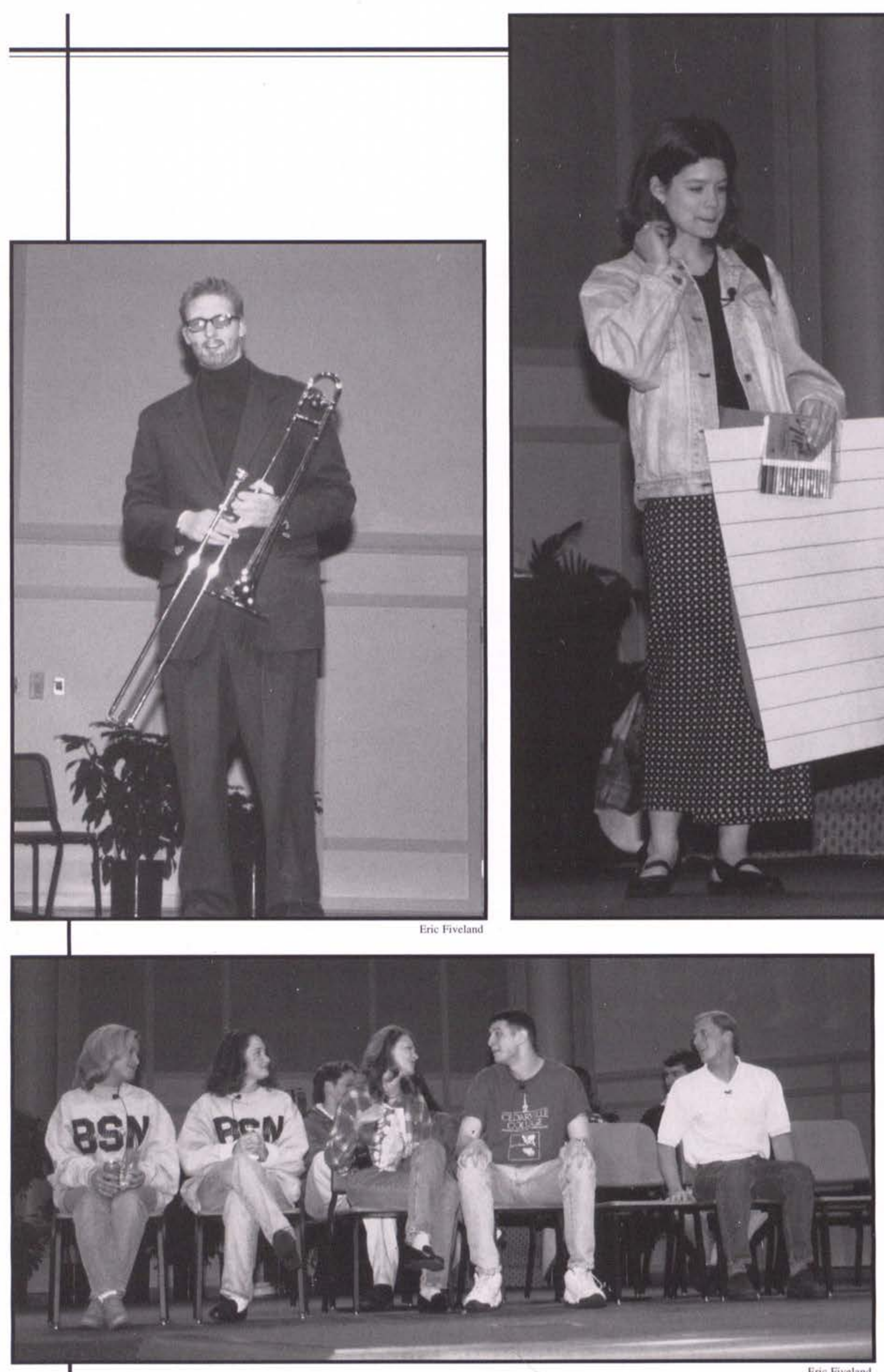

Above left: Jamin Ferner poses as Mike DiCuirci. Above right: Doug Bayler, posing as a eager freshman, tries to make an appointment with Liz Roseboom. Below left: Seniors remember when they had to go to Wittenburg to see a movie. Below middle: Valerie Ashurst, Sarah Holesovsky, and Travis Mulanax sit in Foundations as Derek Green poses as Dr. Allen Monroe. Below right: Eric Sorensen and Bill Workman are old men remembering their Cedarville College days.

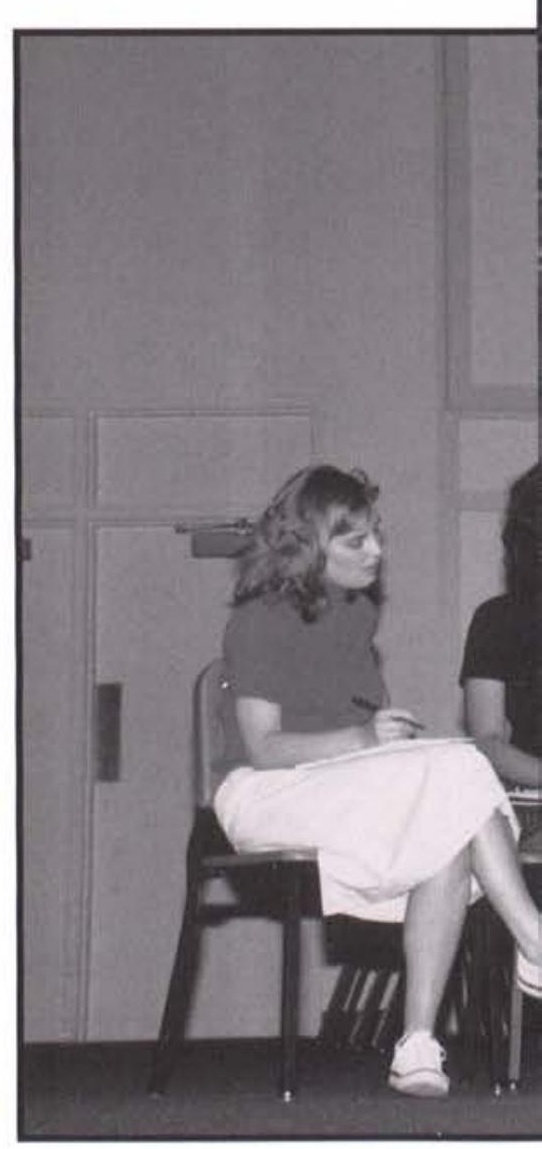


(1) n May 22, photographs quickly flashed on the chapel screen just as the last four years have passed rapidly for many of the class of 1997. Projected 80 years into the future, Bill Workman and Eric Sorensen, patients at the Dandelion Acres, recounted their four years at Cedarville College and the numerous changes the college had since experienced.

Bill and Eric first looked back to their freshman year, 1993-1994. Students were shown falling asleep in Christian Life. An enthusiastic professor threatened a Physical Activity and the Christian Life class with the fabled five-mile run. Derrick Green portrayed Professor of Social Sciences Dr.
Allen Monroe in his notorious Foundations of Social Sciences class.

The sophomore year was marked by a trip to the Wittenberg Theater. Witha 12:45 a.m. curfew alarm, the Cedarville students rushed back to avoid Public Safety's wrath. This led to the Public Safety video accompaniedby the "Cops" themesong. In the video, Junior Tim Simon searchedadormforthe Dr.Dixon portrait.

The junioryear waschronicled by the dreaded satellite chapel services. The video connection with the main auditorium failed, leaving the seniorstosnooze with the audio service in the background. Last year's recording of the Prism of Praise was remembered.

The remainder of the service focused on the senior year. The nostalgic move to the new Jeremiah Chapel started the video. The video then showed memorable chapel speakers and events of the last four years: the chapel screen rising with an attached cut-out of Pierce Brosnan from Goldeneye; missionary Ed Lewis' German account of David and Goliath; the Dandelion Speech; Dr. Dixon firing a golfball at yearbook photographer Eric Fiveland from the stage of the Jeremiah Chapel; and Pastor Green's farewell address on “Green Day," May 5, 1995.

This gave way to the senior slide show and senior song. With his guitar, Jason Grills led the senior class in singing The Kry's "IBelieve in You.” Dr. Dixonclosed the serviceexpressing his love for all seniors.
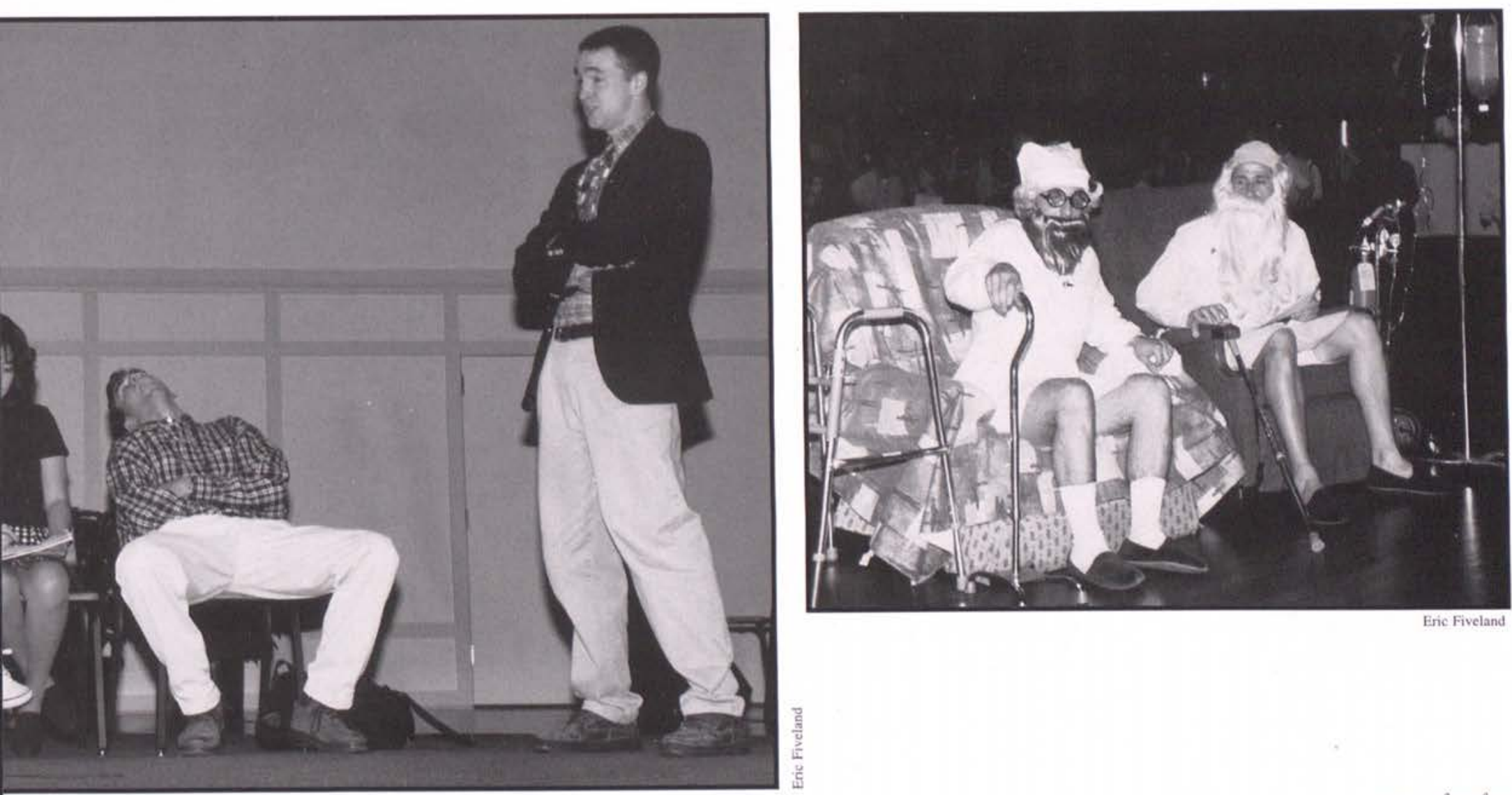

Eric Fiveland 


\section{An Impressive Group}

Gy $\mathcal{P}_{\text {ets }}$ Sutton

Some may wonder how one gets to be in "Who's Who." While many arrive at the 'Ville with visions of being class president, or a varsity athlete, not too many students set out with aspirations of being in "Who's Who." For most students, Who's Who comes as a surprise.

The selection process begins every year with a committee of faculty and students gathering together to narrow down the five hundred and some graduating seniors to a group of about fifty. This group is evaluated on the basis of accademic excellence, extra curricular activity, ministry involvement, and campus leadership. The fifty students chosen at the end of this project become members of the Who's Who Among Students in America's Colleges and Universities.

Among the honors that ac- company membership in this organization are a beautifully calligraphied certificate, the opportunity to purchase an $\$ 80.00$ volume listing every other Who's Who member, and the chance to participate in one of the most memorable chapels at Cedarville College.

This year's Who's Who chapel was orchestrated by Krista Price, Senior communication arts major. Planning for this event began early during Winter quarter and continued right up until the week before, as skits were revised to more thoroughly convey this year's theme, "sieze the day."

"Sieze the day" was chosen as the theme in order to challenge underclassmen to get involved in any way they can.

Activities animated throughout this chapel included; Getting Started, ministry opportunities, intramural sports, on- campus employment (namely Chuck's), and more. Lori Anderson and a cast of many entertained us with their rendition of Maria's "goodnight" song from The Sound of Music.

The pinnacle of the morning may be said to have been the "cheerleader skit" by Krista Price and John Maskill. With mentions of Cedars, engineering majors, and the forensics team, Krista and John had the chapel roaring with laughter. Memorable quotes included "Sink or Swim!," "Sidewalk Talk!" and "who's that girl sinkin' next to me?" "It's me, it's me!"

The Who's Who chapel was capped off with a video of every Who's Who member. Senior broadcasting major Paula Farris put in unmeasureable time into shooting and editing this video to Carolyn Arends' song "Sieze the Day."

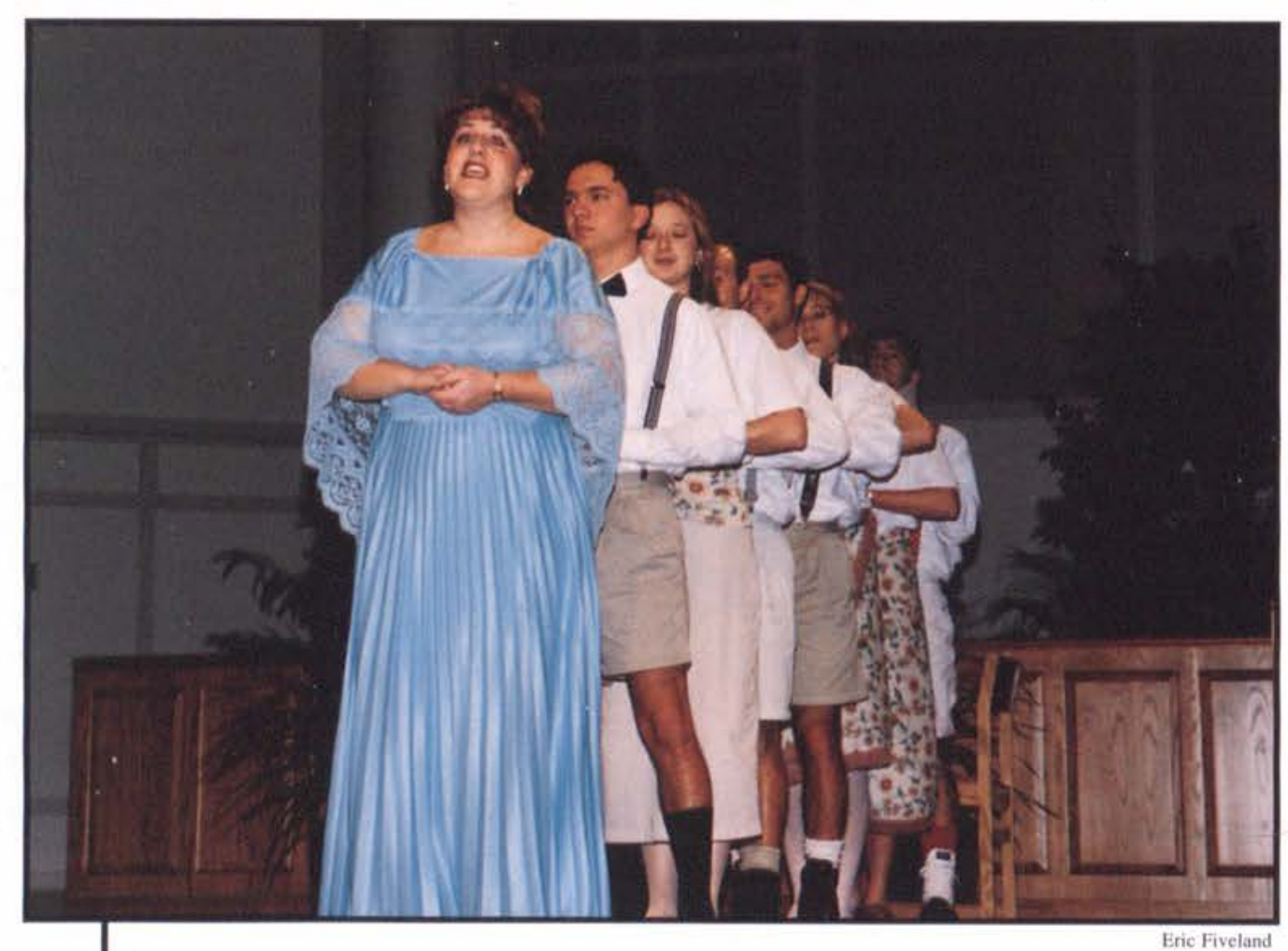

Above: Fraulein Maria, Lori Anderson, sings with the von Trapp children.

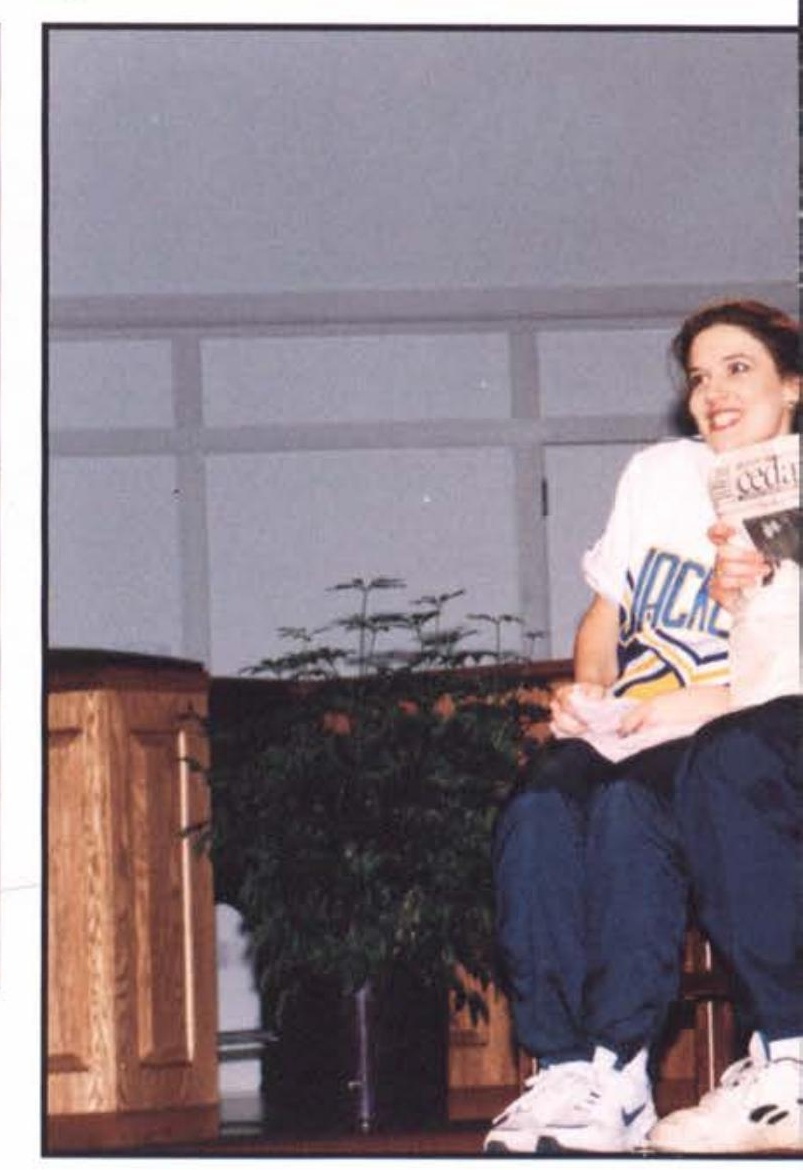



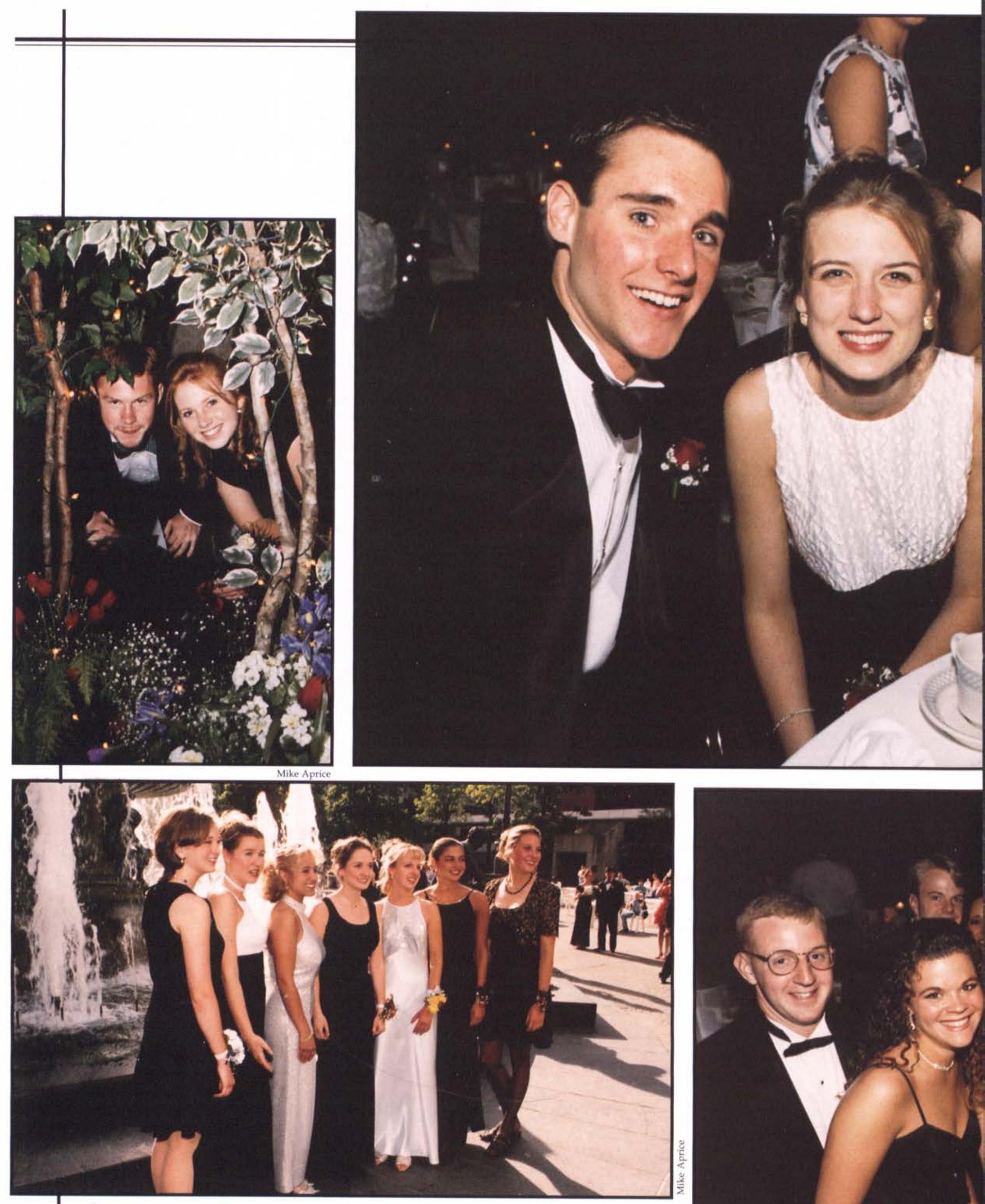

Above left: Cliff Scott and Jen Ingalls peek through the decorations. Above right: Anson Hanbury and his date Joy Webster enjoy their time at the banquet. Below left: Dressing up and posing for pictures is all part of the fun of the evening. Below middle: Good times are had by all at the banquet. Below right: A couple stops to smile for the camera. 


\section{The Story Goes On}

$$
\text { by Strue Garcia }
$$

(1) n May 23, Cincinnati's Westin Hotel hosted the annual Cedarville College Junior/Senior Banquet. Almost 700 well-dressed people loaded their cameras with film and headed to Fountain Square.

At approximately 5:00 p.m., people began to arrive. The entire lobby was reserved and decorated for the pre-dinner reception. Professional photographers took pictures of the couples as well as a picture of the entire senior class.

At 6:30, a dinner of chicken with mustard sauce, white and black rice, salad, carrots and asparagus was served in the Presidential Ballroom, followed by an hour-long intermission. After the intermission, coffee and dessert were served.

Later in the evening, the Junior/Senior committee presented the Senior Tribute, a 17-minute slide show accompanied by various songs. Following theslide show, seniors Krista Price and John Maskill presented the first Senior Hallmark -- a hilarious retrospective look on four years of Cedarville life. They pulled items of memorabilia from abox, each item a symbol of their greatest memories. After pulling out the last item, a cheer-leading outfit, Price and Maskill performed theirinfamous cheerleading act.

AftertheSenior Hallmark, the emcee welcomed Michael O'Brien. O'Brien entertained the audiencewith hislively musicand his genuine humor.He surprised the audience by including a juggling actin his program, one of his lesser-known talents. Senior Chris Rudolph helped with the act.

On a more serious note, O'Brien said, "You may not like my music, but you have to respect my message." His heart for the Lord was evident and was magnified by his moving testimony and gospel presentation. O'Brien closed with the themesong for the evening, "The Story Goes On."

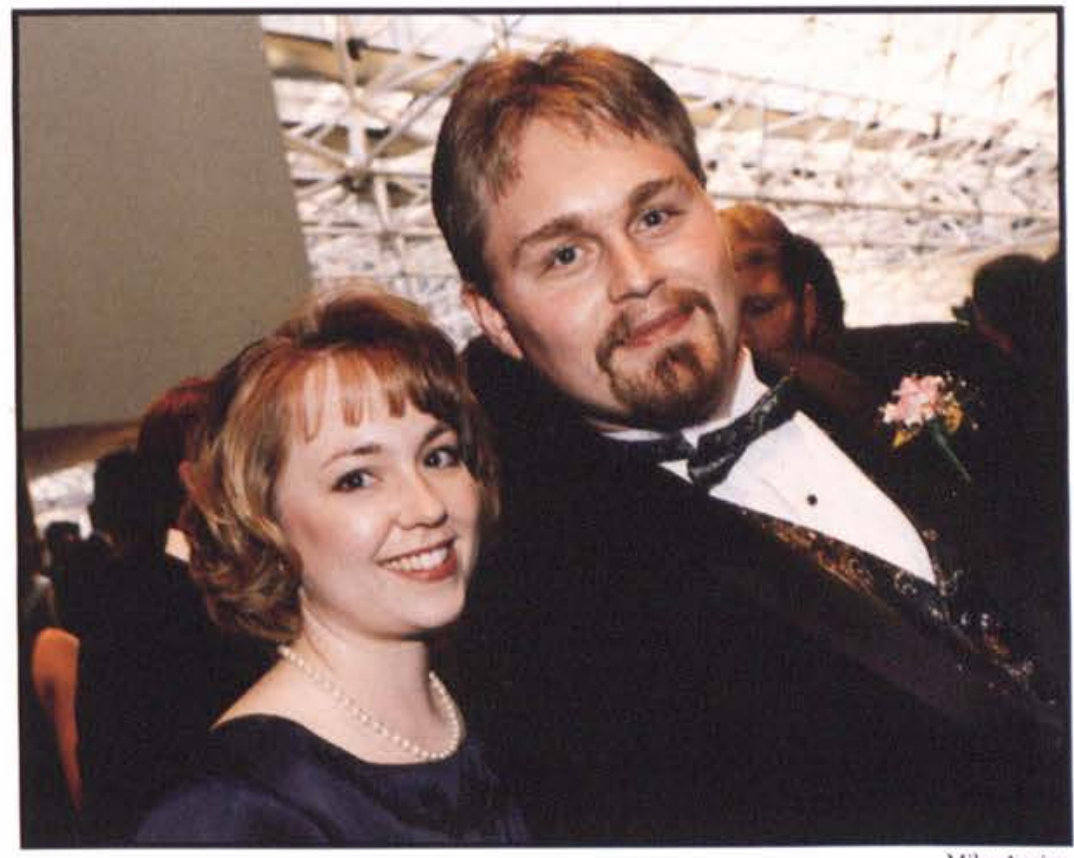

Mike Aprice 


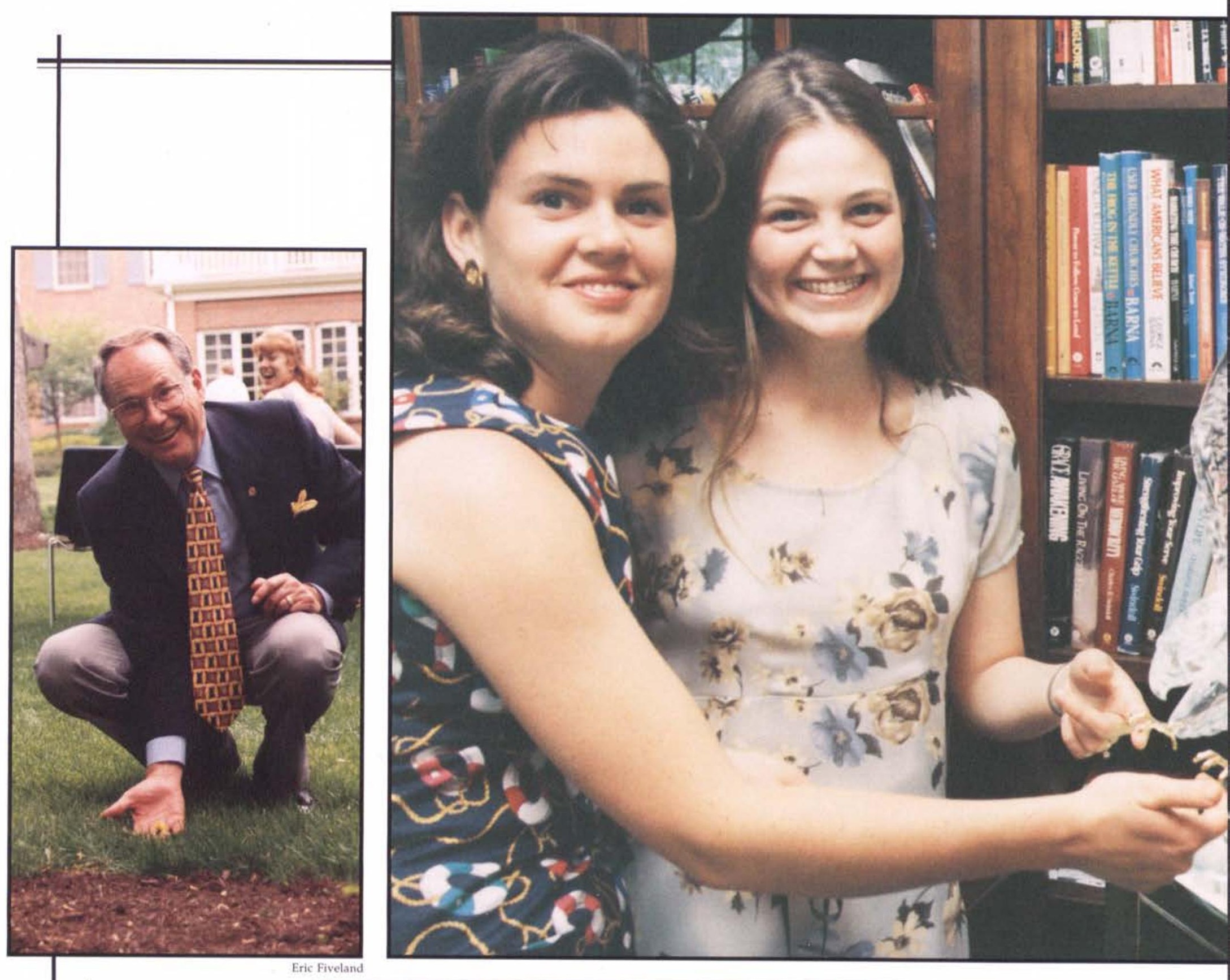

Above left: Dr. Dixon laughs with his guests when he discovers a dandelion in his own yard after having demanded the extinction of the weed. Above right: Sara Howell and Emily Hodel admire an eagle presented to Dr. Dixon in honor of his work on the Dixon Ministry Center. Below left: Doug Bayler tests out Dr. Dixon's chair and plots how to become

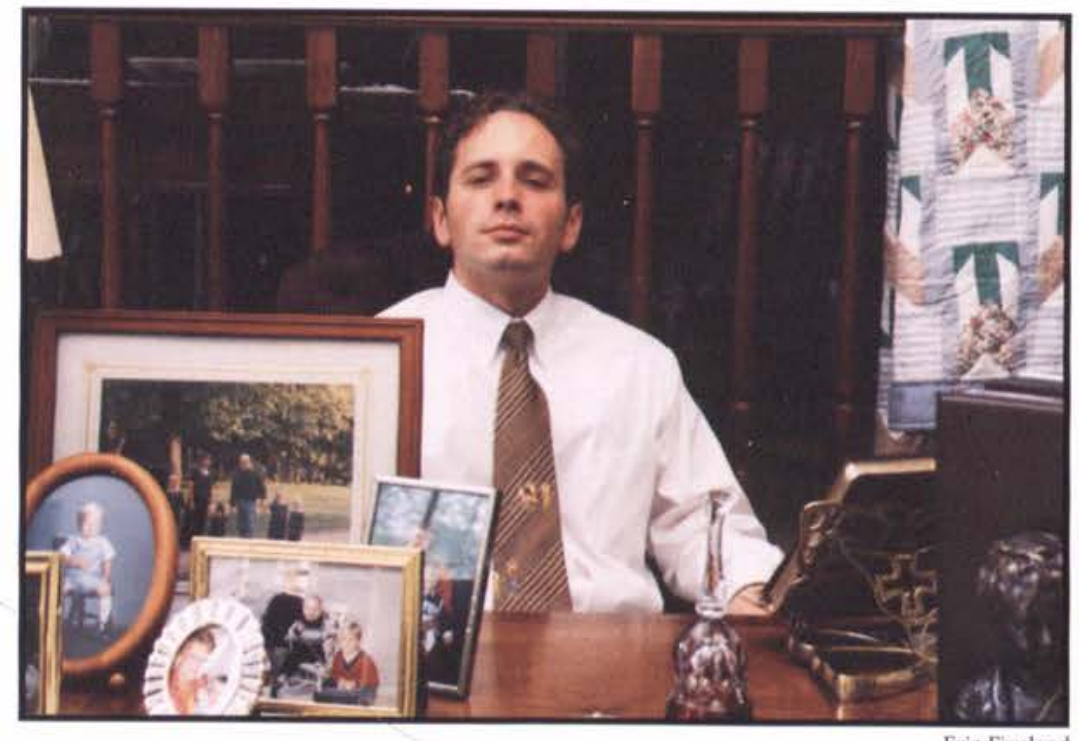

Cedarville's next president. Below middle: Sarah Terpstra, Kerry Allen, Dr. Dixon, Mrs. Dixon, Scott Judkins and Jesse Roberts take one last picture to add to their pile of memories from Cedarville. Below right: Two seniors relax in the Dixon family hammock.

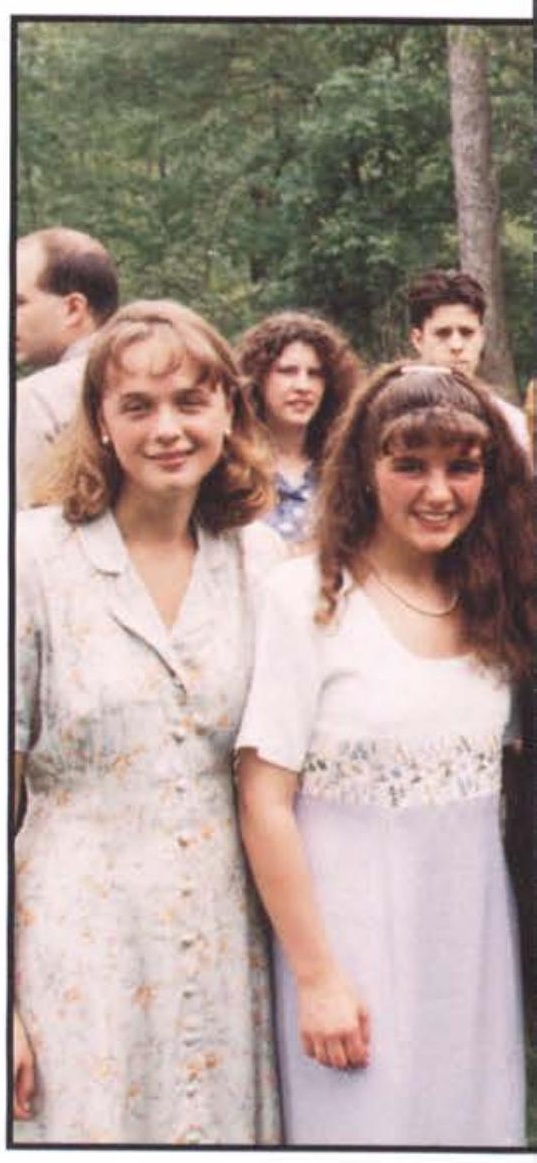




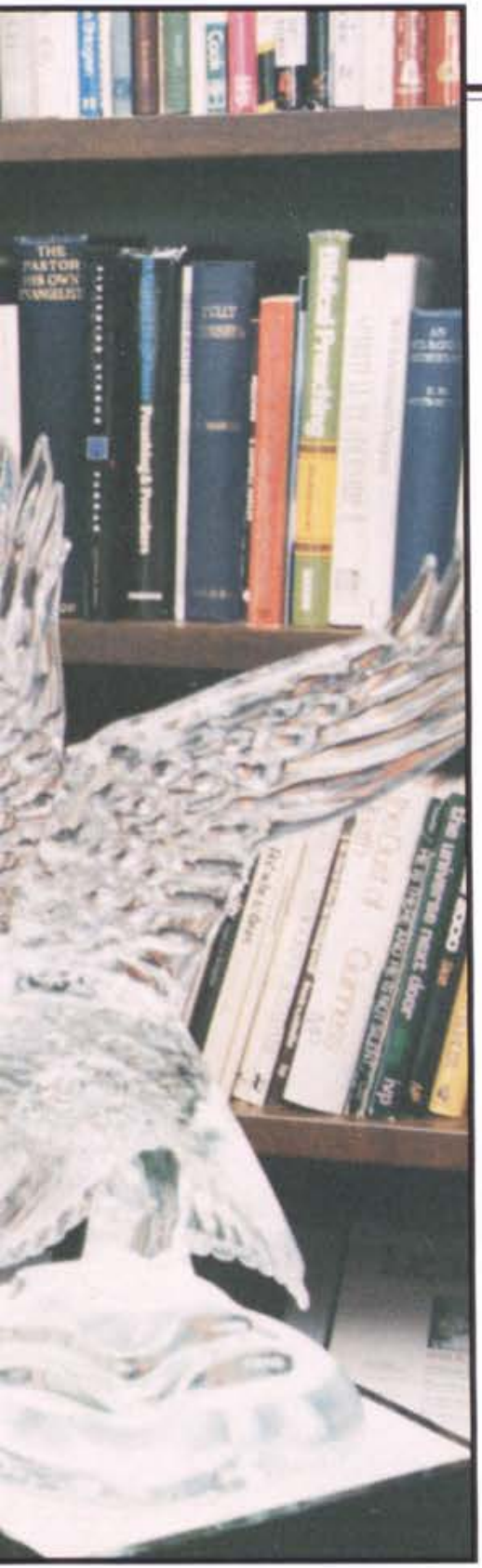

Reception at the Dixon 1 Home

O. and Mrs. Dixon opened their home for the annual reception honoring the graduating class. Over the course of the afternoon, more than 400 seniors rode out to the estate for refreshments on the lawn and the chance to tour the house.

The event was planned by Dick Walker and the Campus Activities Board, and was ca- tered by Chuck's. CAB members drove shuttle vans on a continuous rotating schedule, bringing new groups to the house, and taking previous groups back to campus. All plans went well; even the weather cooperated. It began to rain only as the reception broke up around 5:00.

Between glasses of punch and the occasional quick trip inside to check the basketball scores, Dr. and Mrs. Dixon posed for hundreds of pictures with individuals, pairs, and large groups of students who wanted to commemorate the afternoon and the previous four years. The relaxed atmosphere was also perfect for seniors to gather in groups and chat about past memories and future plans.
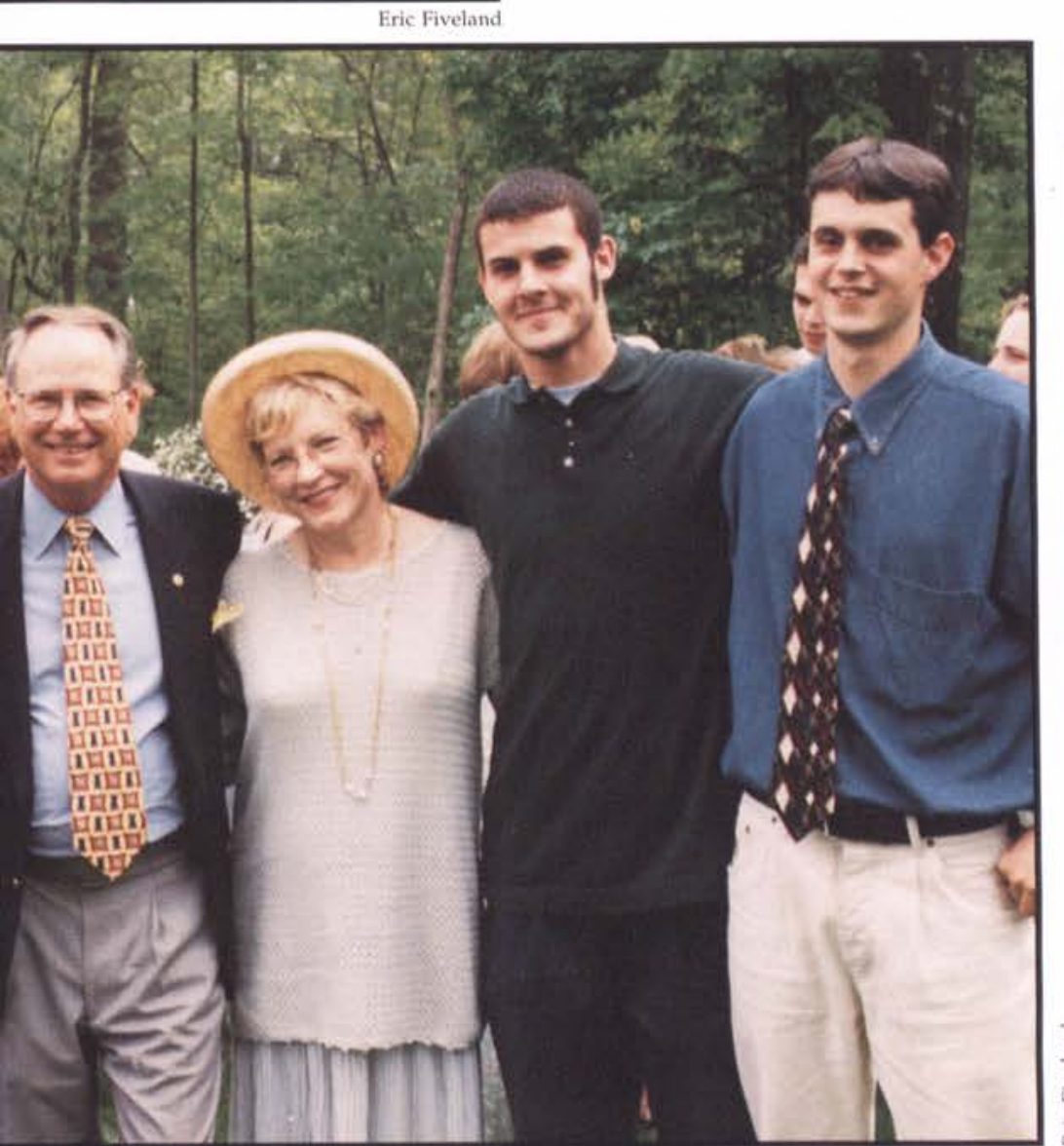

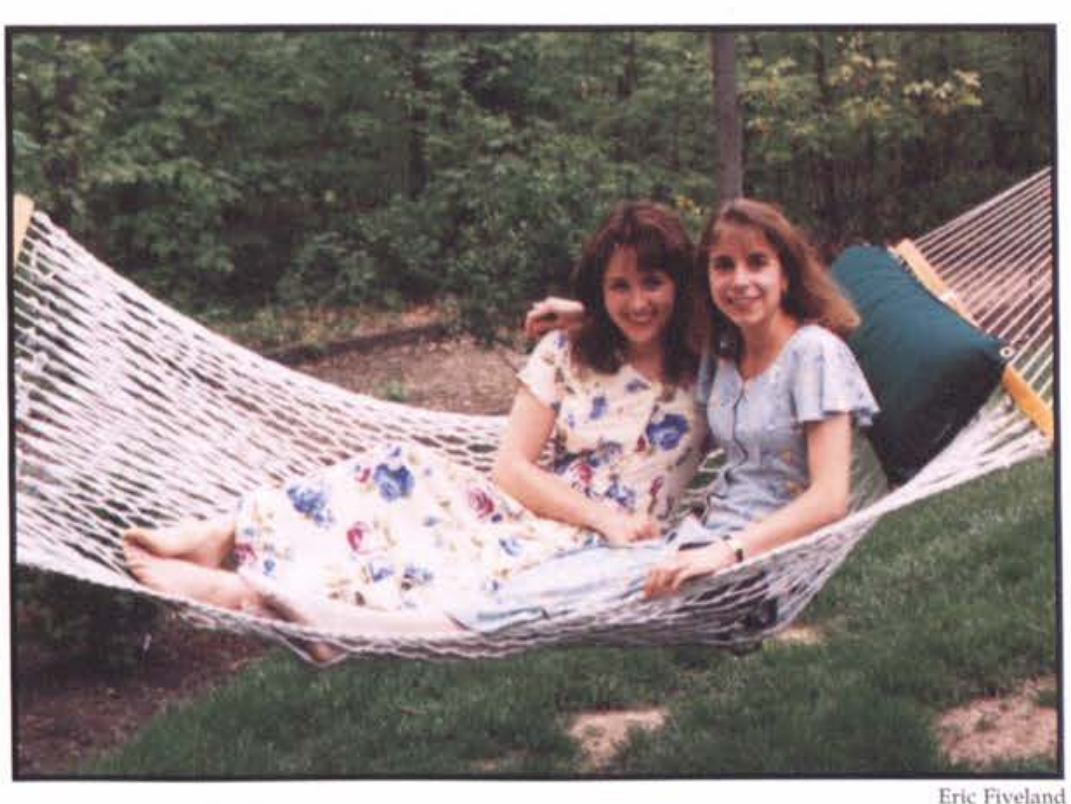

Eric Fiveland 


\section{Been There, Done That}

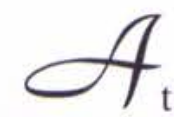
of June 7, 1997, Commencement began for Cedarville's 101st graduating class. More than 500 students were publicly recognized for their years of hard work and study; it was the largest class the college has graduated. The ceremony took place in the Ministry Center, which was filled to capacity with the graduates' family members. Video screens were set up in the Recital Hall and Cedar Hall for additional guests.

Commencement began with the faculty processional, which was played by the Brass Choir, and was followed by the invocational prayer, led by Dr. William Rudd, Chairman of the Board of Trustees.

$$
\text { Gy Matt Sherwood }
$$

After music by the Concert Chorale, President Dixon provided the Commencement Address. He spoke about Cedarville's past, and his vision for the future; he applauded the Class of 1997 for their individual accomplishments, and encouraged all of the graduates to use their education to become Christ's ambassadors in all areas of society.

Following the address, Senior Class President Evan Wood presented the class gift to Dr. Dixon and the Board of Trustees; the gift consisted of the brick welcome sign for the new entrance to the Ministry Center.

During the presentation of awards, Dr. Duane Wood, Aca- demic Vice President, read the list of graduates' names while Dr. Dixon handed the folders to each recipient. In addition to the graduating students, the college administration also bestowed an honorary doctorate on Richard W. Houg, the President of Faith Baptist Bible College and Seminary, where he has served for ten years.

The Class of '97 chose as their verse Jeremiah 29:11'For I know the plans I have for you,' declares the Lord, 'plans to prosper you and not to harm you, plans to give you hope and a future.' Each of the graduates holds onto that promise as the "real world" takes the place of college. As Dr. Dixon said, it was a day of beginnings.
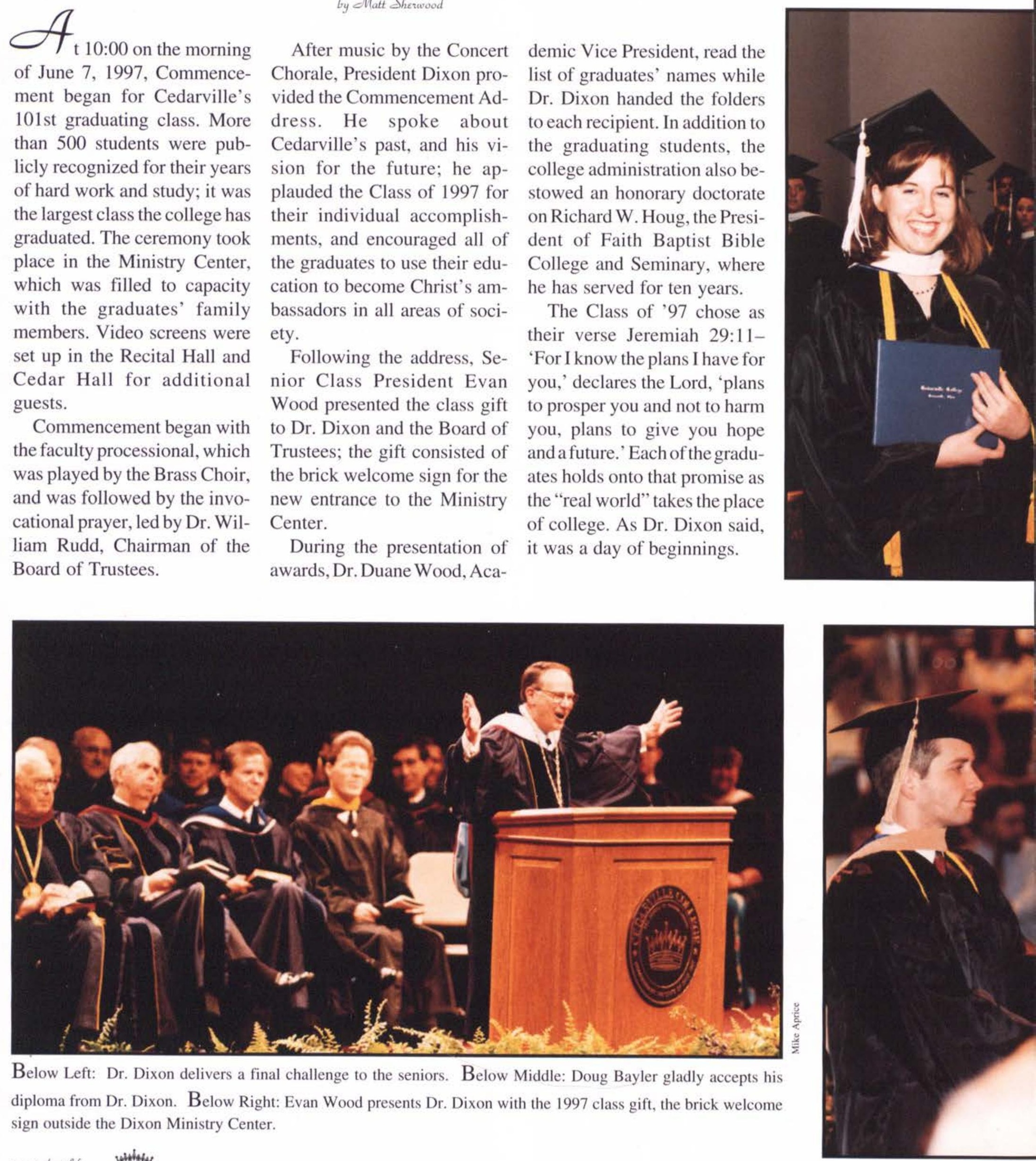

Below Left: Dr. Dixon delivers a final challenge to the seniors. Below Middle: Doug Bayler gladly accepts his diploma from Dr. Dixon. Below Right: Evan Wood presents Dr. Dixon with the 1997 class gift, the brick welcome sign outside the Dixon Ministry Center. 


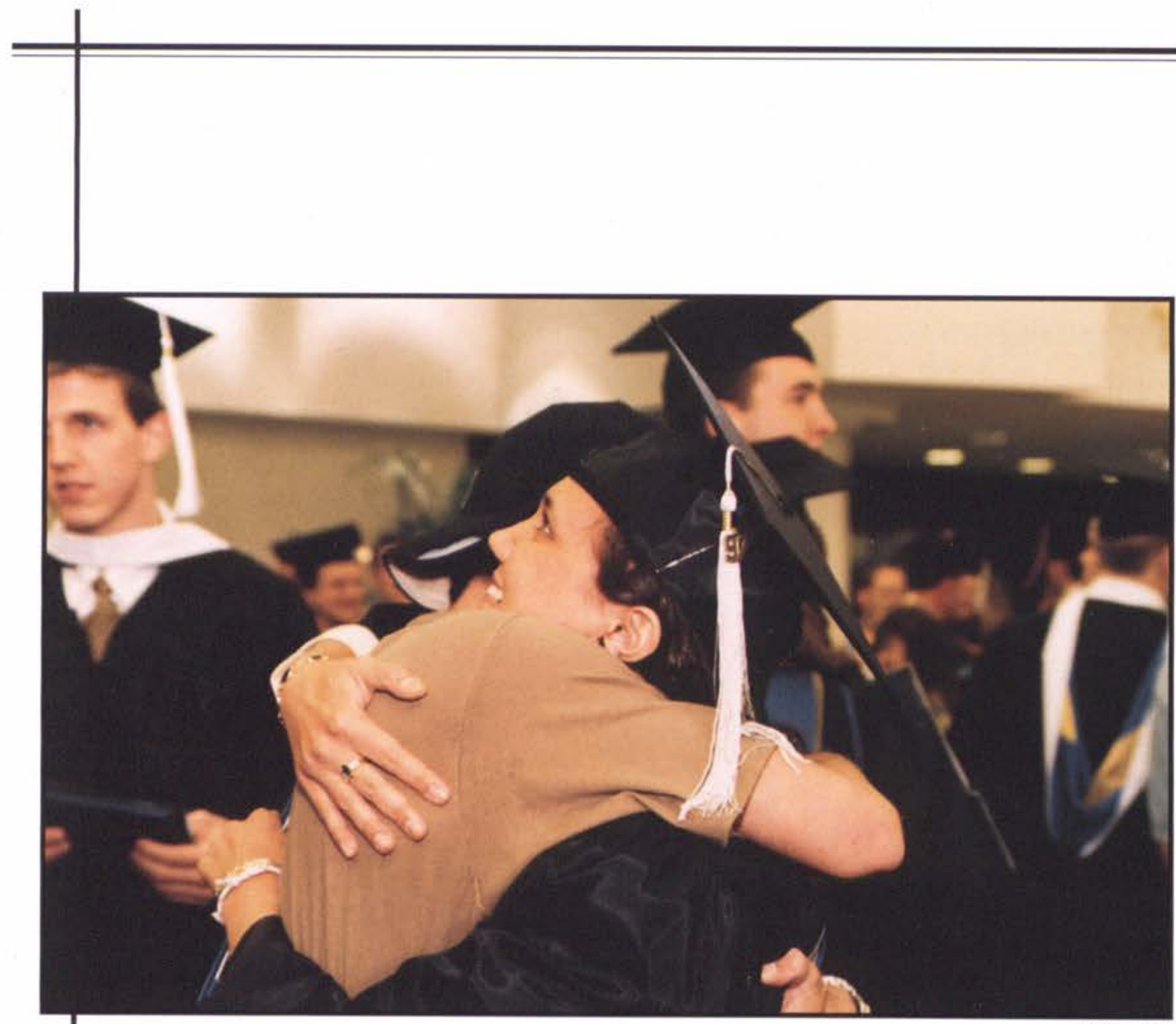

Above: Jennifer Reilly gets a hug after the ceremony. Many friends and family attended the ceremony which was held for the first time in the Dixon Ministry Center. Right: Lisa Van Wormer smiles proudly in her cap and gown.
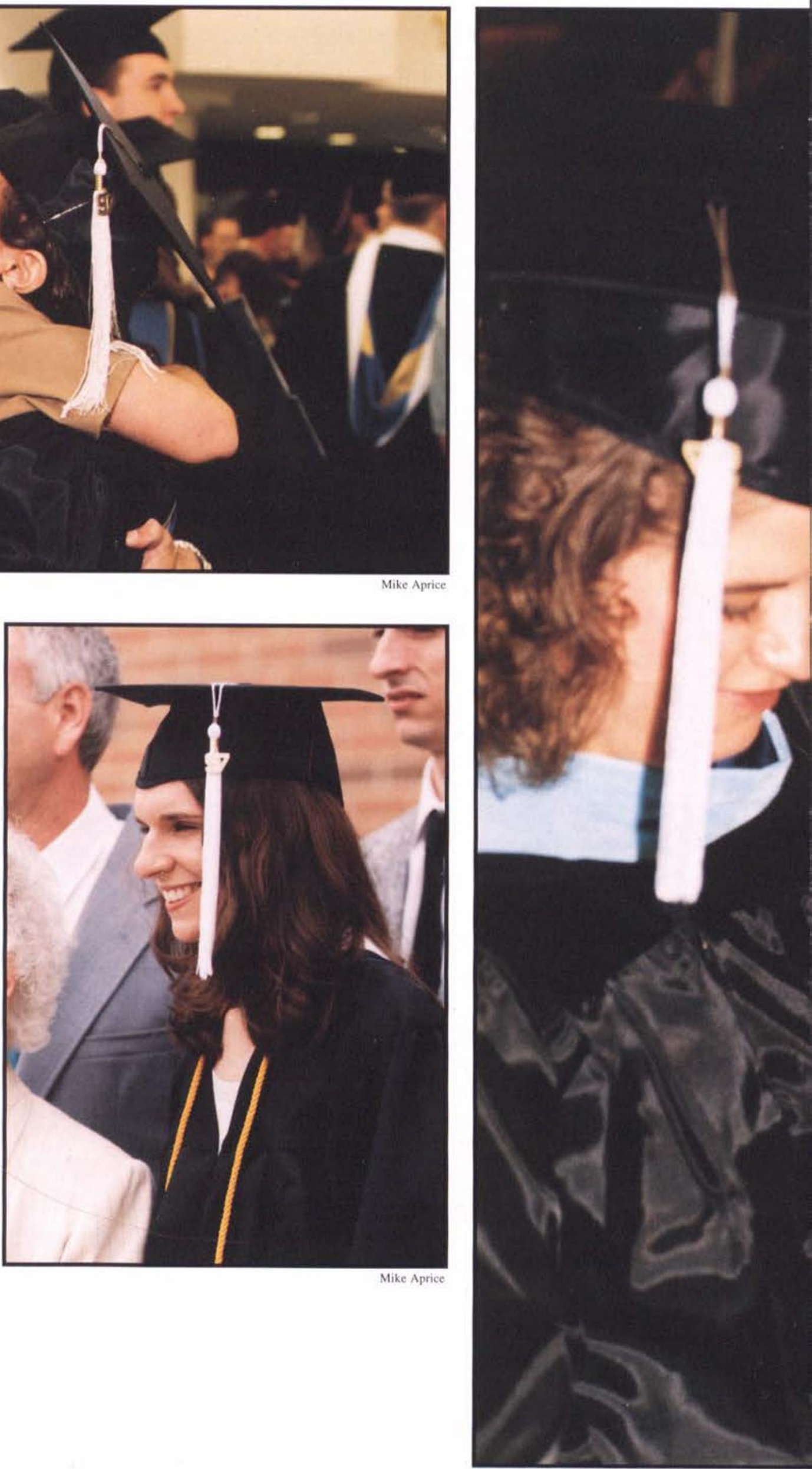


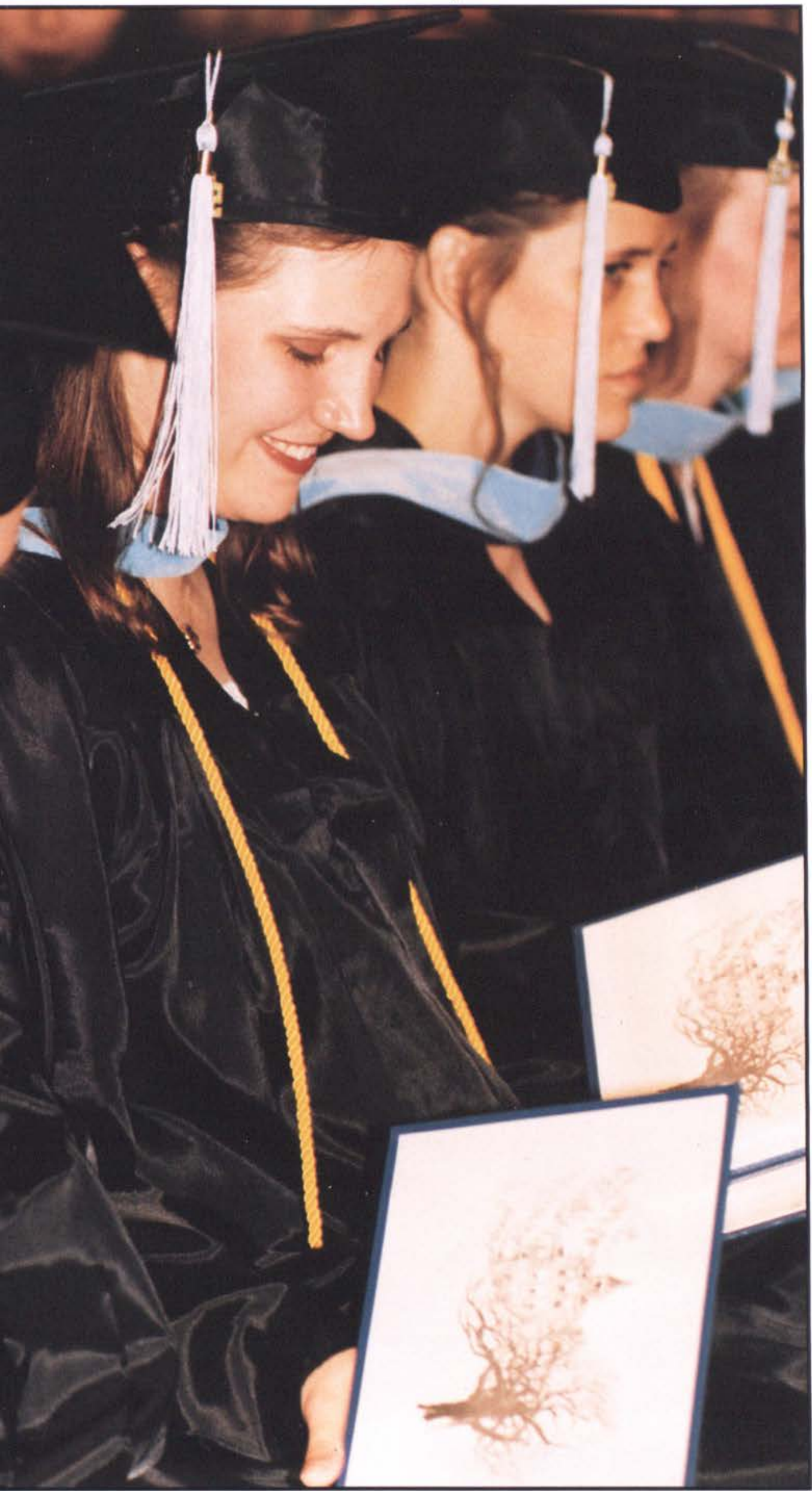

Left: The diploma, the proof of four years hard work. Seniors close this chapter and will soon start a new one. Below: After graduation Gordon Rogers heaves a sigh of relief.

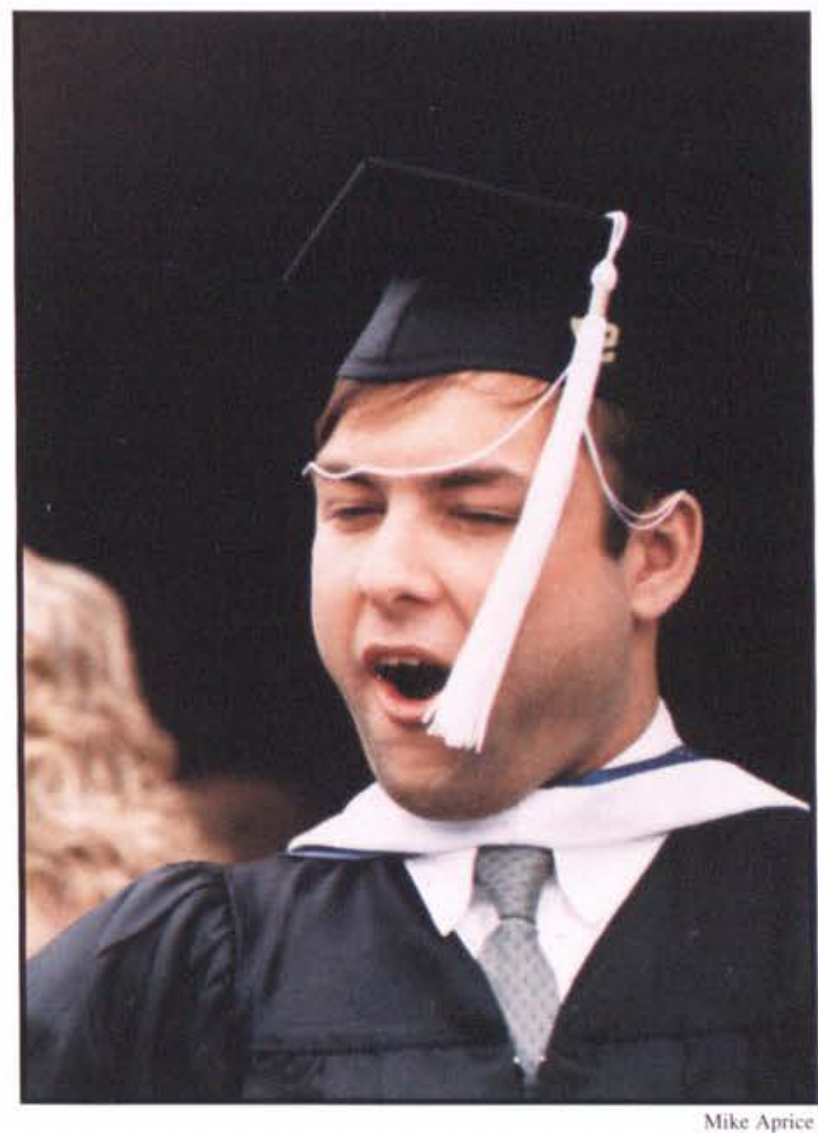



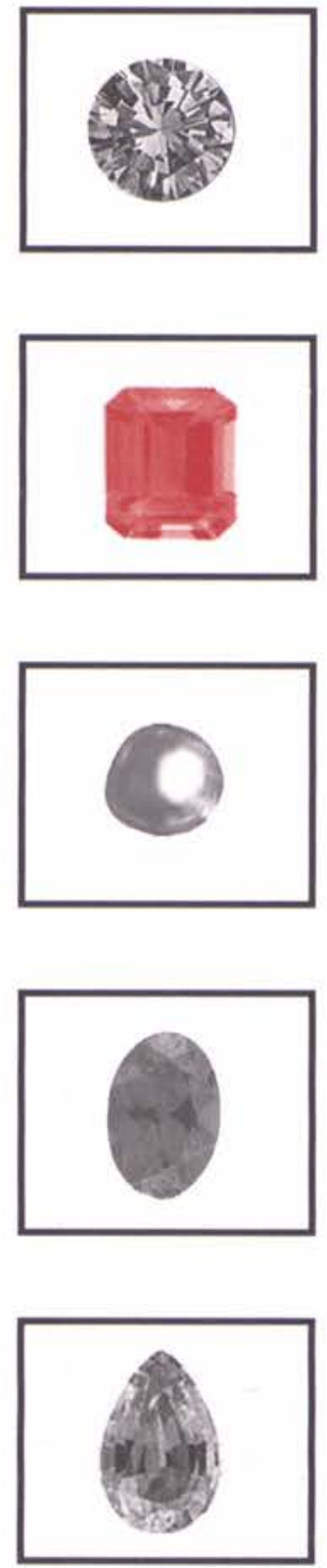

"Jhere is gold and multitude of rubies, Gut the lips of knowledge are a precious jew $\varepsilon$."

Proverfs

20:15

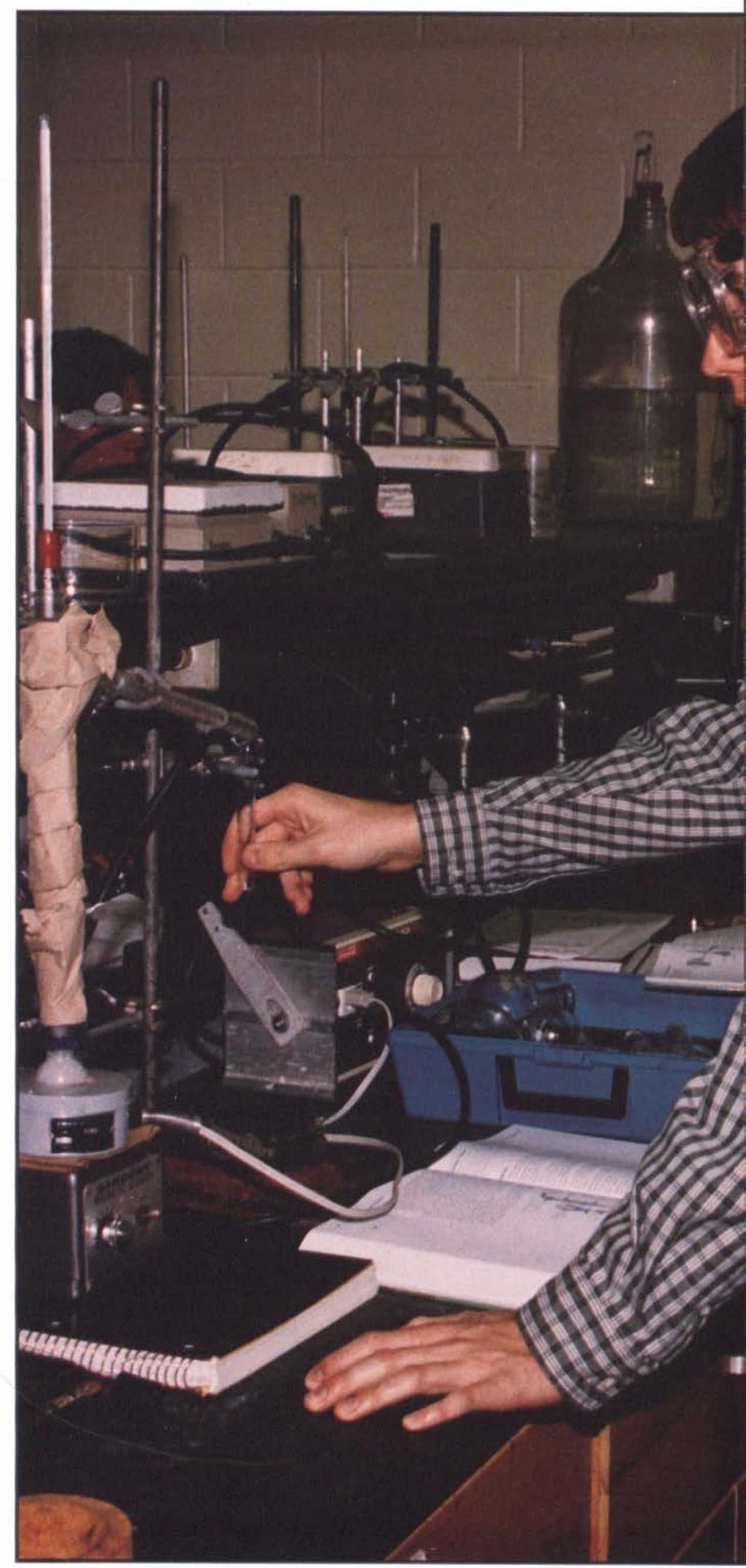




\section{Academics}

Without a doubt, academics pervades the lives of all college students. Some take it lightly while others take it more seriously, but every student must be involved in academics in order to be classified as a student.

Since academics are such a vital part of the college experience, Cedarville places a strong emphasis on this area. The North Central Association of Colleges and Schools, the accrediting agency for colleges in the Midwest, has recognized Cedarville's quality academics by granting Cedarville a $10-$ year renewal of accreditation. Fifty-one major fields of study are offered along with 31 minor fields of study, numbers which are growing at a steady pace with the addition of new majors such as physics and new minors such as art. Bible and mechanical engineering are the top majors for the men while elementary education and nursing lead the way for the women.

Excellent professors who are committed to Christ, their students, and their subject matter desire to see their students succeed both in and out of the classroom. It is such dedication that inspires students to do their best, an inspiration which leads to an education that helps students survive in the world today.

Bonnie Schaeffer 
Board of Jrusters
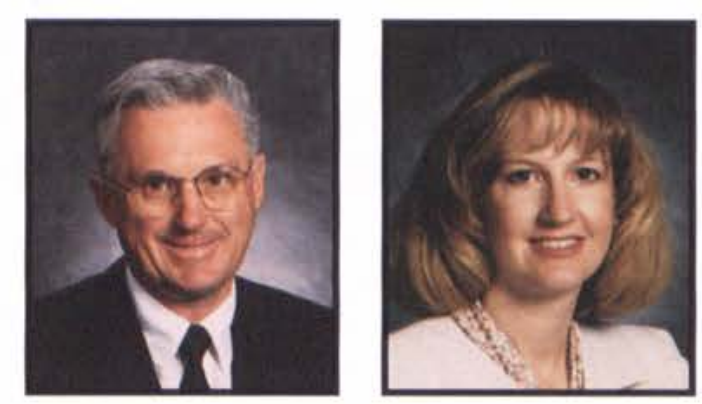

Mrs. Sherry Bouquet

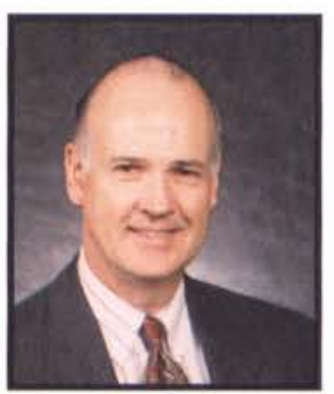

Dr. William Commons

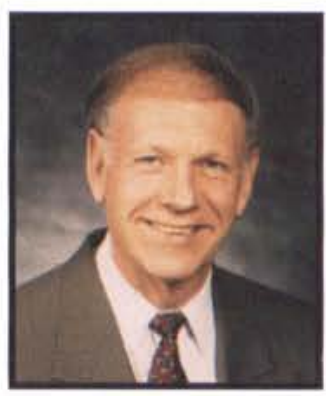

Rev. David Graham

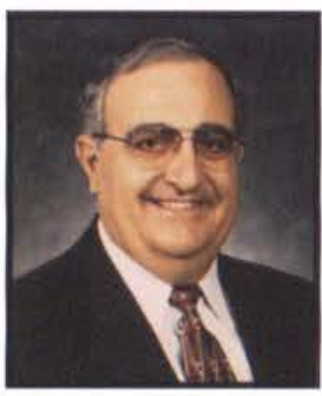

Dr. James Misirian

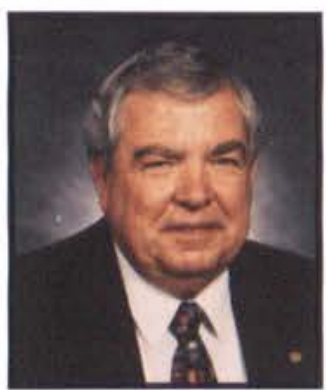

Mr. Bill Smith

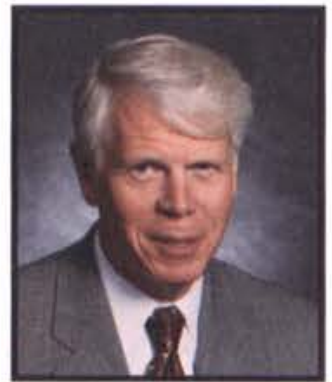

Mr. James DeVries

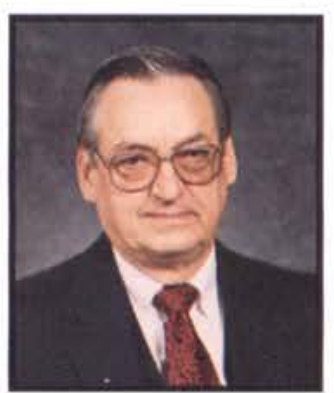

Mr. Roy Guenin

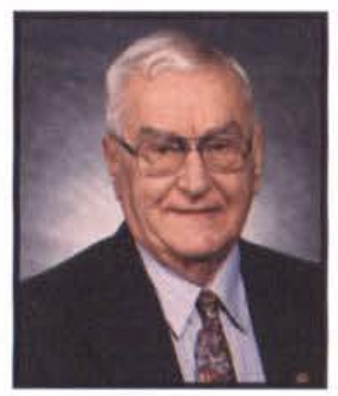

Mr. J. Dale Murphy

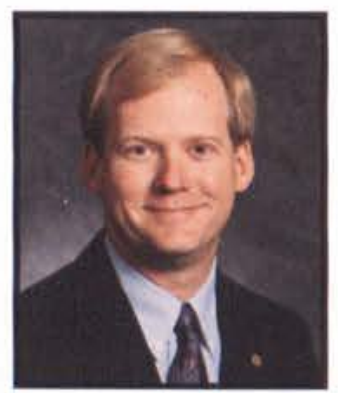

Rev. Bruce Sparks

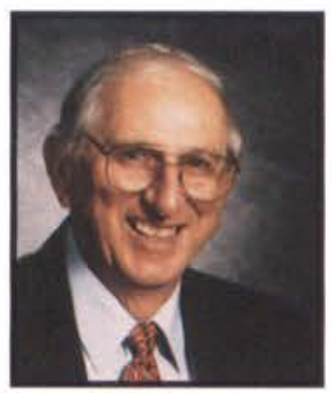

Mr. Francis Bresson

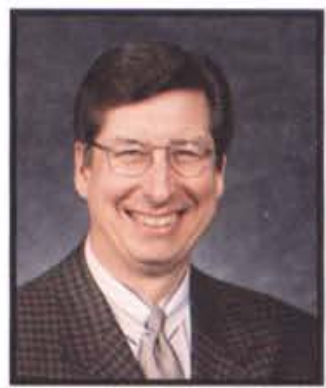

Mr. Daryl Doden

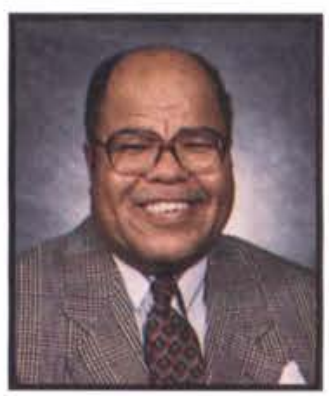

Rev, E. L. Hawkins

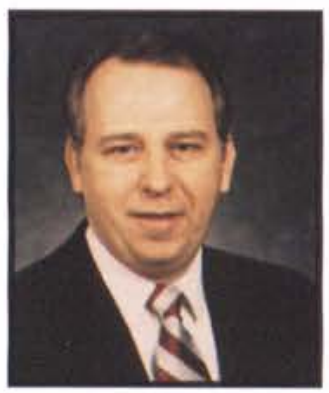

Rev. Randy Patten

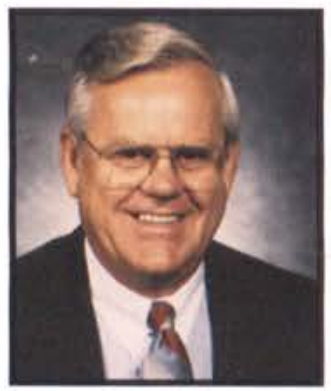

Mr. Albert Stevens

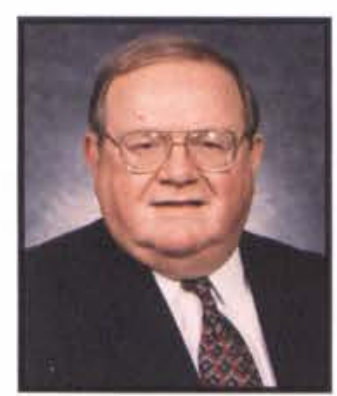

Dr. Eugene Apple

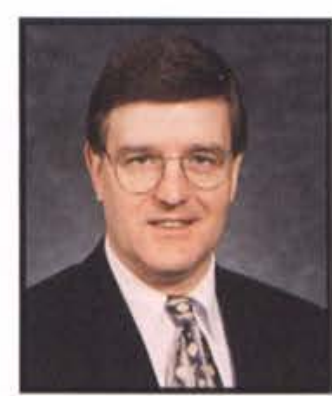

Rev, William Bernhard

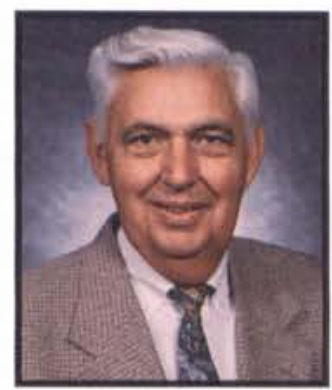

Mr. Gilbert Brueckner

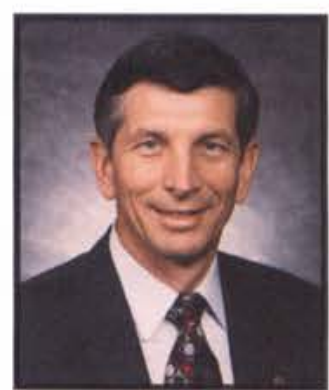

Mr. James Engelmann

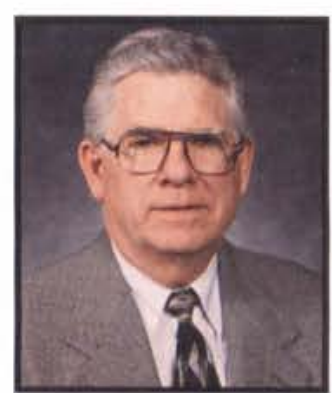

Dr. Jack Jacobs

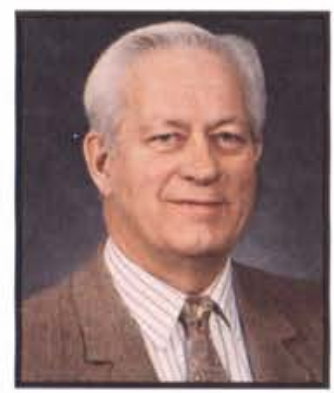

Rev. Lynn Rogers

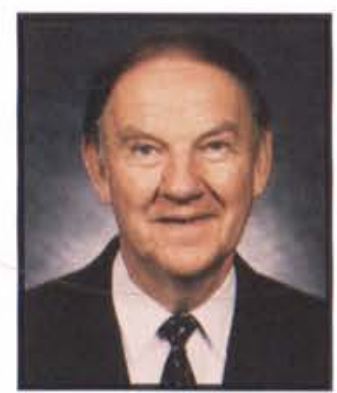

Dr. Donald Tyler

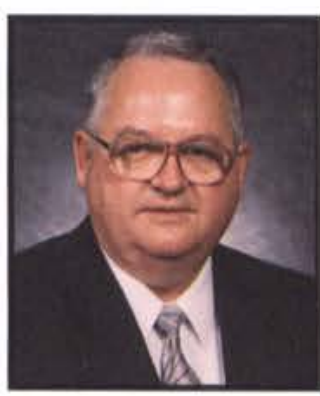

Mr. James Carraher

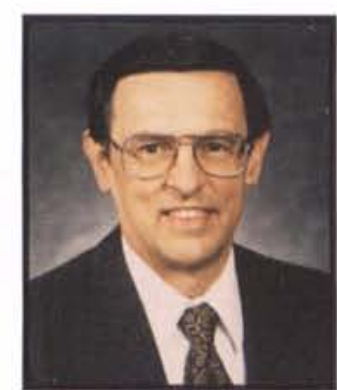

Rev. Lawrence Fetzer

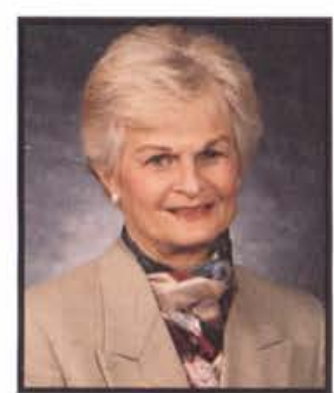

Mrs. Ruth Kempton

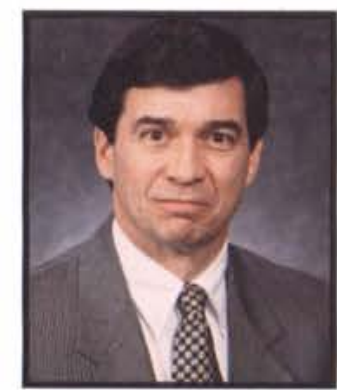

Dr. William Rudd

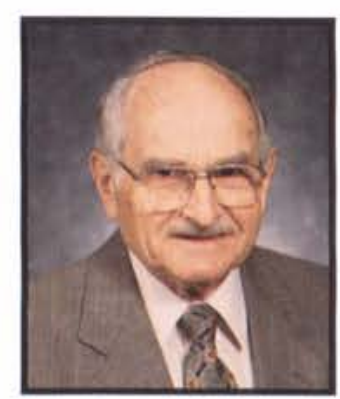

Dr. Paul Vernier

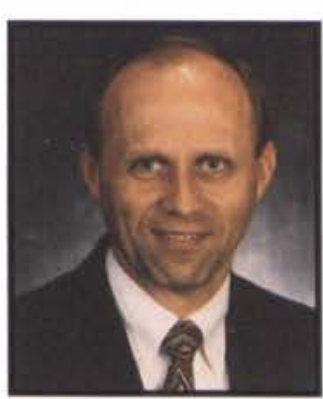

Dr. John Blodgett

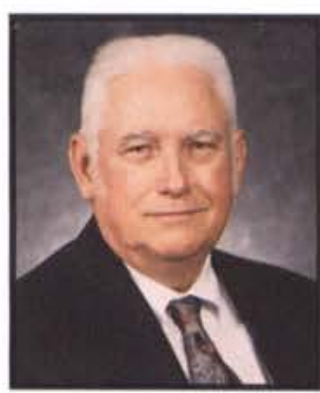

Dr. Jack Cline

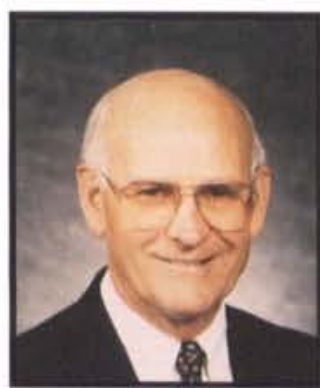

Rev. Joseph Godwin

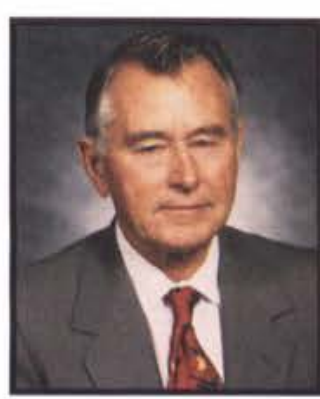

Mr. C. Eugene Miller

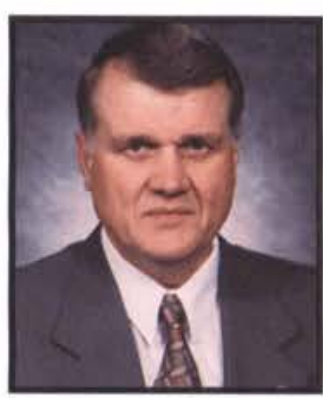

Mr. Lorne Scharnberg

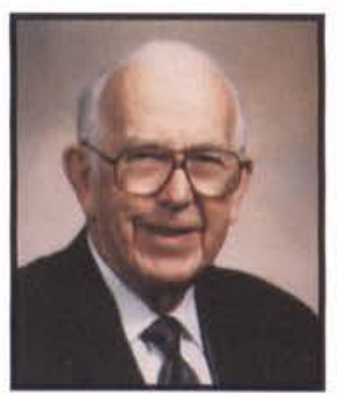

Dr. James Jeremiah Chancellor 


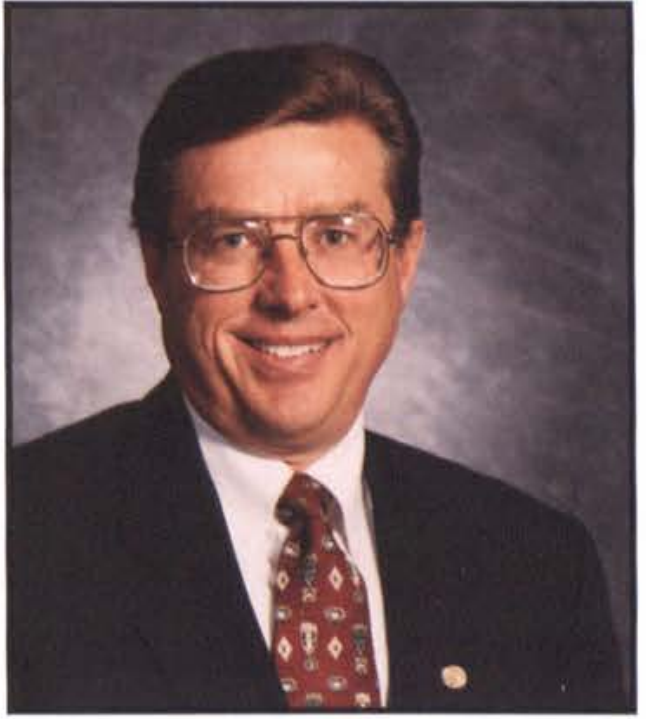

Mr. John Angela

Nier $\mathcal{P}_{\text {usident for }} \mathbb{B}_{\text {usines, }}$

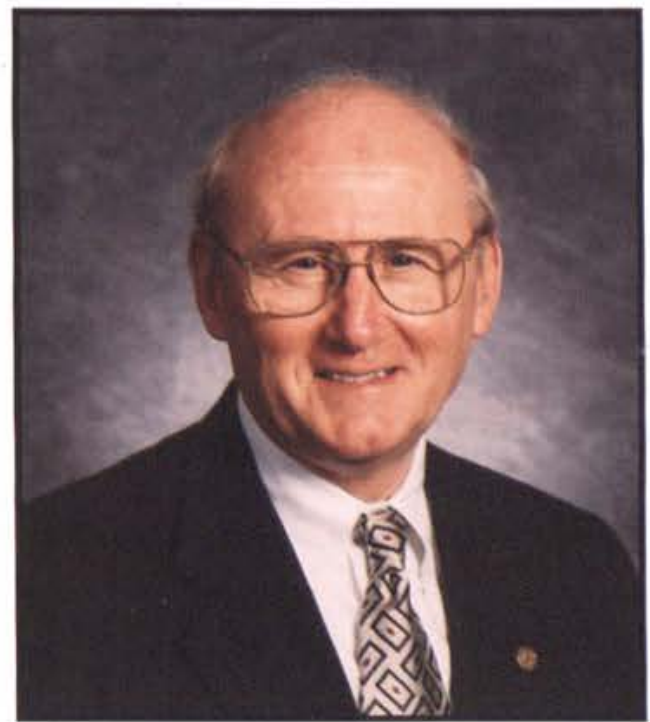

Mr. Donald Rickard Yiec President for Student Servioes

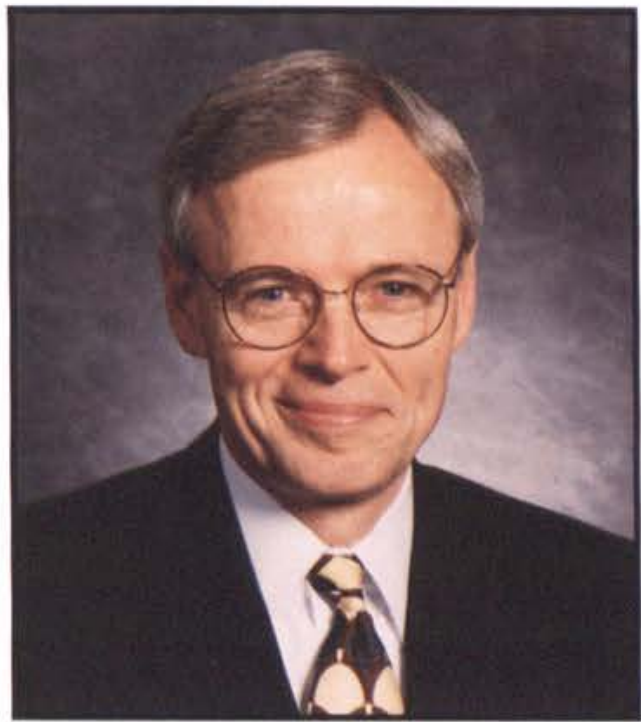

Mr. Robert Rohm Mier Prasident for Christian eMinistries

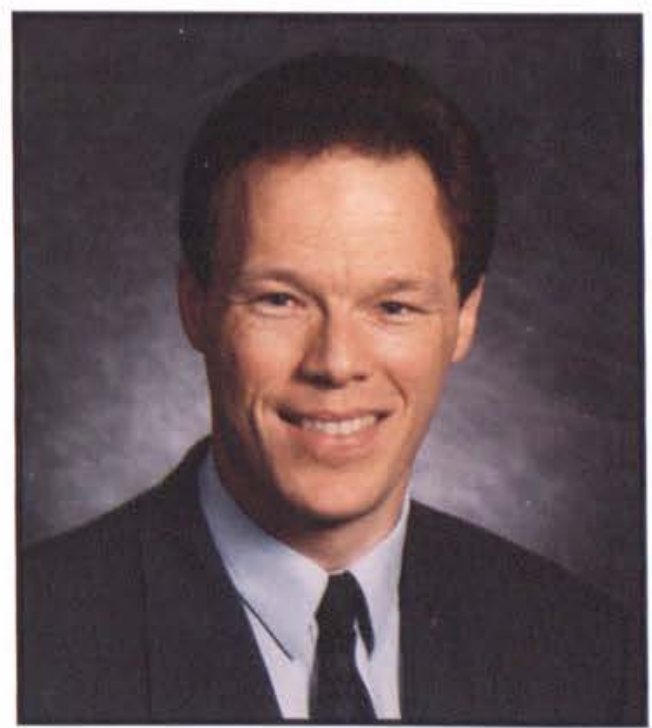

Mr. David Ormsbee

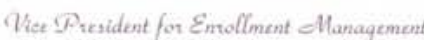




\section{Faculty}

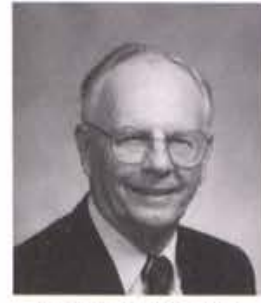

Cifford Johnson, D.EA.

Alustant to the Porrident
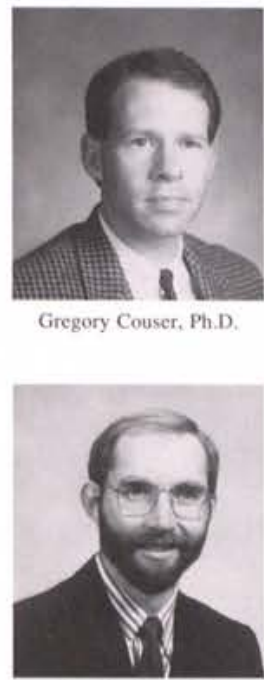

Chris Miller. Ph.D.

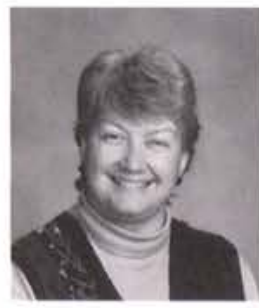

Martha Johnson. M.A.

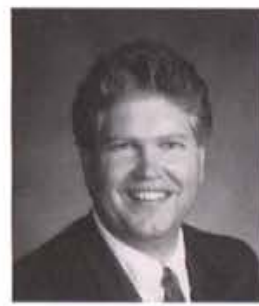

James Kragel, M.A.

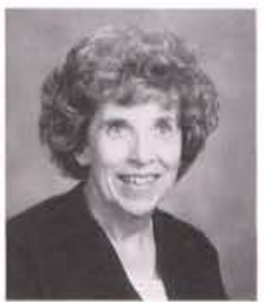

Sandra Entner, M.S.

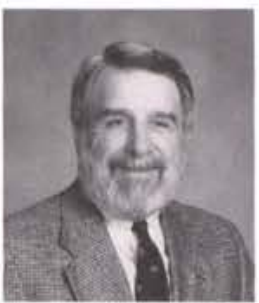

David Drullinger, D. Min.

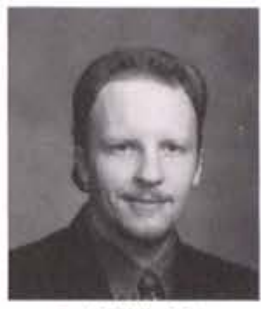

David Mills. M.A

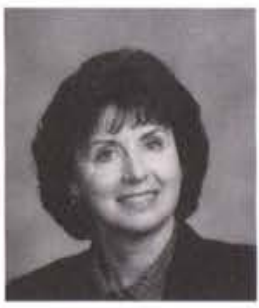

Betty Orme, M.S

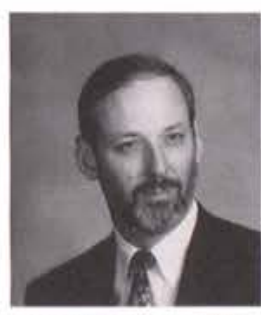

1. Michael Lopez, Ph. D.

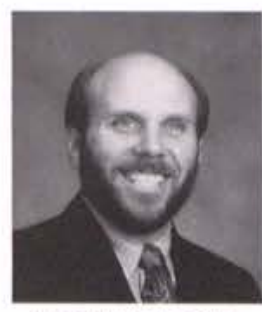

Jonathan Purple, M.Eu

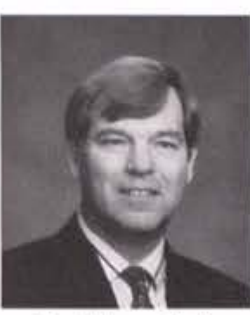

Floyd Flmore, Th.D.

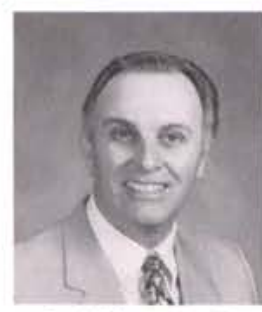

David Warren. Th.M.

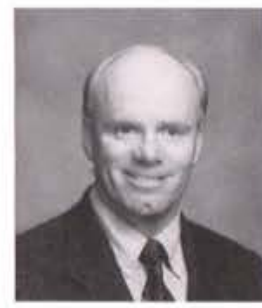

William Ragle, Ph.D.

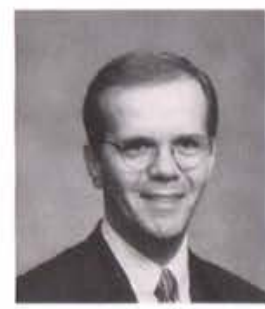

David Robey, Ph.D.

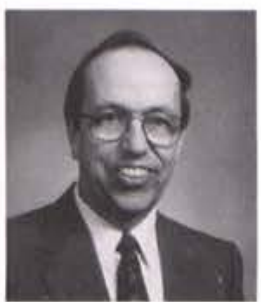

David Rotman, Ph.D

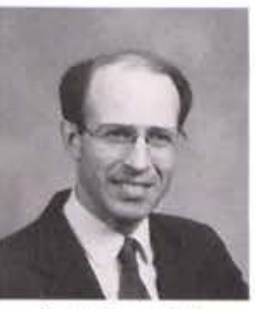

Daniel Estes, Ph.D.

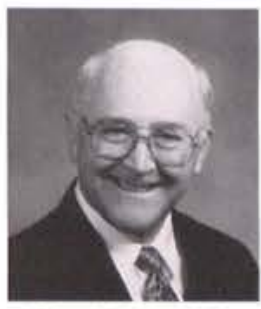

Richard Baldwin, $\mathrm{Ph} . \mathrm{D}$.

$$
\text { Rutimen Adminitation }
$$

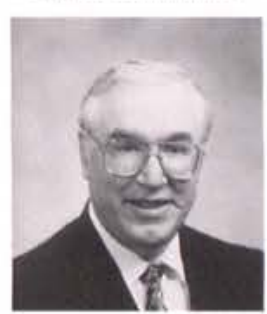

Galen Smith, M.S

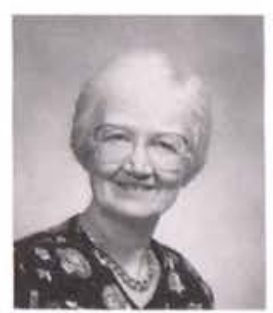

Sue Baker. D.Ed

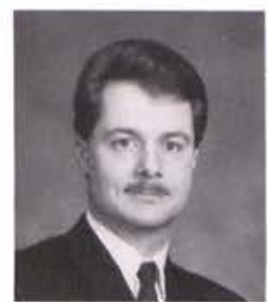

Carl Ruby, Ph.D

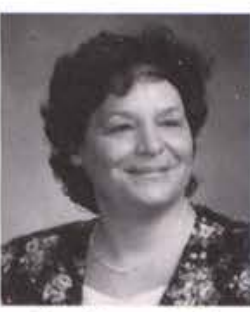

Cheryl Fawcett, Ed.D.

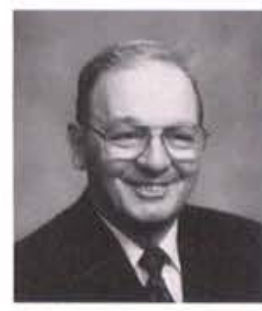

Clifford Fawcett, D.B.A.

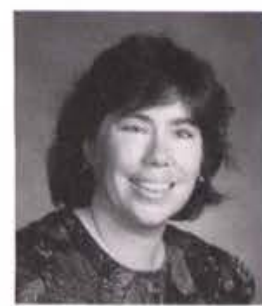

Sarah Smith, Ph.D.

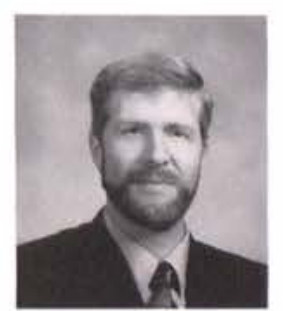

Philip Bassett, Ph.D

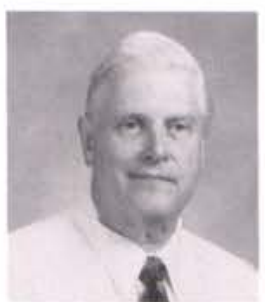

James Bjornstad, M.R.E.
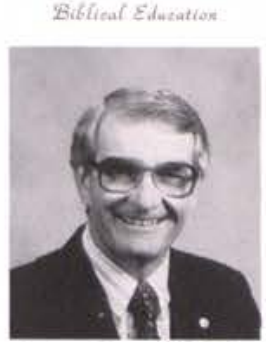

Robert Gromachi, Th.D.

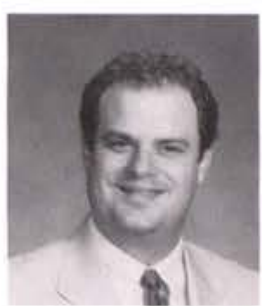

Jeffrey Fawcet, M. B A

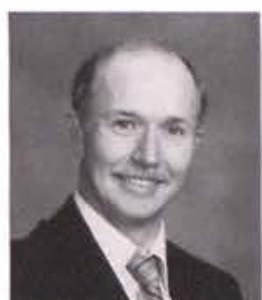

Wesley Sweetser. M.S

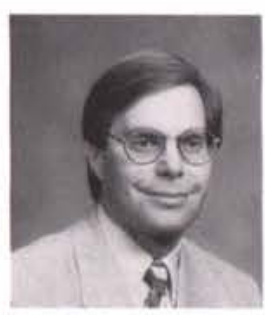

Eddie Baumann, $\mathrm{Ph}, \mathrm{D}$.

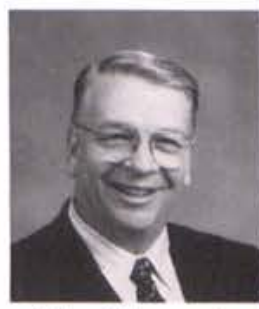

Richard Blumenstock.

D.Min.

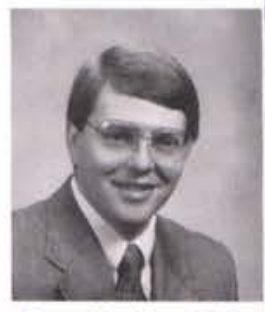

Thomas Hutehison, M.Div.

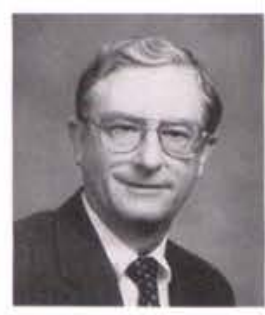

Marinus Hazen, M.B.A.

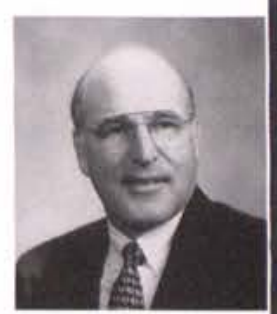

Ronald Walker, DBA.

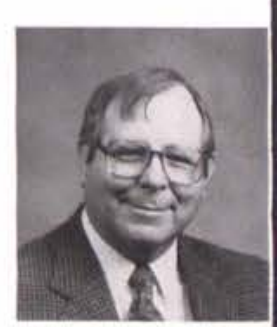

Omer Bonenberger, D.Ed.

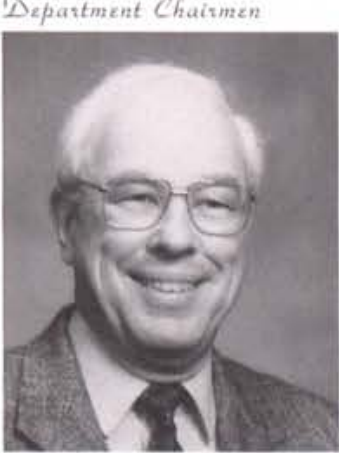

Jack Riggs, Th.D.

Biblical Education

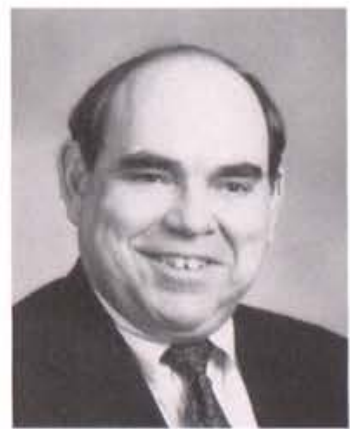

Sharon Johnson, D.B.A.

Rusincss Administration

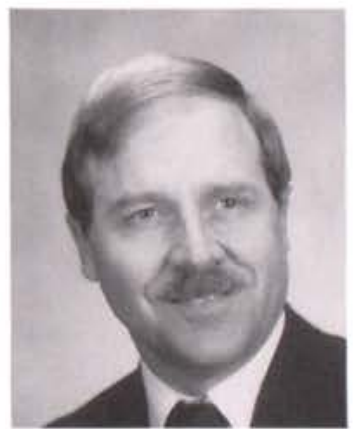

James Phipps, Ph.D.

Communieation -4.ts

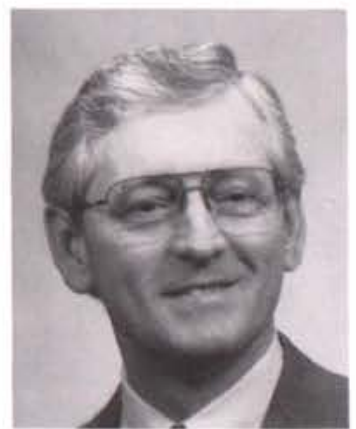

Merlin Ager, Ph.D.

Education

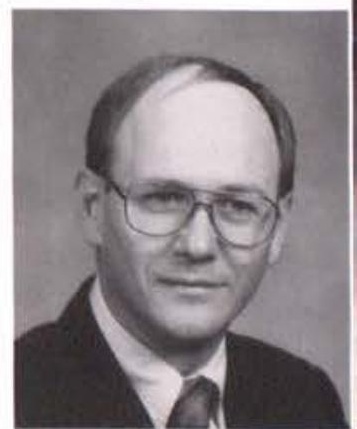

Lawrence Zavodney, Ph.D. 


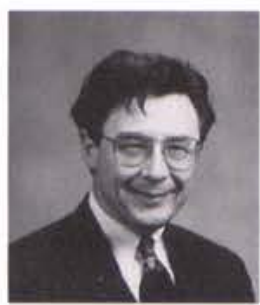

Vernon Whaley, Ph.D.

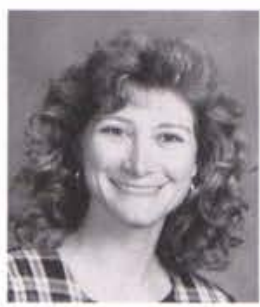

Teena Sanders, M.S.N.

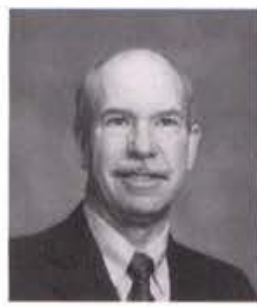

Larry Helmick. Ph.D.

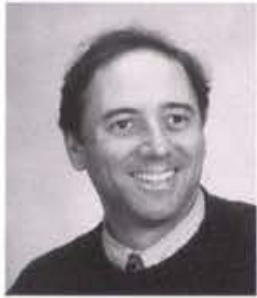

Kevin Roper, Ph.D.

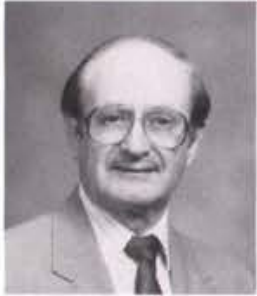

James McGoldrick, Ph.D.

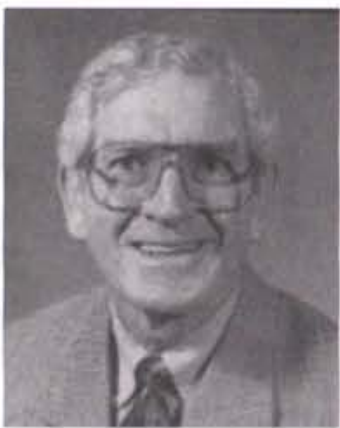

Stanley Ballard, Ph.D

$$
\text { Psychology }
$$

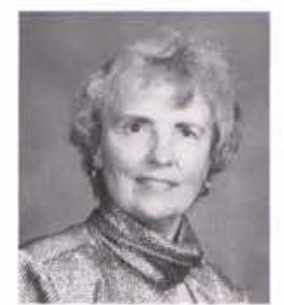

Carolyn Carlson, Ph.D

Datsing

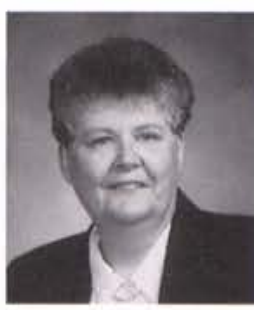

Judith Shrubsole, M.S

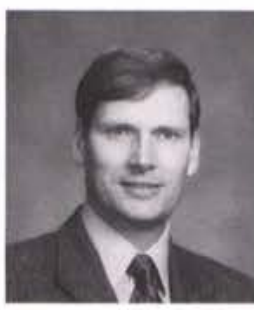

Steven Gollmer, Ph.D.

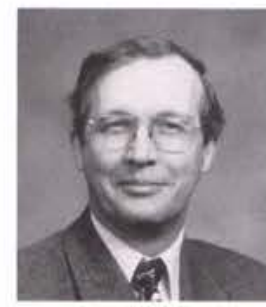

Robert Schumacher, M.S

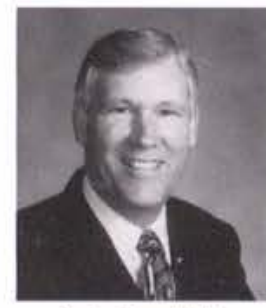

Robert Parr. Ph.D.

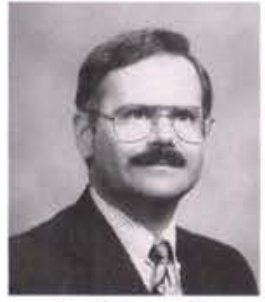

Kevin Sims, Ph.D

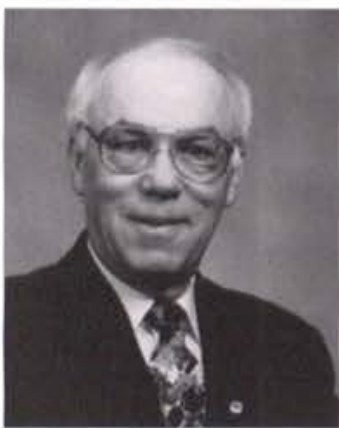

Daniel Wetzel, Ph.D.

Science and Mathrmaties

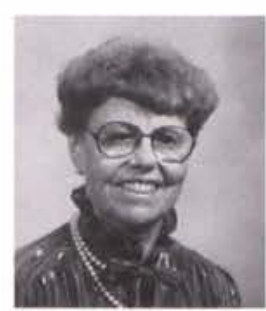

Margaret Ingalls. M.S.N.

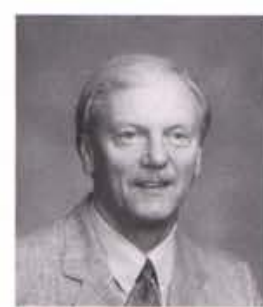

Robert Abbas, Ph.D.

$$
\text { ip sychofogy }
$$

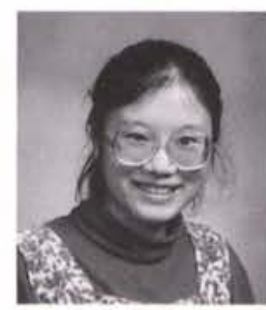

Ling L. Luo, M.D.

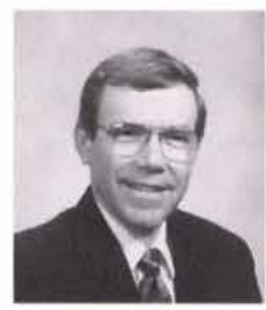

John Silvius, Ph.D.

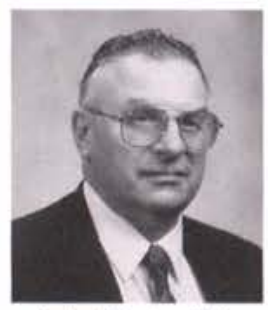

Roben Wiggins, Ph.D.

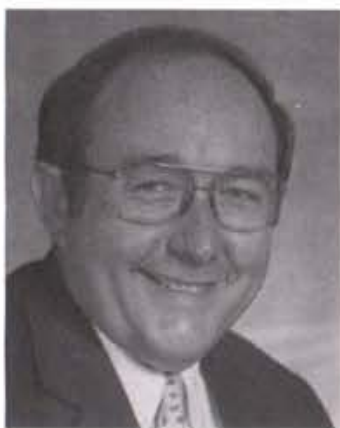

J. Murray Murdoch, Ph.D. Social Science and History

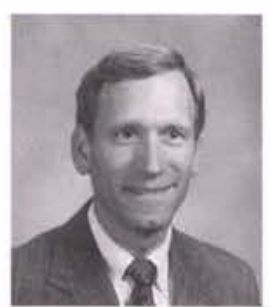

Mark Klimek, M.S.N.

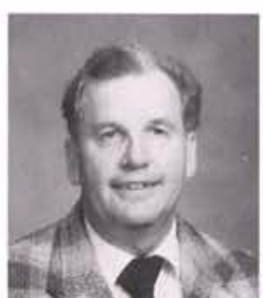

Edwin Braithwaite, Ph.D. Saienar and ellathamation

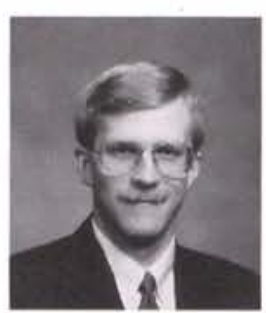

Mark McClain, Ph. D

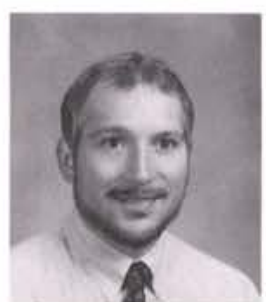

John Whitmore, M.s.

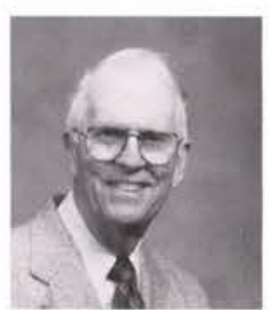

Richard Durham, Th.D.

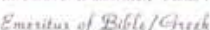

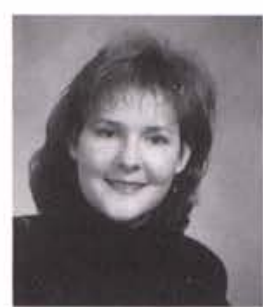

Sandra Pratt, M.S

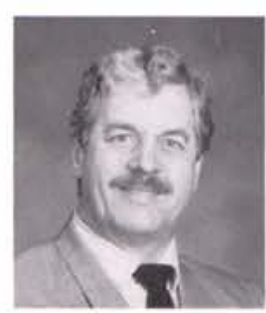

Leroy Eimers, Ph. D.

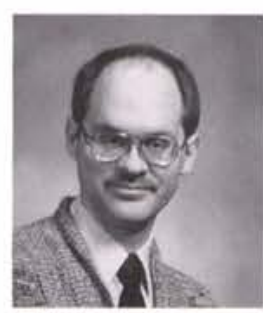

Douglas Miller, Ph.D

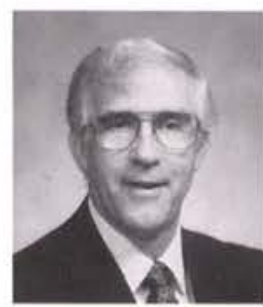

Joseph Halsey, Ph.D. Social Sesener and Histeny

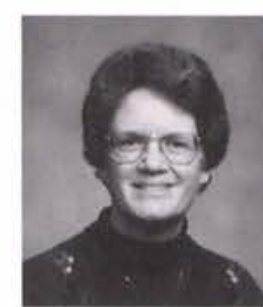

Sharon Rahilly, Ph.D.

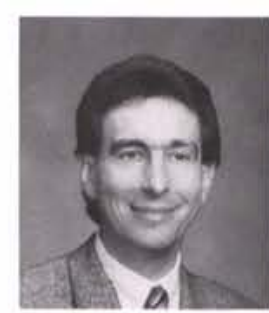

Dennis Flentge, Ph.D.

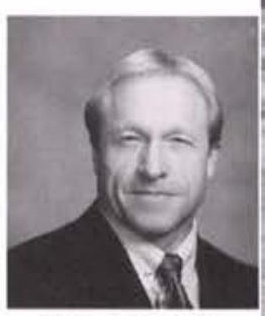

Terry Phipps, Ph.D

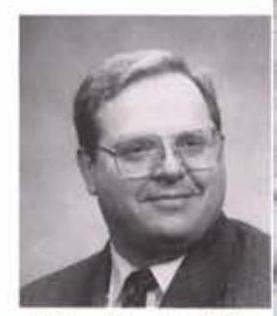

Nelson Henning, Ph.D. 


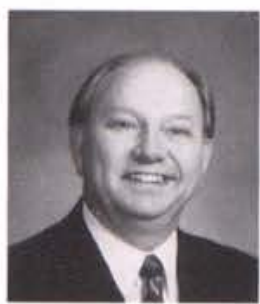

Paul Gathany

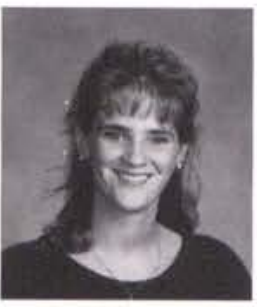

Kristy Griffith

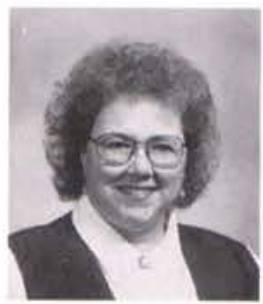

Carolyn Hodson

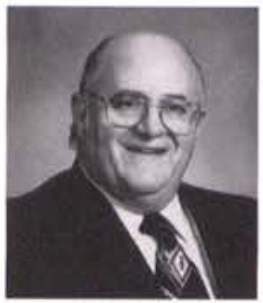

Leigh Hunt

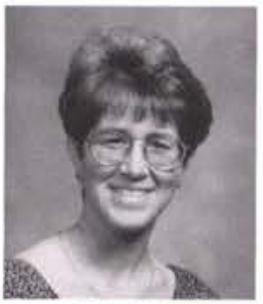

Elaine Keller

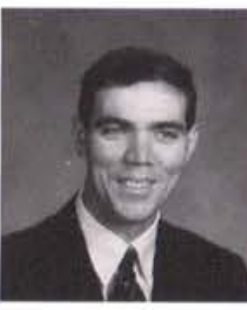

Chad Larr

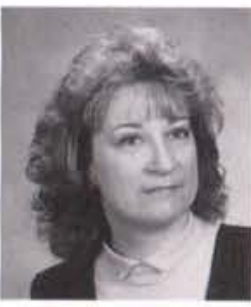

Debra Mc Donalu
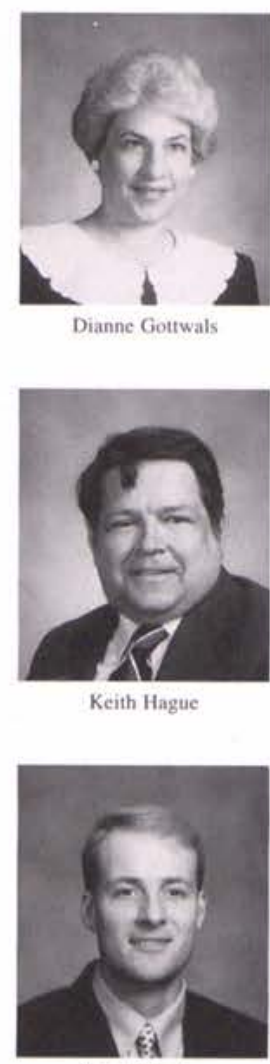

David Hoecke

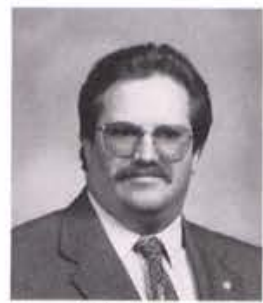

Robert Hunt

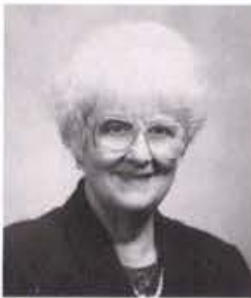

Joanne Kendall

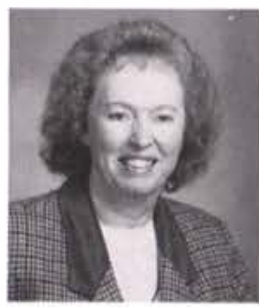

Patricia Lee

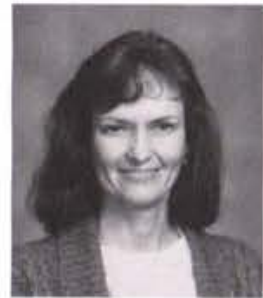

Mishell McIntosh

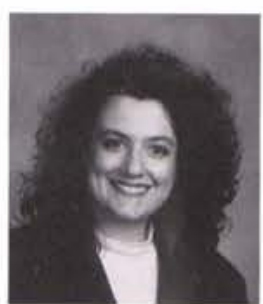

Anna Mari Green

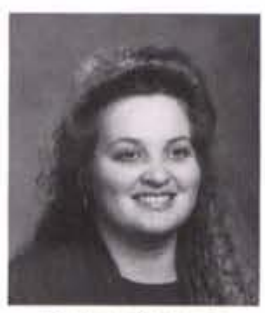

Stephanie Halverson

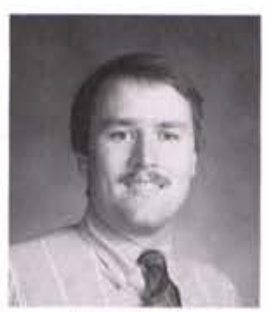

Scot Howder

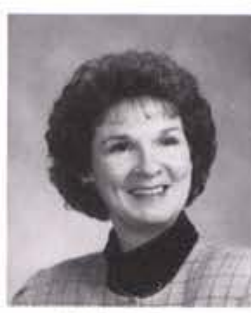

Shirley Johnson

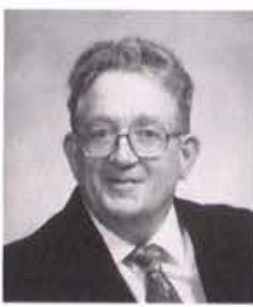

Glenn Knauff

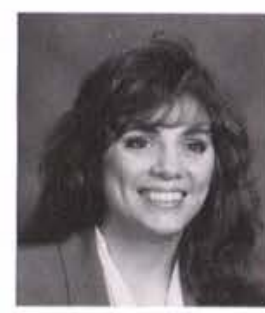

Kimberly Longe

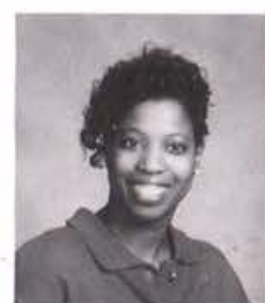

Kezia Mc Neal

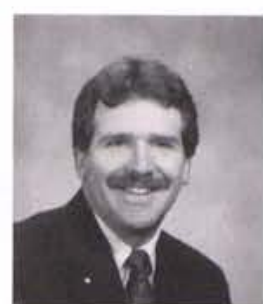

Philip Grafton

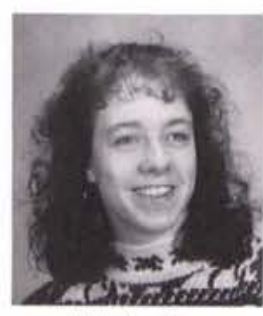

Ruth Hamiltot

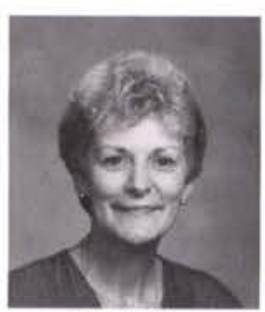

Patricia Huber

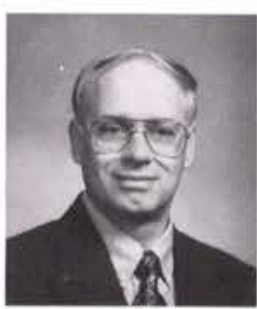

Timothy Johnson

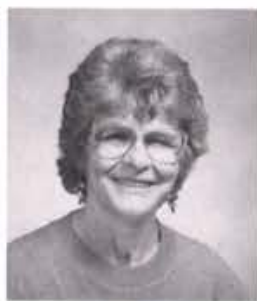

Nancy Knauff

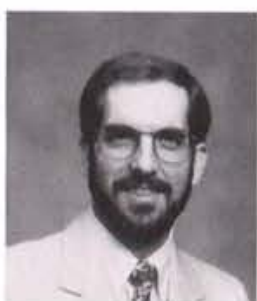

Mark Mathews

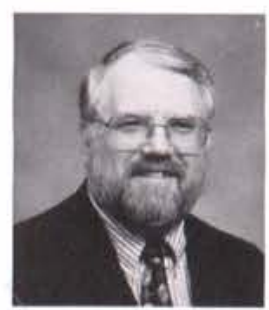

Fred Merritt

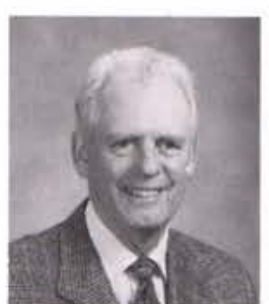

Harold Green
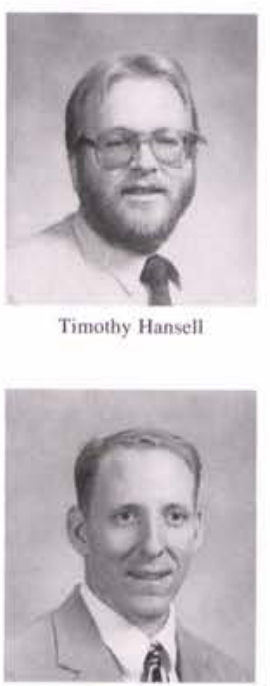

Scott Huck

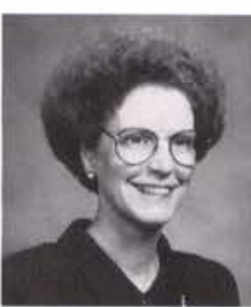

Murtha Kaercher

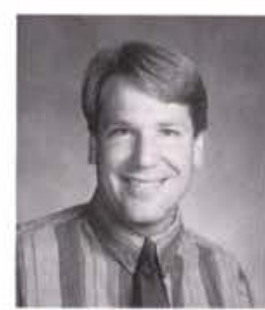

Mark Kordic

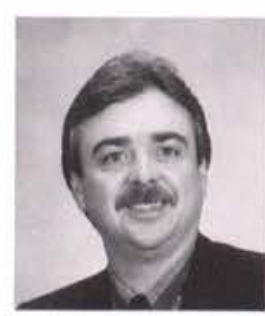

Wayne Maxie

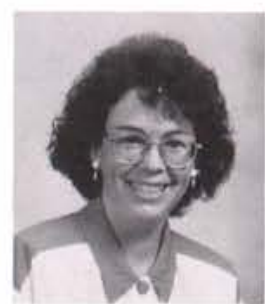

Cheryl Miller
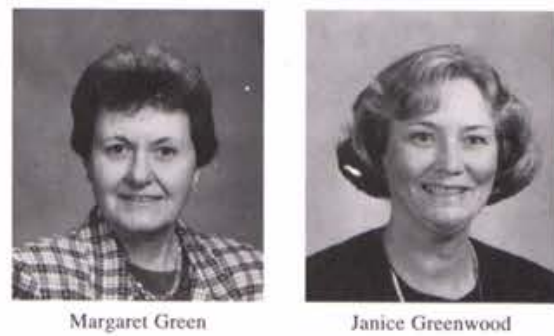

Janice Greenwood

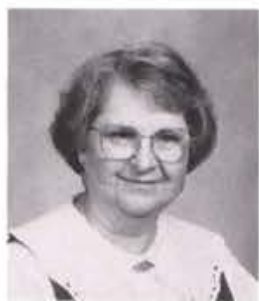

Barbara Helmuth

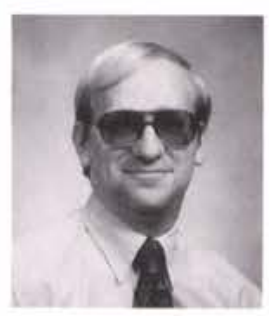

Gary Hull

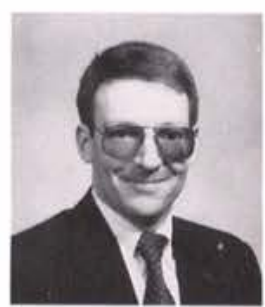

Davis Kammeyes

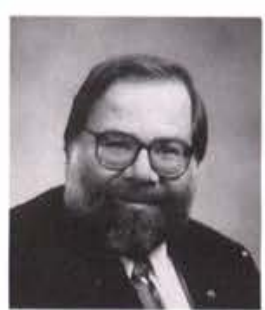

Gregory Kunz

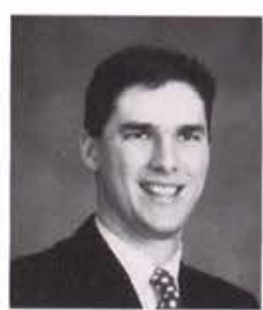

Mark Mazelin

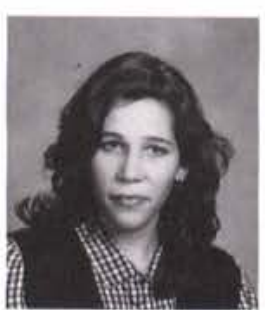

Tiffany Neison

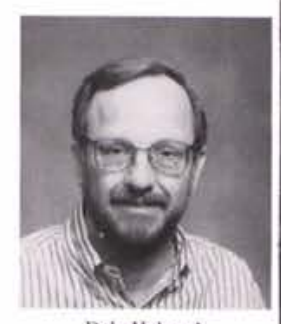

Dale Helmuth

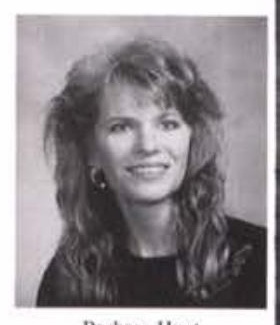

Barbara Hunt

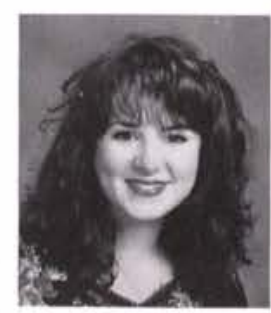

Lori Keim

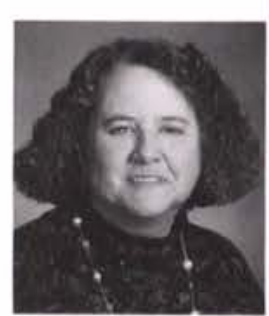

Corime Lakes

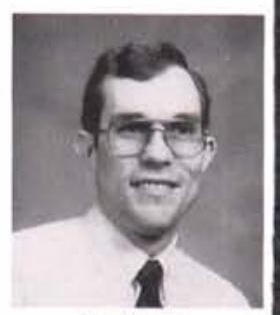

Alan Mc Cain

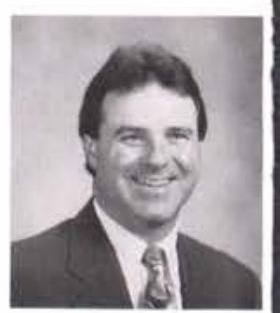

Brian Nester 


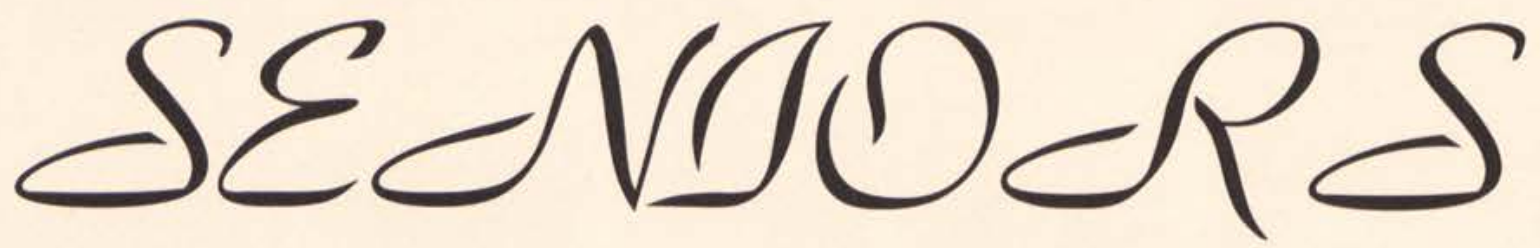

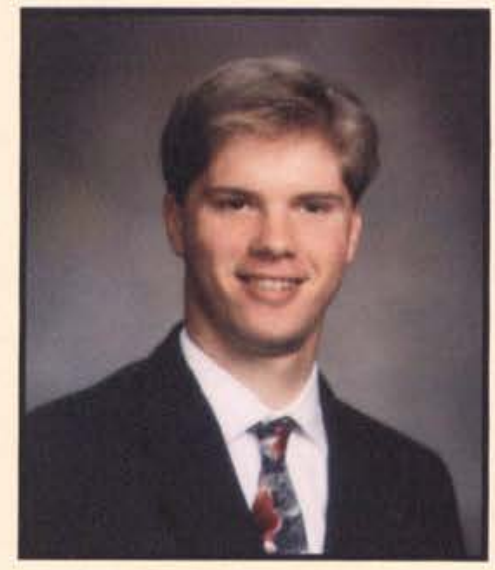

Andrew J. Alderfer

Criminal Justice

Whippany, NJ

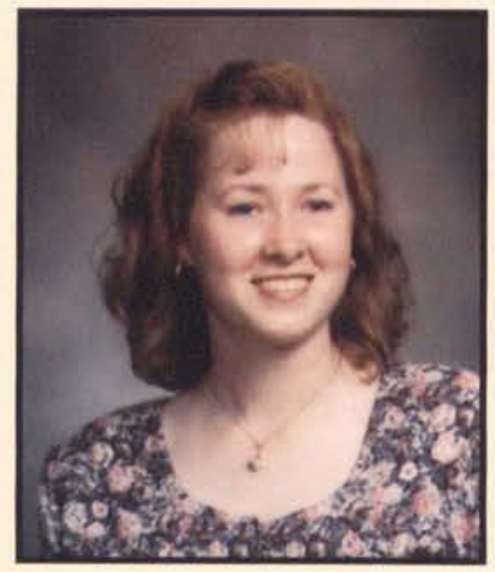

Beth Amos

English

Lancaster, $\mathrm{OH}$

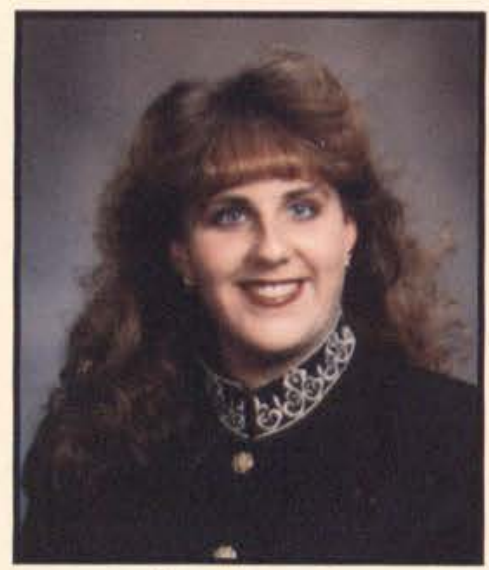

Lori Lyn Anderson

Elementary Education

Cedarville, $\mathrm{OH}$

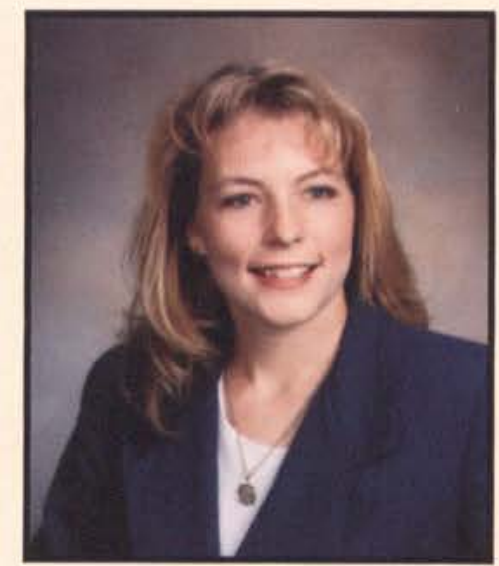

Heather L. Alexander

English Education

Norwalk, $\mathrm{OH}$

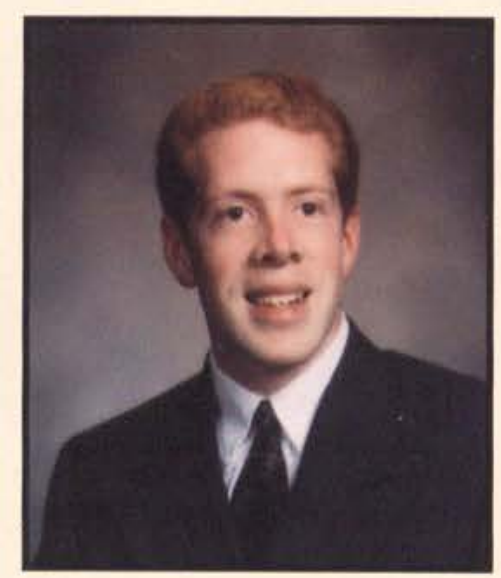

Douglas M. Amundson Finance

Evans City, PA

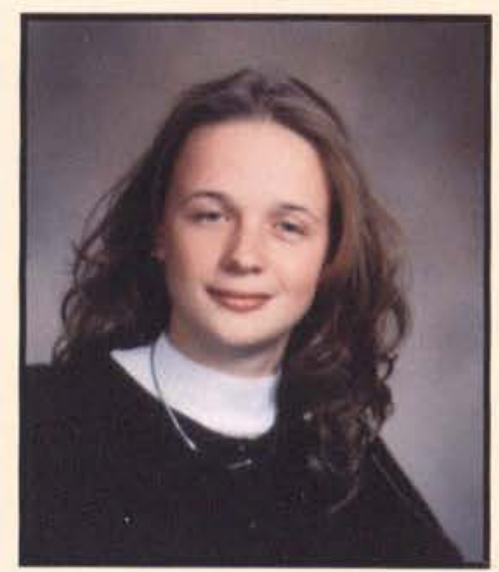

Adria Andrews

Elementary Education

Watsontown, PA

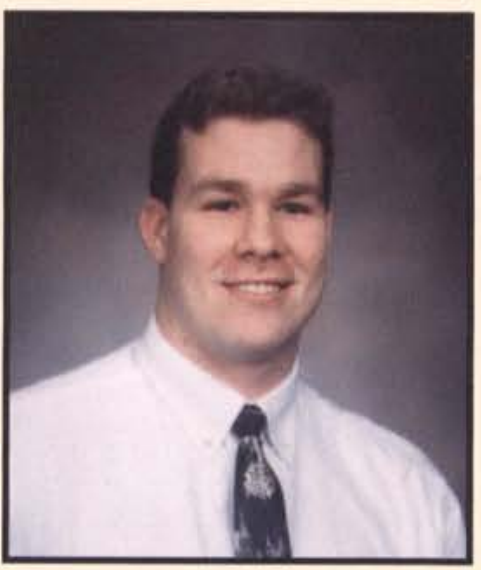

Barton Allen

International Studies/Missiology Chittagong, Bangladesh

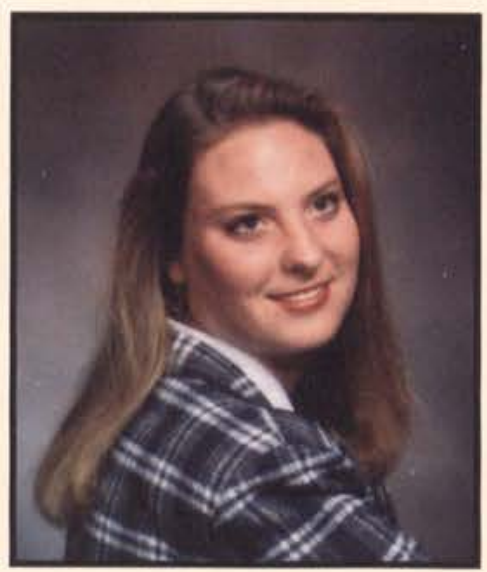

Amy Anderson

Organizational Communications Lone Tree, IA

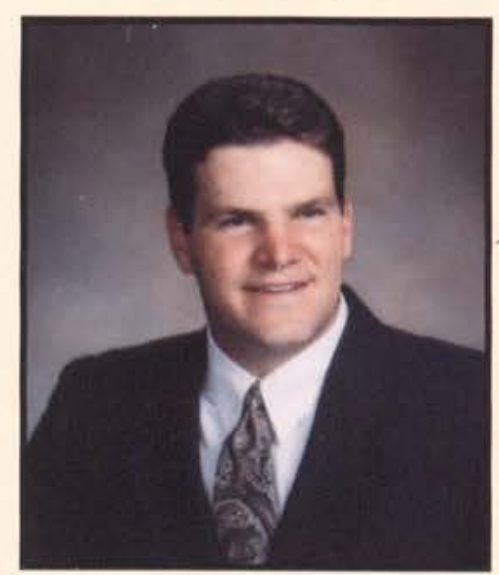

Erik Anglund

Mechanical Engineering

Longmont, $\mathrm{CO}$

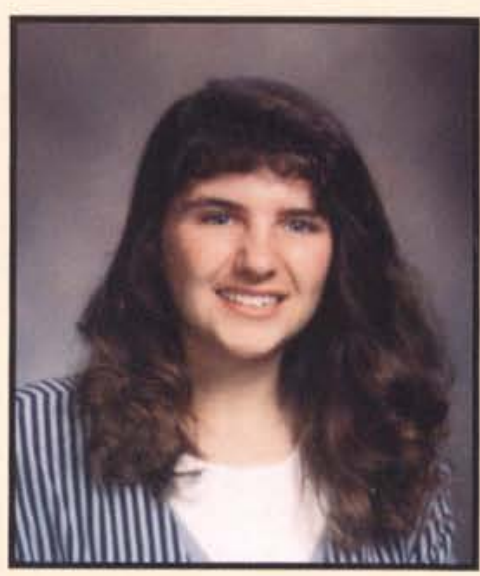

Kerry M. Allen

Human Resource Management Yarmouth, ME

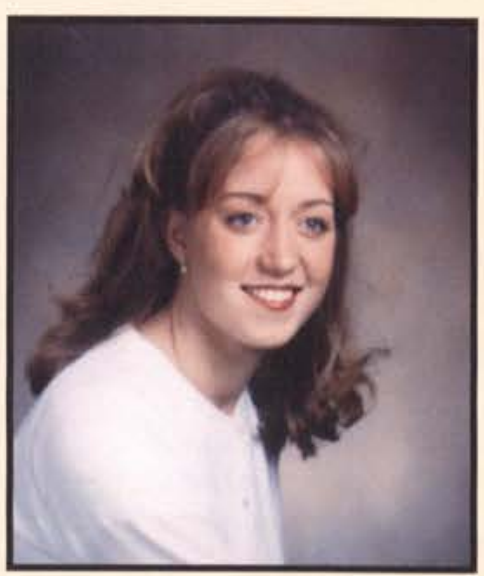

Angie Anderson Professional Writing Cumberland, MD

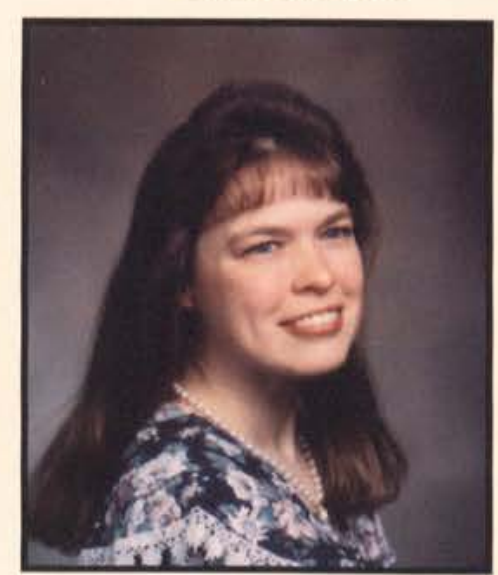

Darla Lynn Anthony Elementary Education Downingtown, PA 


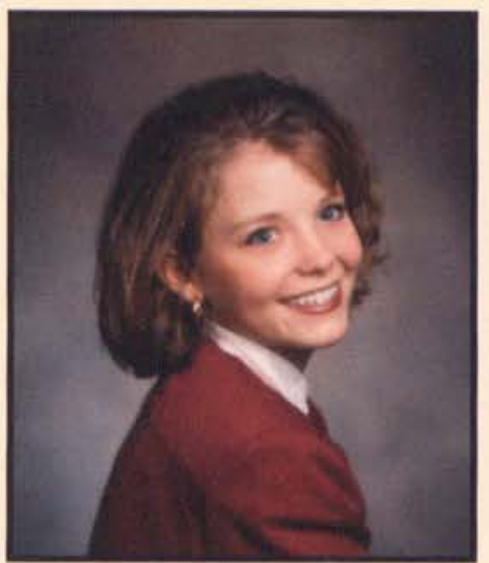

Virginia Barrett

Computer Information Systems Xenia, $\mathrm{OH}$

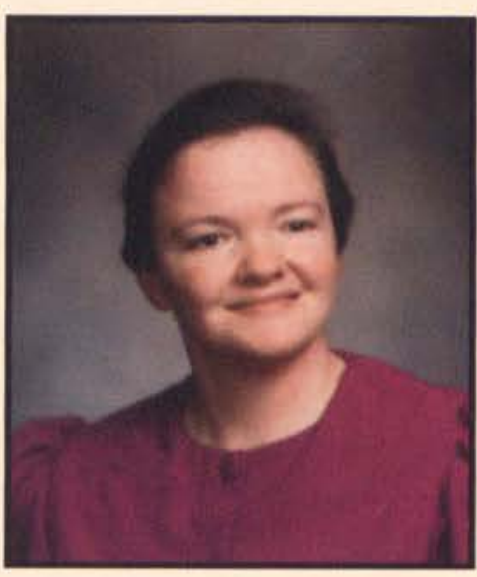

Susan Beachy

Elementary Education

Plain City, $\mathrm{OH}$

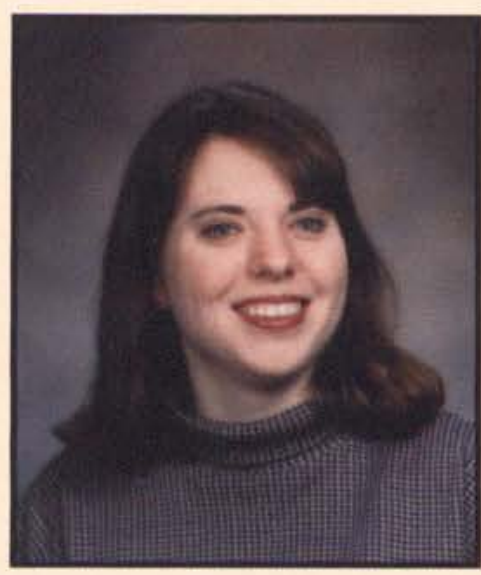

Rachel Beecher

English Education

Fishers, IN

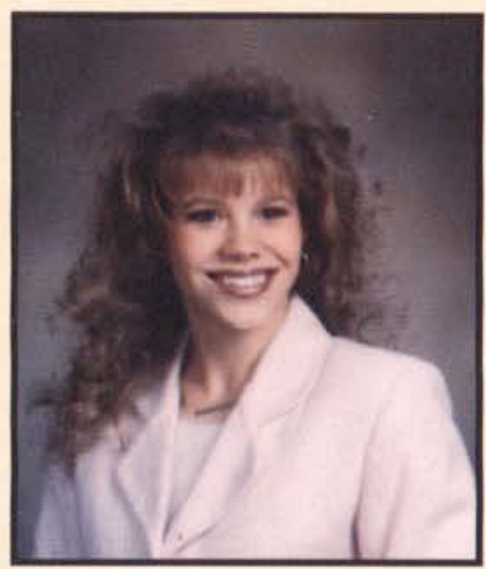

Jennifer Beres

Applied Psychology

Tipp City, $\mathrm{OH}$

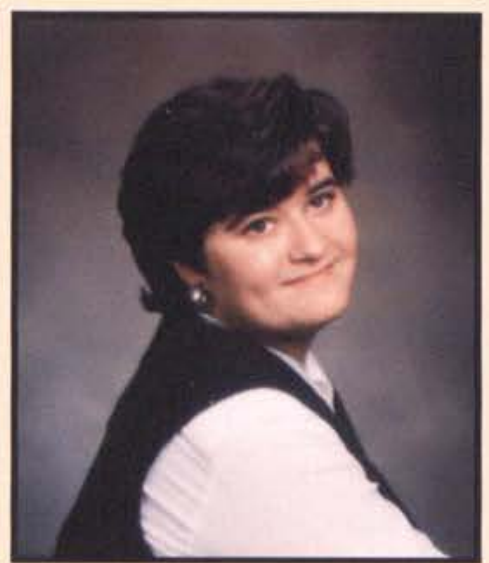

Rachel Batchelder Nursing

Alburg Springs, VT

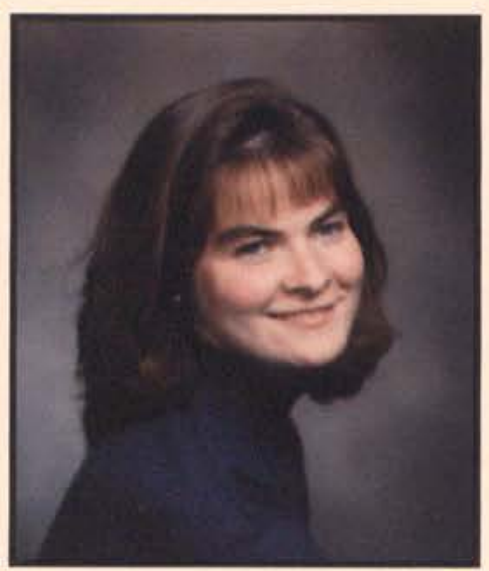

Mary Beth Beal

Math Education

Londonderry, NH

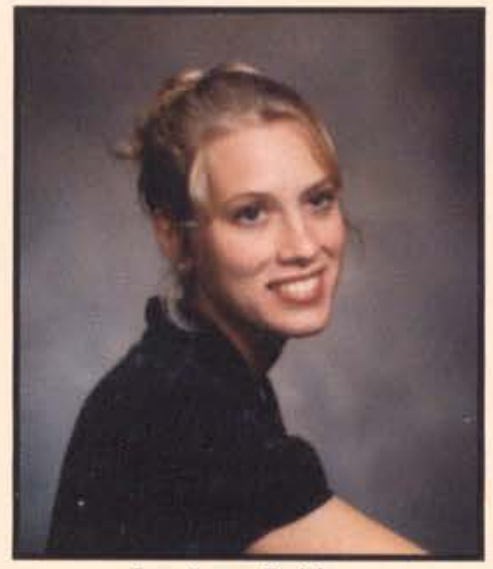

Joy Anne Beitler

Bible Comprehensive

Communication Arts

Forest, $\mathrm{OH}$

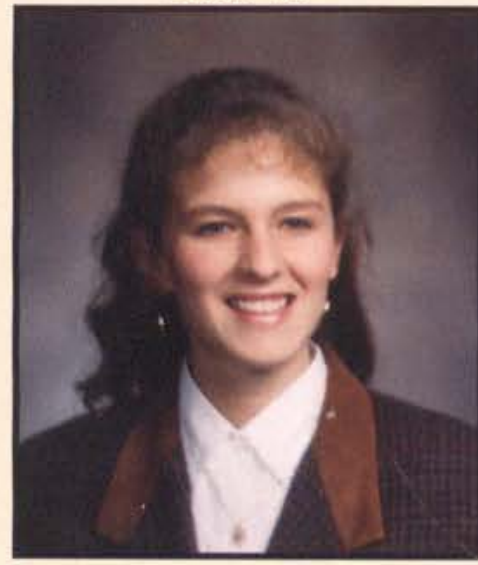

Heather Bethel

Elementary Education

West Buxton, ME

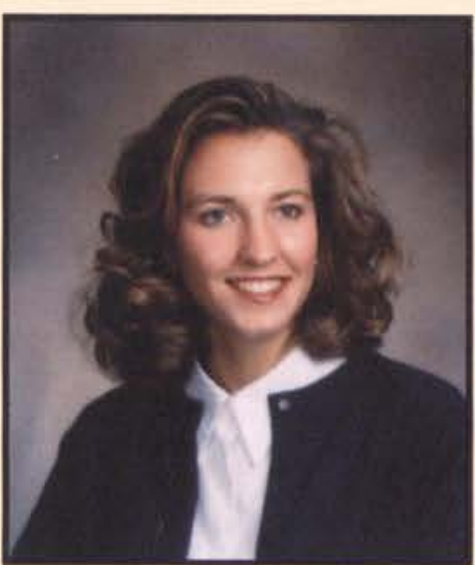

Amy Joy Bathrick

Elementary Education

Cadillac, MI

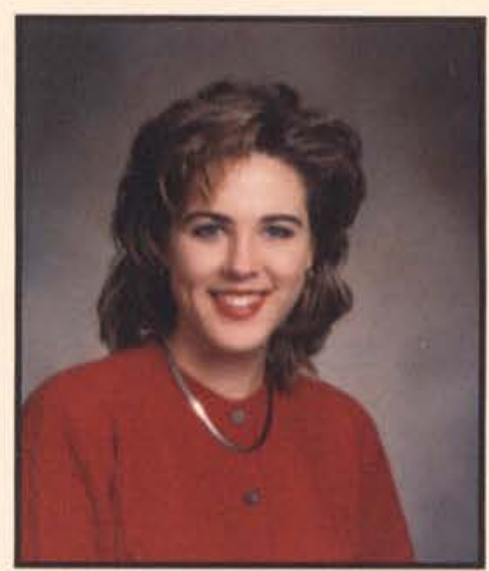

Shannon Bean

Psychology

Ripley, NY

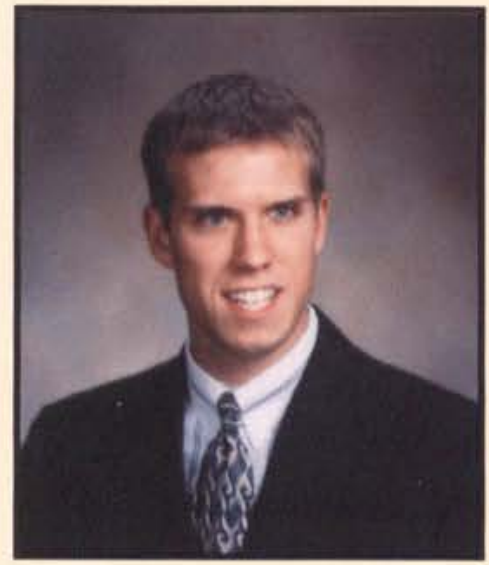

David Joshua Bell

Elementary Education

Worthington, $\mathrm{OH}$

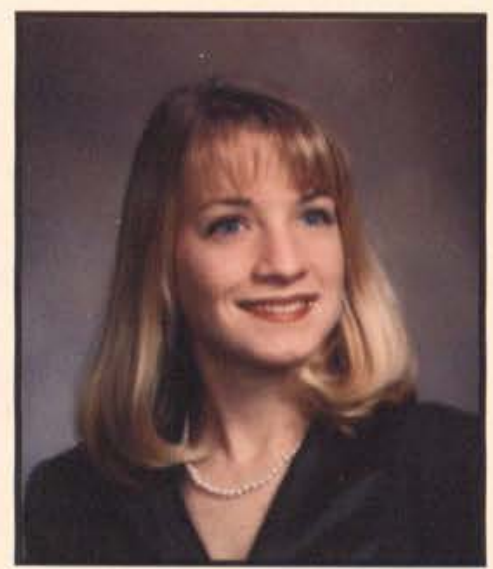

Almeda Bierly

Applied Psychology

Gallup, NM

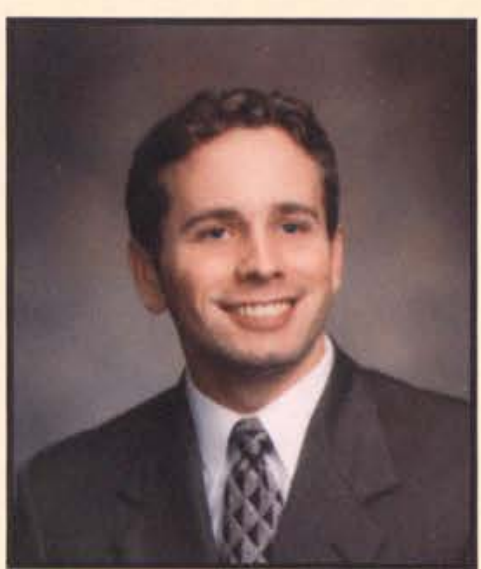

Douglas Bayler

Marketing

Michigan City, IN

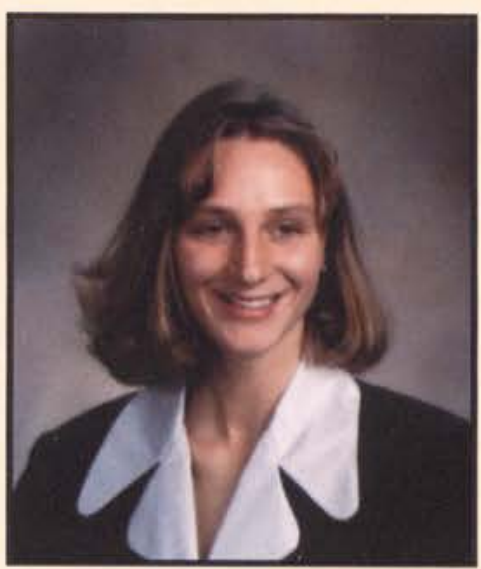

Jamie Beck

Applied Psychology

Galion, $\mathrm{OH}$

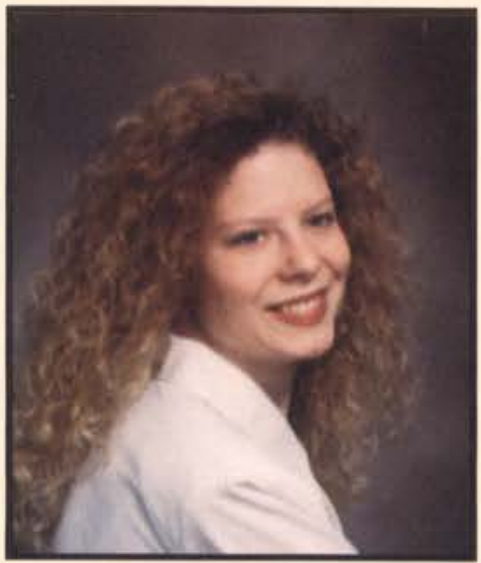

Jennifer Benson

English/Professional Writing

Dryden, NY

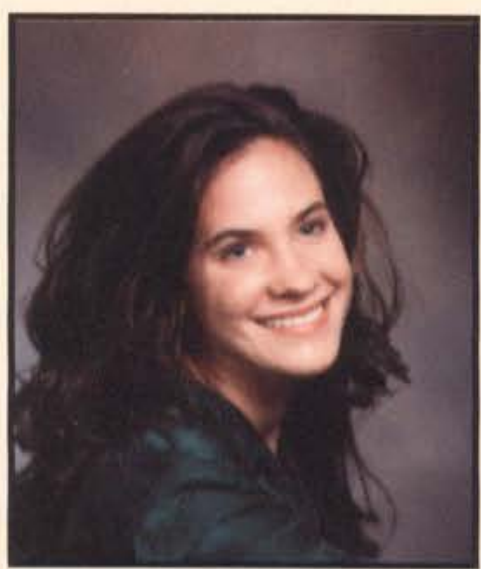

Sandra Birmingham

American Studies

West Jefferson, $\mathrm{OH}$ 


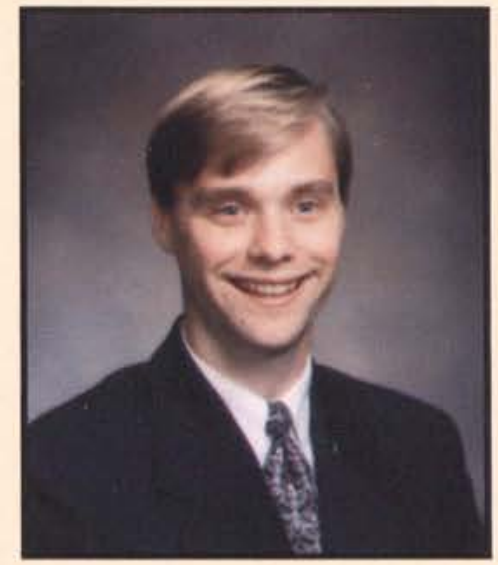

Joel Bjerke

Mechanical Engineering

Rochester, MI

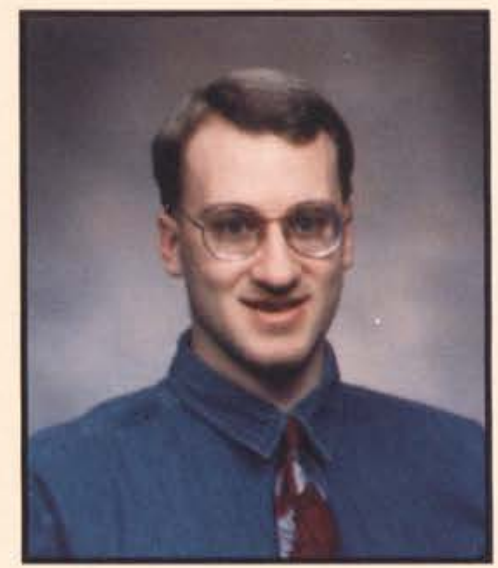

Scott A. Borling

History

Cedarville, $\mathrm{OH}$

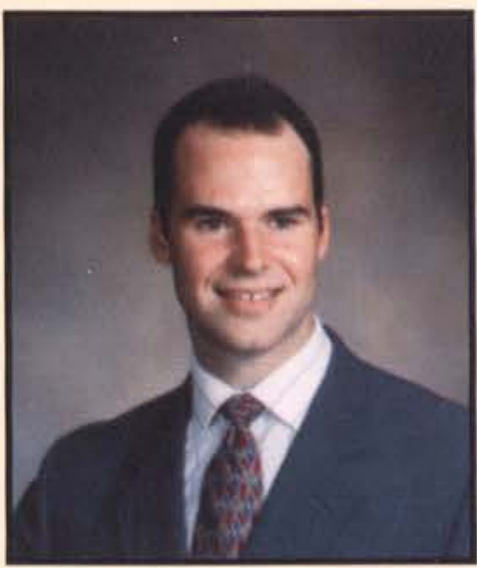

Jeff Breneman

Mechanical Engineering

Roanoke, VA

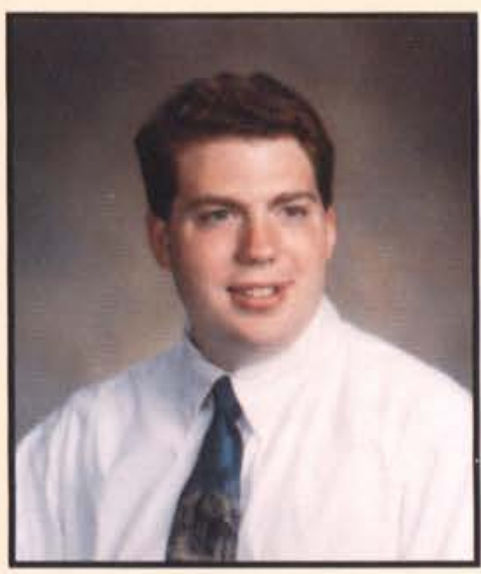

Jason Brown

Communication Arts

Kentwood, M

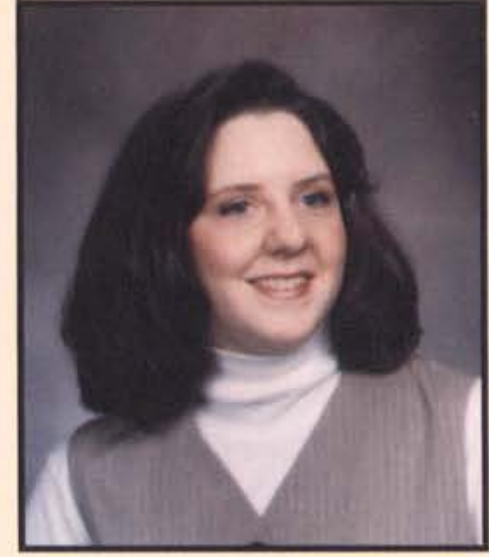

Mary Jo Blanton

Psychology

Urbana, $\mathrm{OH}$

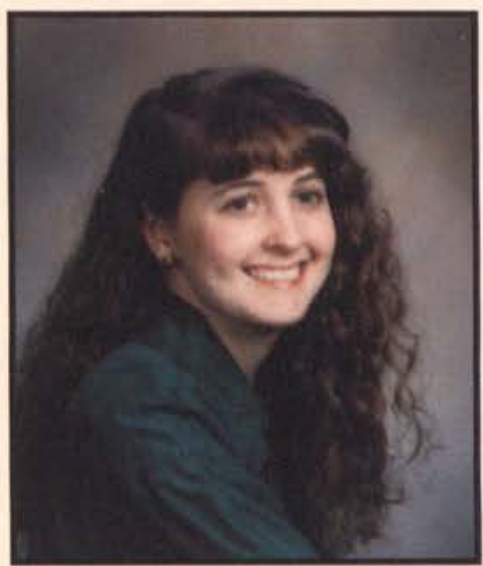

Angela Boyce

Nursing

Titusville, PA

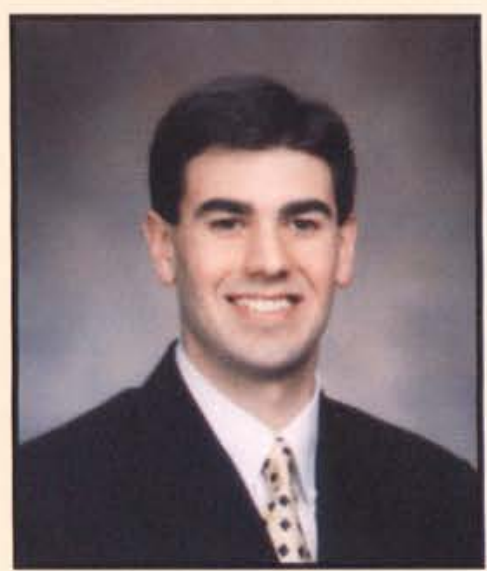

Anthony A. Briggman History

Lake Ariel, PA

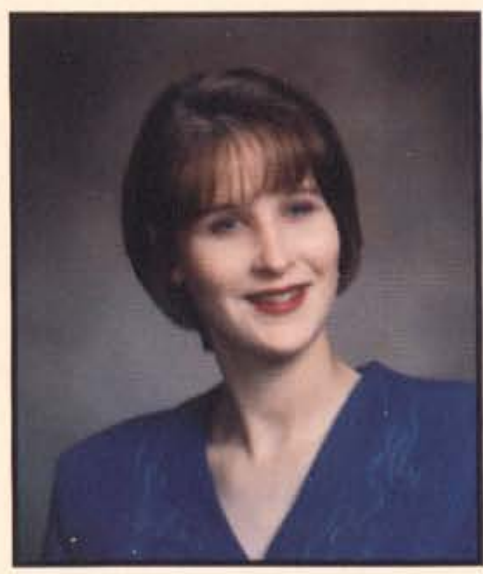

Jill Amber Brown

Music

Sumner, IA

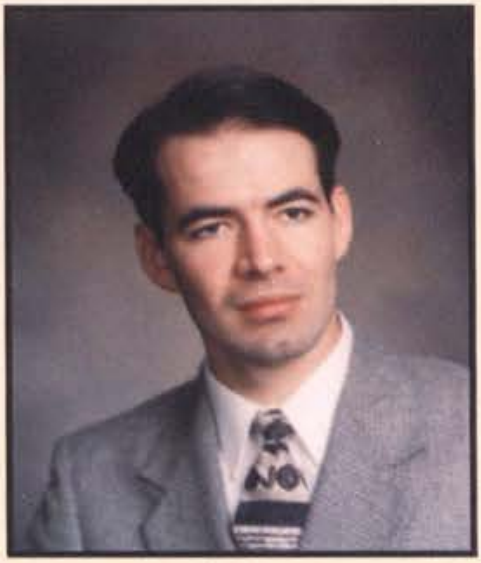

Philip Boggs

Finance/Management

Papillion, NE

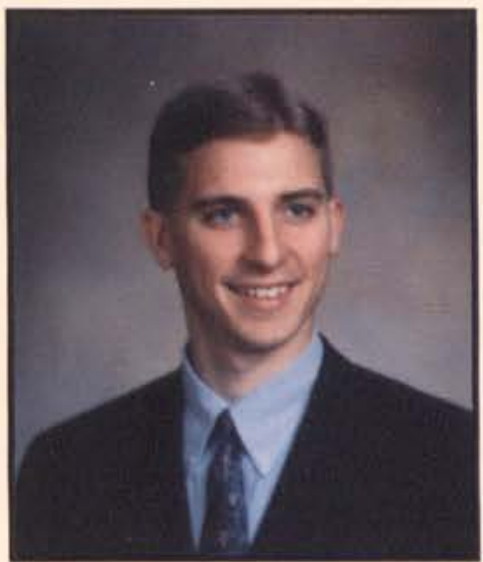

Scott A. Bradley

Chemistry

Centerville, $\mathrm{OH}$

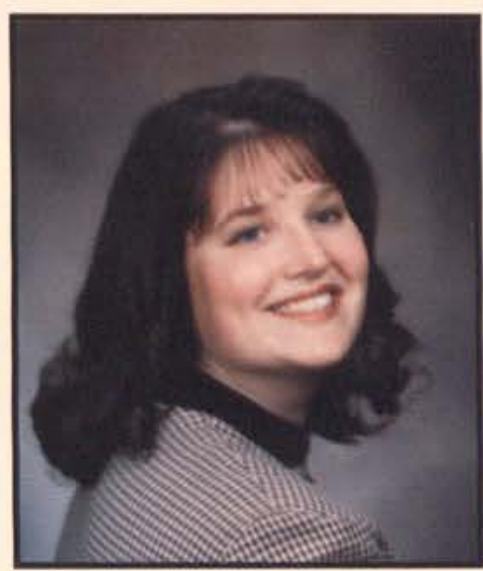

Tonya A. Brockman

Nursing

Huber Heights, $\mathrm{OH}$

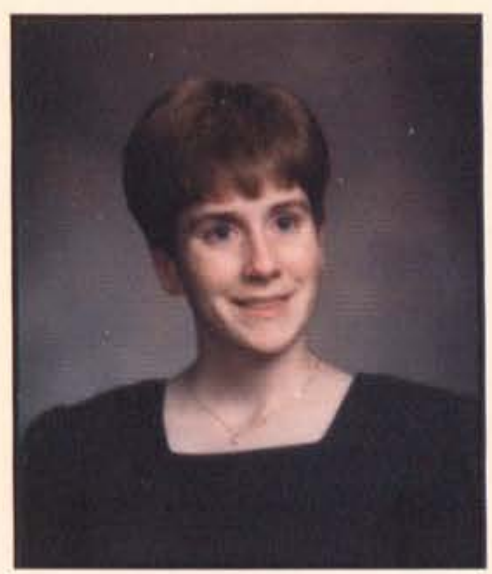

Renee Brown

Nursing

Portland, MI

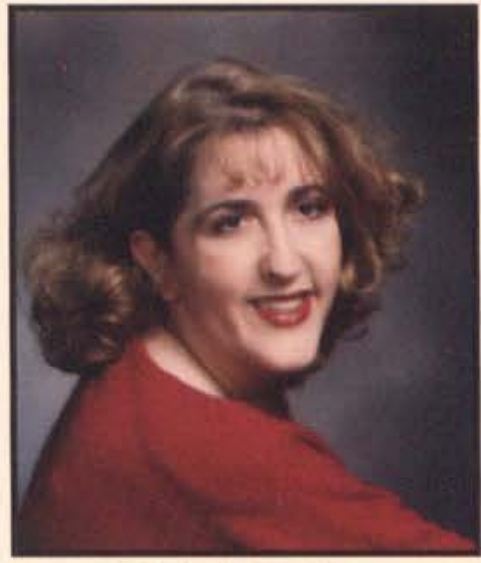

Elizabeth Ann Boone

Communication Arts

Bellbrook, $\mathrm{OH}$

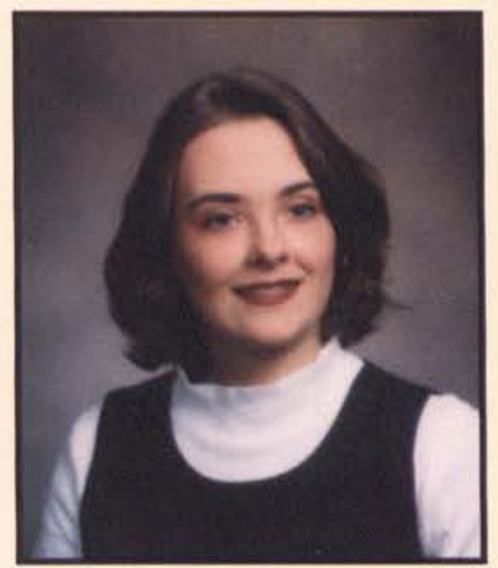

Debbie Brazalovich

English Education Saginaw, MI

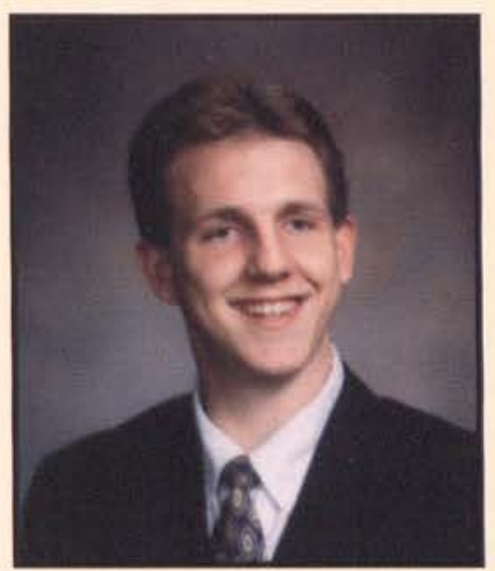

Chad Brown

Pre-Law

Johnstown, NY

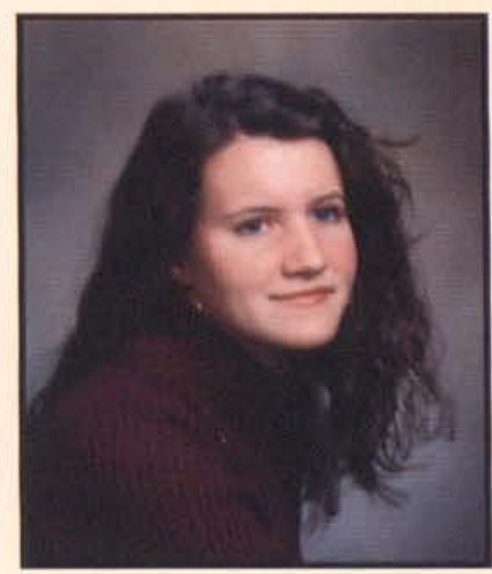

Beth Brubaker

Nursing

Hunlock Creek, PA 


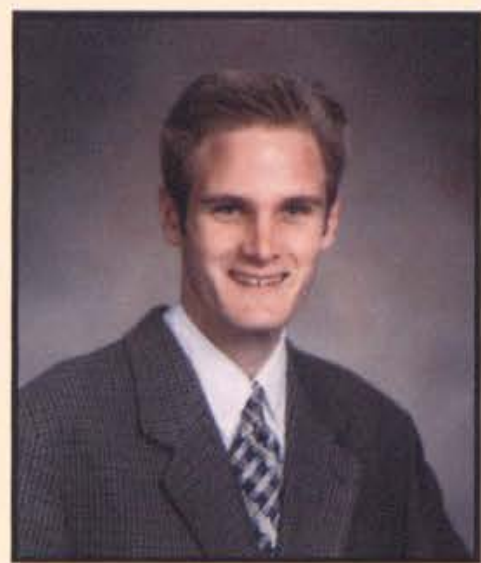

Eric A. Buresh

Mechanical Engineering

Topeka, KS

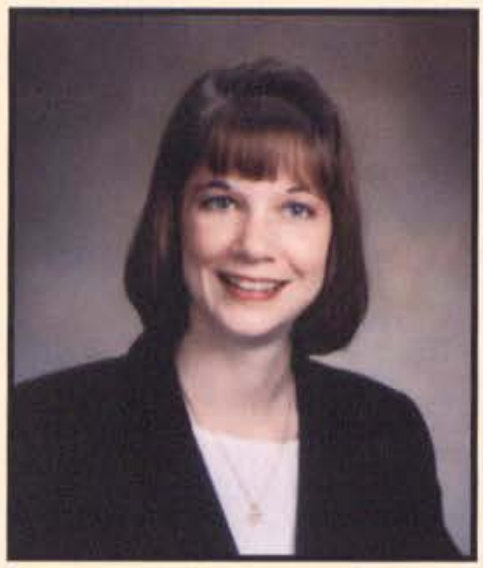

Amy Butcher

Elementary Education

Plantation, FL

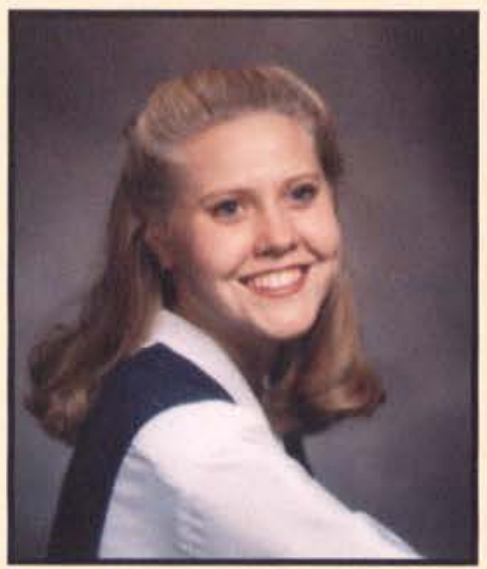

Valerie Calvert

Management

Rochester Hills, MI

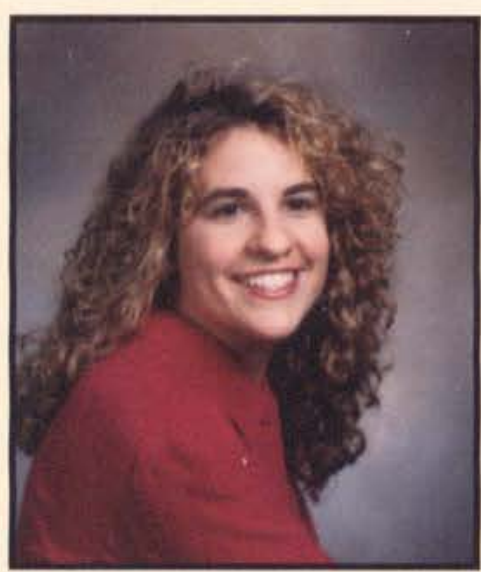

Lisette Anne Cardel

Social Work

Mechanicsburg, PA

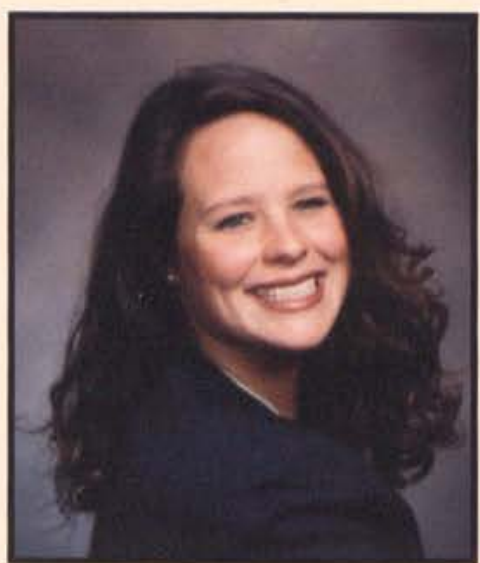

Terah Buresh

Nursing

Topeka, KS

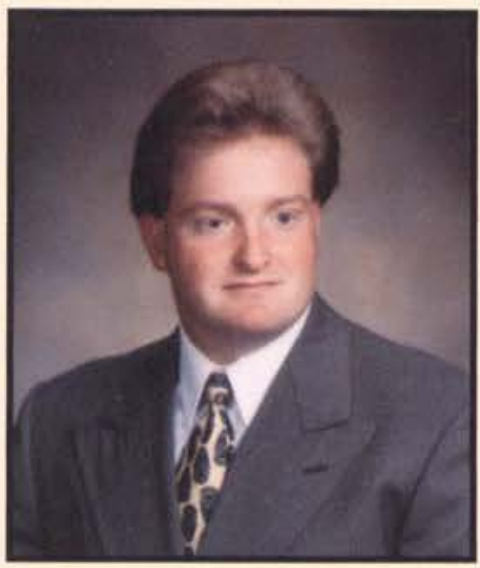

Chad Butler

Bible Comprehensive

Cedarville, $\mathrm{OH}$

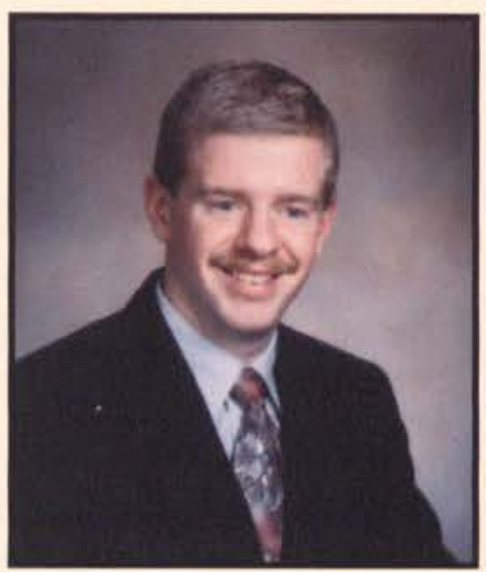

Chris Campbell

Elementary Education

Cedarville, $\mathrm{OH}$

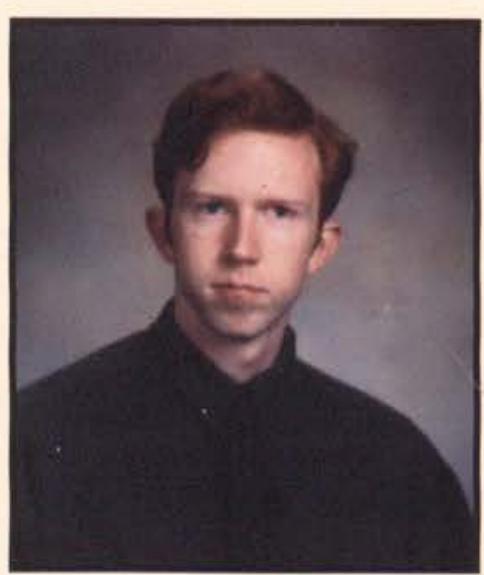

Don Carlson

Multimedia Technology

Wisconsin Rapids, WI

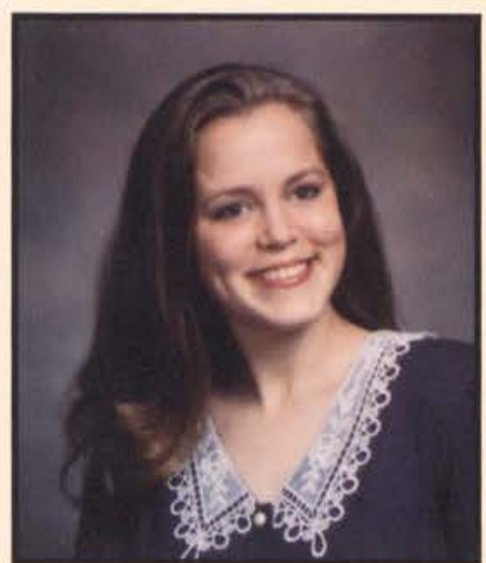

Ellen June Burkhard

Broadcasting/TV Production

Milford, $\mathrm{OH}$

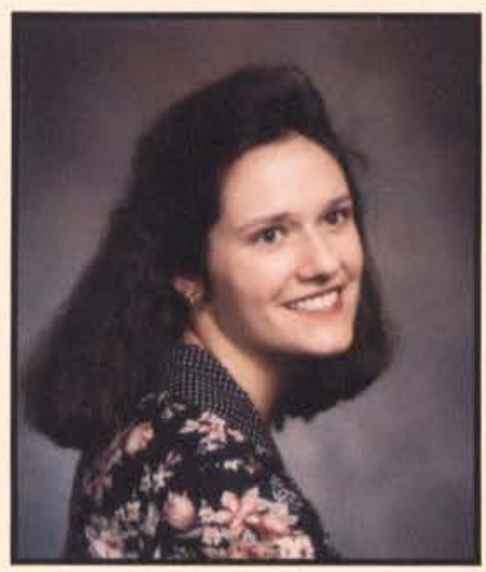

Marnie Butler

Social Work

Cedarville, $\mathrm{OH}$

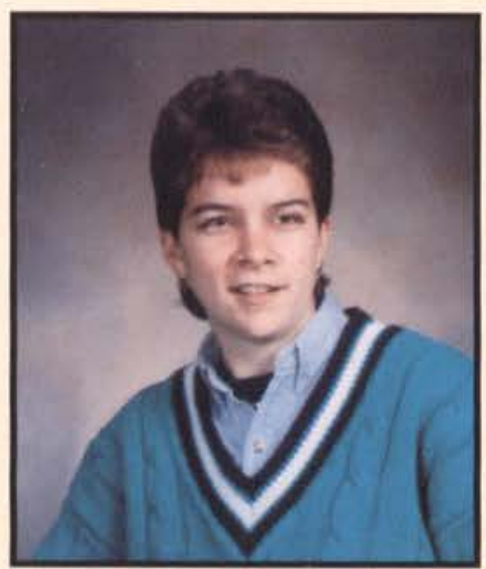

Becky L. Campbell

Physical Education/Health

Warren, $\mathrm{OH}$

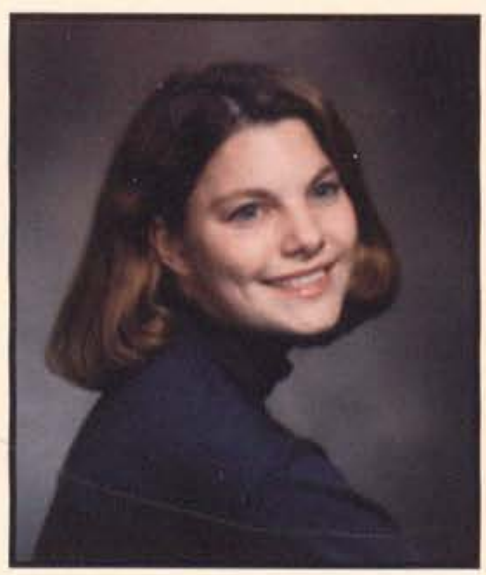

Amy C. Carnahan

Bible/Missions

Clarkston, MI

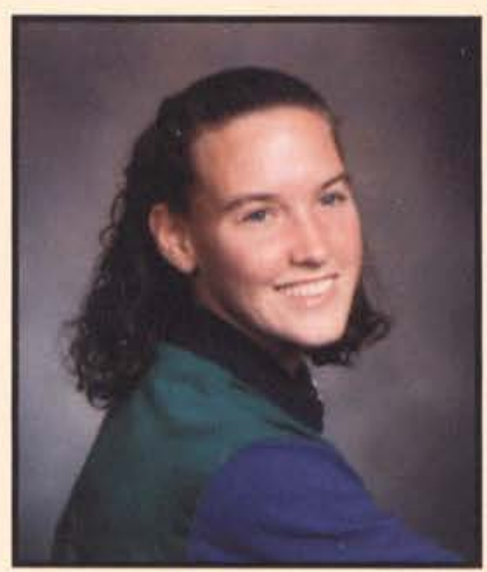

Michelle Kathleen Burson

Comprehensive Physical Education Columbus, $\mathrm{OH}$

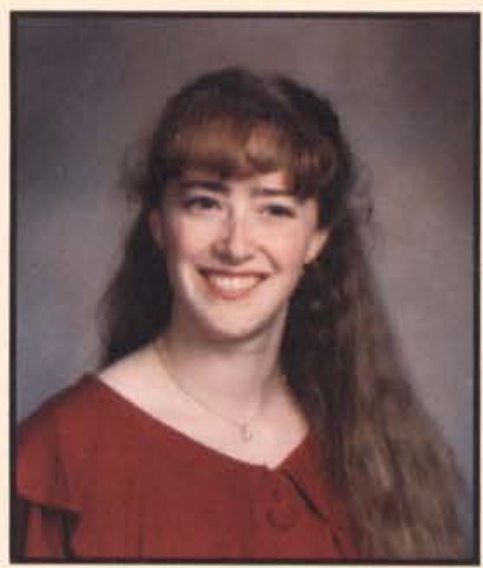

Rita Kimber Call

Nursing

Madison, $\mathrm{OH}$

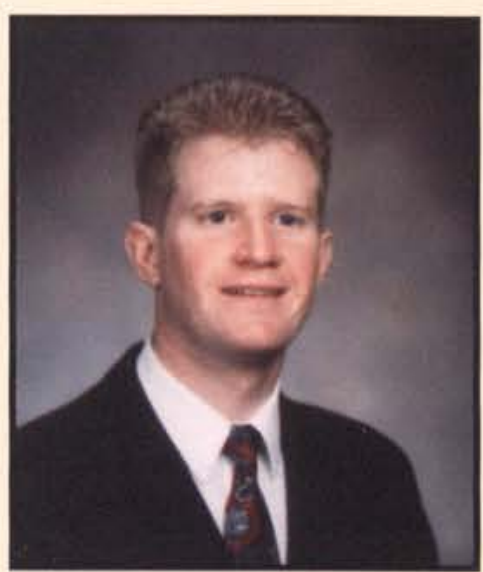

Joseph M. Cantor

Chemistry/Biology

El Cajon, CA

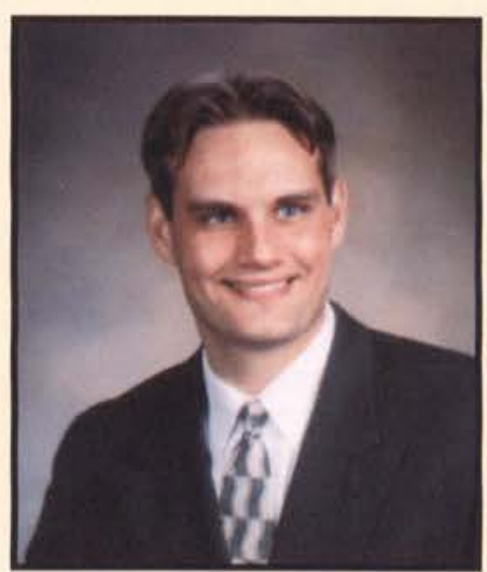

Jason G. Carrier

Bible Comprehensive

Nitro, WV 


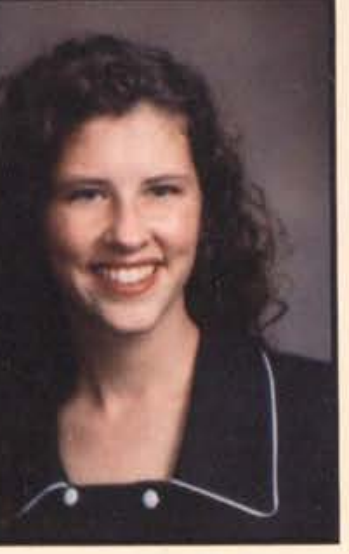

Amy Cartzendafner Broadcasting

Union Bridge, MD

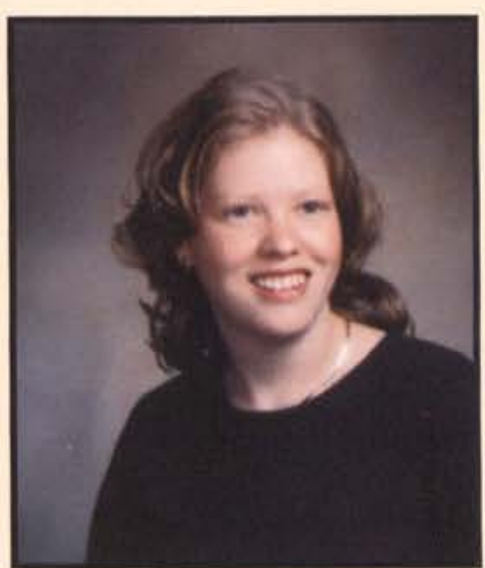

Jennifer Lynn Chesebro Elementary Education Allegany, NY

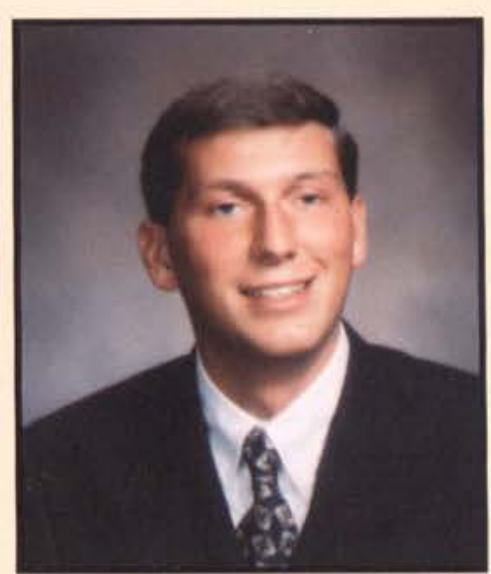

Rodney Wayne Clawson Accounting/Finance South Whitley, IN

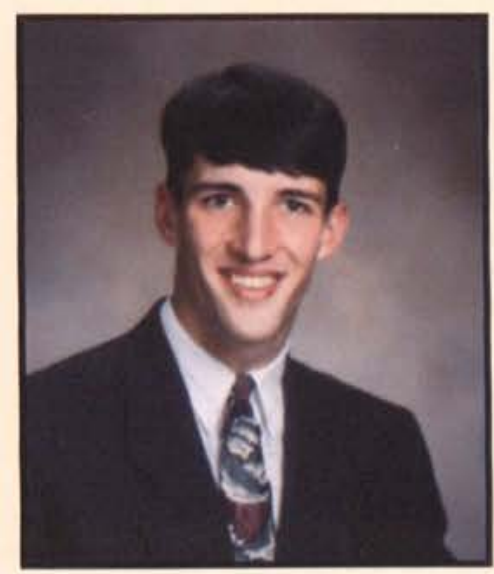

Daniel R. Coleman Mechanical Engineering Hudson, $\mathrm{OH}$

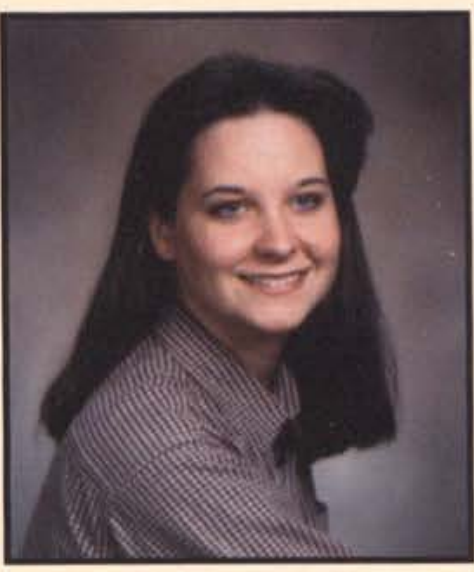

Christine Cassell

Psychology

New Kensington, PA

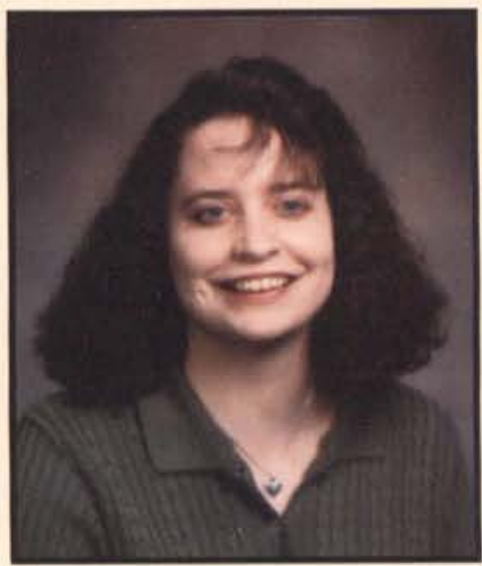

Marjie Chesebro Pre-Law

Portage, MI

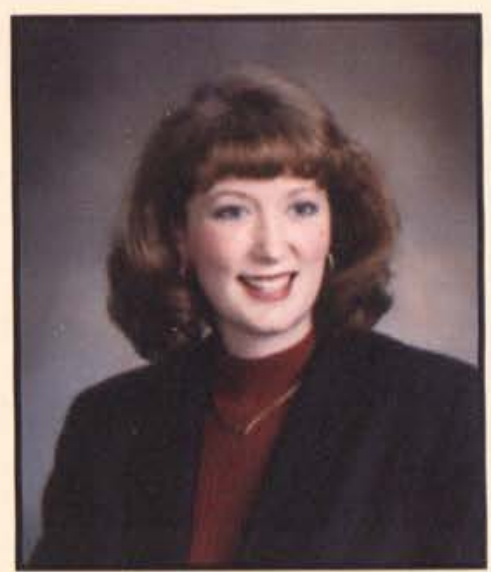

Sara Clayton

Music Education

Creston, IA

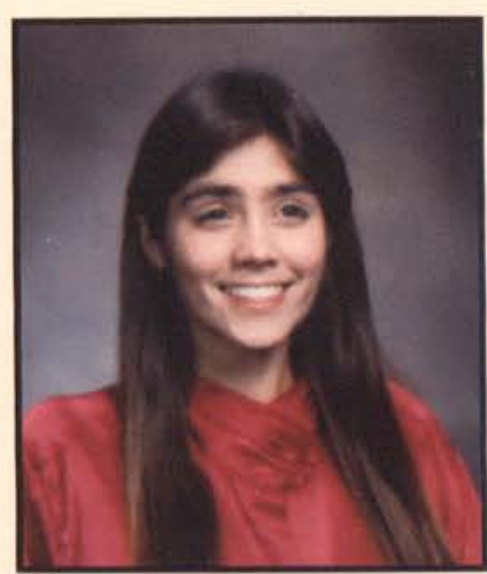

Suzanne Collier Elementary Education Dayton, $\mathrm{OH}$

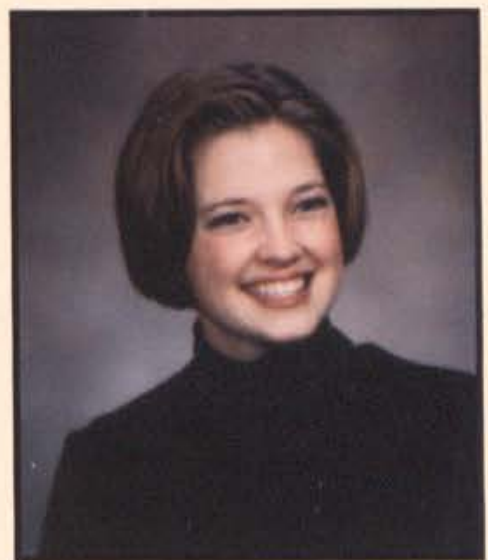

Kelli Cast

English

Medford, NJ

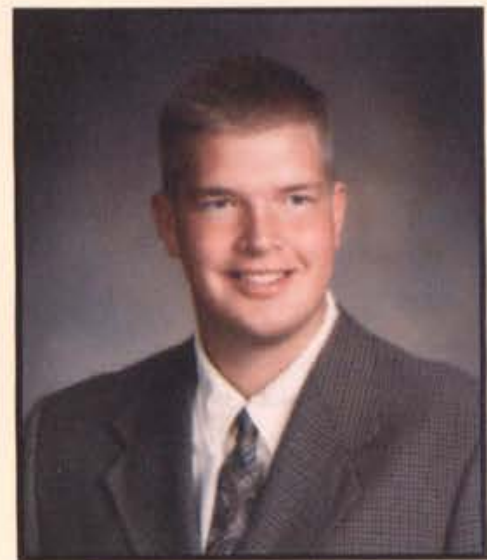

Scott Christner

Finance/Management

Goshen, IN

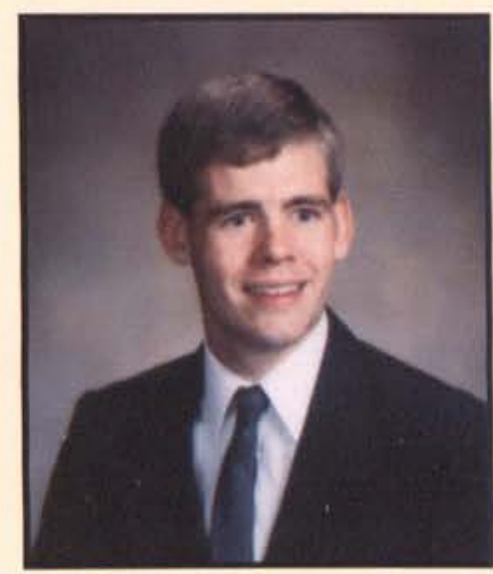

Robert Cobb

Computer Information Systems

Accounting

Sissonville, WV

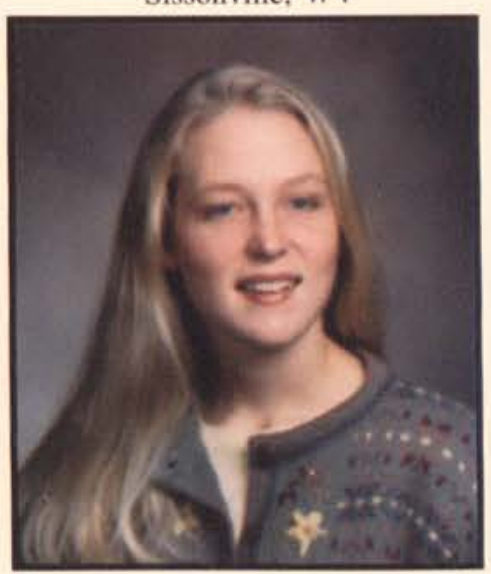

Kimberly Collins Biology

Dollewah, TN

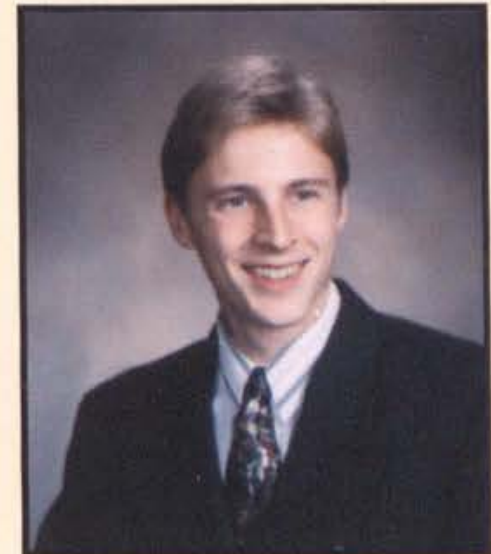

G. Colin Castelow Speech Education Chesapeake, VA

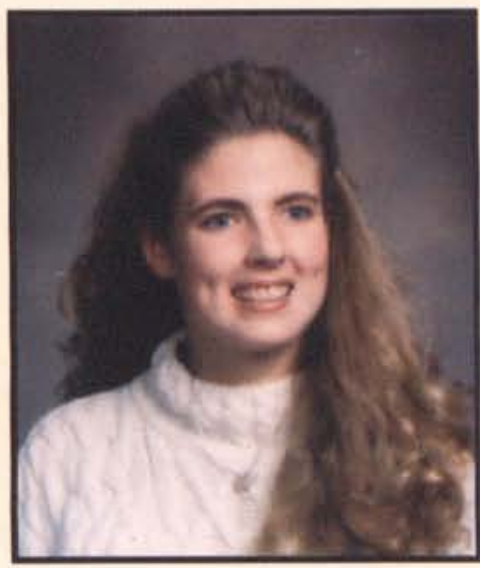

Jennifer Clark

English Education Flint, MI

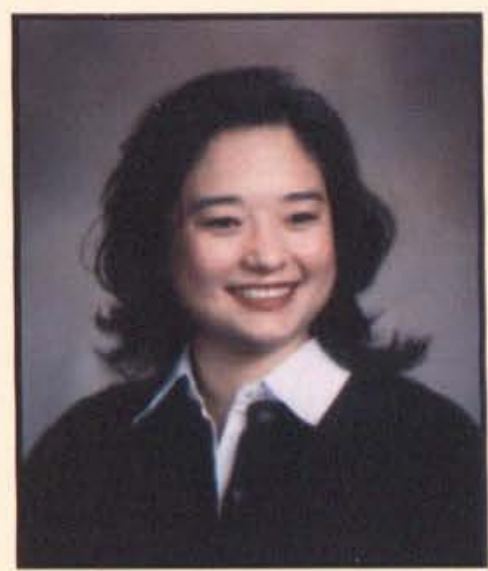

Constance N. Cochren

Professional Writing Houston, TX

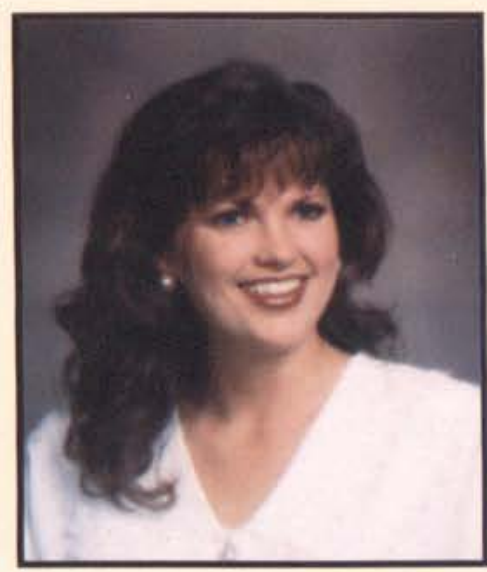

Sharron Lynn Combs Nursing

Jamestown, OH 


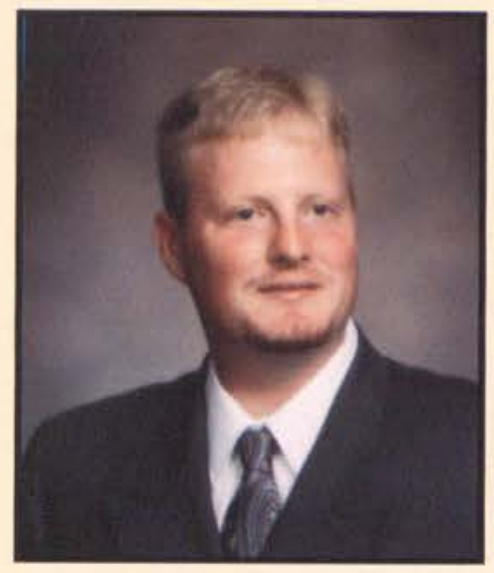

Matthew James Comrie

Communication Arts

Richmond Heights, $\mathrm{OH}$

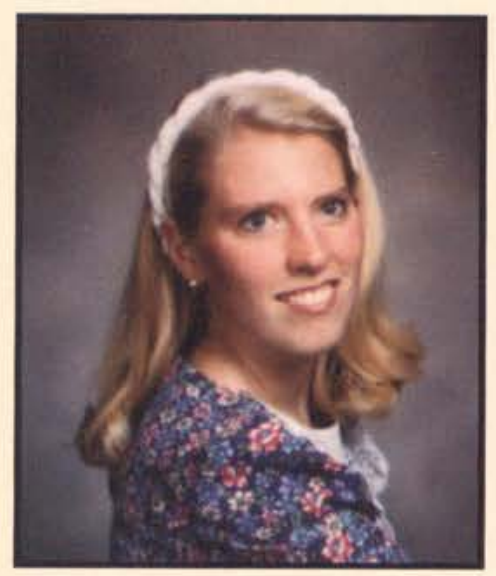

Kim Crawford

Elementary Education

Cedarville, $\mathrm{OH}$

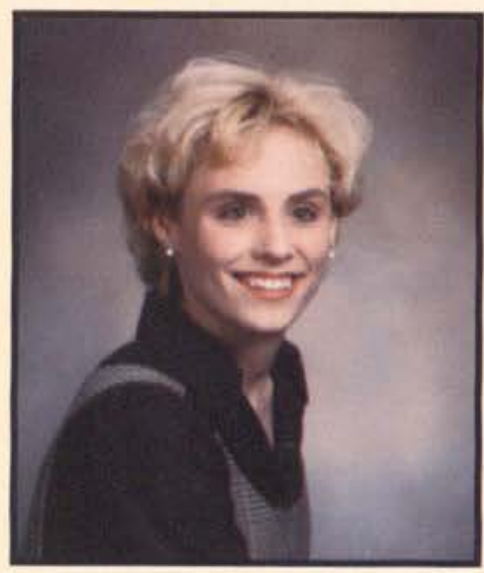

Marcie Curry

English Education

Pekin, IL

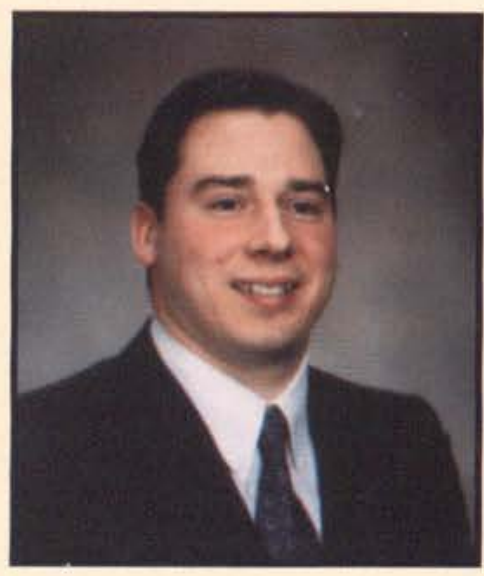

Jason Davies

Physical Education

Shavertown, PA

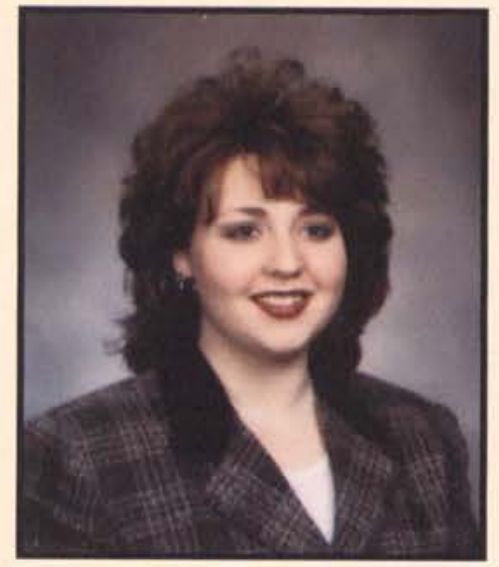

Nicole M. Cooley Music

Cincinnati, $\mathrm{OH}$

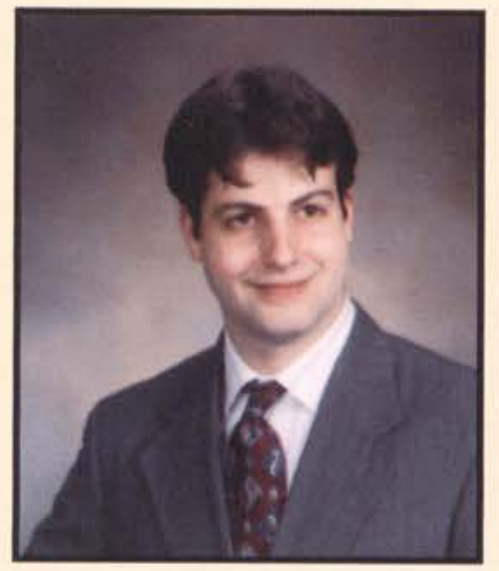

Chad Croft

Political Science

Stormville, NY

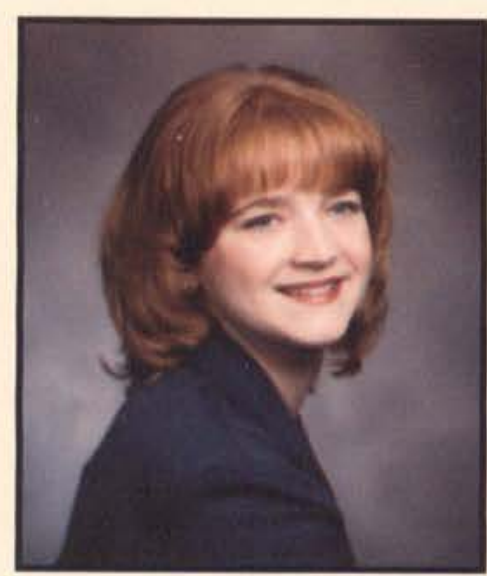

Marie Curry

Nursing

Allen Park, MI

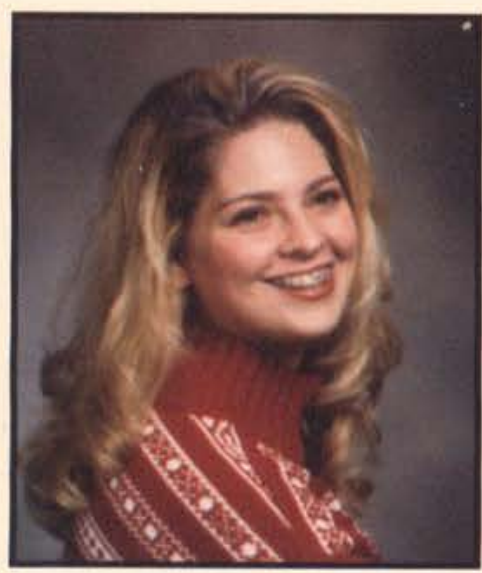

A'Linda A. Davis

Elementary Education

West Chester, $\mathrm{OH}$

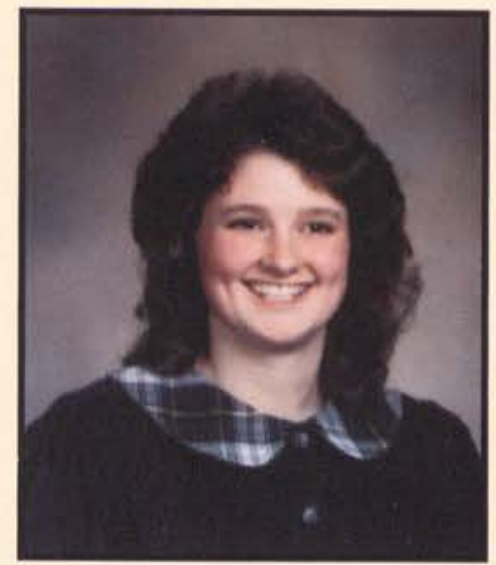

Deborah Copeland

Elementary Education

Mason, MI

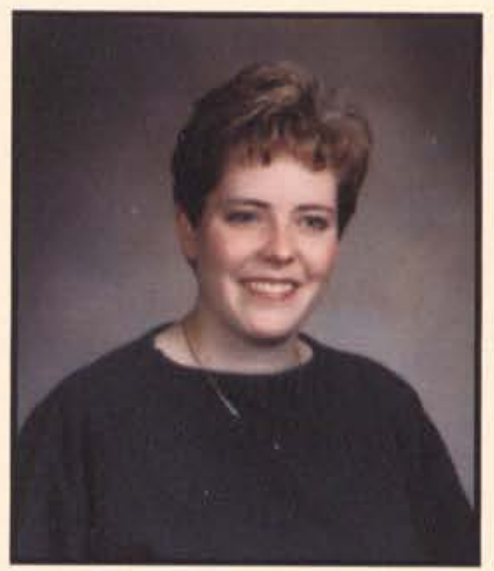

Kori Cronk

Nursing

Freeport, IL

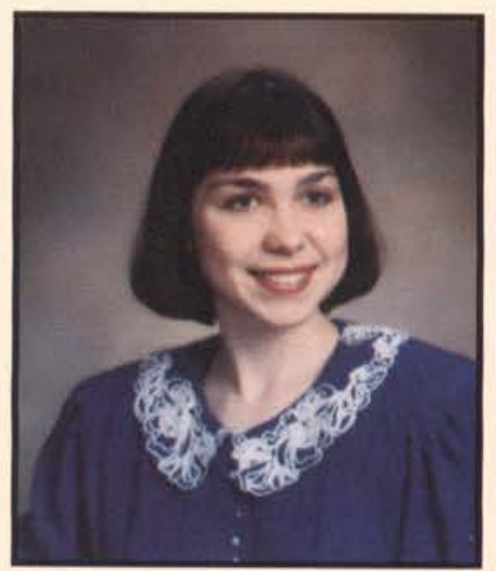

Christine Marie Dabravalskas

Music Performance

Music Education

Grove City, OH

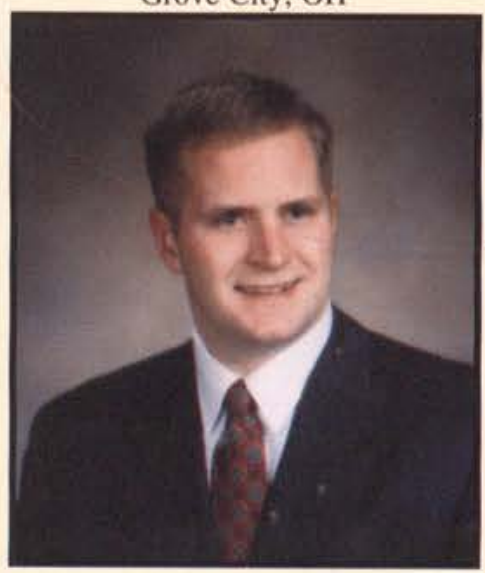

Jeffrey M. Davis

Computer Information Systems

West Bloomingfield, MI

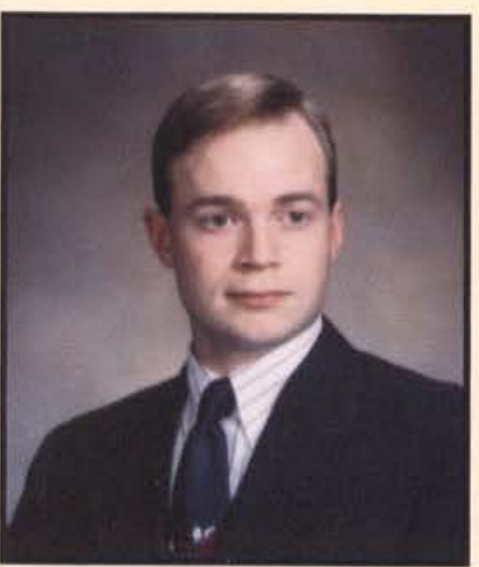

Sean A. Cottrill

Broadcasting

Frankfort, $\mathrm{OH}$

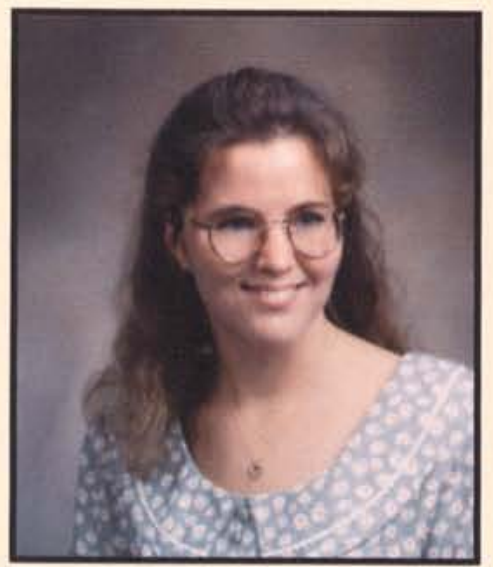

Renee D. Cunningham

Elementary Education

Horseheads, NY

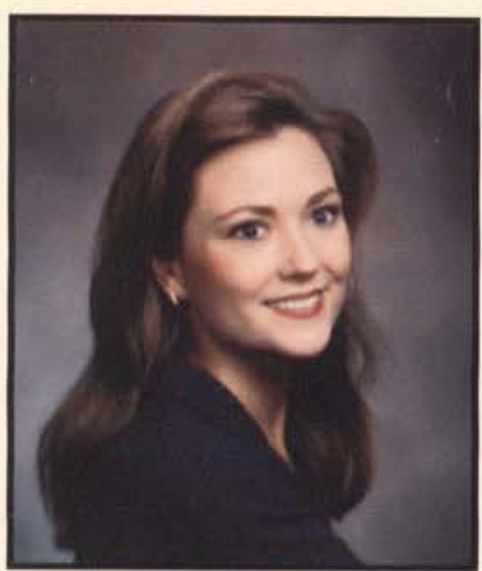

Carey Daniels

Nursing

Ankeny, IA

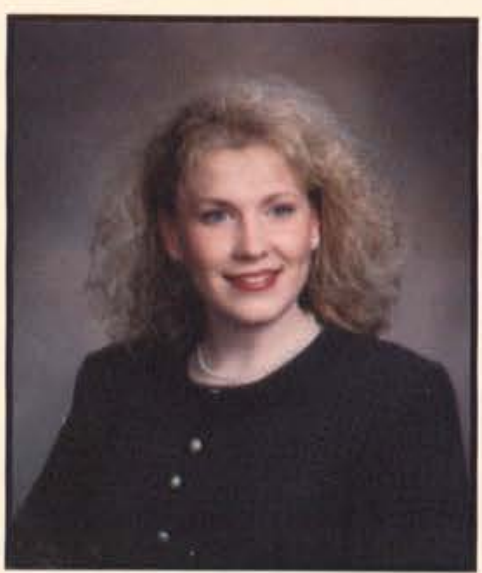

Jerra A. Davis

Nursing

Huntington, WV 


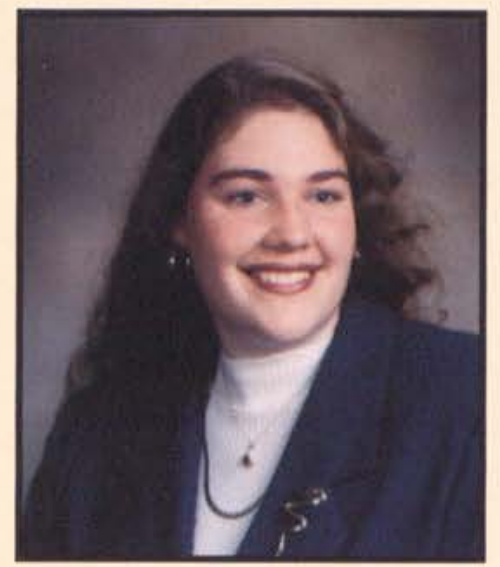

Traci Lyn Durham Elementary Education

Muskegon, MI

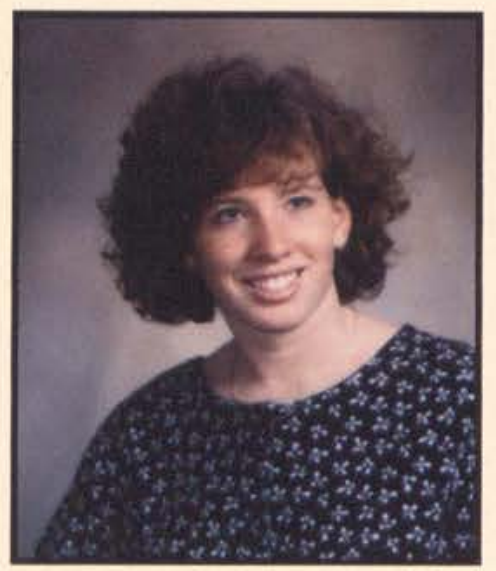

Heather Lynne Edwards

Applied Psychology

Nokomis, FL

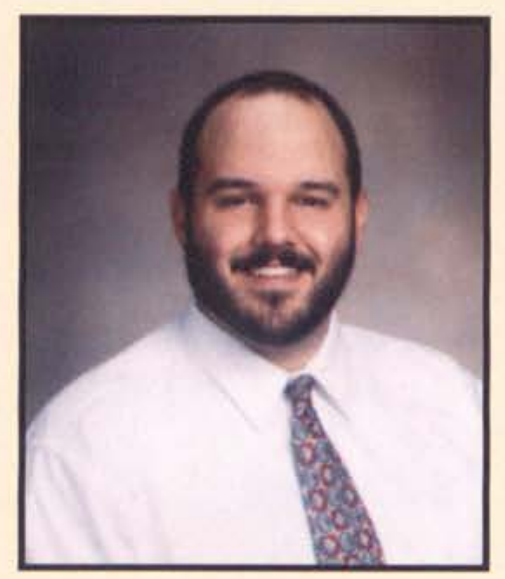

Michael L. Engle

Elementary Education

Springboro, $\mathrm{OH}$

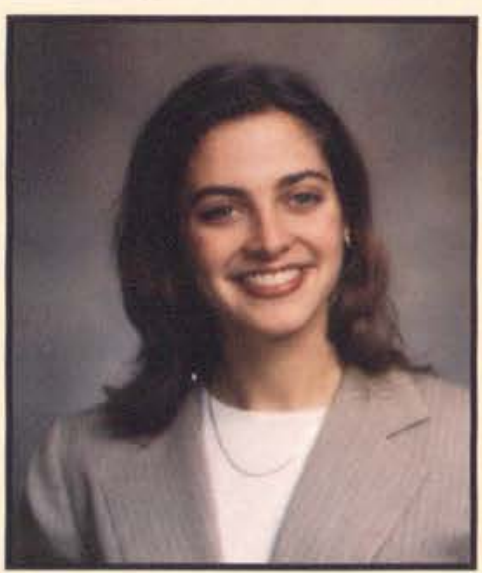

Paula Rose Faris

Broadcasting/TV Production Jackson, MI

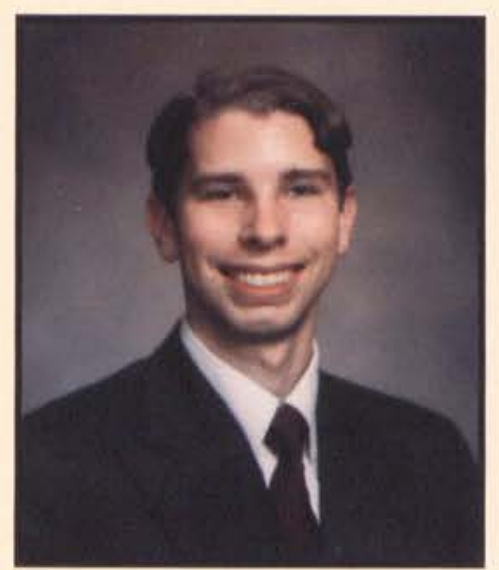

Nathan John Eads

Accounting

Jackson, MI

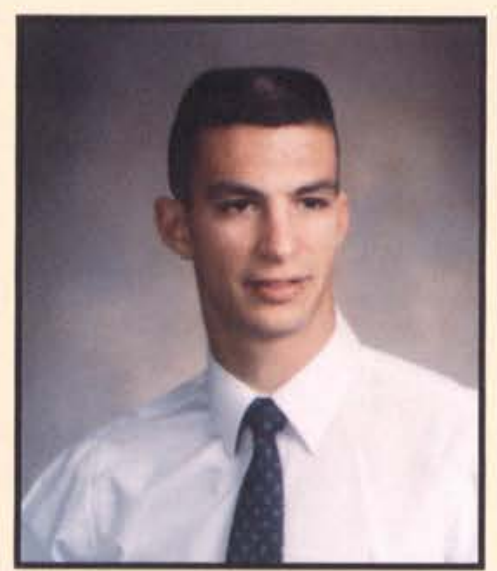

David Eichorn

Electrical Engineering

Lexington, $\mathrm{OH}$

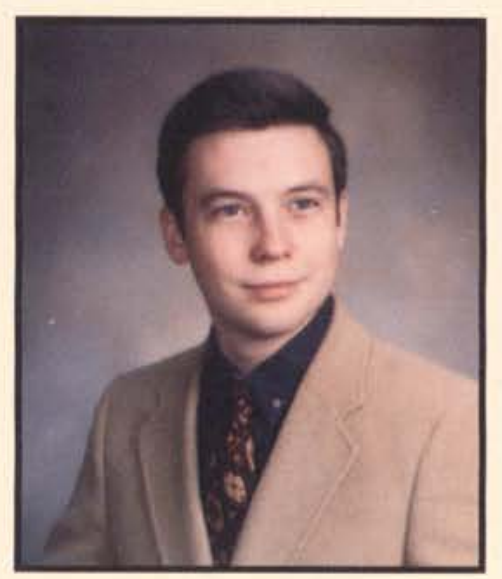

Stephen Estep

Piano Performance

Beavercreek, $\mathrm{OH}$

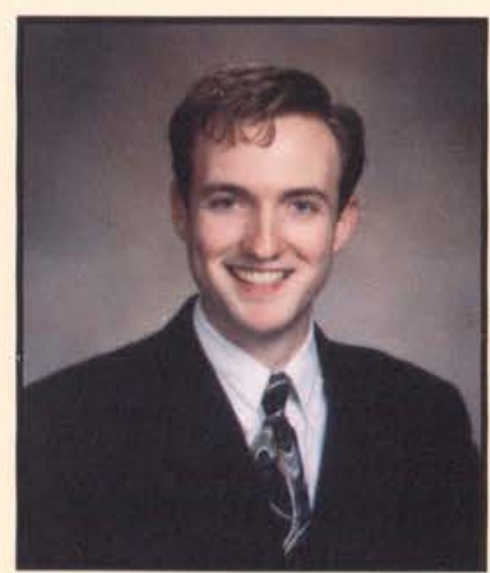

Jeremy Farlow

Mechanical Engineering

Cedarville, $\mathrm{OH}$

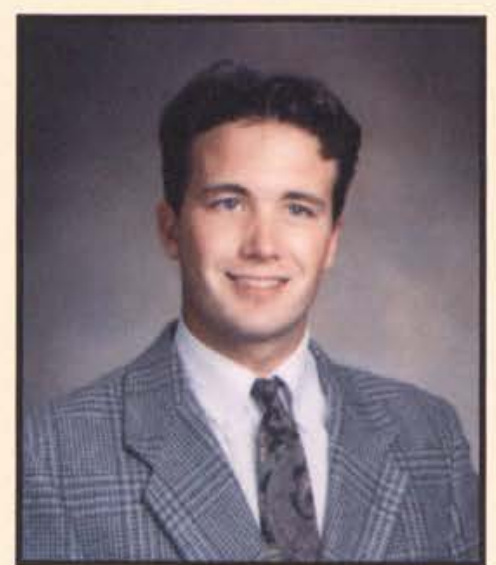

Kipp Edgington

Communication Arts

Fishers, IN

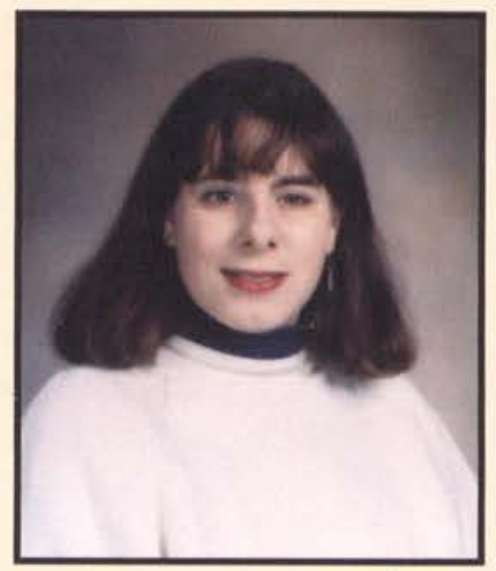

Renee El-Khouri

Nursing

Ponte Vedra Beach, FL

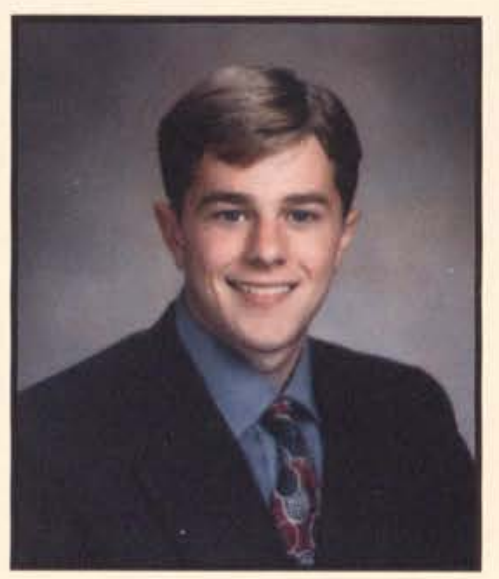

Patrick Estepp

Physical Education

South Webster, $\mathrm{OH}$

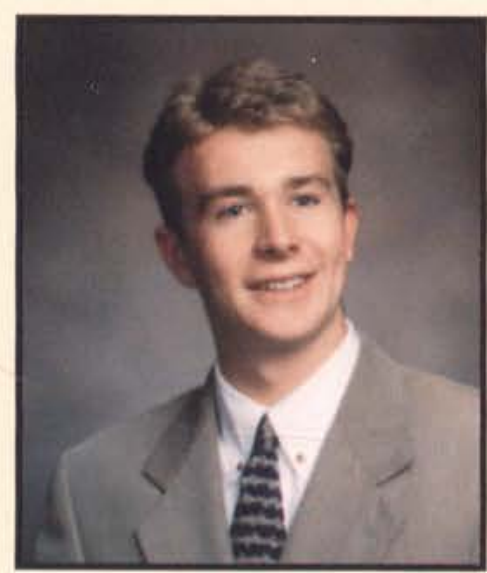

David Farrell

Bible Comprehensive

Covington, $\mathrm{KY}$

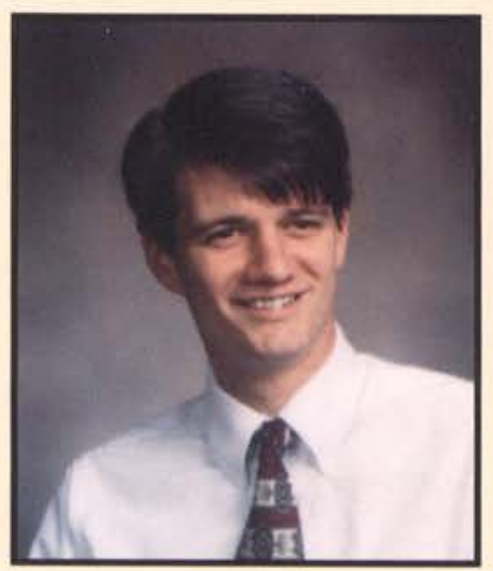

Gregory Alan Edlund

Finance/Accounting Cedarville, $\mathrm{OH}$

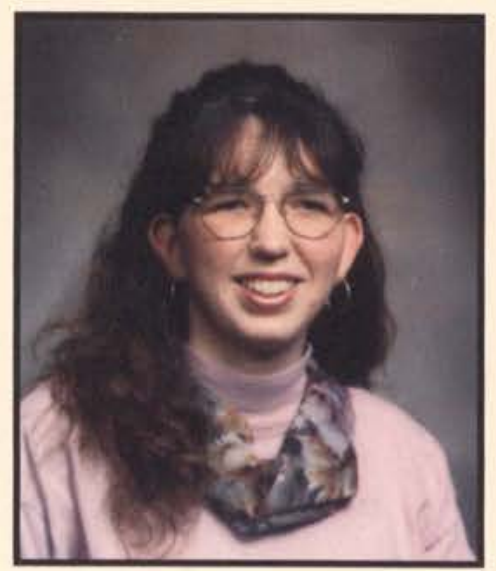

Cheryl Carleen Emmert

Special Education

Huber Heights, $\mathrm{OH}$

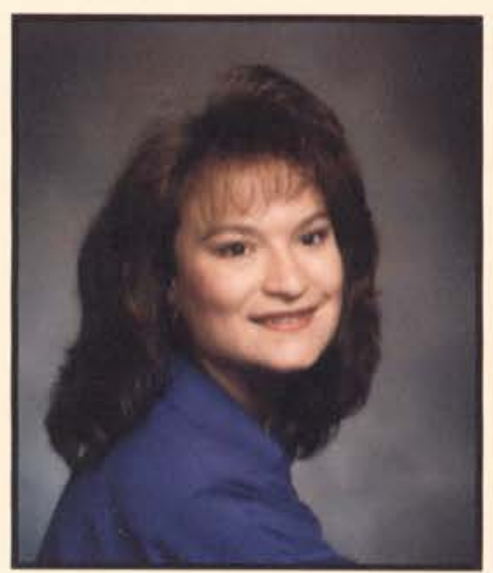

Shellene Everson

English Education

The Pas, Manitoba, Canada

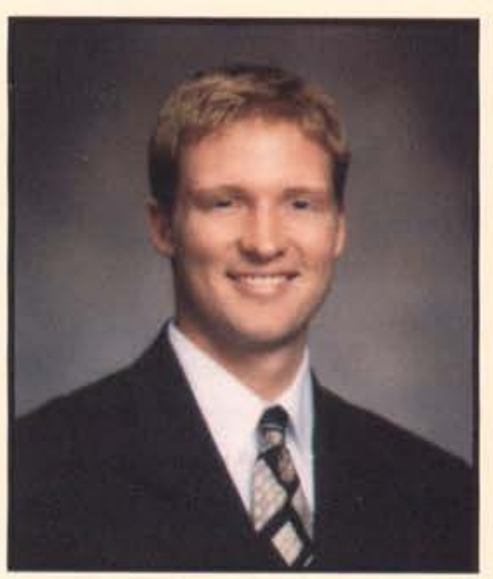

Jamin Worden Ferner Comunication Arts

Moorestown, NJ 


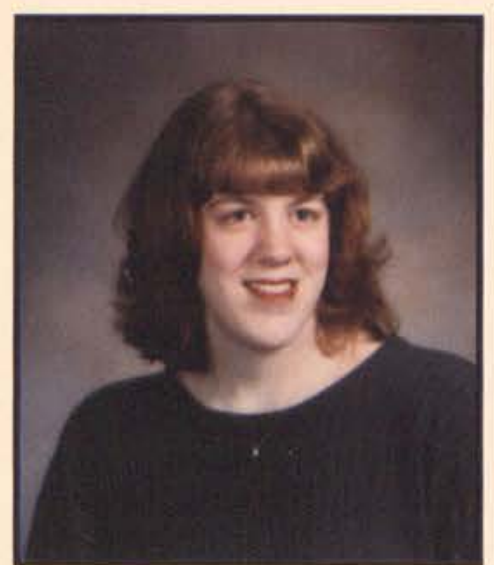

Laura R. Glessner

Biology/Education

Tacoma, WA

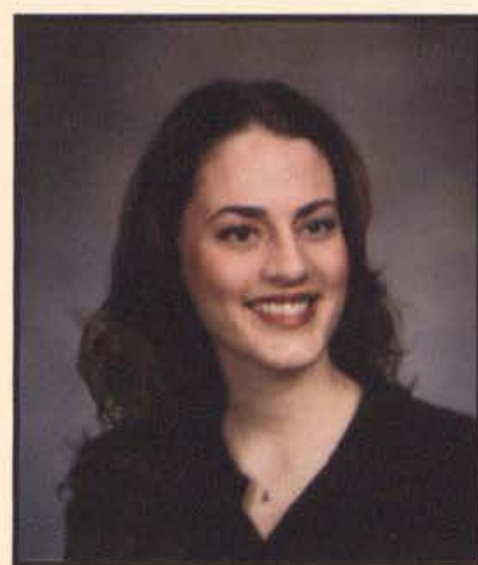

Janna Gottwals

Bible Comprehensive

Cedarville, $\mathrm{OH}$

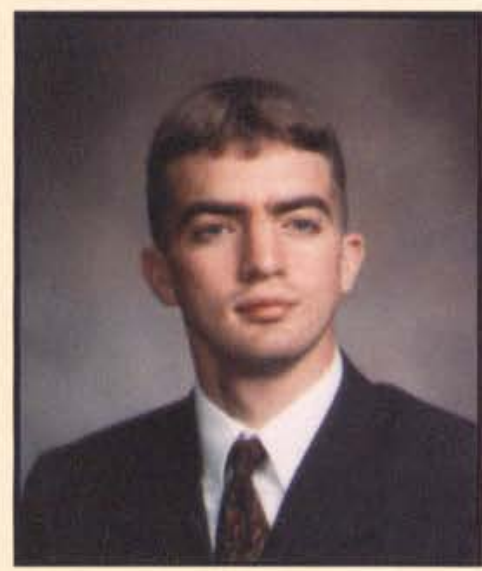

Mike Gribbin

Communication Arts

Mc Kean, PA

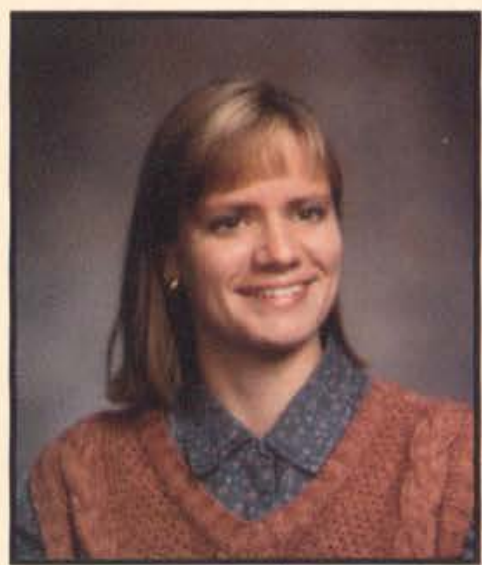

Heidi Karen Gritsavage

Christian Education Youth Macomb, MI

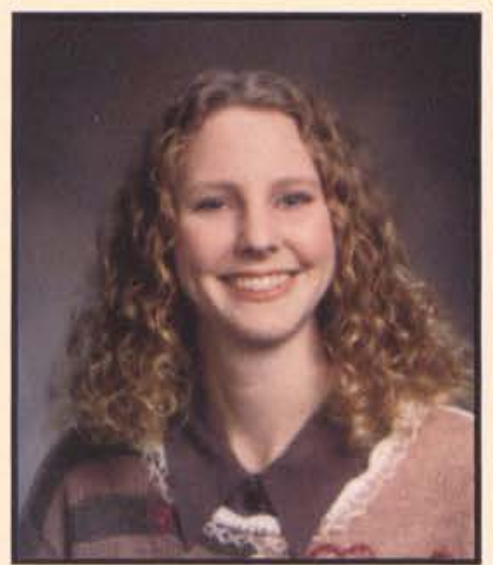

Jolyn Glines

Elementary Education

Danbury, NH

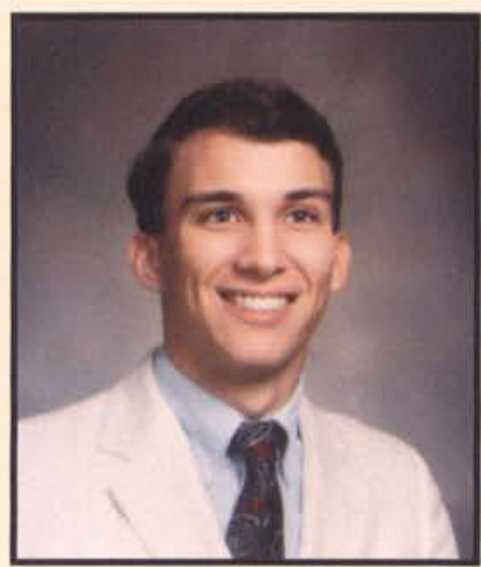

Daniel M. Graham

Mechanical Engineering

Randolph, NY

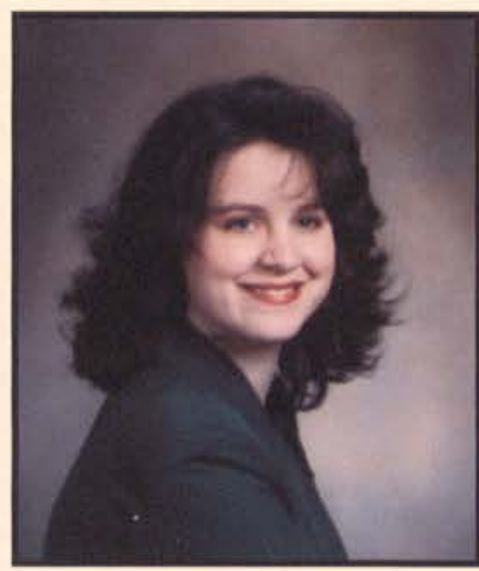

Jessica Griffith

Business Communication

Technology

Liberty. IN

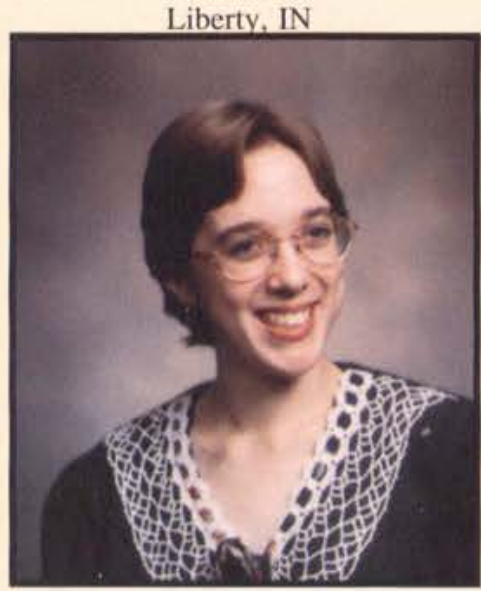

R. Heidi Groves

Nursing

Flemington, N

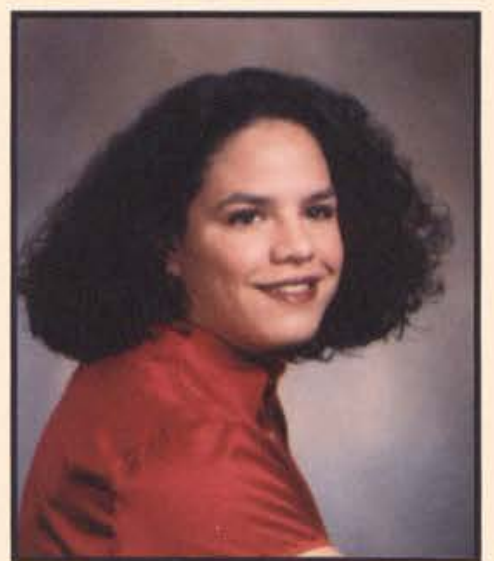

Hasmine Gmuer

Social Work

Trumbull, CT

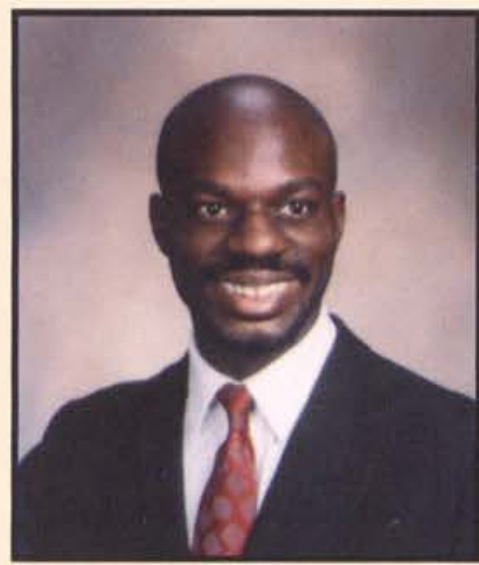

Derrick LeMarr Green

Communication Arts

Cleveland, $\mathrm{OH}$

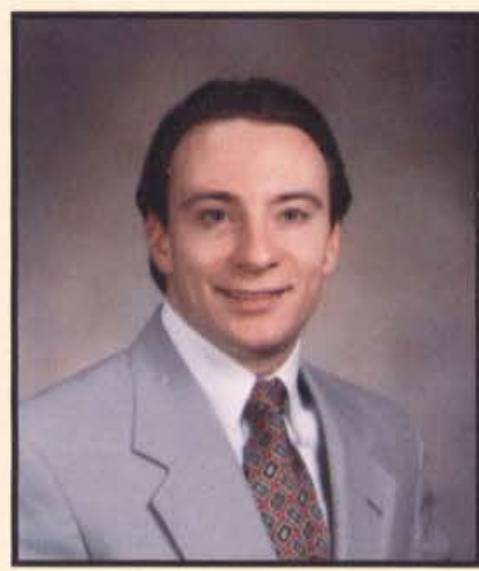

Jason Grills

Communication Arts

Newmarket, Ontario, Canada

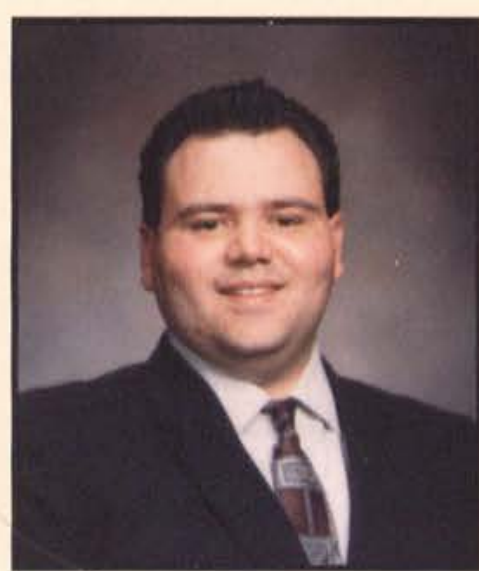

Kevin Grubbs

Bible Comprehension/Missions

Cedarville, $\mathrm{OH}$

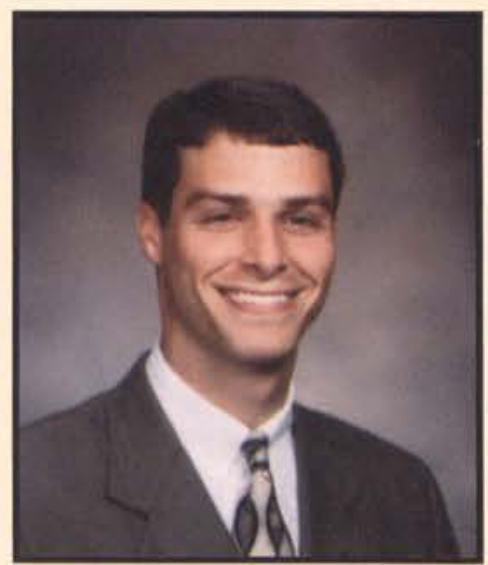

Darrin M. Gosser

Communication Arts

Indianapolis, IN

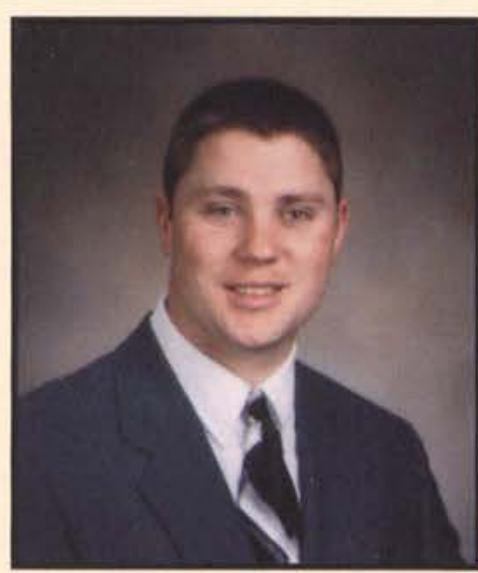

Joshua Green

Bible/Pre-Seminary

Cedarville, $\mathrm{OH}$

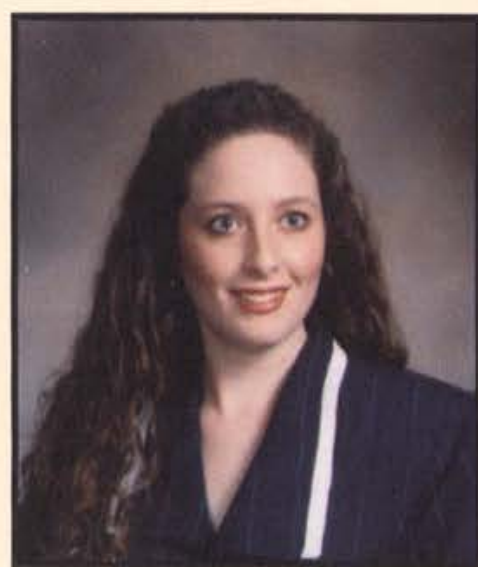

Lisa Dawn Grindall

Elementary Education

Winslow, ME

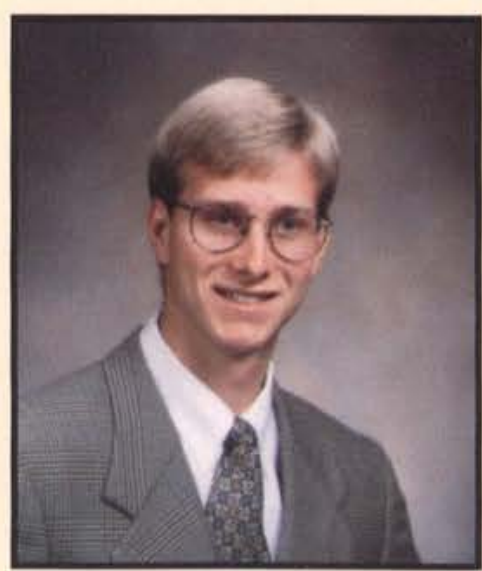

Jeff Gruet

Management

Gloversville, NY 


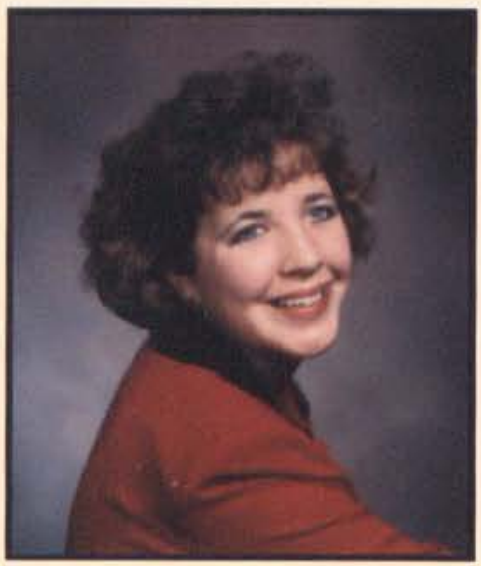

Dawn Henney

Nursing

Flint, MI

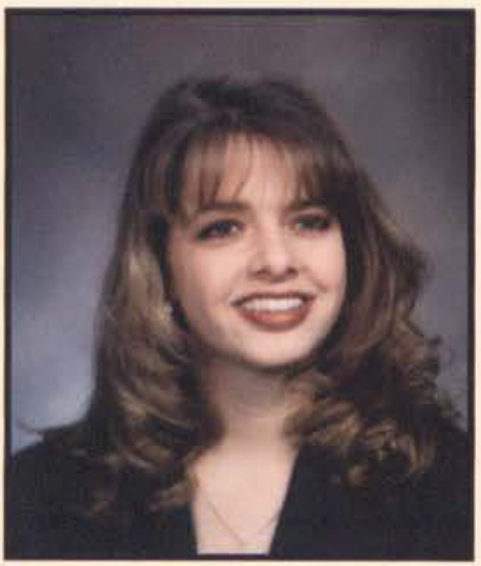

Melissa Hewitt

Elementary Education

Goshen, IN

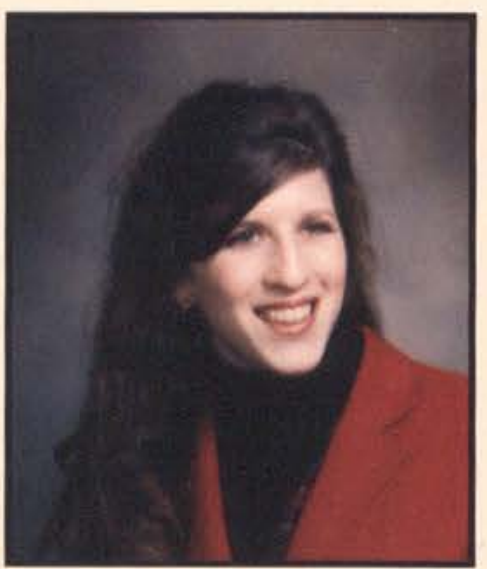

Melissa Hively

Elementary Education

Cedarville, $\mathrm{OH}$

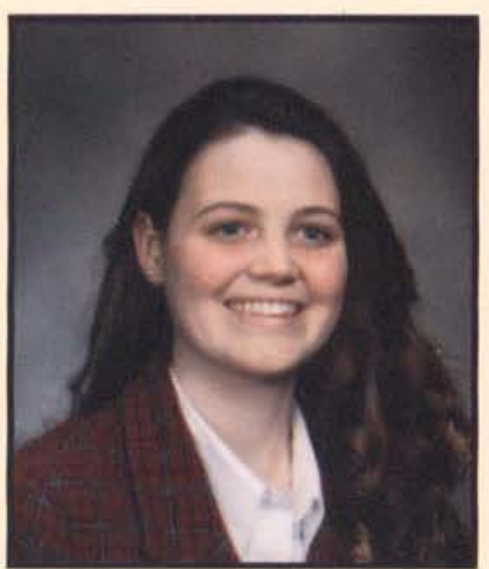

Emily Jean Hodel

Elementary Education

Dawson, IL

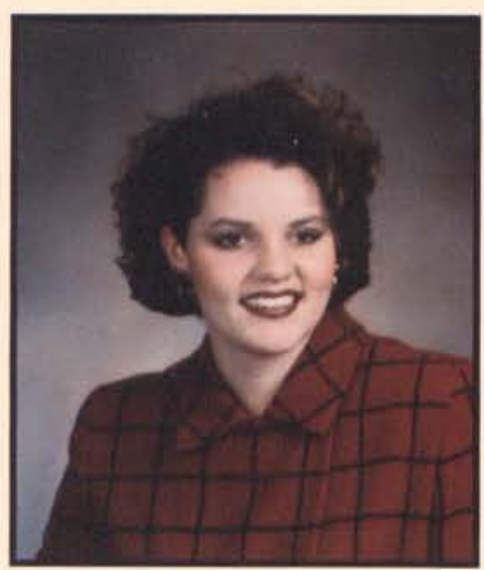

Rachel Henricks

Bible Comprehensive

Archbold, $\mathrm{OH}$

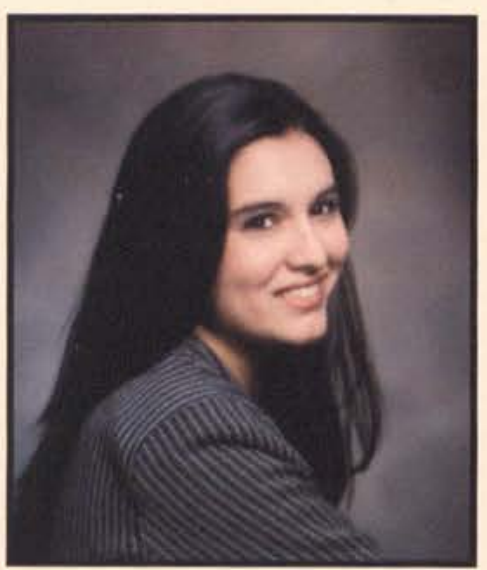

Amy Victoria Hickox

Social Work

Roanoke, VA

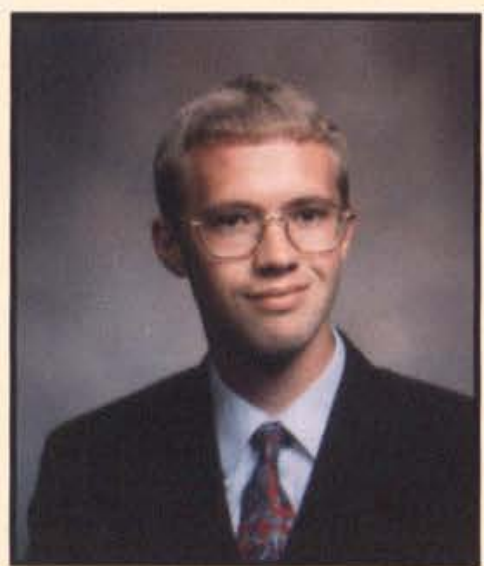

Jonathan David Hjembo

Global Economics/ Int'l Business

New Windsor, MD

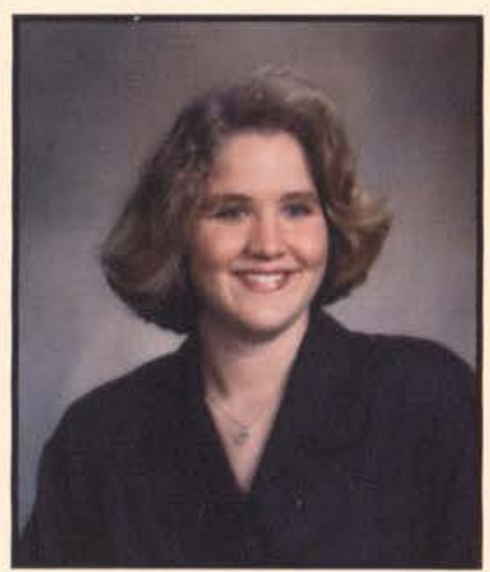

Beth Ann Hoffman Nursing

Clear Spring, MD

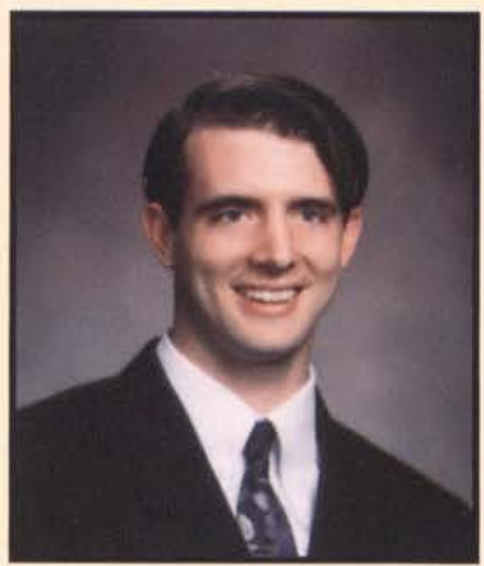

Chad Henry

Business Management/Marketing Frankenmuth, MI

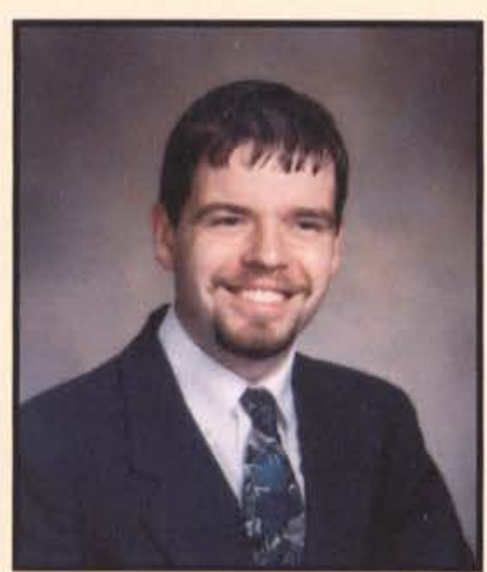

Dallas W. Hill

Broadcasting

Lima, $\mathrm{OH}$

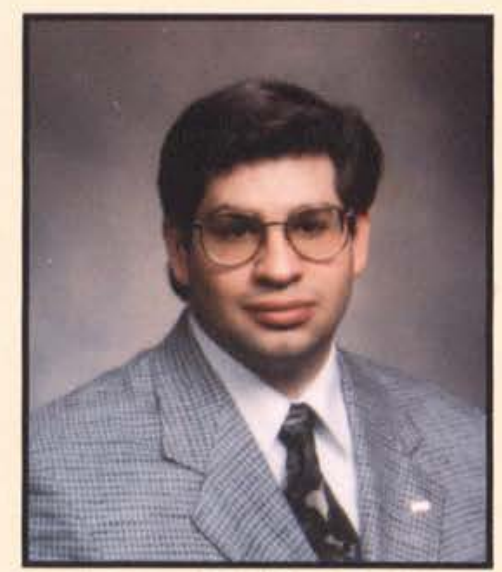

Scott David Hoadley

Electrical Enegeering

Epsom, NH

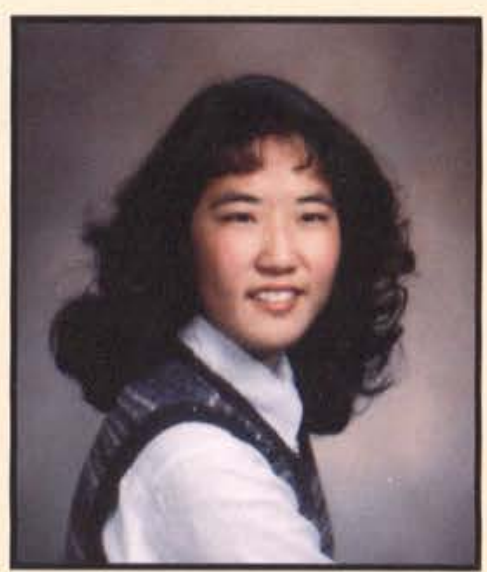

Sarah Lynn Holesovsky Business Management

Madison, WI

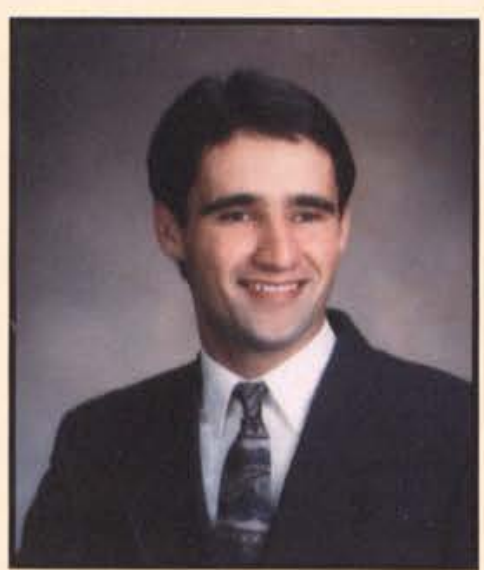

Lowell J. Herschberger Social Work

Sioux Lookout, Ontario

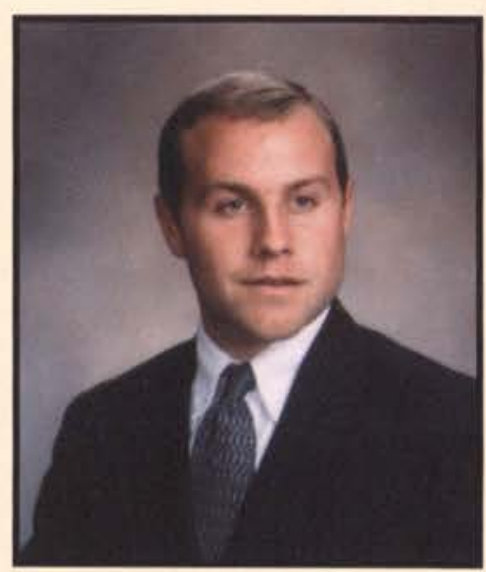

Matthew A. Hintz

Biology

Delaware, $\mathrm{OH}$

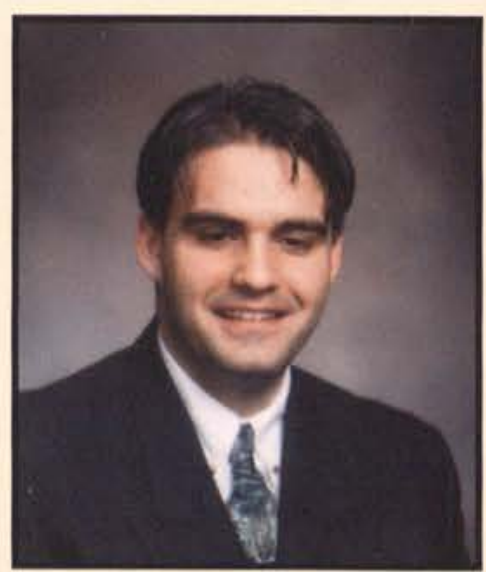

Jeff Hock

Mechanical Enegeering

Newark, $\mathrm{OH}$

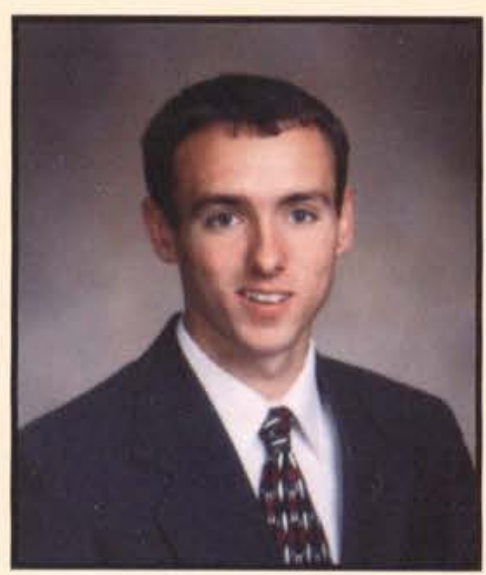

Stephen M. Houg Management Ankeny, IA 


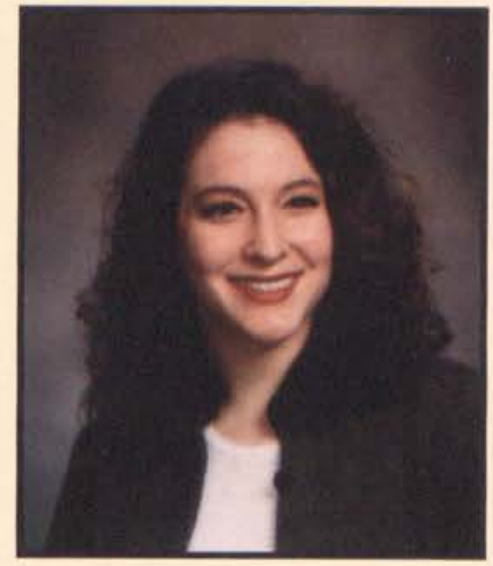

Christina Jackson

Communication Arts

Lewisberry, PA

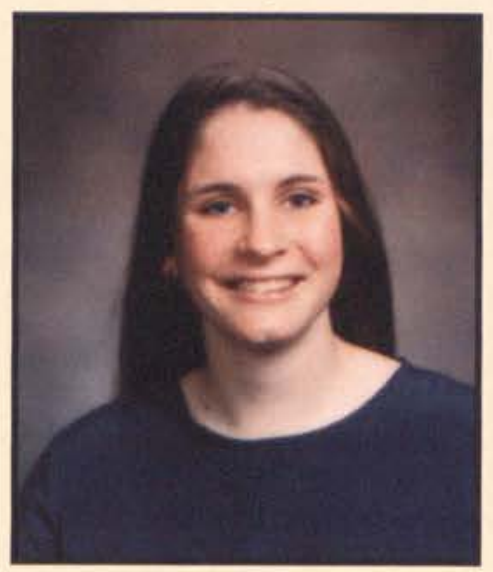

Sarah A. Jenks

Nursing

Bath, NY

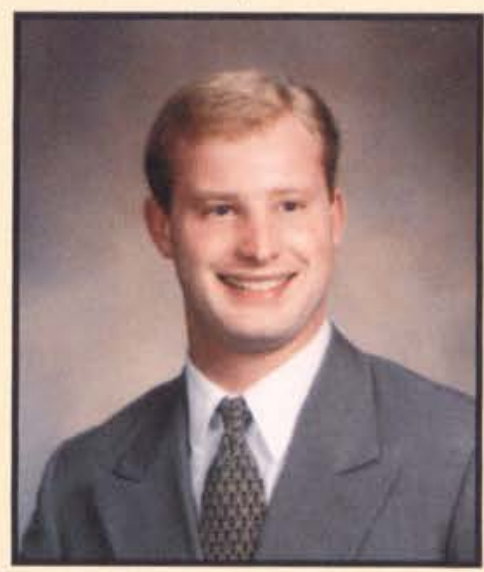

Christopher T. Jones, Jr.

Electrical Engineering

Reading, PA

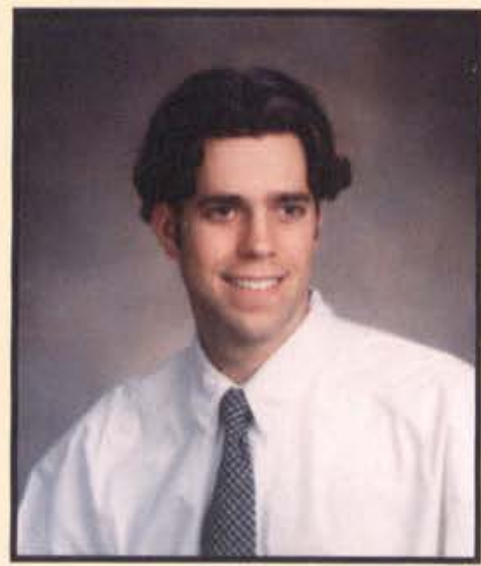

Ryan Junkins

Mechanical Engineering

Downingtown, PA

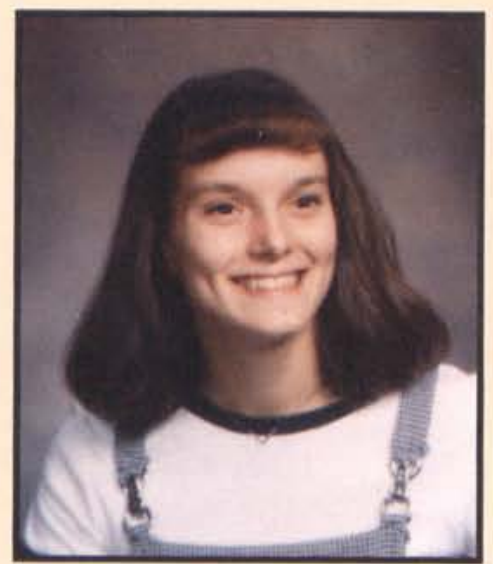

Sarah L. Jackson

Elementary Education

Mason, $\mathrm{OH}$

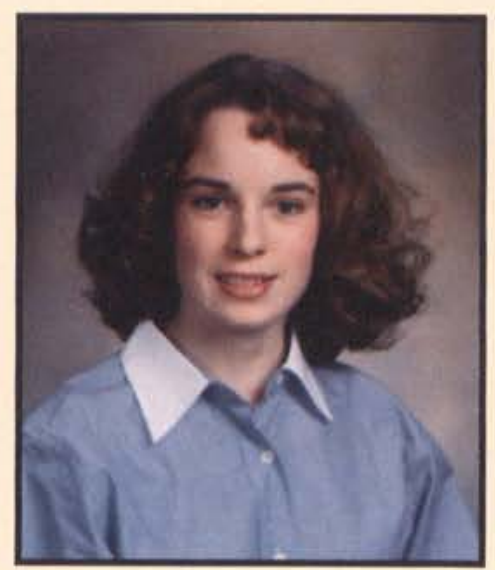

Angela Johnson

Elementary Education

Milford, $\mathrm{OH}$

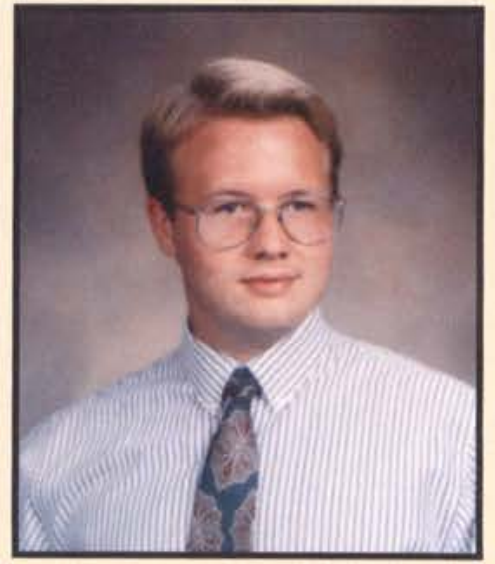

Kevin Jones

Spanish

Madrid, Spain

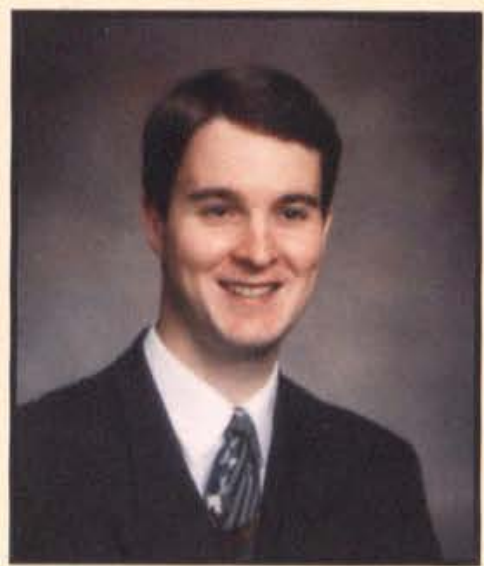

Adam M. Kane

Elementary Education

Daleville, IN

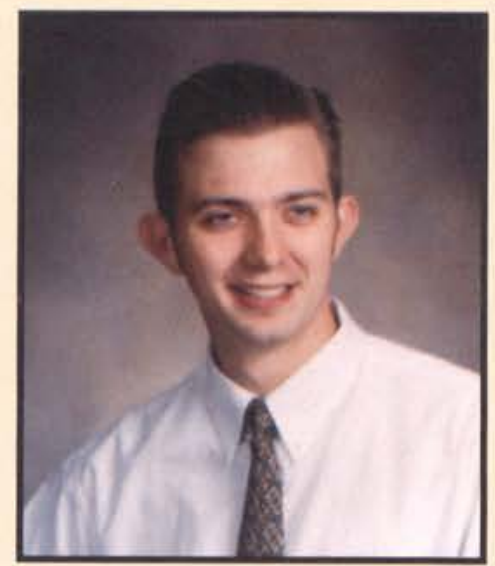

Nathan James

Electrical Engineering

Orem, UT

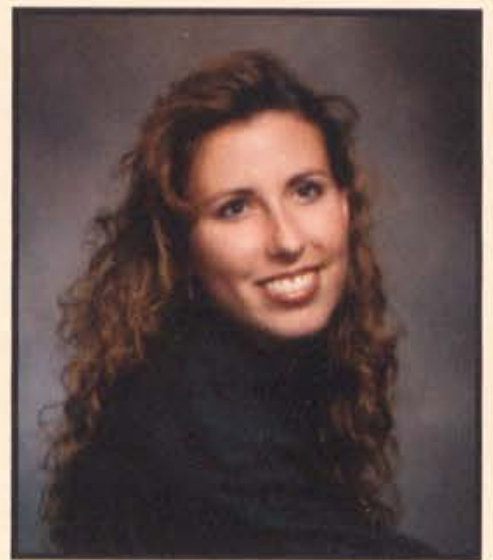

Jennifer Lee Johnson Nursing

Dennison, $\mathrm{OH}$

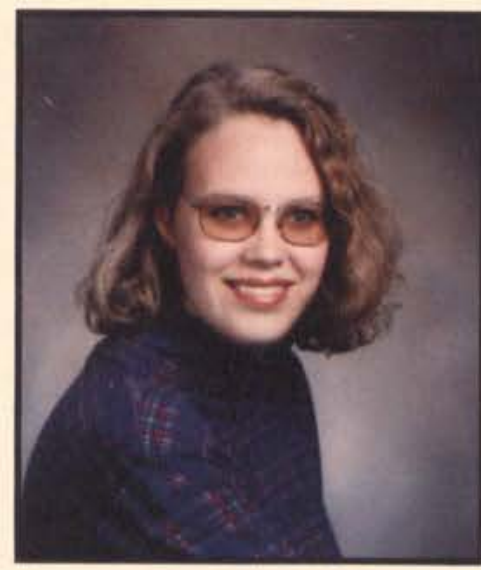

Sofie Jorstad

Elementary Education

Stromstad, Sweeden

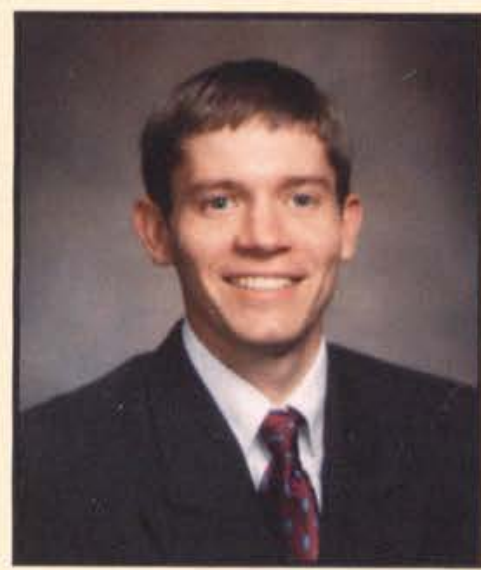

Benjamin M. Kanzeg

Social Science Comprehensive Columbus, $\mathrm{OH}$

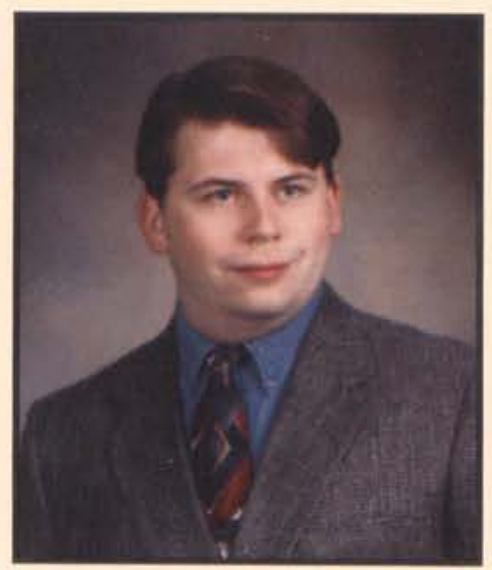

Chester J. Jenkins

Music Education

Anchorage, AK

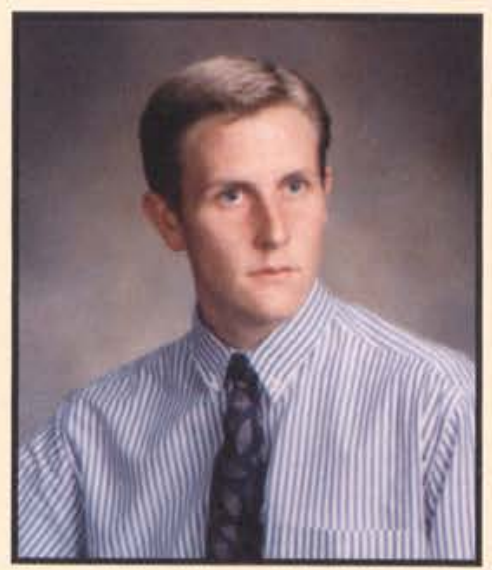

Brian S. Jones

Philosophy/Political Science

Madrid, Spain

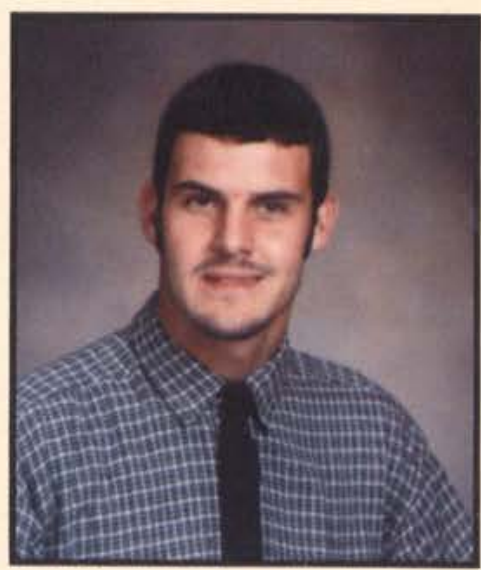

Scott Judkins

Electrical Engineering

Fairfax, VA

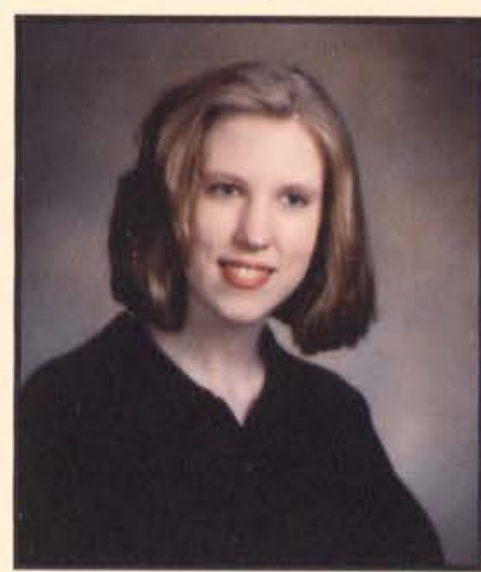

Susan Kauffman

English/Professional Writing

Cuyahoga Falls, OH 


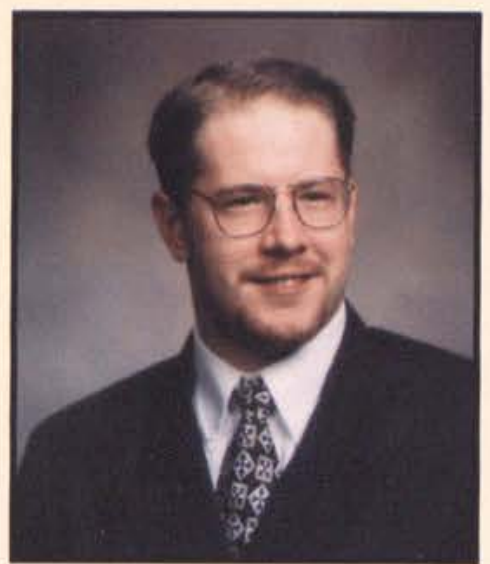

Jason Lehotsky

Finance

N. Jackson, $\mathrm{OH}$

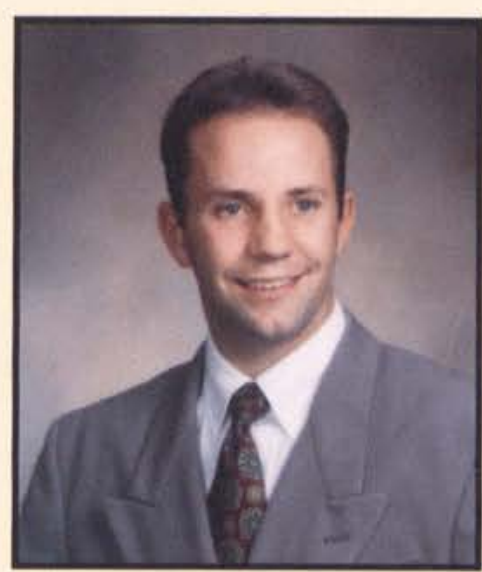

Andy G. Leslie

Physical Education

Belding, MI

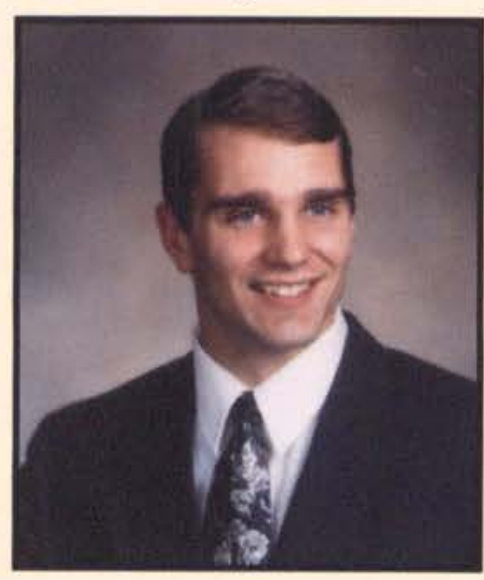

Todd M. Lozier

Bible Comprehensive

Sterling, NY

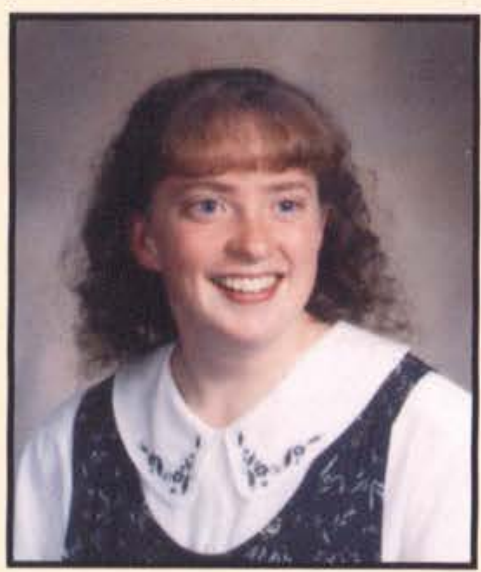

Heather Marie MacLean

Political Science

West Chester, PA

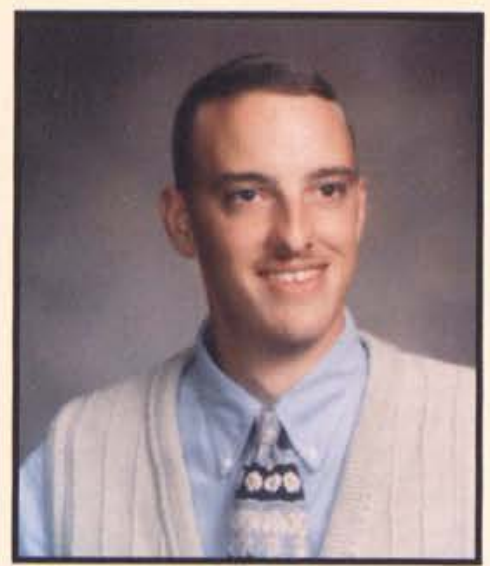

Thomas Leightenheimer

Bible Comprehensive

Cedarville, $\mathrm{OH}$

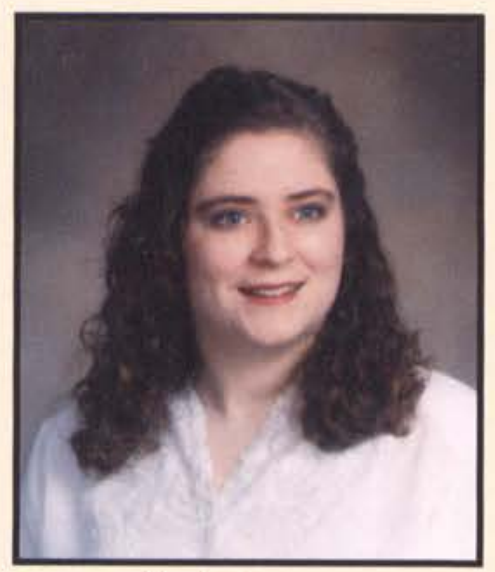

Angie Letson

Accounting/Finance

Computer Information Systems

Essexville, MI

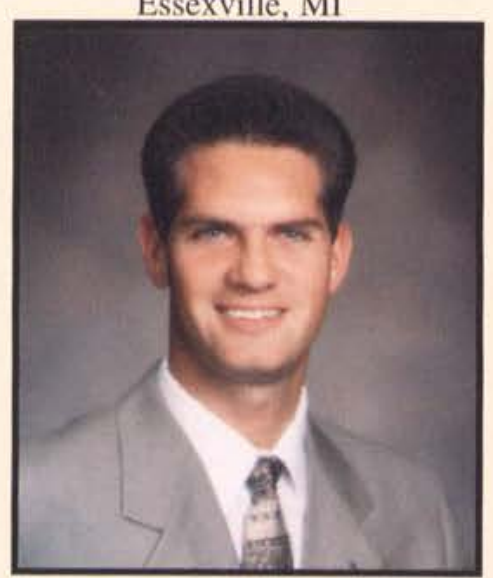

Fred S. Ludwig

Athletic Training

Brooklyn, NY

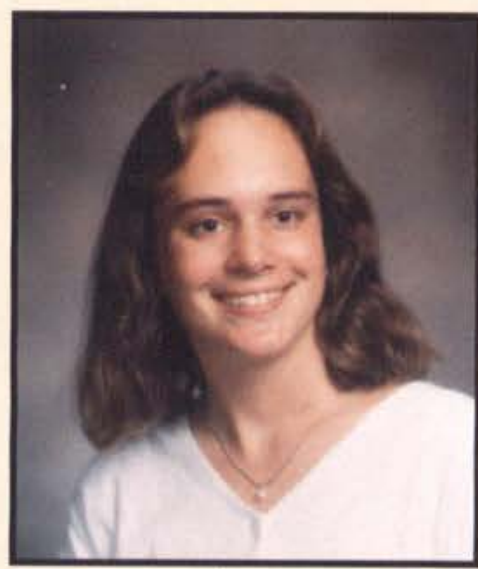

Jessica Erin MacPherson

Bible Comprehensive

Ghent, NY

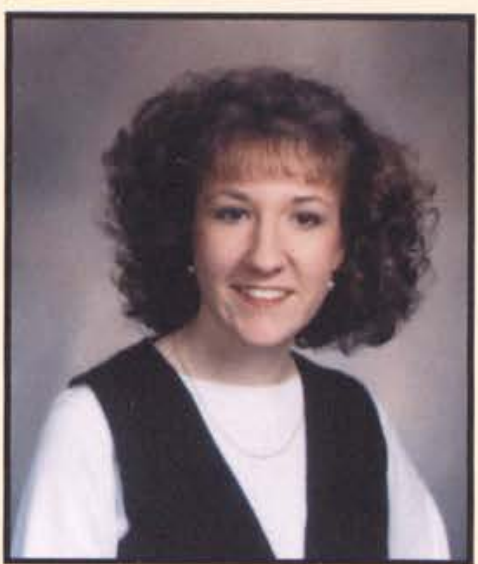

Karrie Lenhart

Psychology

Bible Comprehensive

Cincinnati, $\mathrm{OH}$

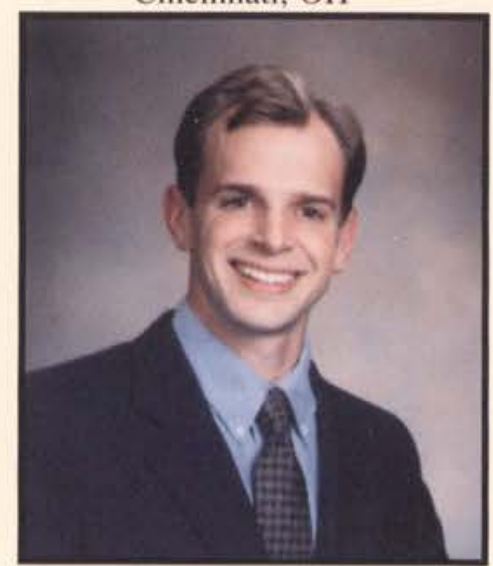

John Linak

Computer Information Systems

Westerville, $\mathrm{OH}$

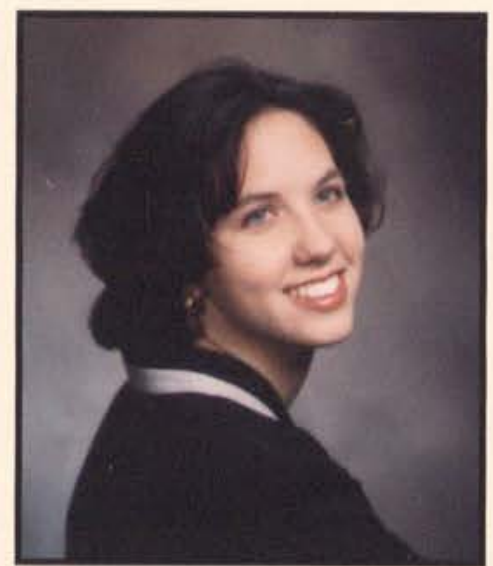

Sara Ludwig

Nursing

Brooklyn, NY

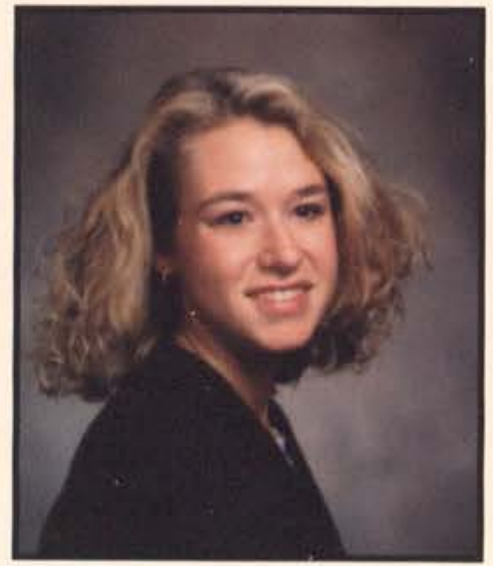

Shari Magin

Nursing

Troy, $\mathrm{OH}$

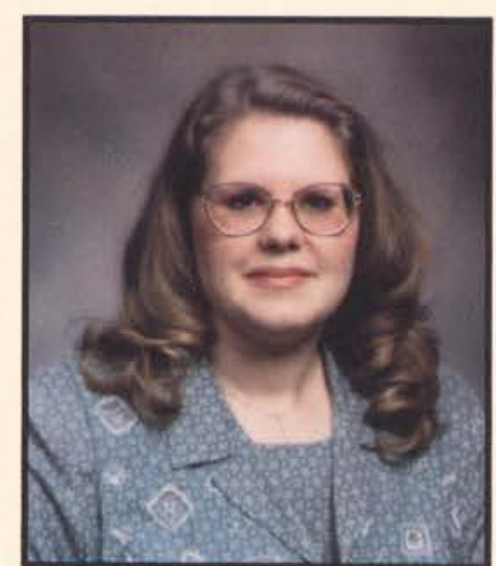

Lesa Andrea Lepak

Elementary Education

Mt. Airy, MD

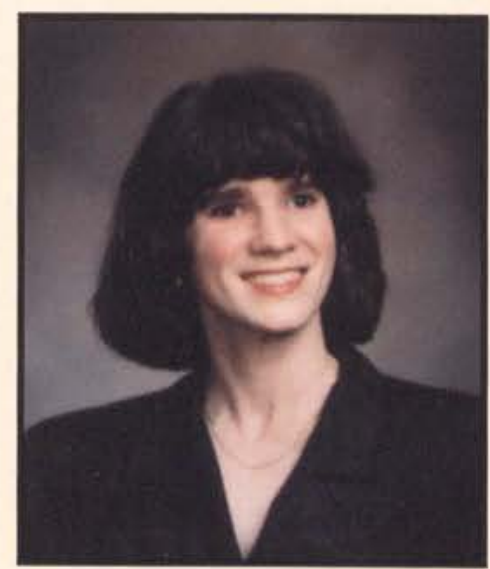

Betsy Lindaberry

Nursing

Clarks Summit, PA

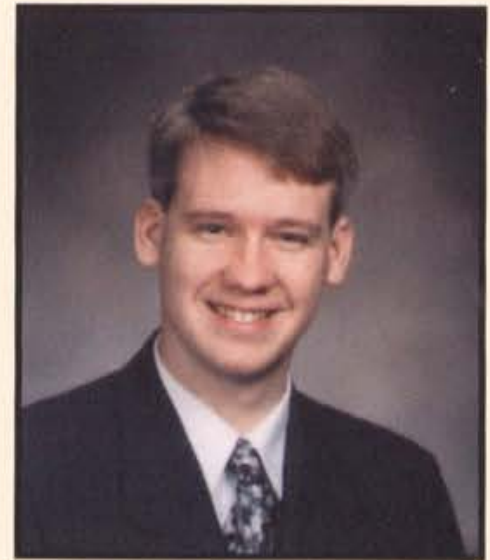

Robert D. MacDonald

Electrical Engineering

Cedarville, $\mathrm{OH}$

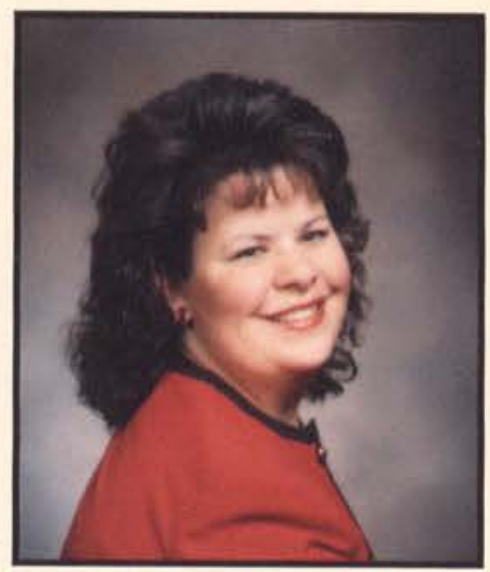

Laura Maki

Music Education

Poland, $\mathrm{OH}$ 


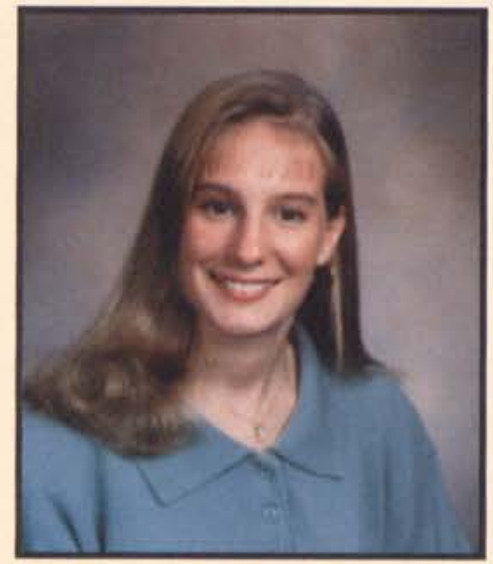

Danette McCullough

Elementary Education

Canton, OH

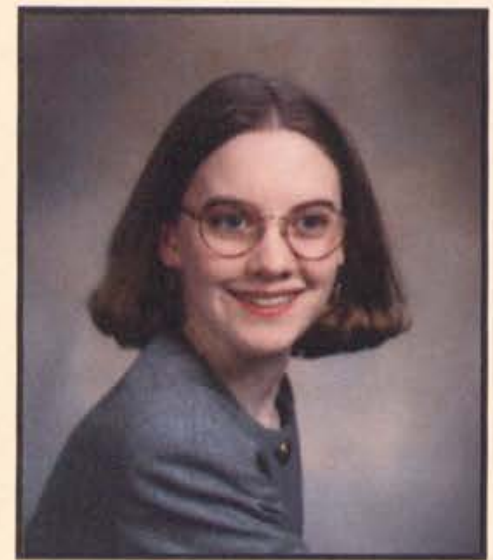

Megan E. McMacken

Professional Writing

St. Louis, MI

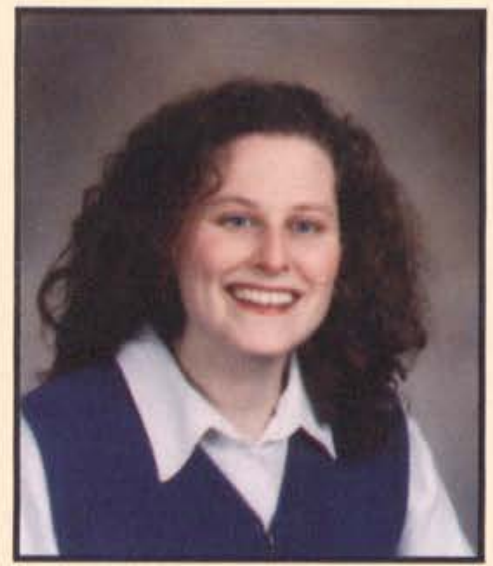

Julie R. Meeden

Nursing

Alton, IL

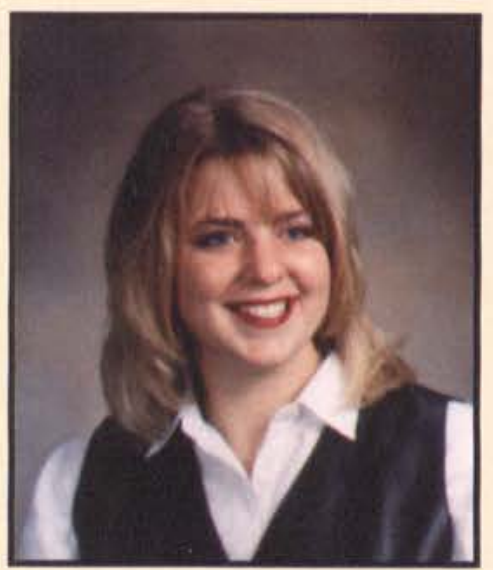

Billie Sue Michael

Nursing

Morgantown, WV

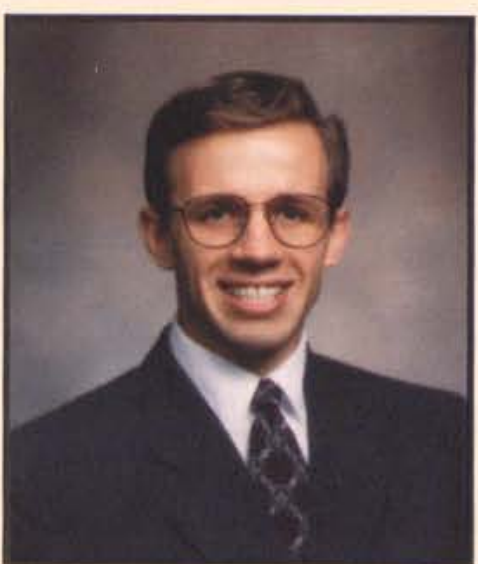

Chad R. McFadden

Bible/Pre-Seminary

Fortville, IN

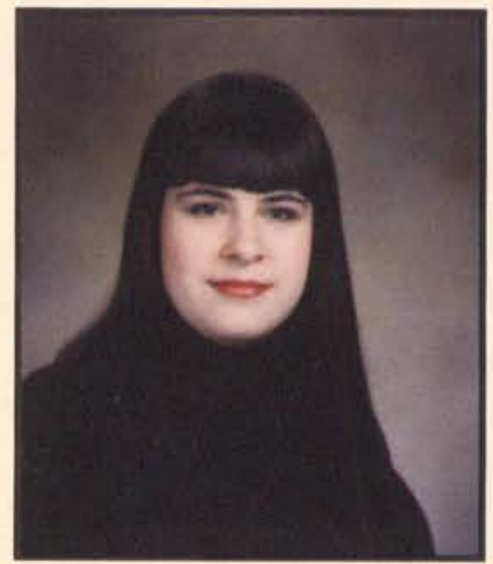

Nancy McMillan

Broadcasting

Maple Valley, WA

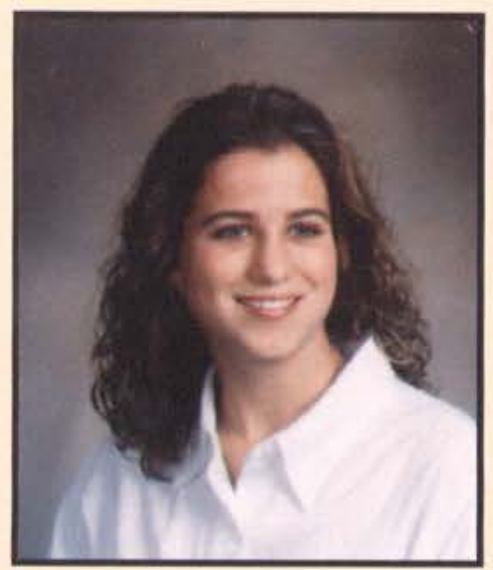

Sharla Megilligan Biology

West Chester, PA

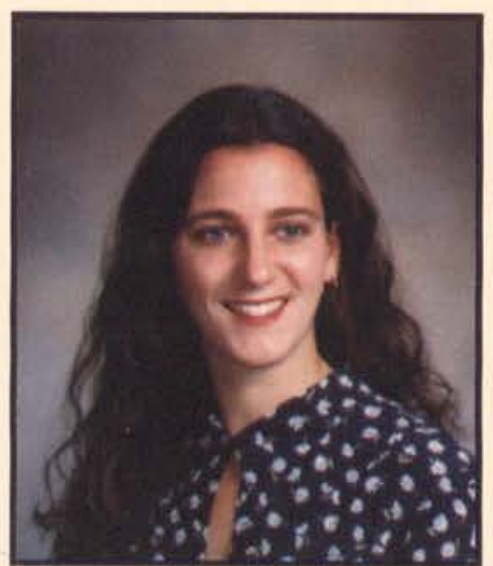

Amy Middleton

Elementary Education

Madison, $\mathrm{OH}$

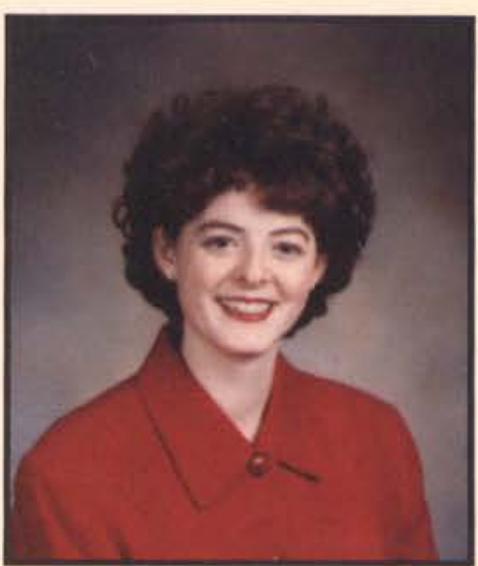

Dawn McGlothin

Nursing

Winona Lake, IN

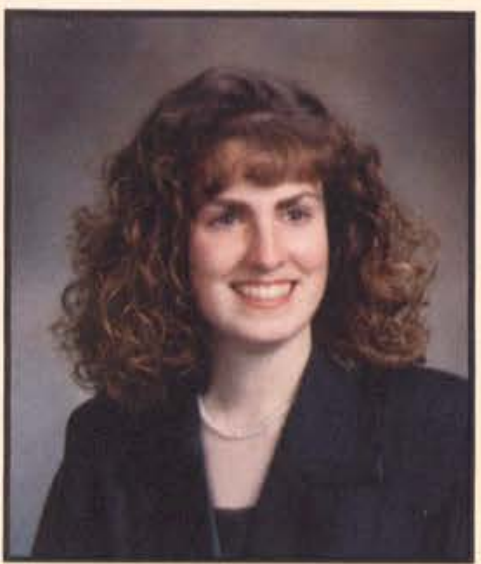

Stephanie Mead

Nursing

Fort Wayne, IN

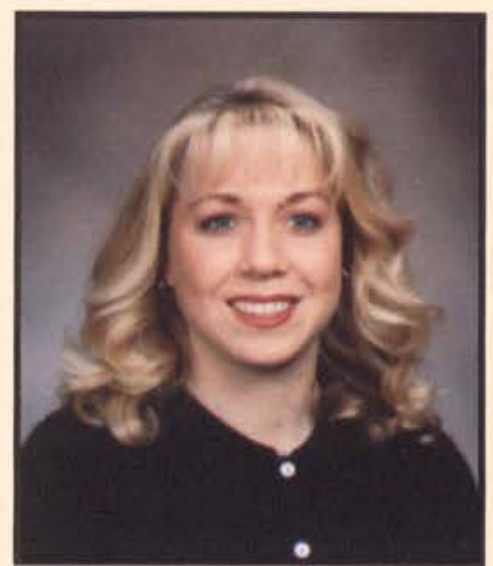

Monica Mennenga

Physical Education

Winter Park, FL

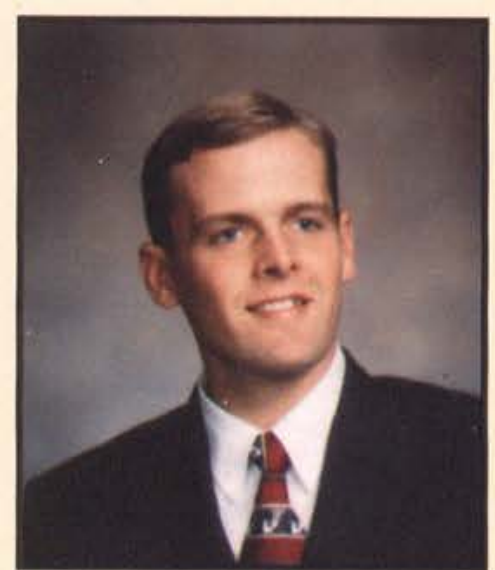

Andrew Stephen Miller Finance/Accounting

Gurnee, IL

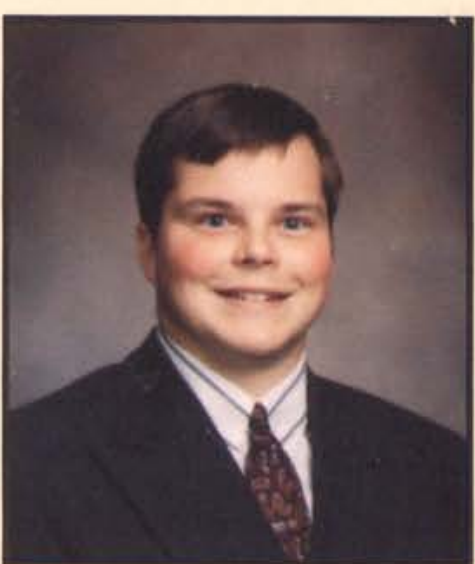

Brandon McGuire

Finance/Accounting

Roanoke, VA

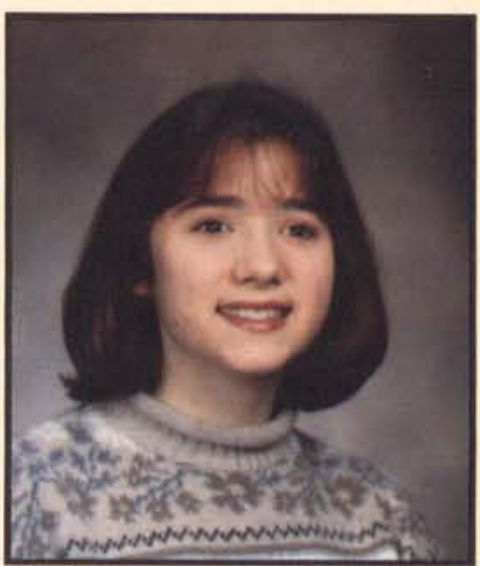

Sarah Medlong

Elementary Education

Parma, $\mathrm{OH}$

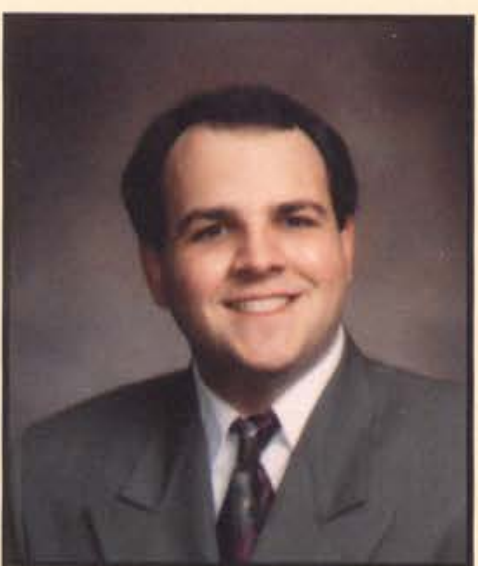

Matthew Seth Meyer

Multimedia Technology

Millerstown, PA

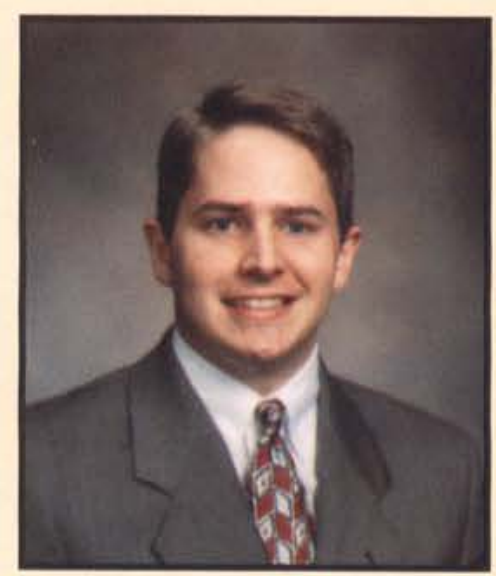

Ben Miller

English

Westerville, $\mathrm{OH}$ 


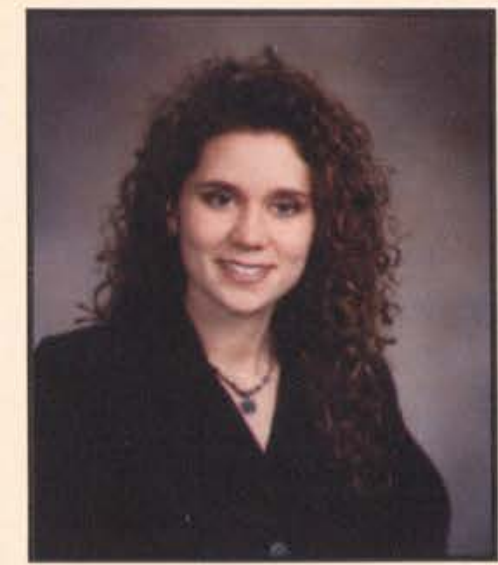

Cara A. Miller

Nursing

Van Wert, $\mathrm{OH}$

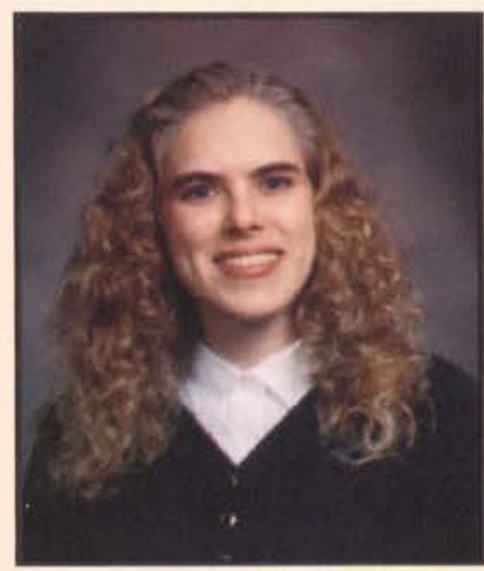

Libby Miner

Professional Writing

Cedarville, $\mathrm{OH}$

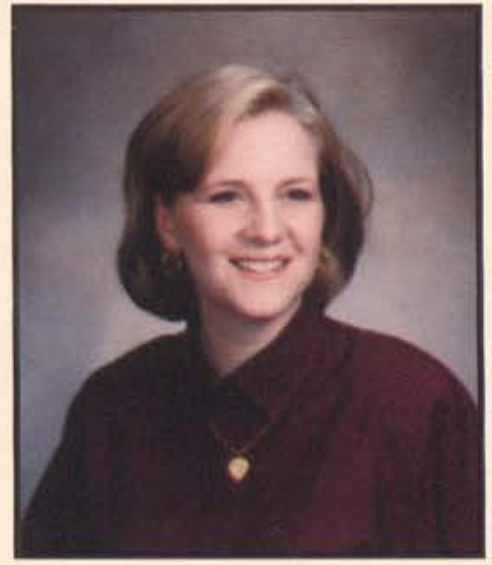

Karin P. Moon

Nursing

Akron, $\mathrm{OH}$

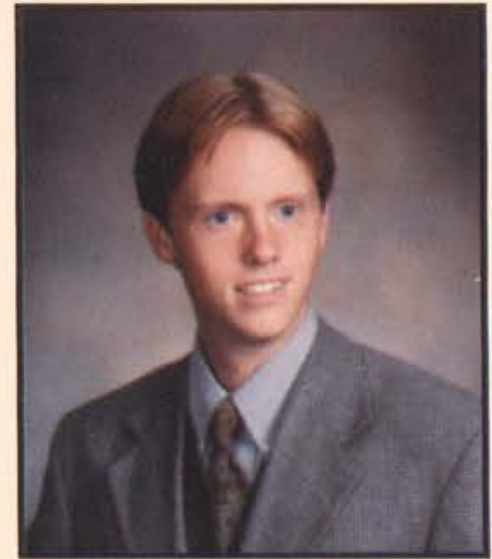

Steve Moulson

Bible/Pre-Seminary

Konyers, GA

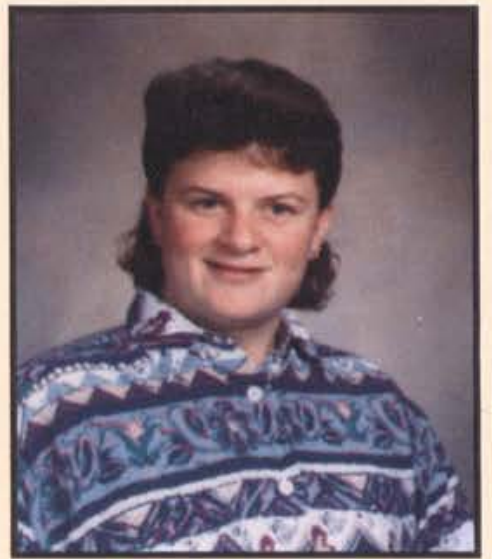

Erika D. Miller

Physical Education

Monroeville, $\mathrm{OH}$

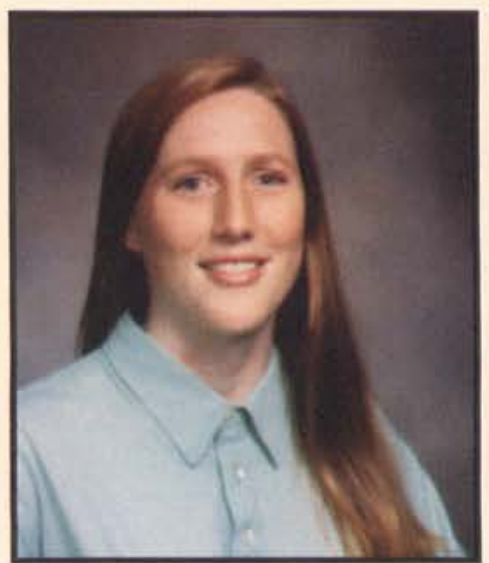

Donna Mitchell

Nursing

Calpine, CA

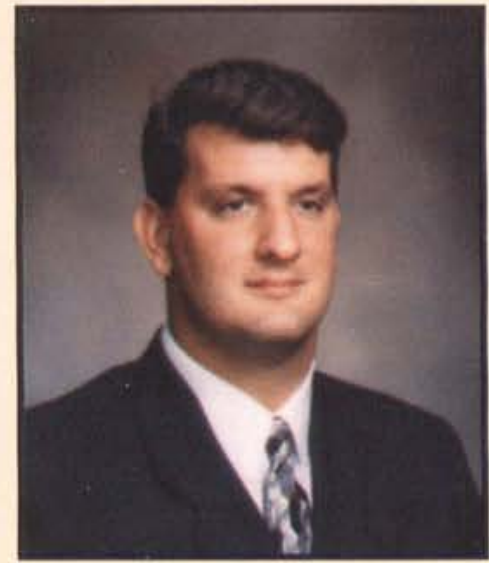

Michael M. Morris, II

Bible Comprehensive

North Collins, NY

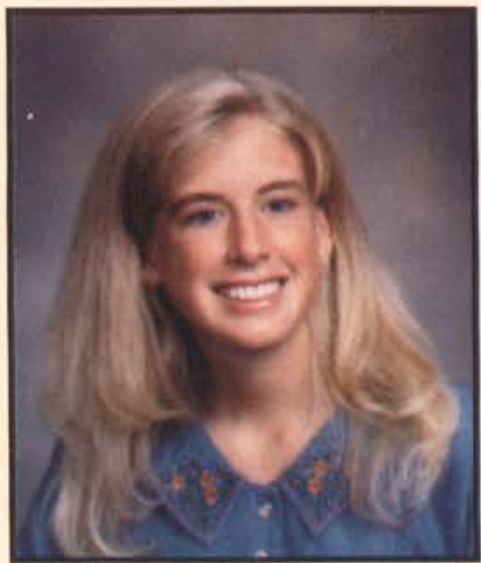

Amanda Mudrey

Biology

Ormond Beach, FL

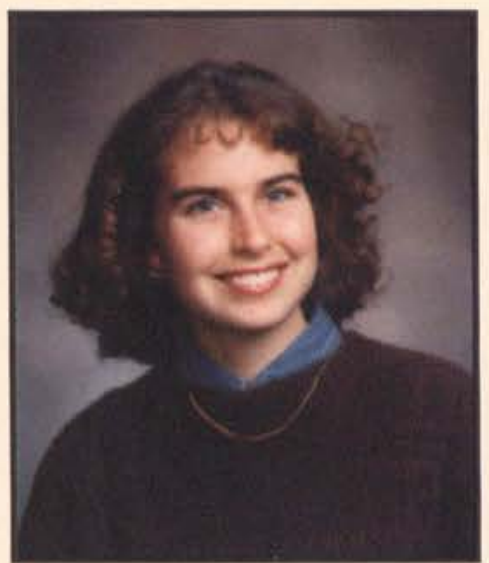

Kimberly Joy Miller

Music/Keyboard Pedagogy

Sioux Falls, SD

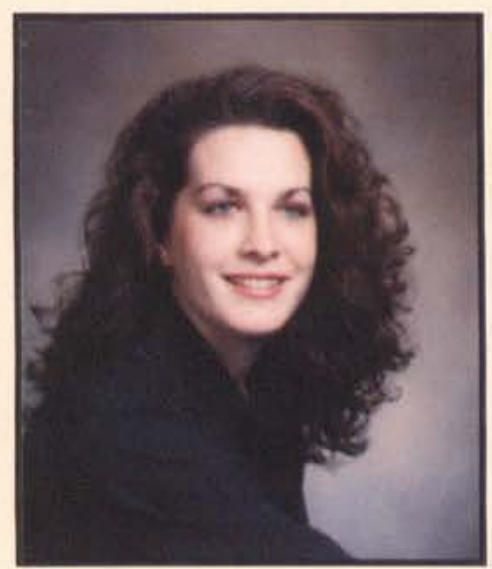

Kristi L. Mitchell

Nursing

Jamestown, $\mathrm{OH}$

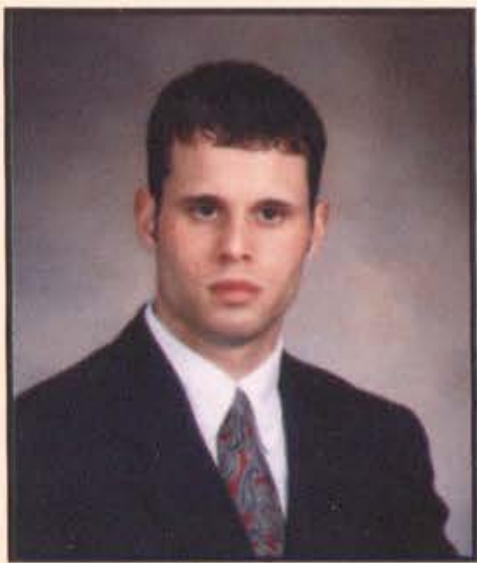

Mark G. Moseley

Chemistry

Lima, $\mathrm{OH}$

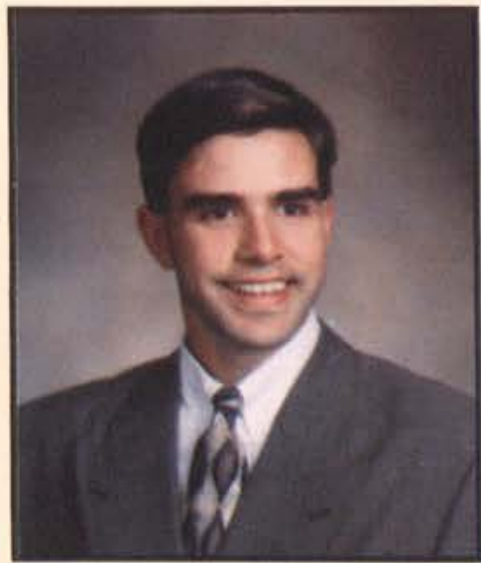

Travis Mulanax

Math Education

Canton, $\mathrm{OH}$

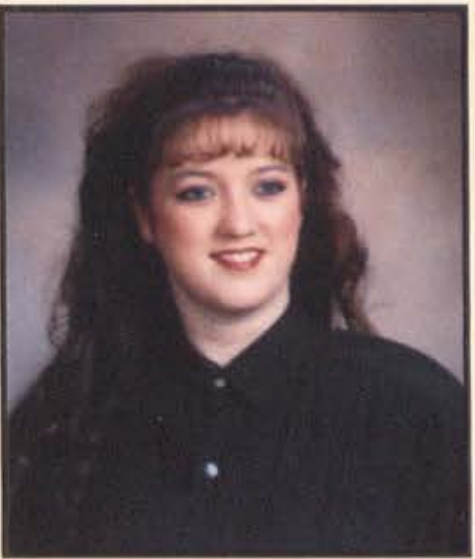

Linda Miller

Elementary Education

Caro, MI

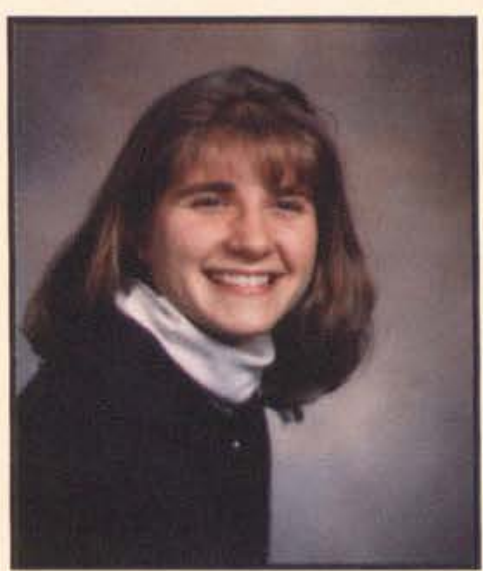

Winona J. Monge

Nursing

Woodstock, IL

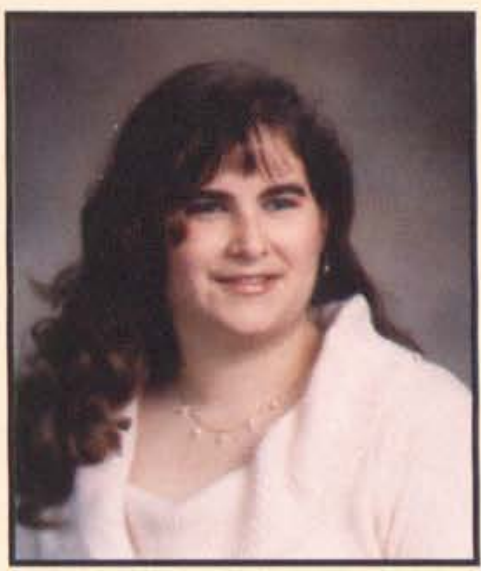

Brenda Sue Moss

Elementary Education

Cedarville, $\mathrm{OH}$

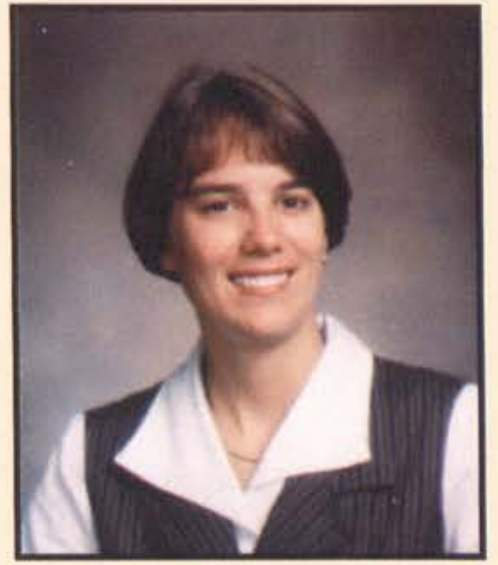

Amanda K. Murray

Bible/C.E. Youth

Gallipolis, $\mathrm{OH}$ 


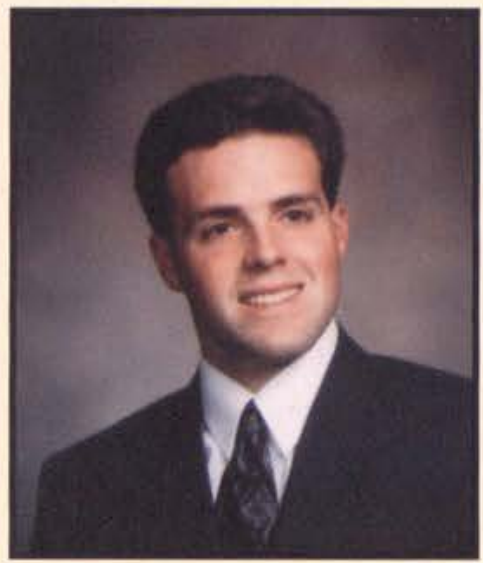

Bradford Nelson

Accounting/Finance

Rochester, MN

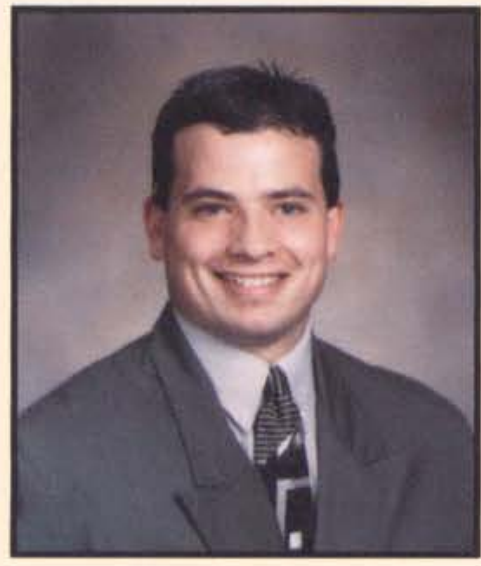

Jason J. O'Dell

Elementary Education

Evart, MI

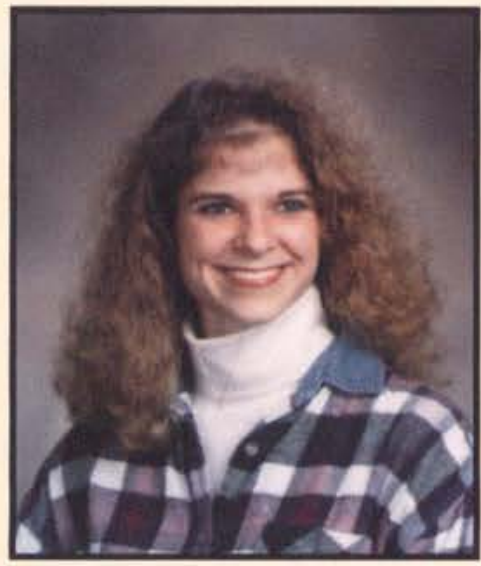

Rachel (Rogers) Olson

Elementary Education

Cedarville, $\mathrm{OH}$

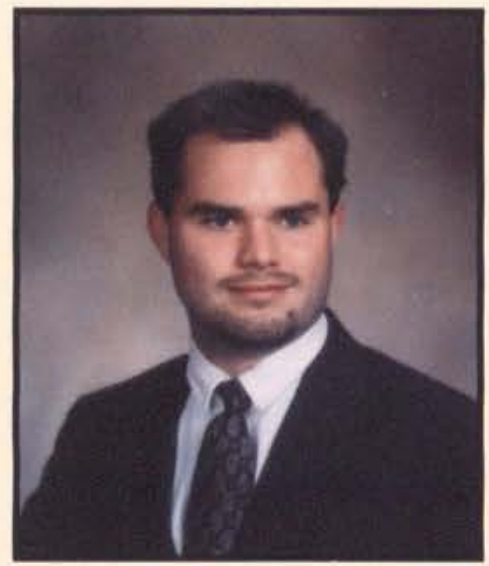

Tim Otton

English

Xenia, $\mathrm{OH}$

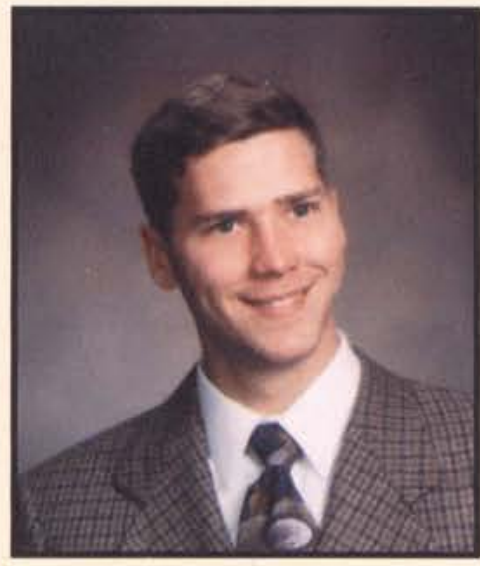

Joshua Lincoln Nelson English Education

Ithaca, NY

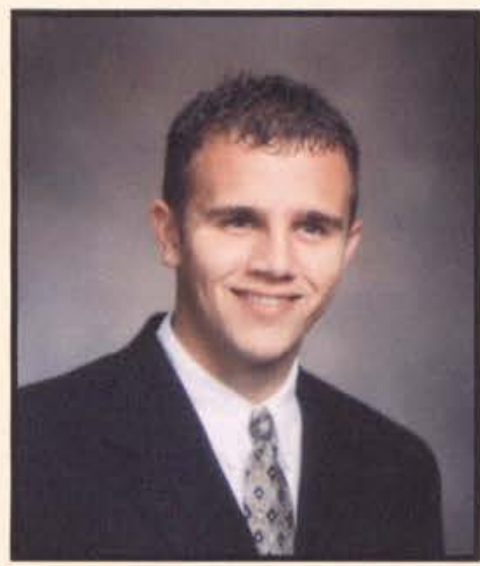

Nathaniel D. Ober

Marketing/Finance

Des Moines, IA

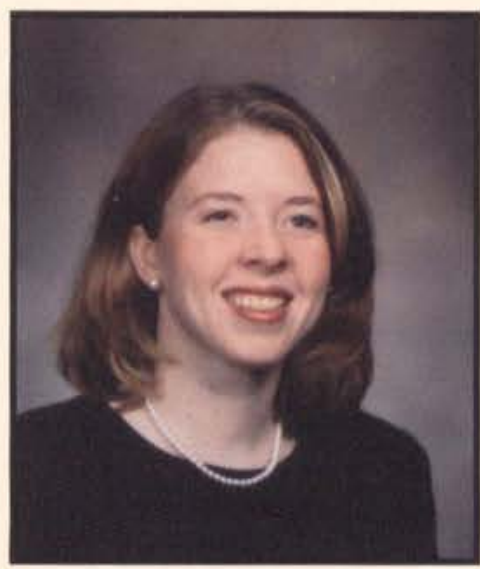

Ellen Oncu

Elementary Education

Lakeland, FL

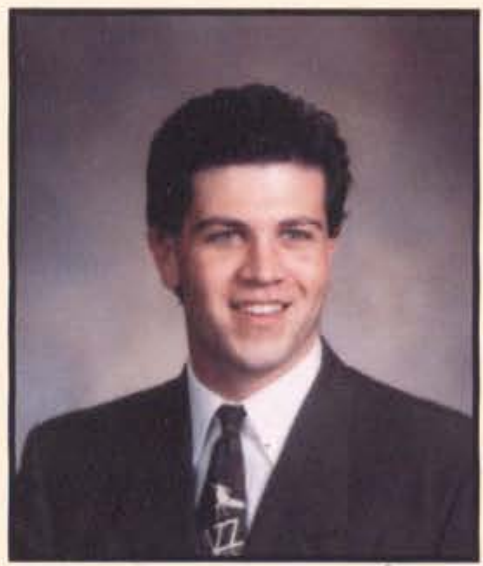

Christian Pagnard

Music Performance

Centerville, $\mathrm{OH}$

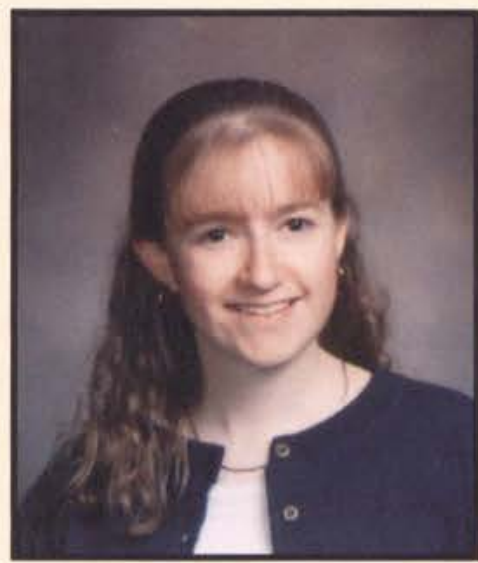

Traci L. Niehaus

Accounting

Maytown, PA

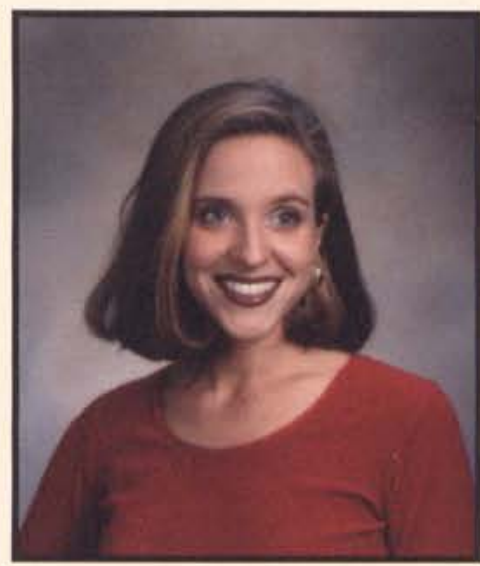

Jennifer Anne Olexa English

Westerville, $\mathrm{OH}$

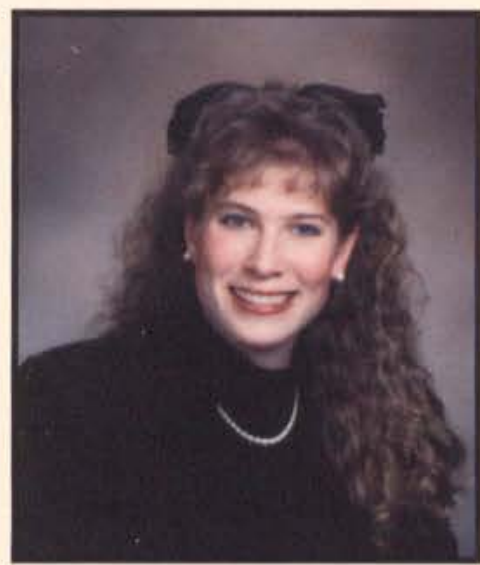

Amanda Orme

Nursing

Chattanooga, TN

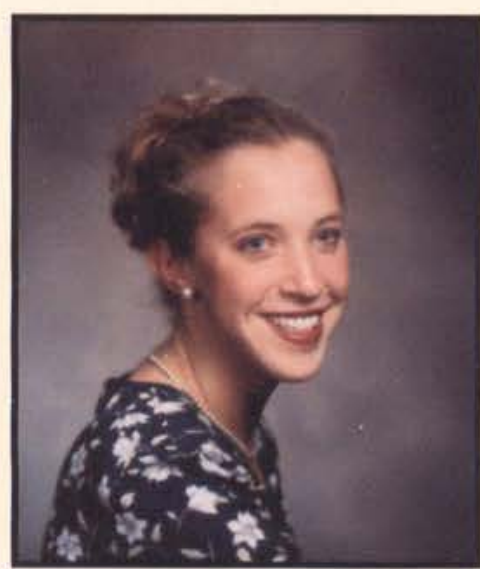

Marianne Elizabeth Palmer

Nursing

Columbus, $\mathrm{OH}$

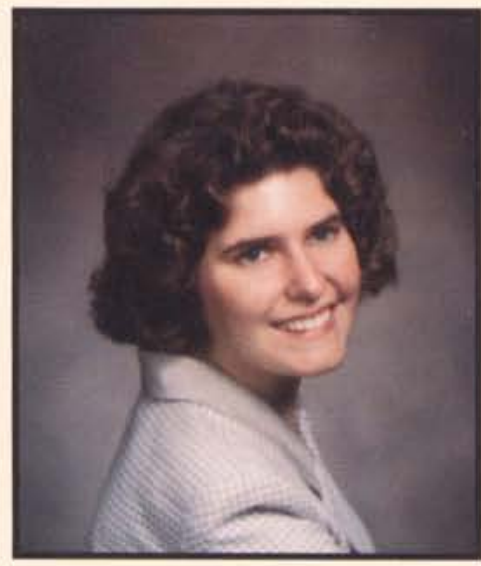

Laura E. O'Bern

Elementary Education

Waterford, CT

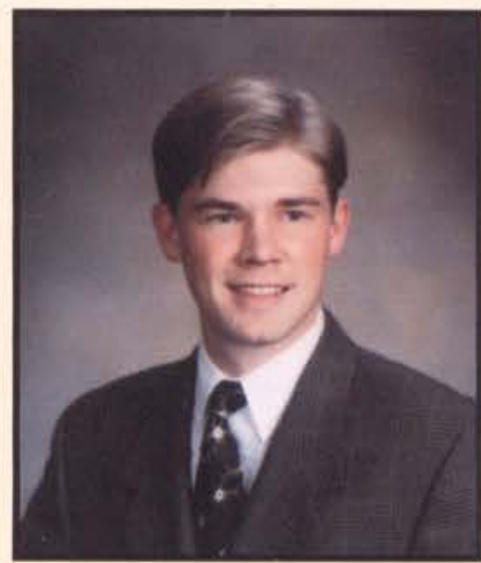

Samuel J. Olsen, II

Biology

Dayton, TN

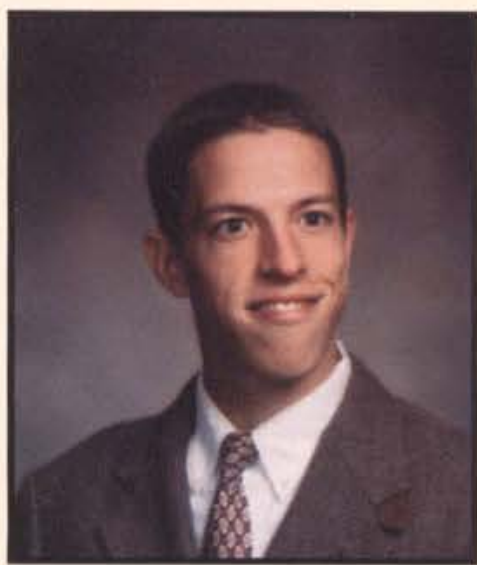

Jason Ostrander

Pre-Seminary

Southampton, PA

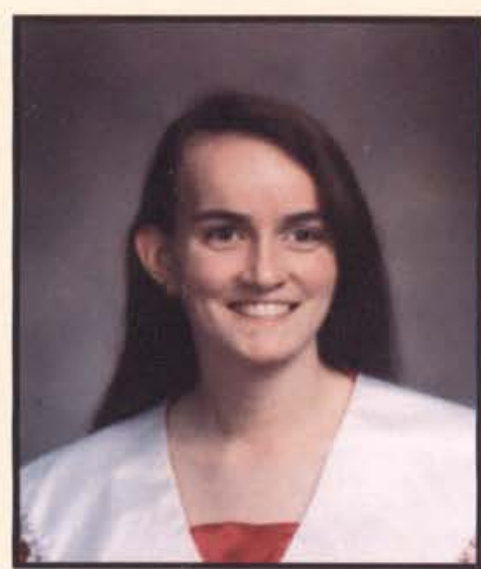

Debra Palmier

Elementary Education

Broadalbin, NY 


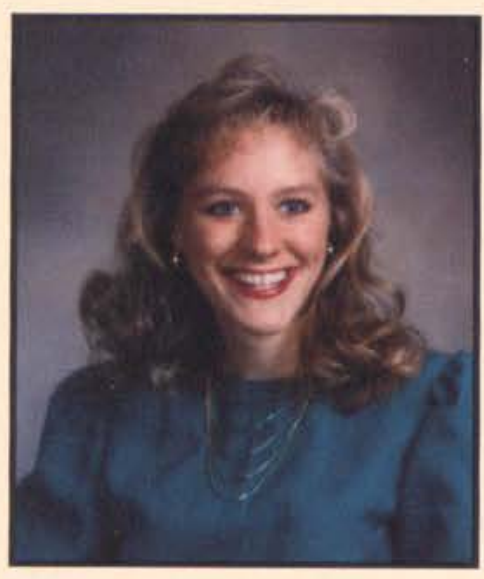

Julianne Pletcher

Elementary Education

Tacoma, WA

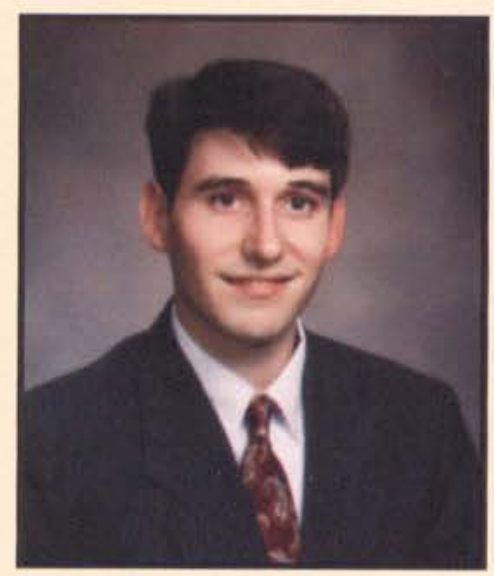

Edward Powers

Electrical Engineering

Glen Burnie, MD

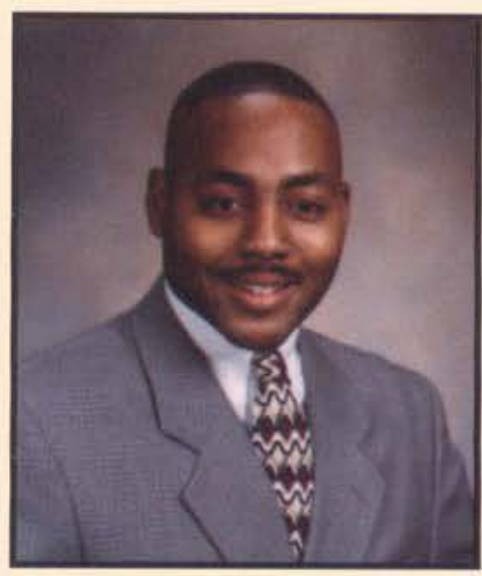

Charles Quarles

Music/Communication Arts

Richmond, VA

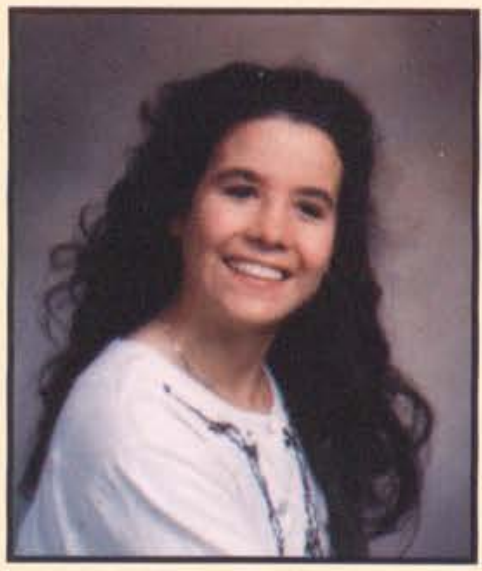

Janelle Reis

Elementary Education

Haslett, MI

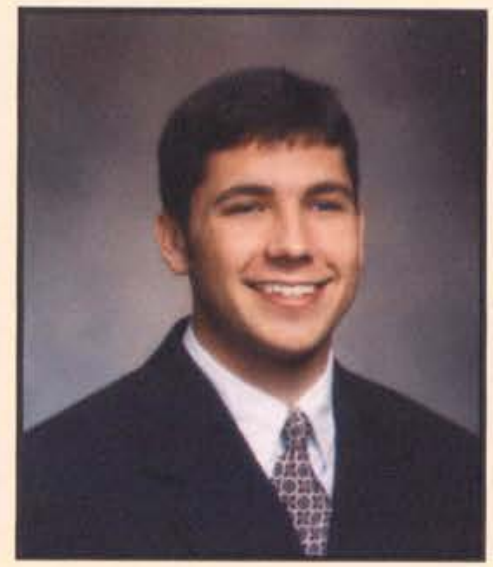

Jason Poling

Communication Arts

Troy, $\mathrm{OH}$

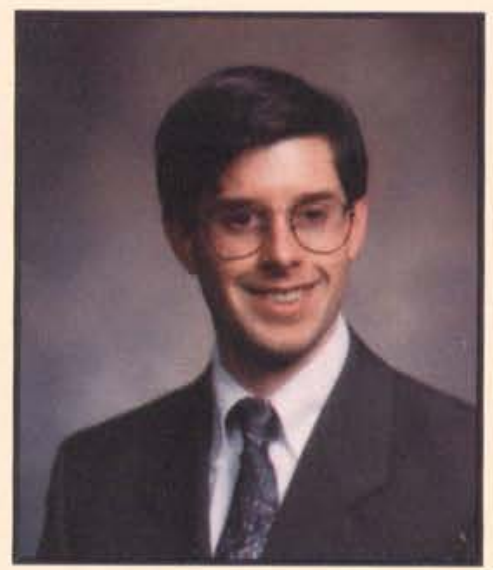

Chris Prediger

Marketing

Dublin, $\mathrm{OH}$

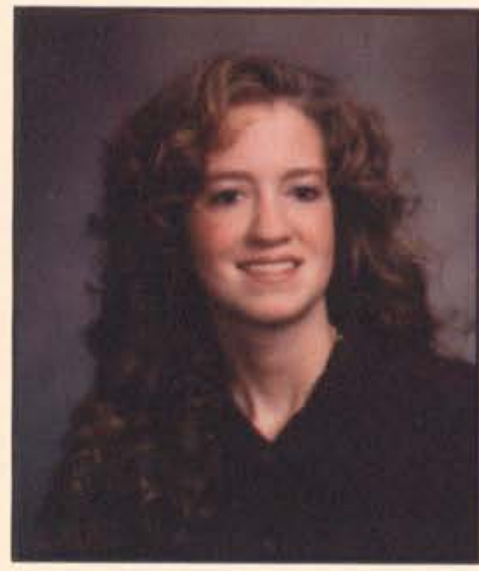

Shari Lynn Read

Nursing

Curwensville, PA

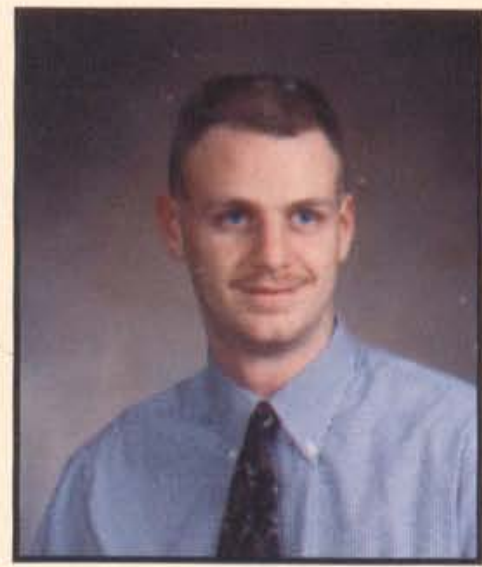

Stephen Reutlinger

Multimedia Technology

Cedarville, $\mathrm{OH}$

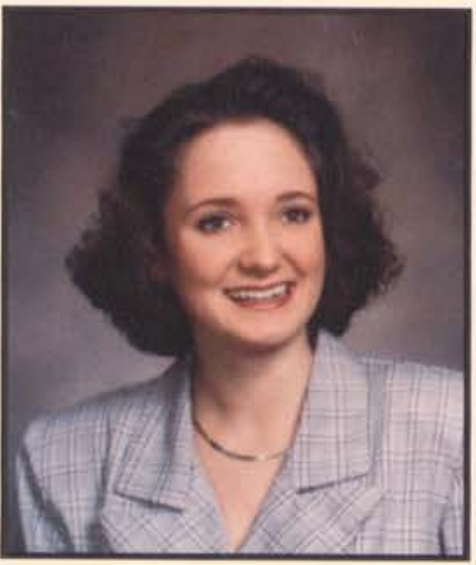

Meredith Pollard Nursing

Gallipolis, $\mathrm{OH}$

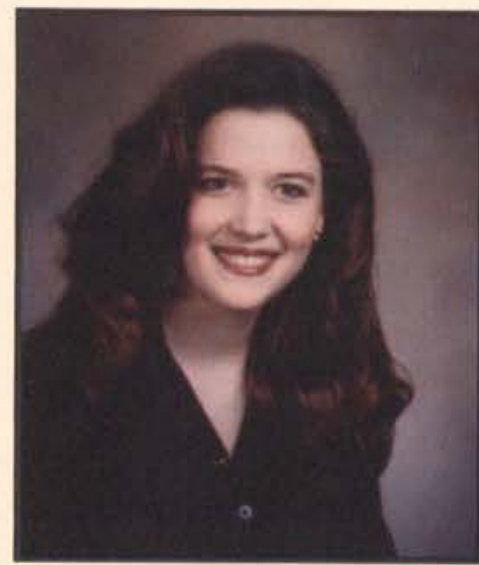

Krista Price

Elementary Education

Caledonia, MI

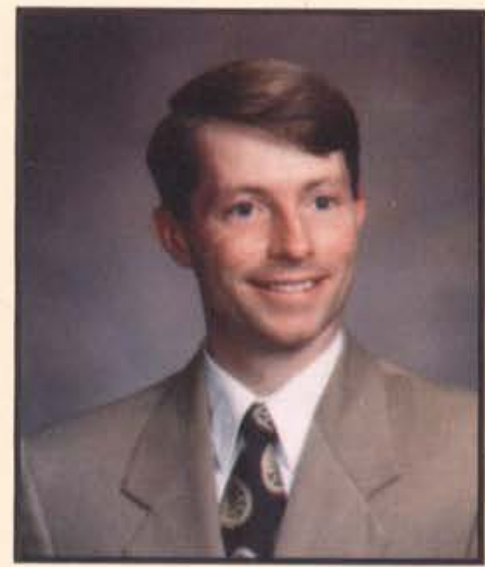

Robert W. Reich

Biology

Quito, Ecuador

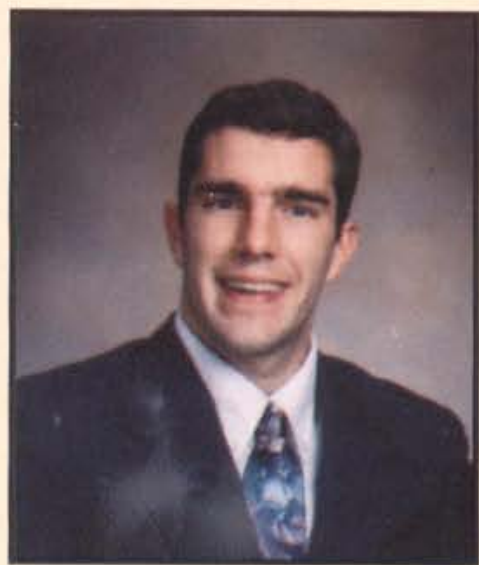

John Dayton Richard

Biology Education

Pre-Physical Therapy

Havertown, PA

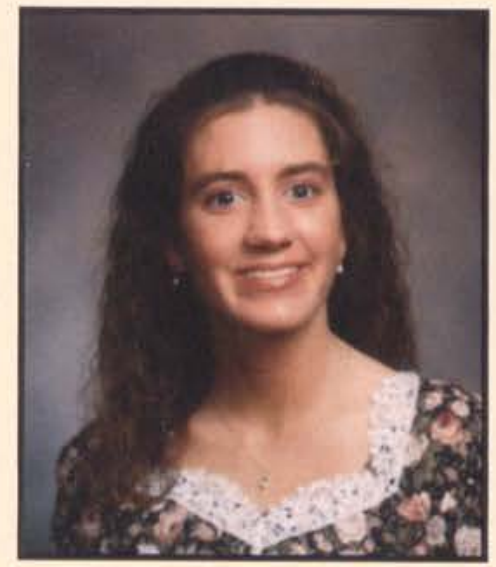

Sarah Powell

Elementary Education

Fort Wayne, IN

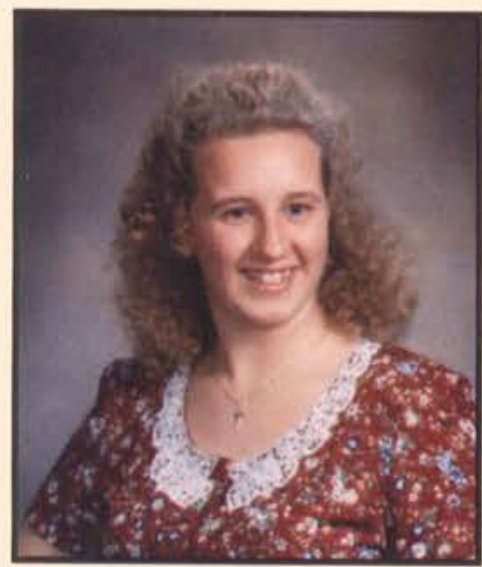

Nicole Pryor

Accounting

Jefferson, MI

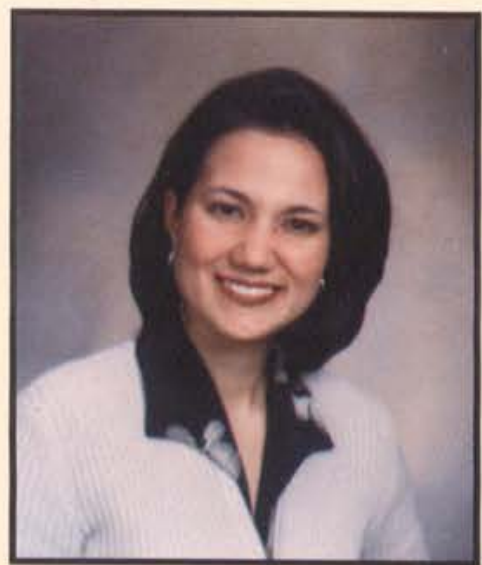

Jennifer Leah Reilly

Communication Arts

Randolph, NJ

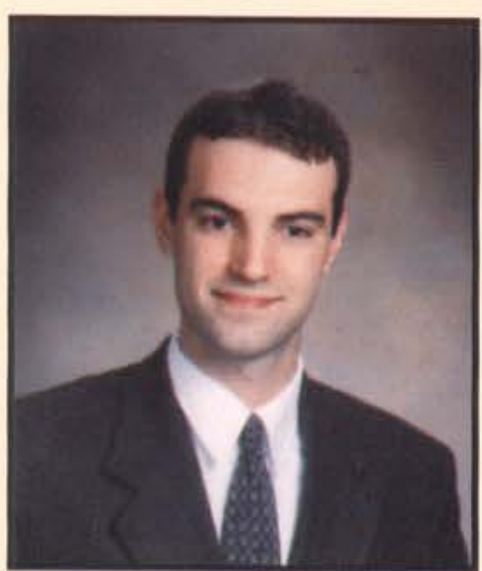

Thomas Noah Richards Bible Comprehensive

Muscatine, IA 


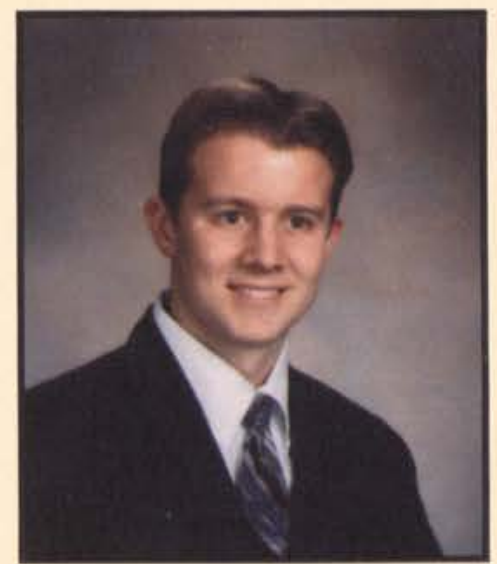

Brent Ridley

Mechanical Engineering

Flint, MI

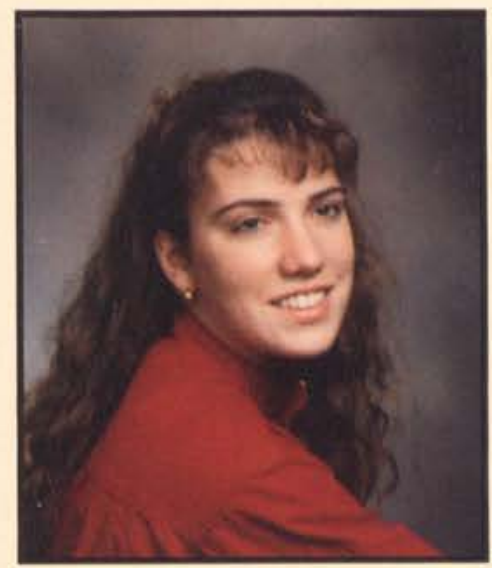

Jennifer L. Rogers

Mulitmedia Technology

Vestal, NY

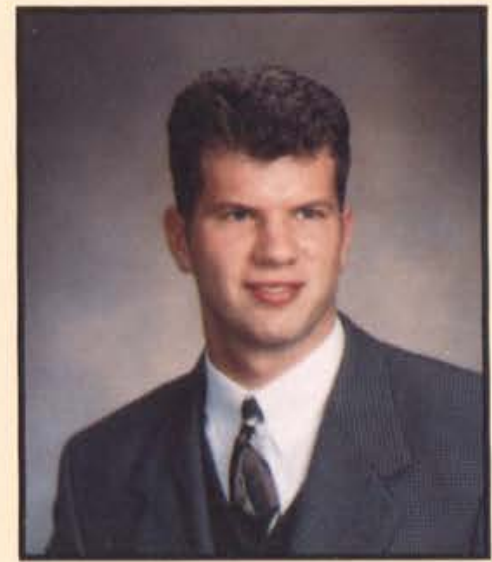

Daniel Ruba

Mechanical Engineering

Philadelphia, PA

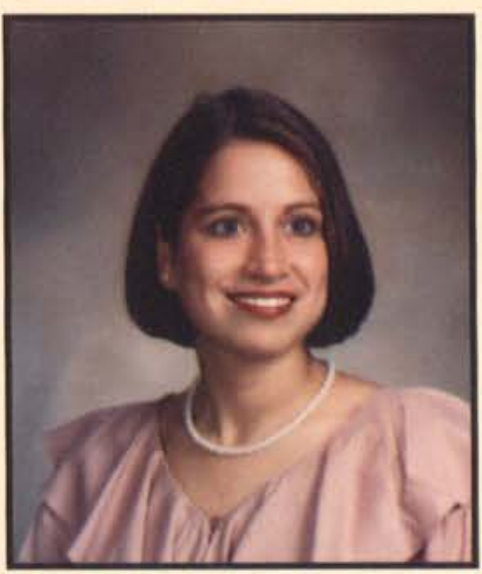

Monica Ruth

Elementary Education

Marion, $\mathrm{OH}$

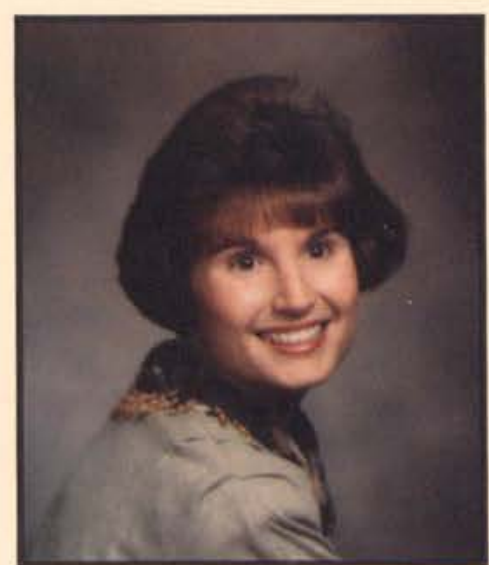

Heather J. Ritchey

$$
\text { Nursing }
$$

Swiftwater, PA

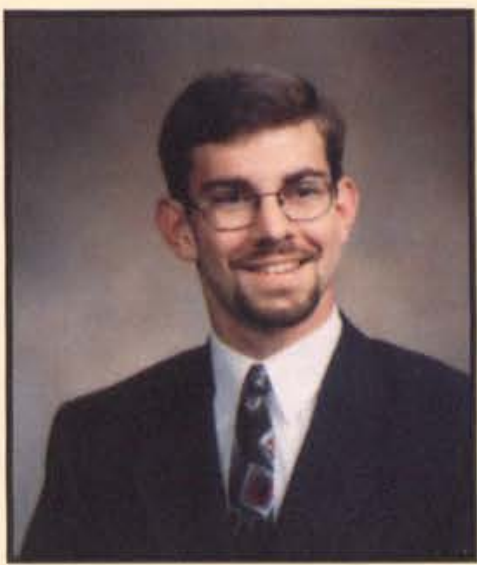

Douglas James Romaine

Social Science

North Plainfield, NJ

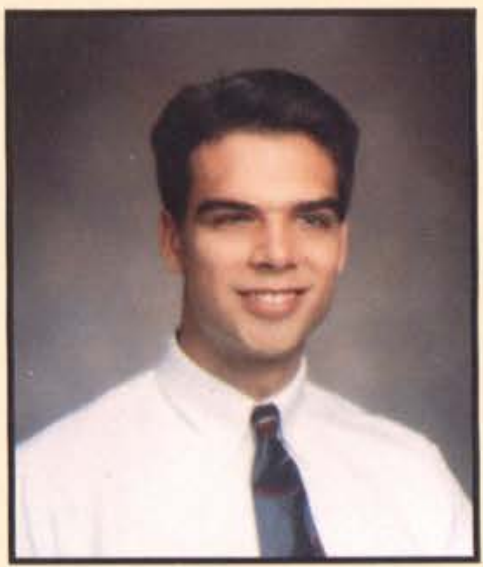

Christopher C. Rudolph

Accounting/Finance

Clarks Summit, PA

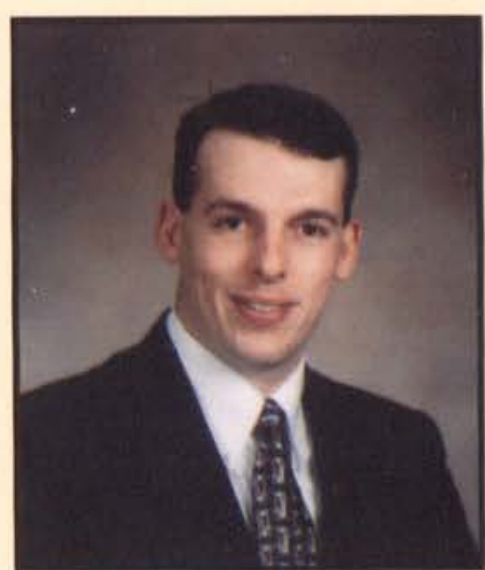

Thomas R. Sagraves

Bible Comprehensive

Springfield, $\mathrm{OH}$

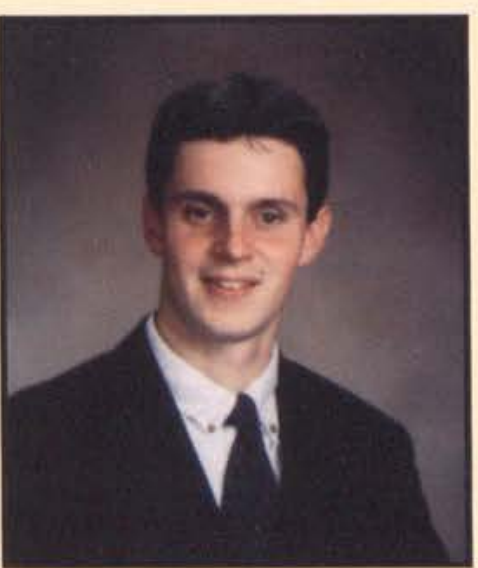

Jesse A. Roberts

Mechanical Engeneering

Milton, VT

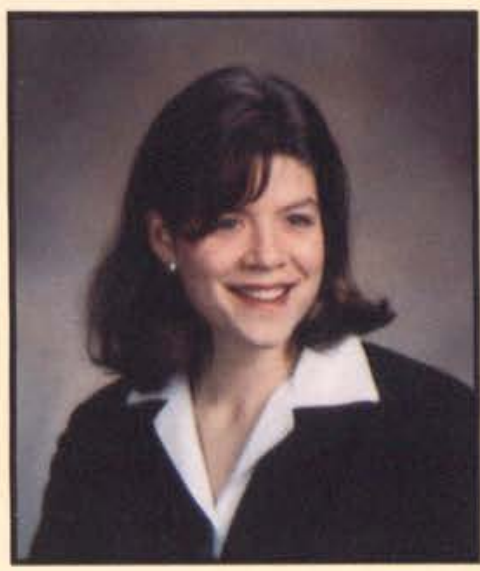

Elizabeth Roseboom

Organizational Communications Peoria, IL

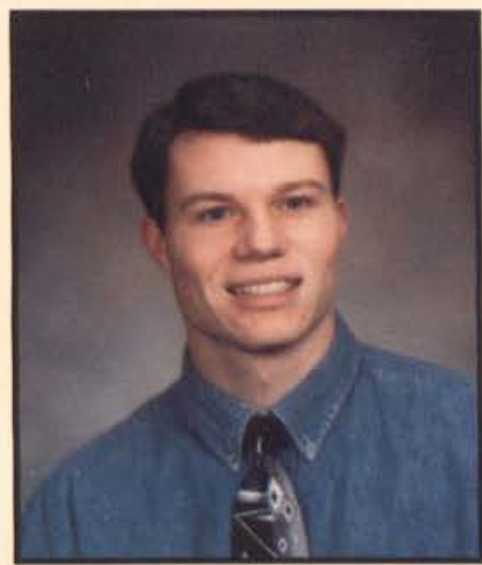

Zach Ruffin

Electrical Engineering

Grand Lake, $\mathrm{CO}$

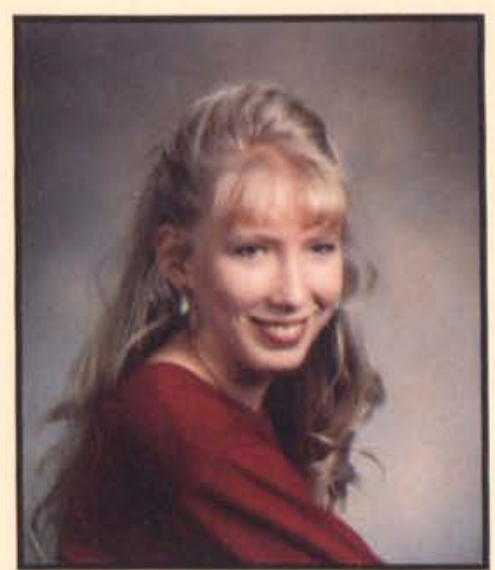

Melinda M. Salyers

$$
\text { Nursing }
$$

Springfield, $\mathrm{OH}$

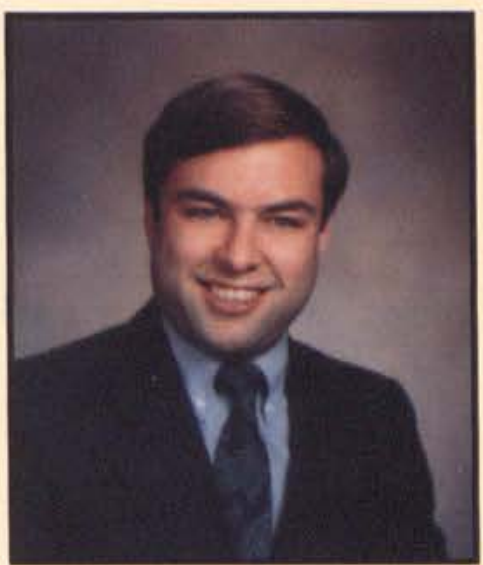

Gordon Rogers

Health Psychology

Westmont, IL

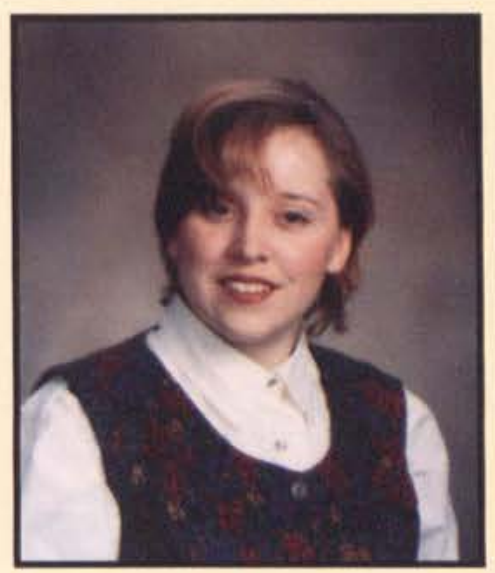

Rachel Elizabeth Ross Biology

Wellington, $\mathrm{OH}$

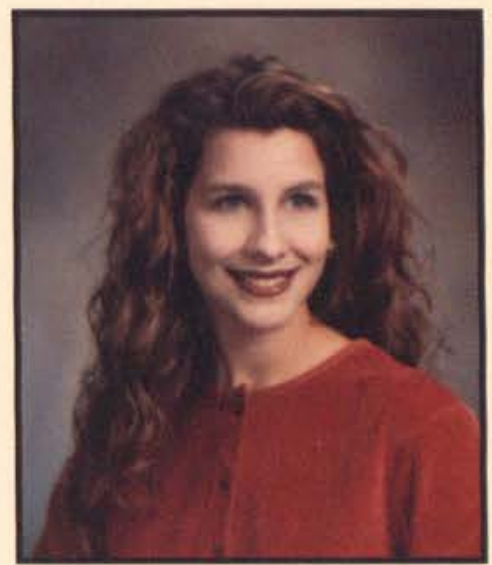

Kimberly Ruhl

Computer Information Systems Derry, NH

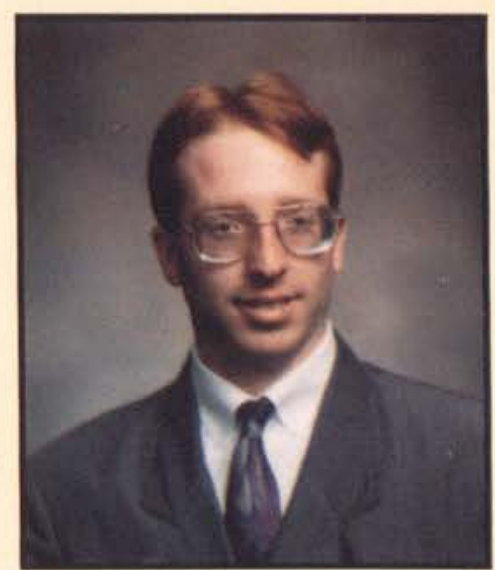

Jeff Saunders

Electrical Engineering

Racine, W 


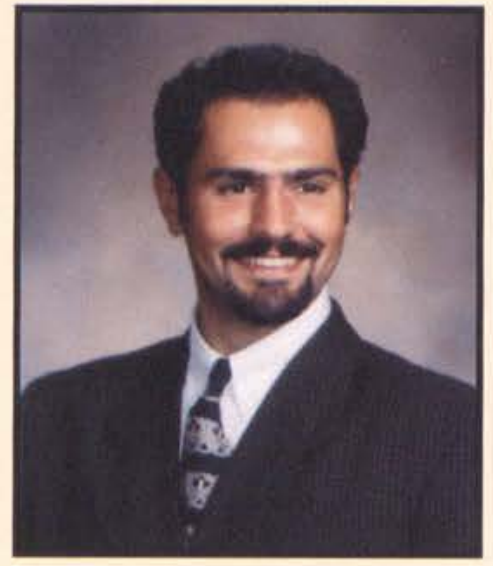

Gabriel Sava

Mechanical Engineering

Woodbridge, VA

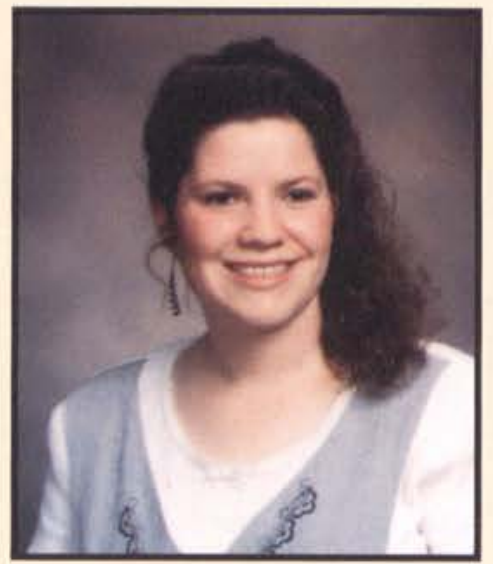

Ivy Schlesener

Nursing

Laurel, MT

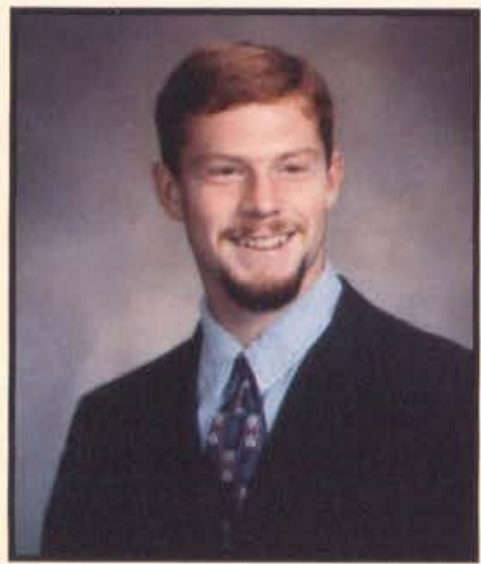

Cliff Scott

Accounting/Finanace

Olean, NY

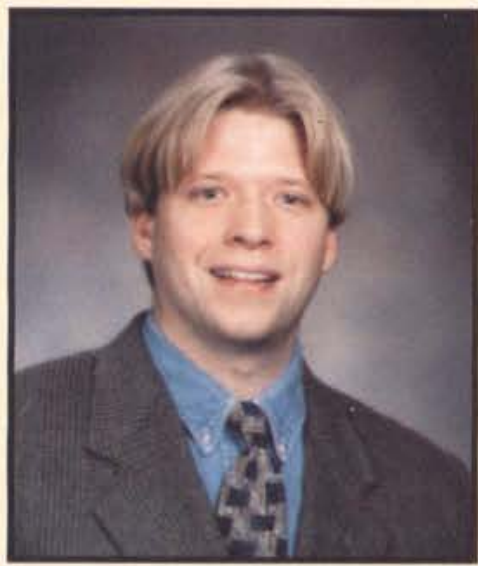

Jason P. Shaffer

Political Science

Lima, $\mathrm{OH}$

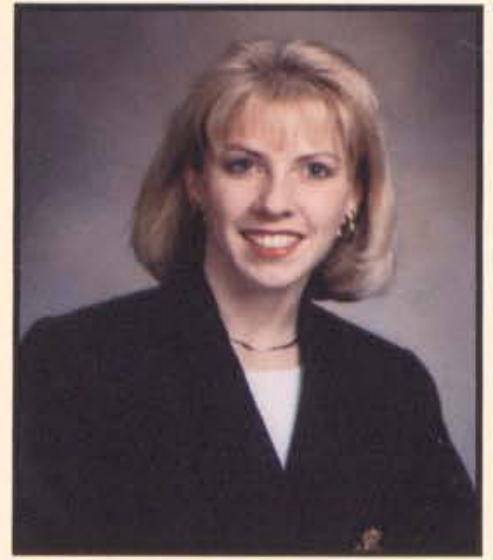

Rachel Savage

Nursing

Germantown, TN

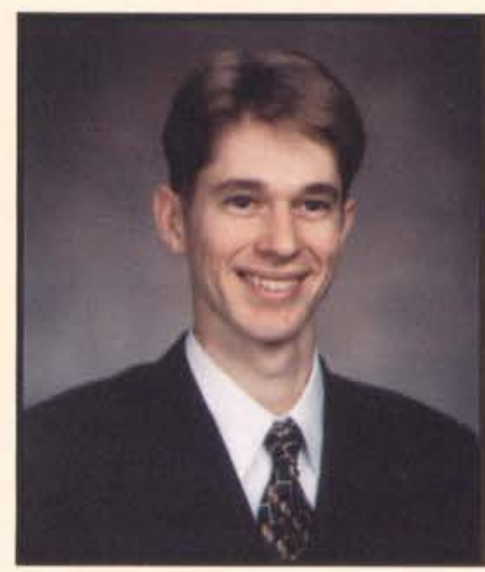

Aaron Jon Schradin

Mechaincal Engineering

Hamilton, $\mathrm{OH}$

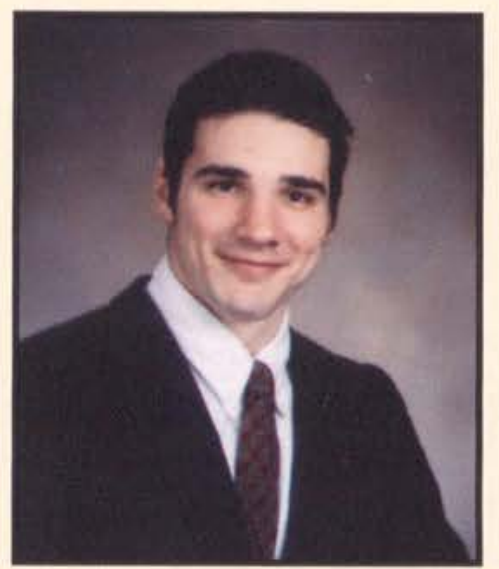

R. Andrew Seefried

Biology

Cedarville, $\mathrm{OH}$

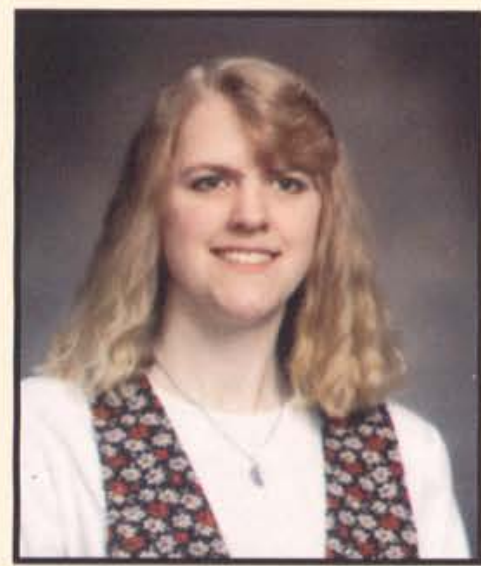

Rachel A. Sharp

Finance/Accounting

Aurora, $\mathrm{OH}$

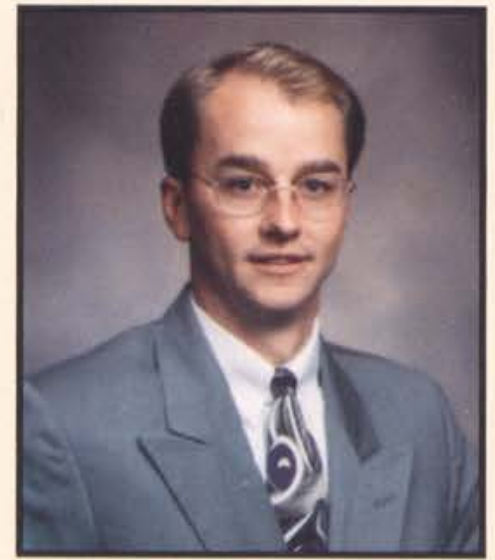

Troy Schaneman

Athletic Training

Douglas, WY

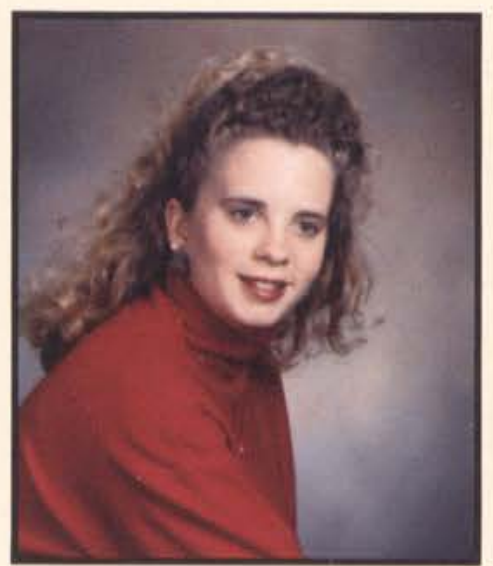

Holly Schulmeyer

Nursing

Columbus, $\mathrm{OH}$

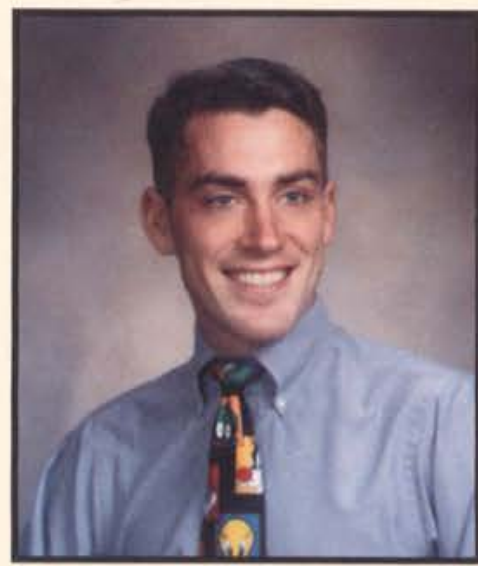

Denver William Seely

Electrical Engineering

Hilliard, $\mathrm{OH}$

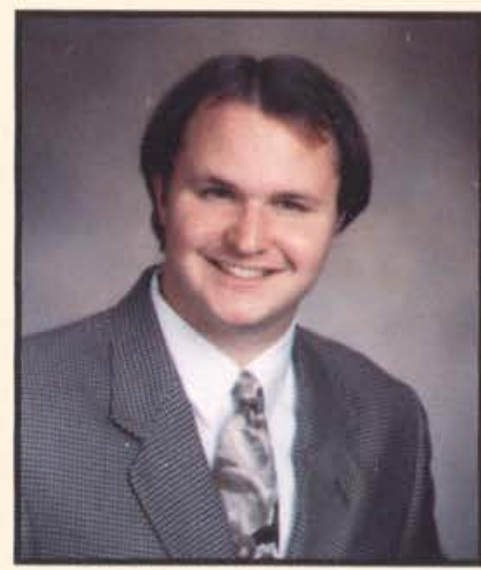

Paul Shaver

Multimedia Technology

Little Rock, AR

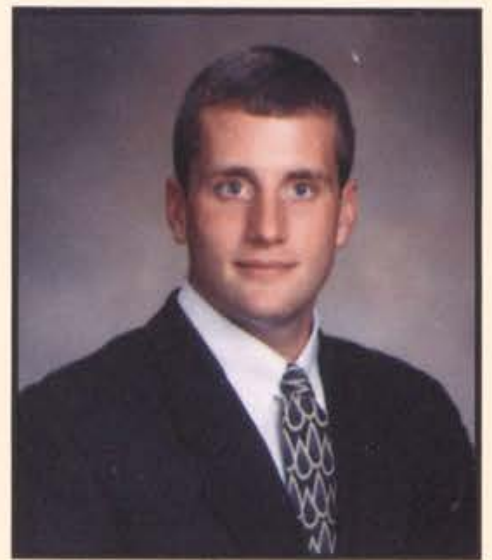

Mark Scharnberg

Marketing/Management

Cumming, IA

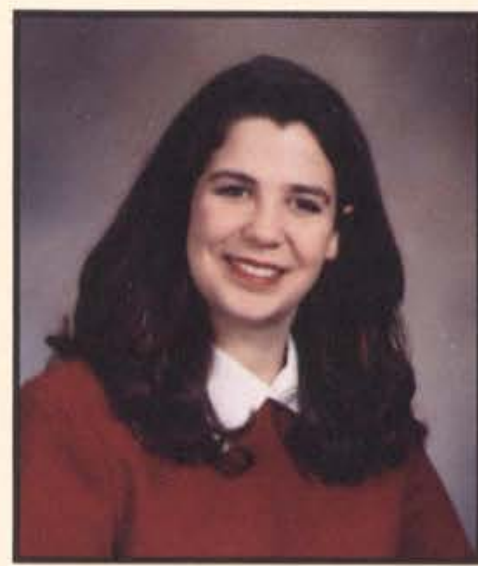

Jessica Schuring

Music Education

Portage, MI

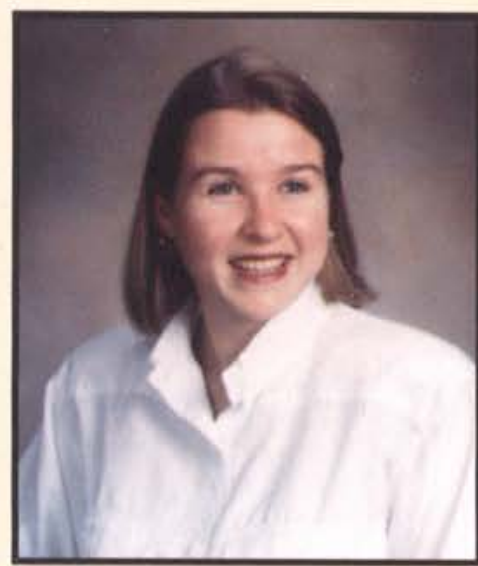

Melissa Sepkovich

Elementary Education

Solon, $\mathrm{OH}$

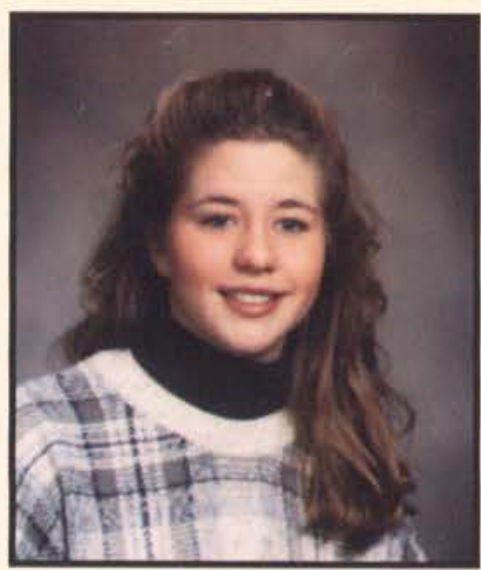

Lori Shelly

Business Communications

York, PA 


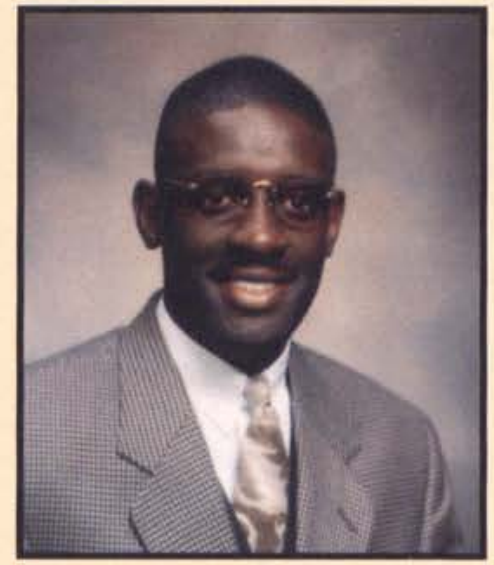

Vance LaBaron Smith

Biology/Pre-Medicine

Pembroke, Bermuda

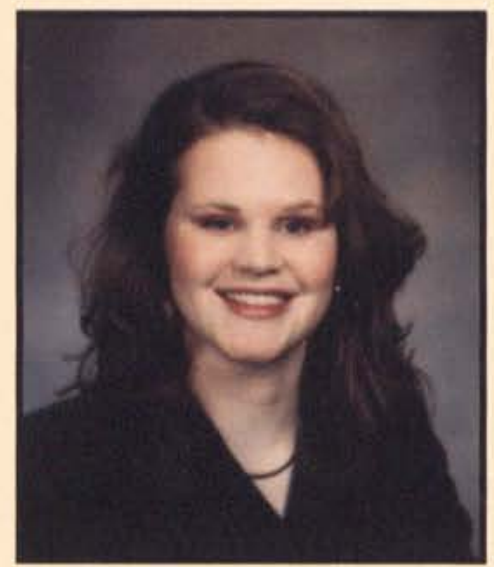

Heidi Sorensen

Marketing

Corning, IA

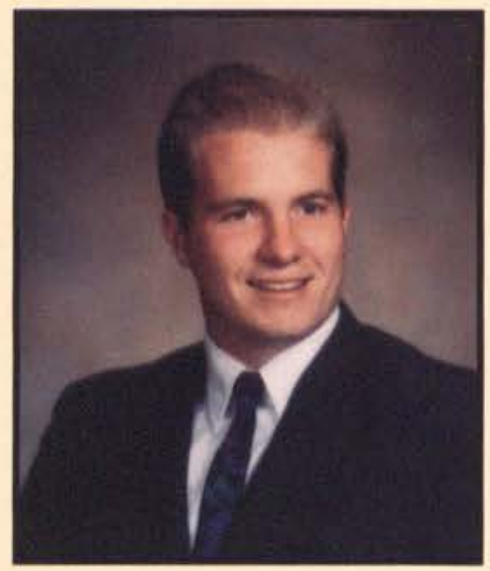

Jeremy Staley

Electrical Engineering

Harrod, $\mathrm{OH}$

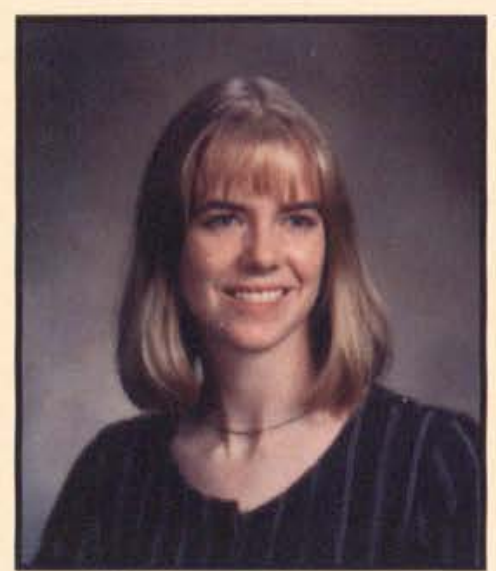

Christina S. Stauffer

Nursing

Narvon, PA

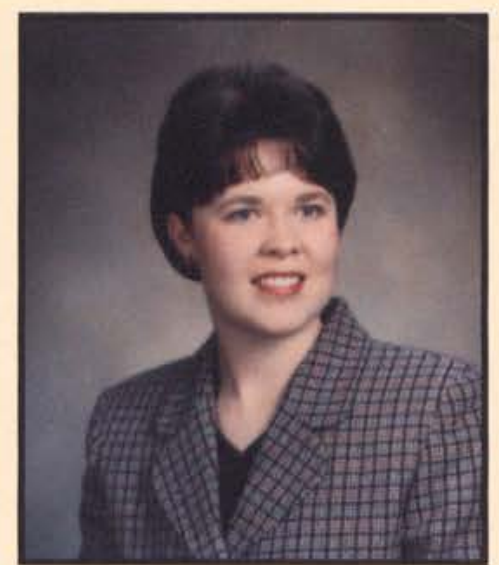

Angela L. Snook

Accounting

Forest, OH

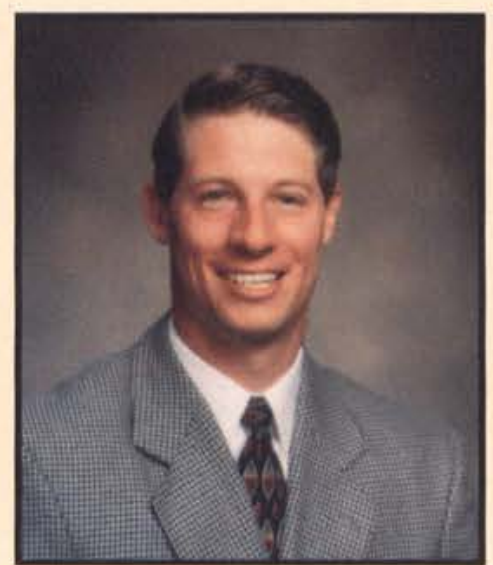

David J. Southwell

Bible Comprehensive Etters, PA

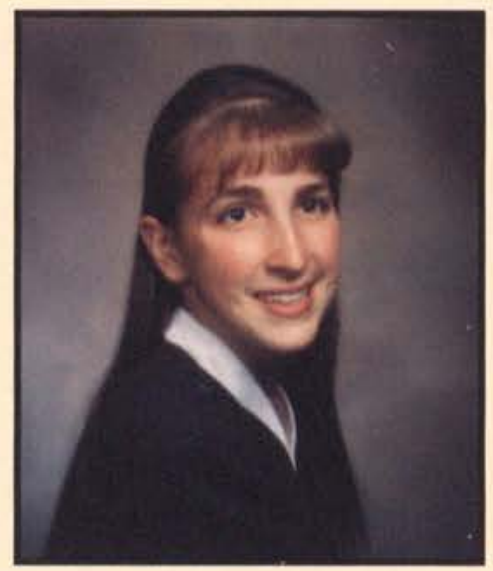

Gwenyth Stambaugh Elementary Education

Hanover, PA

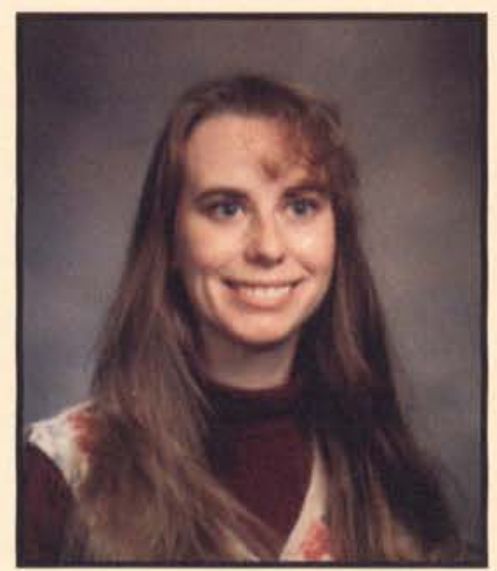

Jacqueline Stauffer Elementary Education Cedarville, $\mathrm{OH}$

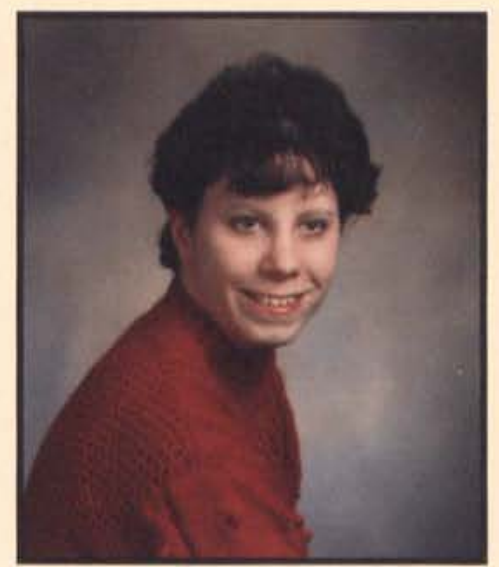

Joanna Snowden

Elementary Education

Mason, $\mathrm{OH}$

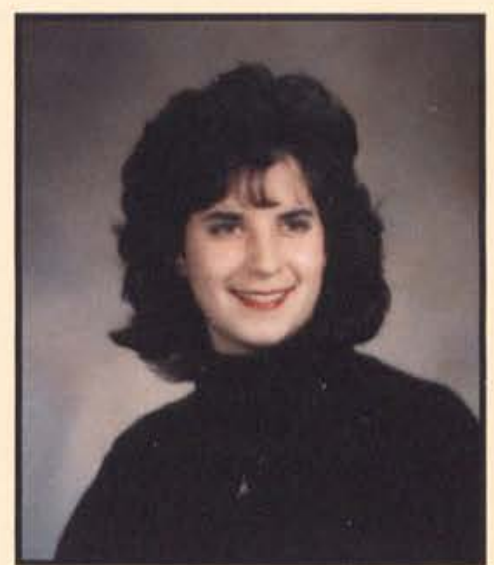

Melissa Sprankle

English Education

Chesapeake, VA

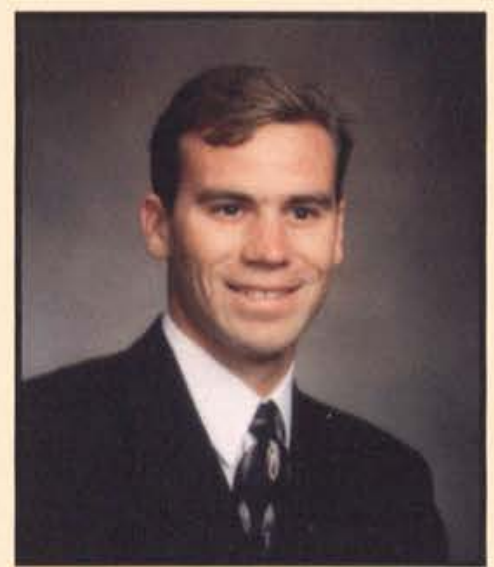

Jeffery Michael Stanton

Computer Information Systems

Defiance, $\mathrm{OH}$

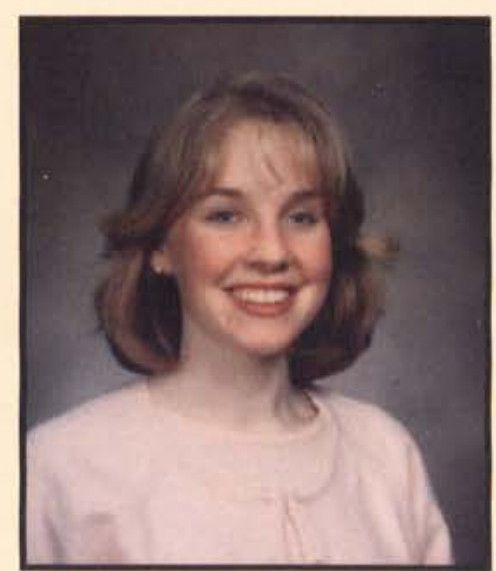

Edith Steele

Elementary Education

Roseburg, OR

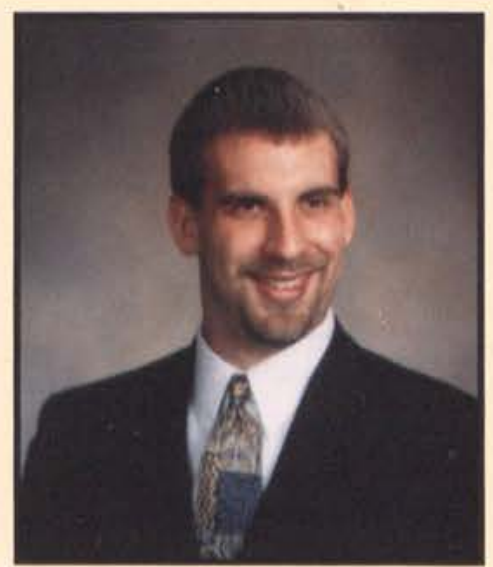

Eric R. Sorensen

Communication Arts

Hoffman Estates, IL

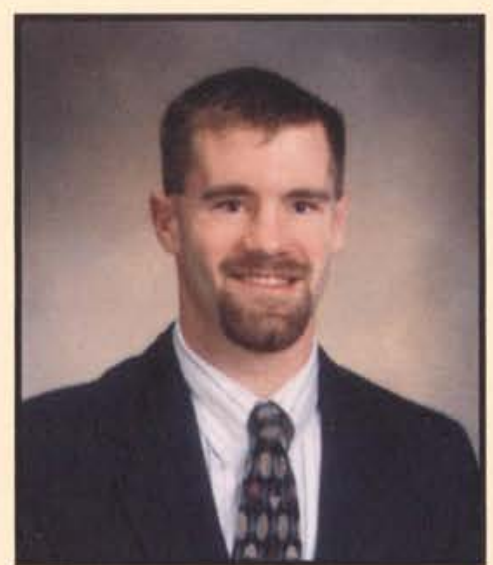

David Staedtler

Markeing/Management

Langhorne, PA

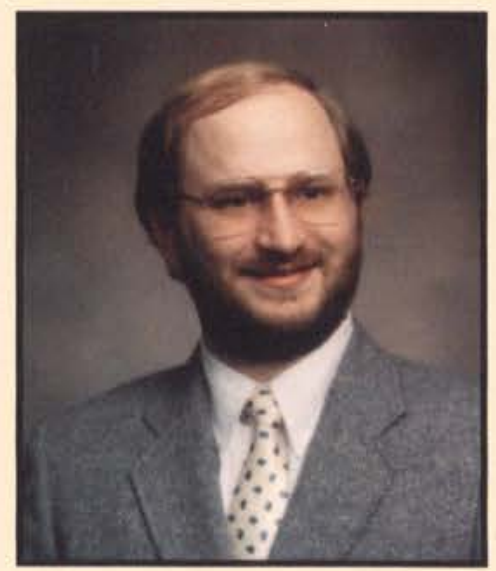

Jeffrey Starkey

Bible Comprehensive

Cedarville, $\mathrm{OH}$

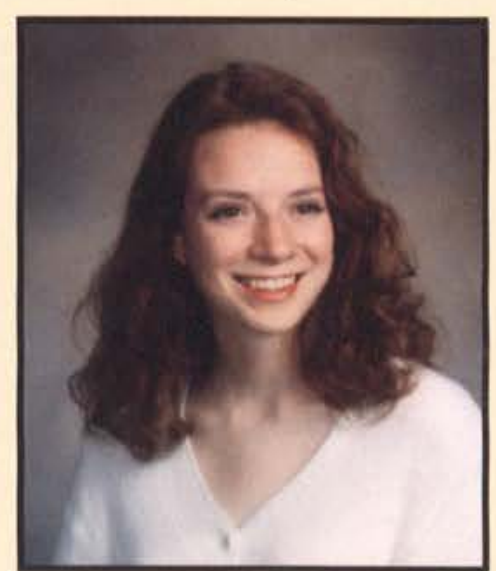

Samantha Sternad Professional Writing Pickerington, $\mathrm{OH}$ 


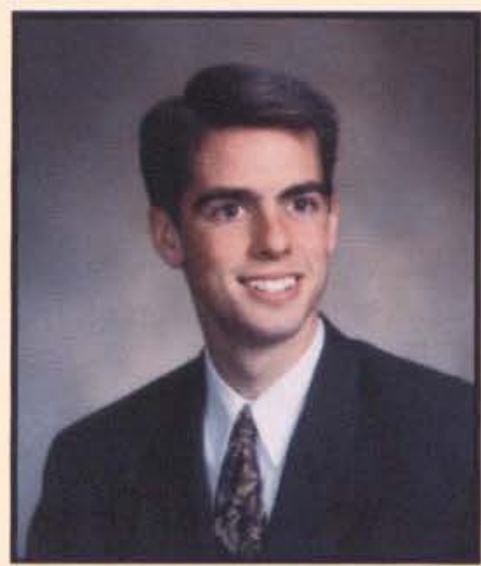

Shane B. Tenny

Broadcasting

Waxhaw, NC

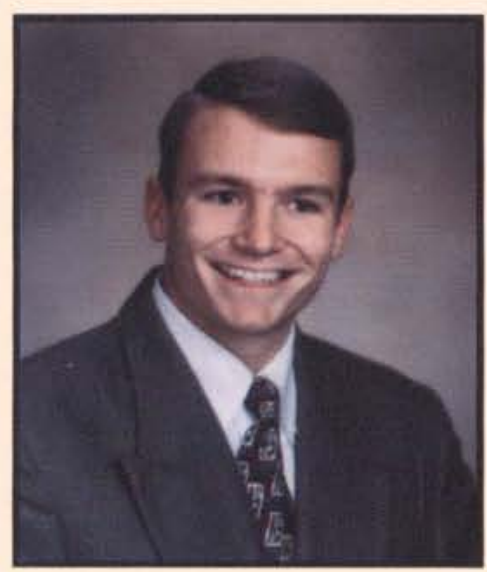

Scott A. Thompson

Finance/Accounting

Monroeville, PA

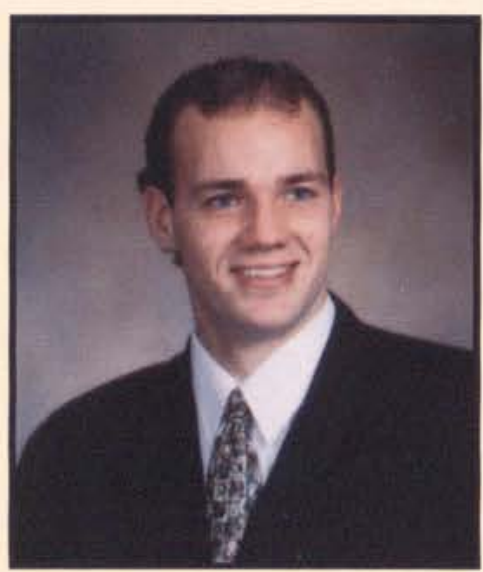

Matthew Towle

Athletic Training

Aurora, NY

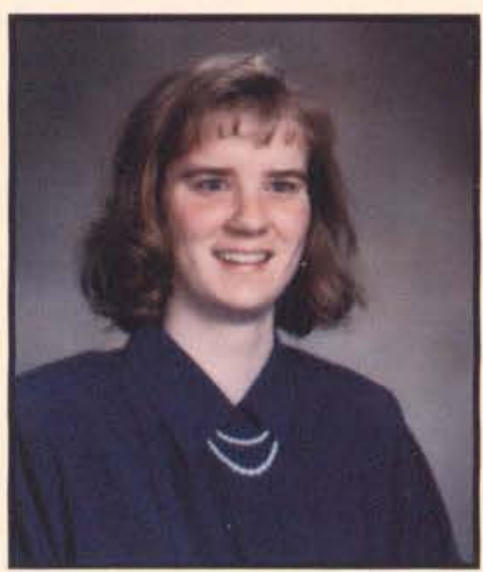

Renee Lynn Tuinstra Elementary Education Bangor, MI

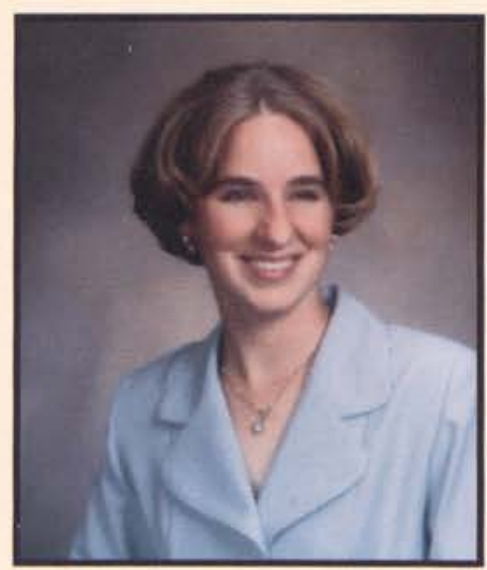

Sarah J. Terpstra

Nursing

Potosi, MO

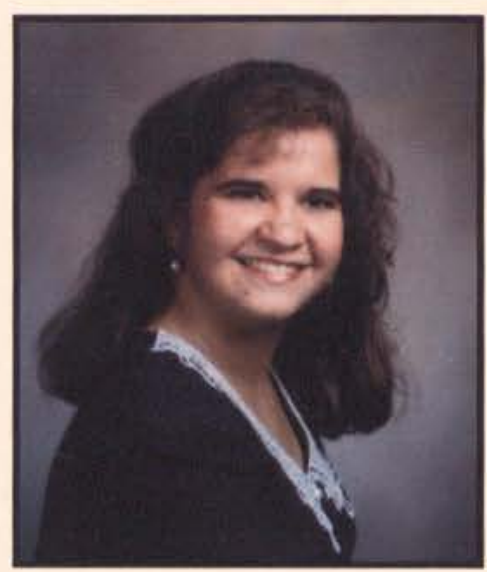

Angela Toms

Elementary Education

Marion, IN

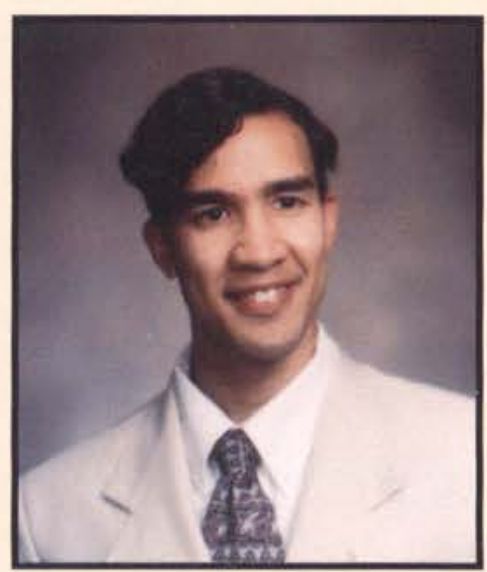

Ray Townsend

Pre-Seminary

Huber Heights, $\mathrm{OH}$

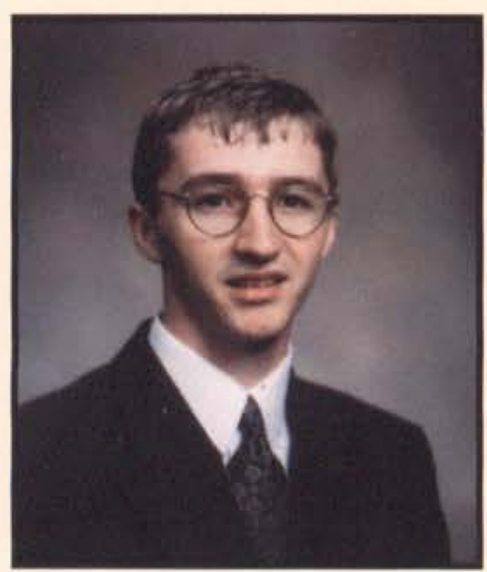

Virgil Vaduva

Computer Information Systems Cedarville, $\mathrm{OH}$

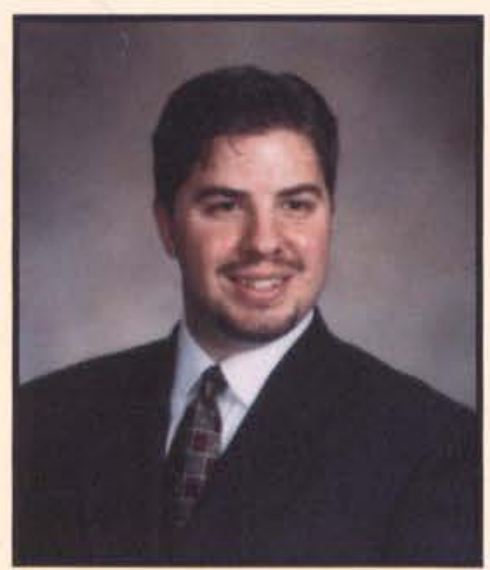

Robert B. Thomas

Marketing

Milford, $\mathrm{OH}$

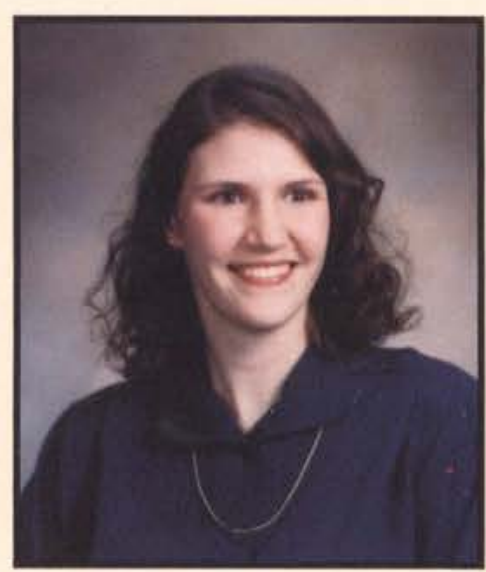

Nadine R. Tomsa

Elementary Education

Johnson City, NY

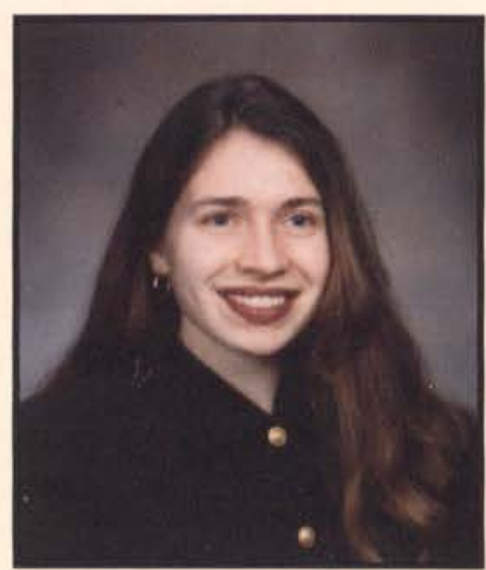

Deborah Trimble

Social Work

Cedarville, $\mathrm{OH}$

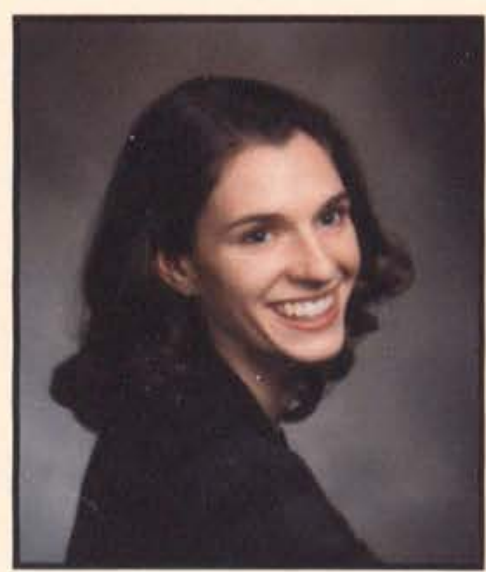

Lisa Kelly VanWormer Professional Writing

Schenectady, NY

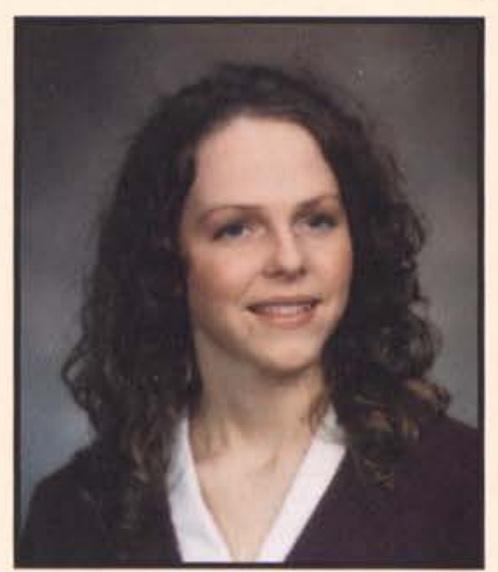

Debra Thompson

Finance/Accounting

Apalachin, NY

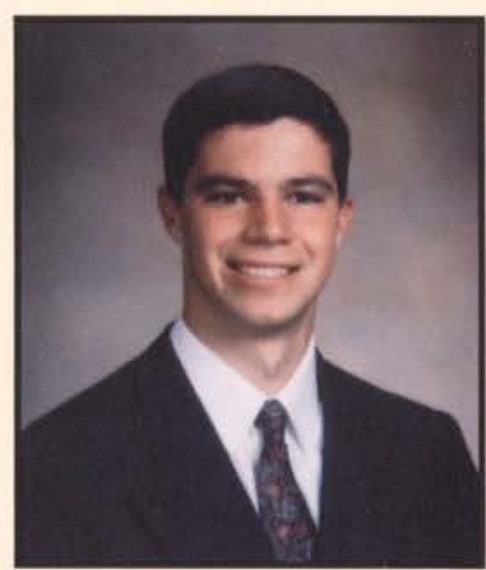

Anthony Torlone

Communication Arts

Westerville, $\mathrm{OH}$

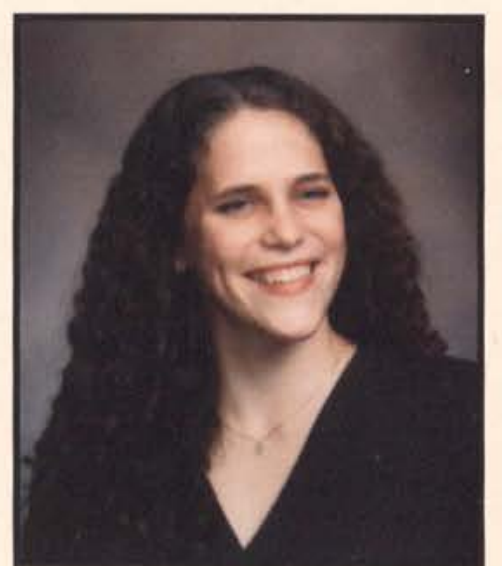

Melany Glisten Trimble Elementary Education

Cedarville, $\mathrm{OH}$

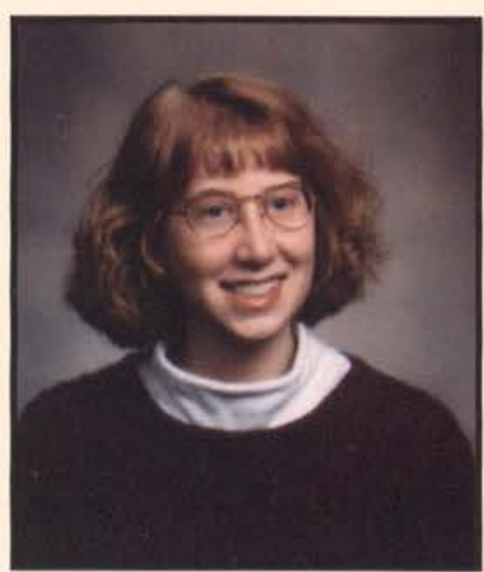

Natalie Vandemark

Electrical Engineering

Grand Blanc, MI 


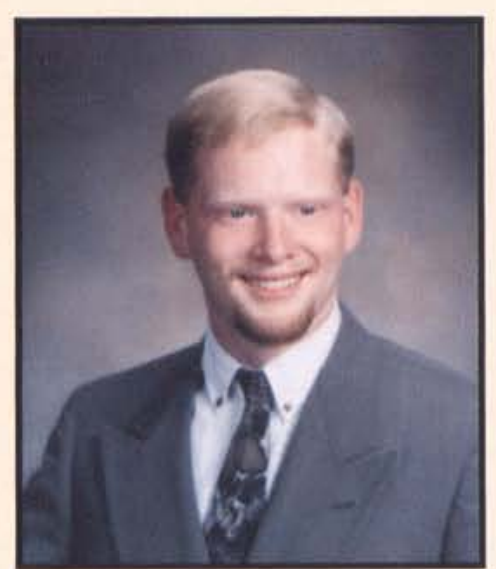

Paul Weaver

C.E. Youth

Danville, IN

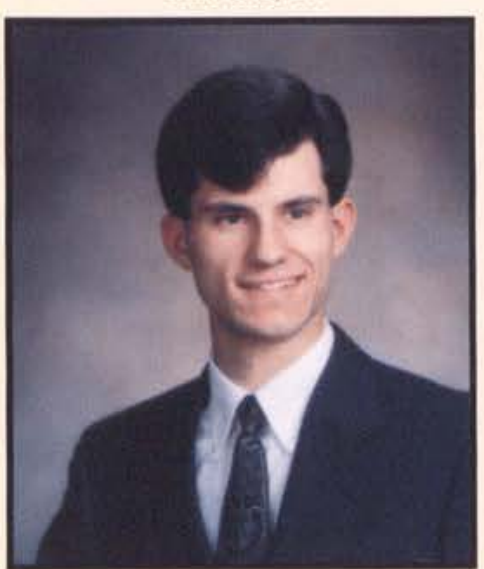

Nathan A. Weh

Electrical Enginnering

Mifflinburg, PA

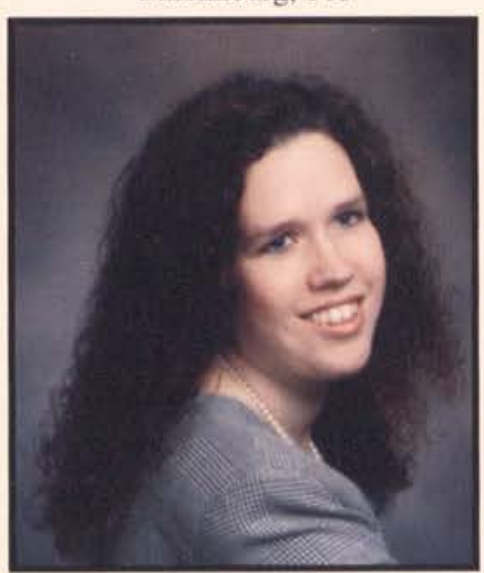

Jodi Whims

Business Communications

Technology

Swea City, IA

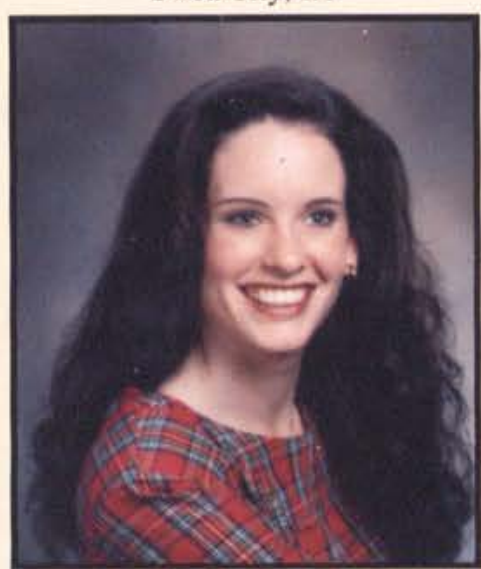

Alissa Whitten

Organizational Communications Romeo, MI

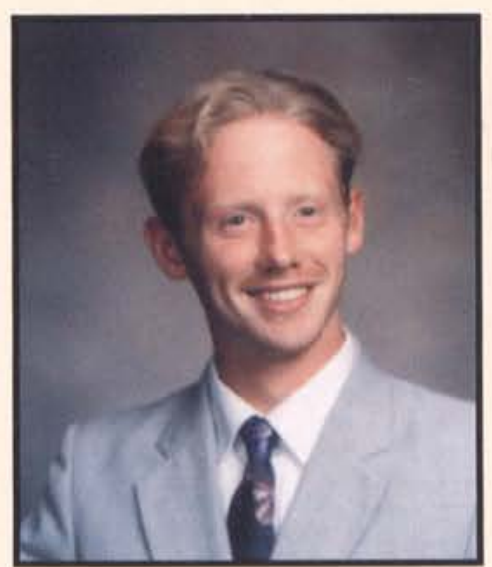

George F. Weber III

Sports Management

Cedarville, $\mathrm{OH}$

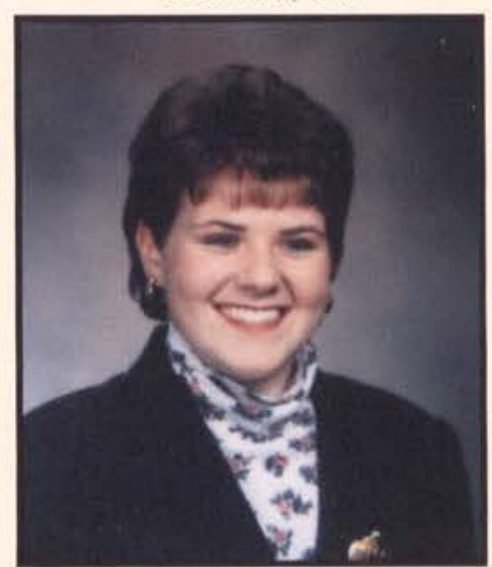

Stephanie L. Weldy

Elementary Education

Springfield, $\mathrm{OH}$

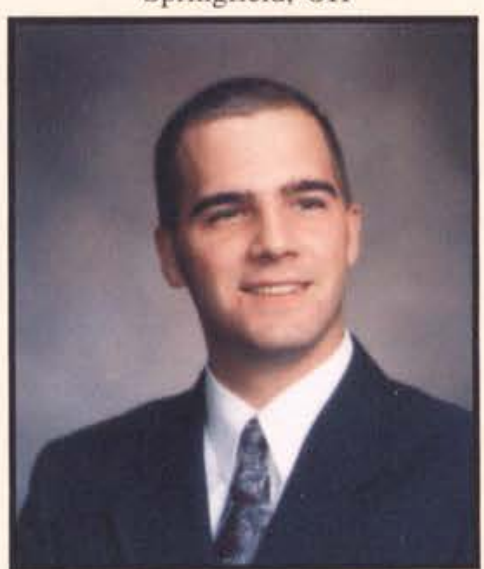

Matthew White

Criminal Justice

Brookville, $\mathrm{OH}$

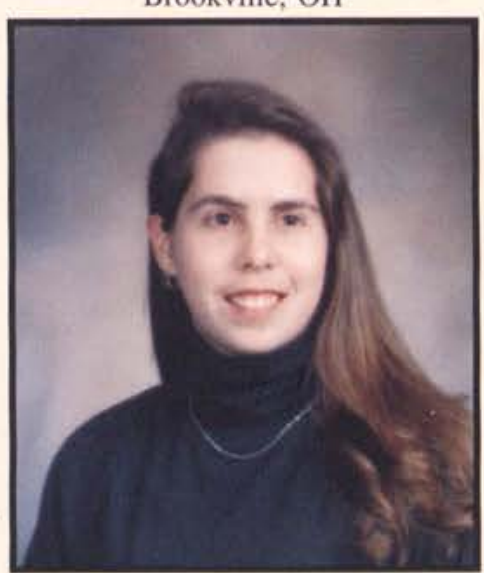

Natalie Wiewiora

Social Work

Wheaton, IL

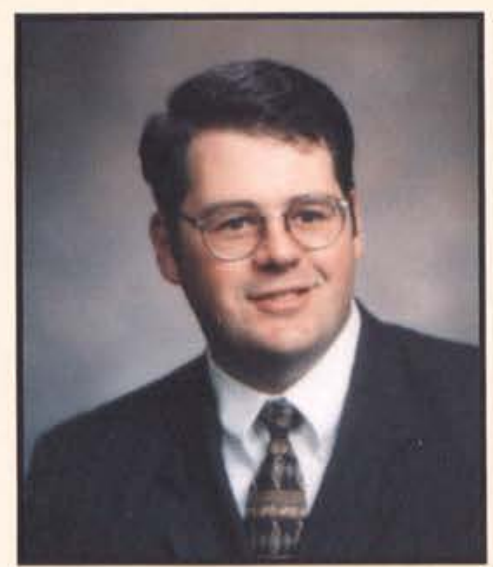

Jonathan B. Weber

International Studies

Meadville, PA

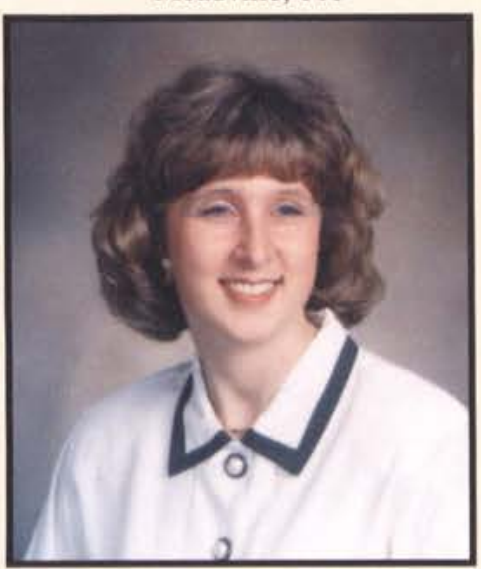

Susan E. West

Music

Temperance, MI

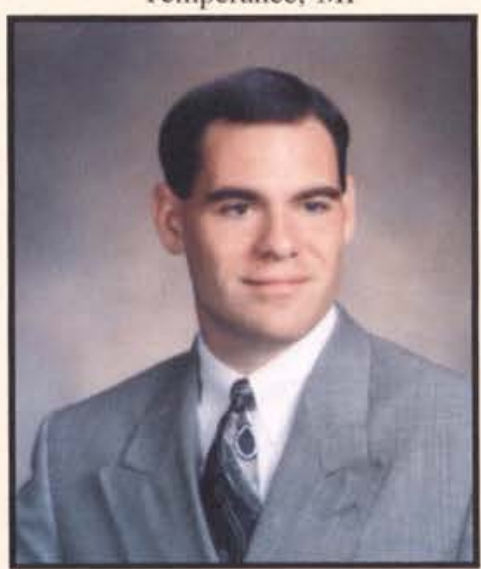

R. Brian White

Communication Arts/Speach Ed. Chesapeake, VA

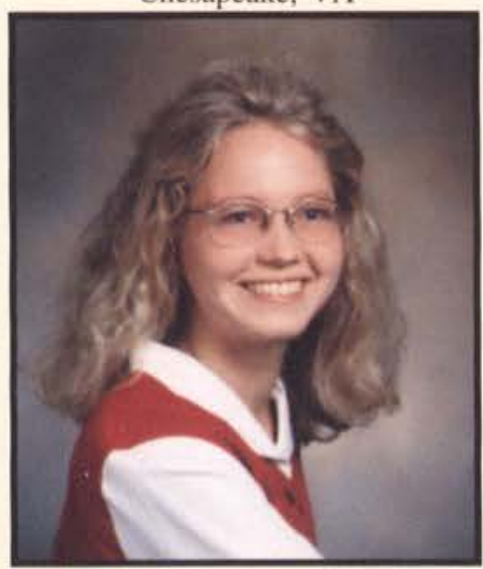

Emily Lynne Williams

Broadcasting

Neenah, WI

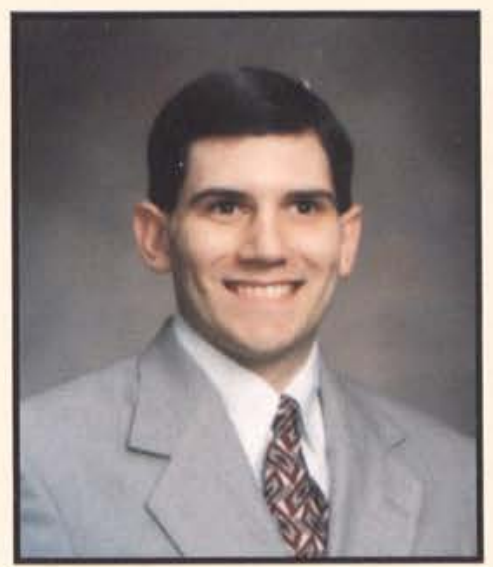

Todd Paul Webster

History

South Euclid, $\mathrm{OH}$

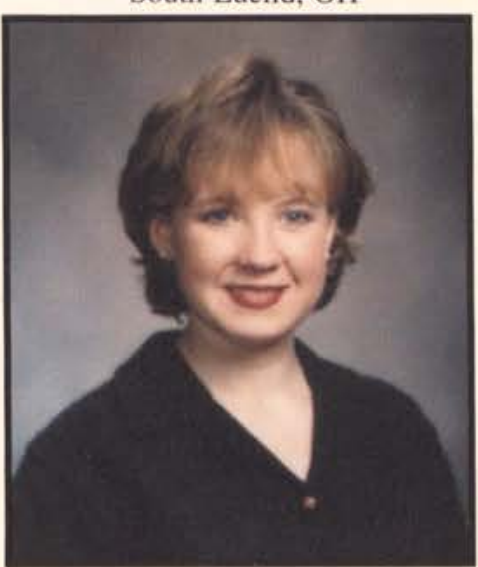

Laurie Elizabeth Whaley Communication Arts

Springfield, $\mathrm{OH}$

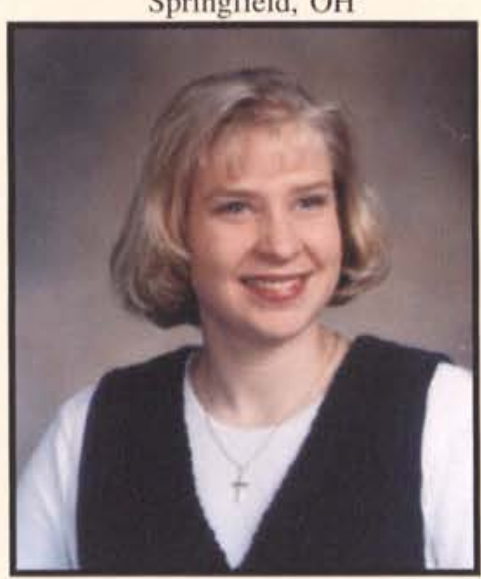

Jennifer E. Whitman

Elementary Education

Dearborn, Ml

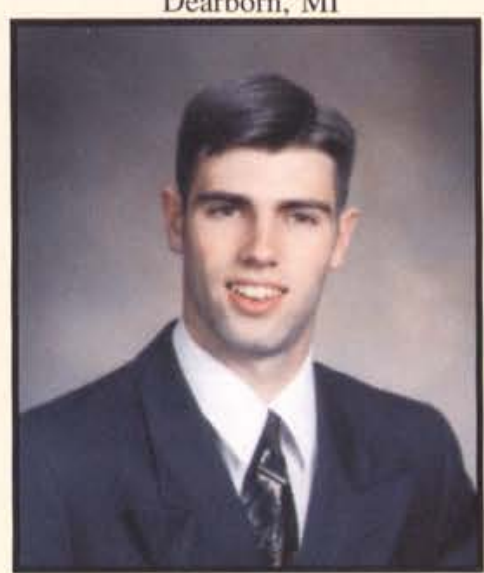

Jon Williams

Bible Comprehensive

Ozone Park, NY 

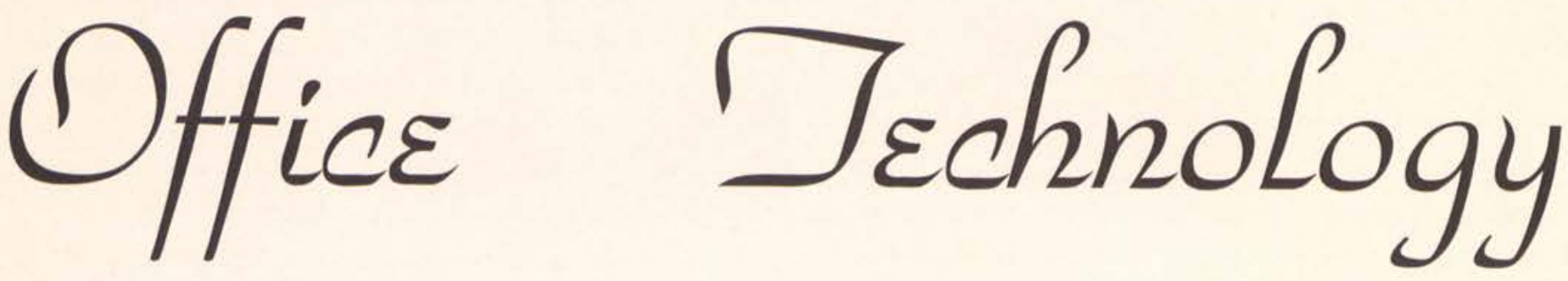

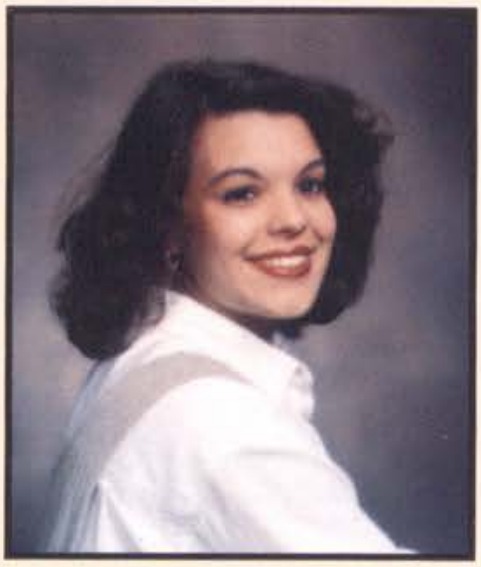

Amy Elizabeth Dietz

Office Technology

Reynoldsburg, $\mathrm{OH}$

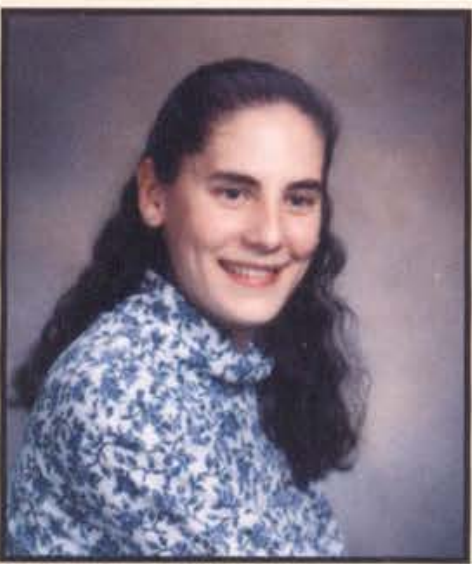

Amy E. Meckley

Office Technolgy

Clayton, MI

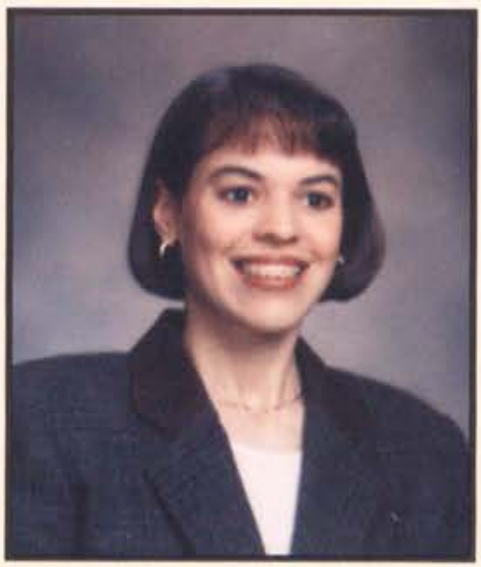

Joanna Ruth Halsey

Office Technology

Brownsburg, IN

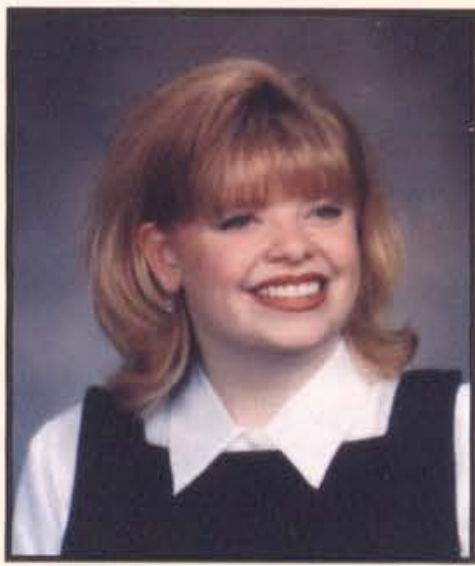

Jamie Weiss

Office Technology

Centerville, $\mathrm{OH}$

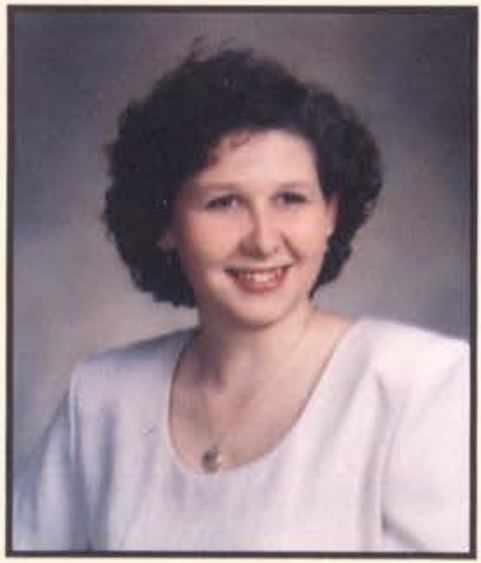

Sarah A. Mattke

Office Technology

Menomonee Falls, WI

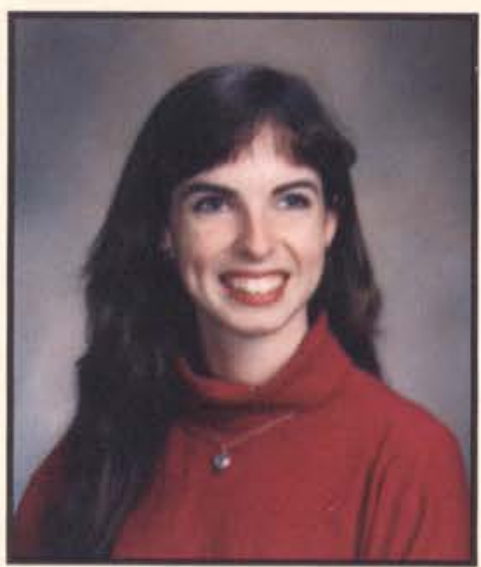

Jennifer J. McCarty

Office Technology

Perth-Andover, NB Canada

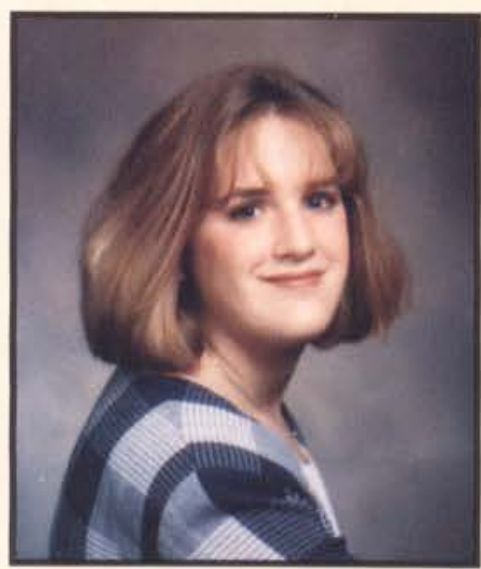

Julie K. Wit

Office Technology

Troy, MI 


\section{Leaders of the $\operatorname{Pack}$}

Right: Senior Class advisor Jane AdamsSmith with their youngest son Shane.

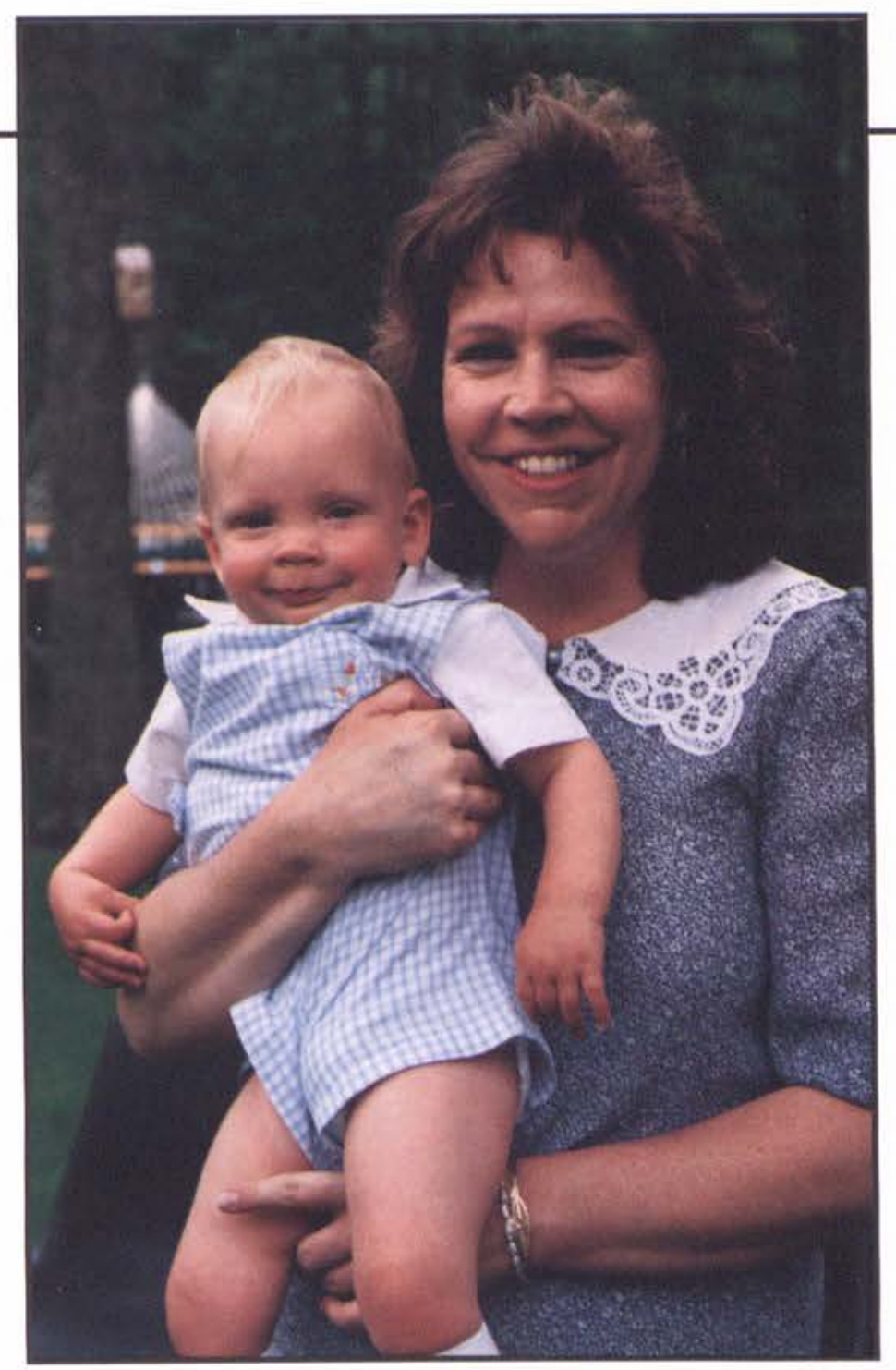

Eric Fiveland

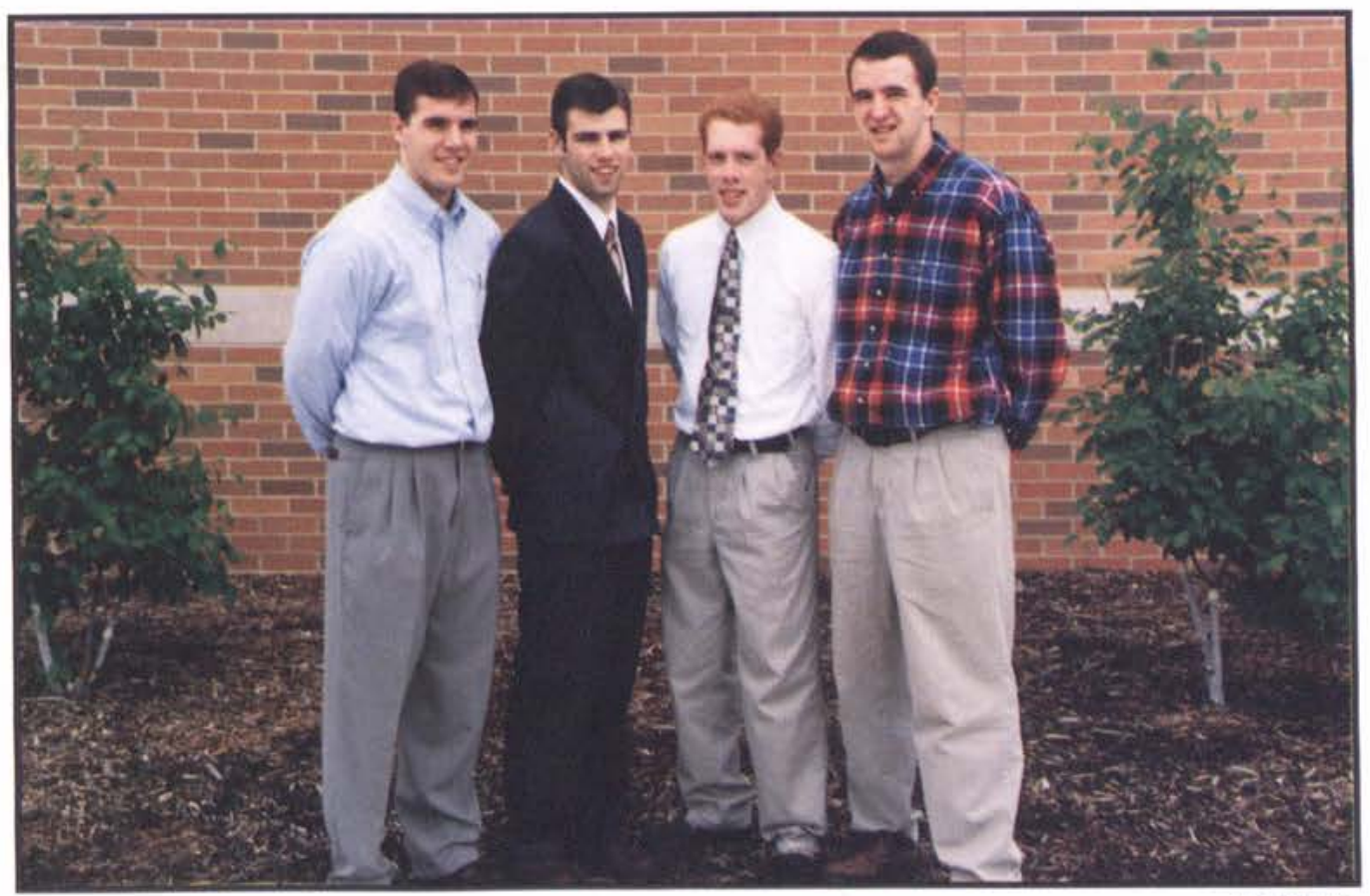

Fric Fiveland
Left: Senior Class Officers; President, Evan Wood; Chaplain, Jon Williams; Treasurer, Doug Amundson; SGA representative, Dave Kaynor. 


\section{- Nurses Drade Smodks for Pins}

$$
\text { Gy Carolyn Simon: }
$$

The Nursing Department held a convocation for its 14 th graduating class on June 6 . The two-hour ceremony took place in the Dixon Ministry Center.

Before the ceremony, faculty, seniors, family and friends mingled at a reception given in their honor by the department. Senior David Butgereit provided music on his classical guitar.

The convocation was opened in prayer by Academic Vice President Duane Wood, and a charge to the class was given by Dr. Irene Alyn, Chair of the Department of Nursing, who reflected on the class Bible verse, John 15:5.

Unique to graduation from the Nursing program is the tra- dition of pinning, the part of the convocation in which the graduate receives the BSN pin. During the pinning ceremony, each student was also awarded a specific character quality which the nursing students and faculty chose as descriptive of that graduate.

Three awards were presented: the Academic Award went to Amy Stewart, who maintained a GPA of 4.0; Kathy Petersen received the Practice Award, given to the nurse who performs above expectations in the clinical setting; and the Character Award, voted on by classmates and faculty, went to Katie Smith.

A high point for the graduates came in the final part of the ceremony when they took off their blue student smocks, leaving only their white nursing uniforms. Lois Baker, Professor of Nursing, said, "this is when it really hits them that they are nurses!"

For their class song, the seniors selected "Any Road, Any Cost." Graduate Renee ElKhouri explained, "we chose this song because it was a long road that we followed to get us to this point--years of lab reports, all-night study sessions, and early morning clinicals-sometimes all in the same week. But God got us through and $\mathrm{He}$ will continue to guide us as we serve Him in our professional careers."
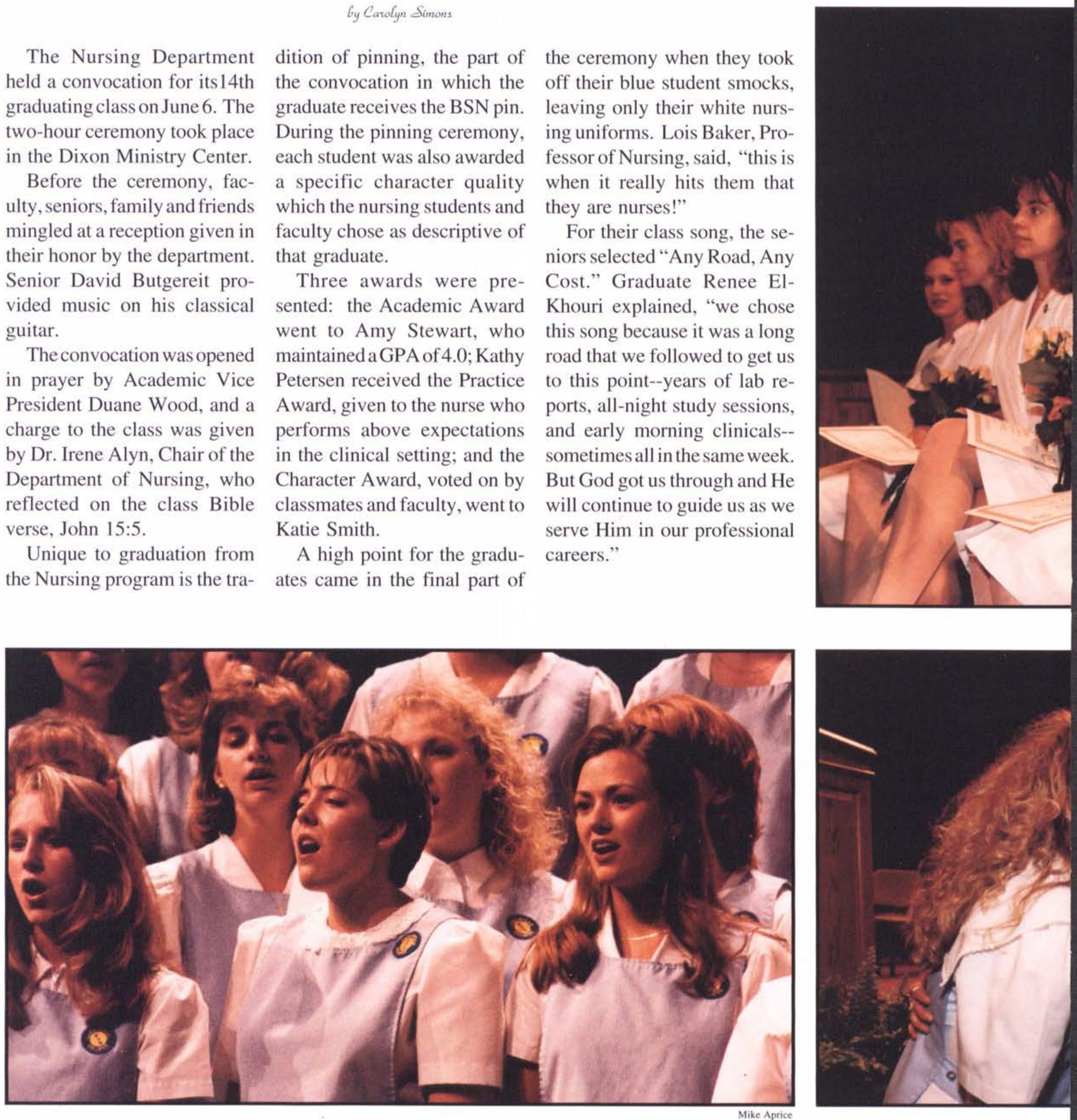

Mike Aprice 


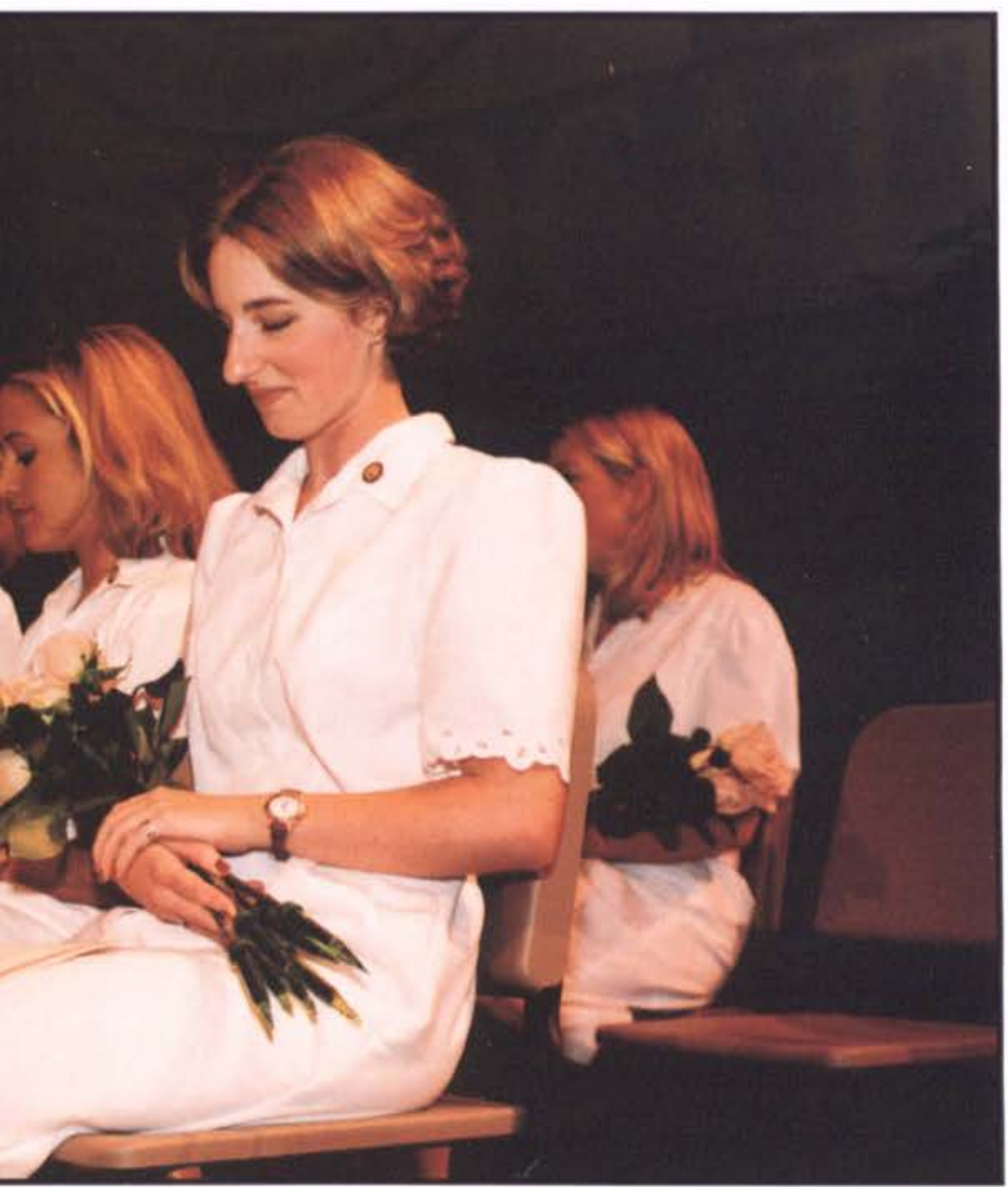

Mike Aprice
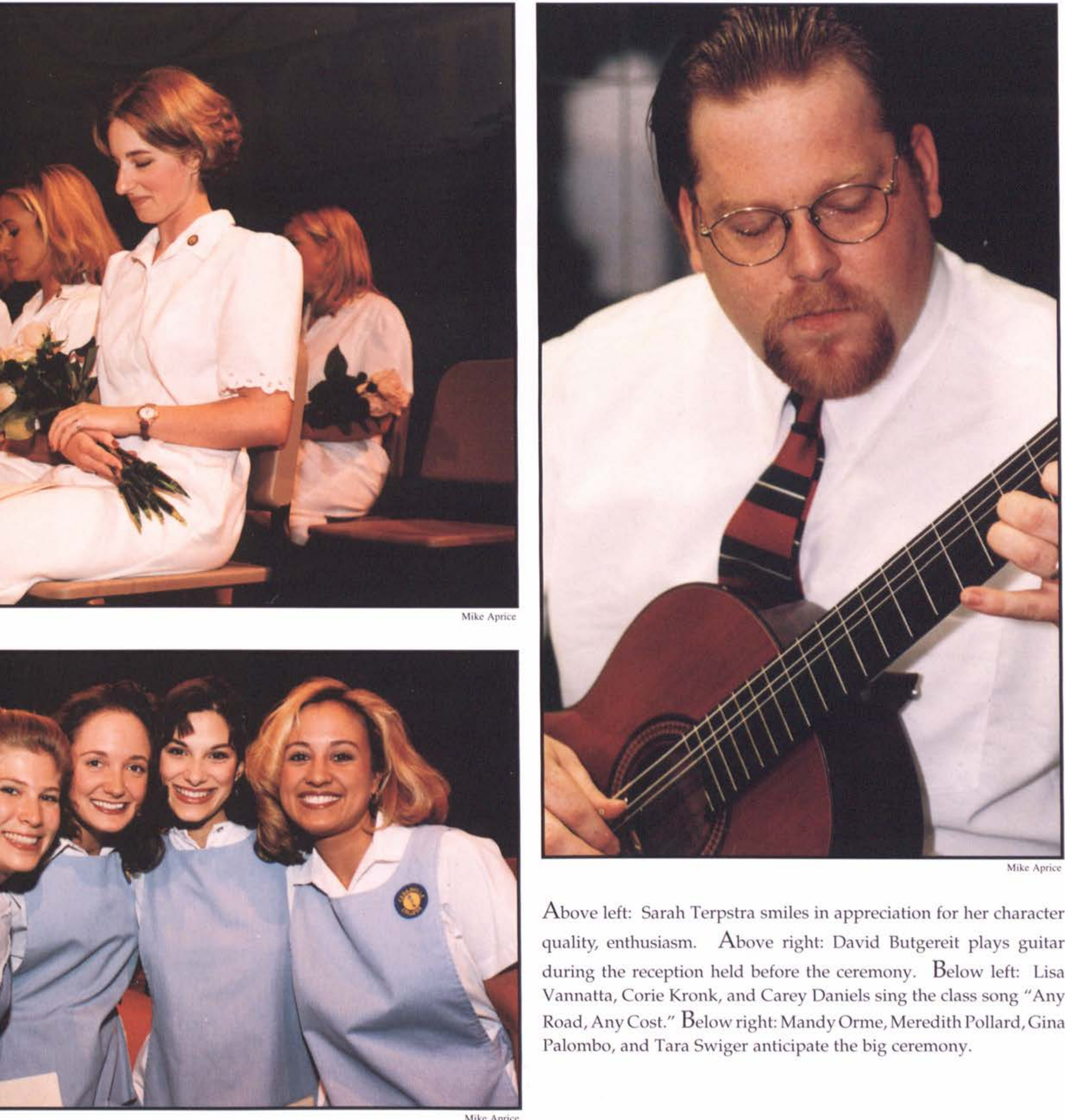

Above left: Sarah Terpstra smiles in appreciation for her character quality, enthusiasm. Above right: David Butgereit plays guitar during the reception held before the ceremony. Below left: Lisa Vannatta, Corie Kronk, and Carey Daniels sing the class song "Any Road, Any Cost." Below right: Mandy Orme, Meredith Pollard, Gina Palombo, and Tara Swiger anticipate the big ceremony. 


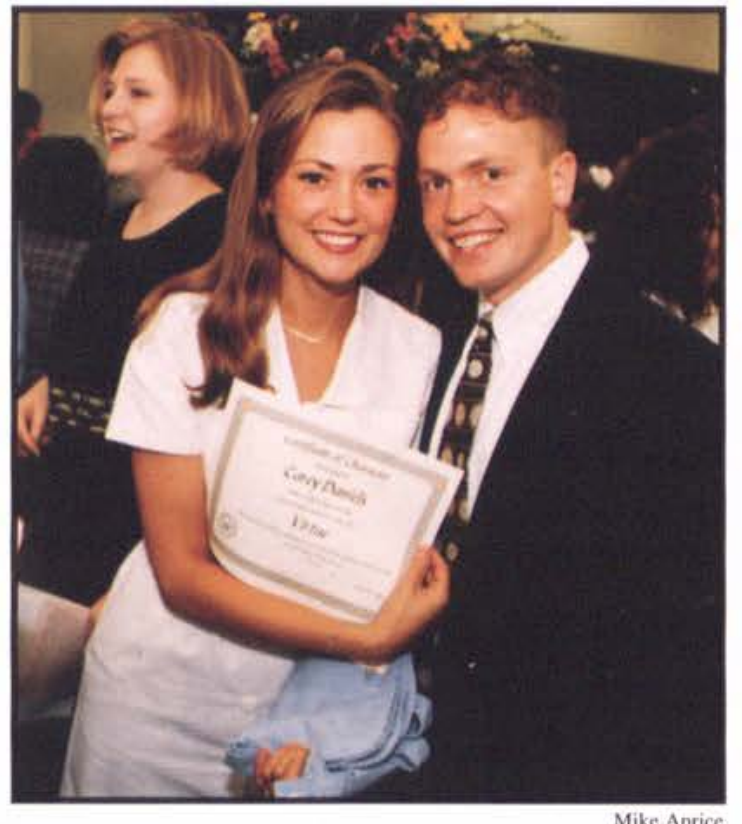

Left: Carey Daniels and her fiance, Matt Clark pose for a shot after the ceremony. Middle: Tonya Brockman receives her official BSN pin. Above right: Betsy Lindaberry, Janet Kollmeyer, Heidi Groves, and Rachel Batchelder display their Certificates of Character. Below right: Billie Michael smiles at Cara Miller. Nursing students develop many strong friendships during their four years in the program.

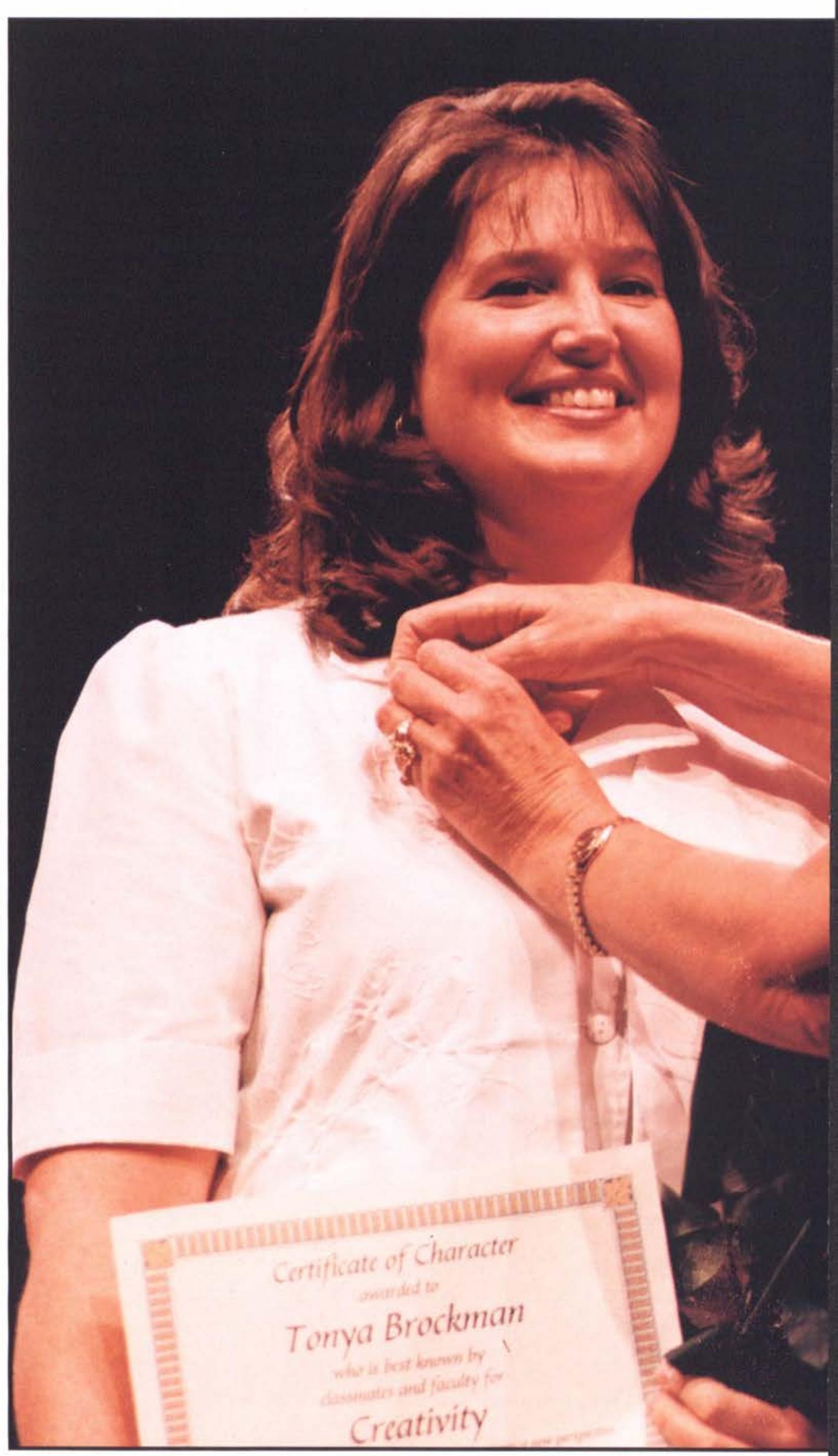



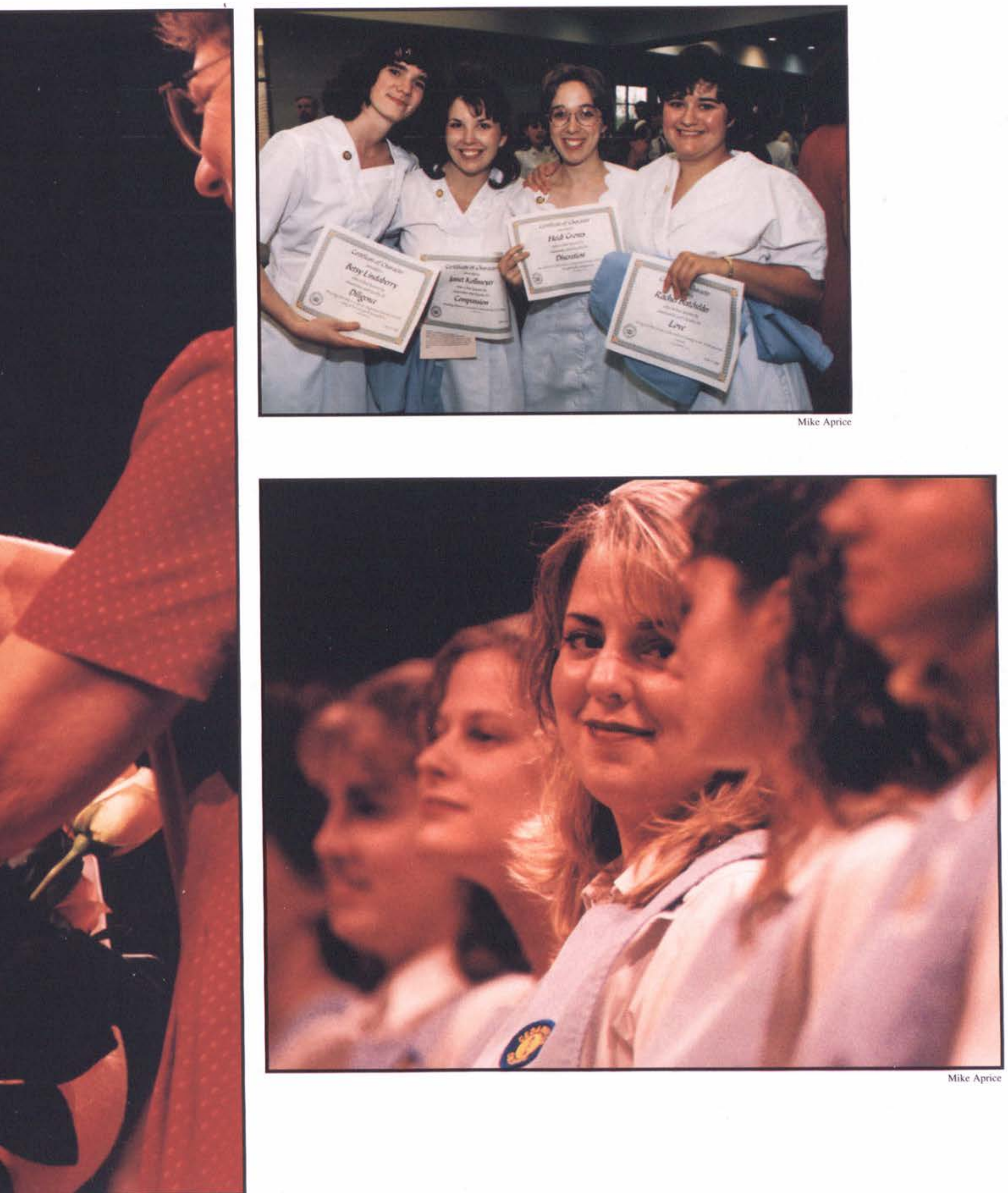


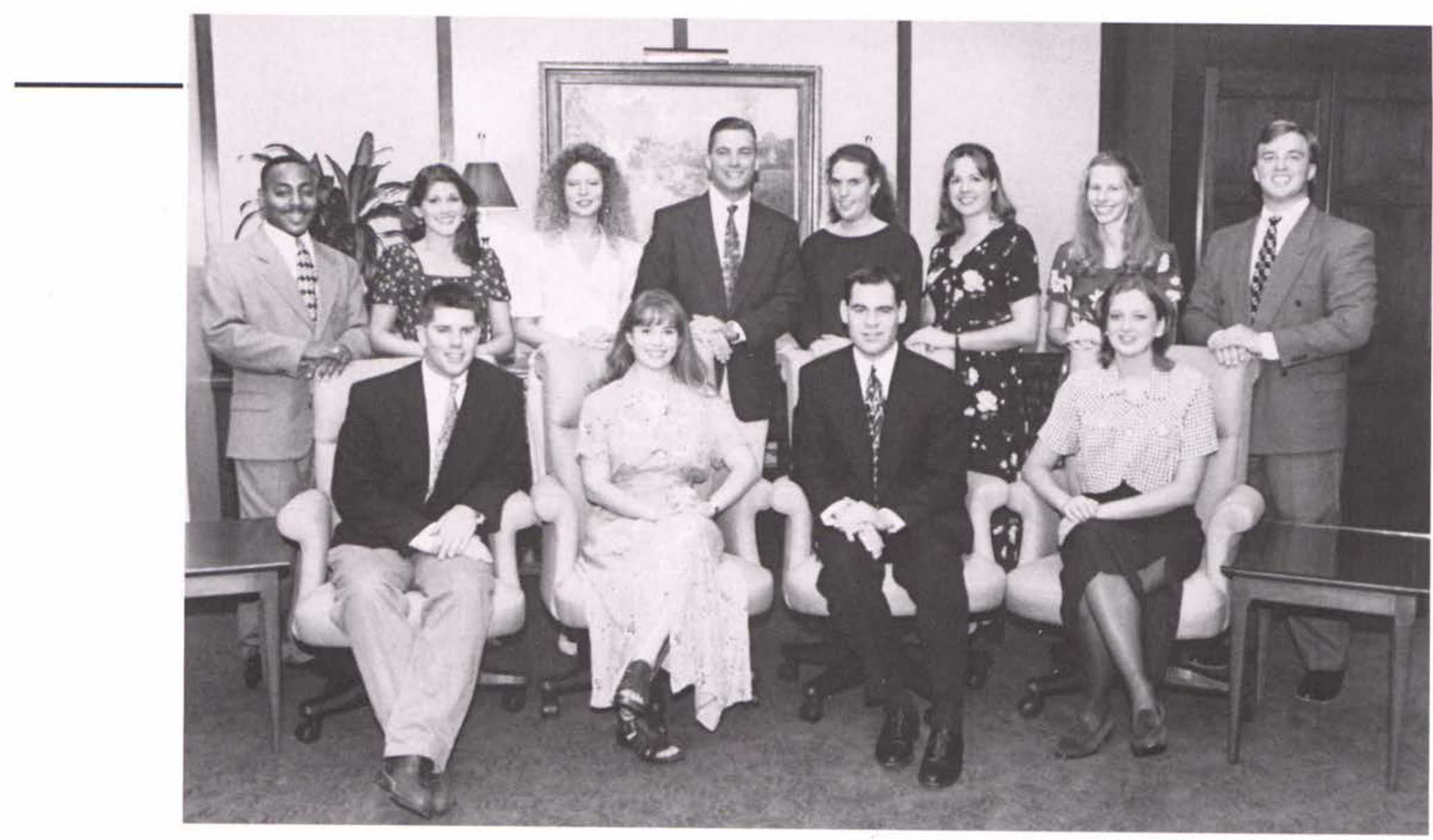

Mike Aprice

SGA Exroutive Council

row 1 (1-r): Scott Kennedy, Vice President Christy Farris, President Brian White, Krysty Bailey, row 2: Chuck Quarles, Laura Refior, Jennifer Benson, Scott Vandergrift, Becky Woelk, Meaghan Lemke, Christina Baley, Jeff Motter.

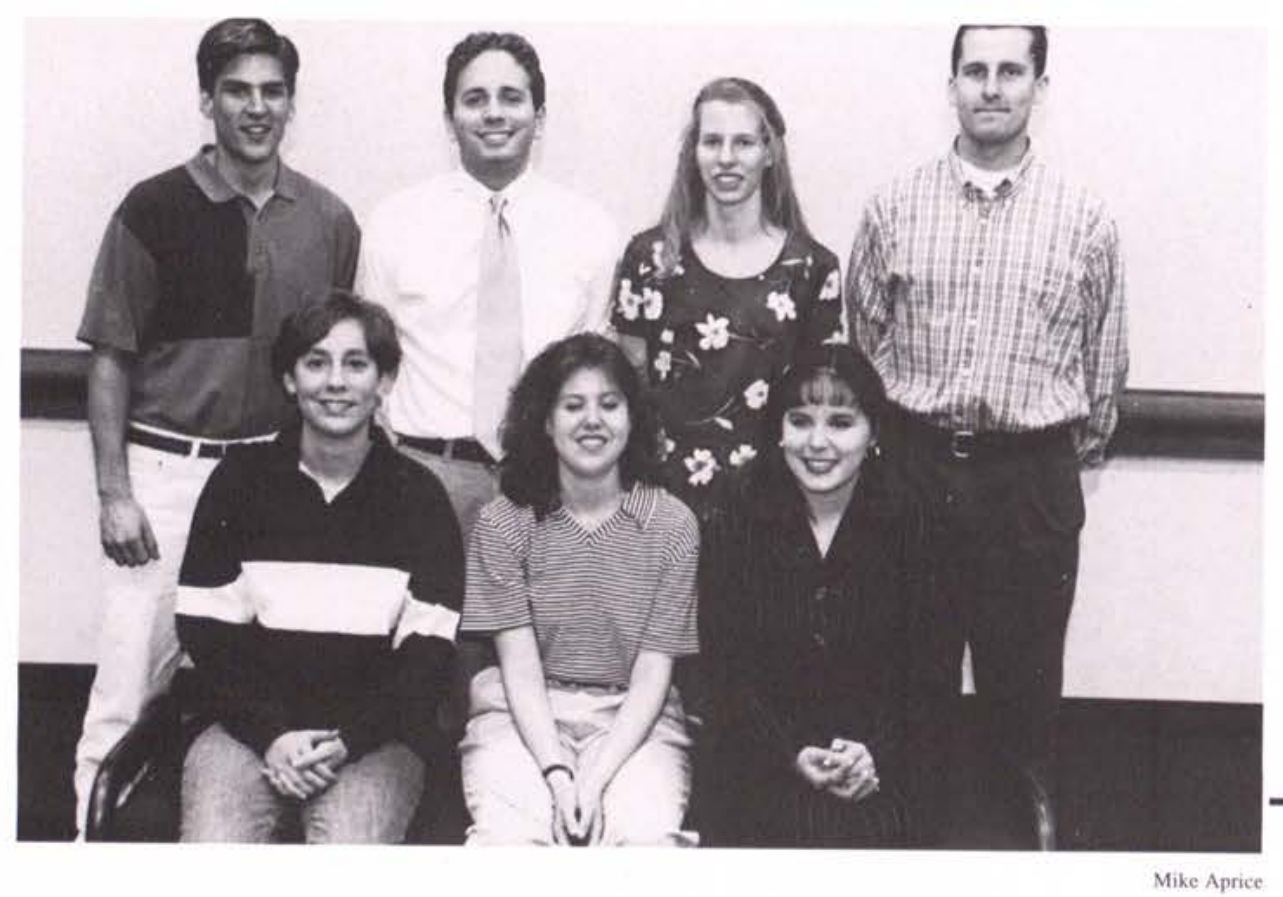

Student Court

row I (1-r): Heather Fourman, Erin Johns, Sara Romang. row 2: Kirk LeBlanc, Doug Bayler, Christina Baley, Erik Larsen. 


\section{Brian}

Student $\mathcal{L}_{\text {eader }}$

\section{White}

Ronald Brian White, or Brian as we all know him, was born in Garland, Texas, October 21, 1975. He lived there for three and a half years with his mother, father and older sister W e n d i . Then after a six month stint in Raleigh, North Carolina, the White family settled in Chesapeake, Virginia where they have lived

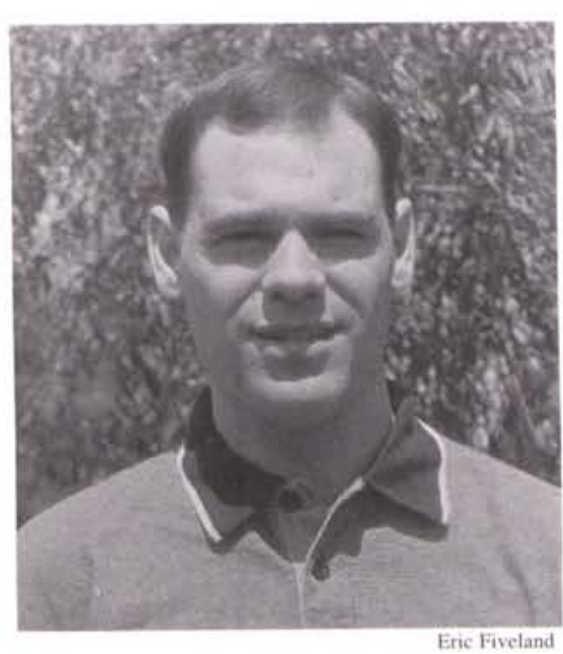

ever since. Brian comes from a family of educators. His father is an administrator of Greenbrier Christian Academy and his mother is a language arts therapist. His sister is a junior high school teacher, and Brian is a Communication Arts major with an emphasis in Speech Education. $\mathrm{He}$ would like to go to graduate school and pursue a Master of Arts degree in Communication, and eventually teach high school or college. Aside from academics, Brian loves sports. Basketball is a favorite: not just watching, but also playing and coaching. He also enjoys politics. In ten years, Brian sees himself teaching or in an administrative position at either the high school or college level. He says that marriage and children are possibilities, as is a dog. He would like to live in Virginia, but is willing to go wherever the Lord leads him. While enrolled at Cedarville, Brian was involved in a wide array of activities. He was on the 1994 Australia Team, and was a

Swords Extension team leader. His participation in student government has been broad, and has ranged from membership on the Constitutions and Elections Committee his freshman year to his election as Student Government Association President. He was on the Campus Activities Board his junior year, and is a three-time small group leader for Getting Started and Winter Games Orientations. Brian is also a three year debate team veteran. Brian hopes that he will leave something at Cedarville that people will remember him by: a legacy of good leadership, and the fact that he tried to make a difference.

Meaghan Lemke 


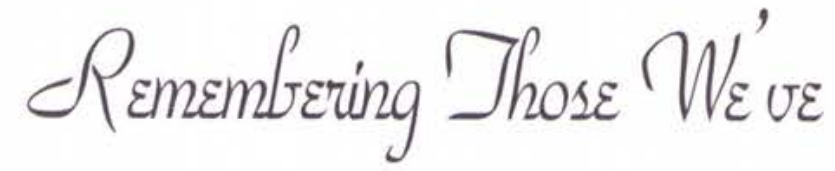

Charles Spurgeon, a nineteenth-century minister stated in his book Morning and Evening: "Oh death, why do you touch the tree where the weary seek shade and rest? Why do you snatch away the fruitful trees? If you must use your axe, use it on the trees that yield no fruit. ... But why do you cut down the good cedars of Lebanon? ... Why? Because of Jesus' prevailing prayer: 'Father, I desire that they also whom You gave me may be with Me where I am.' (John 17:24) It is this prayer that bears our loved ones on wings to heaven. Every time a believer leaves this earth for paradise it is an answer to Christ's prayer." For the Cedarville College family, the 1996-1997 school year was a time for reflection upon such questions because of the sudden deaths of three students: Stephen Michael Brown, Stephen Joel Wildasin, and Nathan Michael Battaglia. On June 18, 1996, Brown was killed in a car accident while on his way home from an outing with the youth group of his church in Elyria, Ohio. It was while attending Open Door Christian School in Elyria, that Brown first came into contact with Cedarville College. He was twice selected to participate in the Cedarville Music Showcase, and it was during these visits to the college that he grew to love the atmosphere of Cedarville and knew it was the place for him. Brown gathered a close group of friends about him his first year at Cedarville, and they were all shocked to hear the news of his death. Paul Yuen, Brown's roommate, said, "When I found out I thought it was a bad joke. The realization that he was dead didn't hit me until the funeral." Joseph Mellish, who lived near Brown in Marshall Hall said, "To me, losing Steve was just like losing a brother." Though Yuen and Mellish both said that they know Brown is in heaven and that they are looking forward to seeing him again, they still feel grief because of his absence. Mellish said, "People shouldn't be afraid to grieve; even though the world goes on around you, your world has stopped, so you need to take time to express your grief."
Brown's parents, Michael and Judy Brown, and his sister Alison, remember Stephen as outgoing and funny, and as a peacemaker among his friends. They said, "He promoted harmony among people and would always encourage others to put God first in their lives, something he also tried to do." Yuen described Brown as having a crazy yet serious personality saying, "I never met someone who could be so silly yet so caring and serious at the same time." Mellish said that Brown "had to be himself no matter what," that he kept his life open like a book for his friends to read. Stephanie Mace, another close friend of Brown's, agreed with this description saying that he was "childlike," very trusting in his manner towards others. During his freshman year, Brown enjoyed singing in the Men's Glee Club and volunteering at the Dayton Gospel Mission. His friends agree that he loved music and they laugh remembering their experiences at forming a band. Yuen was quick to say that Brown only loved music that was glorifying to God. He said, "Steve
Cost

was a real godly example to me, and at the time, I really needed that in my life."

On November 3, 1996, Stephen Joel Wildasin died of injuries he received in a car accident on October 30th Stephen, who accepted Christ into his life as a child while attending a Christian school, will be remembered for his sweet and thoughtful nature. His parents, Harry and Elaine Wildasin, who reside with their three children in Palmyra, Pennsylvania, recall that their son was active in their Community Bible Church where he was well-loved among the children and the elderly alike. "Stephen made every effort to be friendly to all," his parents said, in that he demonstrated genuine interest in what was happening in others' lives. Wildasin's family also remember his "dry wit," and that he was always popular among his coworkers at H.B. Reese where he worked in the summers making those famous peanut butter cups. Kelly Hoppe, friend of Wildasin's at Cedarville said that he always tried to avoid being judgmental but 


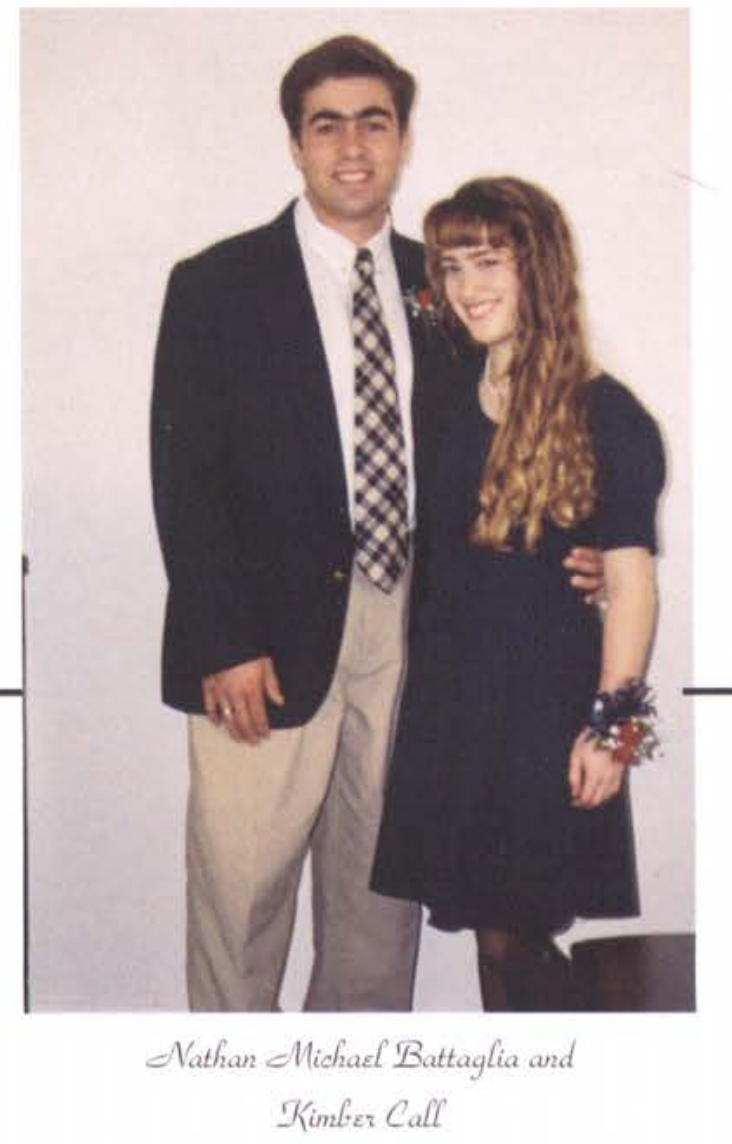

vanted to accept others for who hey were. She said that Wildasin made those around im feel at ease and was also isually trying to make them augh. Kelly also remembers his ability to think of small vays to encourage others, like pringing her favorite desert to er one evening after knowing he had had a bad day. "He vould do anything for anypody," Hoppe said, and she emembers his willingness to e available for his friends vhenever they needed to talk. Although Wildasin was a seior biology major planning to to to graduate school, he enoyed literature and writing and ad a library that included many lassics. Assistant professor f English, Mr. James nowden, enjoyed having him $\mathrm{n}$ world literature class and njoyed even more the friendhip they were able to develop

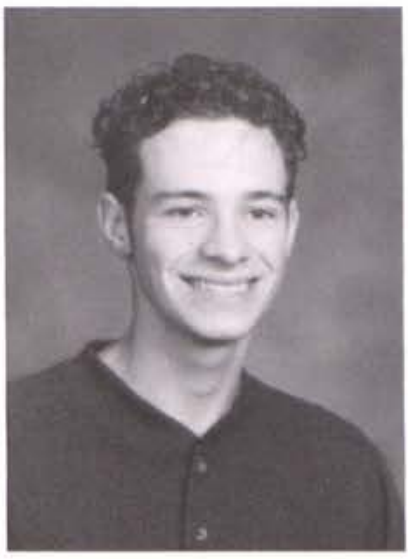

Stephen Michael Brown

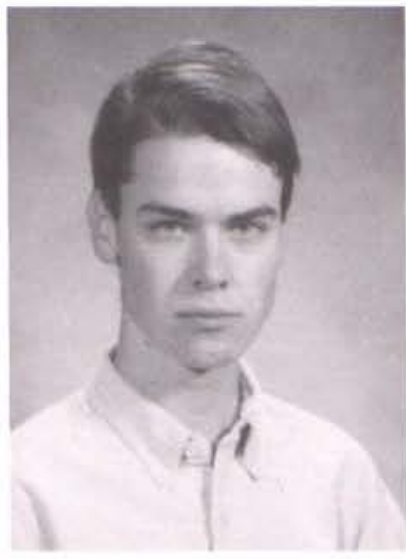

Stephen Joel Wildasin and in comforting Battaglia's family and his friends who would feel his absence most keenly. Dr. Wheeler, who had been his pastor for more than twenty years, testified how Battaglia's life and death had glorified God, saying that his commitment to God was a model for all of us. He described the height disadvantages Battaglia faced as a teenager and how he turned his experience into compassion instead of bitterness. He said, that because Battaglia suffered, "he was sensitive to others who were unlovely, who had also been rejected." Pastor Wheeler further described Nathan as unselfish, giving his time, energy, resources-his whole self to others. "Nate had all the ingredients of a servant's heart; he was driven to serve and had a capacity to love that seemed unlimited." The climax of Pastor Wheeler's testimony came when he described how Battaglia's friend Shawn, to whom Nathan had been witnessing since junior high, accepted Christ as his Savior as a result of Nathan's death. Pastor Wheeler then prayed for the void left by Battaglia to be filled by those who would commit their lives more fully to God and exhibit the kind of compassion that Battaglia had for people. Dr. Dixon closed the chapel service with a challenge to the College family to get serious about living for God all the days that $\mathrm{He}$ has given us, reflecting on our own mortality and remembering to number our days. As the Apostle Paul wrote in Philippians 1:20, "I eagerly expect and hope that I will in no way be ashamed, but will have sufficient courage so that now as always Christ will be exalted in my body, whether by life or by death." Chapel ended with Dr. Dixon leading the College family in singing, "Christ is All I Need."

Charles Spurgeon concluded his discussion about the deaths of God's children by saying that our prayers for our loved ones need not strive against the prayer of our Lord, but in conforming to the will of the One who desires them to be where $\mathrm{He}$ is, we can pray, "Lord, You shall have them. By faith we let them go." family in expressing grief at the loss of one of our members and Dr. Bill Wheeler, pastor of Emmanuel Baptist Church in Xenia, Ohio, led the College 
Laura Abraham Matt Adams

Debbie Allen

Mark Allen

Meredith Allgrim

Josh Amos

Jim Amstutz

Nikki Anderson

Ryan Anderson

Laurie Anna

Becci Appel

Julie Armour

Kevin Armstrong

Christopher Ashcraft

Dena Assid

Jason Atwell

Joshua Ausfahl

Allison Baer

Erin Barker

Jill Barnes

Holly Barnett

Brad Bartlett

Jonathan Basner

Sara Bathrick

Matthew Beck

Jaime Bedford

Eric Bedillion

Summer Bennington

Aaron Berning

Edwin Bett

Allyson Bielo

Stacey Billing

M. David Blackburn

Michelle Boehm

Julia Boertje

Jill Bollman

Michael Bonner

Rob Bouwens

David Boyd

Matthew Brainard

Lisa Branon

Beth Brentlinger

Stacy Brewer

Melody Brickel

Chris Brinkley

Derek Brinkmeier

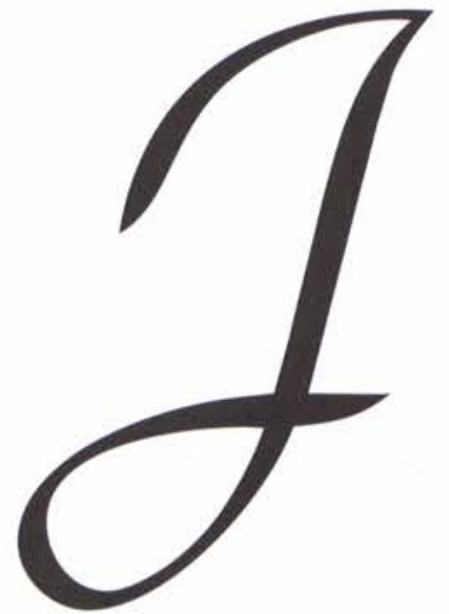

\section{uniors}
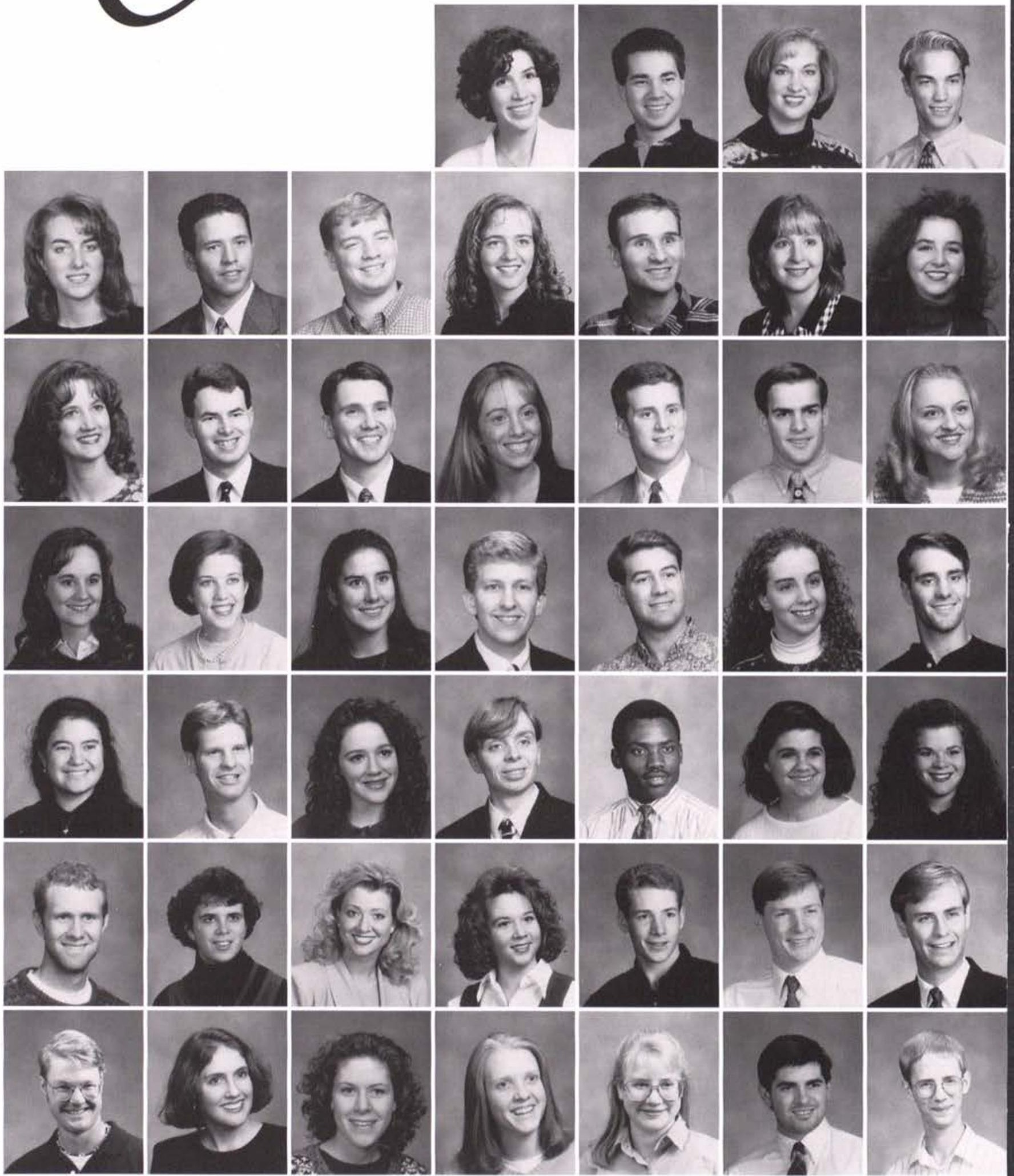

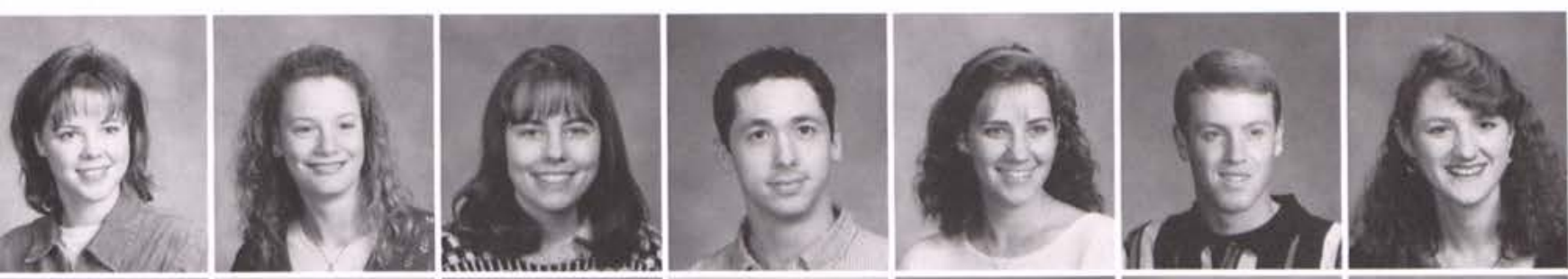

Heather Brodie

Andrea Brooks

Evelyn Brown

Jason Brown

Amanda Bruckner

Steve Burchett

Margaret Burgess

David Burke
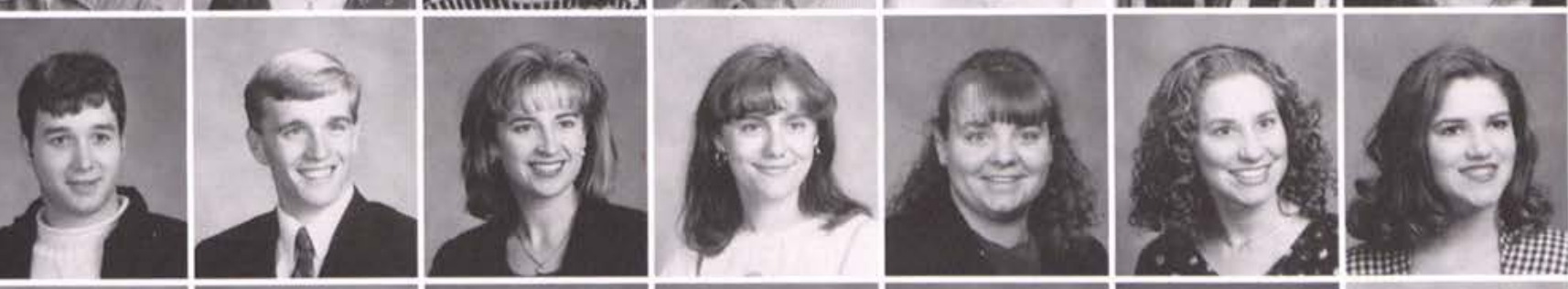

Ryan Burkhard

Sharon Burns

JoHanna Byrer

April Carter

Kristyn Carter

Kristine Chamberlin
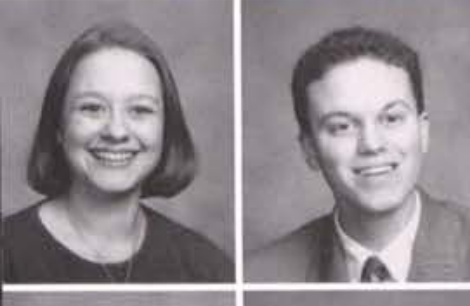

2
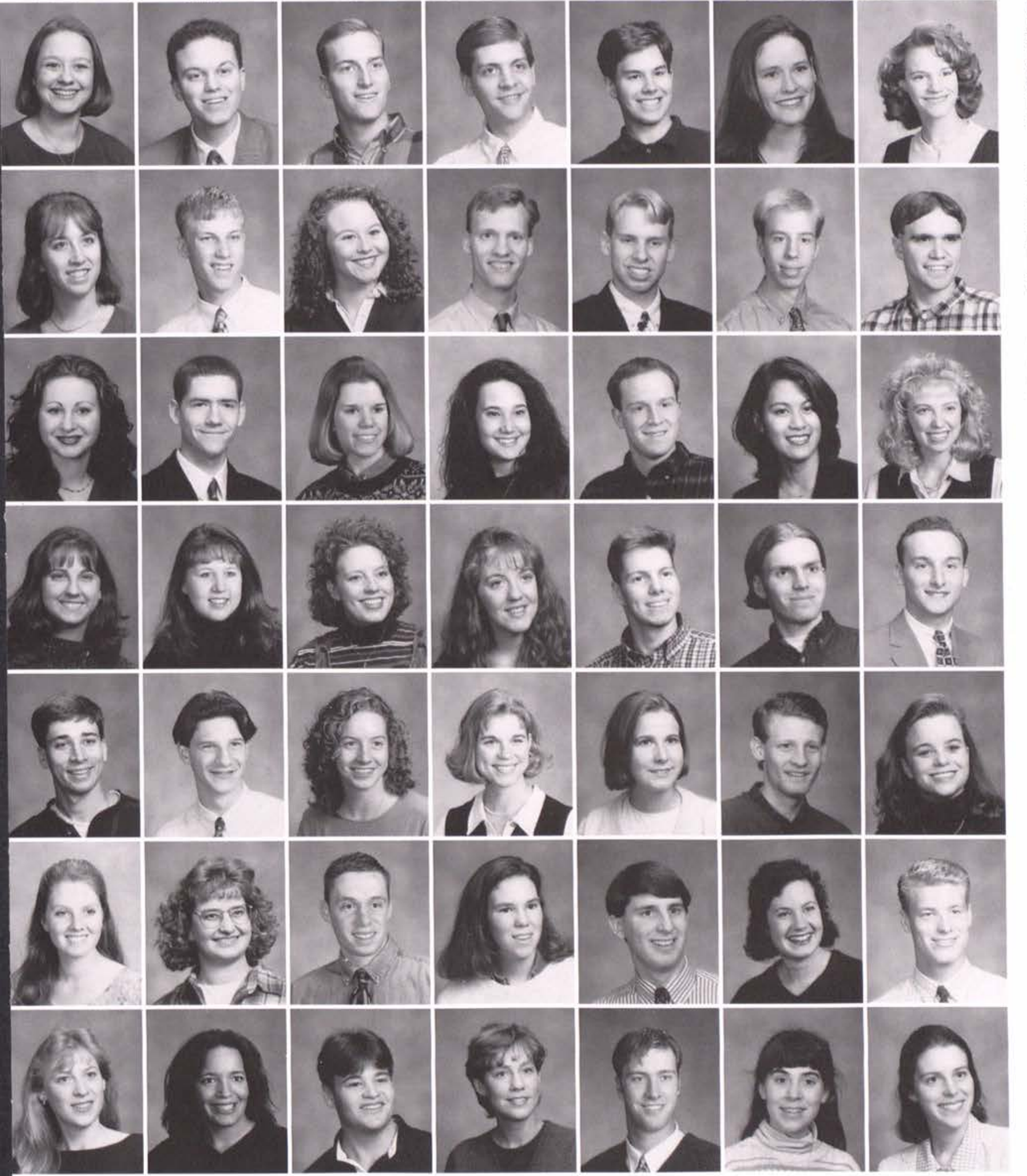

Lea Anne Churgovich

Jason Cirone

Ernest Clark Jr.

Paul Click

Daniel Cochrane

Beth Comer

Rebecca Comfort

\section{Lisa Cook}

Andrew Cooper

Brandi Covert

Eric Crawford

Michael Crawford

Jim Creswell

Gabriel Custer

\section{Gina Davidson}

Clay Davis

Carrie DeKock

Toni DeUsanio

Richard Dewalk

Anna delRosario

Jennifer Decker

Joey Decker

Stephanie Dickerson

Jamie Dodson

Cheri Douglas

David Duhaime

Aaron Dunham

Ryan Dyer

Joel Eaby

David Edwards

Amy Egolf

Stacie Eldeen

Rachelle Elder

Jeff Elliott

Debbie Ellison

Jean Estes

Martha Failor

Bryan Falk

Jennifer Fisher

Timothy Flowers

Laura Foeldvari

Joel Foerch

Amanda Foote

Kelly Ford

Kevin Fountain

Heather Fourman

Matt Fourman

Renee Francis

Kristen Frederick 


\section{Jen}

Praying Dogether

\section{Lutz}

At almost any time one can find a group of three female students sitting together with heads bowed in prayer. They may be in a dorm room or a $\mathrm{st} u \mathrm{de} \mathrm{n} \mathrm{t}$ lounge or an e $\mathrm{m} \mathrm{p} t \mathrm{y}$ chapel. The loc ation does not matter, nor does the size of the group. What excites them is the opportunity to spend time in prayer at a

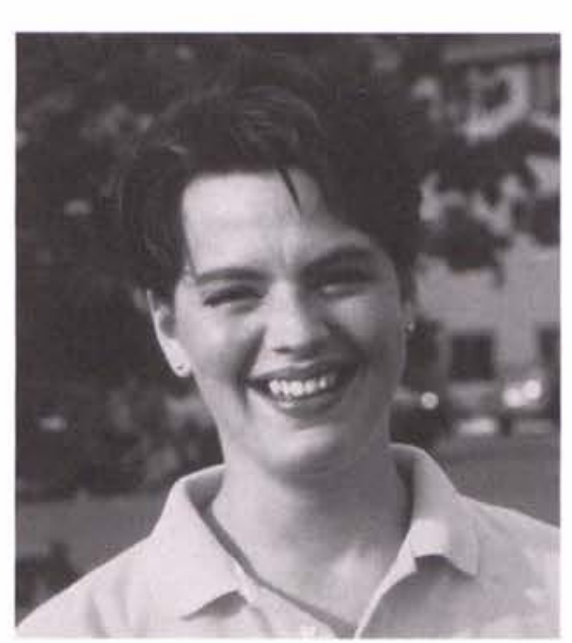

entire campus. The desire spread to other groups, and soon several nursing students began to meet at their own time. Several RAs expressed how God moved in the hearts of those in their units, and several hundred became involved in the Experiencing God Bible study. The desire for women to pray and felset time each week with others who hold the same vision, bringing personal requests and campus concerns before God's throne. Groups like these have committed to similar half-hour time slots throughout the week.

Cedarville junior Jenny Lutz first had the idea for organized campus prayer during this past summer. Throughout that summer, God "rearranged" her life, as Jenny puts it, causing several struggles to increase her desire to know God more. Other friends from her home in Orlando, Florida, were also experiencing His change, and shared with Jenny what God was doing on their secular campuses.

The Prayer Force began in September as eight women met one morning at seven o'clock for prayer. Turnouts on these Monday mornings increased, and gradually the focal point turned to revival across the covering the campus in prayer each day. What no one expected was the willingness in the hearts of those gathered; almost one hundred groups signed up immediately to pray for one half hour per week. Since then, the system has improved, with boxes filled with prayer cards now situated around campus. Each prayer group can pick up the current requests and bring those needs, and any additional ones they have, to God. All involved express an eagerness to see what God will do when people humble themselves in prayer (2 Chronicles 7:14) and wait to see what He reveals (Jeremiah 33:3). These two verses have become the desire of Prayer Force.

Keturah Stork
Victoria Frederickson Heidi Fuller

Bethany Gaffner Christiana Gain

Betsy Galovits Heather Ganly

Stephen Garcia Kristen Gault

Melody Gbur Rebecca Genovia

Jill Gerber Stephen Gerber
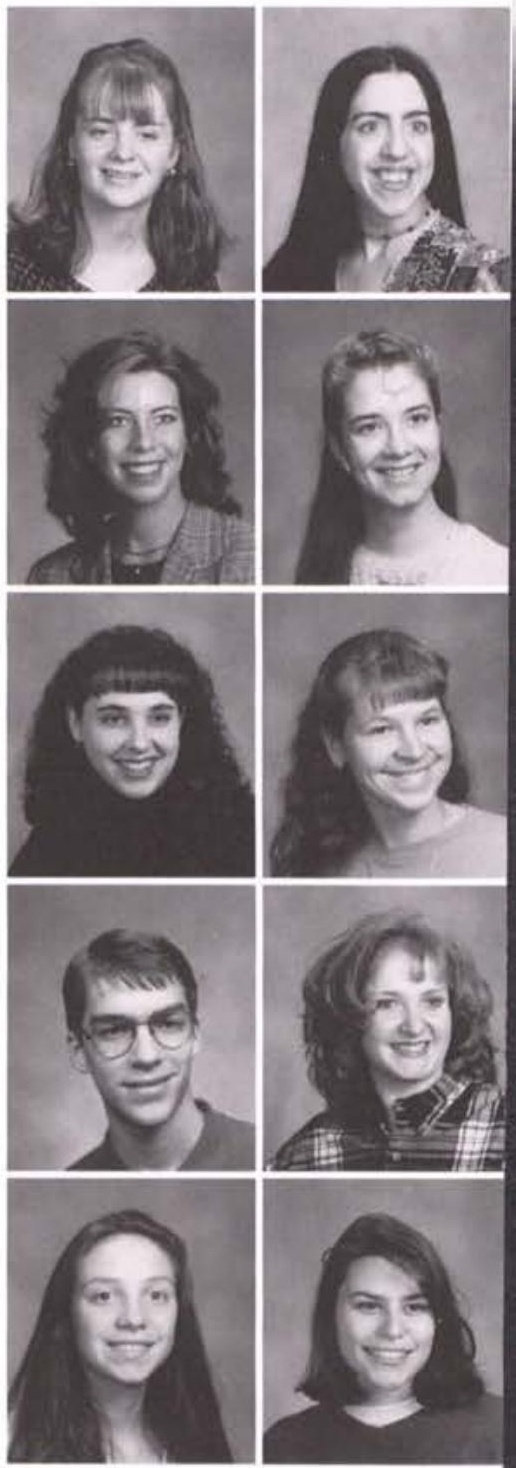

Cathy Gifford Linda Gilbert
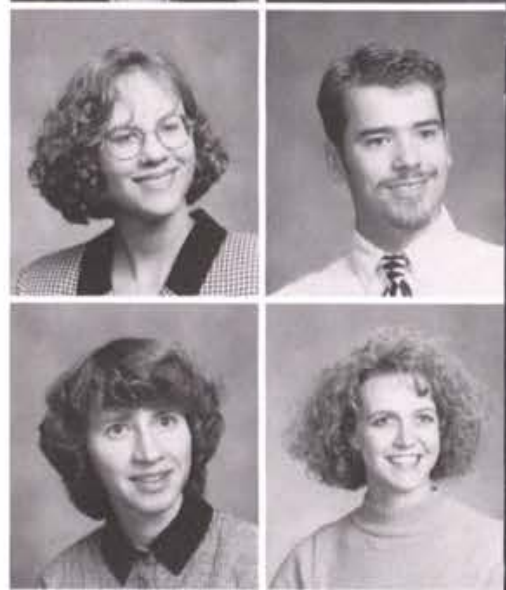

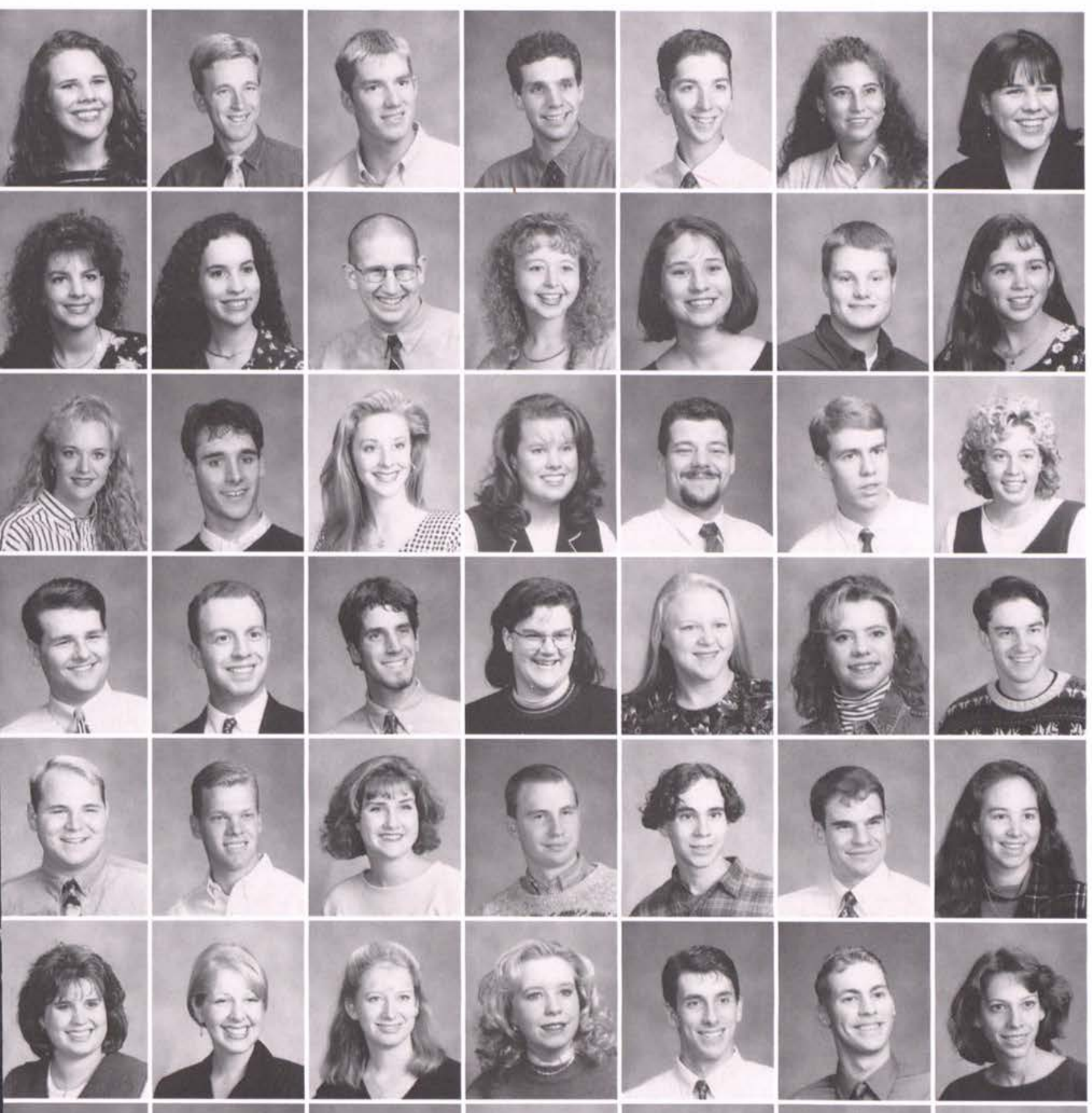

Sarah Gross

Carrie Gwilt

Benjamin Haffey

Jodie Hager

Sara Hall

Josh Halulko

Lori Hamilton

Tiffany Hamilton

Anson Hanbury

Jennifer Hangosky

Cara Harju

Michael Harsh

Nathan Hart

Joy Hasty

\section{David Helton}

Daniel Hicks

Michael Hidalgo

Brooke Higgins

Michelle Higgins

Alicia Hill

Dusty Hill

\section{Geoff Hillman}

David Hofert

Julie Hoffman

James Holz

David Hoskins

Tim Hotchkiss

Nancy Houck

Kathleen Housten

Jody Hovis

Abigail Howe

Patricia Hubbard

Dan Hudson

Brent Hughes

Erica Hunter

Amy Hurst

Scott Husband

Aaron Hutchison

Jeff Hyatt

Brenda Inion

Becky lvey

Julie Jaskilka

Rebecca Jenks

Erin Johns

April Johnson

Jaime Johnson

Julien Johnson

Rebecca Johnson

Todd Johnson 

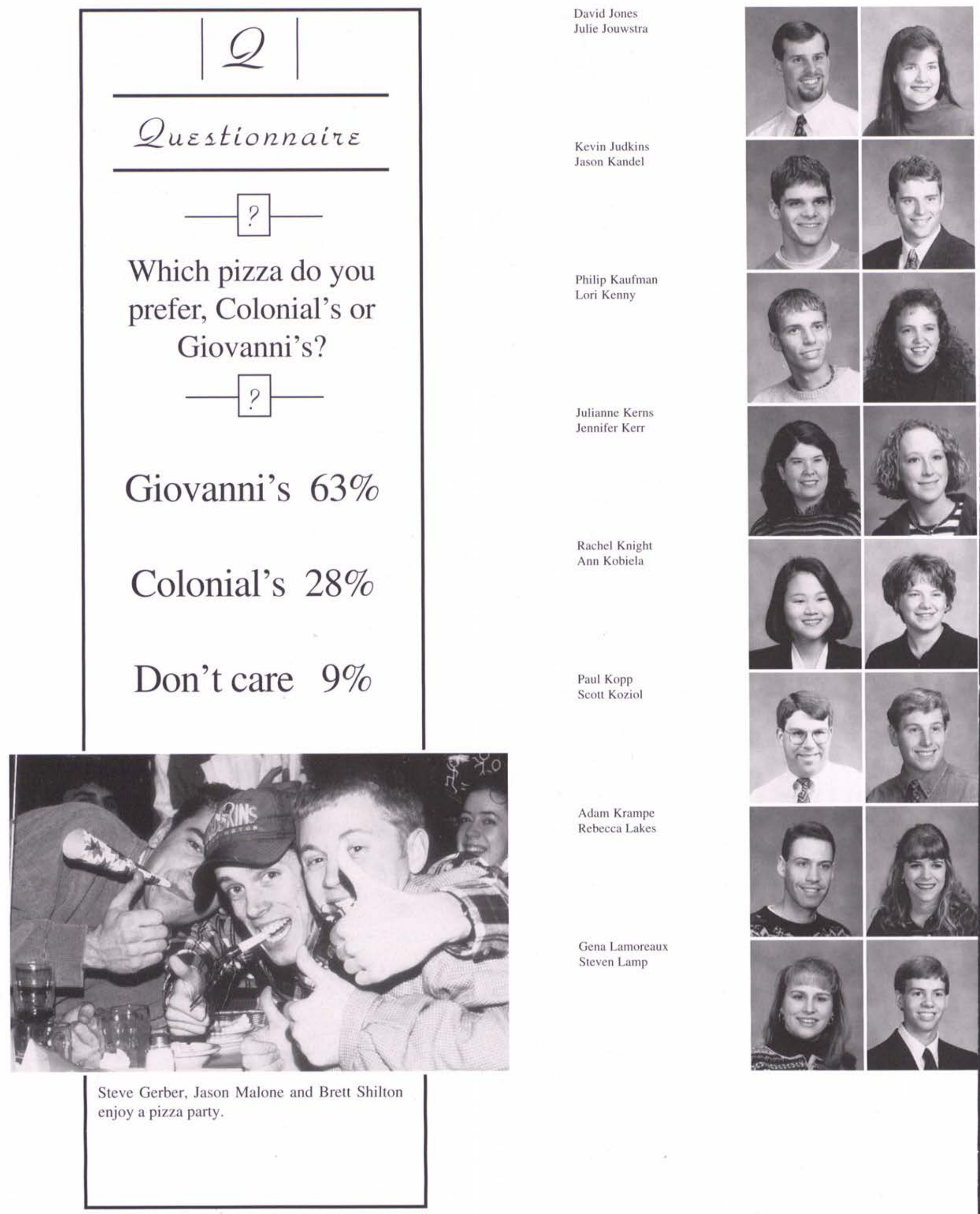

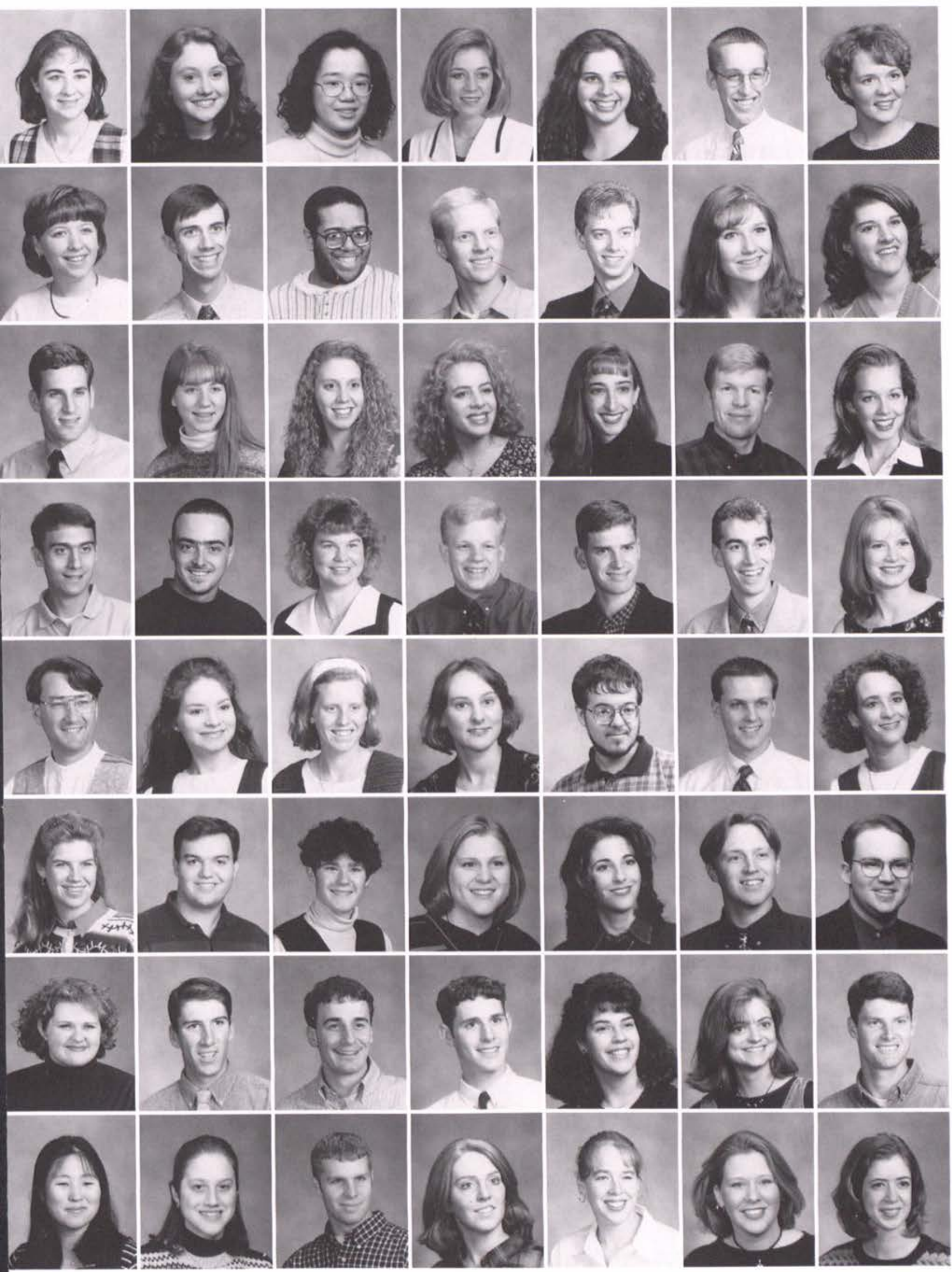
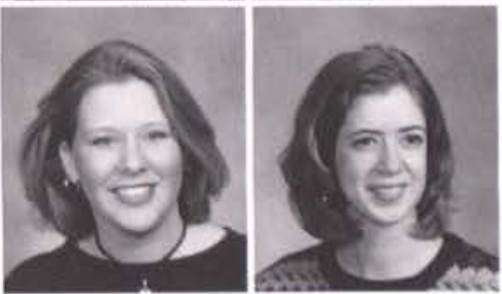

Anna Lankhorst

Jaime Larson

Sonwoo Lee

Angela Lenhart

Nellie Leuck

Christopher Leverette

Sarah Lightly

Karisa Linafelter

Joe Lloyd

Hugo Lopez

Derek Luke

Craig Magrum

Amy Martin

Christine Martin

Neil Massai

Tamara Matula

Kim Maynard

Annie McClintock

Julie McCoy

Dale McCrory

Noel McDermitt

Stephen McGillivray

Brian McGuinness

Becky McIntyre

Pete McLeod

David McPherson

Ethan McQuinn

Emily Meeks

Steve Merchant

Angela Meredith

Angela Meyers

Tammy Michaels

Jeff Mick

Kyle Miller

Mindy Miller

Sara Miller

J. Timothy Miller

Stephanie Moody

Katie Moon

Kathryn Moore

Tom Mullins

Rob Mulvaney

Heather Murdoch

Mathew Murphy

Brad Murray

Jeff Neuman

Amy Nichols

Patricia Noble

Ben Nordaas

Atsuko Ohtake

Andrea Osterc

Dan Parlin

Elizabeth Parr

Wendy Passineau

Rebecca Patten

Jessica Patton 
Mitona Pel

Charis Perez

Holly Peterson

Melinda Pickel

Ruthanne Pierson

Hilary Pifer

Christopher Pittenturf

Samantha Polgardy

Aaron Ponzani

Richard Porter

Cynthia Potter

MaryBeth Powell

Elizabeth Price

Nathan Radford

Seth Rafferty

Jacob Rambo

David Rea

Jamin Reda

Laura Refior

Jesse Reibson

Rebecca Ribeiro

Patricia Rice

Eric Riddle

Chrissy Rising

Charity Rizer

Micah Roberts

Shawna Roberts

Charles Robinson

Carrie Robyck

David Rooke

Rene Rosencrantz

Julie Ross

Chrystie Ruba

Daniel Rudd

Ann Ruegsegger

Paula Rummel

Jason Runnion-Gray

Joshua Rupp

Carla Salvaggio

Stacy Saville

Michele Schafer

Mark Schleith

Bethany Schloegel

Stephen Schoon

Laura Schulz

Lana Schwinn

Dana Scott

Will Scotton

Tami See

Amanda Senior

Andrew Shaw

Scott Shaw

Karin Shilling

Courtenay Shoaff

Lesley Shover

Erick Shumaker
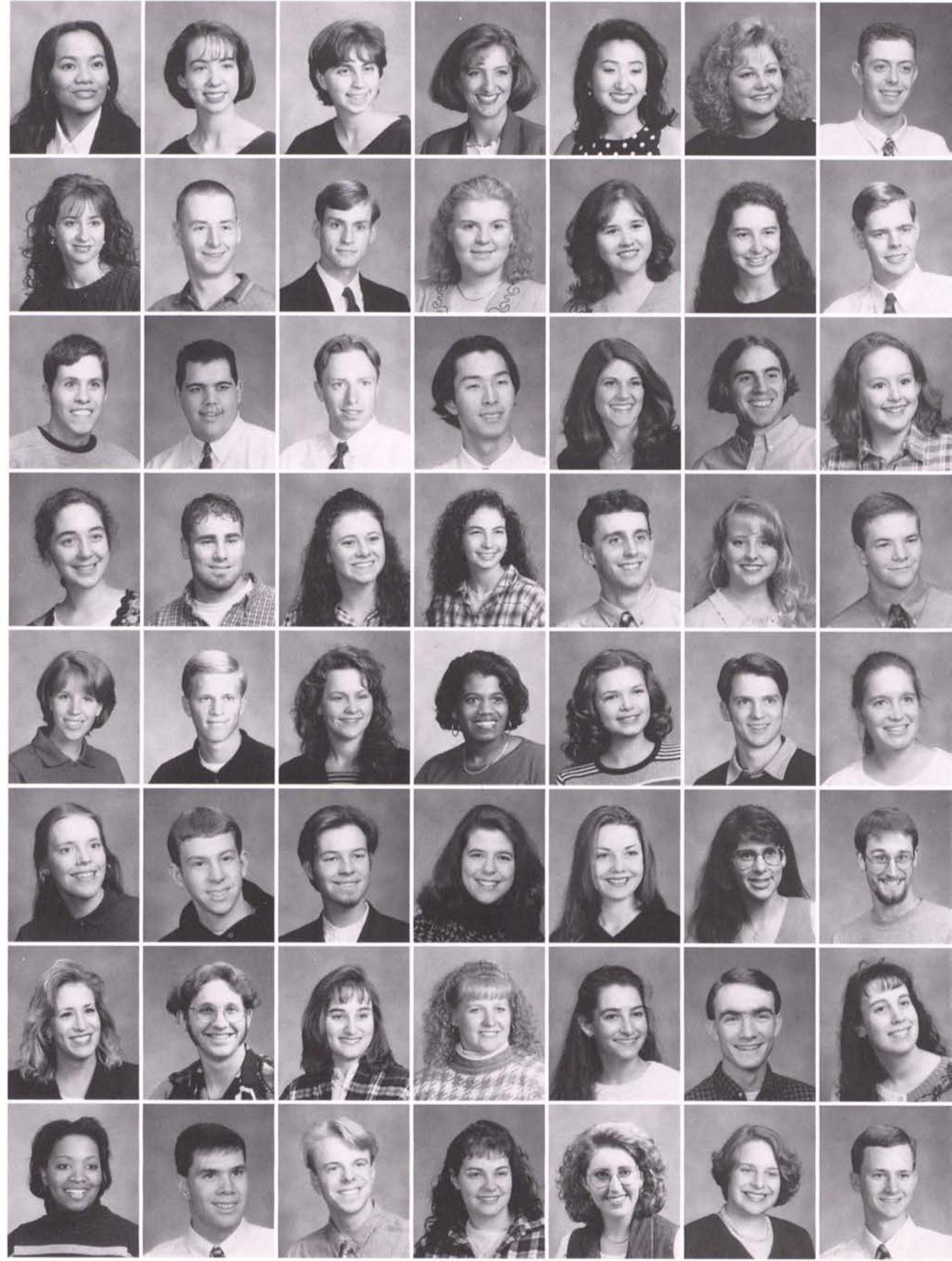
Nikki Streit

Randall Strong Abby Stroven

Christopher Strychalski

Jessica Stuenzi

Matt Stutzman

Sarah Svendsen

Sharon Swank Brenda Tabberer

Brooke Taylor

Daniel Temmesfeld

Amy Thayer

Greg Thompson

Matthew Thompson

Sarah Townsend

Jeremy Toyer

Trisha Trost

Scott Van Loo

Scott Vandegrift

Jennifer Vander Bush

Ben Vawter
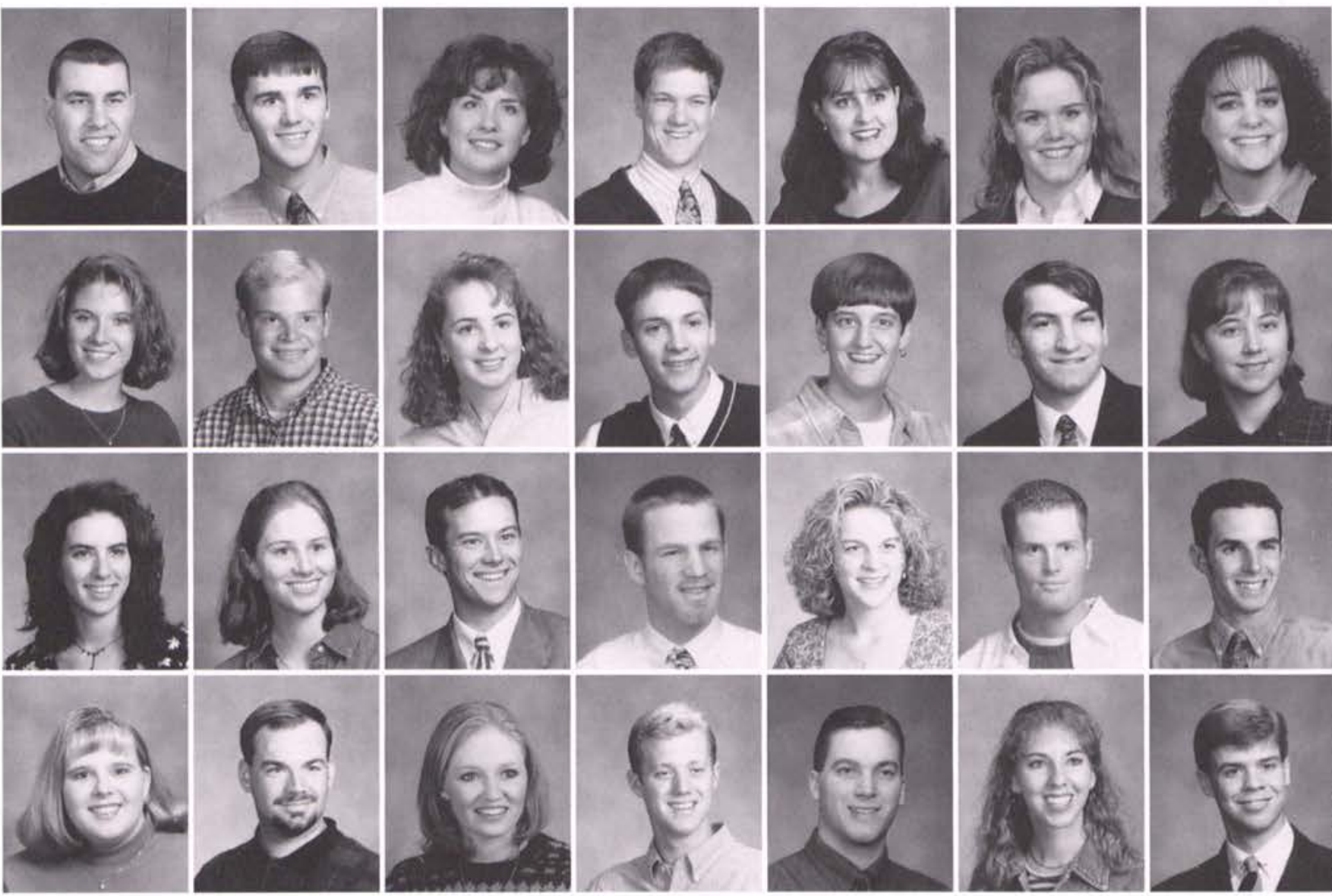

\section{The Invasion of Art}

$$
\text { Gy Heather Jourman }
$$

For years, one could enter the Fine Arts Building several blocks from the main campus without finding any evidence of the visual arts. But all of that changed three years ago with the arrival of Mr. Terry Cham-

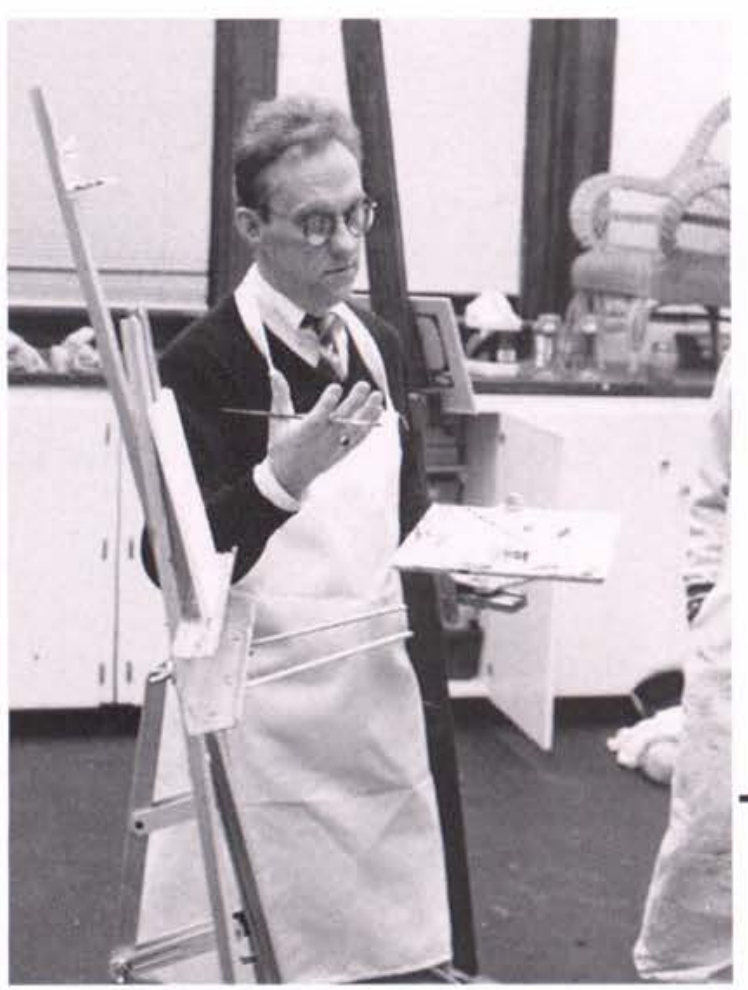

berlain, Assistant Professor of Art. Ever since he began offering art courses, student interest has grown, so that now, for the year, art is being offered as a minor.

Before coming to Cedarville, Chamberlain taught art classes at Dayton Christian School for 23 years. About five years ago, Dr. $\mathrm{C}$ h a $\mathrm{r} l$ e s Clevenger, chairman of the Department of $\mathrm{Mu}$ sic, asked him to come and teach one art course at Cedarville as a means of gauging student interest.
Chamberlain said that students responded positively, and courses were added one by one until now. For the first time, there are enough courses to establish art as a minor.

Chamberlain is encouraged by students' interest in art. He said, "I strongly hope that the college can build toward offering art as a major within the next few years." Chamberlain also said that the first majors in art would be in graphic design and art education.

Chamberlain's hopes of an art major are well founded. Academic Vice President Dr. Duane Wood said that he is glad to have the art minor in place, and is optimistic about the college's future goal of establishing an art major. $\mathrm{He}$ said that the timing of the nex step depends largely upon how well the art minor grows.

Chamberlain said that it is important for Cedarville to de velop an art program becaus so many other Christian col leges do not have quality ar courses. Students who want to study art but also want to at tend a Christian college ar often forced to choose betweer the two.

"On a secular campus Christians face the potential o their spiritual lives deteriorat ing, which, of course, will af fect their art," said Chamber lain. "So what better place t train a Christian artist than in Christian college?" 
Jennifer Abas

Daniel Adams

Dawn Albertson

Jason Alexander

Emily Alt

Jennifer Alt

Al Anderson

Ehrin Arimura

Mark Armstrong

Alyssa Arndt

Wayne Arnold

Rachael Ayres

Jennie Bahn

Alicia Baisley

Allister Baldwin

Rebecca Barber

Elizabeth Barker

Emilio Basa

Lezley Bath

Trevor Batt

Rob Bayley

Jennifer Bear

Matthew Beckley

Bryan Bell

Andrew Bergman

Josh Berrus

Joseph Beyer

Ryan Bigelow

Haylee Biggs

Jessica Black

Rebekah Blackwood

Darcie Blakemore

Cynthia Blanton

J. Erik Bledsoe

Melissa Blore

Kevin Boblitt

Kimberly Boesch

Amy Bohn

Michelle Bolt

Rebecca Border

Sara Bosaw

Dan Bosworth

Elizabeth Bowersox

Kris Boyes

Shannon Boynton

Kelly Bozick

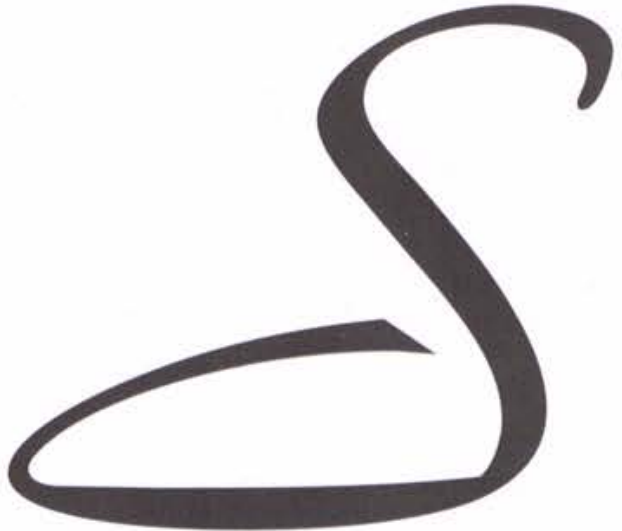

ophomores
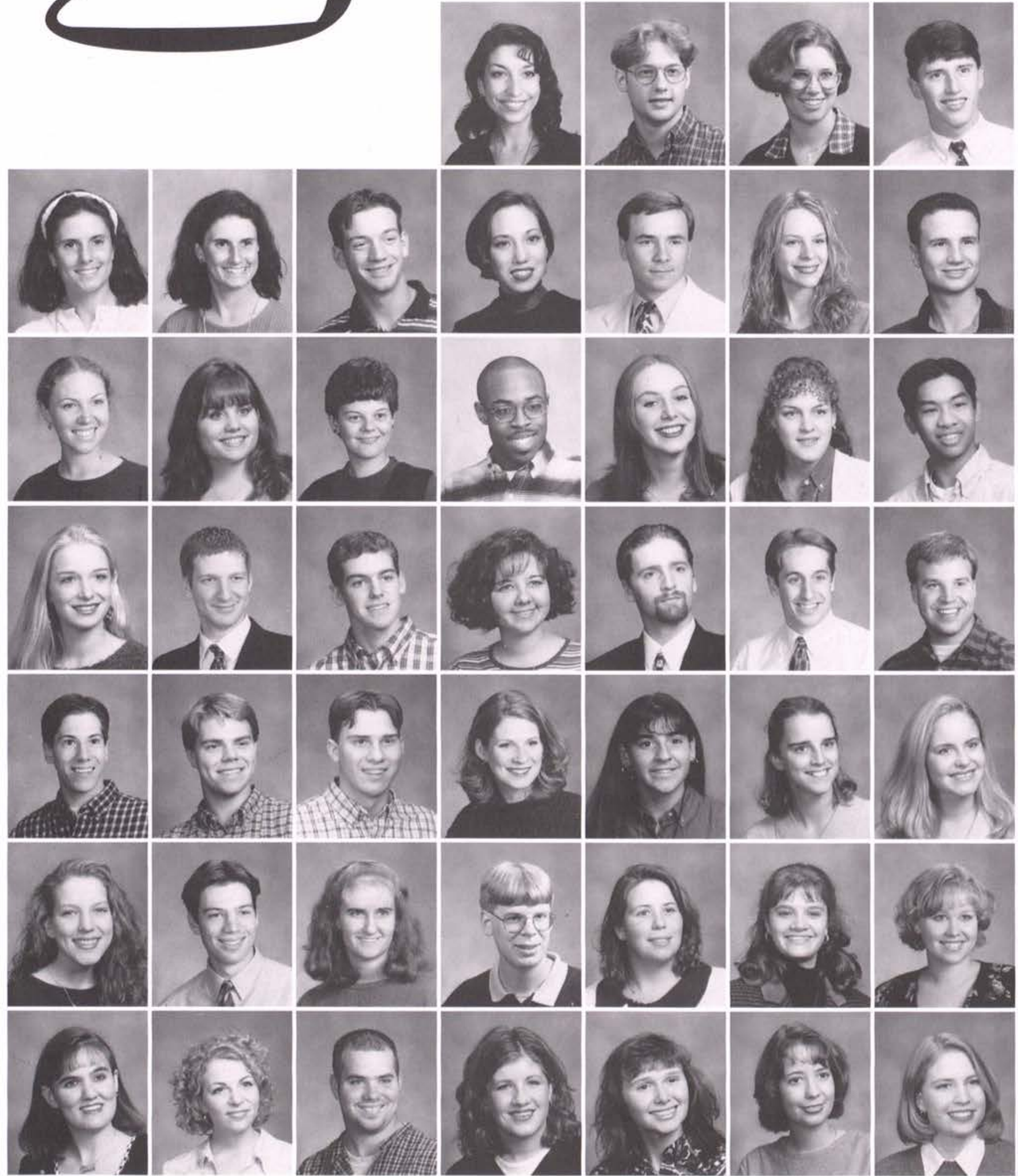


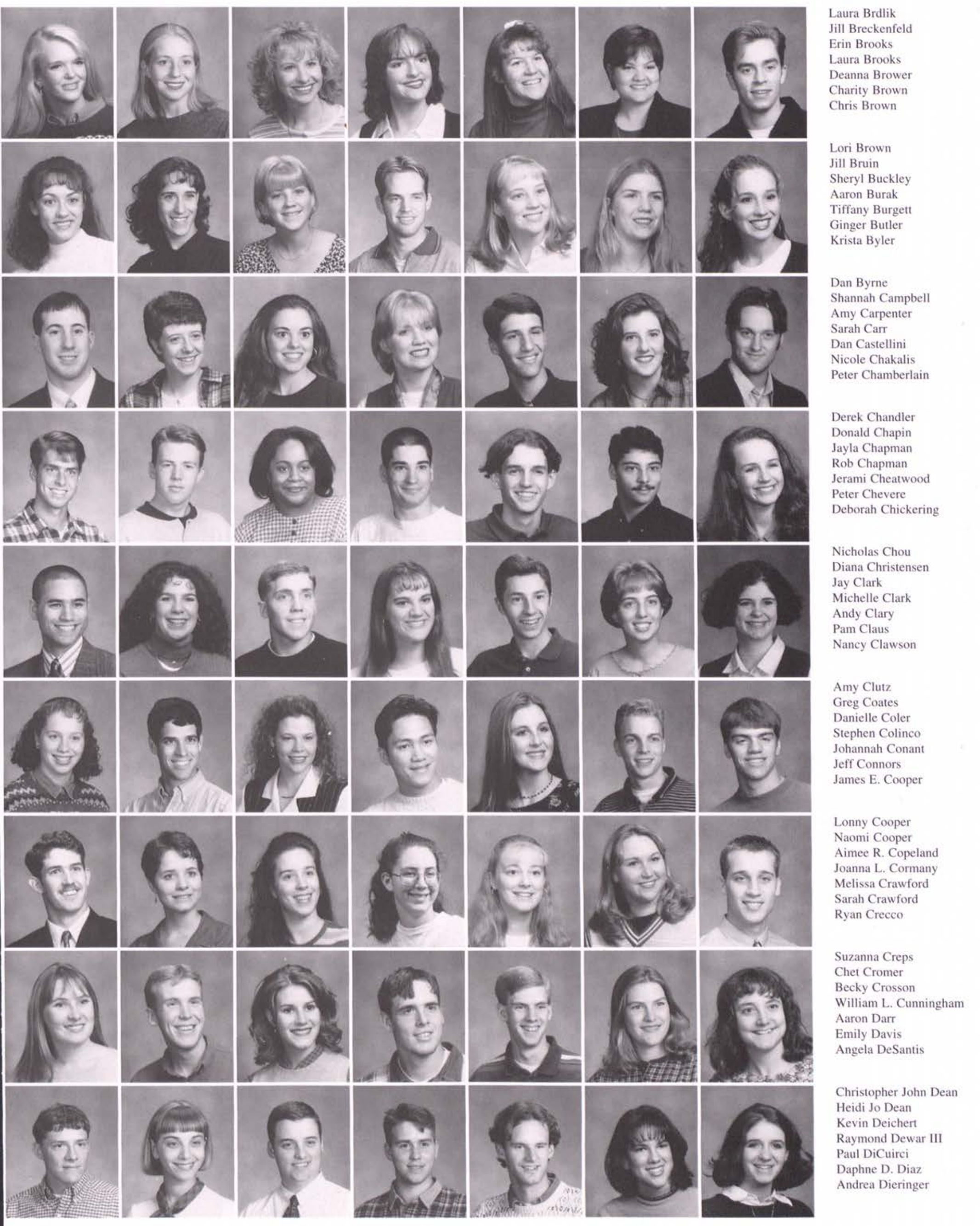


Emily Dizer

Guna Doerr

Jason Doese

Damon Douridas

Kelly Drager

Jodi Lynn du'Monceaux

Susan J. Dunham

Bryan Dye

Rachel M. Dyer

Bryan Emanue

Jon Emery

Jocelyn Endsley

Roxanne Englan

Rachel English

Kimberly Ervin

Rutledge Etheridge

Brent Ferguson

Noella Fisher

Rebecca Fissel

Eric Fiveland

Greg Flory

Sonya Rondelle Fogliani

Daniel Forness

Tedd Forrest

Ben Forshee

Julie Forstrom

Jori Forward

Jolie Fourman

Heather Fraker

Bethany Frank

Nathan Freeman

Stephanie Friemel

Doreen Erika Fuhr

Jess E. Gamiere

Nick Gapinski

Brian G. Gardner

Jennifer Ann Gardner

Tanya Gardner
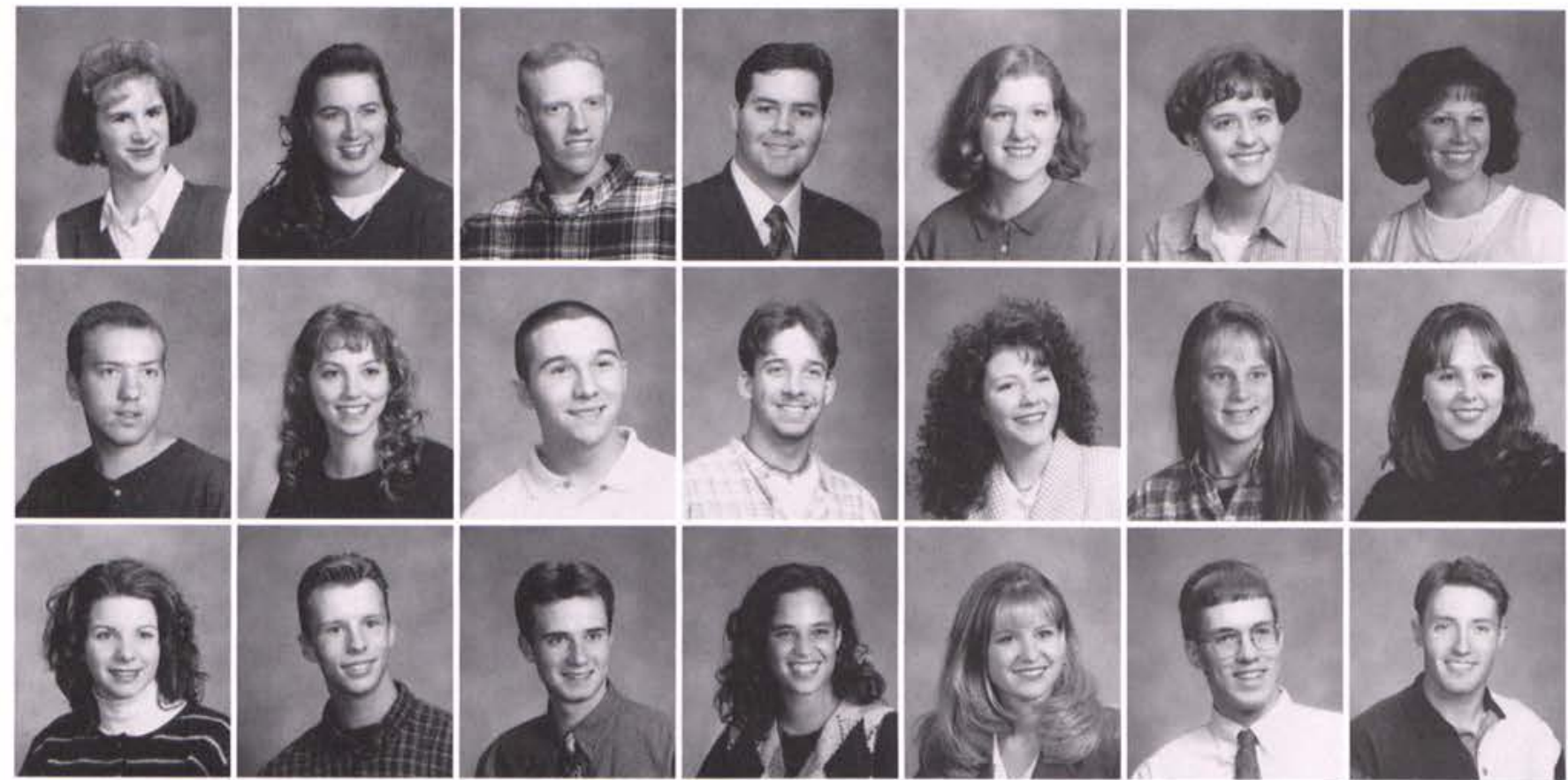

.
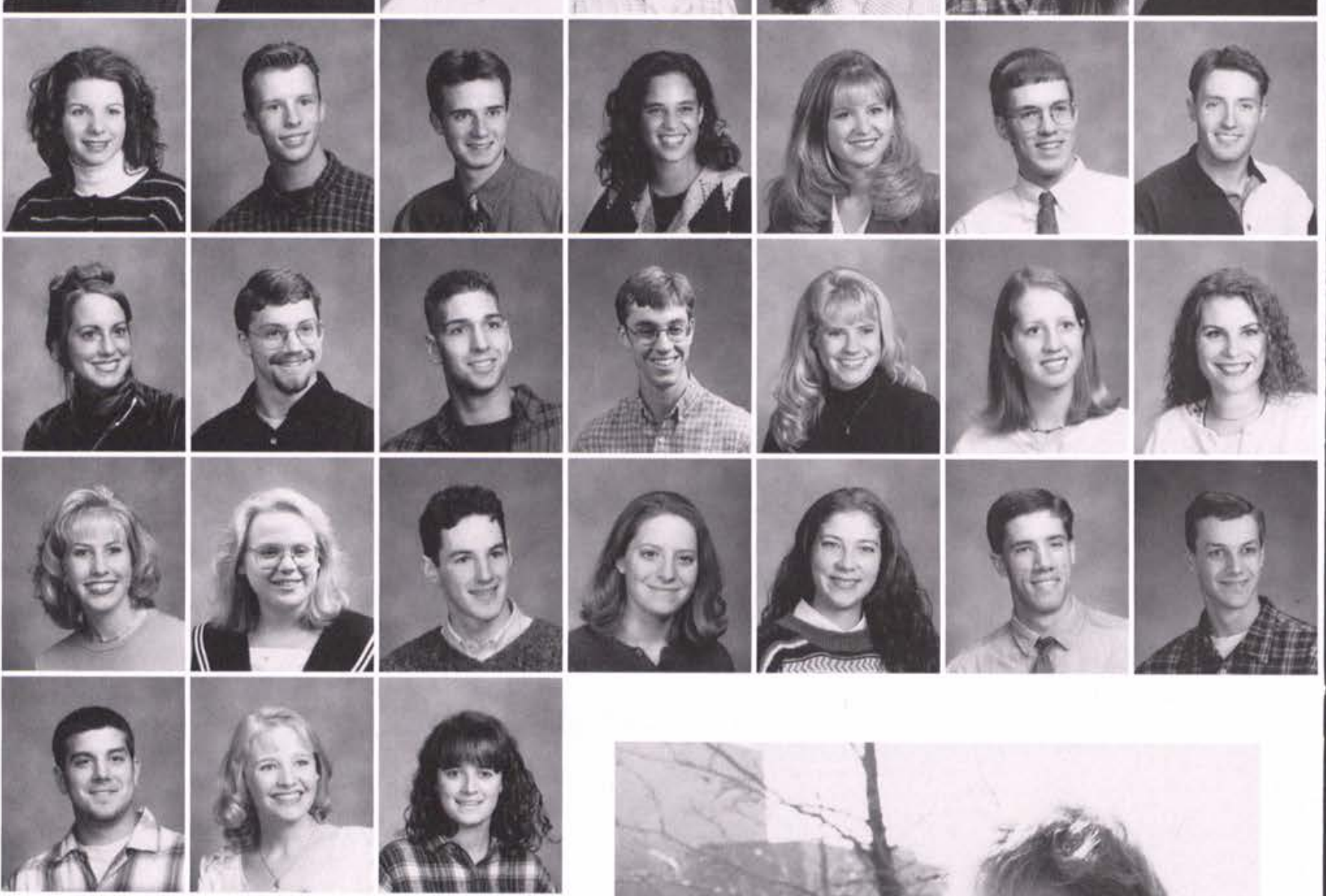

When the sun comes out so do the students. Right: John Grapentine, playing guitar, and Mark Wyse, on the drums, enjoy the nice weather by making music. Below: Christina Lay and Brett Frey tour campus on a bicycle built for two.
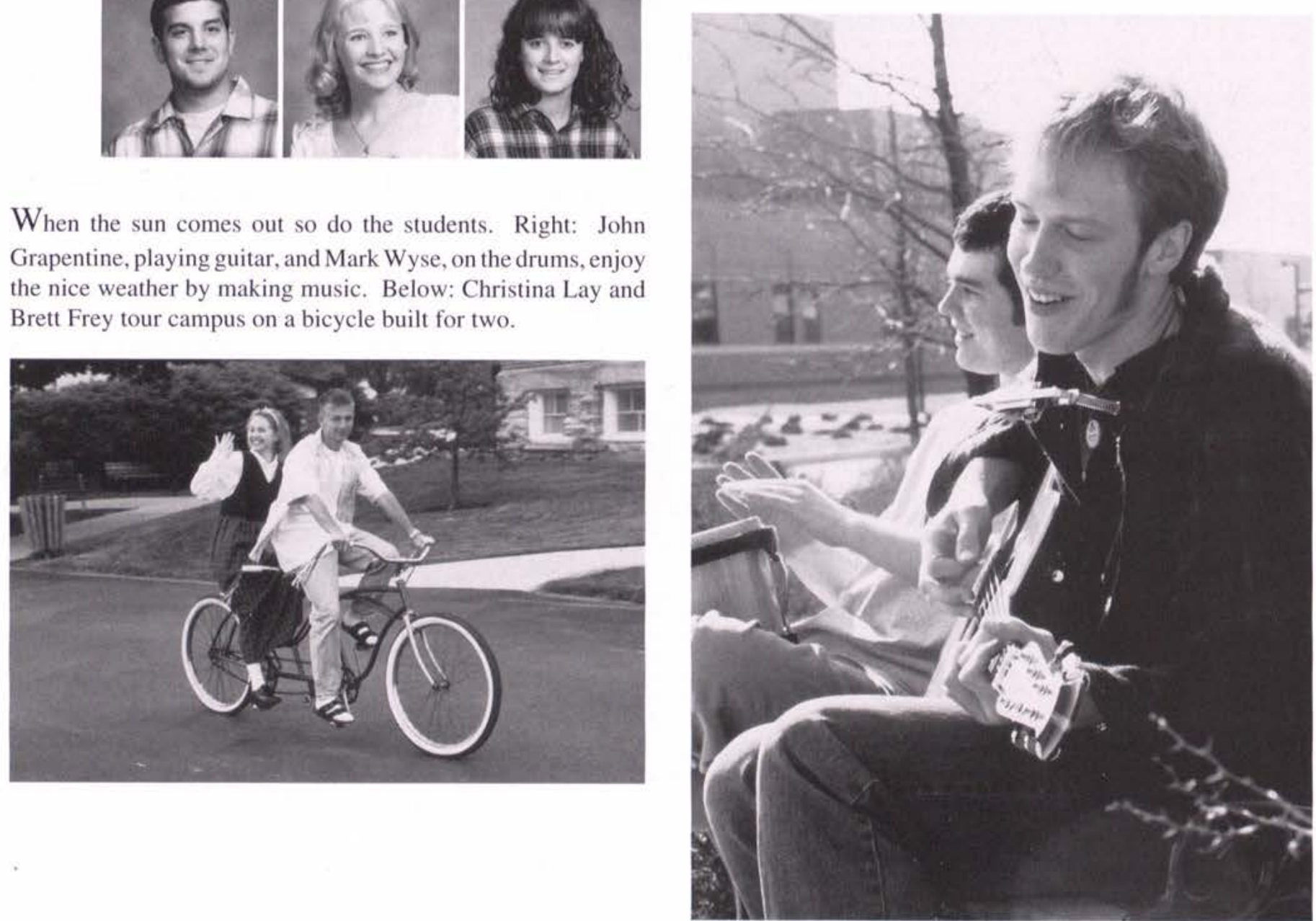


\section{Students Back Dole}

Danny 7. Cook

At the Bloomingburg farm where Bob Dole spoke on Thursday, Oct. 10, the weather was gray and cold. Farmers, who made up a majority of the crowd, kept the flaps down on their hats, some enjoying the ruckus of chants, flags, and placards, some becoming restless. Many waited for more than two hours before the Republican presidential hopeful showed, having to be content with the spectacle of reporters, secret servicemen and CBS crew that set up spotlights in the bleachers.

Among the Bloomingburg crowd were 200 Cedarville students. Trip Radtke, a senior English/Political Science major, organized the campus-wide effort. It was part of his job as one of four state campaign coordinators of Young Ohioans for Dole/Kemp.

"On Tuesday, I had twenty people signed up; by Wednesday evening, after I had sent out the e-mail, I had about 160. A lot of RA's took their whole units. Our students filled up the back half of the whole rally area," Radtke said.

Below: Don Smith, Sara Bosaw, Cara Hines, Sarah Simons, and Veronica Mayer all cheer for Presidential candidate Bob Dole.

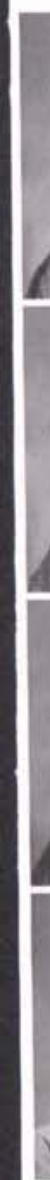
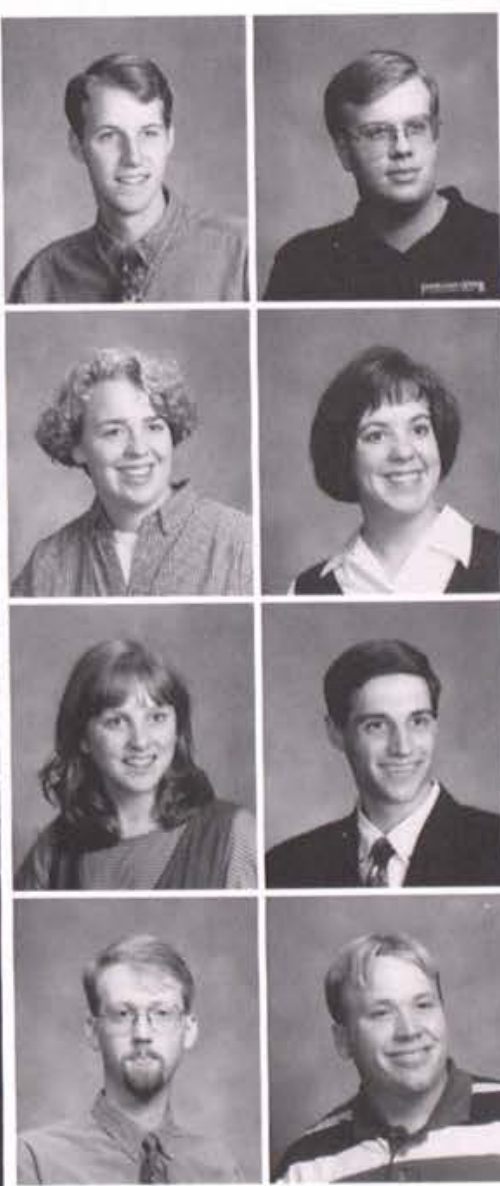
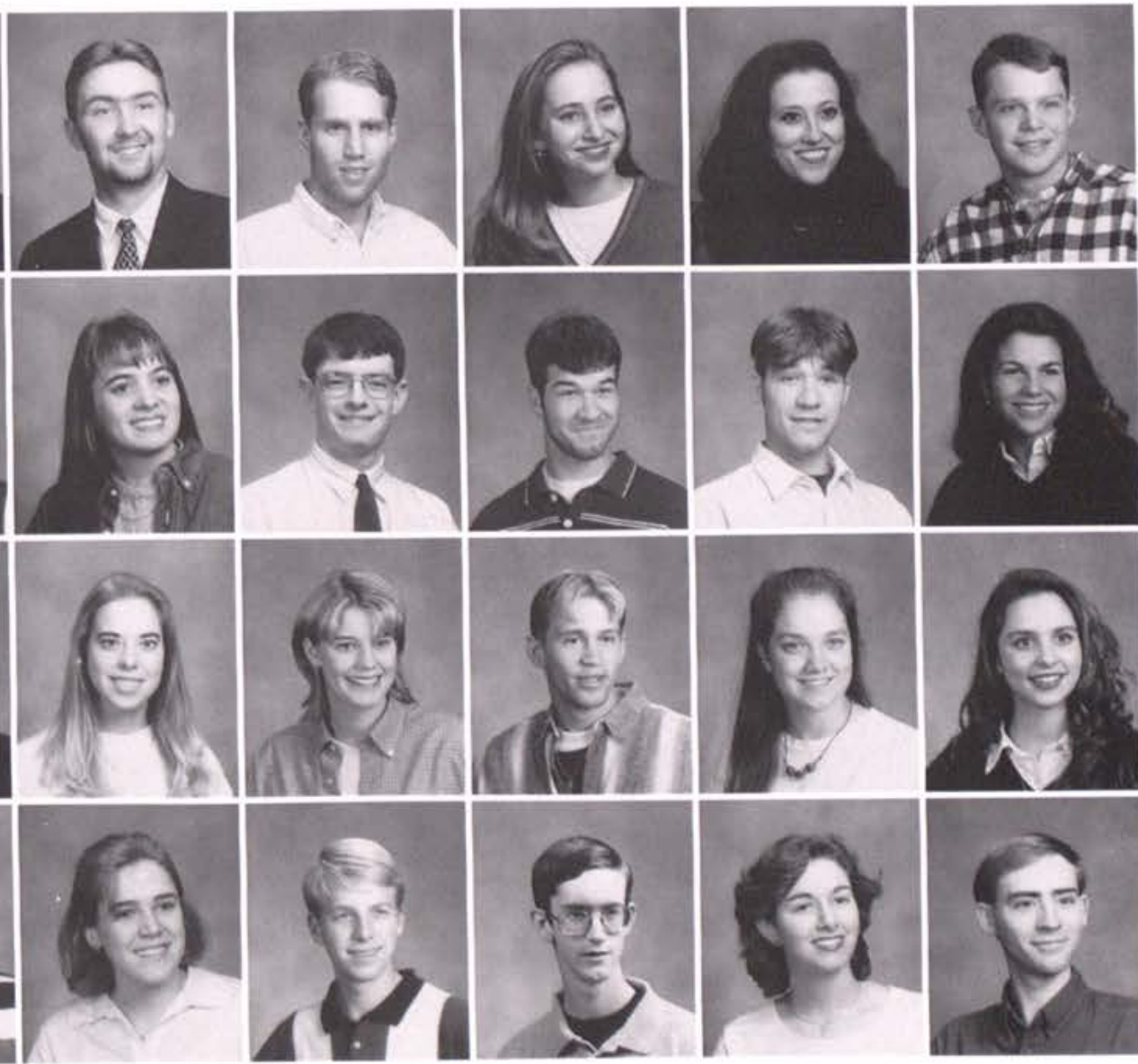
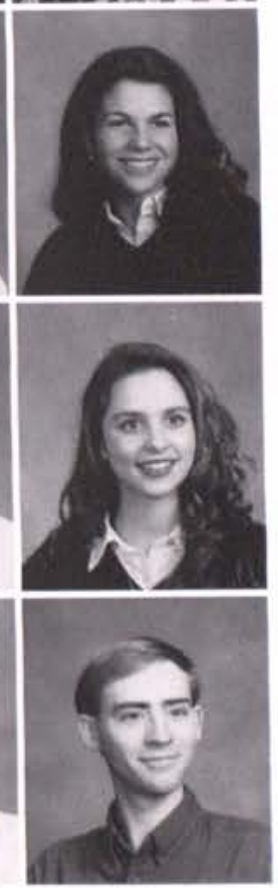

Brian Gault

Peter J. Gauvin Brandon Geiger Jonathan Geiger Amber George Amy Gillespie Dave Gillespie

Shelly A. Gillett Jana Glessner

Erin Goehring Steve Goldsworth Barry Gorsuch Berney D. Gorsuch Kelly Eileen Grady

Monique Gras Kevin Greer Rachel Griffin Lesley Grow Michael Grubbs Carolyn Guerette Lara Gyutik

Kevin Haisch Matthew D. Hamer Elizabeth Hamilton Steve Handel

Lance E. Harkleroad Rachel L. Hart Dave Hassenzahl 

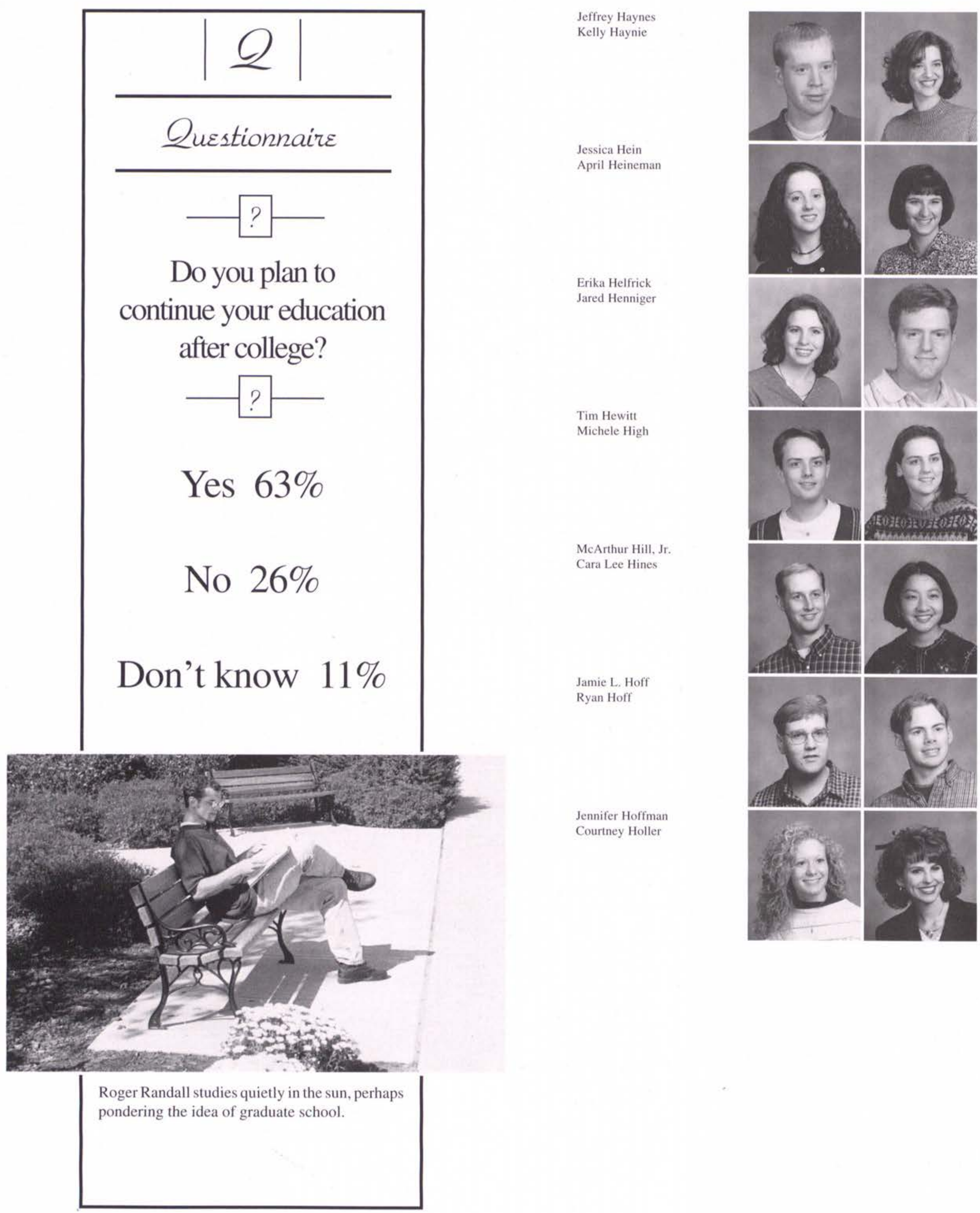
Melanie A. Lehr Meaghan Lemke Jim Leonard

Michael Lewis John C. Lieb, Jr.

Andy Litteral

Matt Loose

Shawn Lundvall Mandy MacKay

Daniel Craig MacLean

Stephanie Mace

Joan Mallman

Carrie A. Mansfield

Samuel Mantracadi

Ghena Marie Marchetti

Rachelle Marcum

Christy L. Marr

Kristin L. Marshall

Sarah L. Martin

John M. Mason

Michael Maurice

Kathy Mayer

Eileen Mc Coskey

John McCaw

Mike McCubbin

Stacie McDaniel

Elizabeth McDonald

Kathy McFarland

Meredith McGahan

Kimberly 1. McKenzie

Erin McLarty

Philip McVey

Joseph Mellish

Kristina Mencarini

Jason Merritt

\section{Amy Mershon}

Ross Meyers

Jamie Michael

Joshua B. Michael

Mark Milec

Jeff A. Miller

Michelle L. Miller

Shawn LaMar Miller Laura Jean Milligan

Lindsey Milne

Jared Mitchell

Lindsey Mitchell

Anastasia Mobley

Chris Moles

Kelly Montague

John Moodie

Eric Morgan

Jeff Motter
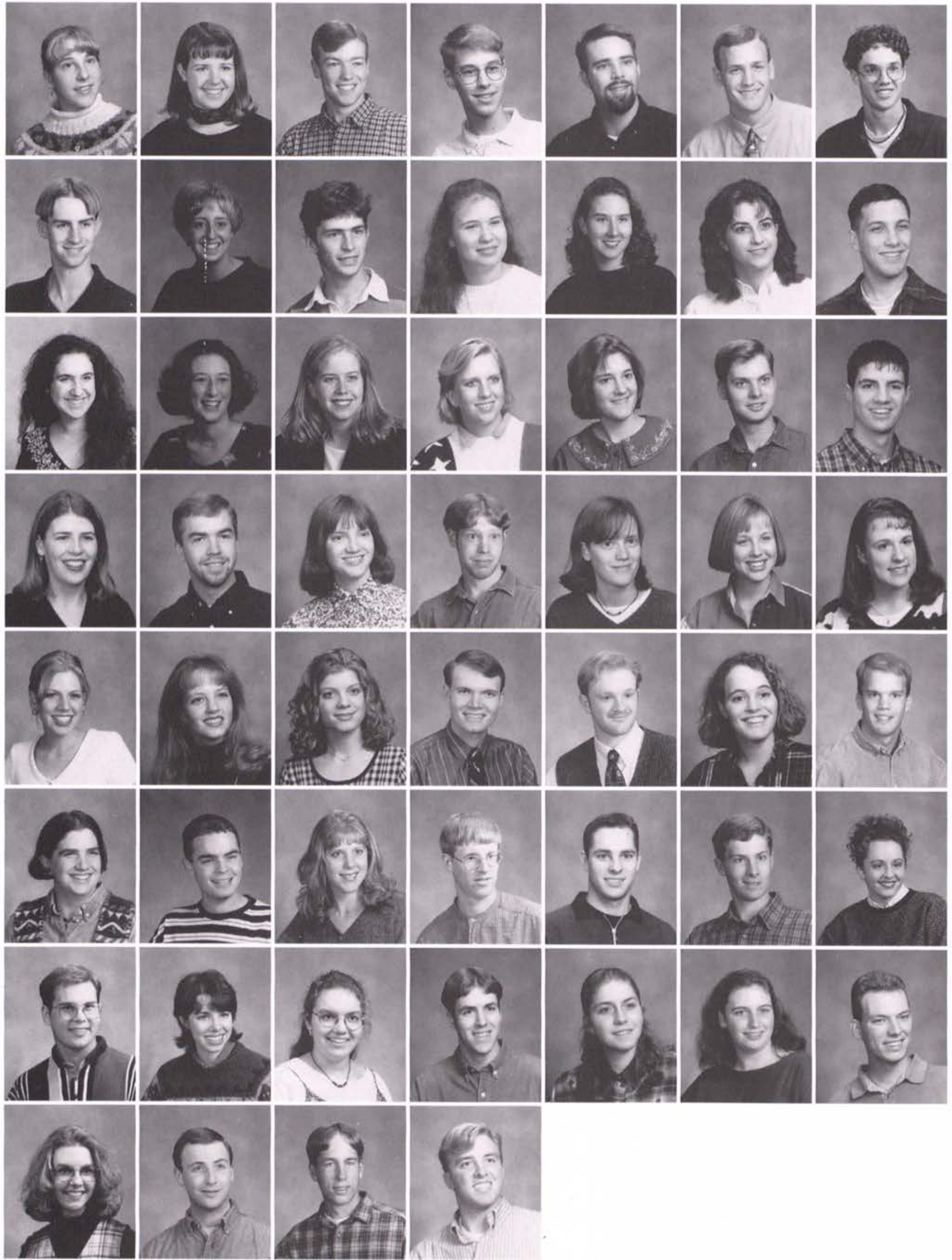

部 


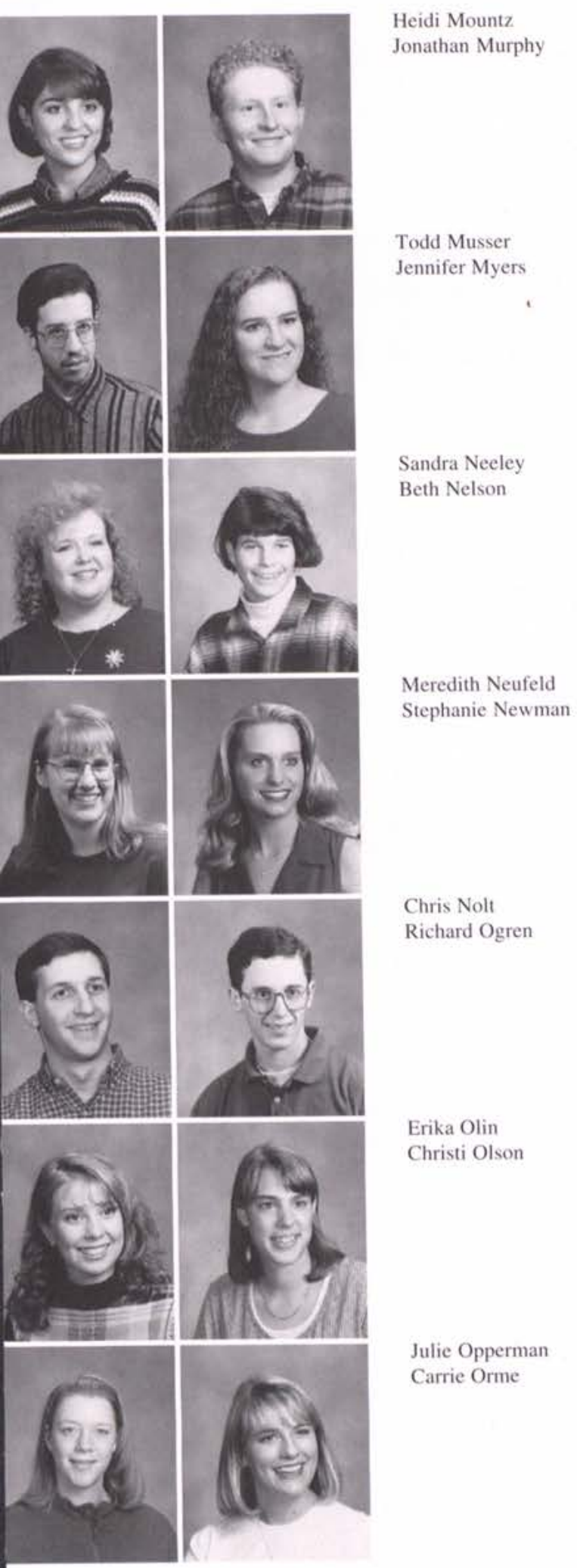

\section{Dr. Vernon}

Church Music

\section{Whaley}

Dr. Vernon Whaley joined the Cedarville music faculty in November, 1995, in order to help create and develop a new major, the Church $\mathrm{Mu}$ sic Ministry $\mathrm{m} \mathrm{a} \mathrm{jor}$, which was approved last May. Previously, this was only available as an emphasis.

Whaley graduated f $\mathrm{r}$ o $\mathrm{m}$

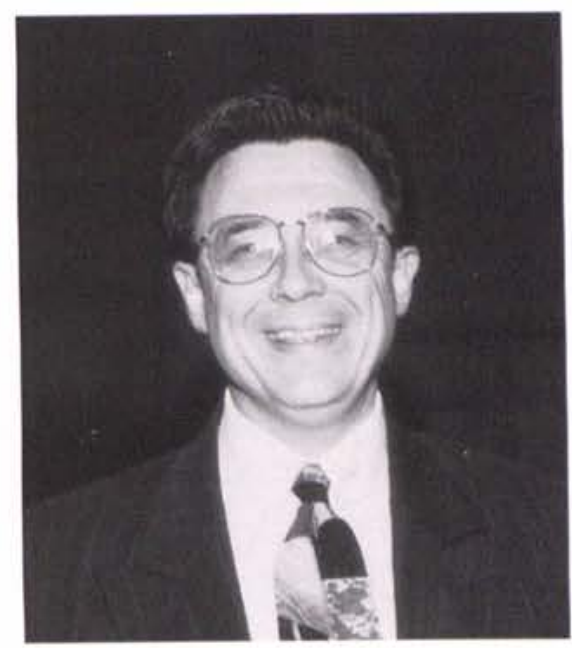
Nashville's

Freewill Baptist College in ulty. 1972 with a Bachelor of Arts in Bible and Music with an emphasis in Voice, and earned a Minister of Church Music and a Doctor of Ministry degree from Luther Rice Seminary in Jacksonville, Florida.

From 1979 to 1985 , Whaley chaired the music department at Freewill Baptist College, while earning his second master's degree, this time in Music Education from Middle Tennessee State University. In 1992, Whaley received his doctorate in Music Education with an emphasis in Composition at the University of Oklahoma in Norman.

Whaley is also involved in the Christian Music industry. In 1990, he started a publish- ing company, Integra Music Group. It was through a publishing project that he met Associate Professor of Music $\mathrm{Ch}$ a rle s Pagnard, and became acquainted with Cedarville. In 1995, Dr. $\mathrm{Ch}$ arles Clevenger, chair of the Department of Music, a s k e d Whaley to join the fac-

In the short year that Whaley has been at Cedarville, he has helped create the new music ministry major, as well as teaching numerous classes. He coordinated a summit on church music, hosted at Cedarville March 3-5, which was attended by pastors, music ministers, publishers, editors, and artists, including Steve Camp, Buddy Green, Warren Wiersbe, Don Wyrtzen, and Dan Milheim.

In addition to his teaching duties, Whaley still works on arranging, recording, and publishing; last spring, he produced two recordings for Cedarville, featuring numerous vocal and instrumental groups from the college.

Erica Chung 
Kerri Osterc

Matthew Palombo

Janel Perkins

El Campos Persons

Leah Peter

Ben Peterson

Tim Phillips

Sheri Phipp

Chris Pierre

Michael Pierson

Dale Pinkley

Deborah Pittman

Yvette Pizano

Amanda Poff

Richard A. Porter

Ross Pound

Melissa Powell

Erin Prentis

Jeremy Preston

Joseph M. Preston

Rachel Puckett

Kathleen Pugh

P.J. Puryear

Jennifer Puterbaugh

Naseem Qussar

Roger Randall

Erin Reagan

Joe Reaper

Brian Reen

Annmarie Reynolds

Gregory Rheam

Kimberly Rideout

Amy Rigg

Brent Rill

Amanda Ringler

Mark Robertson

Erin Ross

Melissa Salyer
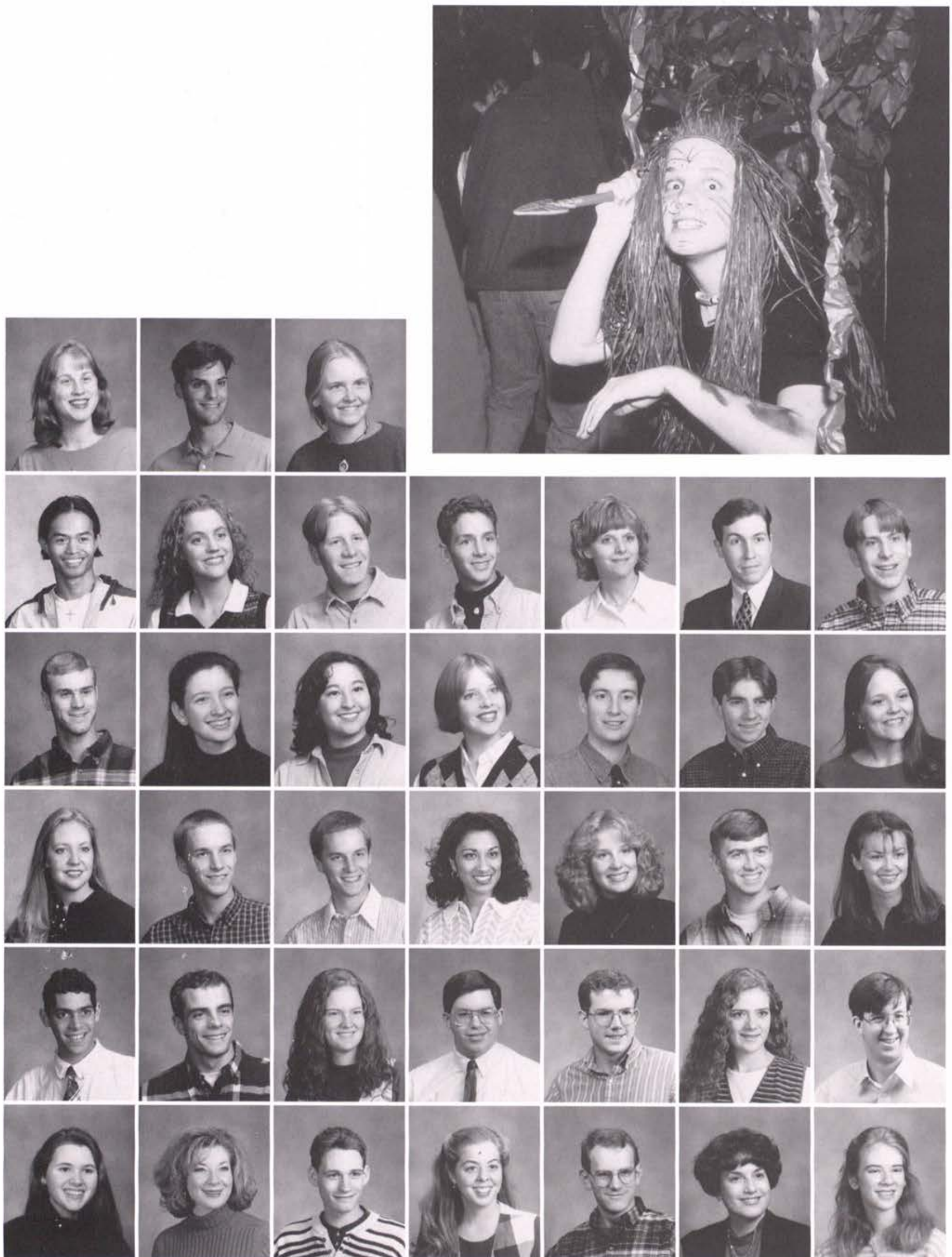

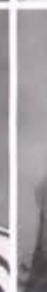

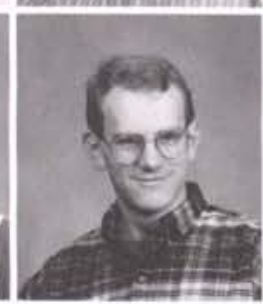
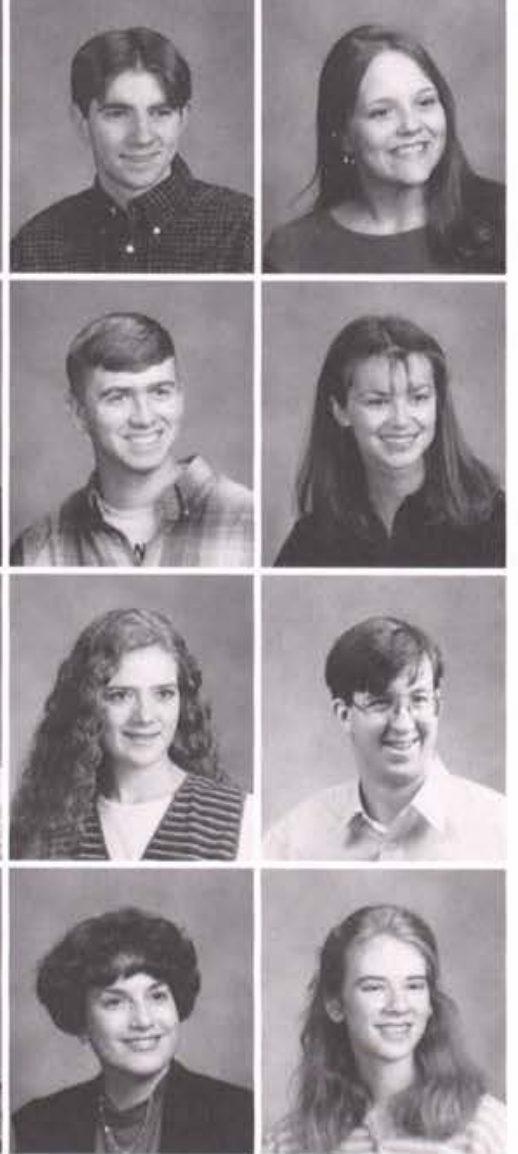

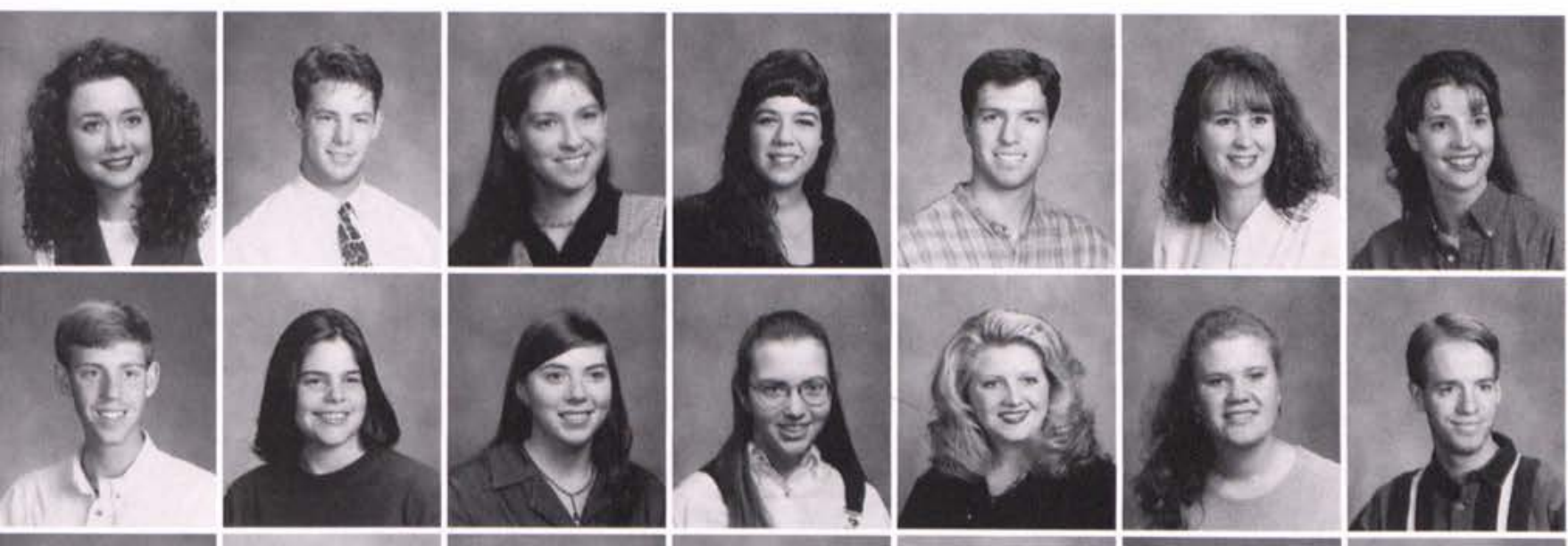

Selena Samuelson

Tom Sanderson

Bonnie Schaefer

Summer Schafer

James Scheid

Megan Schier

Lindsay Schneider
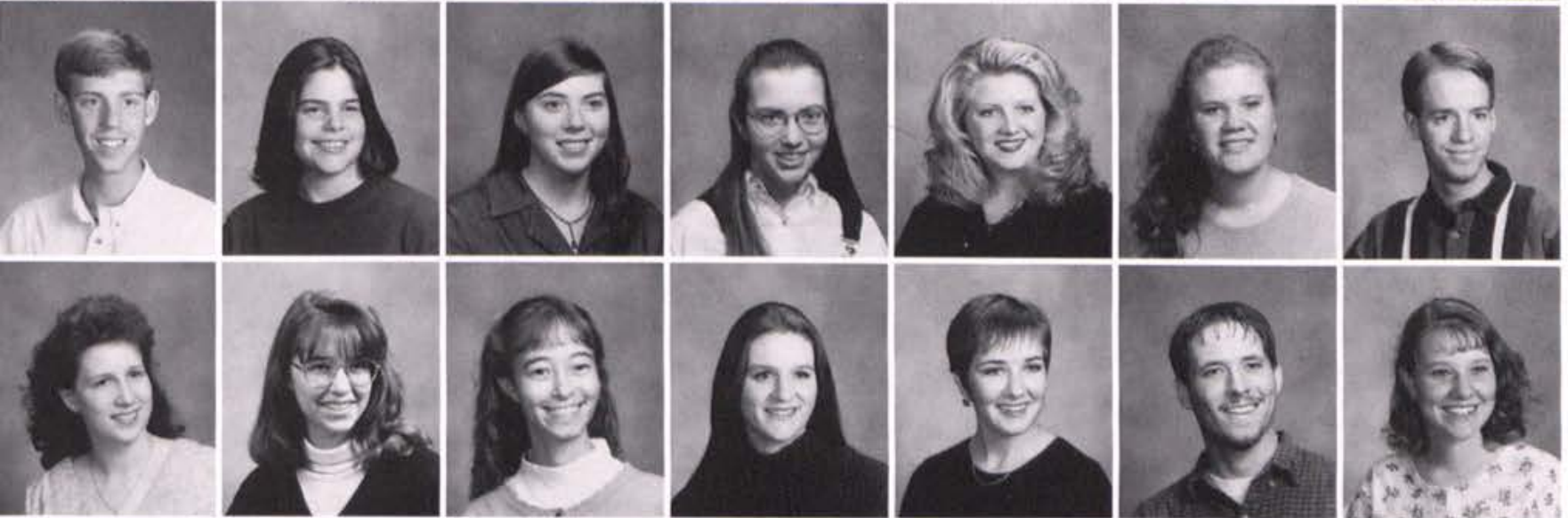

Chris Scott

Kimberly Scott

Amber Seely

Jamie Sell

Bethany Seyfang

Tara Seyfert

Paul Sheldon

Hannah Sherwood Andrea Shihady

Jessie Silvieus

Robyn Simmonds

Sarah Simons

Patrick Skelton

Elizabeth Smith
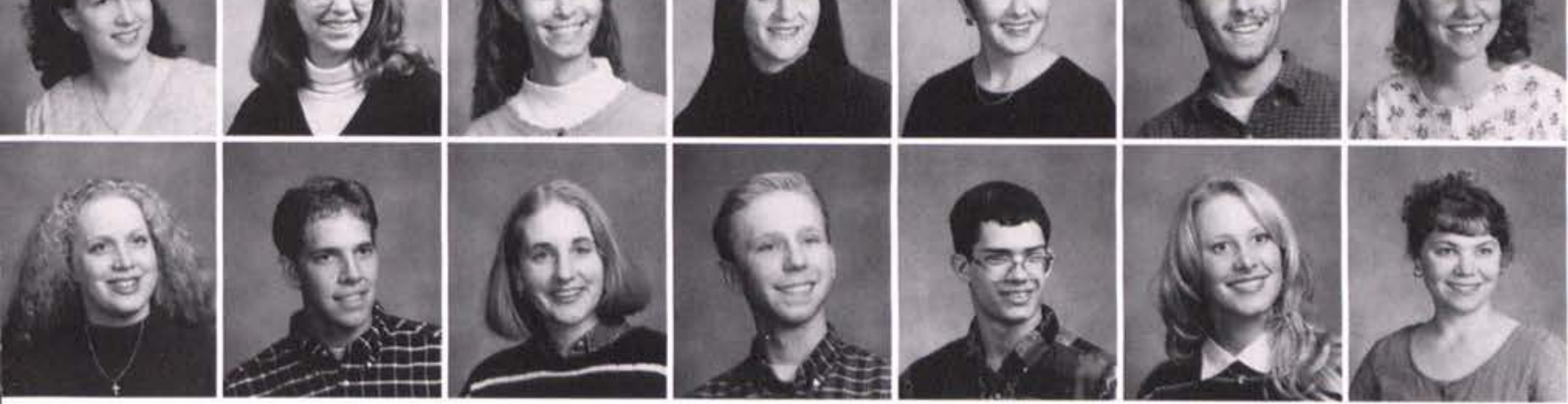

Heather Smith

Jed Smith

Valerie Smith

Josh Snyder

Aaron Sokoll

Holly Sorensen

Valerie Sorg

\section{And The Winner Is}

Several creative halls and inits won the Open House prizes this year. They invested hours of work designing Christnas themes and then over-haulng their living quarters.

The time that Willets 3rd loor Central (Jodie Hagar) pent was well worth it. They put together "A Jingle in the ungle." As guests entered their all, their first stop was the ABWE mission board, who ent them to the nurses' station or shots. They then entered he "jungle," a setup complete vith trees, dirt, darkness, and savage" creatures that jumped ut to surprise passers-by..

McChesney's 1st floor back Tim Simon) won for the guys' alls. They did "An Indiana ones Christmas." As visitors valked through the hall, they encountered leaves, twigs, and even poison ivy. The hall made an Ark of the Covenant room and a pit that everyone had to rope-swing over. As people left, a huge beachball volleyball, decorated as an boulder, chased each group out the door.

Maddox units 15 and 16 (Becky Ivies) won for the ladies' units with a "Cave-girl Christmas." These women went pretty far with their theme, and actually cut a girl's hair and glued it to themselves so they could be hairy cavewomen. Their costumes were Flintstone outfits, and they created a dark cave atmosphere by lining the walls and ceiling with black garbage bags. They had a small pond in their unit and an electric fireplace, around which they sat on stumps of wood. One member of the unit stood outside with a club, directing visitors into the "cave."

Lawlor's units 14 and 18 (Steve Keary) won the prize for their "Mission Impossible Training Course." Here, visitors were fingerprinted, went through interrogation, completed the training course and firing range, and finally were stamped "Eligible." The unit played the Mission Impossible theme song, and stacked their computers to resemble a security display.

Row 1(1-r): Diana Christensen, Nancy Gillespie. row 2: Debbie Ellison, Dr. Dixon, Quincy Kilburn, Haylee Biggs, Amy Gillespie, Gina Doerr.

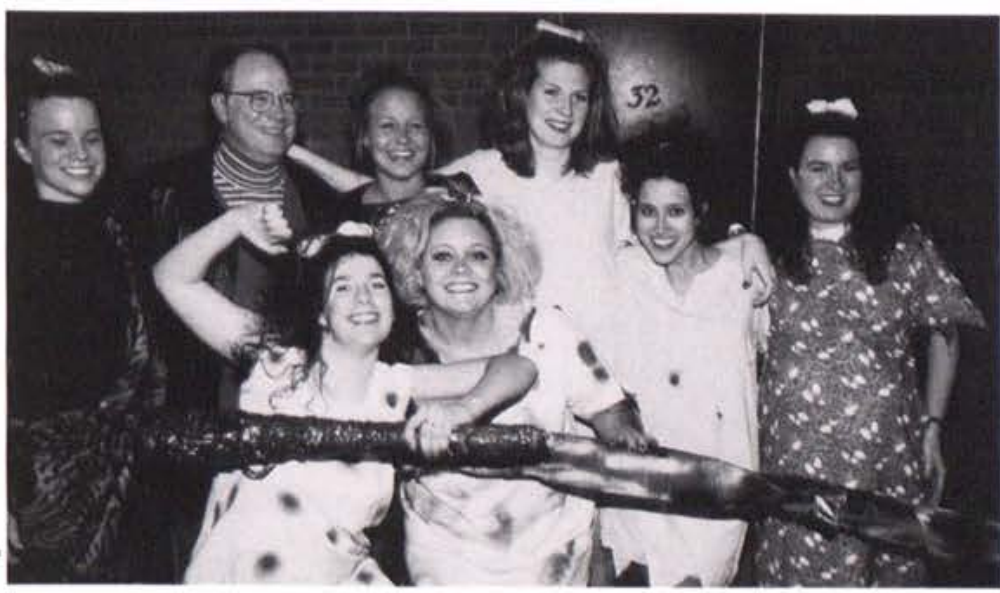




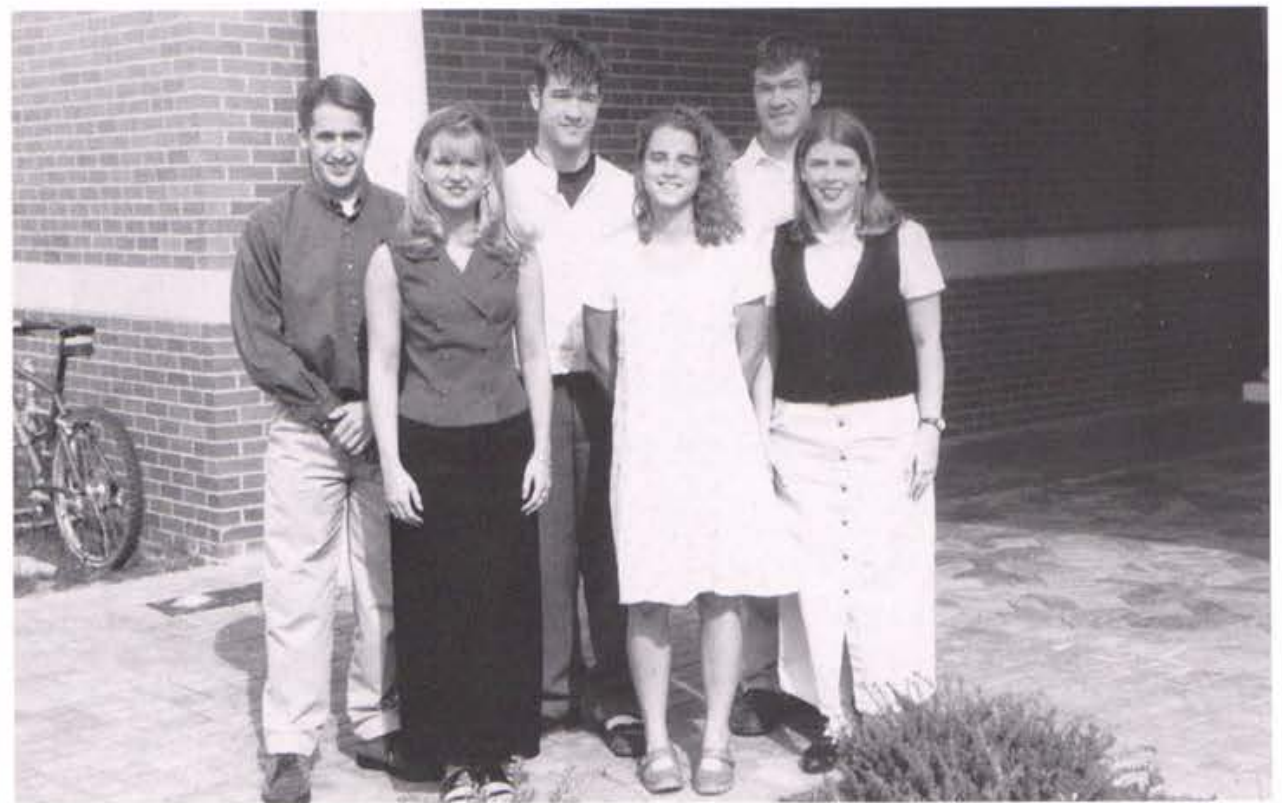

Sophomore Class

Officers

Left to right: Brian Bell, Chaplain; Rebecca Fissel, Treasurer: Berney Gorsuch, Vice-president; Rebekah Blackwood, Secretary; Barry Gorsuch, President; Kathy Mayer, SGA Representative.

Alicea Stachler

Michael Stark

Erin Steelman

Keturah Stork

Jennifer Stover

Stephanie Streetman

Steve Strong

Joshua Strychalski

Erin Stuenzi

Melissa Stumbo

Monica Stutzman

Earl Swartzentruber

Josh Tackett

Christy Taylor

Gretchen Taylor

Jennifer Tegtmeier

Lisa Thomson

Jennifer Tiel

Erin Timco

Stephanie Tomsa

Jill Townsend

Christopher Troupos

Rebecca Upham

Seth Valentine

Rebecca VanLoon

Rebecca Vanderground

Bethany Vaught

Beth Vencill

Bradley Voumard

Melissa Wabeke

Laura Waddell

Catherine Walcott

Bethany Walker

Lisa Walker

Christy Ward
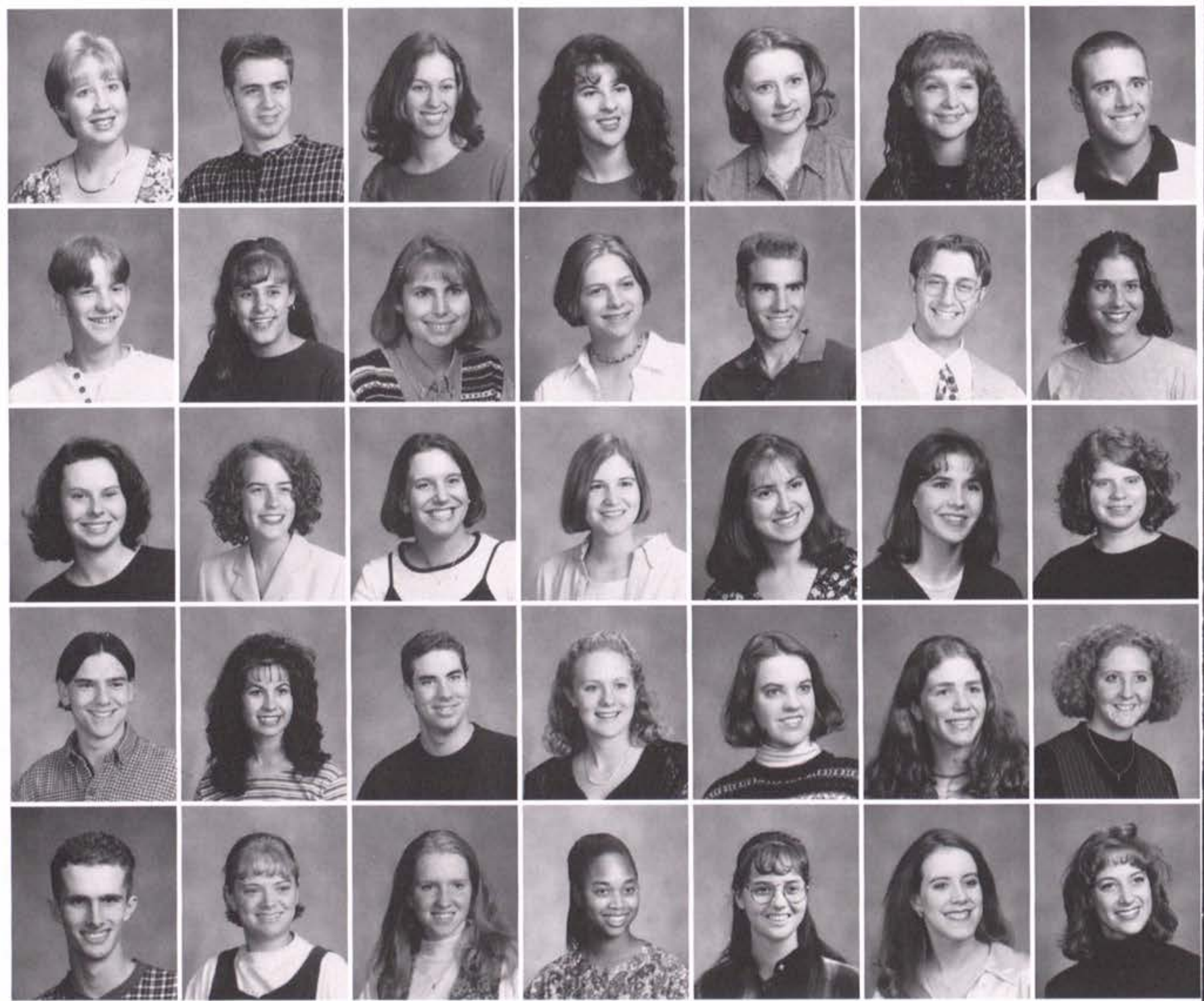


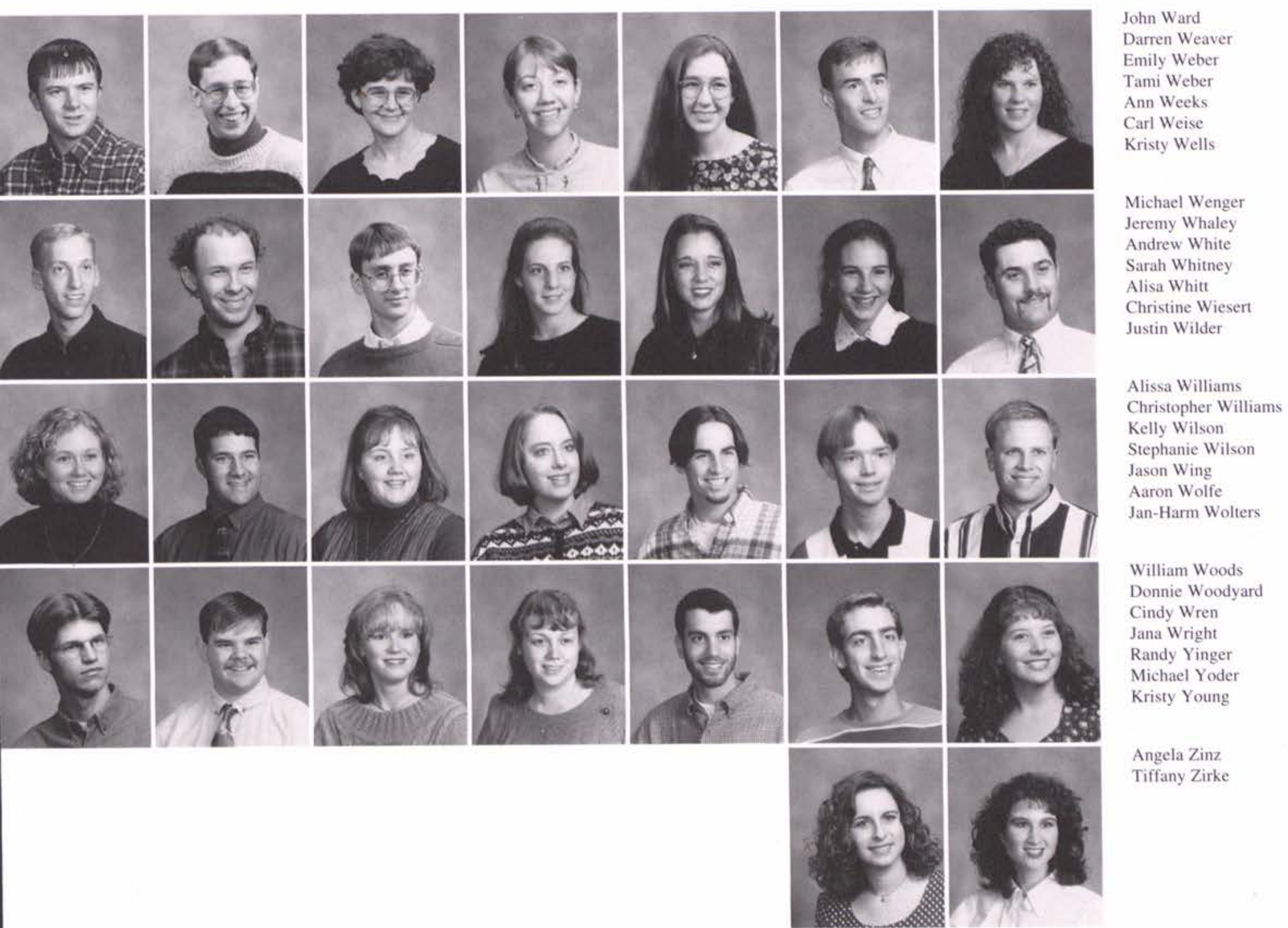

\section{Big Names for Brass Conference}

Chasis Perez

Friday, January 31-Saturlay, February 1, 1997 marked he beginning of the first Cedarville College Christian Brass Players Conference. The evening featured music performed by the Brass Choir and Symphonic Band and their special guests for the occasion, he Carillon Brass and $\mathrm{Mr}$. Robert Nagel.

The Carillon Brass is a quintet that was established in 1976. Each member currently holds a faculty position at Ohio coleges and universities, and the group includes Cedarville's Professor Charles Pagnard.

As a group, the Carrillon Brass have participated in more than 1,500 concerts, recitals, and workshops in the southwest Ohio area alone; they have performed international concerts, as well. Their awards include those won at the Keystone International Brass Ensemble Competition and International Brass Quintet Competitions in Narbonne, France.

Mr. Robert Nagel was the special guest of the evening. Nagel earned his B.S. and M.S. from the Julliard School of Music. He has taught at the Yale and Manhattan Schools of Music, New England Conservatory of Music, and North Carolina School of the Arts. He has taught in China and coached the Asian Youth Orchestra, and has played in radio and television in New York City for over 20 years.

The performers played a wide range of peices, from $\mathrm{Re}$ naissance church music to recent compositions by James

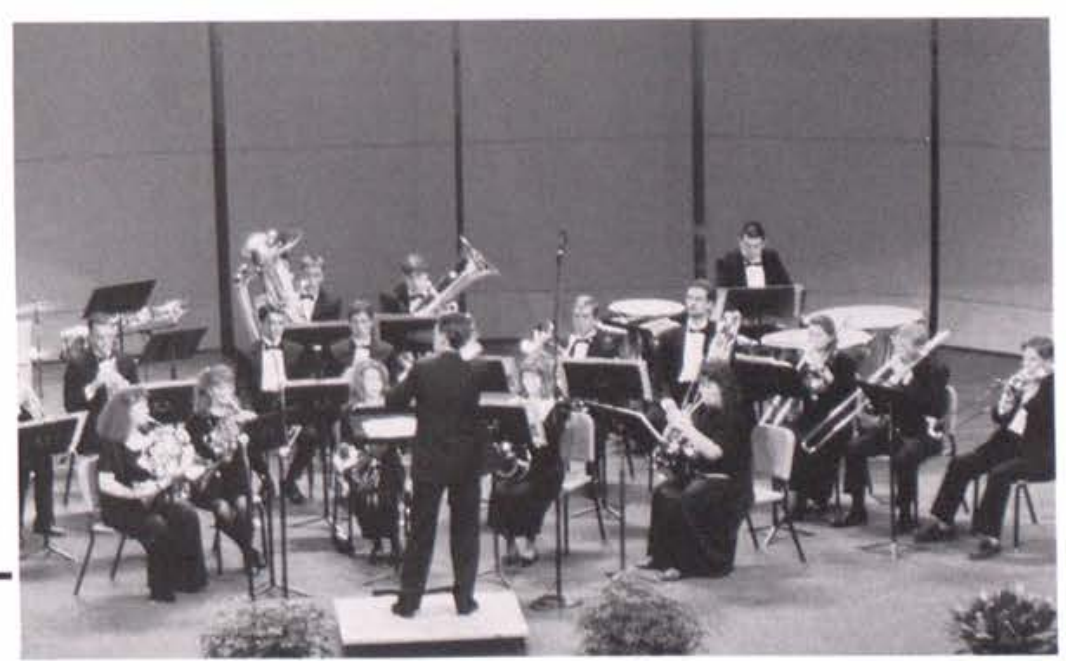

Curnow.

The purpose of the conference was to include Christian musicians from all over the country. The Department of Music hopes that it will become a regular event at Cedarville. 
Andy Abbeg

Elisabeth Abe

Kristina Abraham

Caleb Acton

Melissa Adam Jared Aldridge

Eric Allcorn

Danette Allen

Luke Allen

Titus Allen

Jennifer Ament

Timothy Amstut: Richard Anderson

Dana Anglund

David Anthony

Jenny Armour

Elizabeth Augustus

Philip Ausfahl

Daniel Avery

Stephen Baab

Carolyn Baker

Deborah Baker

Ranee Baker

Matthew Baldwin

Clarissa Band

Bethany Barch

Bradford Barnard

Jaclene Barnard

Jeff Barrons

Molly Bast

David Beary

Heather Bechtol

Ron Becker

Peter Bednarek

Karen Belding

Sandra Bennett

Stacie Bennett

Sarah Bensink

Sabrina Berry

Jim Billock

Thomas Bischoff

Emily Bishop

Christine Bjornstad

Karen Bjornstad

Becca Blackburn

Andrew Blodgett

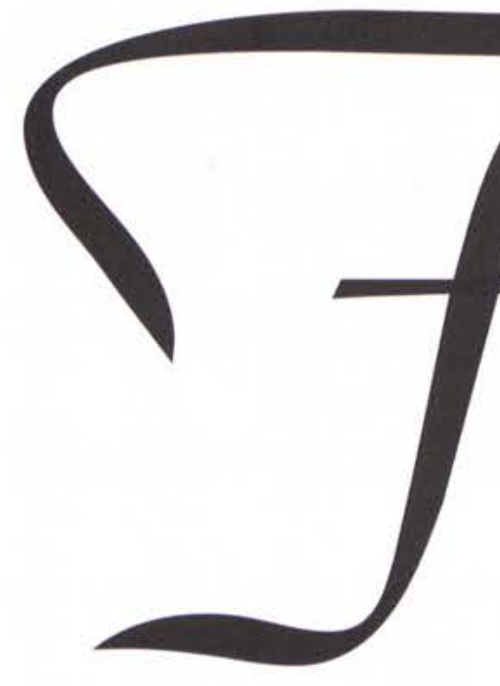

reshmen
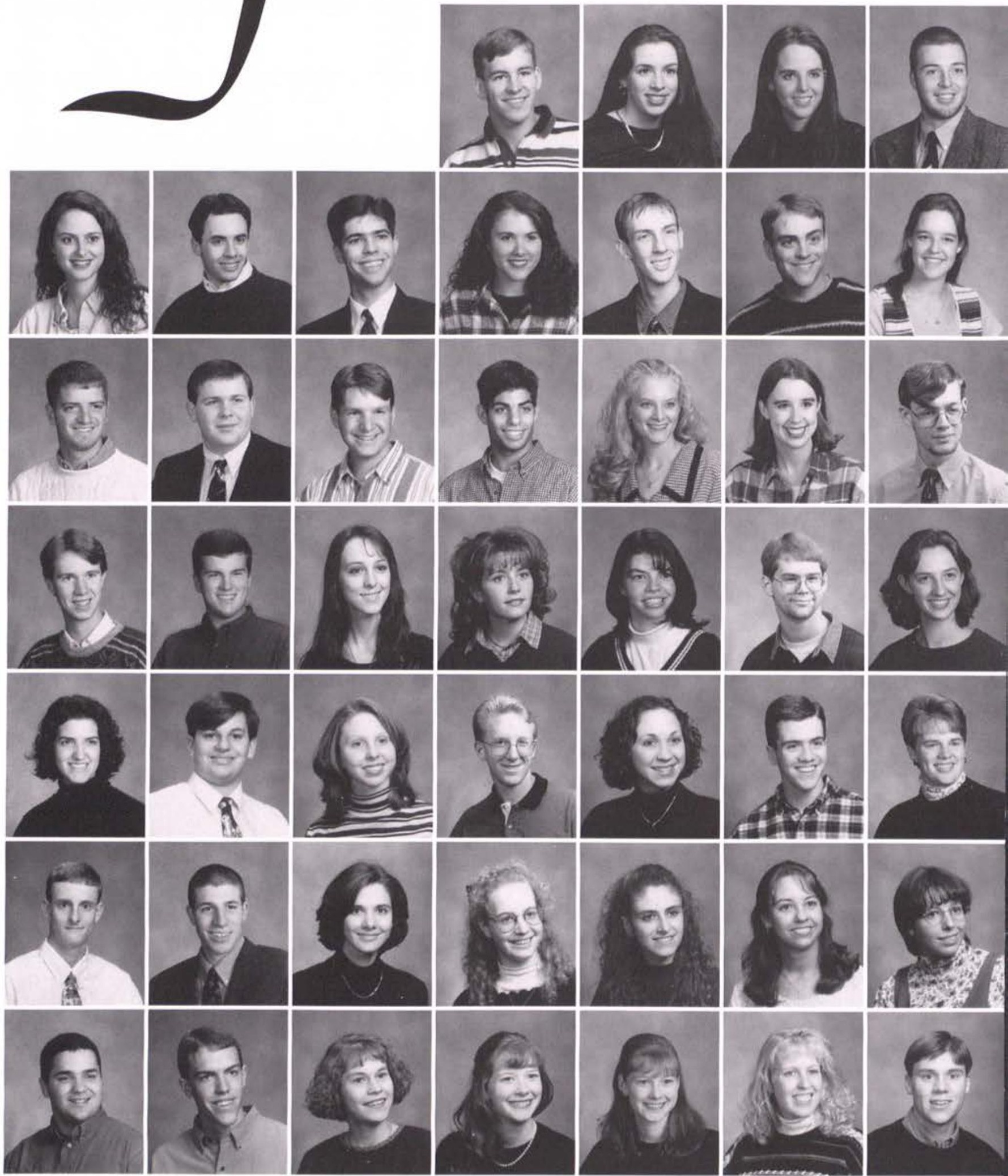


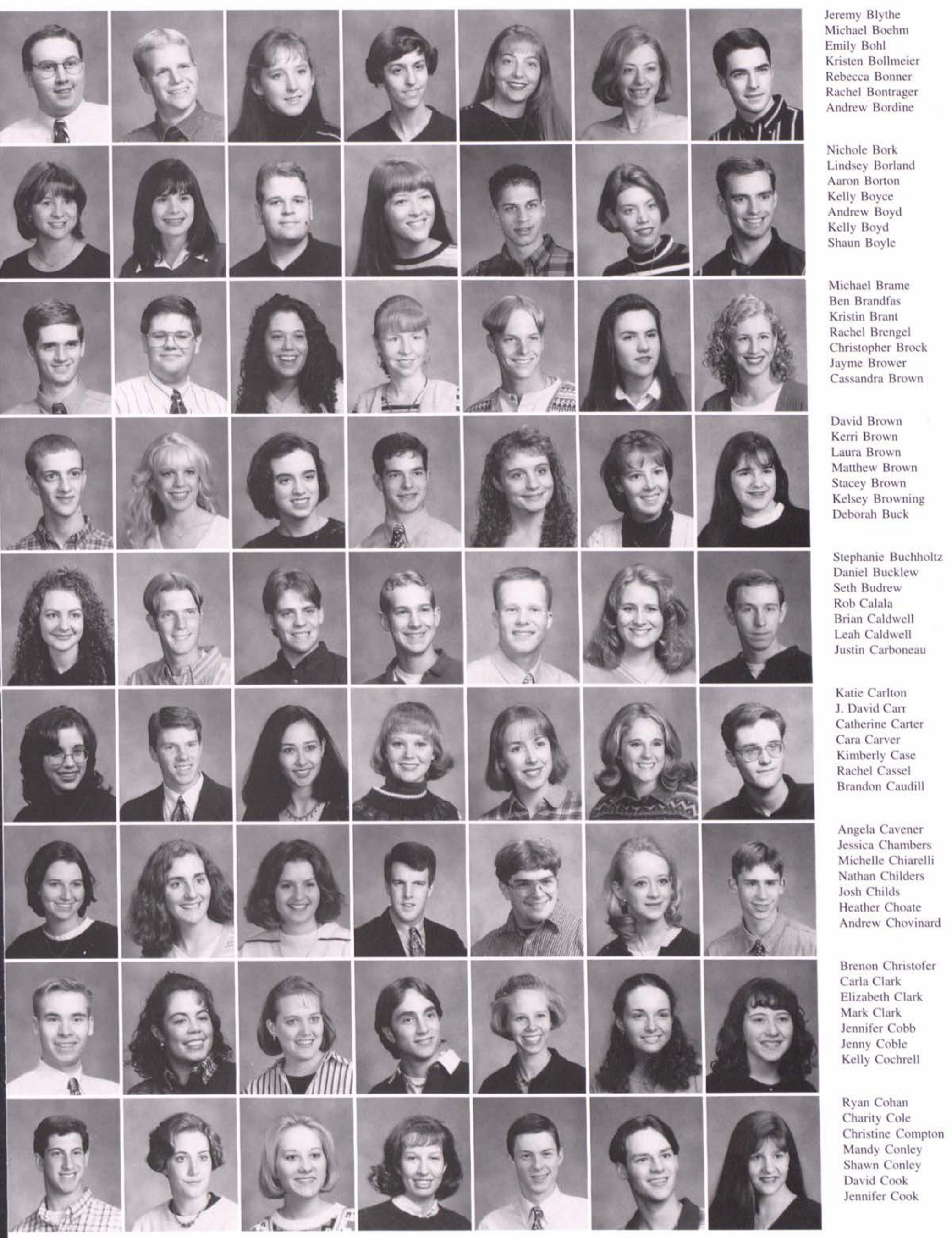




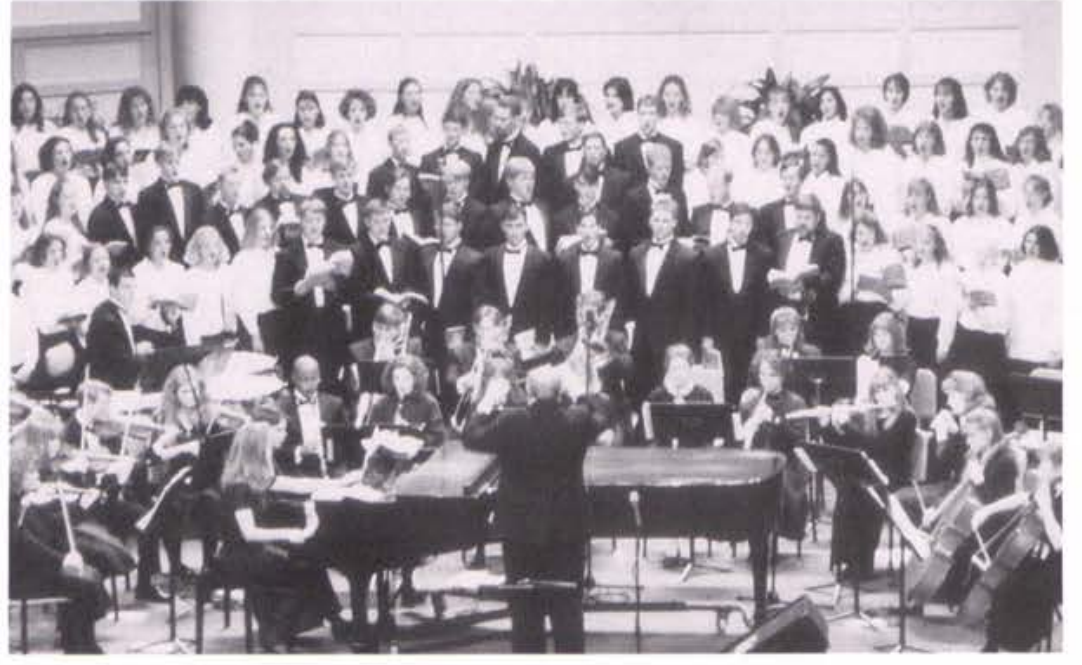

The Oratorio Chorus is a fall quarter tradition at Cedarville. It includes students from many majors, and residents of the village. The 180 member chorus was directed by Dr. Mark Spencer, Assistant Professor of Music. The piece chosen for this year's performance was Handel's Messiah.

The oratorio, Italian for "a place of prayer," originally evolved from sacred themes and was usually performed in a church without costumes or scenery. The Messiah focuses on the life of Jesus Christ, and takes place in three parts, which recount the prophecy of $\mathrm{His}$ birth, His death, and the redemption of the world. The music itself was designed for the modest Dublin orchestra, with the bassoon and oboe,

\section{Christmas Sing-A-Long-}

Charis Perez

added to the performance, to strengthen the choral pieces.

George Frederick Handel, born in Halle, Germany in 1685 , moved to England in 1712 where he wrote operas as well as music for the church and court. In 1742, he produced the oratorio Messiah. The oratorio, composed in twenty-four days, is considered among the finest choral works ever written.

The Oratorio Chorus performed familiar choruses such as And He Shall Purify, Glory to God, and the popular Hallelujah. Also included were recitatives, in which the solo voice follows the natural rhythms and accentuations of speech.

This year's concert was staged in a unique way. Instead of performing the entire concert on the stage, the chorus mingled with the audience on the main floor. The goal was to help the audience sing along on the choruses. Musical scores of the Messiah were provided for purchase, and signs were posted at different sections of the auditorium. dividing the audience and chorus into soprano, alto, tenor and bass sections.

The Cedarville College Orchestra, under the direction of Richard Wright, accompanies the Oratorio.
Matthew Cook Shauna Cook Brett Cooley Tina Coon

Tiffiny Cope Amber Corbin Erinn Cottrill

Sallisha Cowell Andrea Creamer Alex Crum

Amy Crunelle Christa Culler Jordan Culp Aliel Cunningham

Shawn Dabravalskas Hannah Dager Amber Dahms Kevin Damcott Scott Dangelo Cory Davids Carolyn Deltaan

Nate DeHart

Robert Dehnke-Hirschleman Nate De Ridder

Jessica De Vinney Casey Dellinger Paul Dennison John Diggle
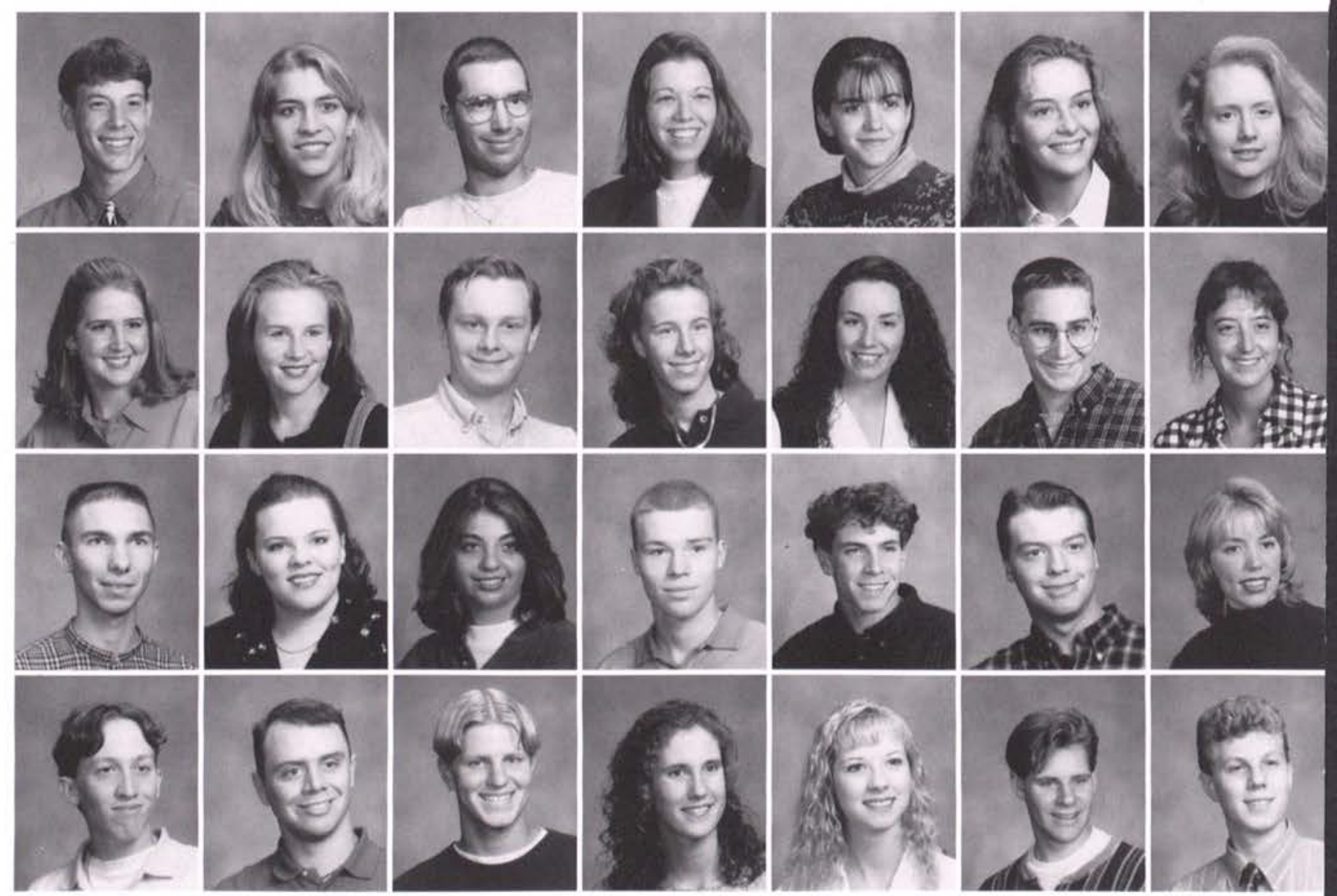


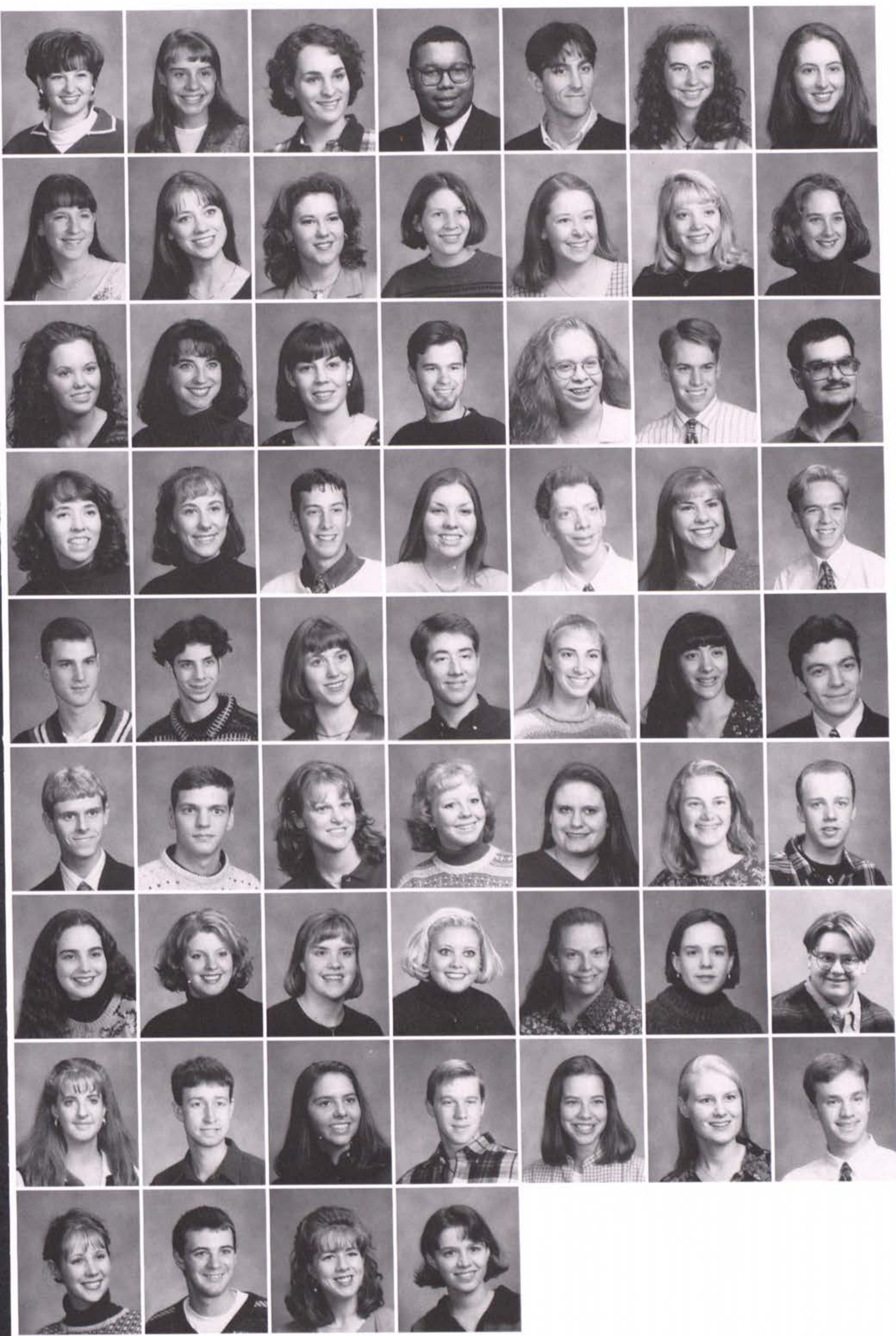

Kara Doden

Jennifer Dolby

Gretchen Dorman

Michael Dorsey

Michael Dull

Adrianne Eads

Melanie Edwards

Michelle Edwards

Sara Eleveld

Andrea Endicott

Christiana Estes

Carrie Fabian

Angie Fancher

Karyn Farkus

Michelle Farley

Andrea Farlow

Jennifer Ferguson

Josh Fetzer

Summer Fielden

William Fischer

Matt Fjare

Denelda Foltz

Sonya Foster

David Fourman

Becky Fox

Steve Fox

Loree Fraley

Jeremy Frank

Garett Freeman

Matt Freitag

Summer Friend

Daniel Fries Jr.

Tracy Friesen

Grace Frishey

Josh Fronovti

Tom Fry

Jonathan Fuge

Christine Fullerton

Caren Furst

Melinda Gates

Sara Gedraitis

Chris George

Lavonna Getz

Jennifer Gifford

Sarah Gilchrist

Nancy Gillespie

Allison Gillet!

Stephanie Gleason

Justin Goehring

Gretchen Goertzen

Matthew Goetz

JeriAnn Goodbar

Justin Goodrich

Jenni Gottwals

Sarah Grapentine

Shawn Graves

Kimberley Greene

Michael Gregg

Julie Ann Gregory

Joy Griffith 


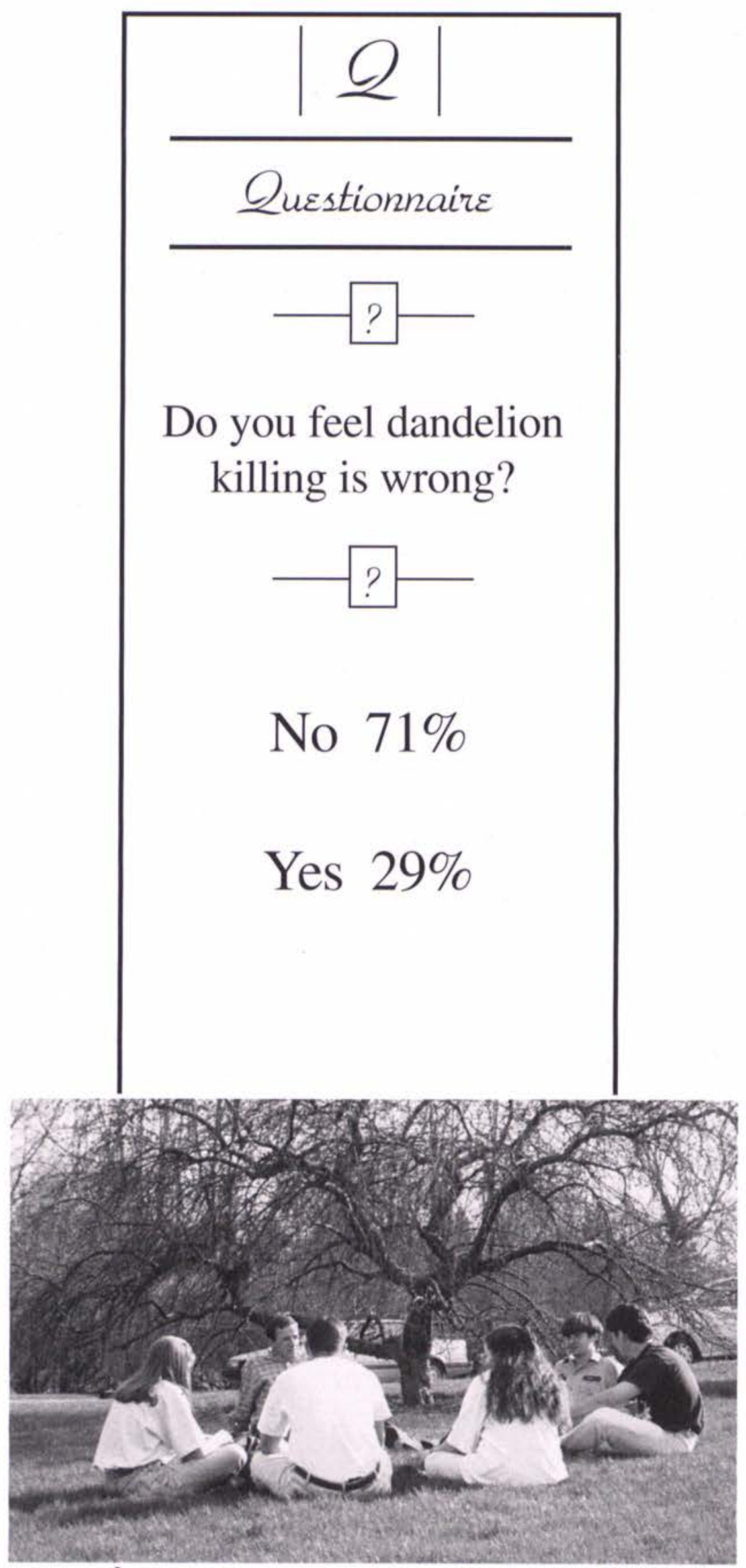

"Did you get permission from Dr. Dixon to sit on that lawn? 'Cause I sure don't see any dandelions!"
Christopher Grigson Kelly Grindall

Jason Groesser

Meredith Gross

Stephen Guenther Jr. Karen Guikema

Paul Gwilt

Daniel Hacker

James Hall

Jonathan Hallsten

Amy Hamilton

Michael Hammond

Jillianne Hancock

Clinton Hardesty

RoseAnn Hassell

Karen Hayes

Mark Hayner

Leanne Heath
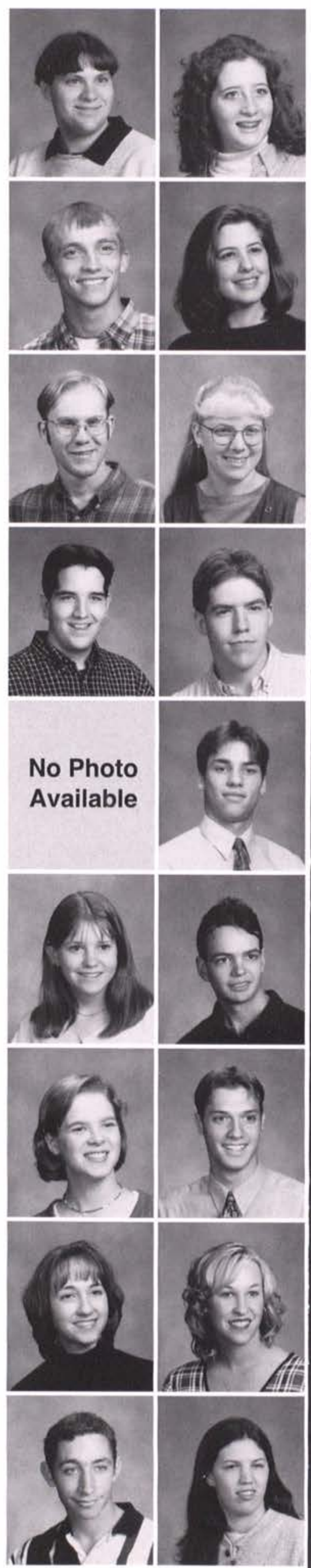


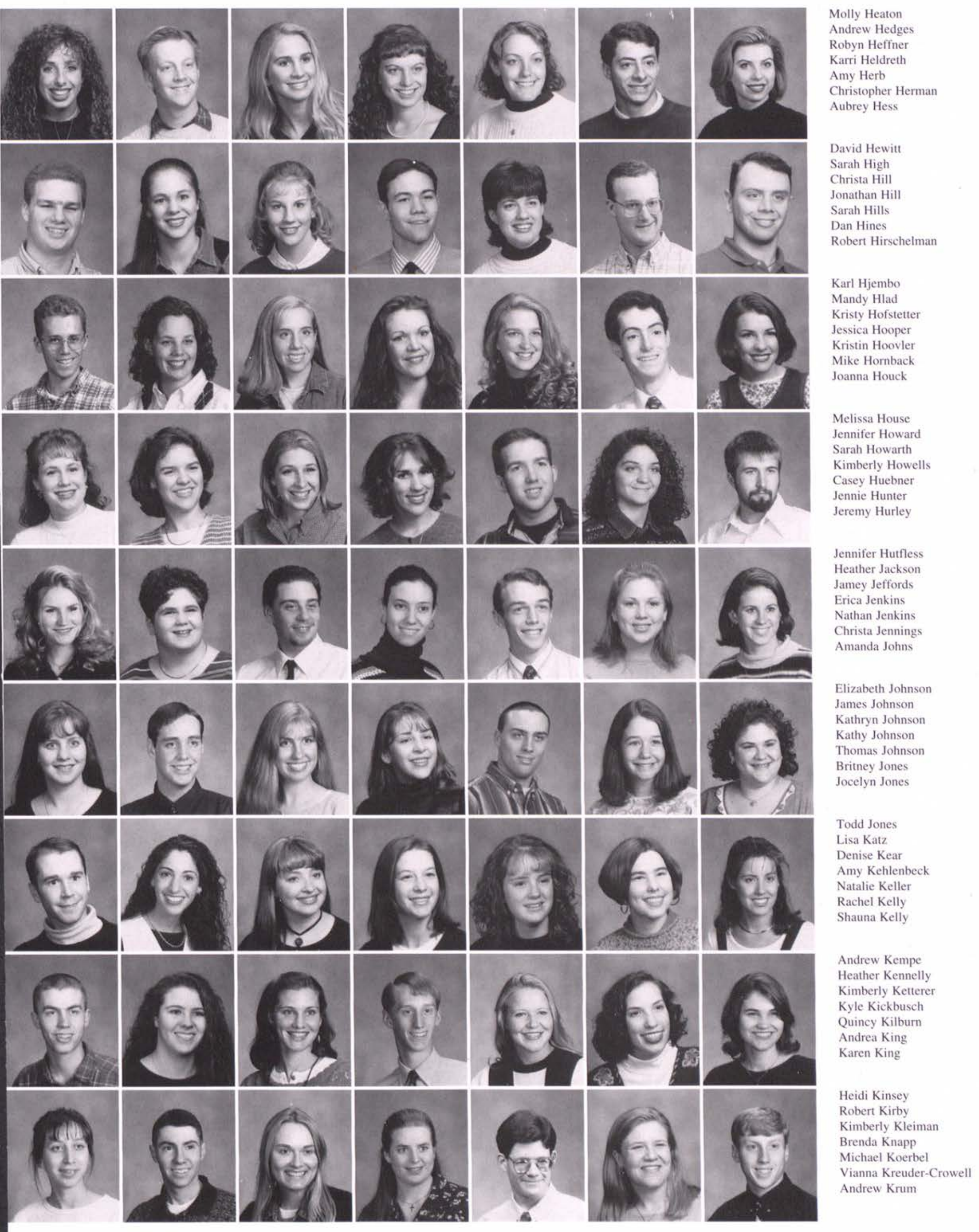


Kristin Kuhn

Barbi Kuvshinikov

Amy Kuyper

Daniel Kwast

Timothy Kwast

Hannah Lakes

David Lambert

Julie Lamborn

Michael Lampton

Sara Laning

Jennifer Larson

Rebecca Leatherman

Jason Lee

Brandi Leeds

Scott Lehr

Shanna Lenhart

Cheyenne Leslie

Kimberly Leverson

Nathan Lewis

Tiffany Linden

Christy Linerode

Betsy Linnell

Jennifer Linton

Katie Little

Tricia Lloyd

Rob Losey

Michael Luck

Nikki Luckmann

Brandon Luke

Jenny Lutz

Jessica Lutz

Lisa Mac Pherson

Kelsey Mahl

Jay Manchesky

Elizabeth Marcy

Aaron Margene

Jere Margene

Carol Marks

Rosemarie Marotta

Justin Martens

David Martin

Jill Massey

Angela Mast

Paul Mattson

Heather Maybury

Sarah Mayer

Dennis McChargue

Molly McClure

B.J. McComas

Tamara McComb

Jennifer McDonald

Dave McGrew

Amanda McGuire

Mandy McLaughlin

Joel McLeod

Emily McMahon

Amy Mead

Scott Meek

Jessica Meissner

Brenda Mick

Andrea Miller

Virginia Miller

David Mills
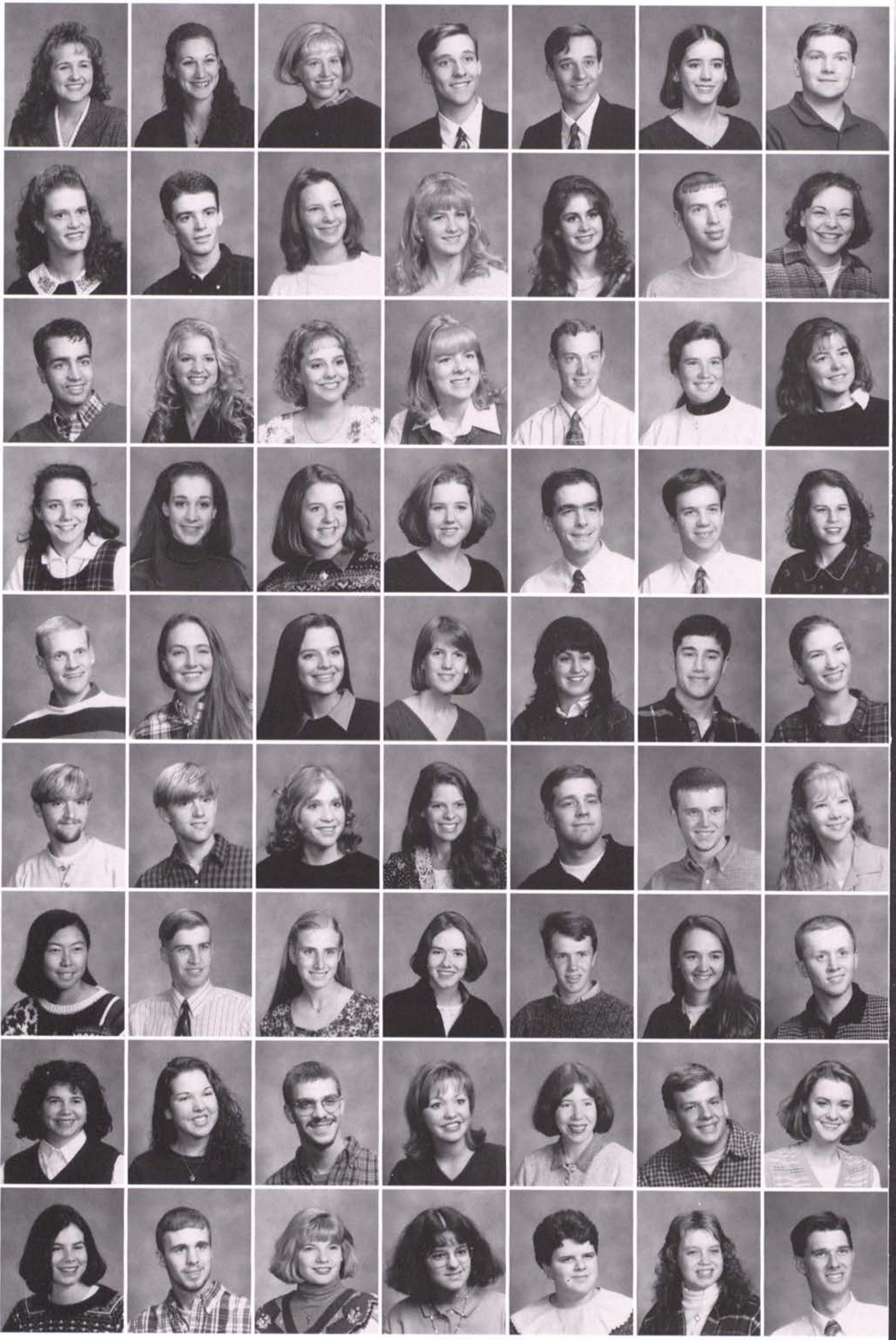


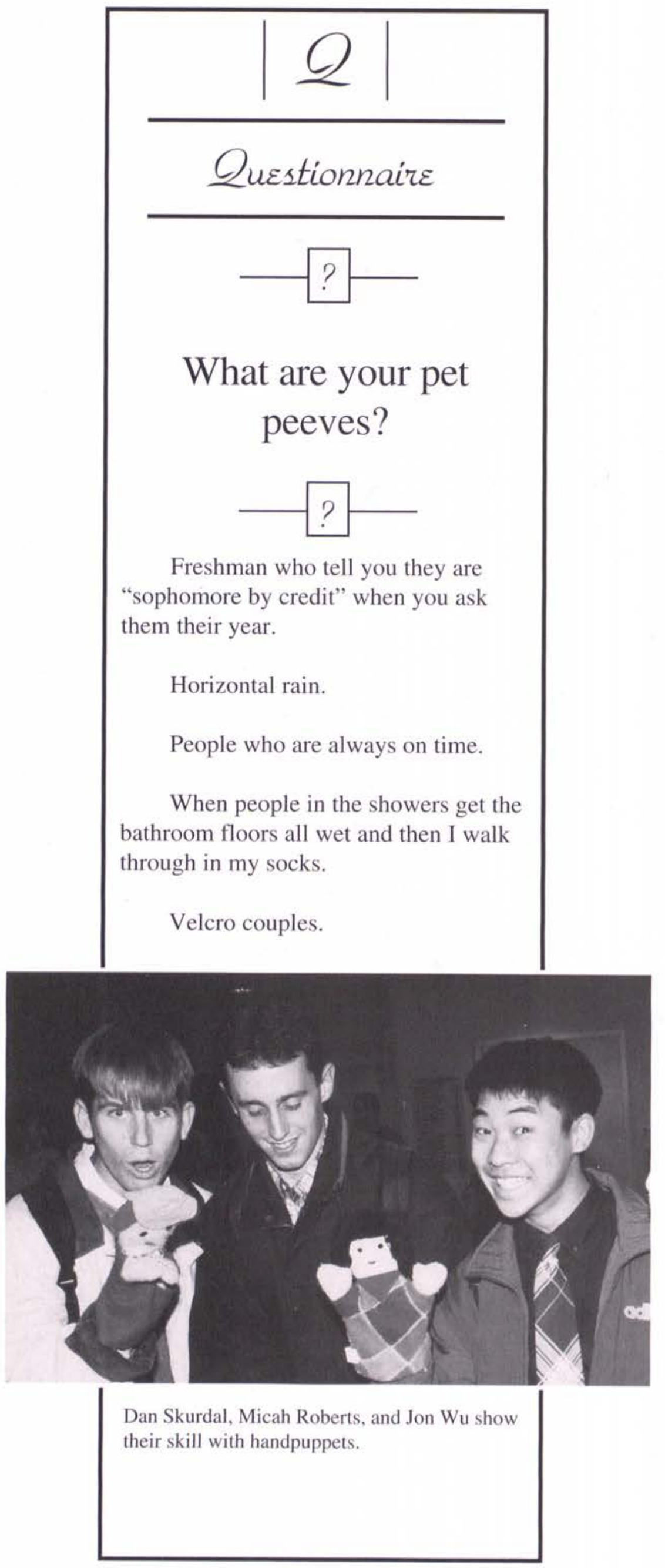

Amanda Mullin

Brent Mulli

James Murdock Angie Musgrave

Brad Neese Jonathan Neu

Bethany Nichols Keiko Noble

Isaiah Noftz

Lon Nolt

Andrew Nyveldt Kurt Oetken

Christine Olbrich Gudrun Olson

Jeffrey Olson Theresa Olson
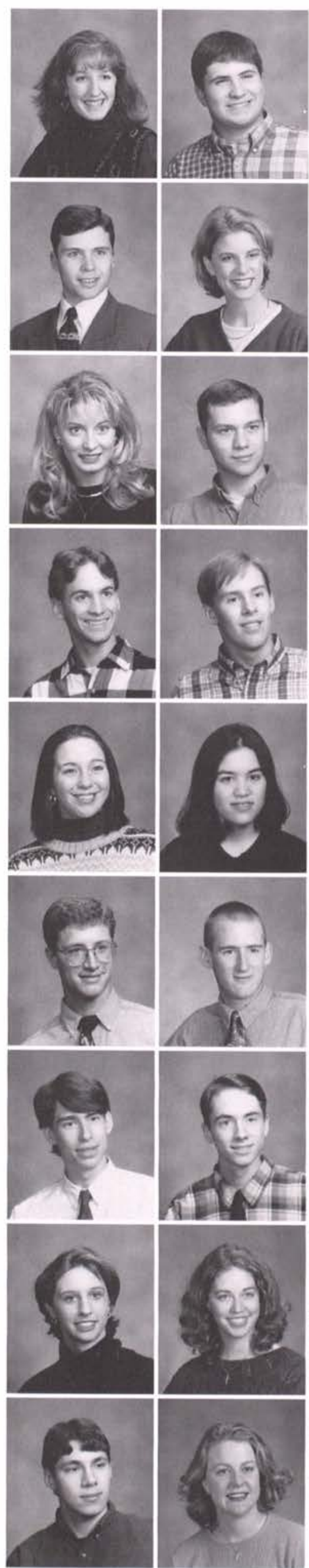
Kimberly Sanderson

Joel Schenk

Gabe Schlappi

Christine Schmid Ben Schroeder

Crystal Schulman Jeff Schumacher Connie Schwartz Lisa Scott

Jennifer Seeley

Jessica Seeley

Stephanie Selin

Danny Sellers

Shane Sevo

Craig Shank

Aaron Shaw

Jill Shearer

Mike Shepherd Josh Sherwood

Micah Shields

Kenny Shierman Christian Sicard

Jared Skillings

Chris Smith

Jaclyn Smith

Joshua Smith

Kristie Smith

Michelle Smith

Stephen Smith

Dale Sorensen

Melissa Sparks

Nicole Sparling

Anna Spears

Jenny Stankiewicz

Tammy Stark

Stacy Stauffer

Susanna Steeg

Nathan Stephens

Ryan Stevens

Rachel E. Stewar

Rachel J. Stewart

William Stewart

David Stone

Amy Stowers

Maureen Strang

Jennifer Strunk

Jenny Stryker

Dale Stupnicki Jr.

Benjamin Stutzman

Alisha Sucsy

Joshua Sucsy

Michael Sullivan

Rebecca Summers

Eric Svendsen

Sandra Swales

Tara Swaney

Justin Swanson

Janelle Swartz

Paul Swenson
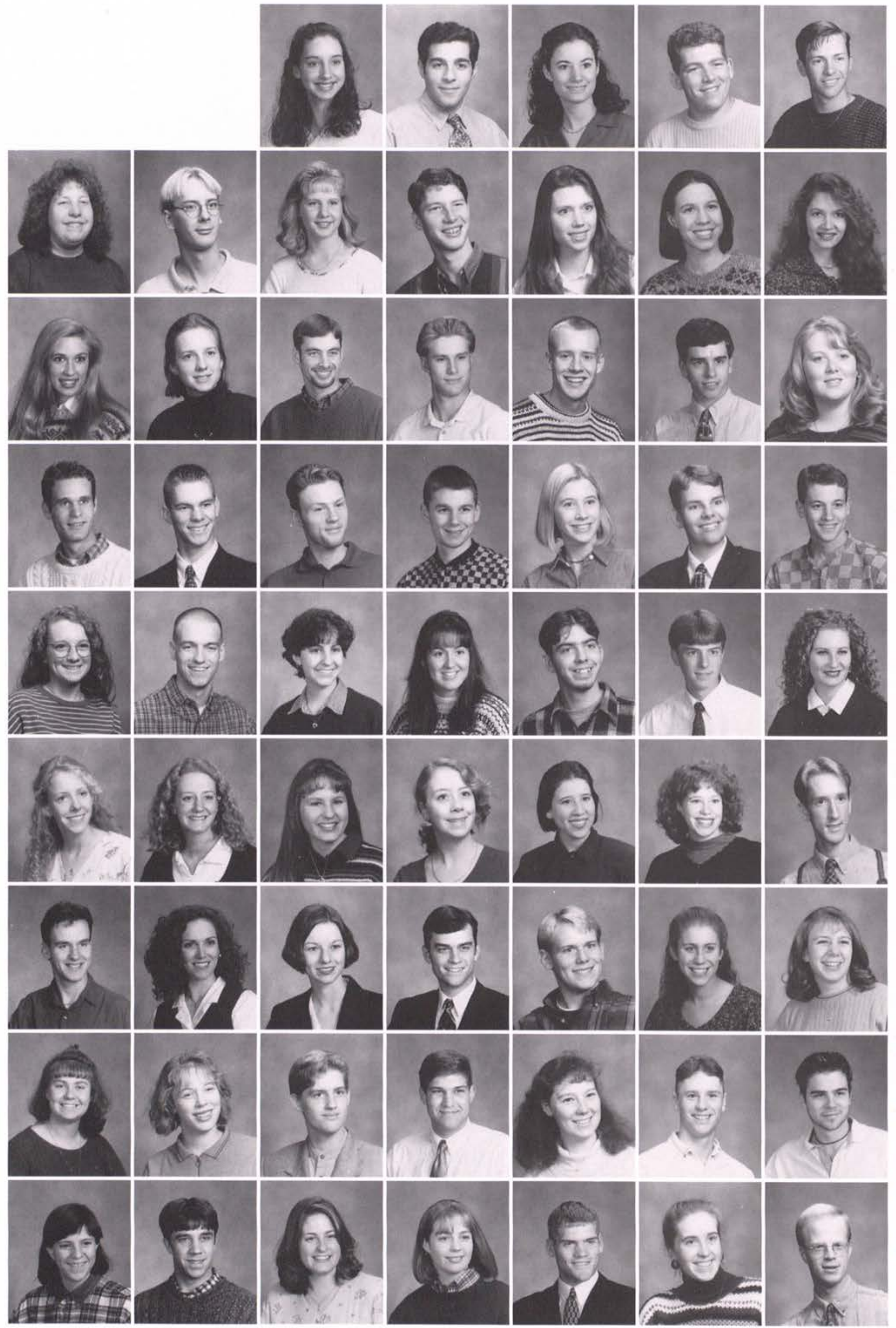


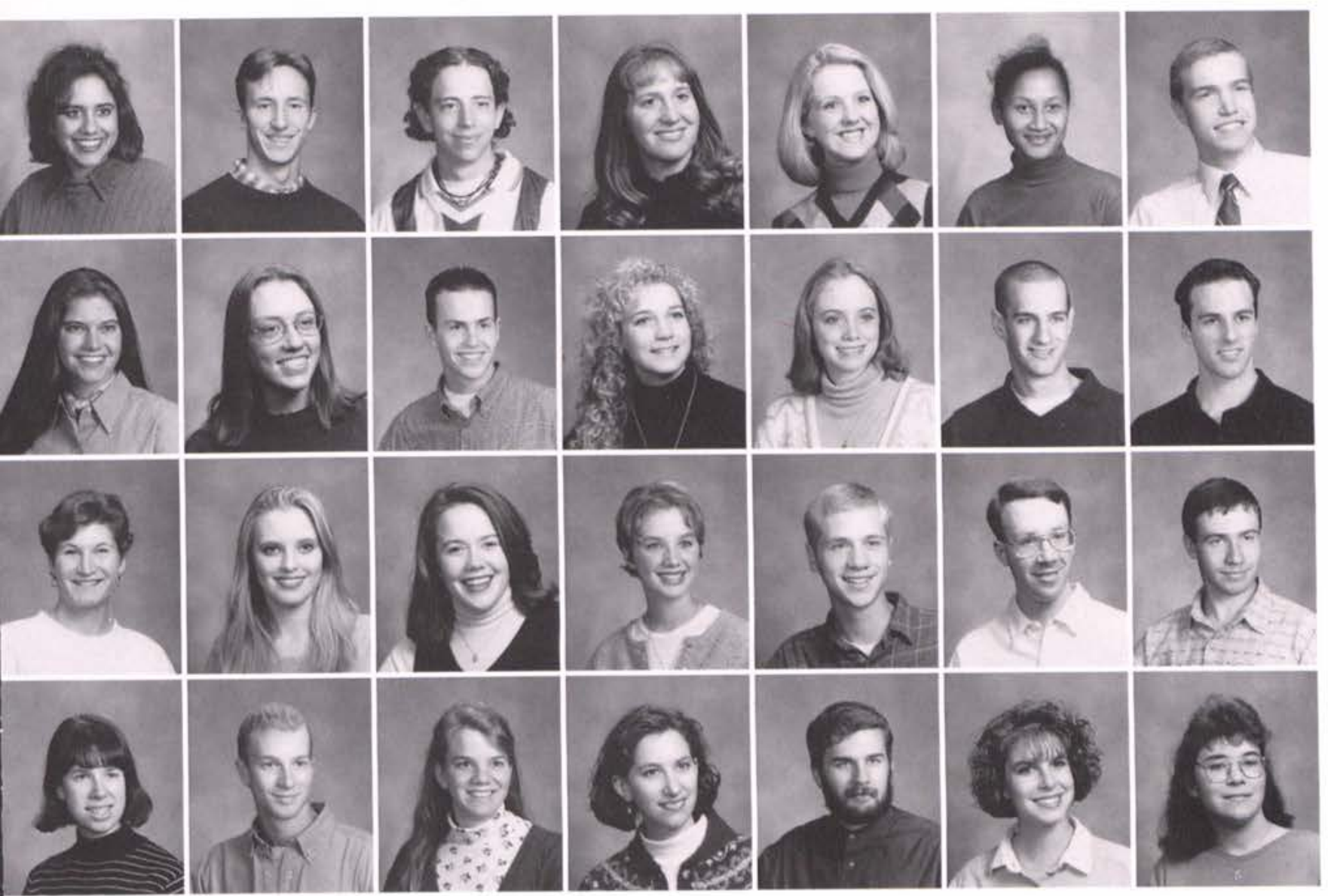

Michael Thure

Rachel Tilton

Ellie Titland

LeeAnne Toyer

Heather Tracey

Joshua Tromp

Mark Treadwel

James Trimble

Julie Trout

Aaron Trylick

Erin Tuinstra

Rachel Turkis

Jonathan Turner

Kristy Turner

Amy Twigg

\section{New Majors Broaden Academic Horizons}

Cedarville College has always offered degrees in minisry as well as non-ministry professions, and this academic year the administration has added three new majors to the baccalaureate program: physics, physics education, and church music ministry.

The physics major is designed much like the engineering major, but emphasis is added in the physics and mathematics areas. Students can pursue either a physics major, which will help them prepare for research careers or graduate school, or a physics education major, which will prepare them to enter either graduate school or a secondary education teaching position.

"These majors have been on the back burner for about thirty years, and it is really exciting to see them come to reality," said Dr. Dan Wetzel, chairman of the Science and Mathematics Department.

Academic Vice President Dr. Duane Wood said the church music program was always emphasized in the music department, but because of the new Ministry Center, it was time to make it a major. It is similar to a combined music and ministry major with a core of electives in any area the student wants to pursue, such as multimedia technology, counseling, or ministry.

Dr. Vernon Whaley, Associate Professor of Church Music, was hired to oversee the new program.

"The whole idea is to aggressively pursue those stu-

Elizabeth Rogers studies diligently in Chuck's. dents interested in church music, because the need for fulltime ministers of music is continually growing.

The church music major is designed to prepare students for church ministry, graduate studies in a music discipline, or seminary.

Wood described the college's satisfaction with the

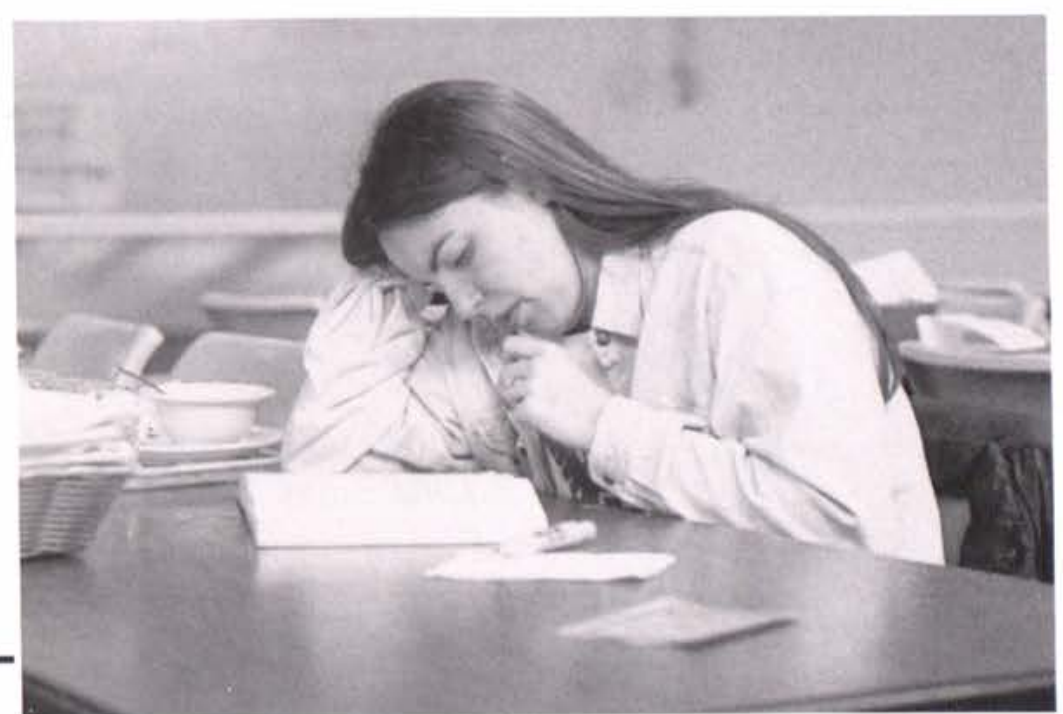

new programs: "We have been in a period of years where we have added new majors, both with a secular profession emphasis in the traditional liberal arts and in Christian ministries areas. These new majors further strengthen our foundation." 

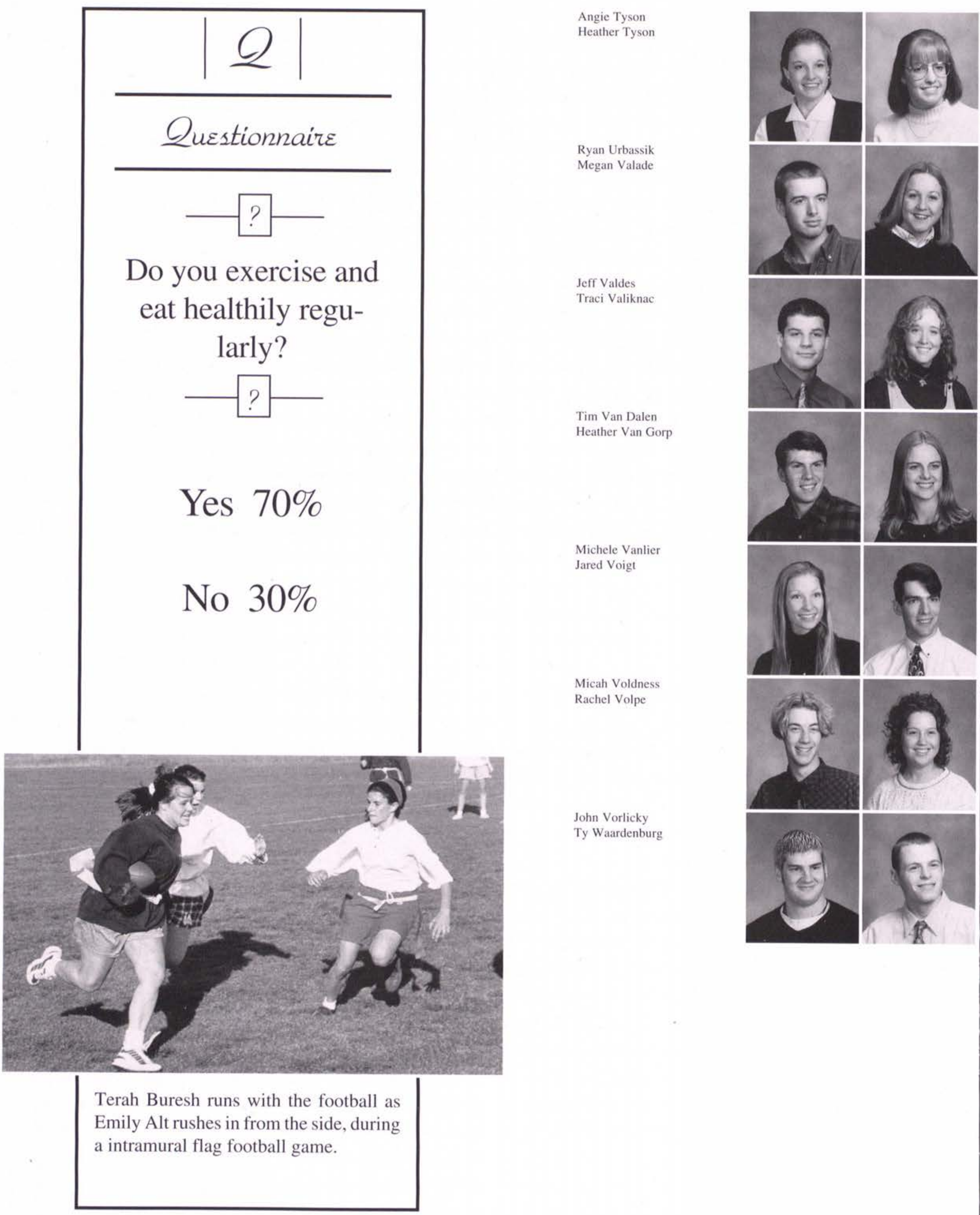

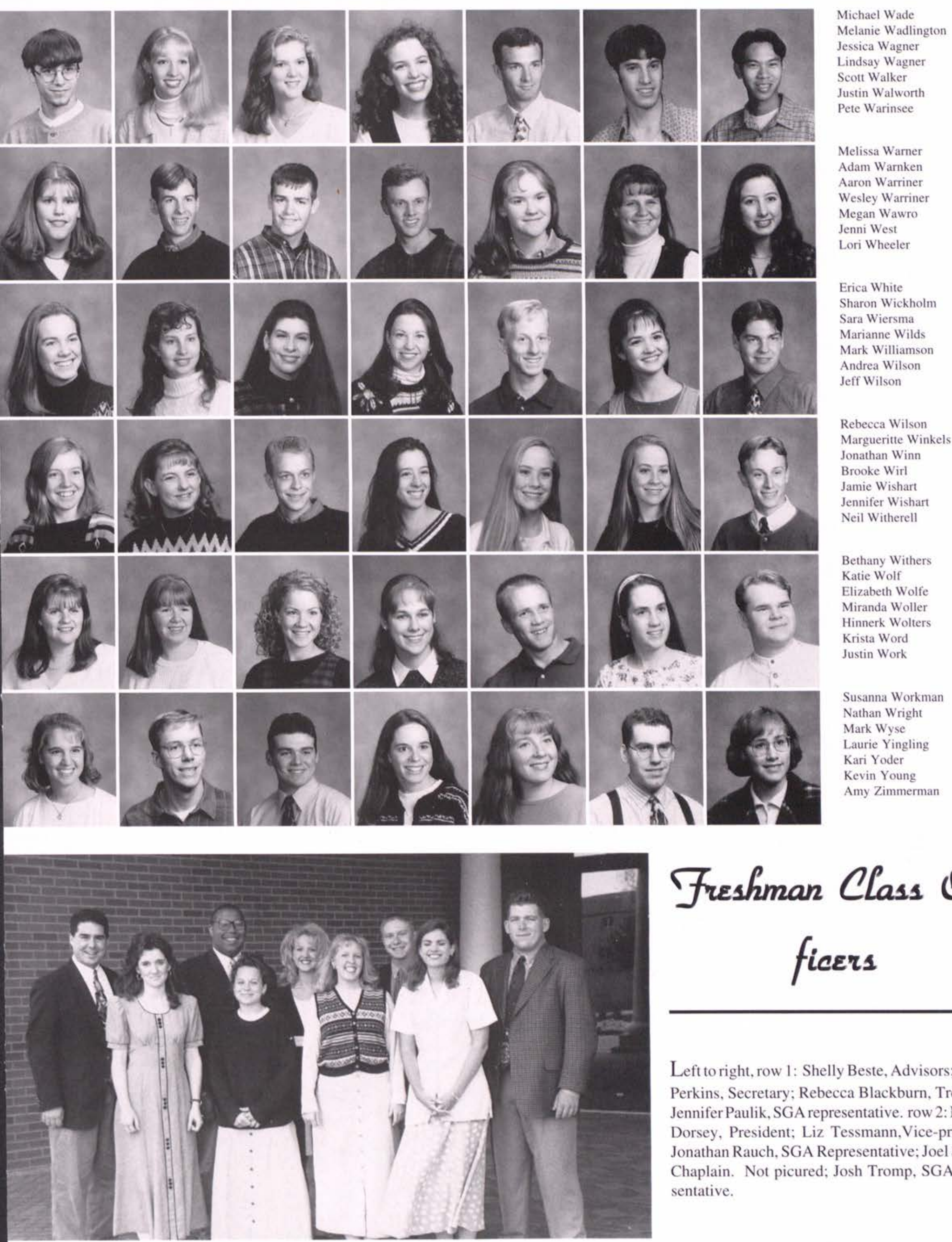

Treshman Class Officers

Left to right, row 1: Shelly Beste, Advisors; Kelsey Perkins, Secretary; Rebecca Blackburn, Treasurer; Jennifer Paulik, SGA representative. row 2: Michael Dorsey, President; Liz Tessmann,Vice-president; Jonathan Rauch, SGA Representative; Joel Schenk, Chaplain. Not picured; Josh Tromp, SGA Representative. 
by Keturah Stork

A very cheery hello greeted me as I called, "Knock, knock" at the door of Mrs. Kaercher's office. The smile and the eager desire to be of assistance welcomed me just as warmly. I quickly found myself wrapped up in a fascinating discussion about her sons and their families and about the joys of working directly with college students. I found myself thinking of how I would one day view my own job. Will I see my day's work as a task to be completed out of duty, and then forgotten at the end of the day? For Mrs. Murtha Kaercher the question is not how she approaches her position at the 'Ville, but how she faces every aspect of her busy life.

She arrived at the college about ten years ago, working part-time with Dr. Allen. Within a few months, she received her present position at Patterson Clinic, assuming the responsibility of all its insurance-related tasks. During a typical day she places numerous calls to local hospitals and doctors' offices to confirm student coverage. She can proudly say that all Cedarville students are adequately insured and therefore obtain reliable health services from area practitioners. "It is a wonderful testimony that our students' bills are paid." She also sends the insurance claims to a thirdparty adjuster, an organization which sifts through the many student bills. However, much of her time is spent in talking with students and with their parents via phone to work out the details and complications of being sick and of obtaining coverage. Mrs. Kaercher values most these moments with the students.

After her husband Richard retired from the Air Force in 1985 , he took a position of teaching math, physics, and computers at Xenia Christian High School. Following this change, they moved to Cedarville and Mrs. Kaercher began working at the college. Both desire to retire in their old farm house in Cedarville because, as she expressed, "We love the small town atmosphere." What is more, she hopes to remain with the clinic and the college. "It is a treasure to be this close to something that is so full of Christ."

Actually, Murtha's involvement with Cedarville College goes back a number of years. Both of her children graduated from here. Today the eldest, Gene, uses his doctorate in chemistry, earned after his years here, at Cargill Corporation in Tennessee. Her other son, Brandon, graduated with the first engineering class from the 'Ville and today works with Prince Corporation in Michi- gan. Murtha has three grand children.

In those possibly rare mo ments when she has the oppor tunity to spend time as she pleases, Mrs. Kaercher enjoy working around her home, botl indoors and outdoors. One car also find her reading a gooc book or doing cross stitch anc other types of handwork. Bu perhaps what she and her hus band find most fulfilling is en tertaining in their home. They of course love to have thei family come to visit. How ever, they also view hosting as a wonderful opportunity to house students who have hac surgery, and also their parents The Kaerchers desire to share the gifts God has given to them

This outlook spills over into Mrs. Kaercher's job. Handling insurance claims is a means for her to have a ministry to col lege students. And what better time than when students are 


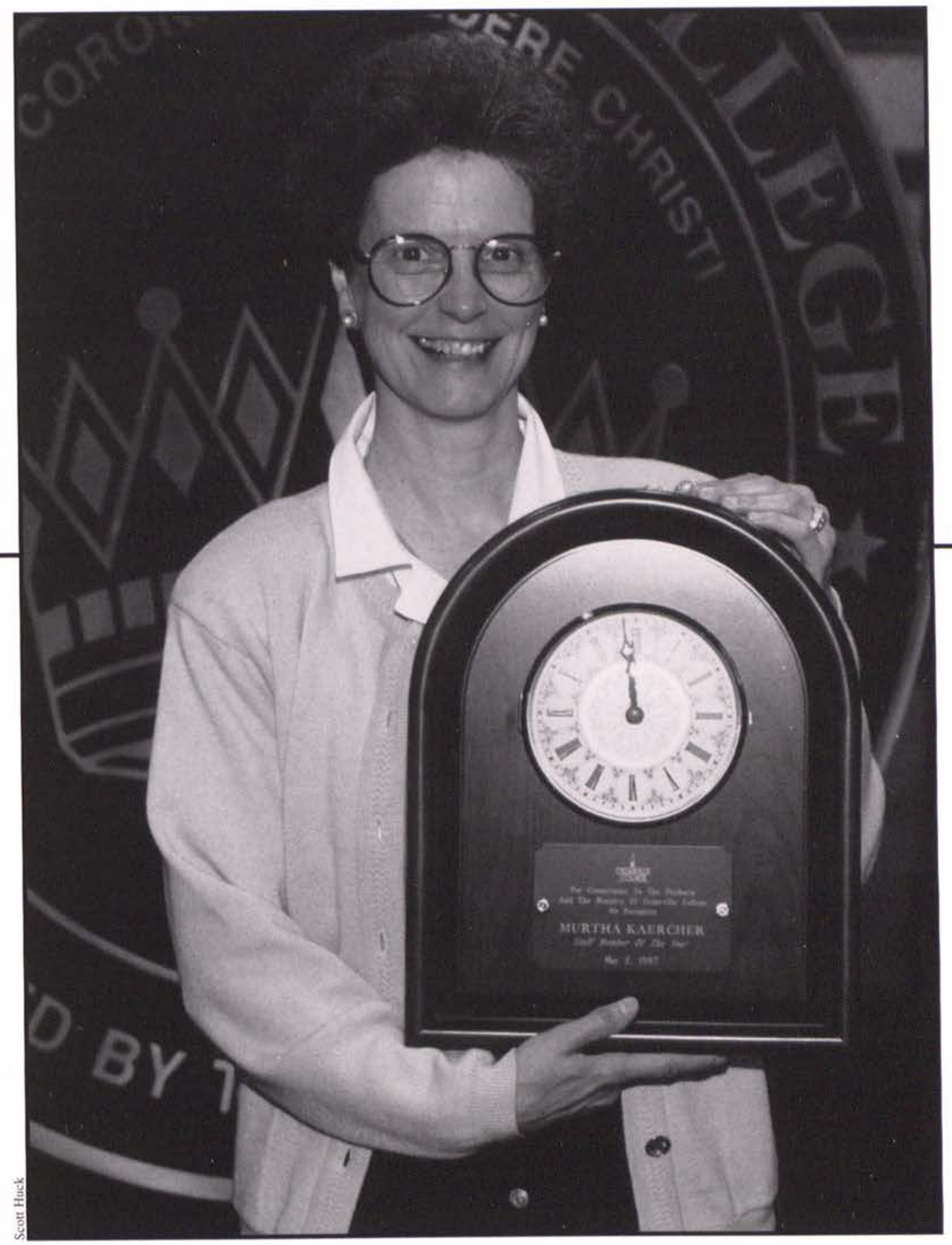

hurting and sick--"It provides a wonderful outlet for parenting." She, along with the other godly, compassionate women with whom she works, has many opportunities to provide spiritual input into the lives of students. She has watched many tears fall, laughed with many students, and encouraged or admonished many others. Kaercher believes "this is the most wonderful age to be in--the mid to late $50 \mathrm{~s}$," because she is around the age of most students' parents. Students feel that they can talk openly with her. However, her ministry does extend to the parents. "Some of our best friends are the parents of students with insurance ques- tions." Through interaction with parents, she can calm their fears and assure them that although many miles separate them from their sick son or daughter, their child is being cared for. Encouragement plays a large role in her daily work.

How does she know how to deal with the circumstances she sees while at work, such as a very ill student or a bill that will not be fully covered? She relies on God to give her words of wisdom in each situation. "Before a word is on my tongue, You know it completely, O Lord," as Psalm 139 says. She often shares her life verse: "So do not fear, for I am with you; do not be dismayed, for I am your God. I will strengthen you and help you; I will uphold you with my righteous right hand" (Isaiah 41:10). She and her husband pray for the students that each one meets at work. After all, what better way to impact lives and to "keep our perspective young," than to work with young adults and to help influence them in a godly direction. 
by Heather Fourman

"We have different gifts, according to the grace given us . ... if it is serving, let him serve ..." - Romans 12:6a,7a

On May 2, 1997, Dr. Daniel E. Wetzel, surrounded on the chapel platform by his wife Phyllis and other members of his family, told the audience of applauding faculty and students that being honored as Faculty Member of the Year was a very special moment in his life, one that ranked high on his list of other special days like when Christ came intohis life or when his wife told him, "I do." Later, Dr. Wetzel said, "I was surprised and humbled to be chosen by the students to receive this award; it's always a thrill to be honored by those you serve. That was truly a special day I'll remember for the rest of my days." Dr. Wetzel, who has served as chairman of the science and mathematics department for eight years, first came to Cedarville in 1963 when there were only about 500 students enrolled at the college. At that time, all the science classes were in Collins Hall. Dr. Wetzel said, "We taught physics on the top floor, chemistry on the first, and biology was in the basement, and five of us professors shared what is now Dr. Murdoch's office." In 1973, the science department moved from its tight quarters to the newly constructed Science Center which was later expanded to the Engineering, Nursing, Science Center in 1990. Dr. Wetzel was instrumental in planning for the college to add an engineering program and in planning for the college to construct the ENS, serving as chairman of the Engineering Feasibility Committee in 1987-88 and serving as chairman of the ENS Building Task Team in 198889. The science and math-

\section{Faculty Member of the Year Dr. Daniel Wetzel}

ematics department is currently the largest academic department at the college, and Dr. Wetzel desires to serve each of the professors in the department and maintain a relationship of accountability with them. He said, "I want to be a good servant - to my family, to students and faculty. I know that I have the gift of service... I don't see myself as a 'boss.' Some people introduce me to others as their 'boss,' but I see myself as more of their colleague. We are all workers together." Dr. Wetzel said that one of his greatest responsibilities as chairman is to help provide the means for the professors in the department to accomplish their goals. Each year, Dr. Wetzel writes out goals he wants to achieve in the next year: scholarly and teaching activities, and service goals related to the college, the community, and his church, Grace
Baptist in Cedarville. He shares his list of goals with all the professors in the department and also keeps a list of their goals for the year. At the end of the year, Dr. Wetzel and all the science and math professors evaluate each other on how well they each achieved their goals. Dr. Wetzel said, "I think the professors in the department really appreciate this accountability system. . . . It also promotes a real sense of camaraderie. There is such diversity in this department because we all have such different interests, whether it be physics, biology, math, chemistry. Yet we all are able to work so well together." Dr. Wetzel graduated from Morehead State College in 1955 and earned his masters in nuclear engineering from the University of Cincinnati in 1963. In 1971, he earned his doctorate in physics from Ohio State University. Before 


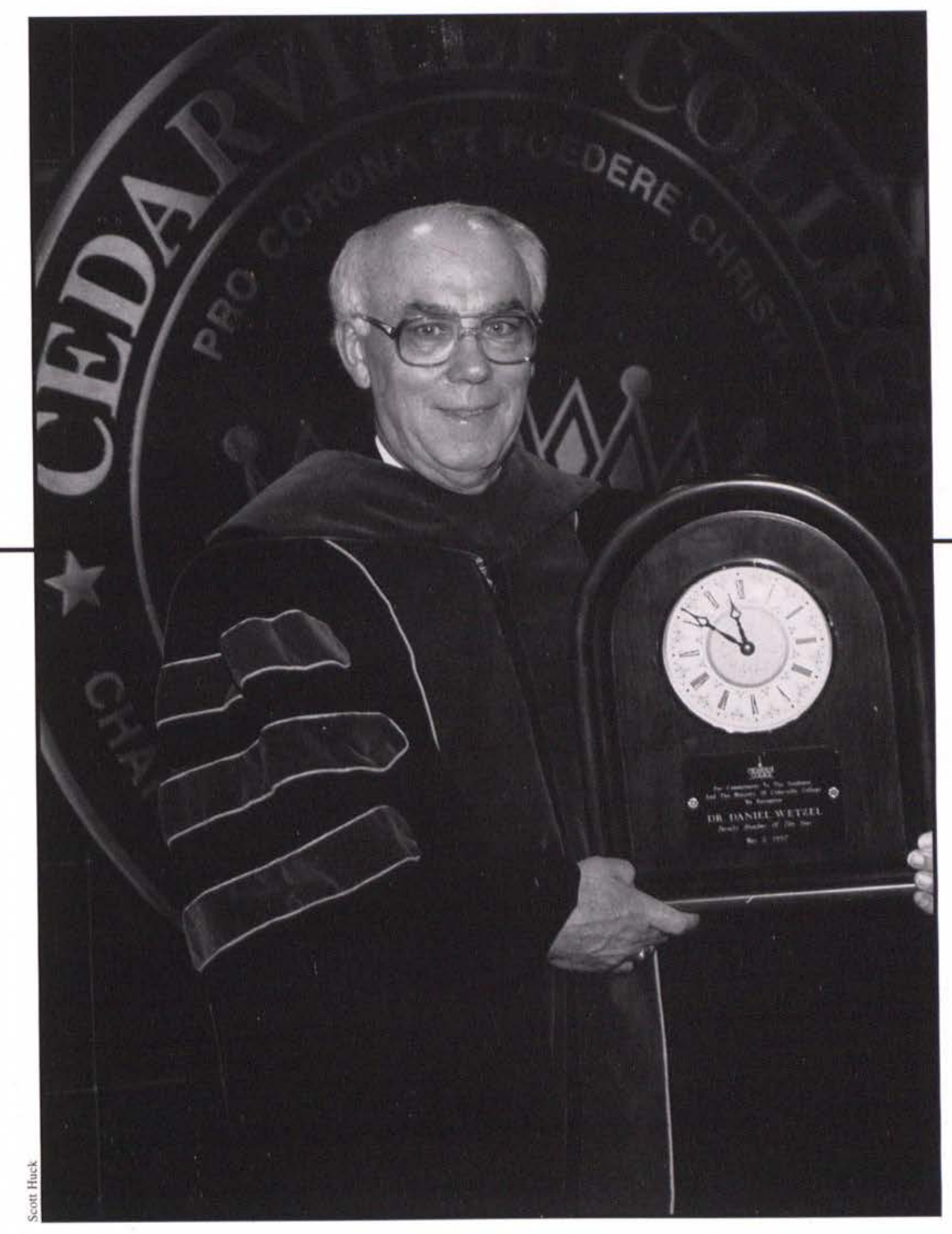

joining the faculty at Cedarville, he worked from 1955-61 at General Electric Company in Cincinnati as a mechanical and nuclear engineer, designing and testing jet engines and trying to develop a nuclear powered airplane. From 196163 , he worked at Batelle Memorial Institute in Columbus as a nuclear physicist analyz- ing nuclear reactors. Dr. Wetzel made the switch to teaching at a time in his life when he was experiencing setbacks and disappointments and was searching for the direction God wanted him to take. It was then that Psalm 27:14 became a very important verse to him: "Wait on the Lord; be of good courage, and he shall strengthen thine heart. Wait, I say, on the Lord." Dr. Wetzel said, "I was being impatient; I had to realize that God didn't want my tithe, my time, or talent as much as He just wanted me." Dr. Wetzel felt God calling him to serve in some Christian ministry, and Pastor Bill Brock of Emmanuel Baptist Church in Columbus directed Dr.
Wetzel's attention towards Cedarville College. Dr. Wetzel has no plans for retirement. He said, "How do you retire from ministry? When I'm told that I've stopped being effective at my job, that's when I'll step down. God called me here to Cedarville, and God will call me away." 


\section{Concert Chorale}

Soprano: Lori Anderson, Jenny Armour, Noelle Bertelson, Lisa Branon, Jill Breckenfeld, LeaAnne Churgovich, Jennifer Cook, Sara Eleveld, Denelda Foltz, Amber George, Carolyn Guerette, Rachel Henricks, Jody Hovis, Joanna Houck, Melissa Kaufman, Hannah Lakes, Sarah Lightly, Erin Prentis, Mandy Prusha, Selena Samuelson, Jessica Schuring, Nikki Streit, Rebecca Upham, Melanie Wadlington, Lisa Walker, Alisa Whitt. Alto: Jennifer Abas, Julie Armour, Allyson Bielo, Jill Brown, Leah Caldwell, Lisa Cook, Shauna Cook, Nicole Cooley, Sallisha Cowell, Kelly Grady, Brenda Inion, Julie Jaskilka, Tricia Johnson, Becky Kayser, Jennifer Myers, Susan Perry, Jessica Peterman, Kathy Petersen, Hannah Rives, Nicole Sparling, Beth Stewart, Janelle Swartz, Jessica Seeley, Nadine Tomsa, Jamie Weiss, Susan West, Sarah Whitney. Tenor: Jason Atwell, Matt Beck, Josh Berrus, Erik Bledsoe, Kevin Boblitt, David Boyd, Anson Hanbury, Dave Jackson, Jason Naill, Jason Overturf, Dan Scott, Matt Sherwood, Stephen Slates, Vance Smith, Jonathan Weber, Steve Weber, Chris Williams, Jonathan Winn, Jason Atwell. Bass: Aaron Armour, Mark Armstrong, Ryan Burkhard, Shawn Dabravalskas, Jesse DeConto, Brent Ferguson, Dan Forness, Ryan Freed, Chad Jensen Jerry King, Greg Larson, John Maskill, Brian Naess, Josh Nelson, Aaron Ponzani, Chuck Quarles, Dan Rainsberger, Ben Robinson, Josh Rupp, Scott Shaw.

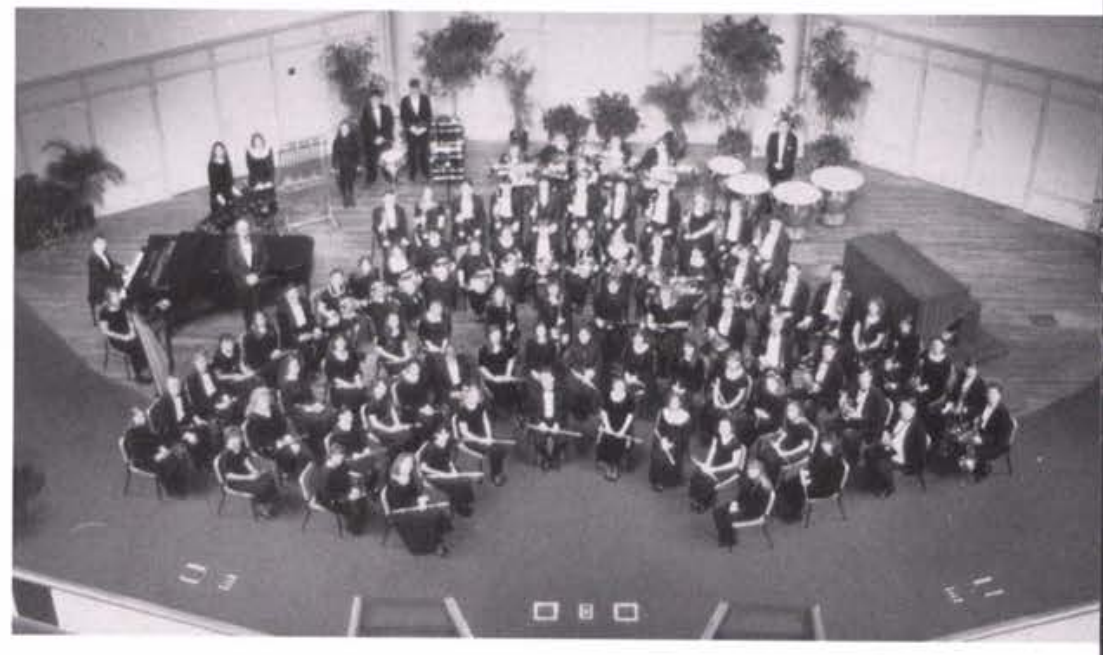

\section{Symphonic Band}

Mr. DiCuirci - Director. Piccolo: Becky Lakes. Flute: Sarah Clayton*, Faith Buchanan, Lezley Bath, Jason Brown, Sarah Mayer, Tammy McComb, Tracy Friesen, Amy Morse, Nichole Bork, Jennifer Rogers, Ruth Jackson. Oboe: Elizabeth McDonald* Gretchen Dorman, Amy Kehlenbeck, Leanne Heath. Bassoon: Deborah Kruse*, Cindy Potter, Amy Zimmerman. Bass Clarinet: Liz Parr*, Rachel Schafer Saxophone: 1st Alto: Chet Jenkins*, Andy Heyd. 2nd Alto: Shawn Conley. Tenor: Jim Dyer, Denis Jordan. Baritone Sax: Paul Shelton . Clarinet: 1st: Michelle Delaney*, Rachel Soderstrom, Angela DeSantis, Cindy Lawry, Allister Baldwin, Karisa Linafelter. 2nd: Heather Smith, Ann Weeks, Amanda Bruckner, Elizabeth Rogers, Susan Hunsaker, Rebecca Kniowski 3rd: Holly Waechter, Nathan Lewis , Nathan Hart, Michelle Edwards Suzanna Creps, French Horn: 1st: Eileen McCoskey*, Rachel Wirt. 2nd: Julie Saucier, Amy Clutz. 3rd: Lane Dykman. 4th Melody Brickel, Summer Schafer, Becky Voigt. Baritone: John McCaw* Julie Palmer, Chris Robertson. Trumpet: 1st: Chris Pagnard*, Brian Rowley, Israel Barr, James Scheid. 2nd: John Filson, Mark Warren, Jeff Brown, Debbie Frank, Andrew Nyveldt. 3rd Jared Voigt, Heather VanGorp, Chris Smith, Andy Clary: Trombone: 1st: Tom Mullins*,. Damon Titus. 2nd: Jill Townsend, Debbie Pittman. 3rd Kevin Armstrong, Paul DiCuirci. Tuba: Chris M. Brown*, Chris Grigson, Beth Frank, Virginia Miller .Percussion: Ben Vawter*, Eric Perkey, Steve Estep, Carol Marks Brent Mullin, Mark Wyse. Harp: Jenny Stryker.

*Section Leader 

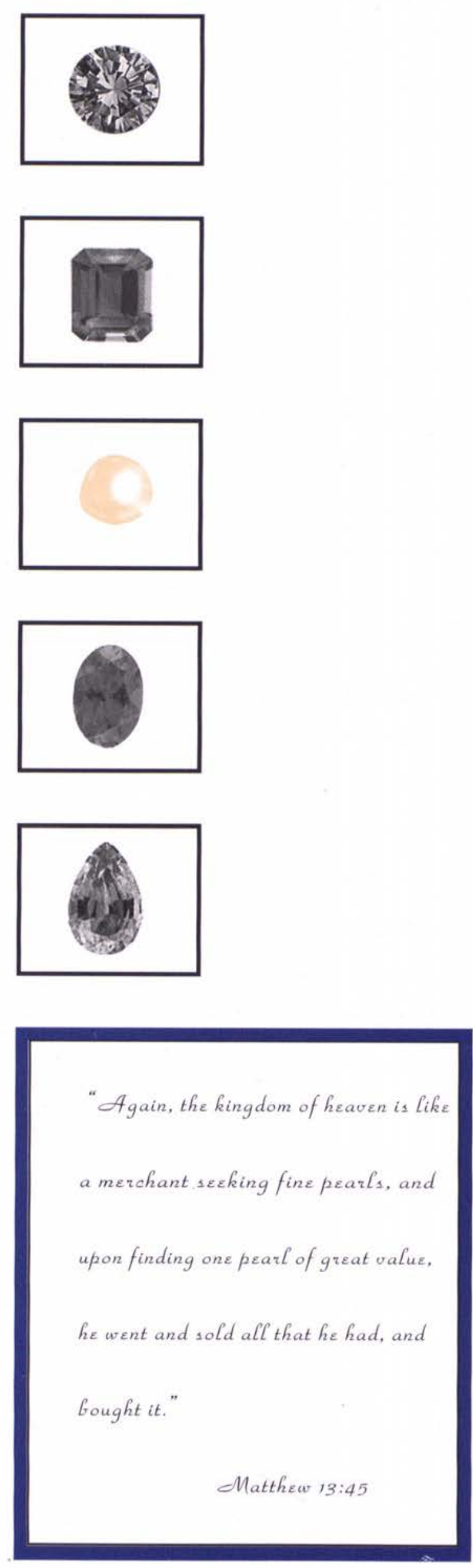

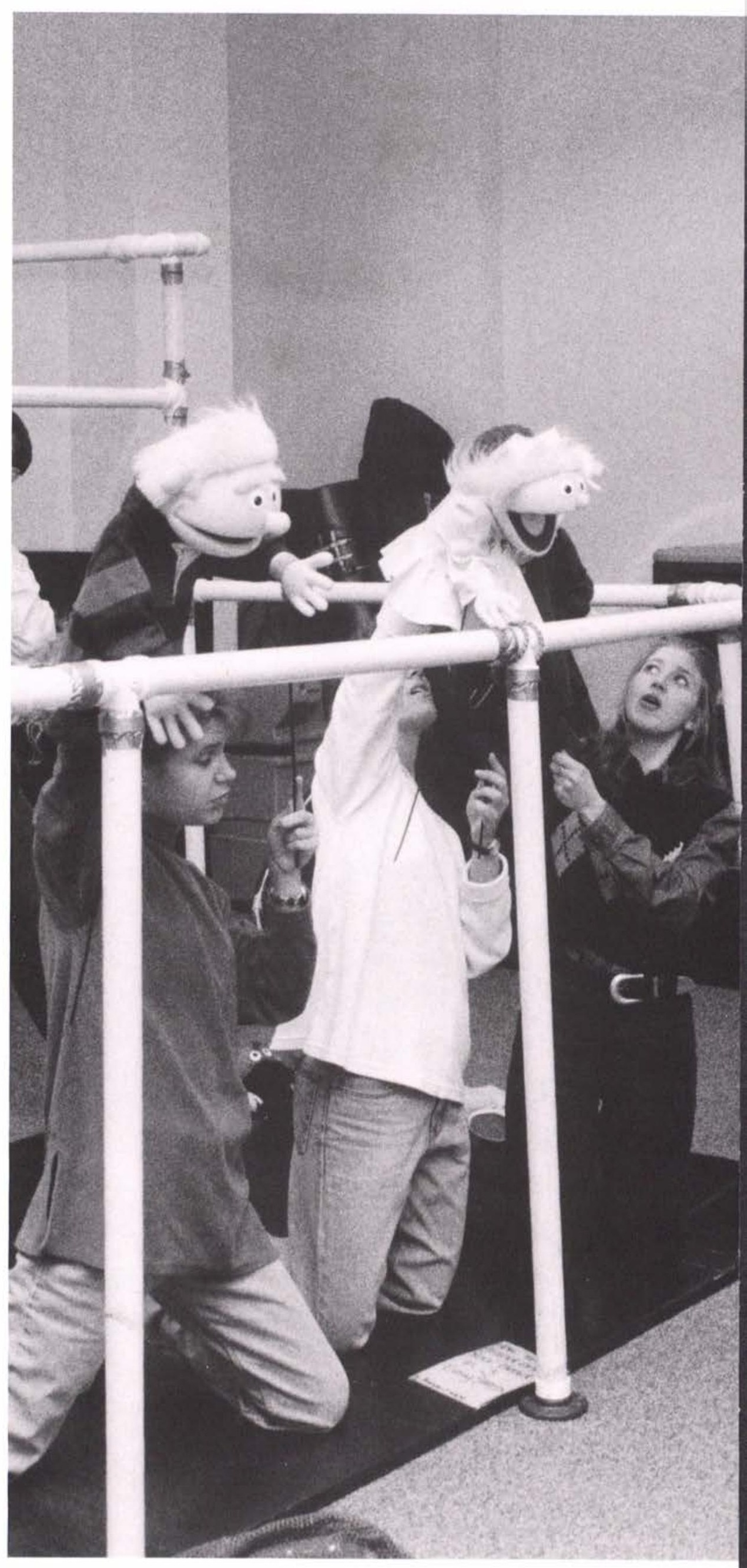




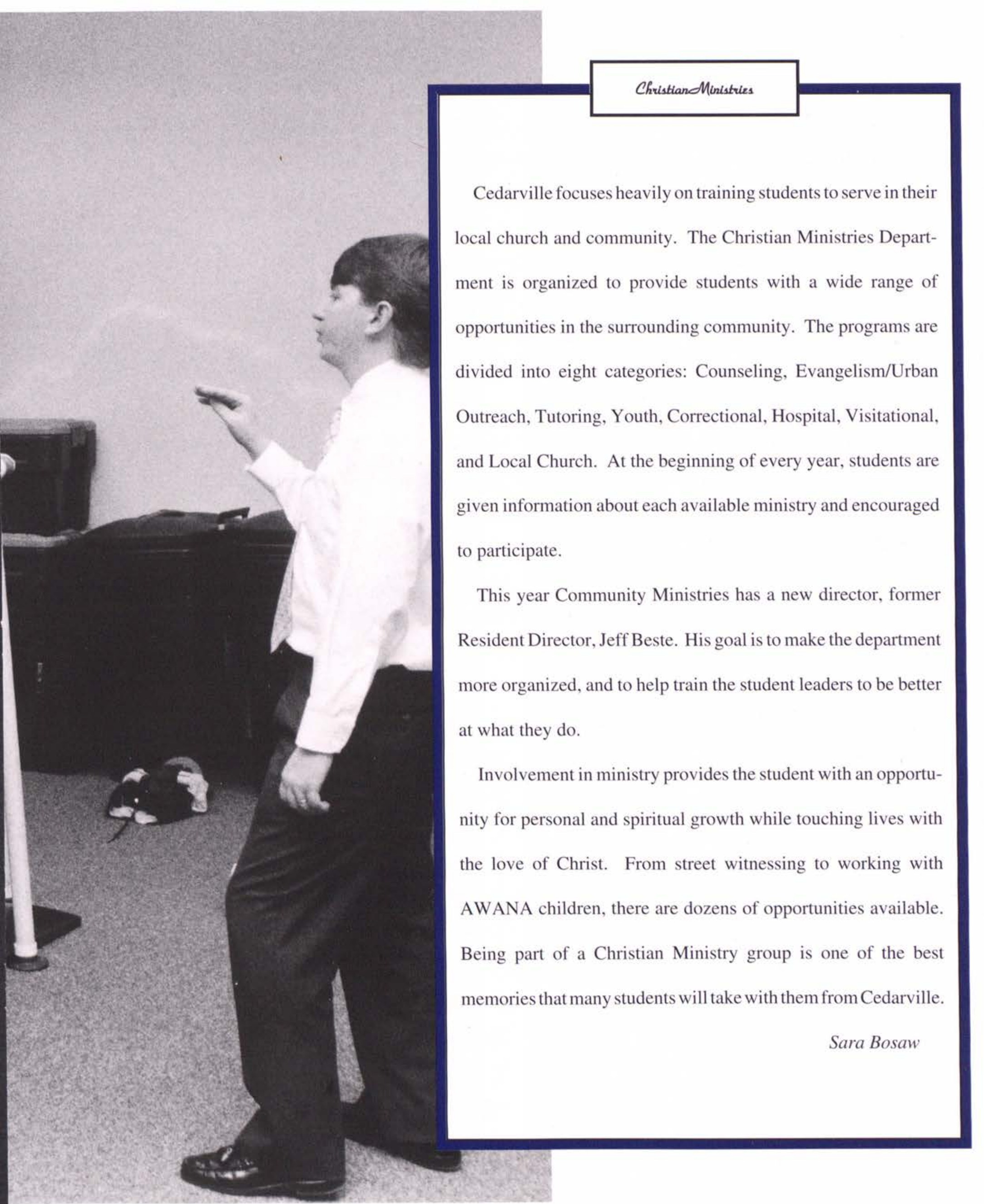



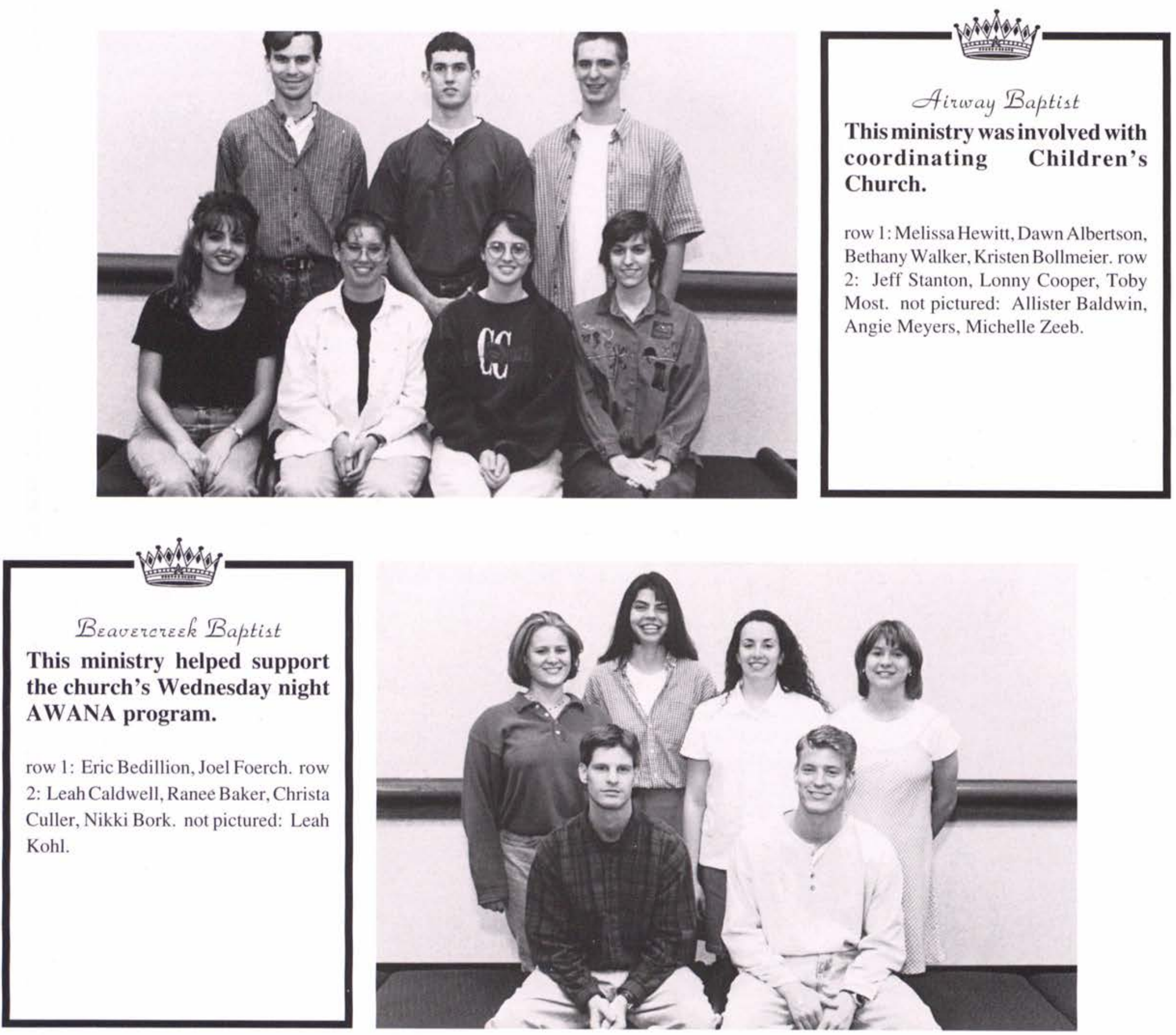

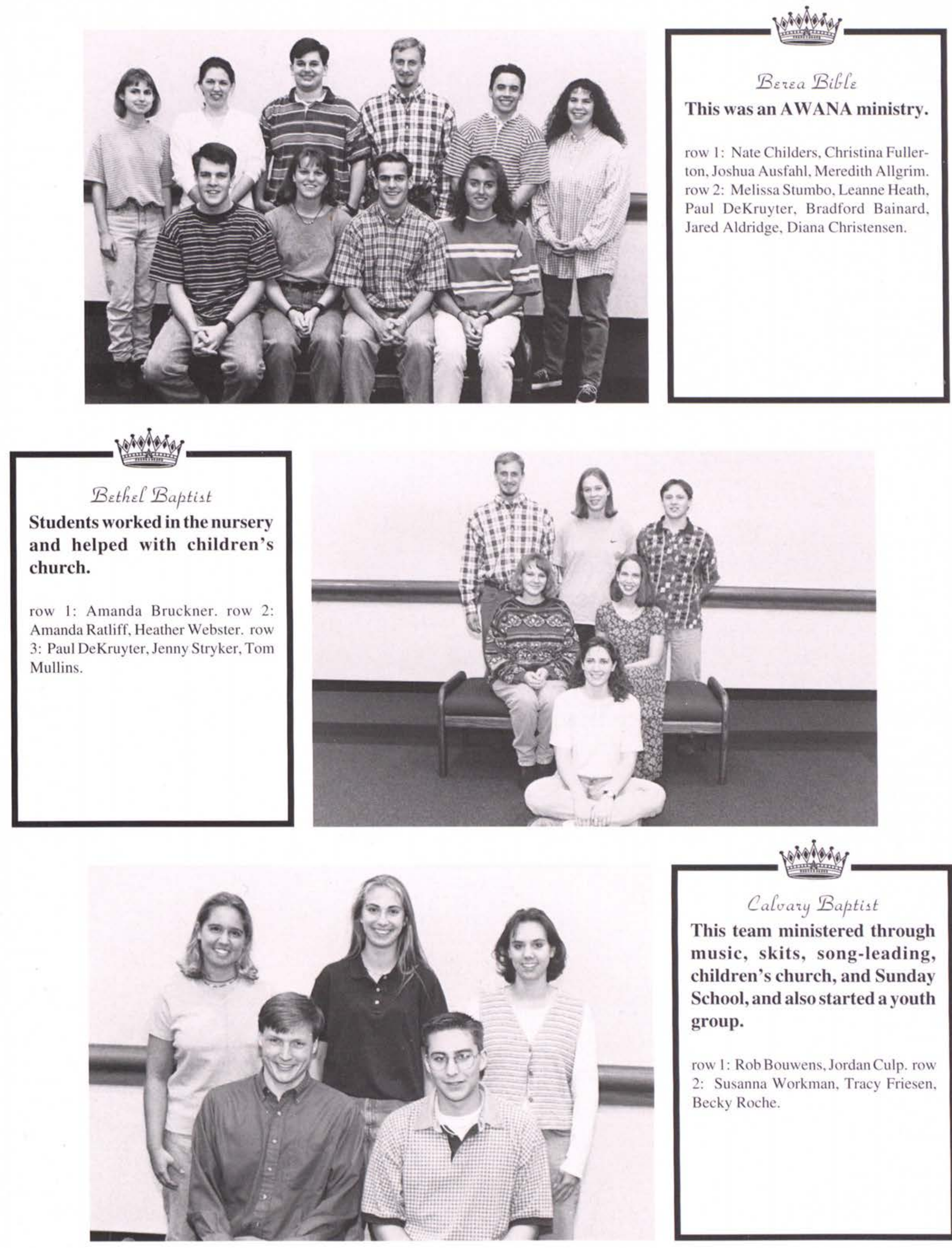

This team ministered through music, skits, song-leading, children's church, and Sunday School, and also started a youth group.

row 1: Rob Bouwens, Jordan Culp. row 2: Susanna Workman, Tracy Friesen, Becky Roche. 

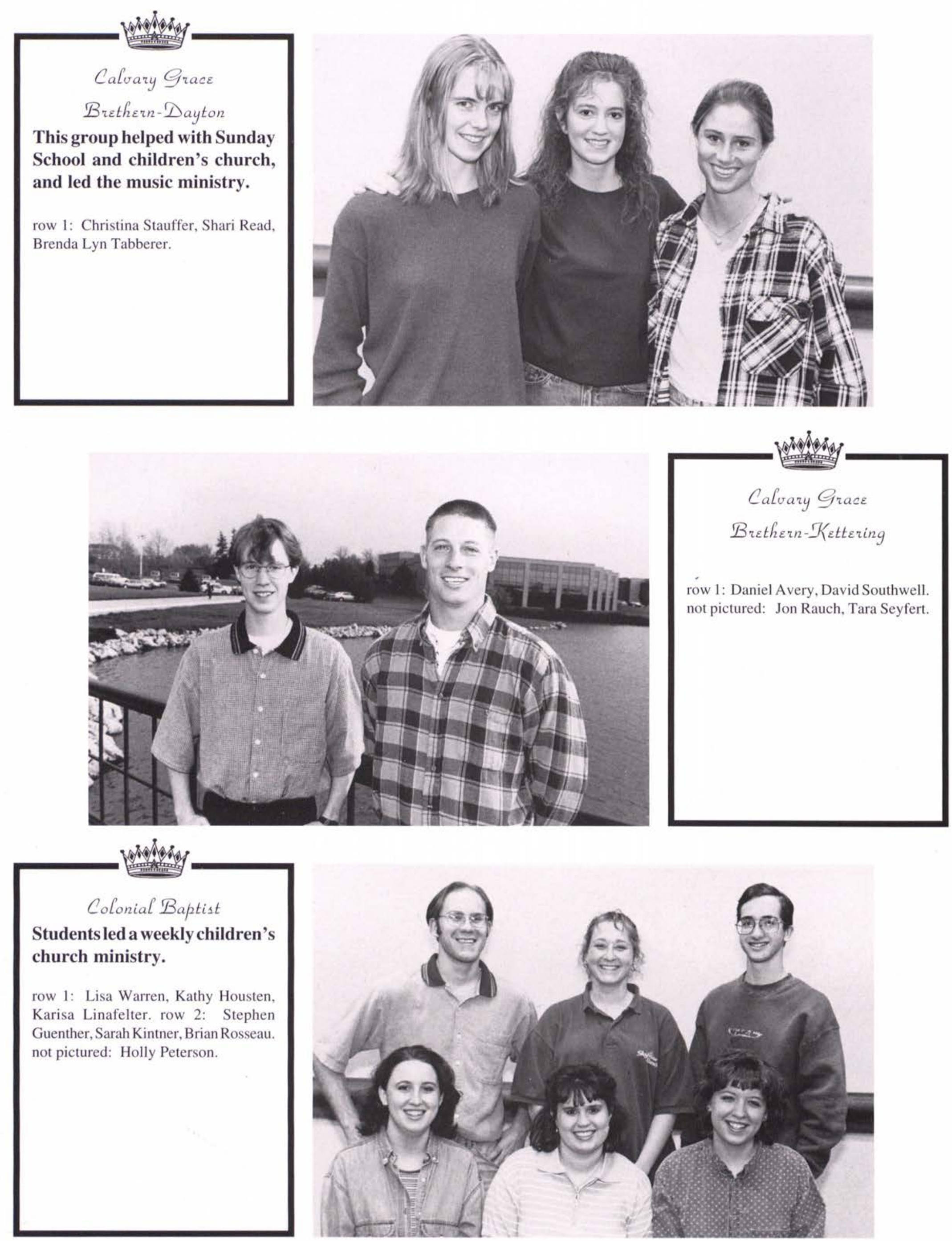

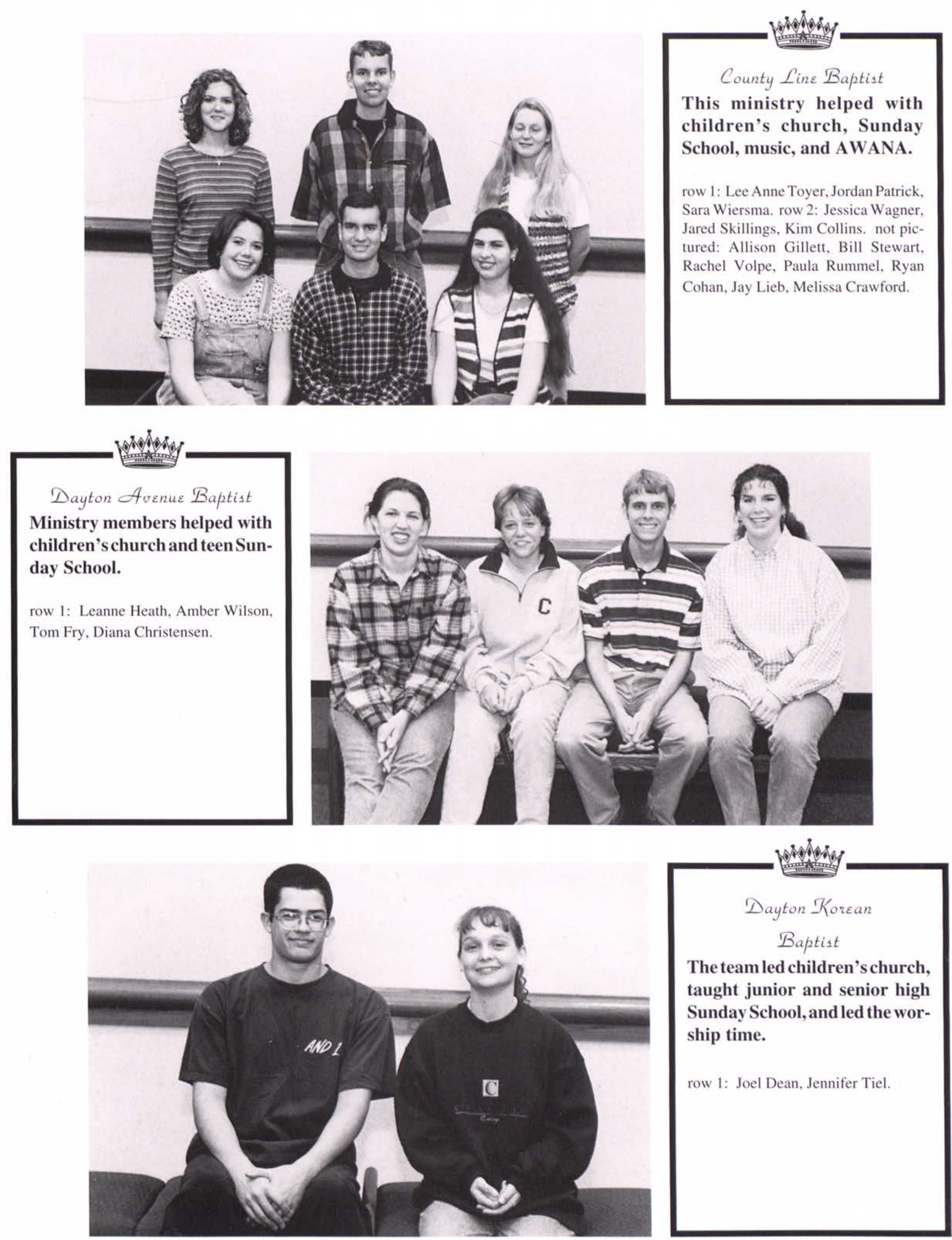

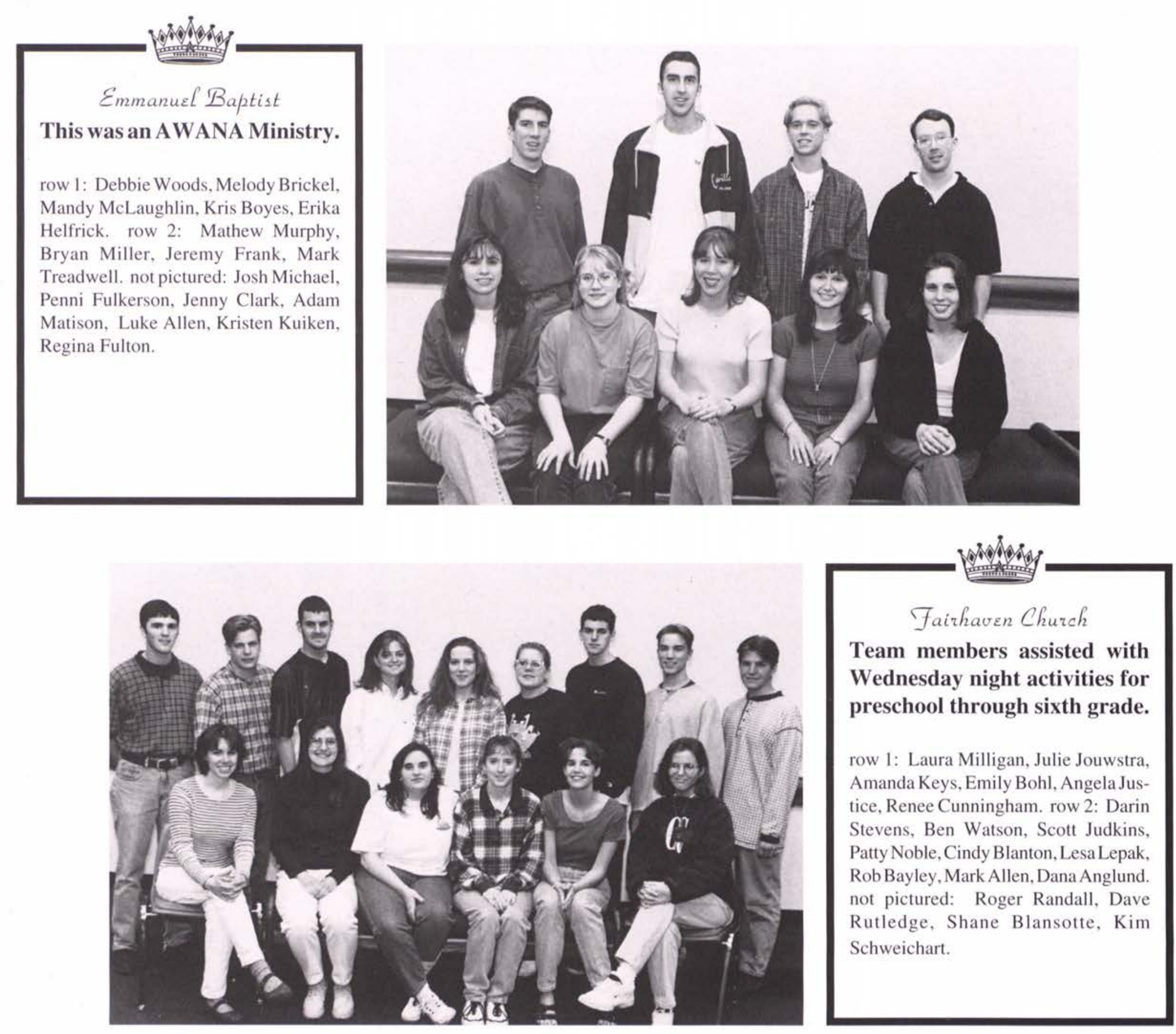

vidy

\section{Jaith Baptist}

Students ministered to Sunday School and primary students, led music, and provided a pianist.

row 1: John Moodie, Annmarie Reynolds, Chris Robertson.

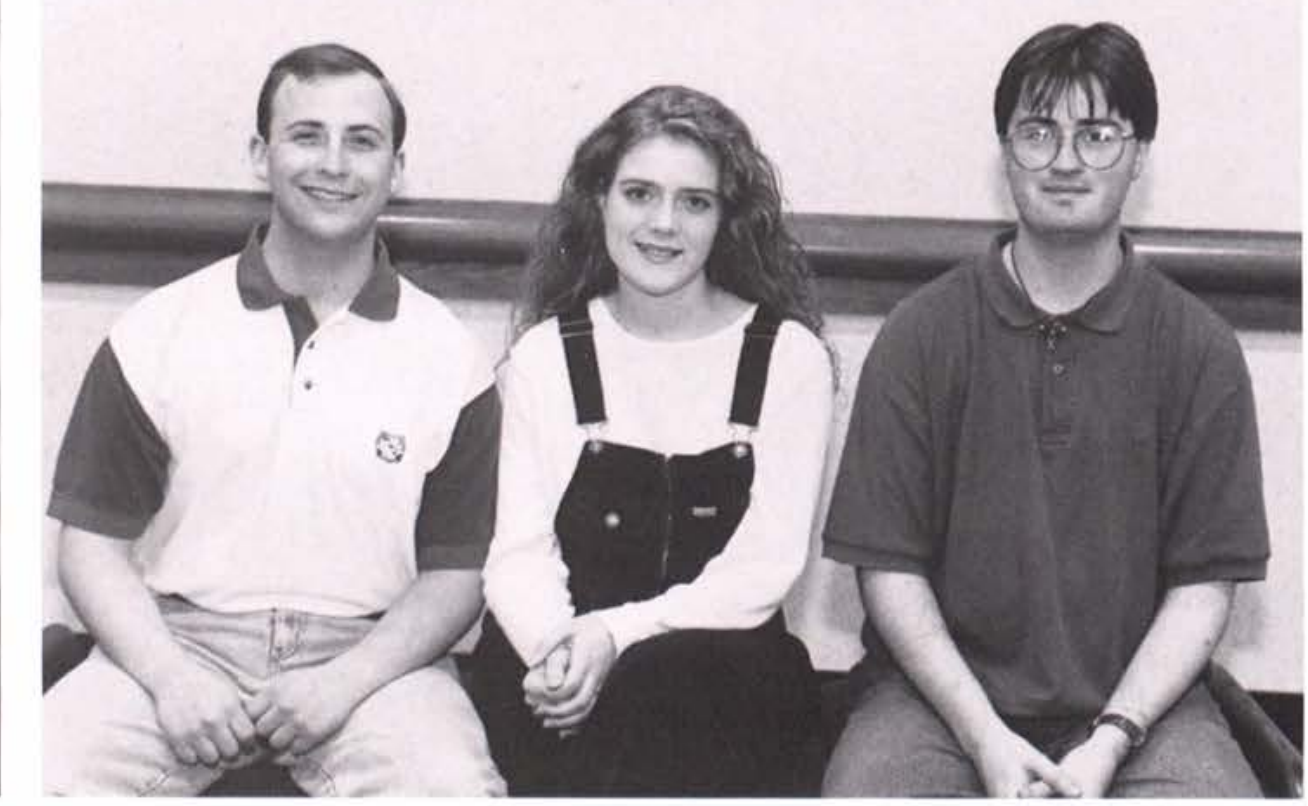



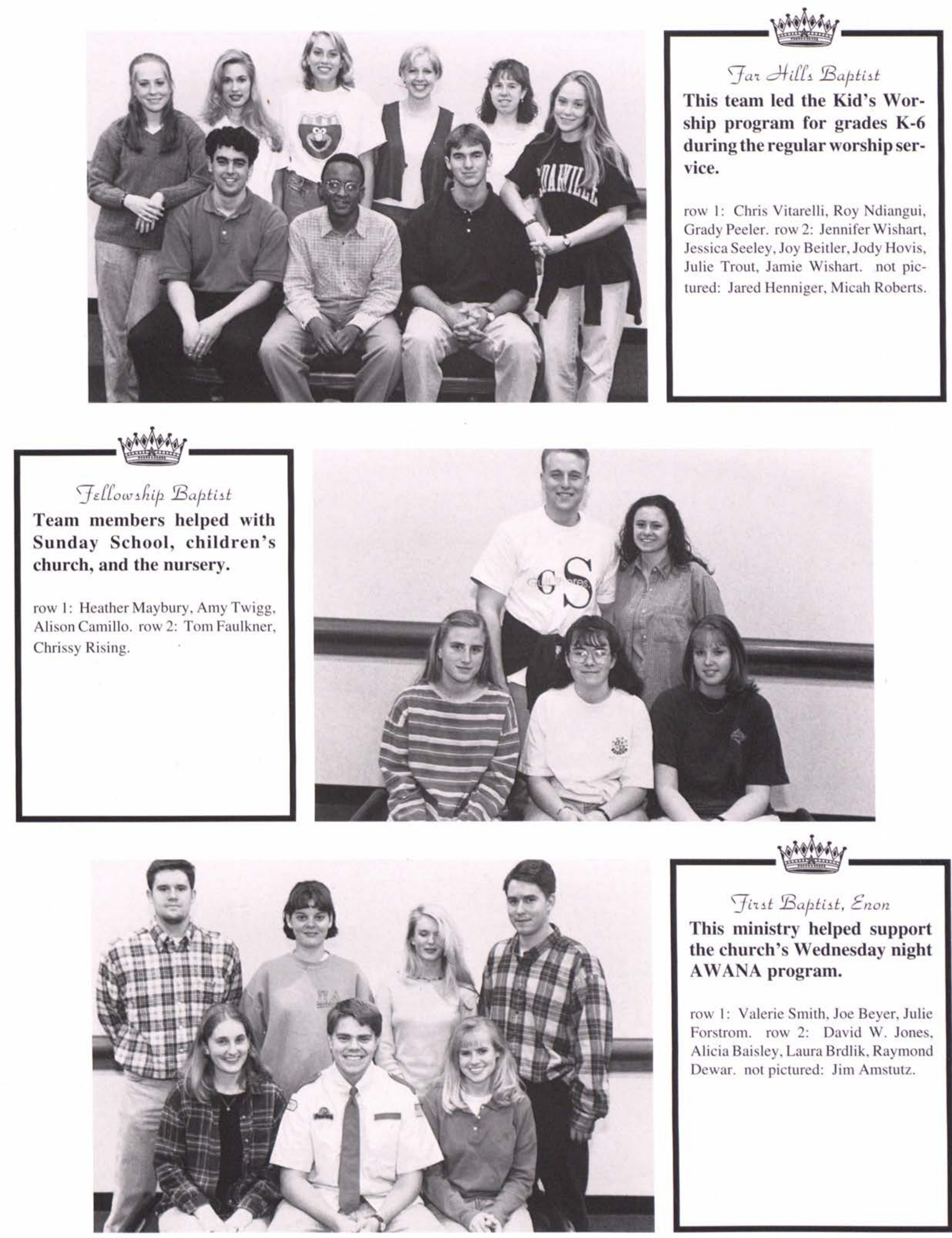
First Baptist, Dipp City

Team members taught Sunday School, youth group, and children's church, and led some music services.

row 1: Tim Flowers, Tim Miller, Nikki Luckman, Mike Lewis.
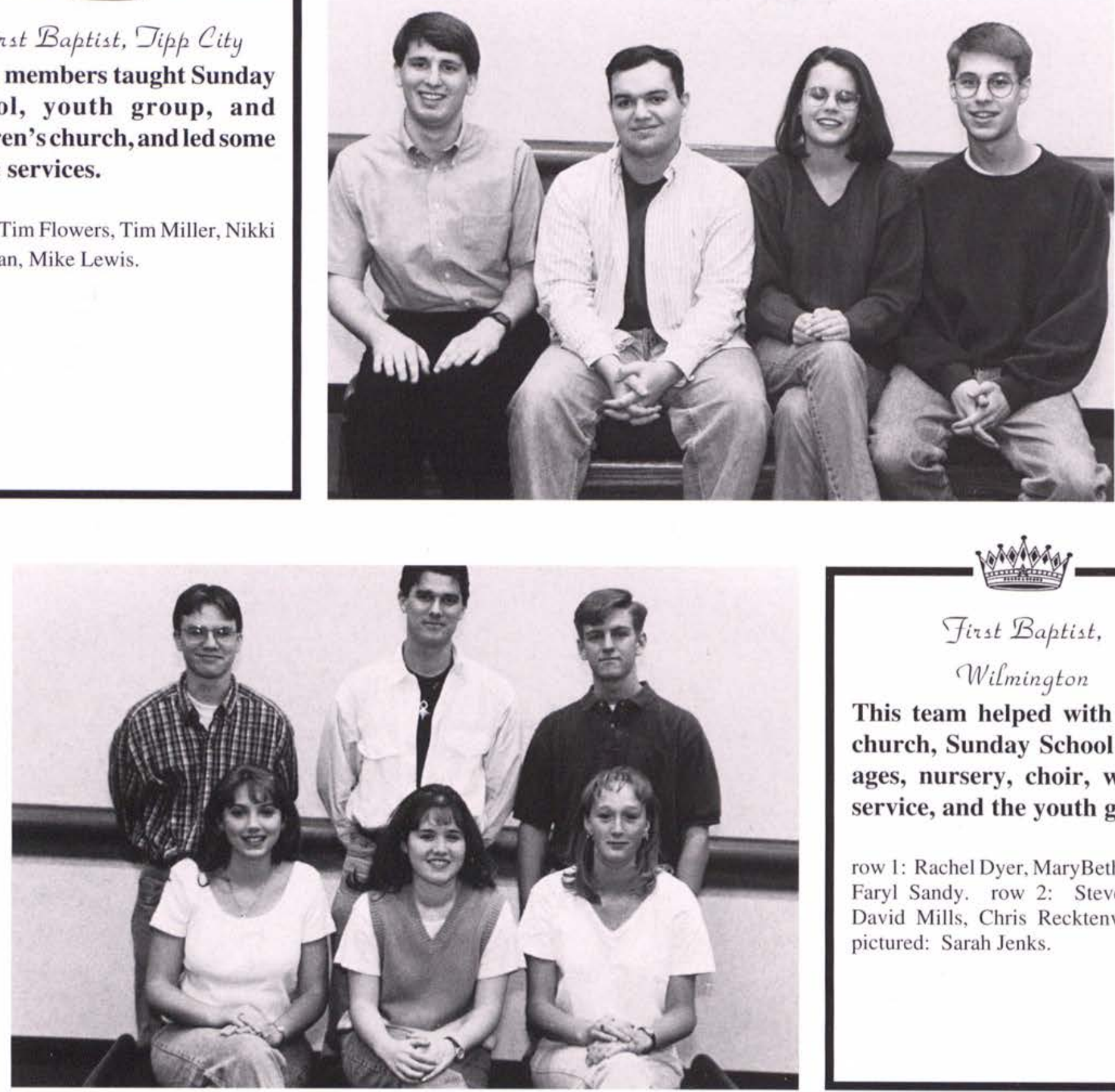

This team helped with junior church, Sunday School for all ages, nursery, choir, worship service, and the youth group.

row 1: Rachel Dyer, MaryBeth Powell, Faryl Sandy. row 2: Steve Slates, David Mills, Chris Recktenvald. not pictured: Sarah Jenks.

. Laura Waddell, Holly Barnett, Rebekah Sorensen. row 2: Emily Bishop, Amanda Mudrey, Raymond Dewar III, Stephanie Gleason, Christina Ream. not pictured: Kim Boesch, Rebecca Leatherman.

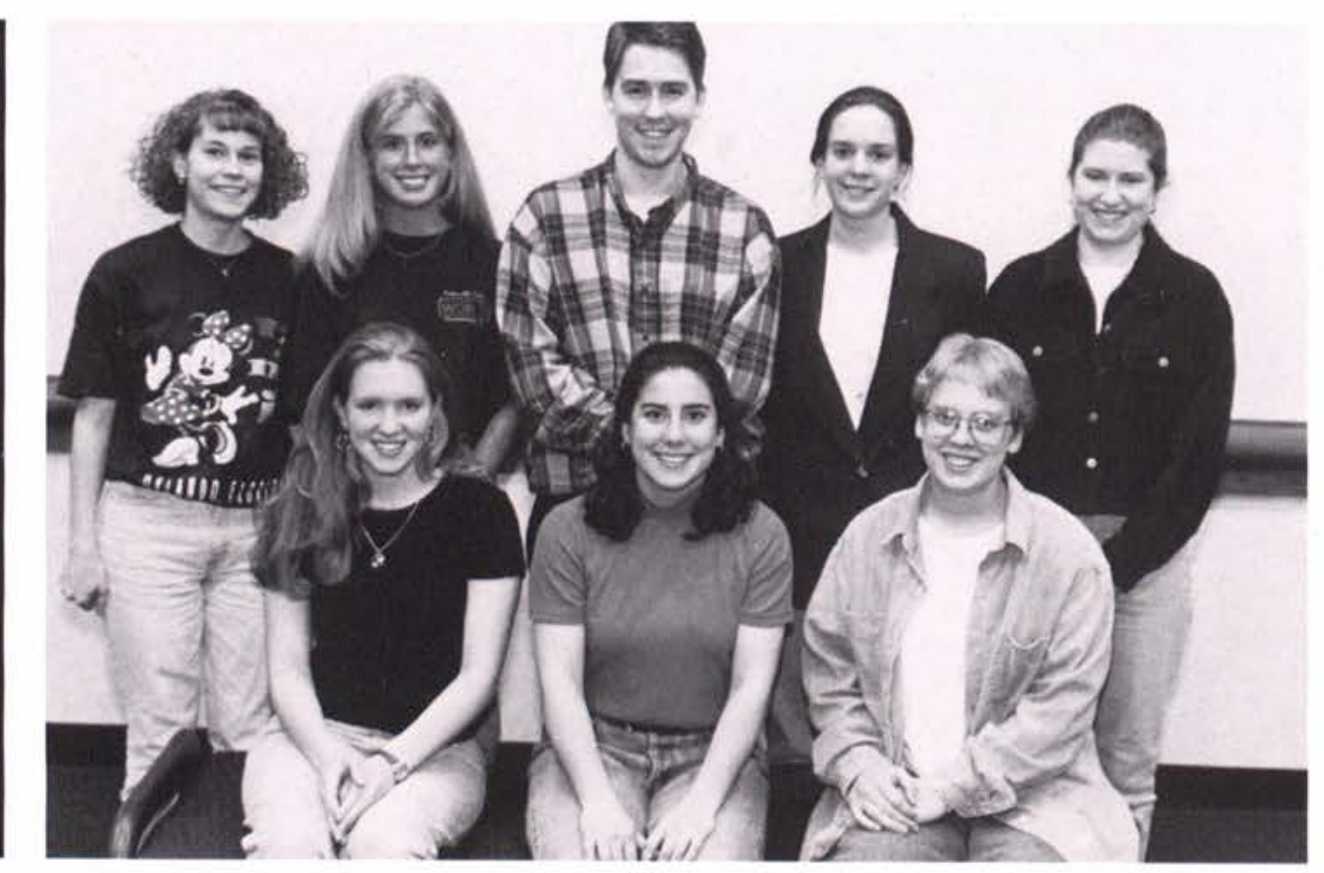




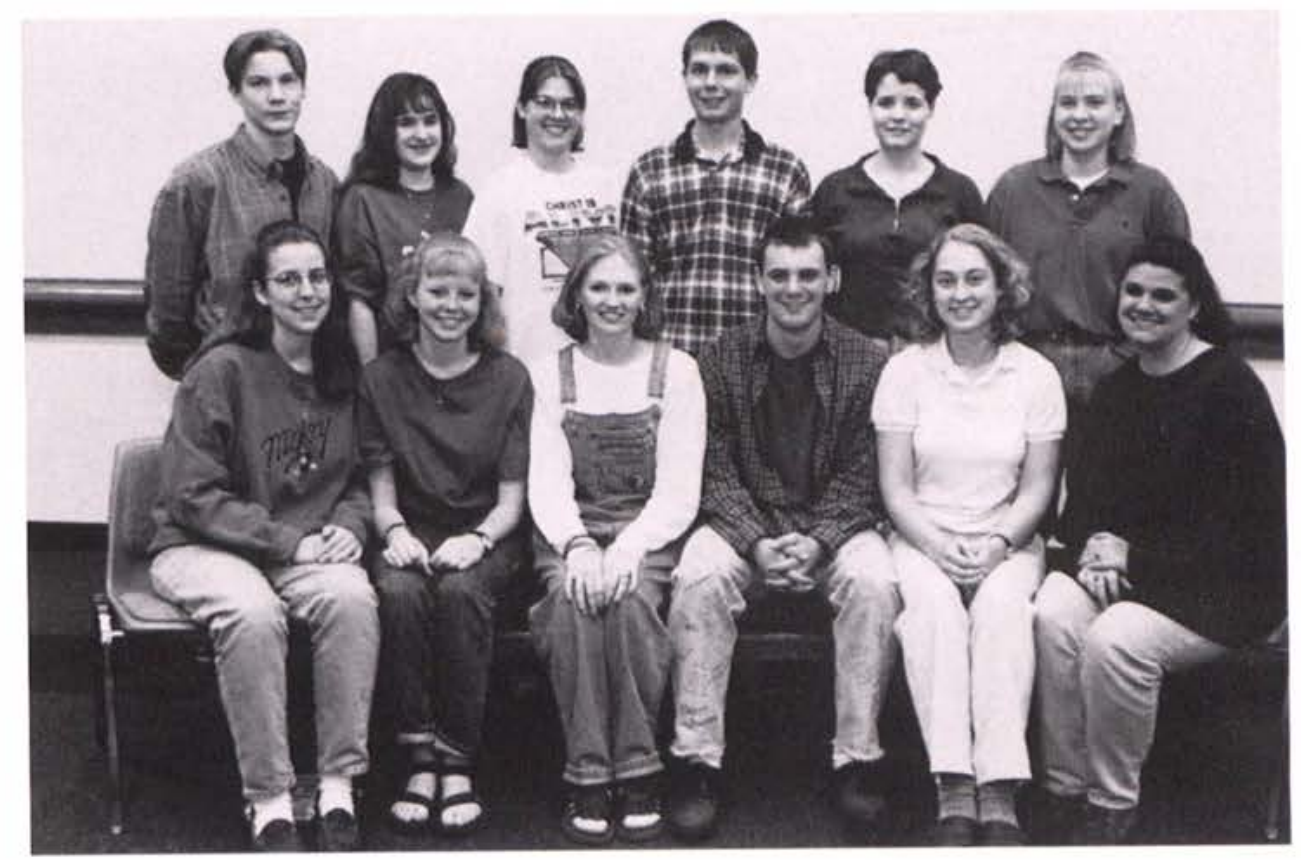

\section{Forest - Ridge Baptist}

This was a small church; team members helped with the new AWANA program.

row 1: Elizabeth Stone, Caren Furst, Trisha Trost, Michael Gregg, Kristin Rosner, Allyson Bielo. row 2: Steve Bruhl, Debbie Buck, Naomi Redington, Steve Weber, Naomi Cooper, Jen Whitman.
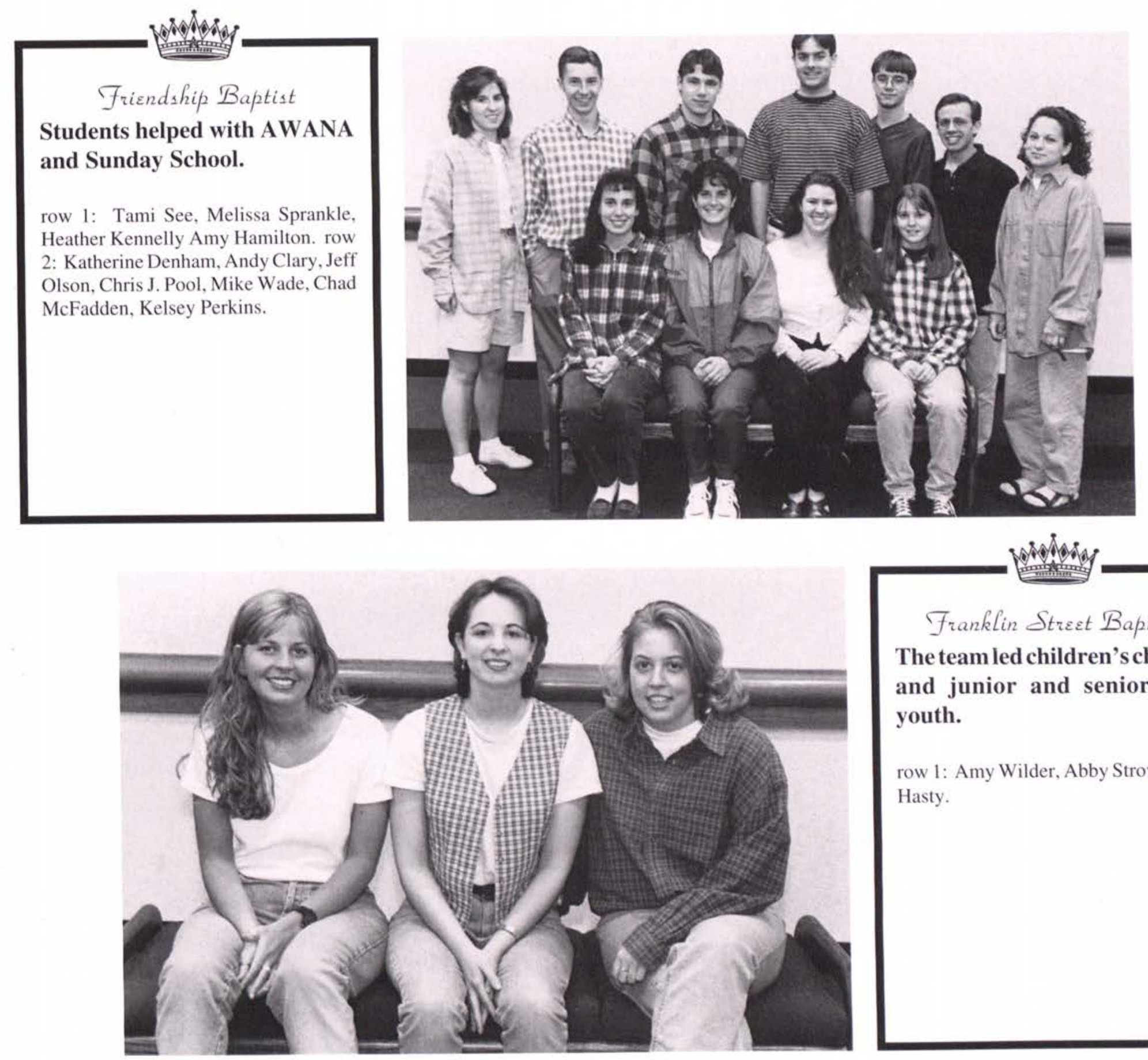

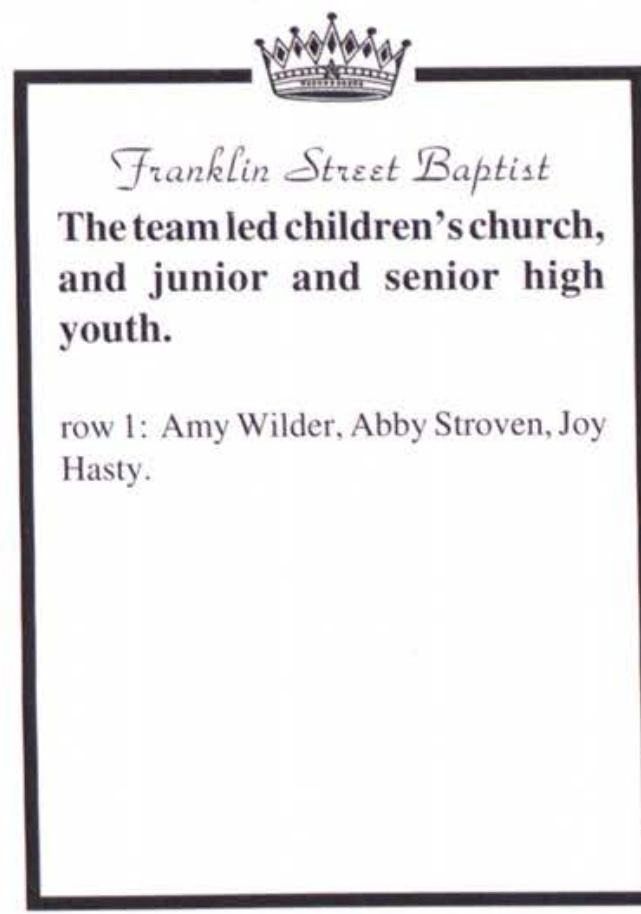



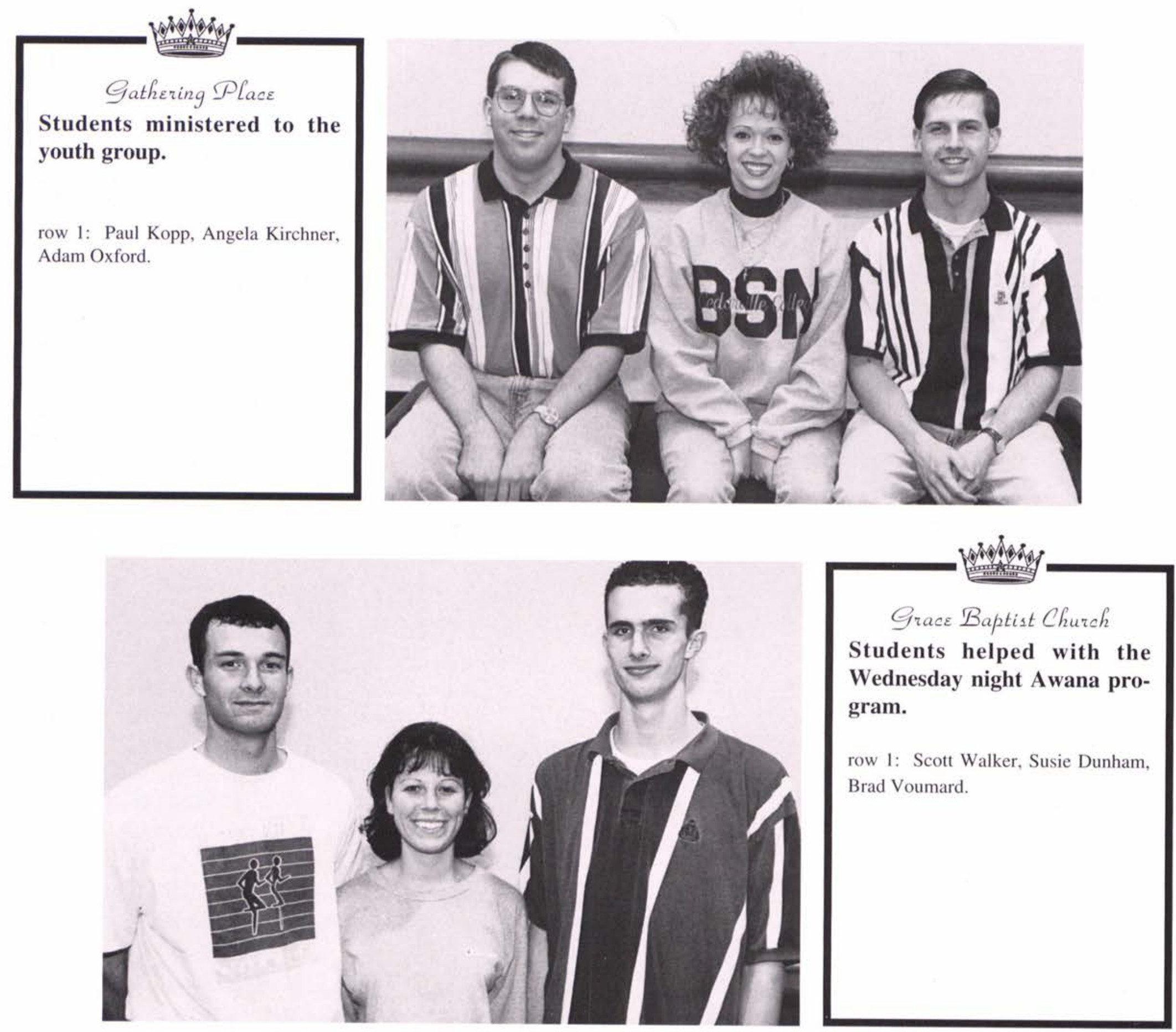
oroty

Grand Heights Baptist

This team helped with the nursery, preschoolers, and first and second grade.

row 1: Joy Peterson, Ray Townsend, Nancy Houck. row 2: Sarah Rittgers, Dawn Albertson, Kristin Hoovler, Brandi Leeds, Deborah Chickering. not pictured: Angela Boyce, Gillian Gombis, Shari Magin, Heather Ritchey, Heather French, Jeff Saunders, Stephanie Mead.

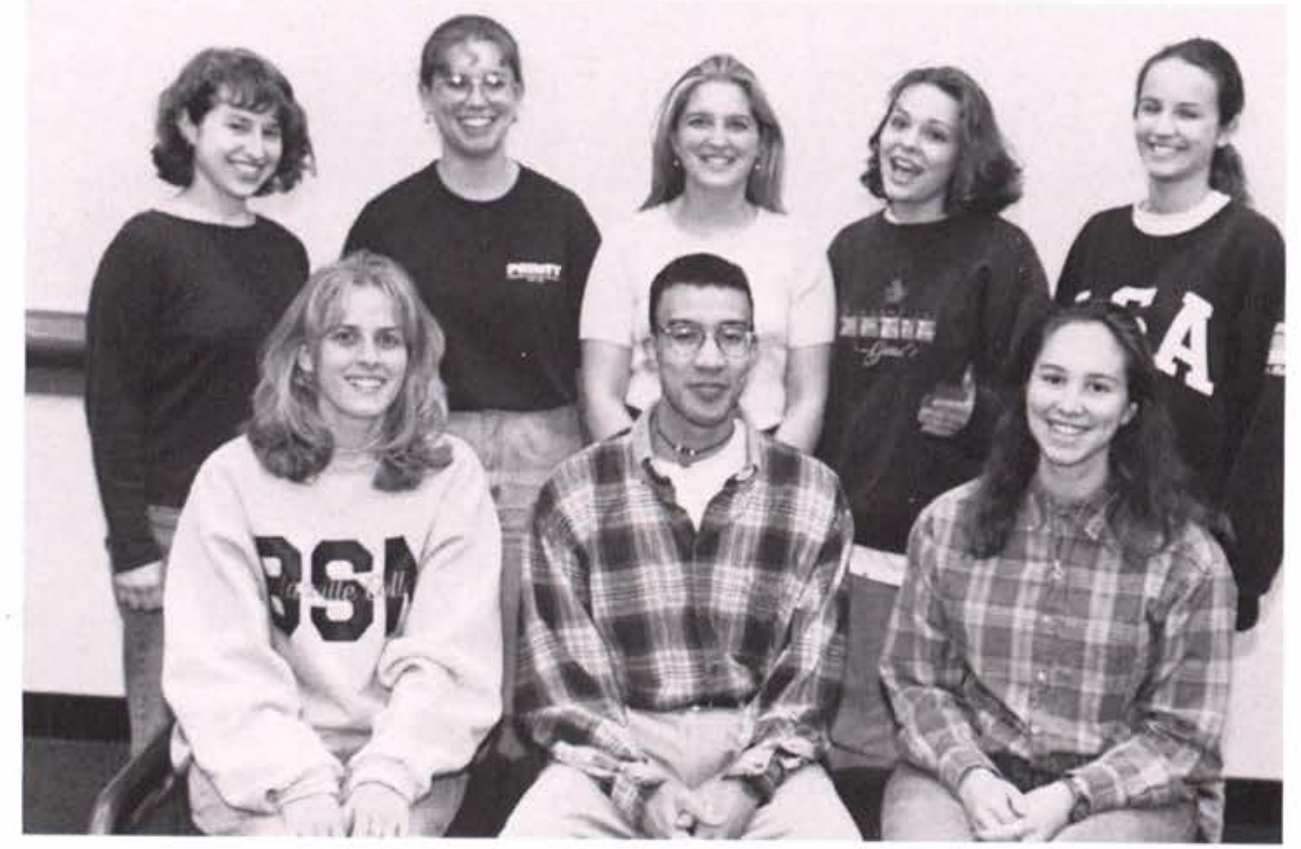



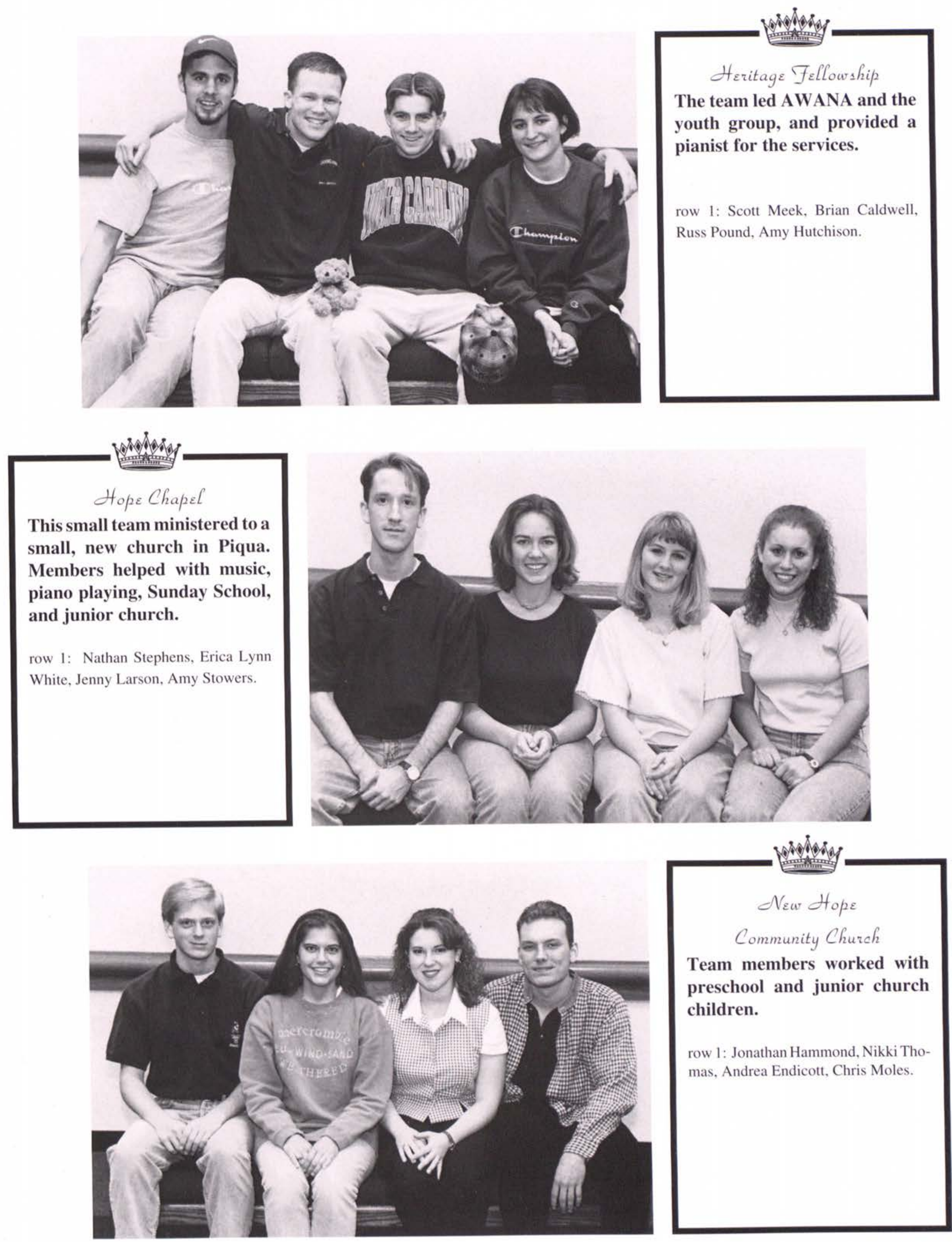
vionsos

\section{Northside Baptist}

Students helped with the Wednesday night A wana clubs.

row 1: Jen Hoffman, Sarah Holesovky, Pam Claus, Amy Thayer, Chrystie Ruba, Ben H. Stutzman. row 2: Joel Howard, Dave Staedtler, Mark Denges, Nate Freeman, Rod Clawson, Dan Douglas, Brad Nelson.
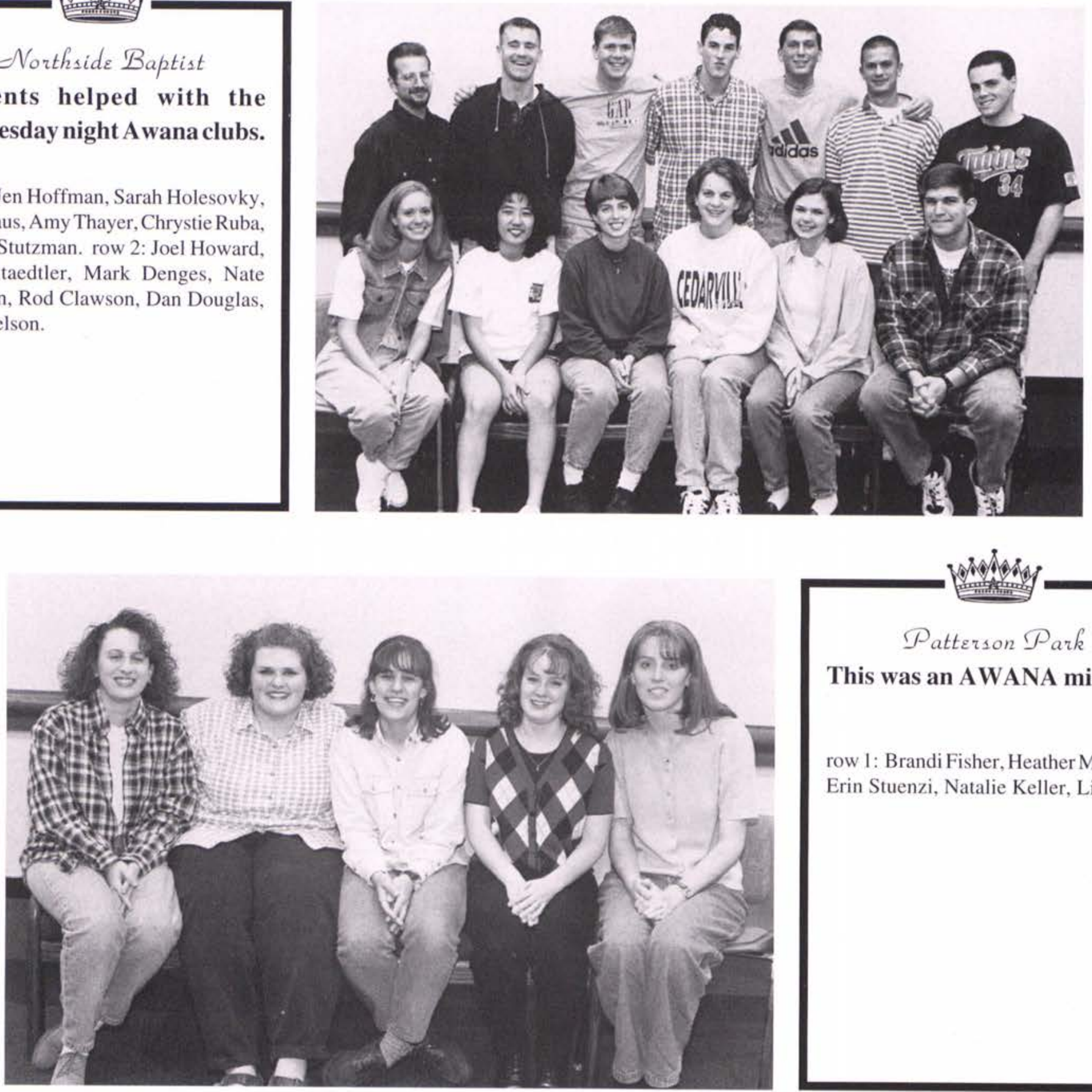

This was an AWANA ministry.

row 1: Brandi Fisher, Heather Murdoch, Erin Stuenzi, Natalie Keller, Liz Parr.
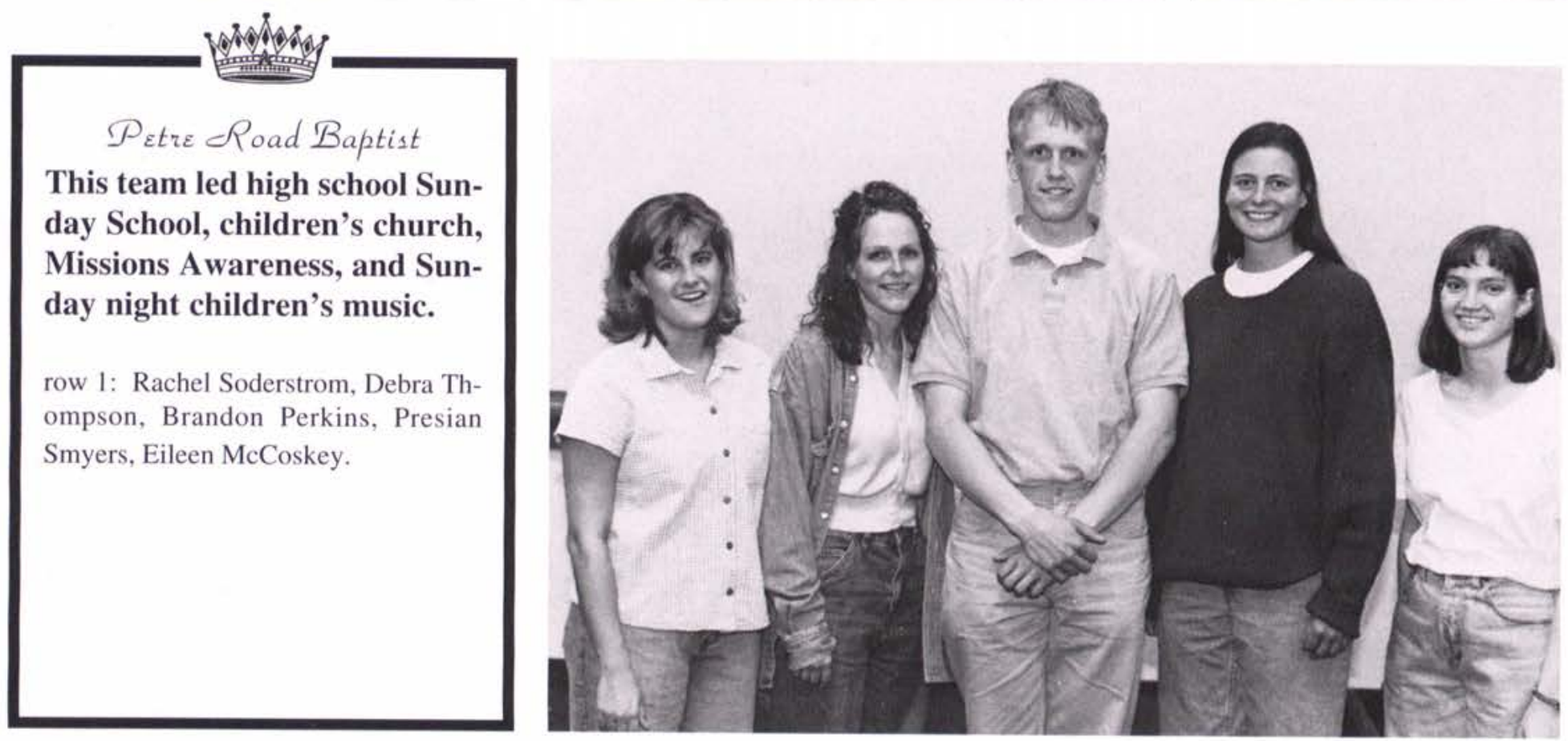

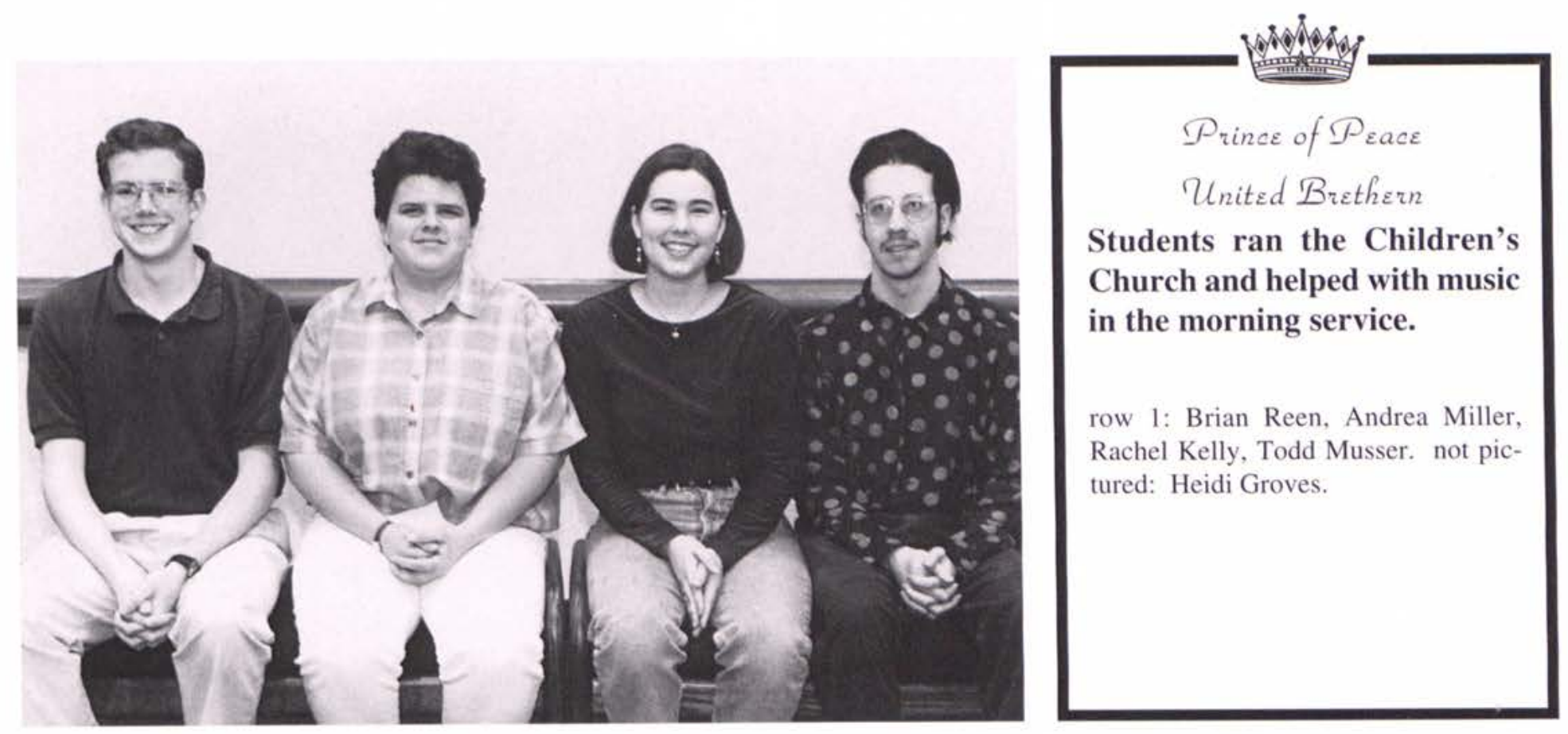

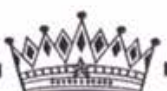 \\ Southgate Baptist \\ Church}

Students made a large contribution to the Wednesday night Awana.

row 1: Rob Mulvaney, row 2: Mary Beth Beal, Laura Kroner, Lori Hamilton, Kathy Housten, Sheri Wilson, Debie Kruse. row 3: Jeanette Irish, Stephanie Tomsa, Joey Decker, Kerry Allen, Adria Andrews, Kristin Kuhn. not pictured: Christi Baldwin, Julie Hastings, J. Micheal Yoder, Troy Page, Andrew Alderfer.
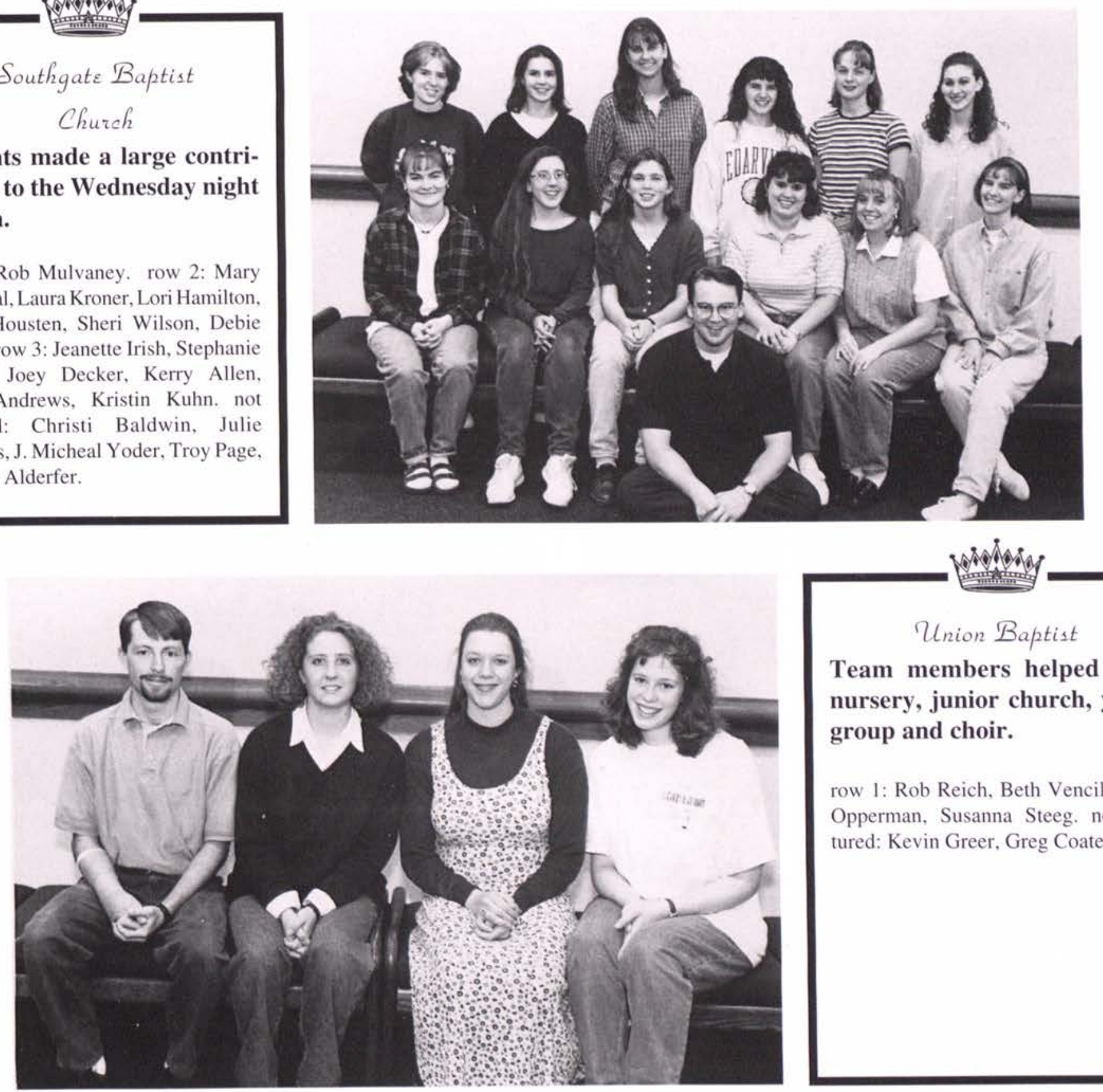

\section{Union Baptist}

Team members helped with nursery, junior church, youth group and choir.

row 1: Rob Reich, Beth Vencill, Julie Opperman, Susanna Steeg. not pictured: Kevin Greer, Greg Coates. 

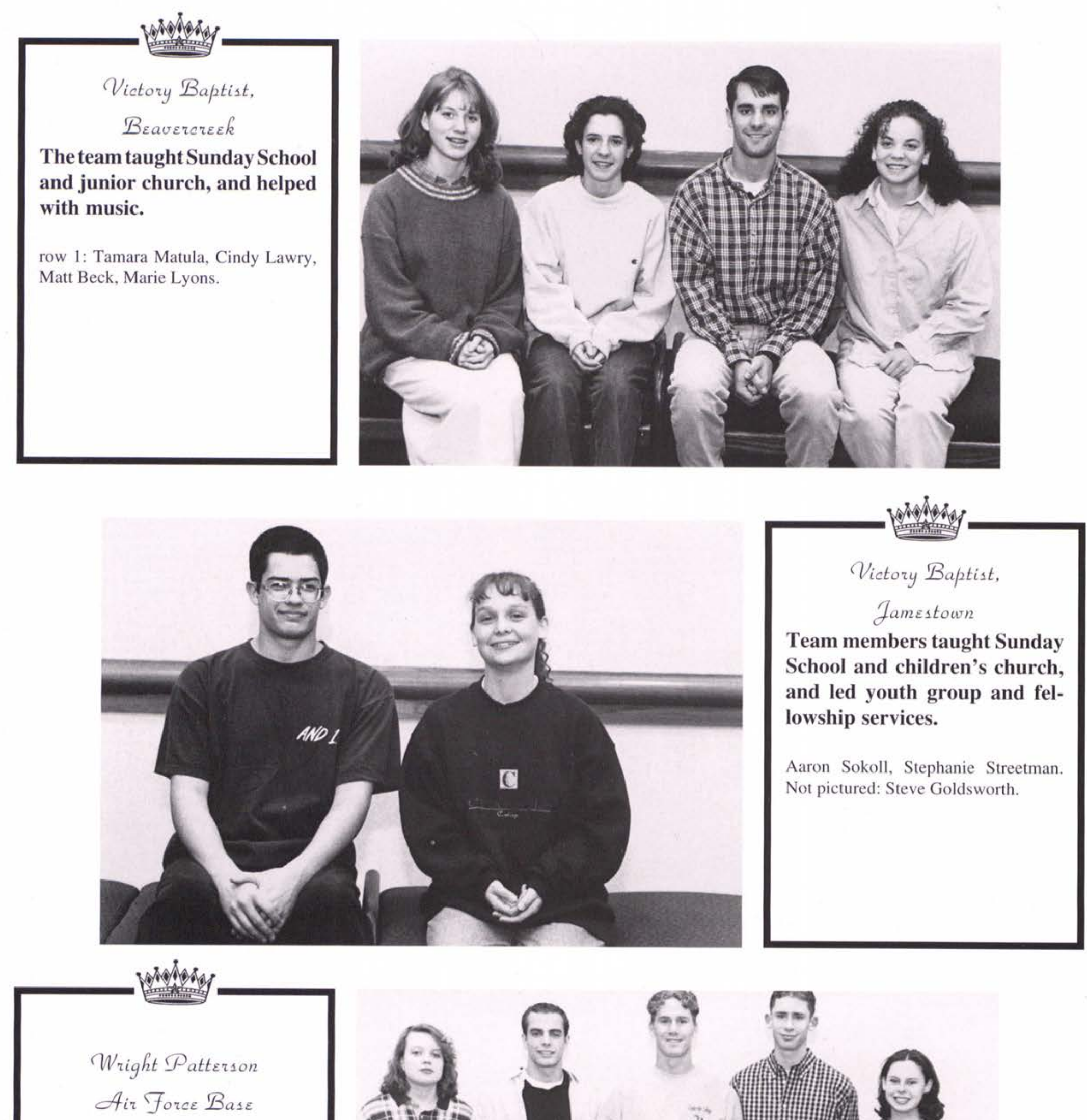

The team was involved in Sunday School and Children's church.

row 1: Kristin Marshall, Rachel English, Lina Ponder, Daphne Diaz, Heather Kunert. row 2: Virginia Miller, Roger Randall, David Rutledge, Andrew Chouinard, Gretchen Taylor.

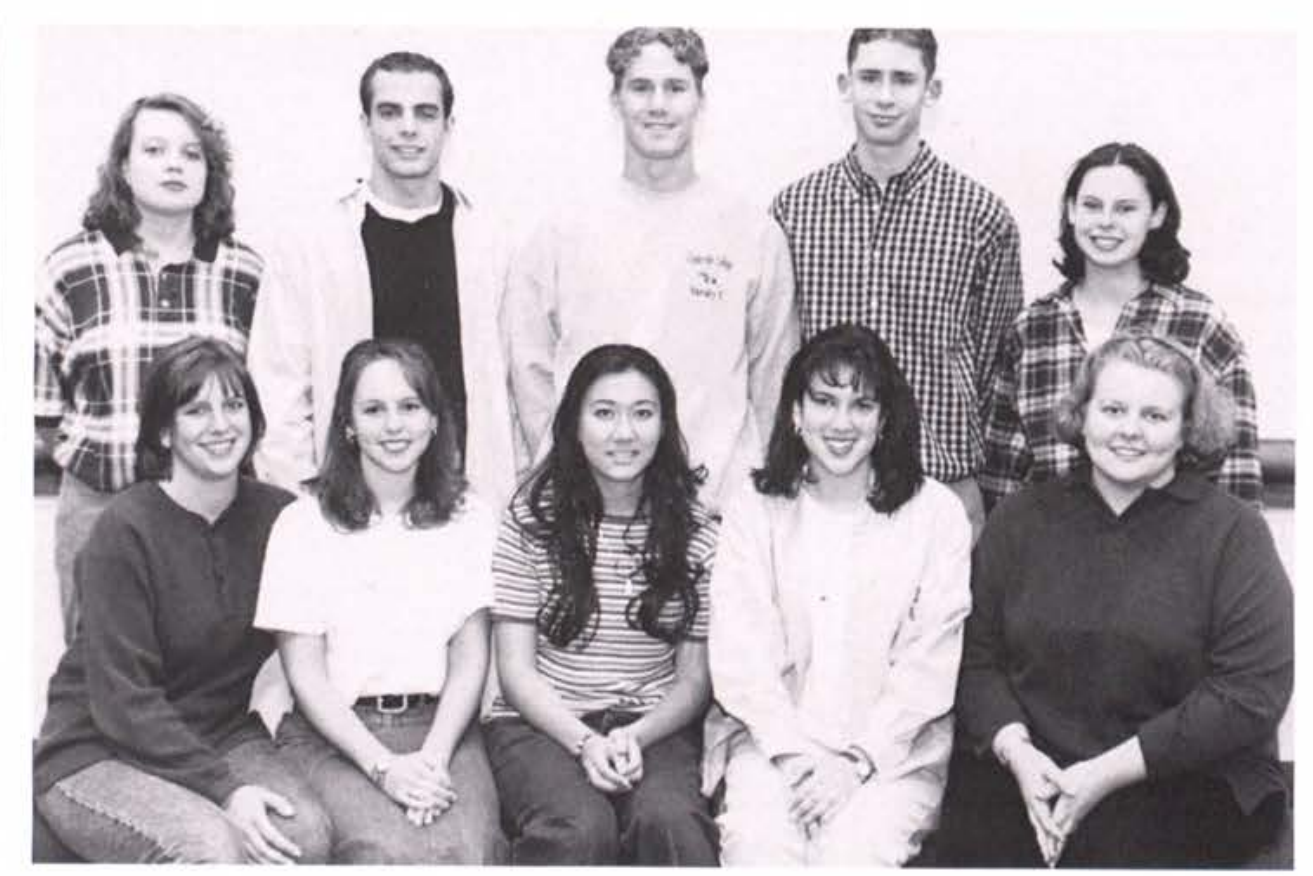



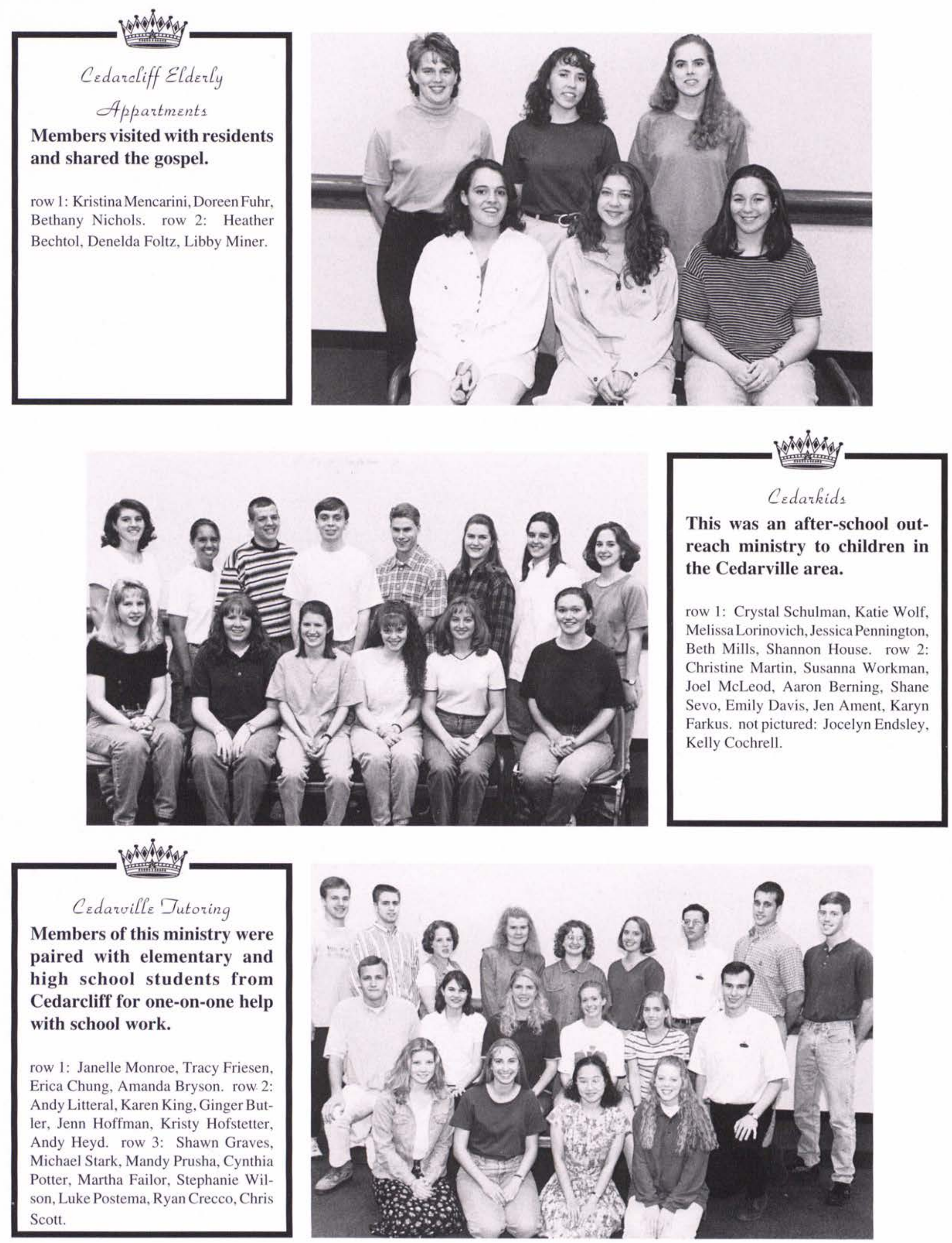


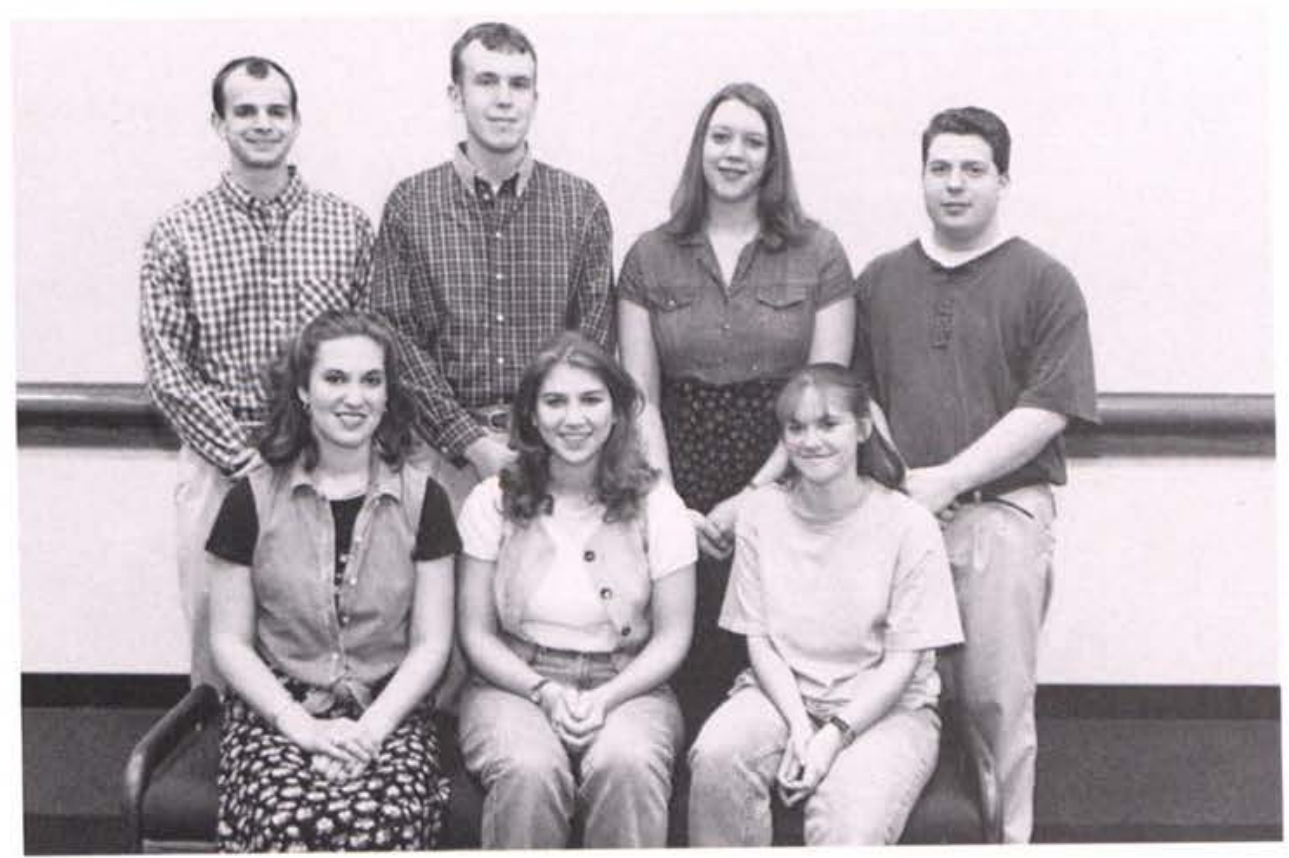

\section{Central State University}

This was an evangelism and Bible study ministry.

row 1: Debbie Allen, Julie Saucier, Megan Wawro. row 2: John Linak, Chet Cromer, Julie Opperman, Jason Brown. not pictured: Doug Bayler, Kara Lehman, Wendy Baker.

\section{Clowns for Christ}

This team served as a resource for other ministry teams. They performed puppets, magic tricks, and other entertainment for other teams' special events.

row 1: Sandy Bennett, Nikki Anderson, Betsy Linnell, Angela Howe, Nathan Radford with Judson, Emily Wilijamaa.
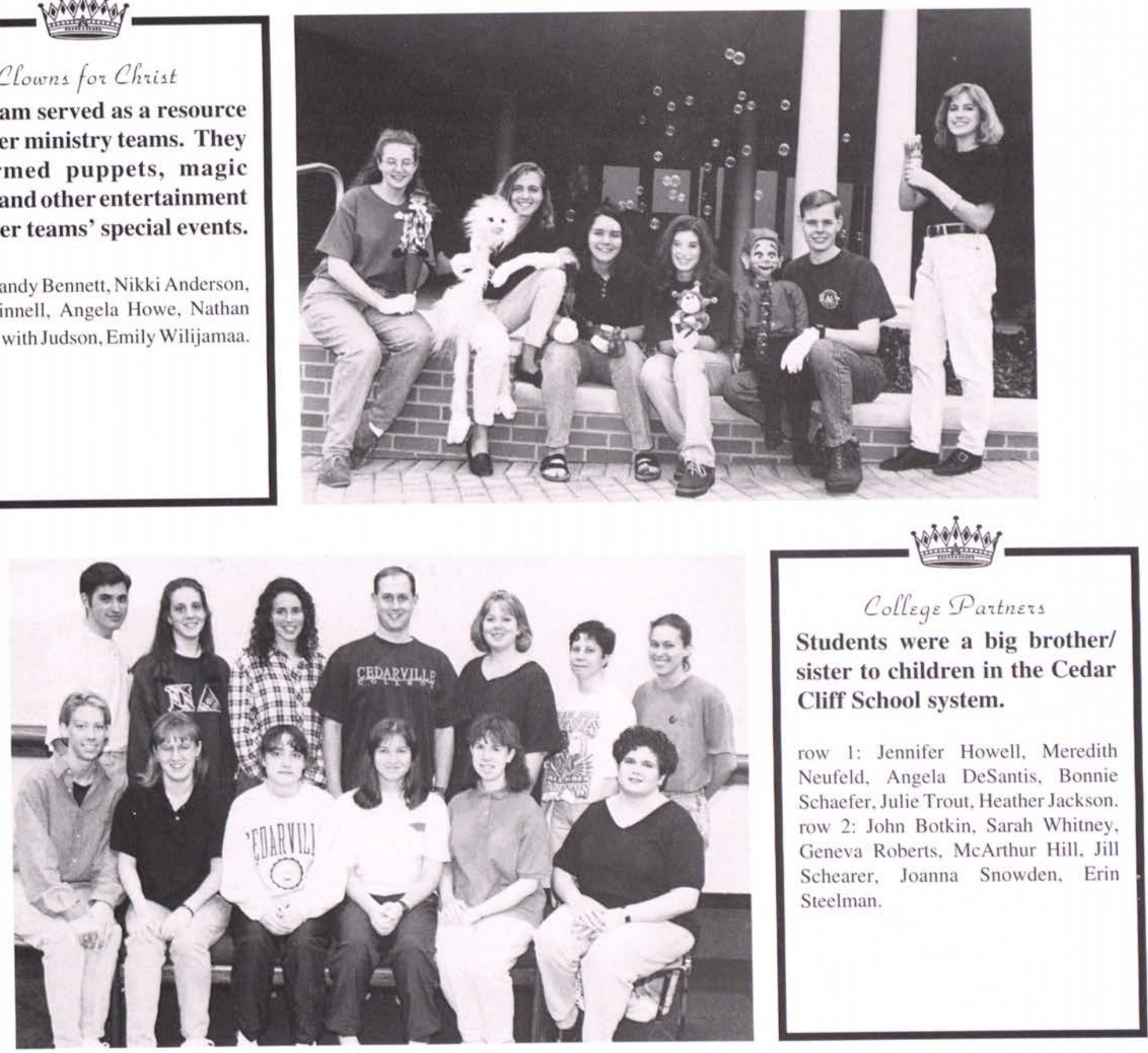


\section{Dayton Detention Home}

Team members led a weekly service and visited individual units in the home.

row 1: Mark Armstrong, Noelle Folkman, Ross Meyers, Greg Johnson. row 2:Hannah Rives, LaVonna Getz, Amanda Bryson, Summer Bennington, Tara Swaney, Melinda Schwartz. row 3: Charity Cole, Jonathan Emery, Jim Johnson, Al Anderson, Jeff Elliott, Rutledge Etheridge, Evelyn Brown, Lewis Pulley, Jeff Motter.
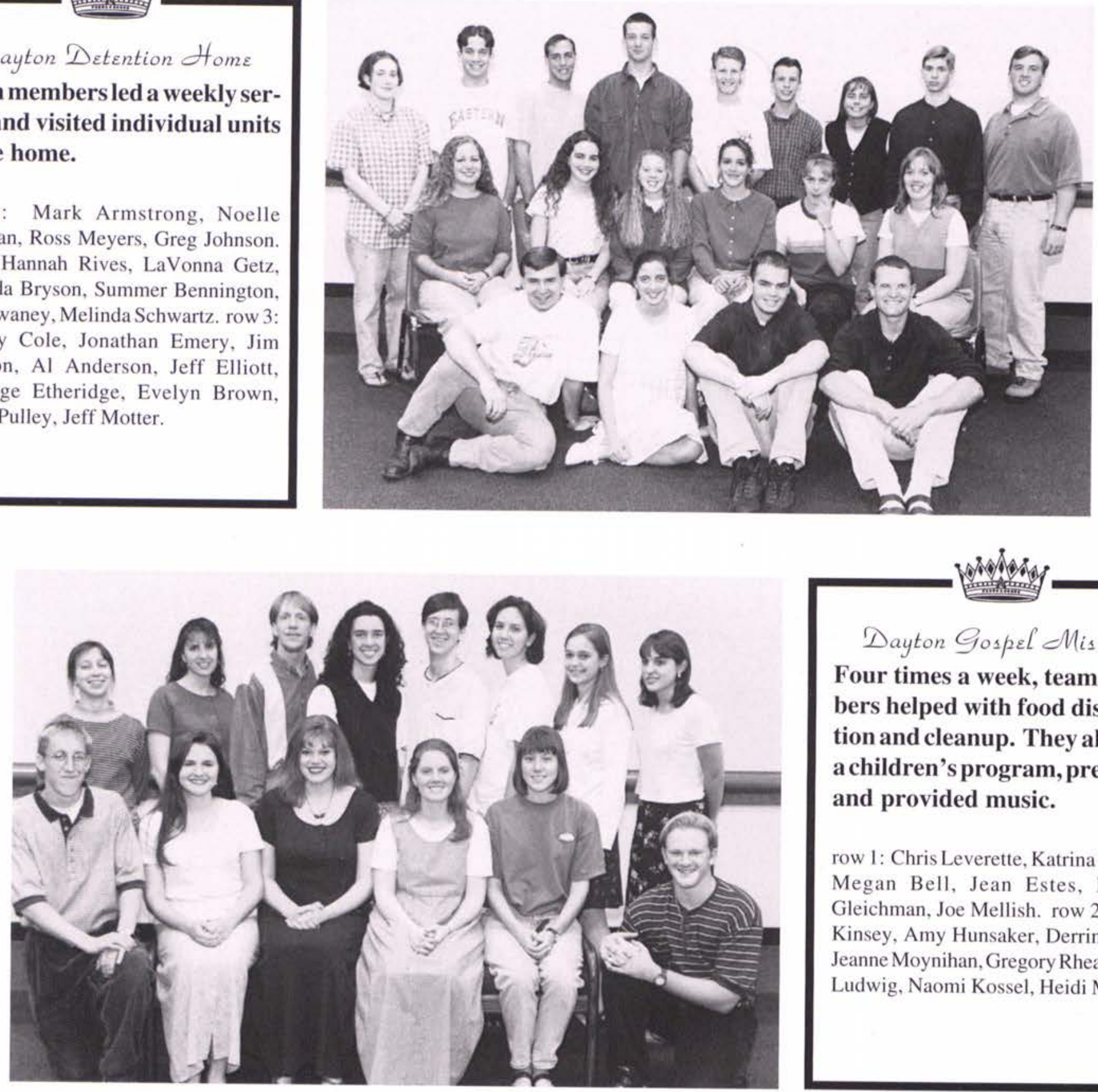

voros

\section{$\longrightarrow$ ining \\ Dayton Gospel Mission}

Four times a week, team members helped with food distribution and cleanup. They also ran a children's program, preached and provided music.

row 1: Chris Leverette, Katrina Thayer, Megan Bell, Jean Estes, Debbie Gleichman, Joe Mellish. row 2: Heidi Kinsey, Amy Hunsaker, Derrin Smith, Jeanne Moynihan, Gregory Rheam, Sara Ludwig, Naomi Kossel, Heidi Mountz.

Students worked with adults who had physical/developmental needs.

row 1: Joel Rose, David Duhaime, Will Sutton. row 2: Rebecca Thangan, Jana Wright, Kristin Jacoby, Sara Clayton, Julianne Kerns. not pictured: Jeremy Hurley, Becky Story.

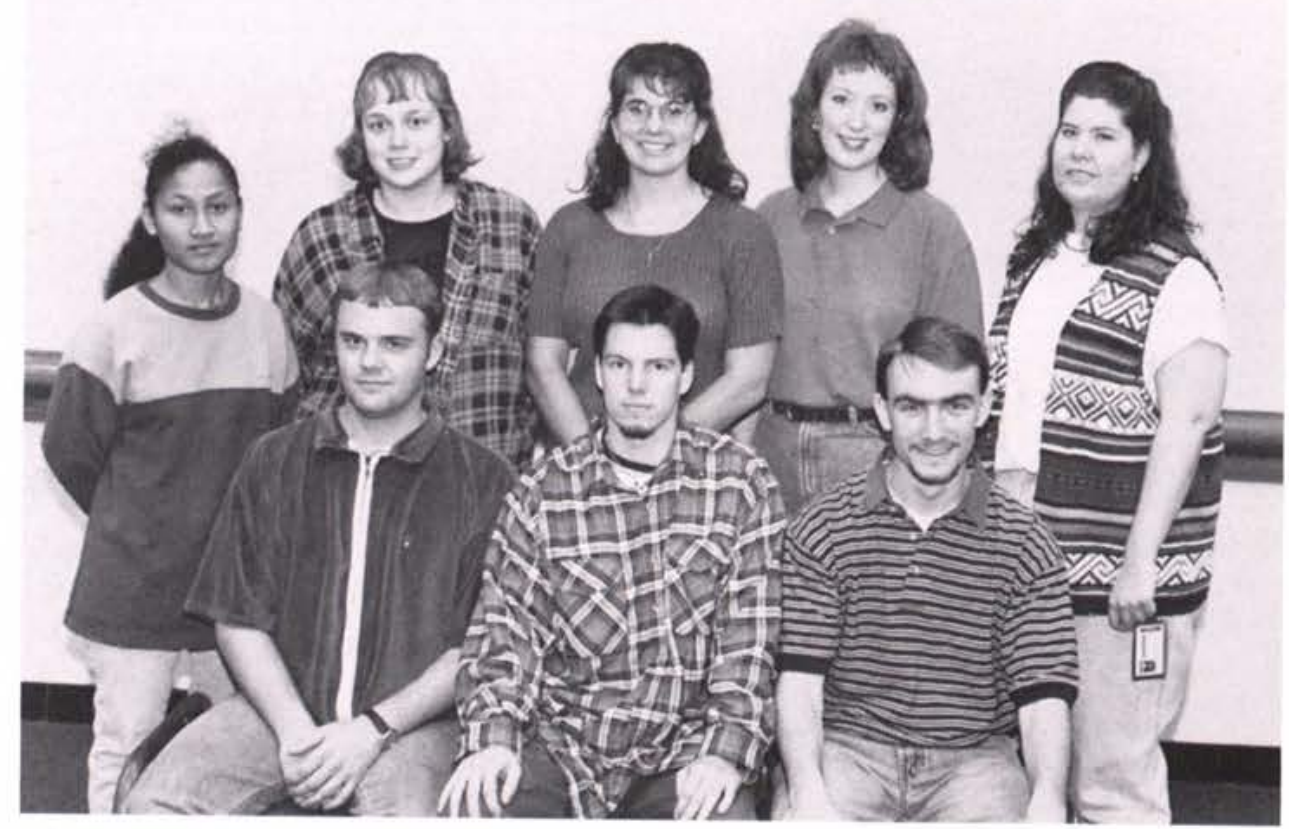



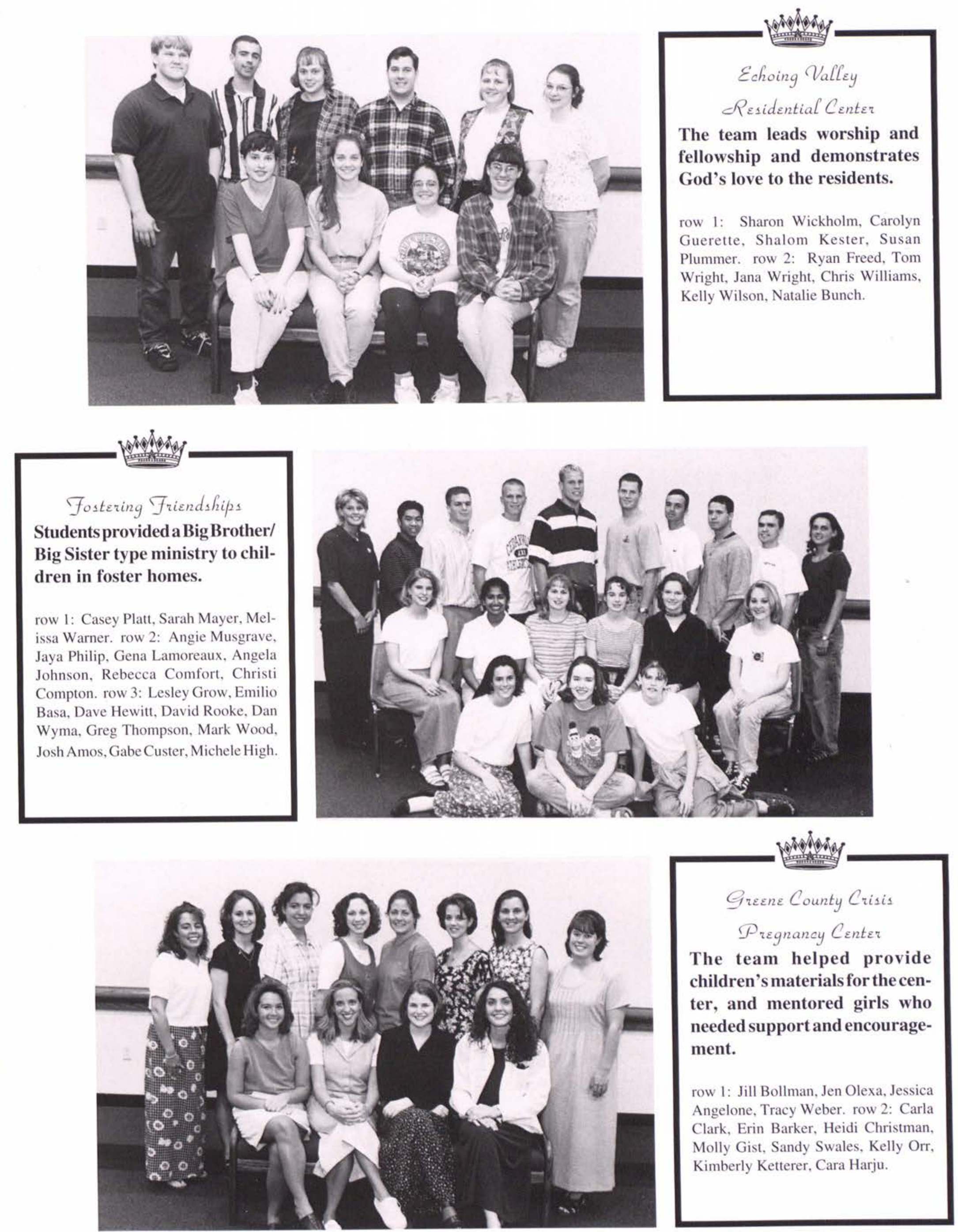


\section{Grenn County Jail}

This was a one-on-one ministry with the inmates.

row 1: Jared Wick, Tamara Shrader, Kelsey Browning, Melissa Wabeke, John McCaw. row 2: Ken Howard, David Hofert, David Cook, Michael Wenger, Jason Alexander, Brad Neese.
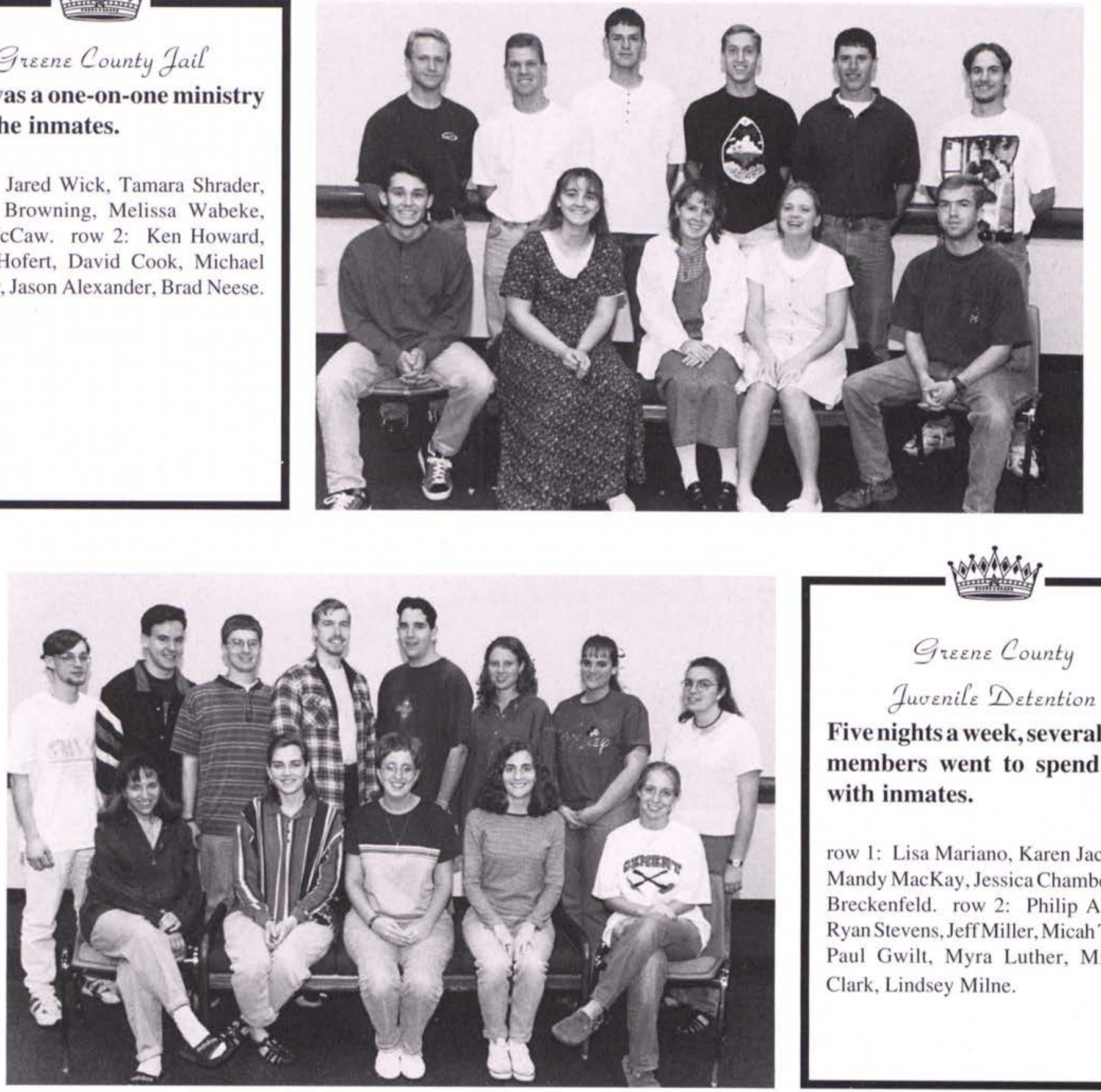

Five nights a week, several team members went to spend time with inmates.

row 1: Lisa Mariano, Karen Jacobsen, Mandy MacKay, Jessica Chambers, Jill Breckenfeld. row 2: Philip Ausfahl, Ryan Stevens, Jeff Miller, Micah Thirey, Paul Gwilt, Myra Luther, Michelle Clark, Lindsey Milne.

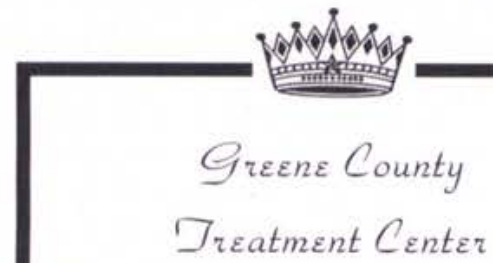

Students encouraged and built friendships with young men/ women at a rehabilitation center.

row 1: Brooke Frazier, Haylee Biggs, Holly Peterson, Gina Doerr. row 2: Jonathan Rauch, Jonathan Witmer, Jonathan Williams. not pictured: Jonathan Wu, Scott Christner, Steve Gerber, Jeff Davis.

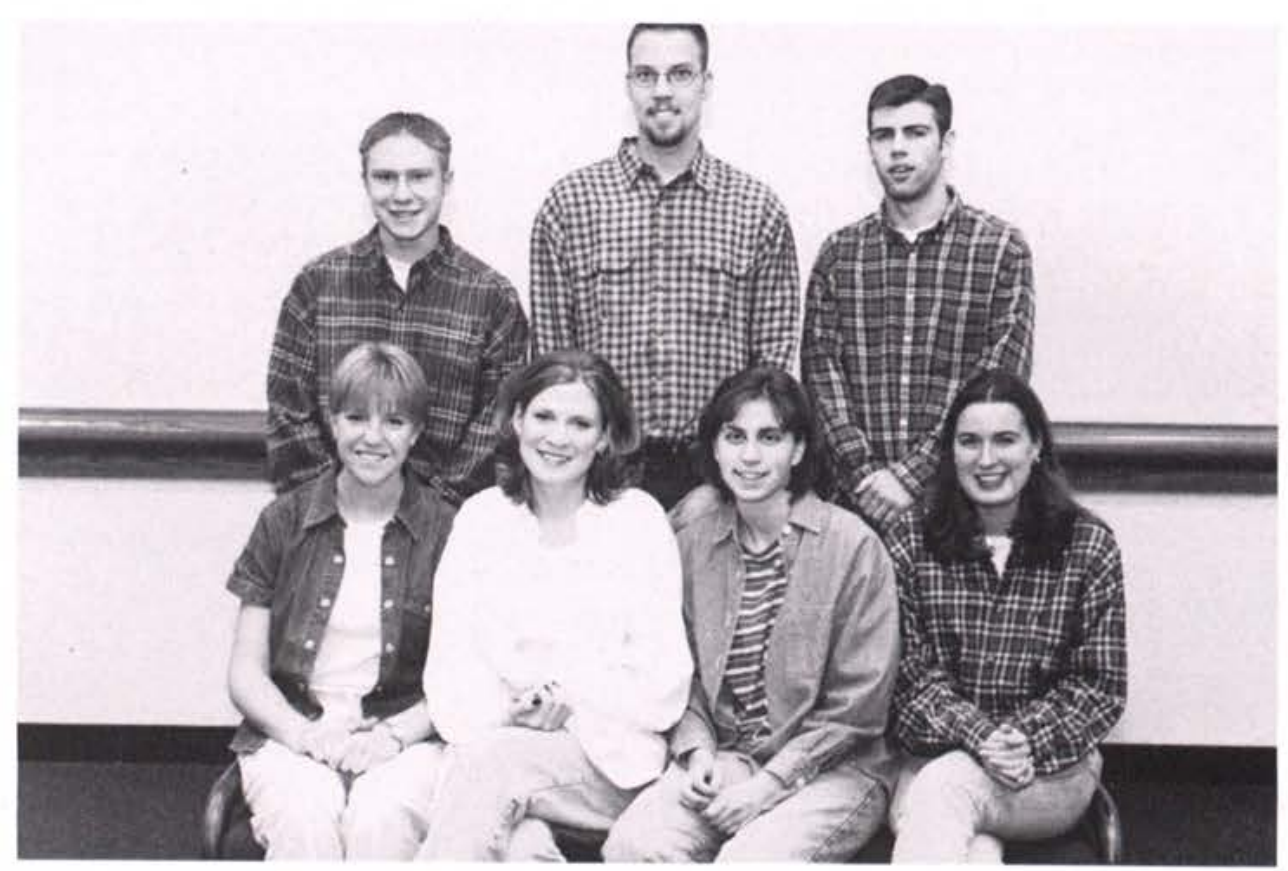



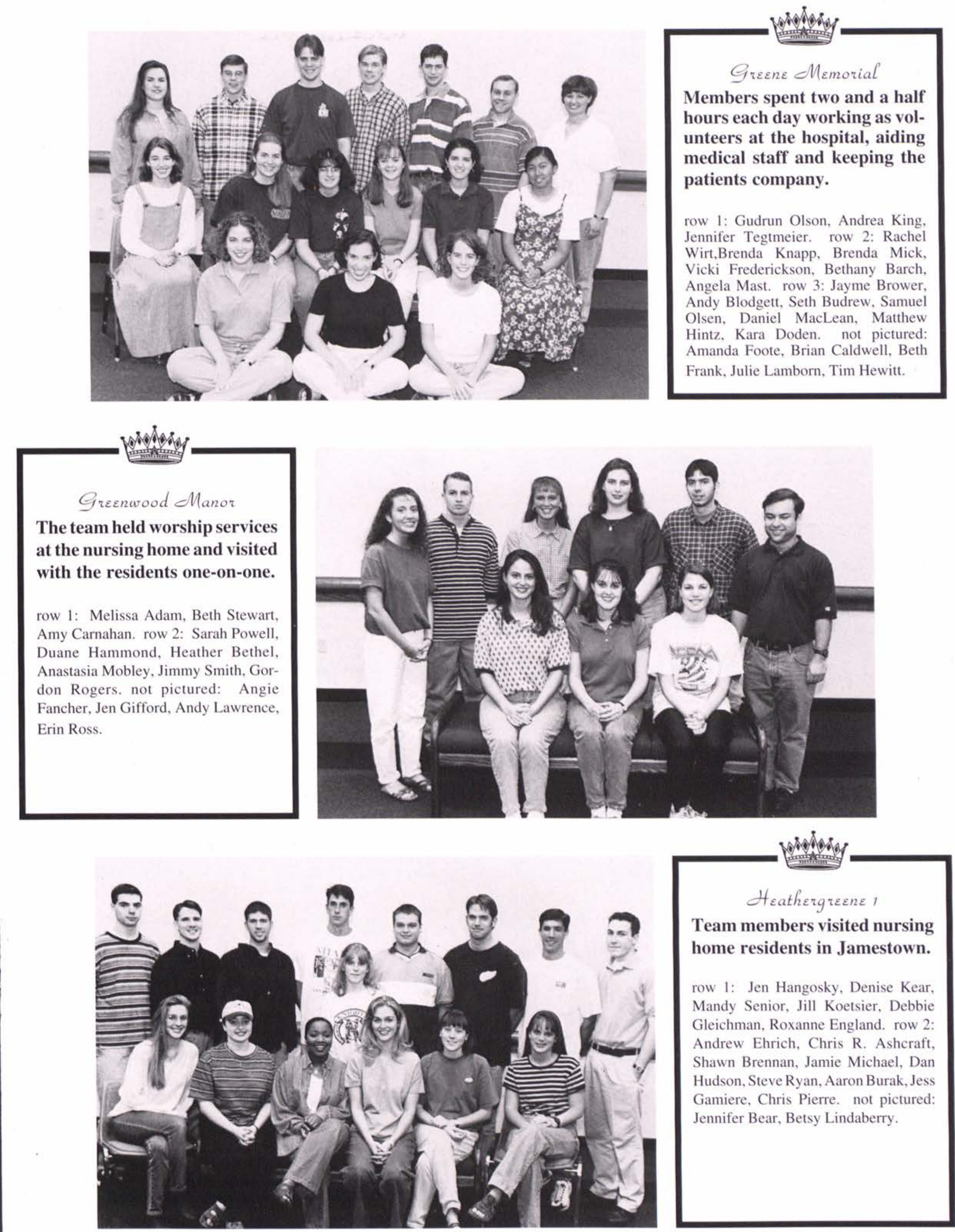

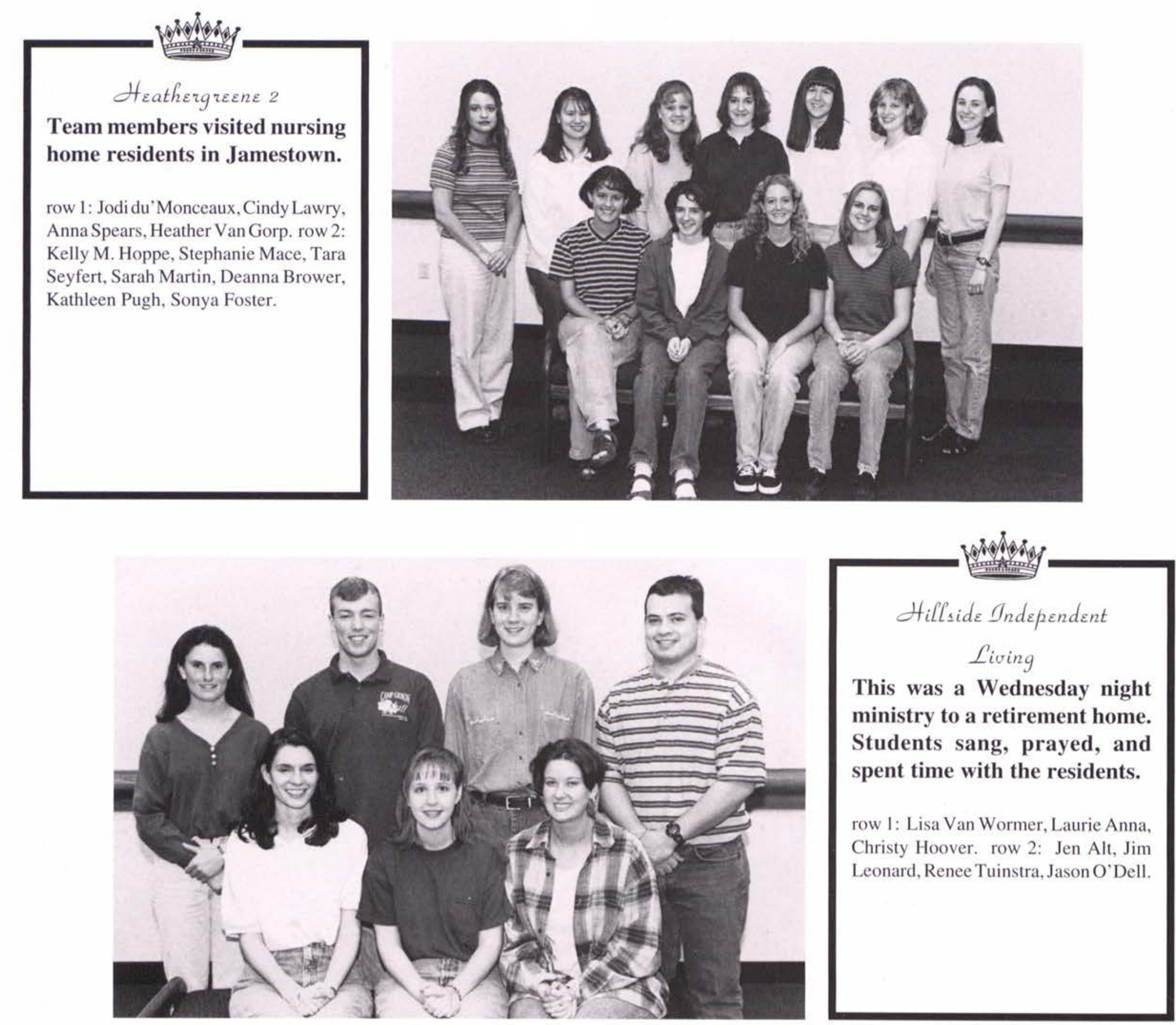

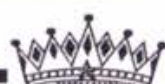

\section{International Friends}

Team members led a Sunday School type program for international students at $\mathrm{OSU}$ in Columbus.

row 1: Jamie Sell, Carrie Mansfield, Heather MacLean, Monique Gras. row 2: Rebecca Tharngan, Angie Tyson, Sonwoo Lee, Edwin Bett, Ben Nordaas, Kouichi Ikeda, Kari Lynn Yoder.

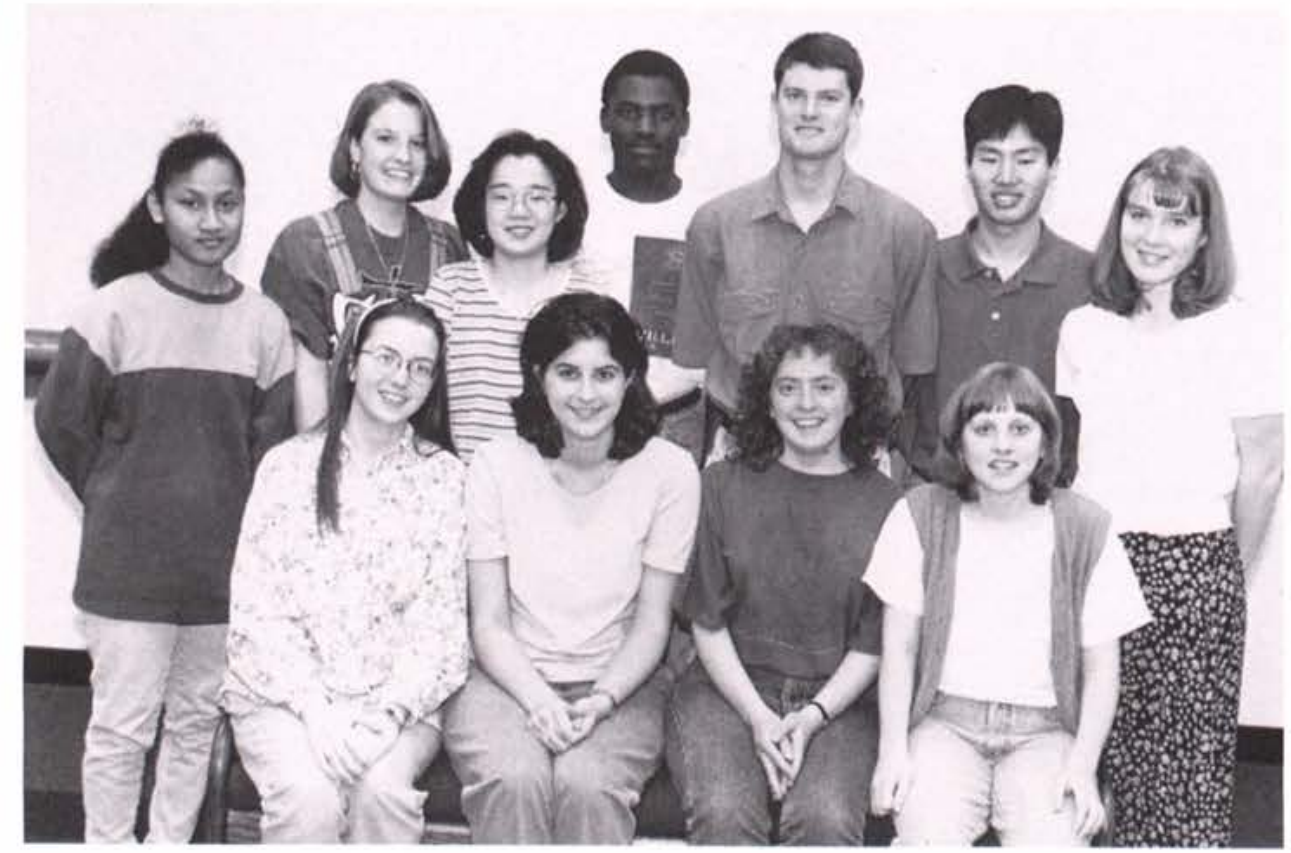




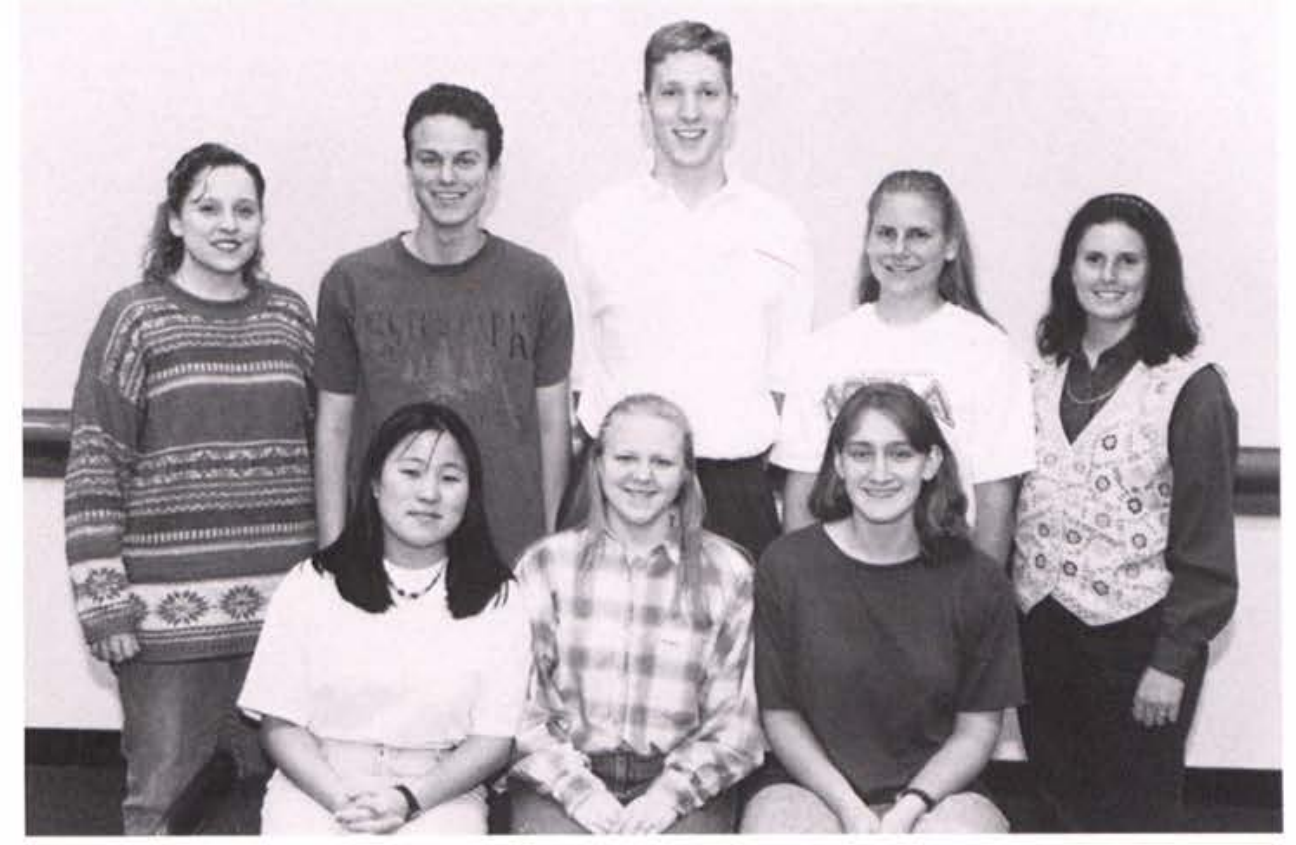

9007

Team members visited with the elderly one-on-one.

row 1: Atsuko Ohtake, Michelle Higgins, Suzanne Lehman. row 2: Rachel Ross, Jason Cirone, J. Michael Perkins, Kelly Reitz, Emily Alt.

vingery

Knights of Pythias

This team conducted Sunday and Wednesday services for residents of a nursing home.

row 1: Michelle M. Smith, Aimee Copeland, Bethany Nichols, Tina Coon, Allison Justice, Melissa Kaufman, row 2: Philip Boggs, Paul Click, Nathan Houk, Kevin Boblitt, Brent Ferguson, Sara Laning, Tiffany Peterson, Elizabeth Smith. not pictured: Keith Hodel, Robert Cobb, Jenifer Gardner.
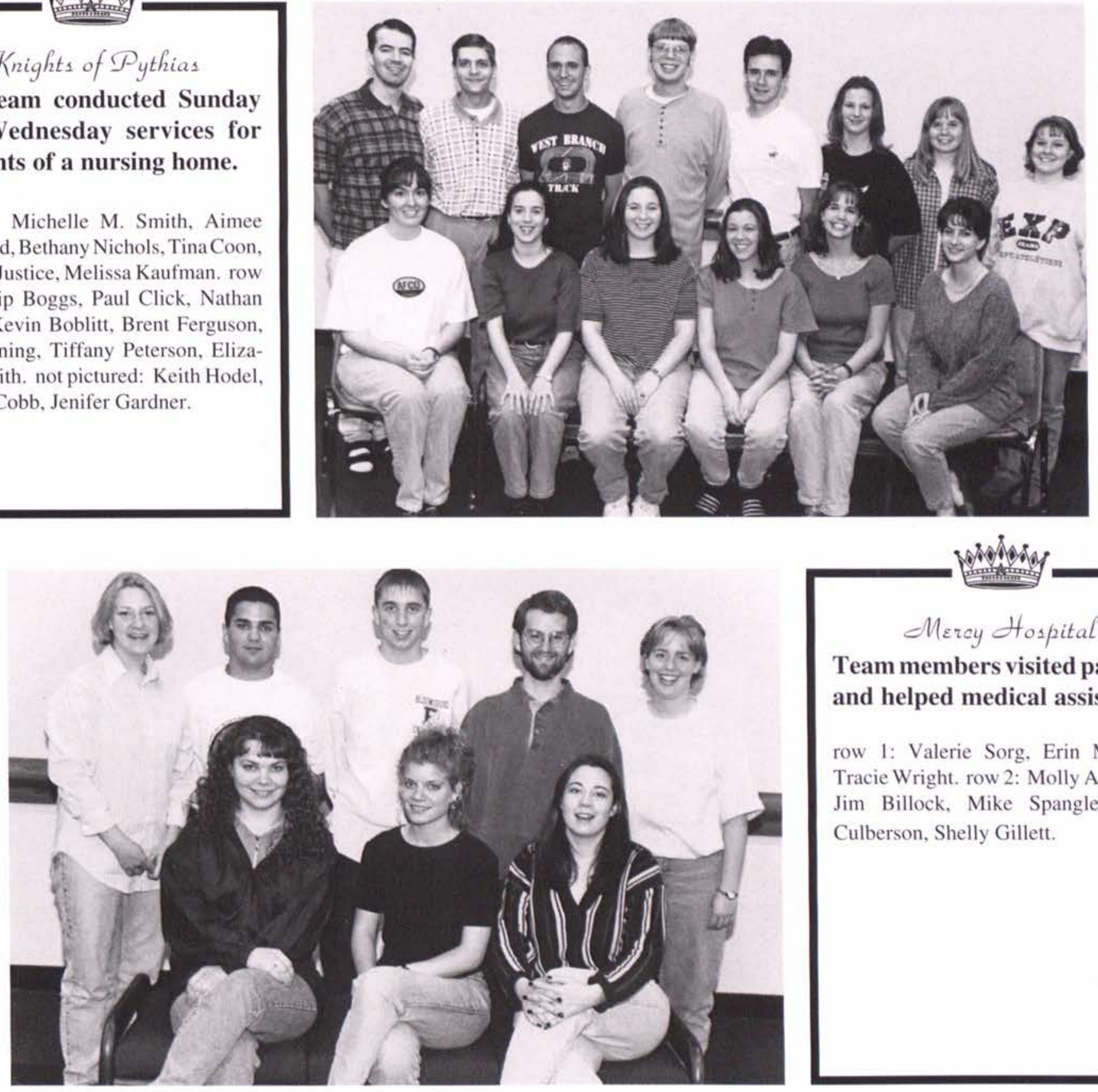

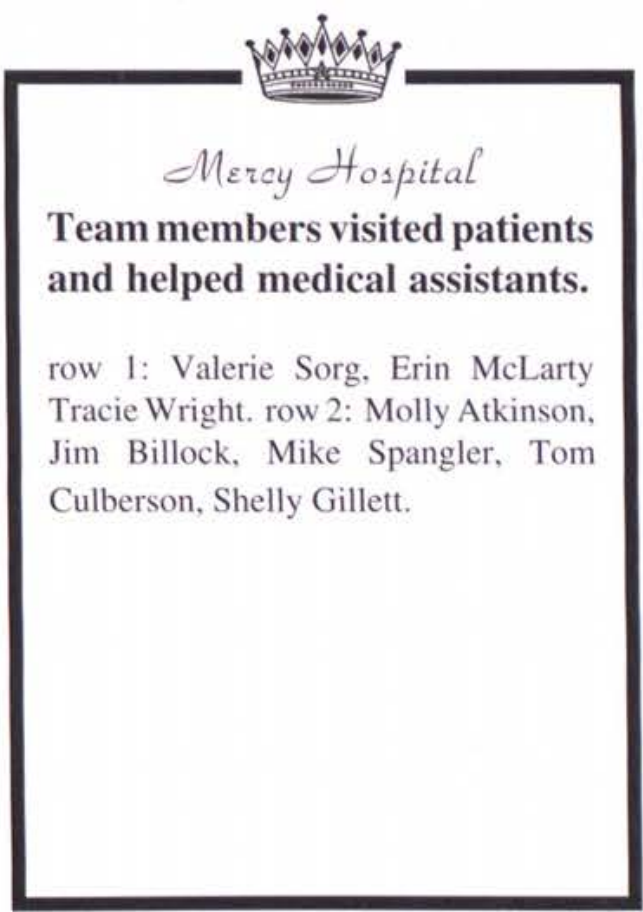



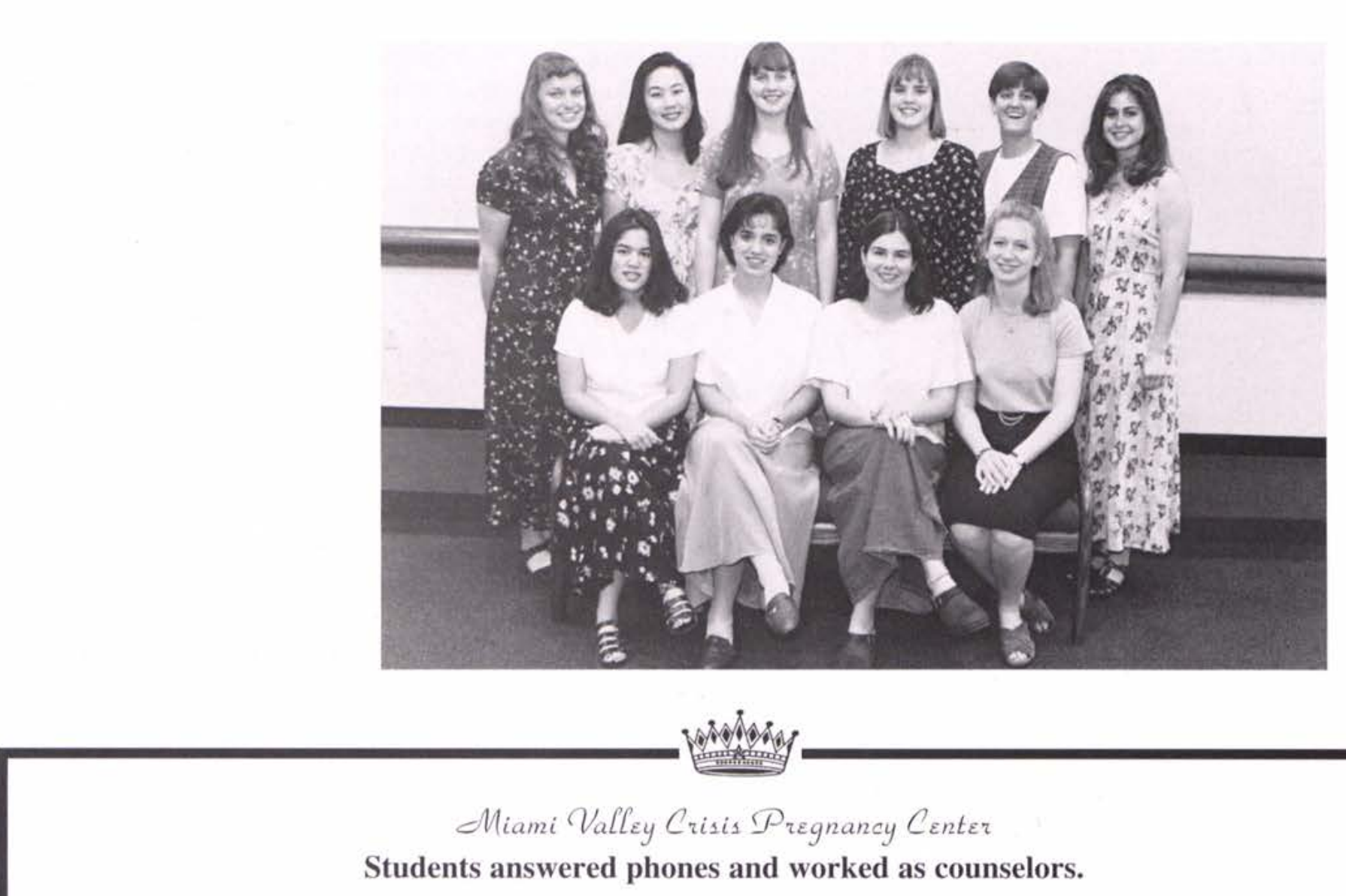

row 1: Keiko Noble, Laura Brown, Amy Mead, Abigail Howe. row 2: Karri Heldreth, Kathy Sohn, ElizabethJohnson, Sarah Gilchrist, Jessica Stuenzi, Rebecca Leatherman.

\section{0. \\ $\mathrm{O}_{\mathrm{p} z \mathrm{n}}$ Heirs}

A street evangelism ministry to Columbus, Cincinnatti, and Dayton.

row 1: Kim Kleiman, Jaya Philip, Elizabeth Clark, Ernest Clark, Jr., Joshua Ausfahl, Nate Childers, Barry Gorsuch, Erin Johns, Dan Hicks. row 2: Jessica MacPherson, Megan Bell, Amy Stowers, Josh Halulko, Lori Hamilton, Amy Nichols, Jean Estes, Steve Kline, Brian Rosseau, Ross Meyers, row 3: Lisa MacPherson, Christina Riley, David Boyd, Tim Johnson, Heidi Kinsey, Heather Maybury, Michelle M. Smith, Erin Stuenzi, Andrew Alderfer, Darrin Smith. row 4: Catherine Walcott, Steve Lamp, Richard Porter, Matt Rose, Rob Kocher, Kyle Kickbusch, Berney Gorsuch, Ryan Coverdell, Jon Schultz, Jennifer Abas, Dan Johansen, Brett Shilton.

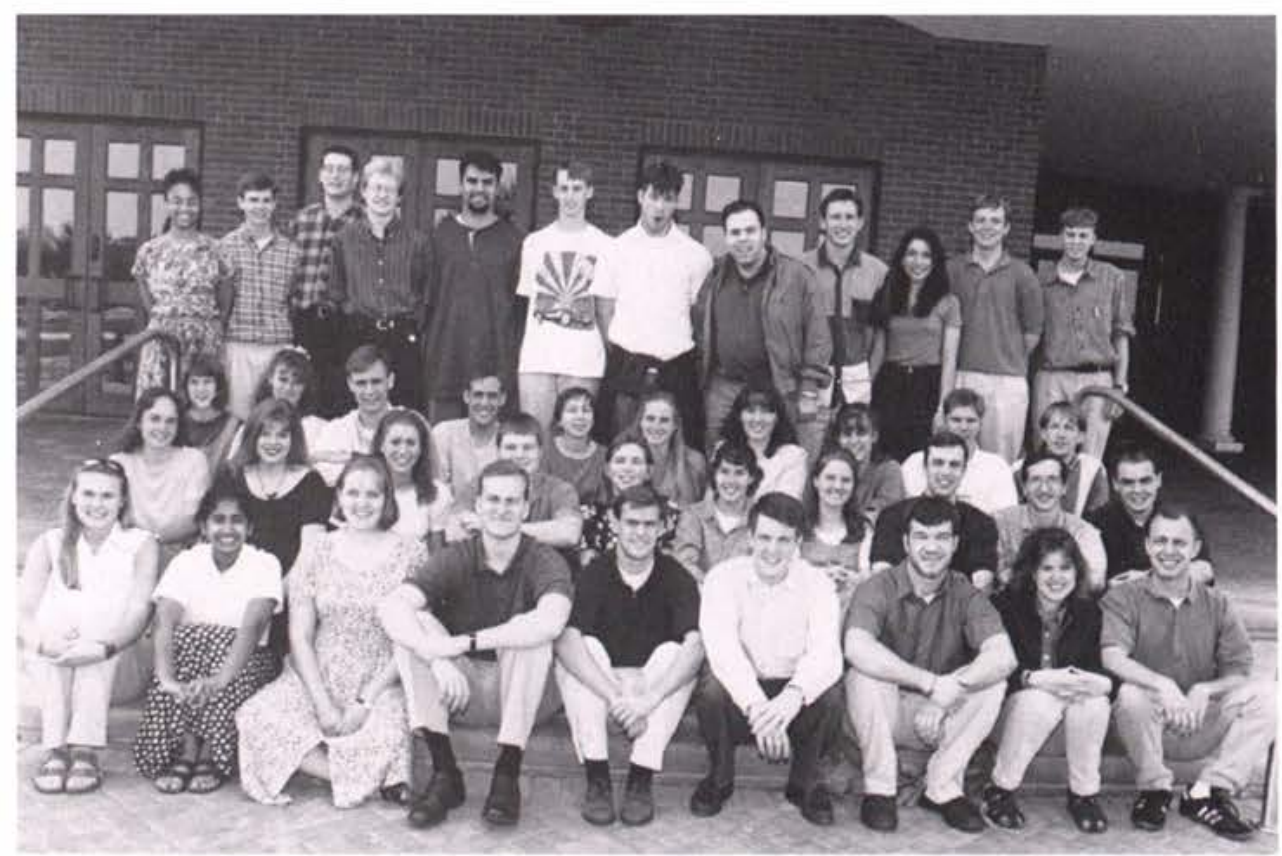




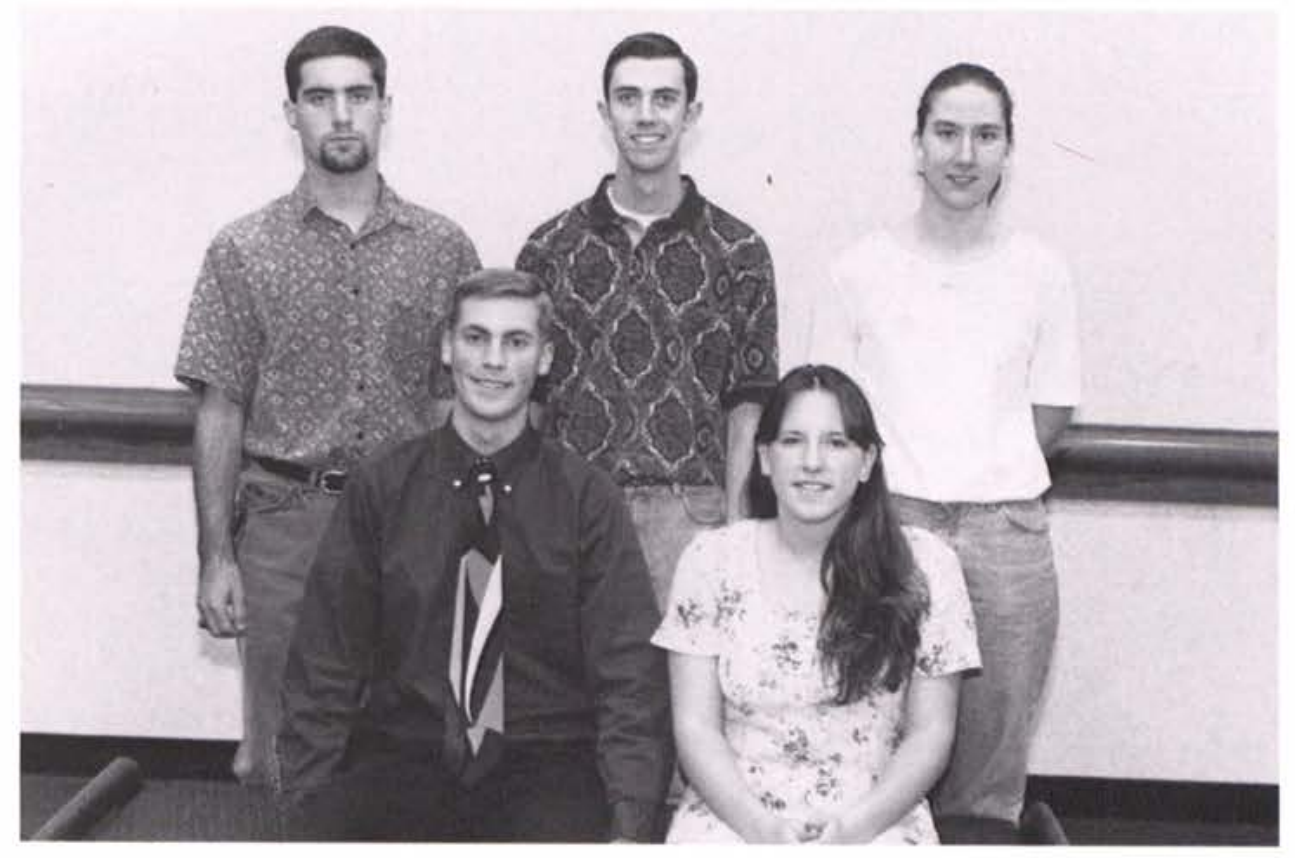

ratosy

\section{The Other Place}

The team went to a homeless shelter in Dayton to meet the physical and spiritual needs of the homeless and the people who run the shelter.

row 1: Brent Hughs, Michelle Edwards, row 2: Kevin Modin, Joe Lloyd, Joan Mallman.

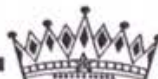

$$
\text { Ronald McDonald House }
$$

RMH is a place for parents of hopitalized children to spend the night. The team members answered phones, cleaned, checked people in and out, and provided a listening ear to people who needed to talk.

row 1: Tiffiny Cope, Tamara Shrader, Christine Bjornstad, Ann Kobiela, Christy Cleaver, Kim Kleiman. row 2: Kim Leverson, Meredith Gross, Sarah Glupker, Shannon Snow, Stephanie Buchholtz, Brian Thomson, Holly Waechter.

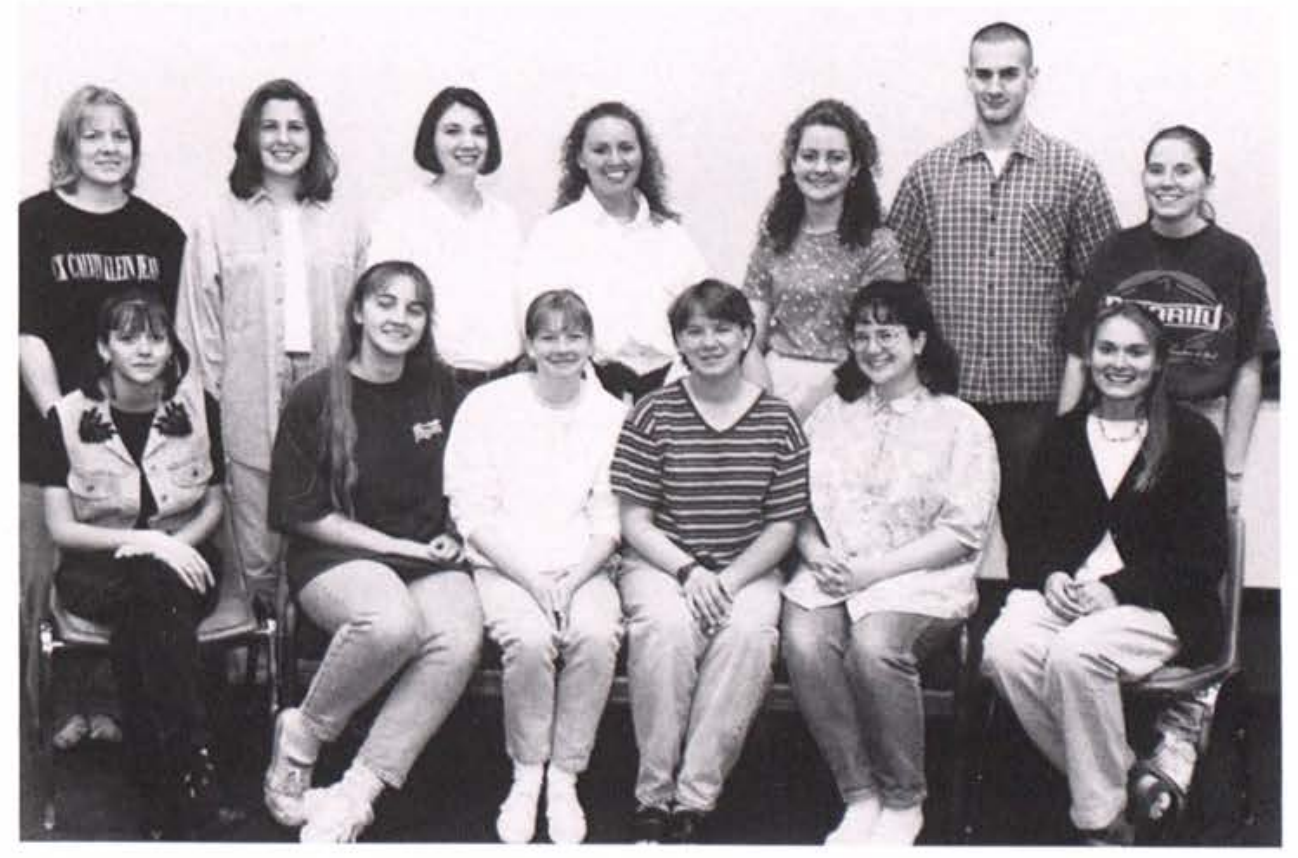



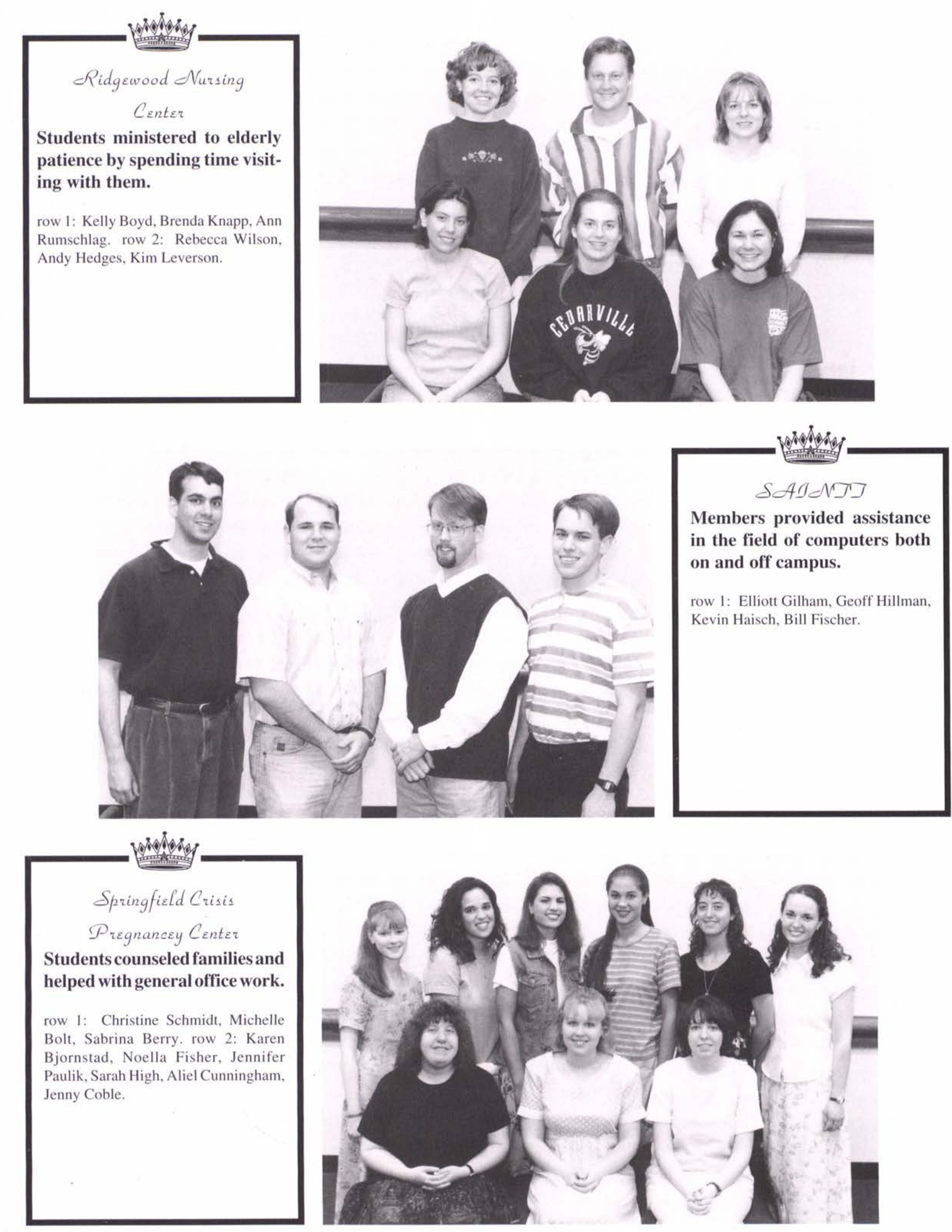


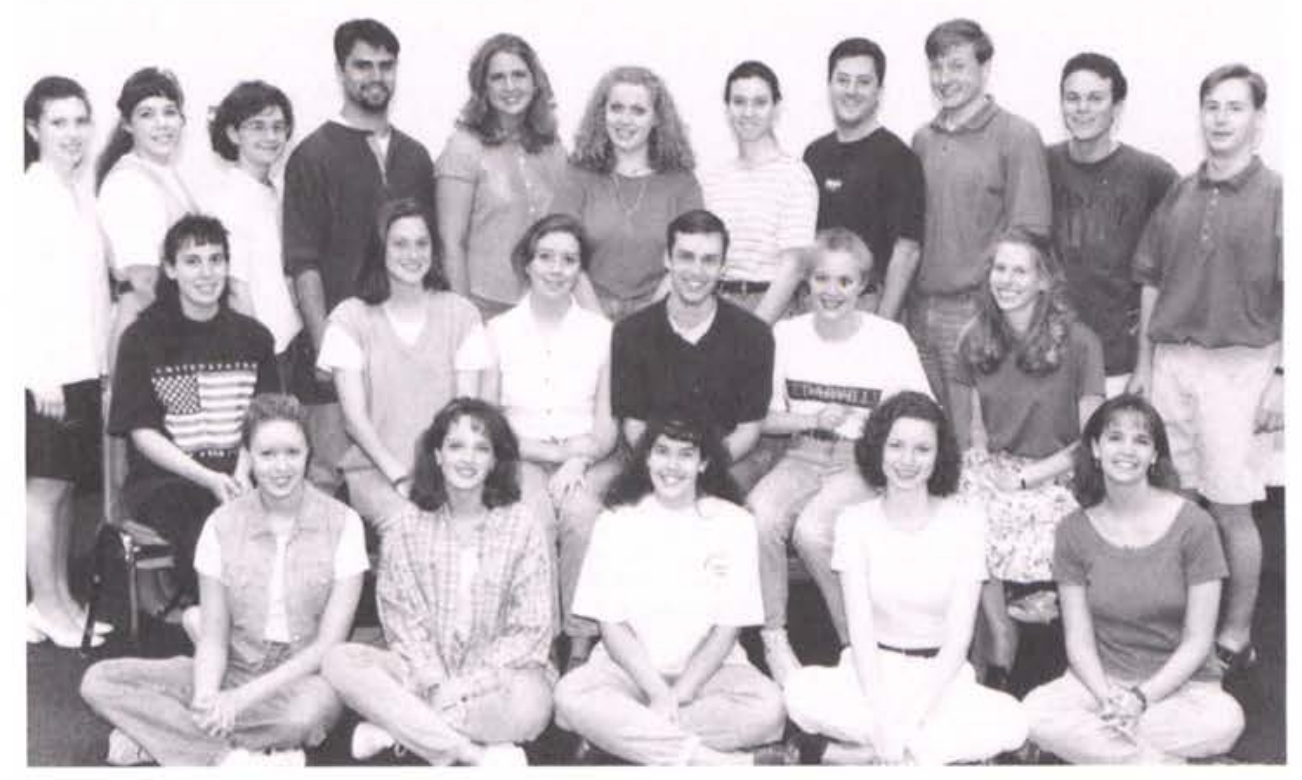

Springfield Dutoring

Members participated in innercity tutoring and mentoring programs at Hayward Middle School and Springfield South High School.

row 1: Bethany DeGraw, Kim McKenzie, Amy Nichols, Rachel J. Stewart, Allison Justice. row 2: Tami See, Andrea Osterc, Tammy Stark, Stephen Kline, Tiffany Hamilton, Christina Baley, row 3: Connie Schwartz, Summer Schafer, Emily Weber, Rob Kocher, Sallisha Cowell, Heather Smith, Erica Jenkins, Denis Jordan, Tim Flowers, Jason Cirone, Aaron Ponzani.

Rebecca Ritzel, Tiffany Burgett, Philip Lehman, Michele High, Jen Kerr. not pictrued: Sarah Holesovsky.

Twice a week, students helped children and disabled people ride horses.

\section{Yellow Springs Ciding Center}

$$
\text { . }
$$

.
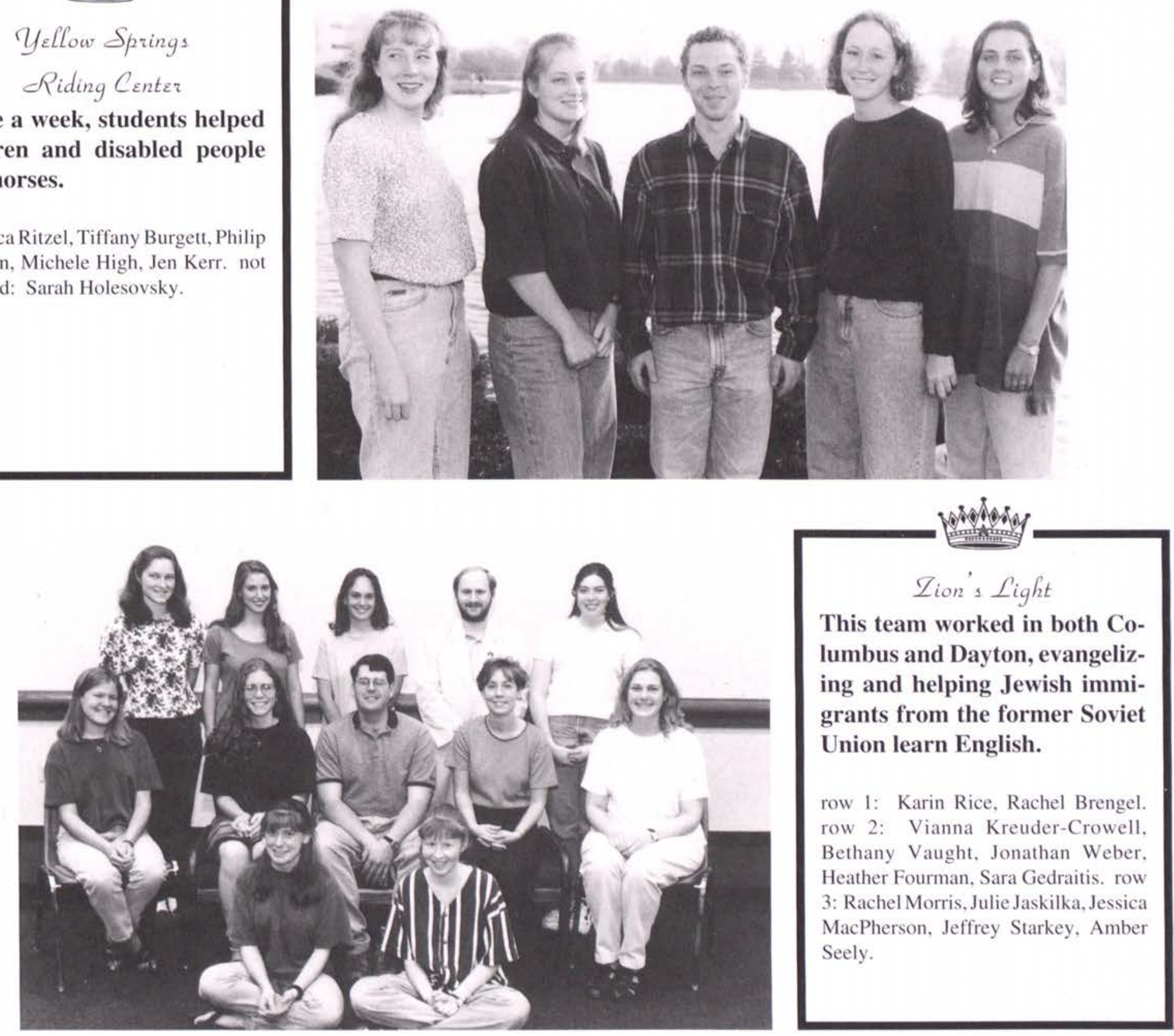

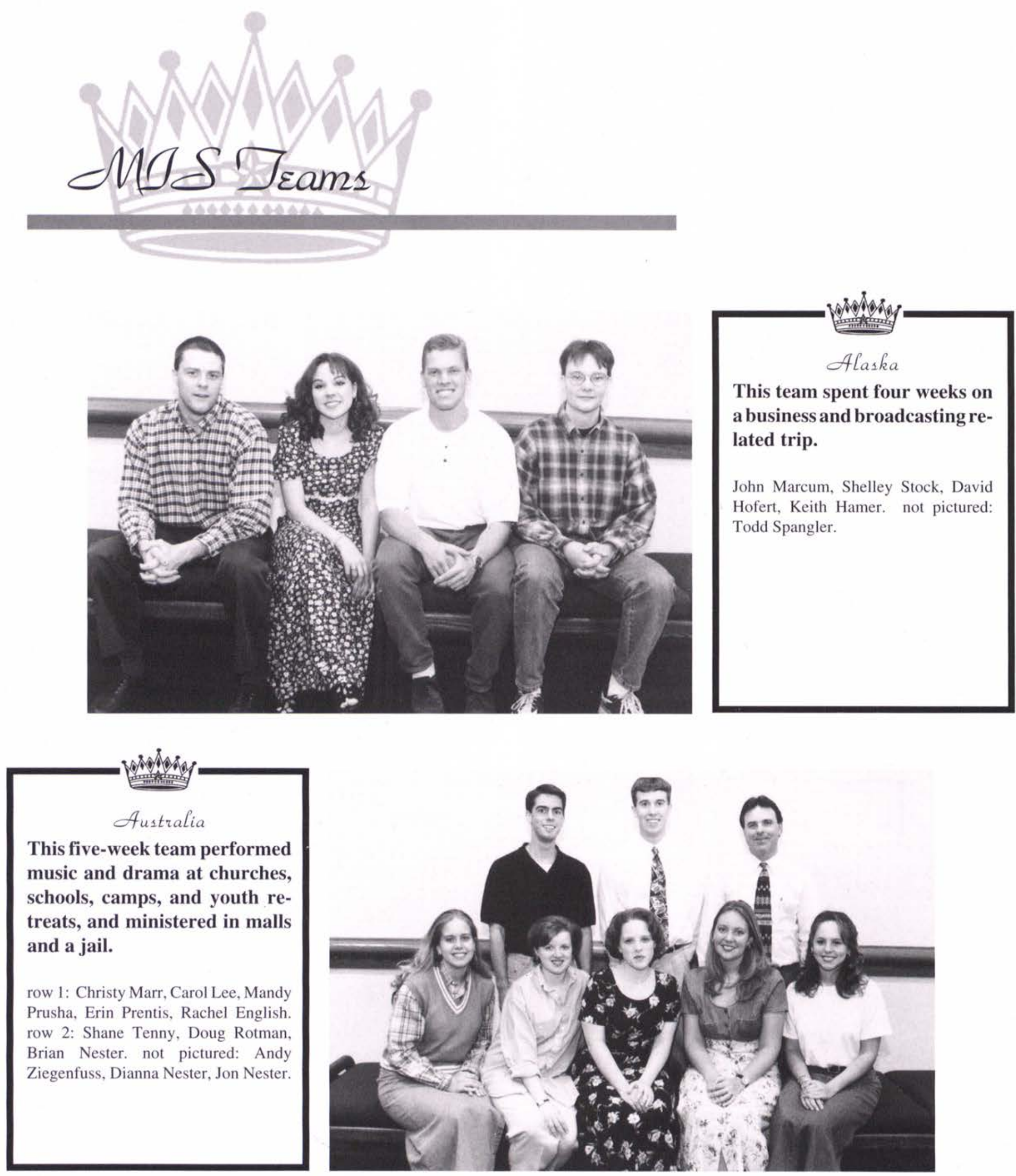

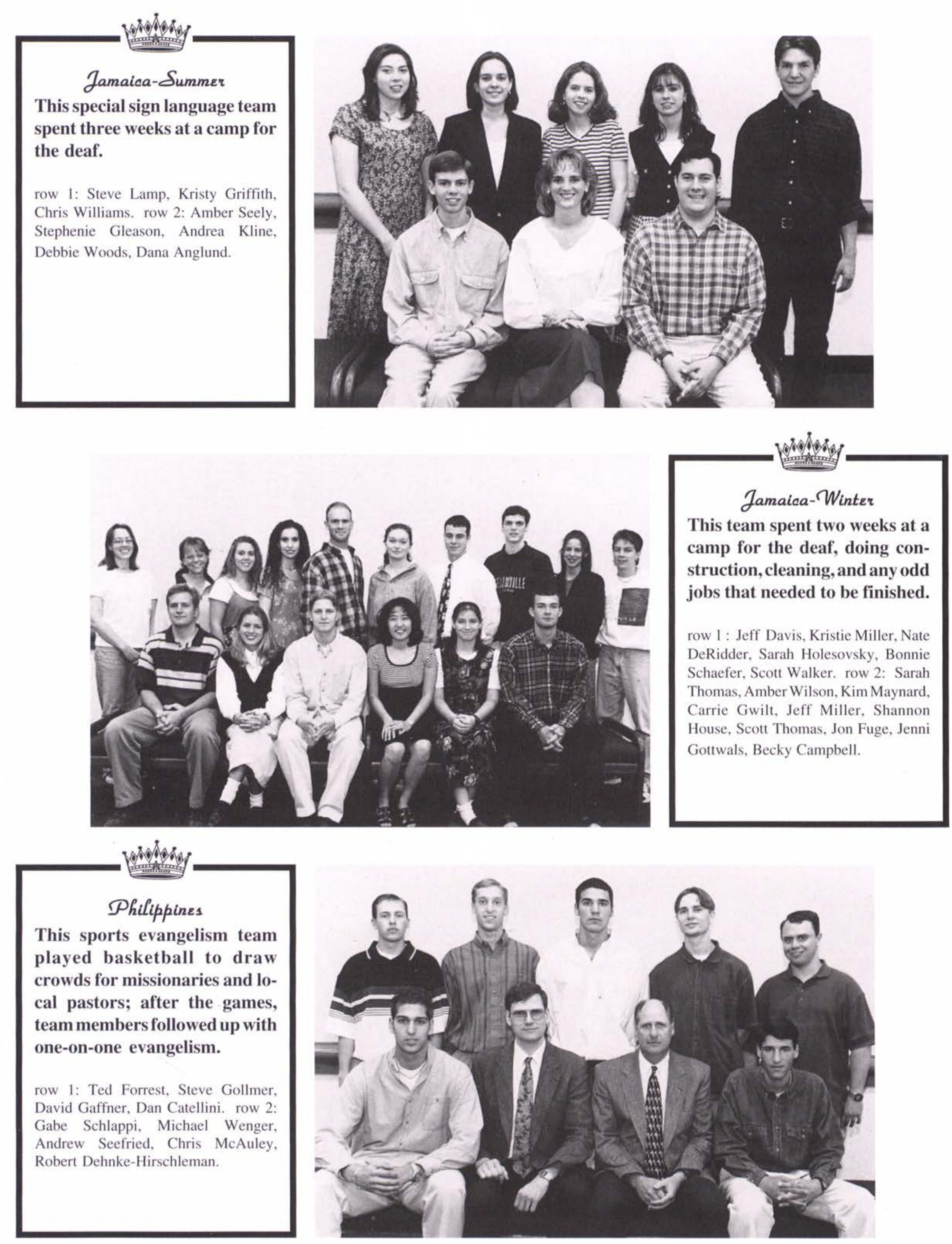

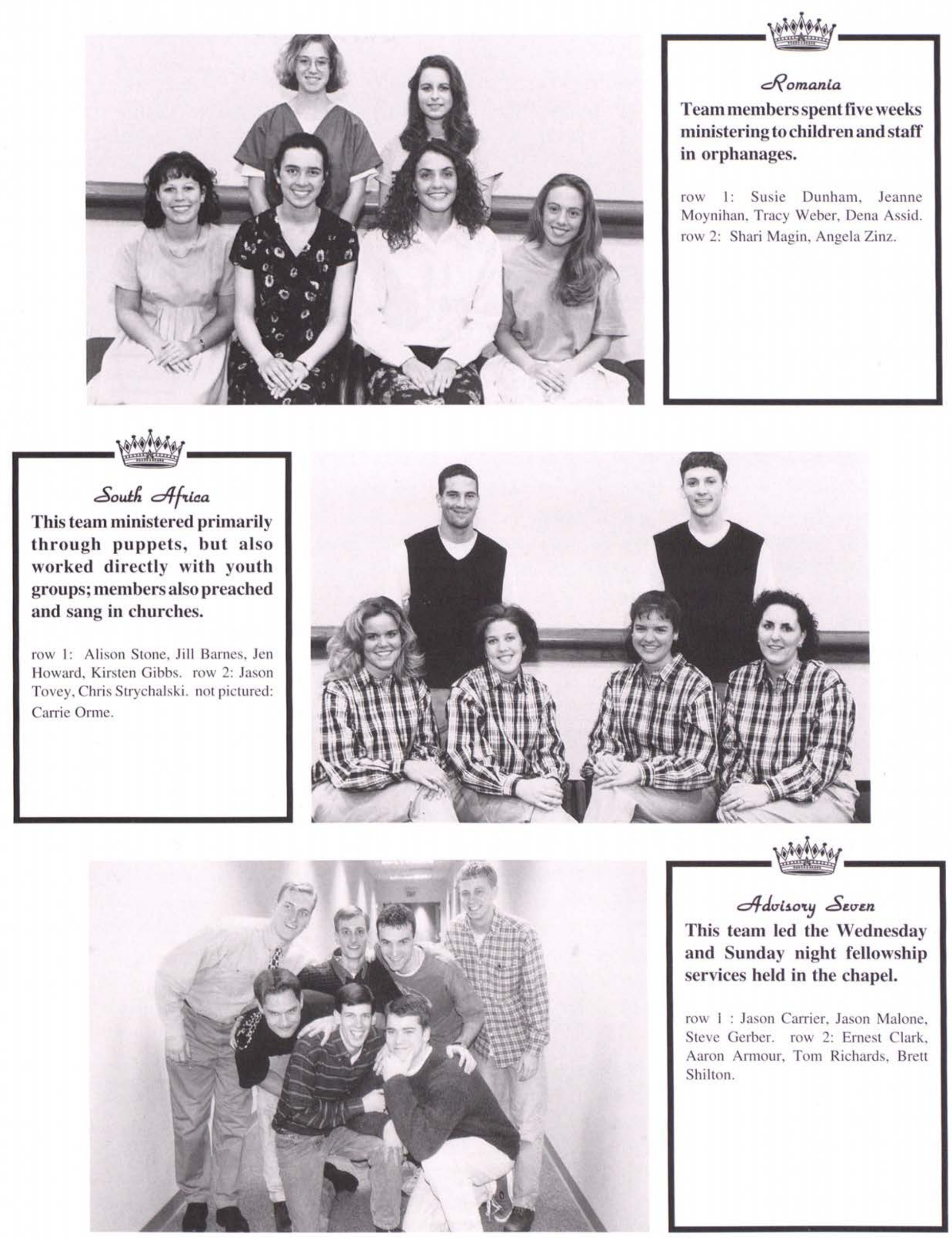

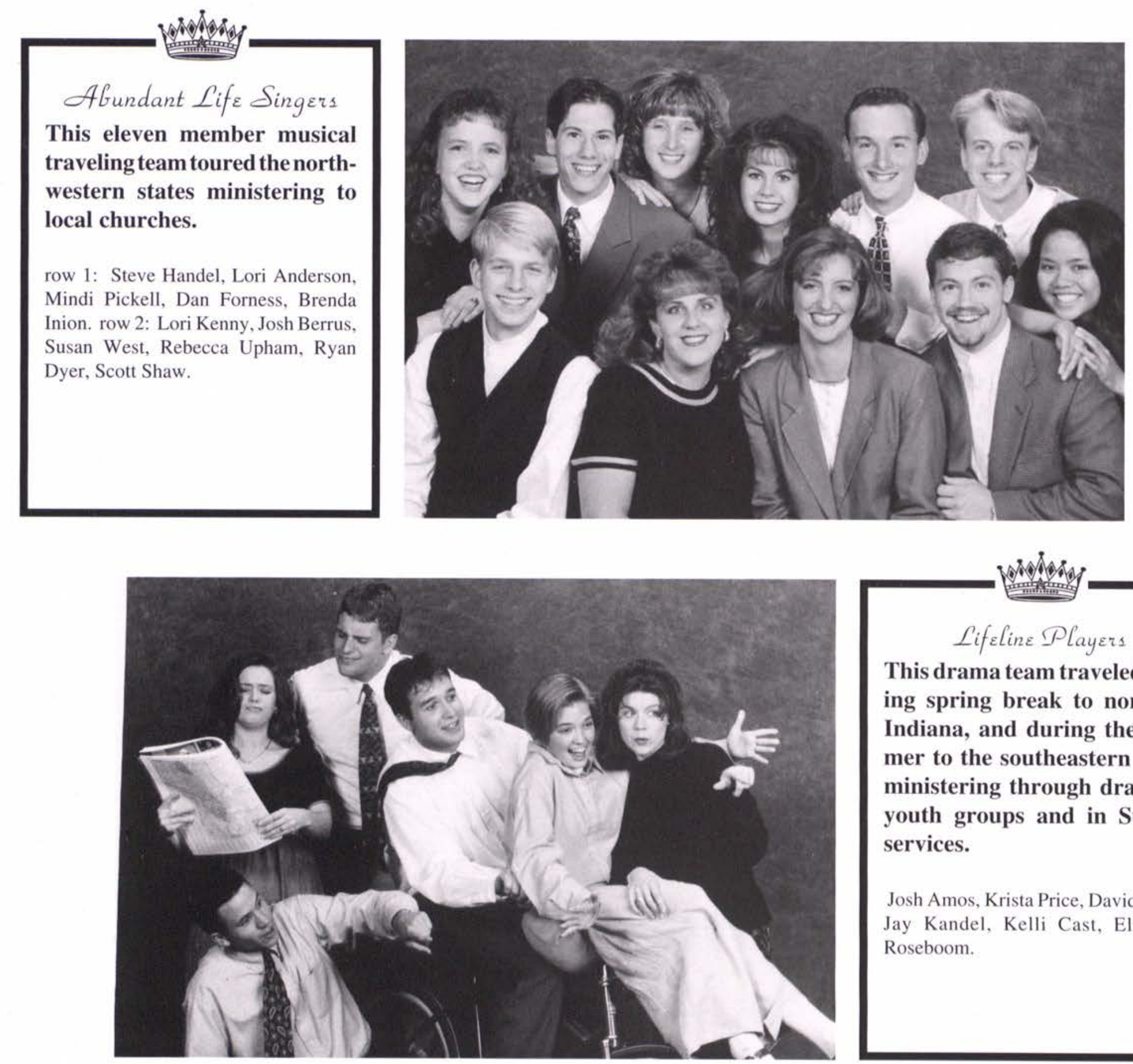

This drama team traveled during spring break to northern Indiana, and during the summer to the southeastern states ministering through drama to youth groups and in Sunday services.

Josh Amos, Krista Price, David Burke, Jay Kandel, Kelli Cast, Elizabeth Roseboom.
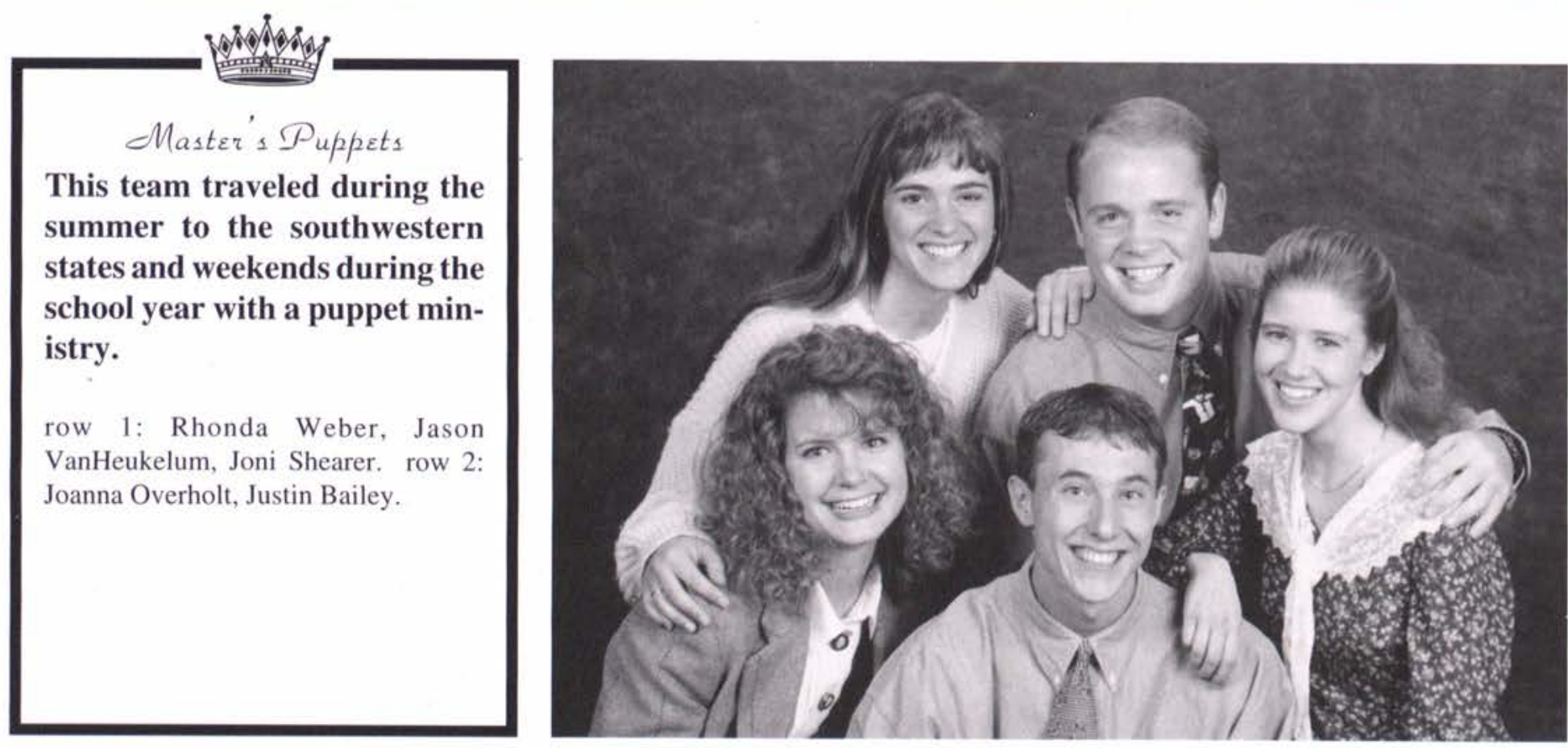

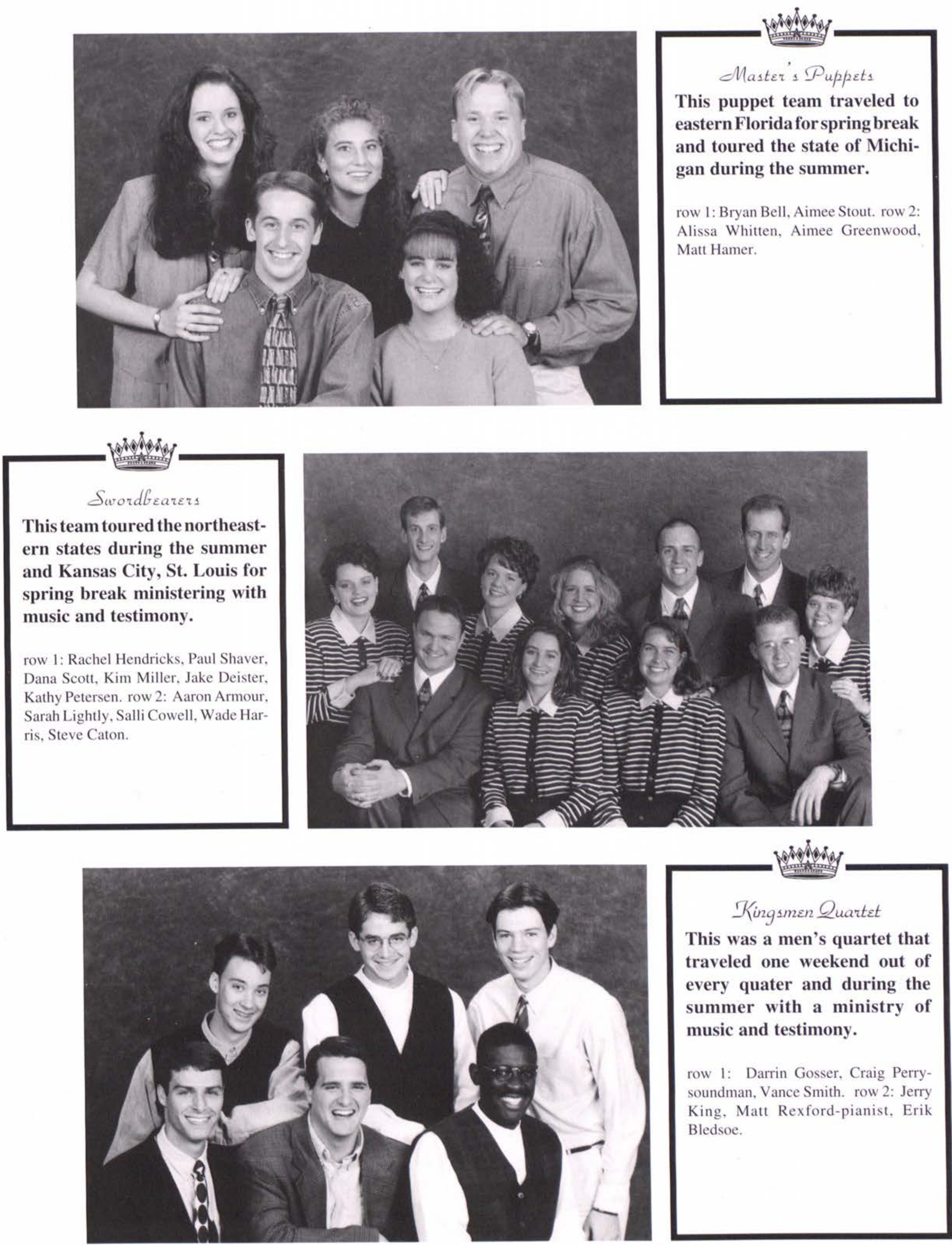

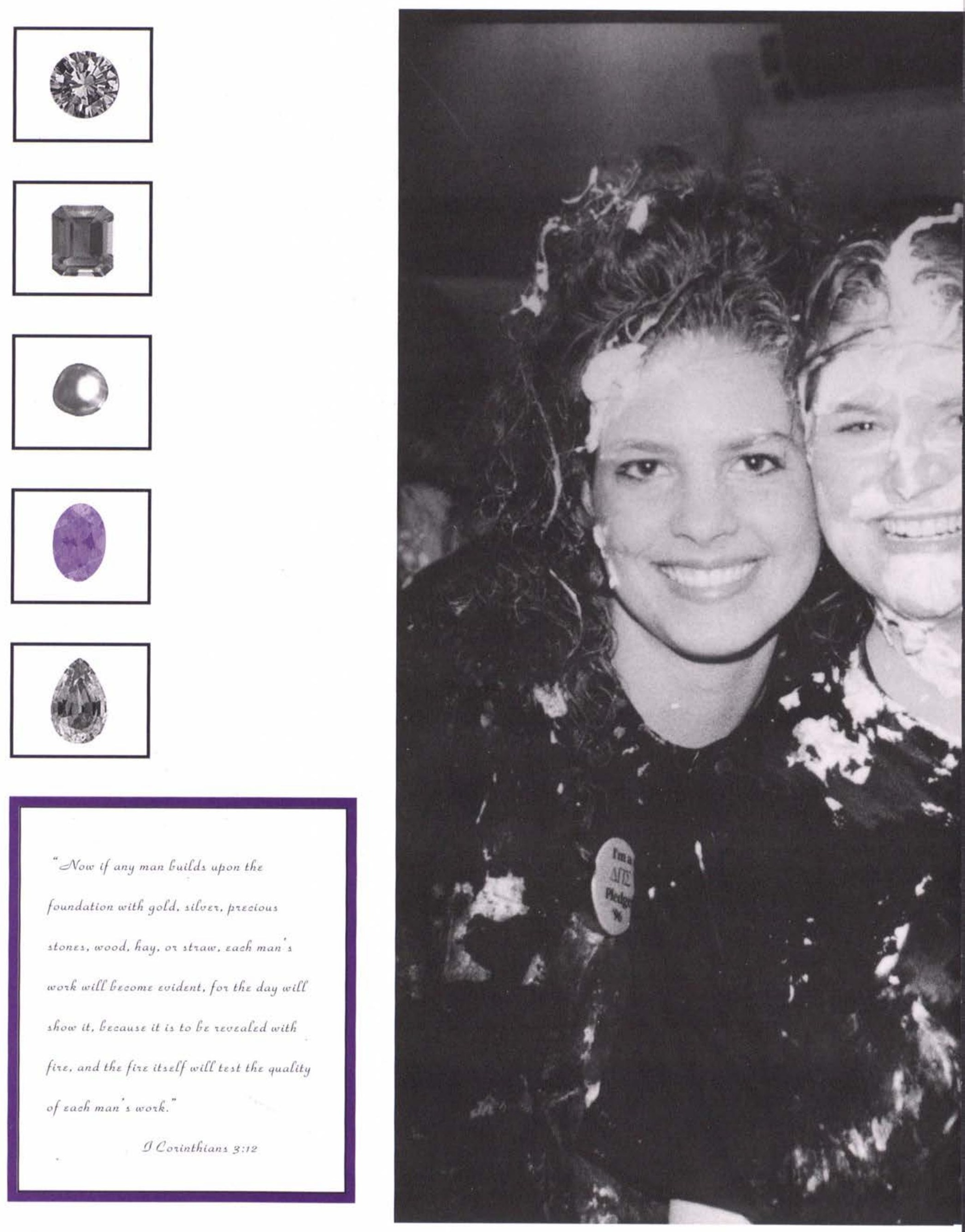


\section{Organizations}

College isn't just about academics. Membership in one of the various campus organizations can offer a chance to get out and do something other than study.

Cedarville has nearly 60 organizations that appeal to the different interests and personalities of the student body. There are organizations for interaction among athletes or across cultures, encouraging communication, or examining social issues. Some are designed to help the community or to touch the individual. The organizations cater to all hobbies and majors, all professional or extra-curricular interests.

The organizations serve several purposes. They provide social opportunities by allowing students of similar interests to become acquainted when perhaps they would otherwise have never met. Many provide opportunities for community service both in Cedarville and around the world. The organizations also encourage professional awareness by giving practical experience and training in different fields of study. Finally, the main goal of each is to encourage its members and challenge them to grow spiritually.

Bonnie Schaefer

Eric Fiveland 
Alpha Chi is a men's service organization. The Greek letters "AX" stand for "Anar Christo," "Men for Christ."

row 1: Gabe Custer, J. Mike Yoder, Dave Hewitt, Dan Byrne, Luke Postema. row 2: Chris R. Ashcraft, Joe Lloyd, Tobin Strong, Derek Luke, Philip Boggs, Brandon Luke, Edwin Bett, Chris M. Brown, Mike Sabella, Ken Howard, Chet Cromer, Chris Knickerbocker, Tim Hewitt, Woody Weaver.
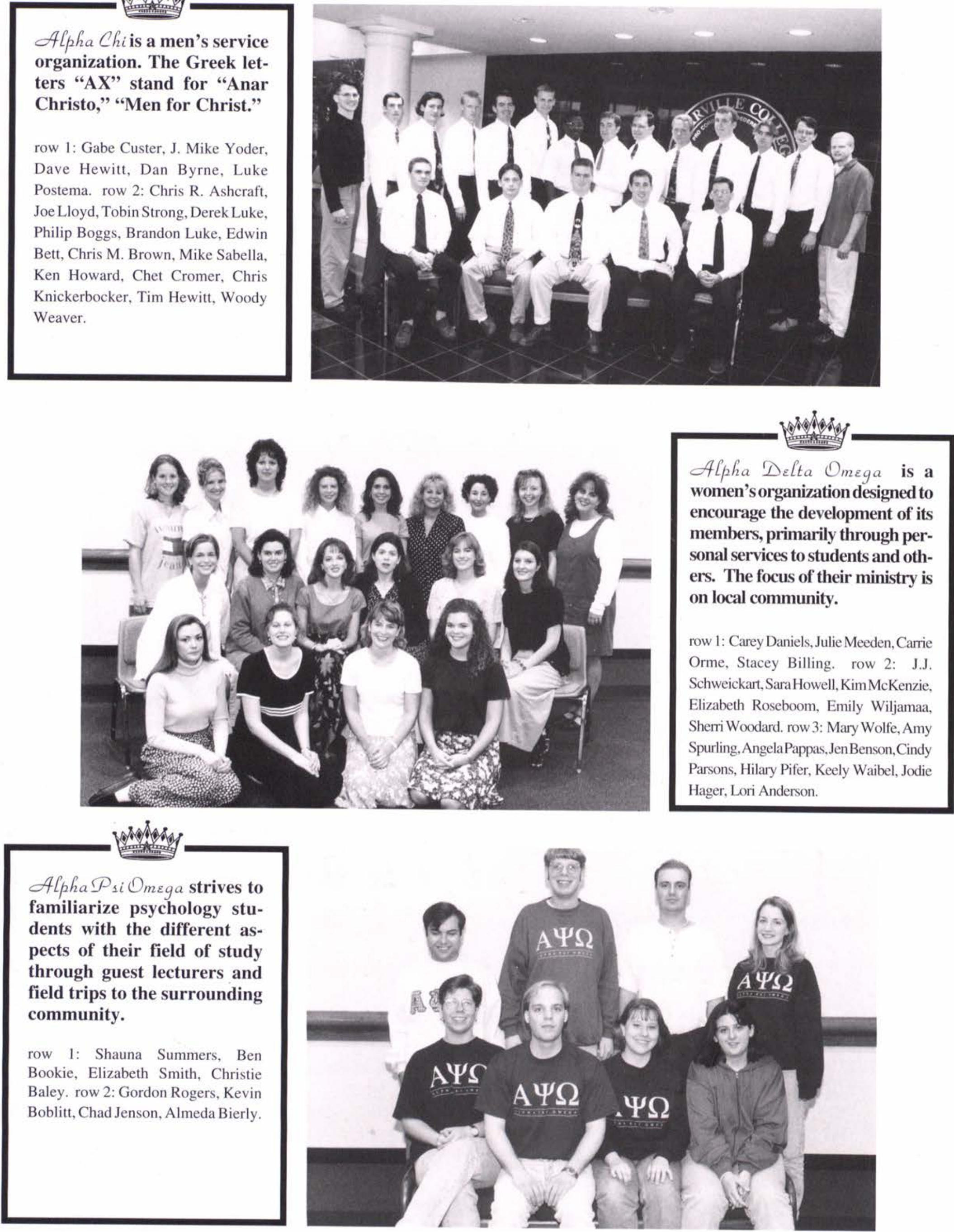

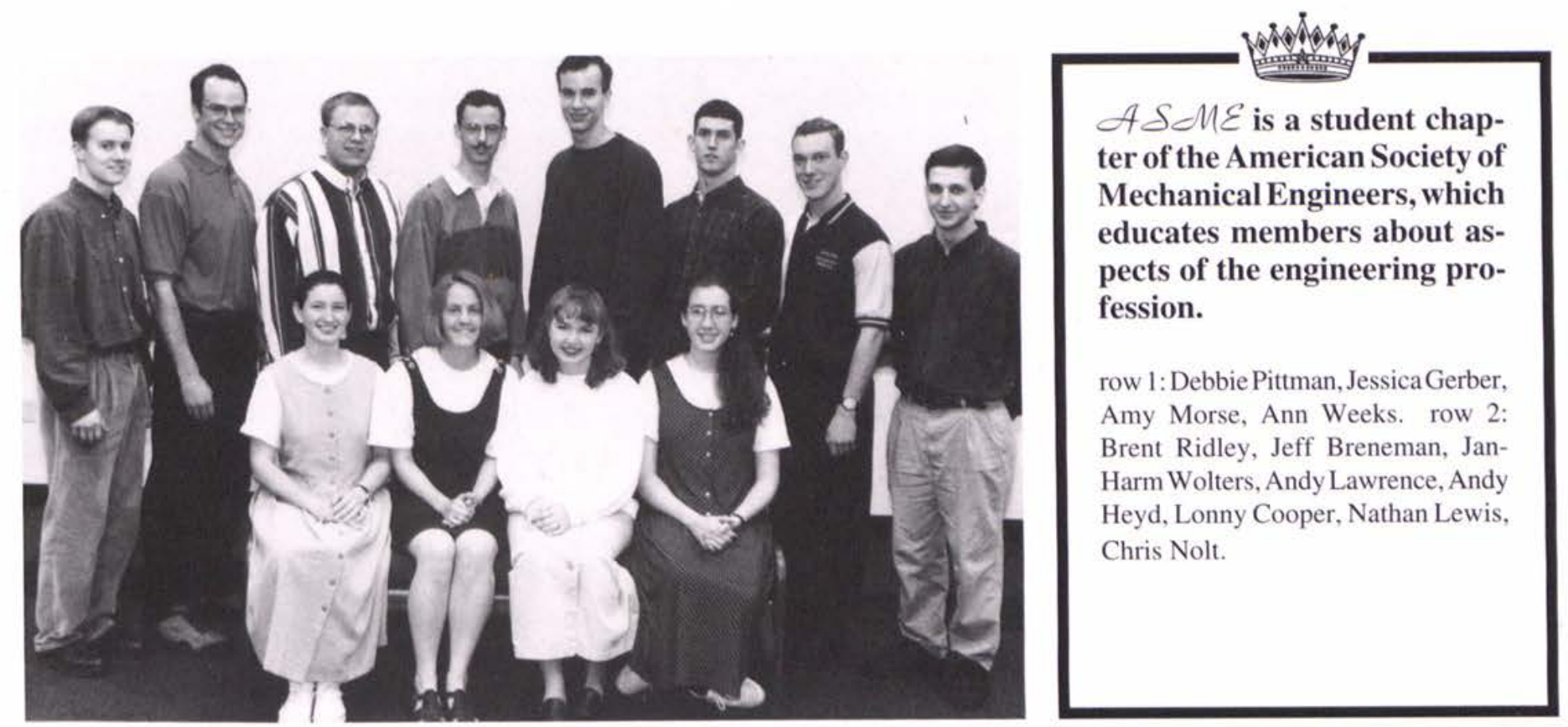

aviosy

Arociation of Information Dechnologies Professionals prepares Computer Information System majors for a career in data processing by acquainting them with professionals in the field as well as with various hardware and software. DPMA is responsible for the Cactus BBS.

Charis Perez, Angie Letson.
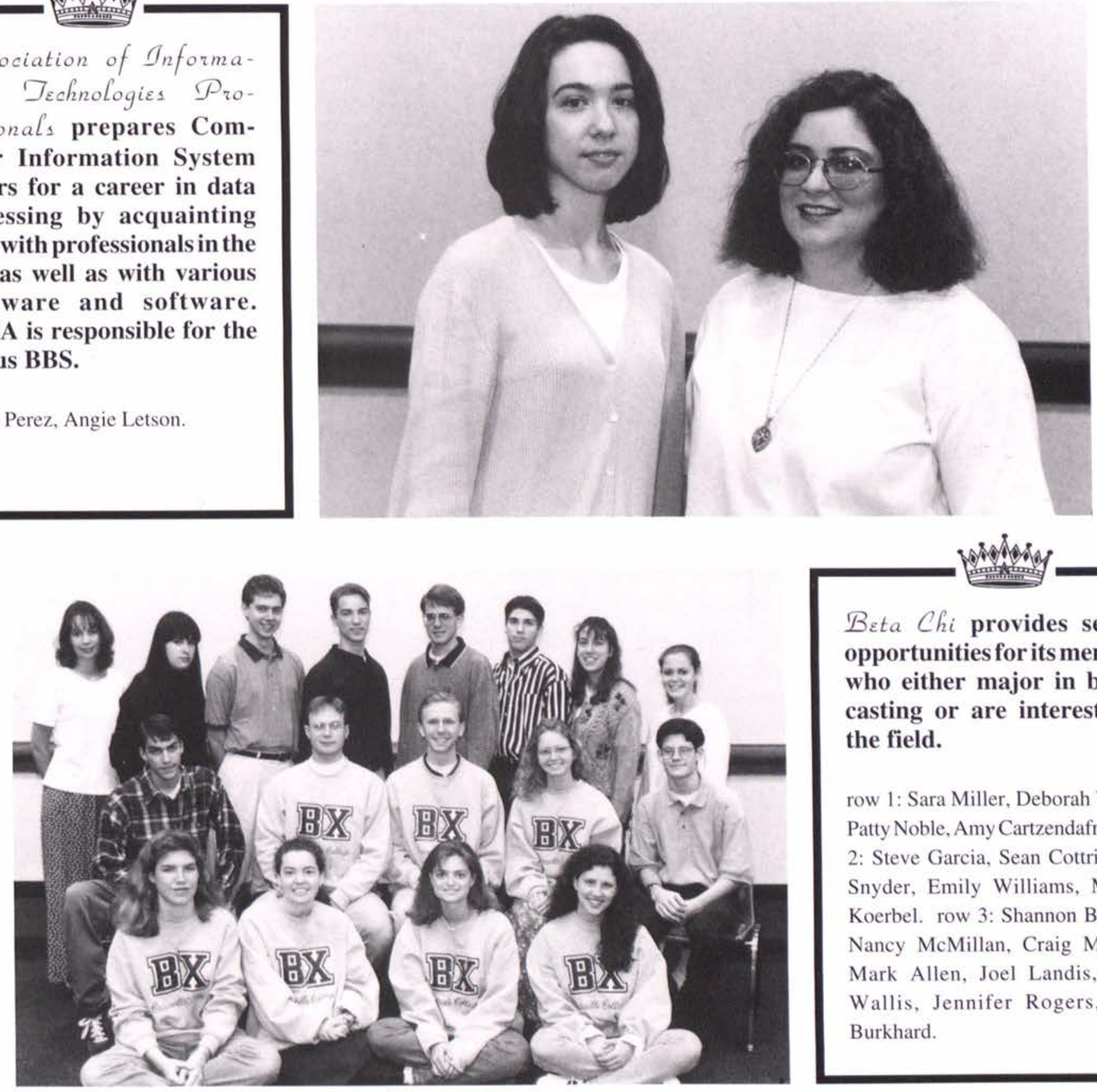

Beta Chi provides service opportunities for its members who either major in broadcasting or are interested in the field.

row 1: Sara Miller, Deborah Woods, Patty Noble, Amy Cartzendafner. row 2: Steve Garcia, Sean Cottrill, Josh Snyder, Emily Williams, Michael Koerbel. row 3: Shannon Boynton, Nancy McMillan, Craig Magrum, Mark Allen, Joel Landis, Philip Wallis, Jennifer Rogers, Ellen Burkhard. 
Beta Pho $_{\text {D }}$ lta, $_{\text {, the Ger- }}$ man Club, encourages interest in and support of missions in German-speaking countries, fosters awareness of the culture, and provides an opportunity for students to practice speaking German.

row 1: Darla Anthony, Sarah Simons, Debbie Pittman, Woody Simons (advisor), row 2: David Hassenzahl, Andrew Marshall, Jan-Harm Wolters, David Carl, Gordon Rodgers.
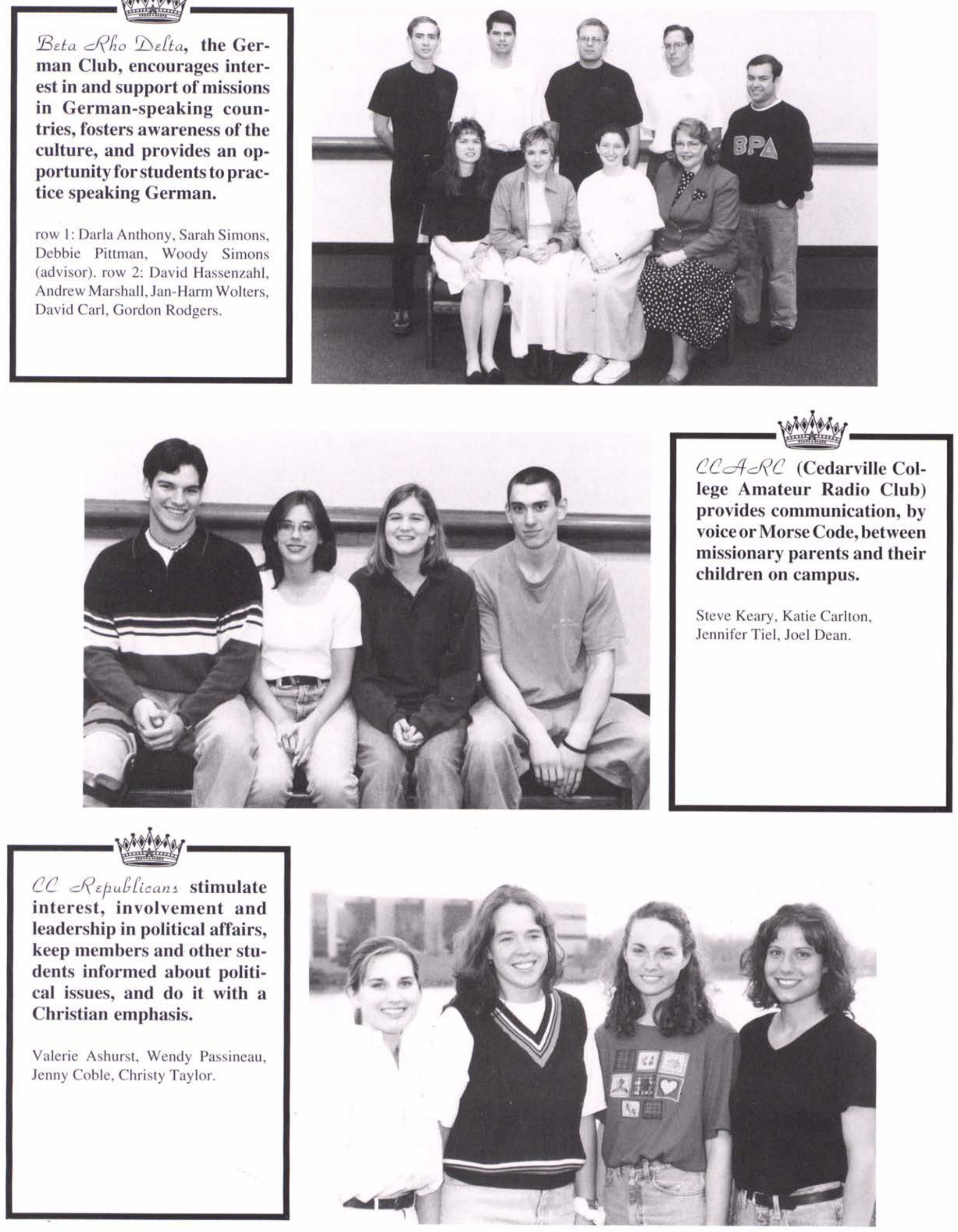


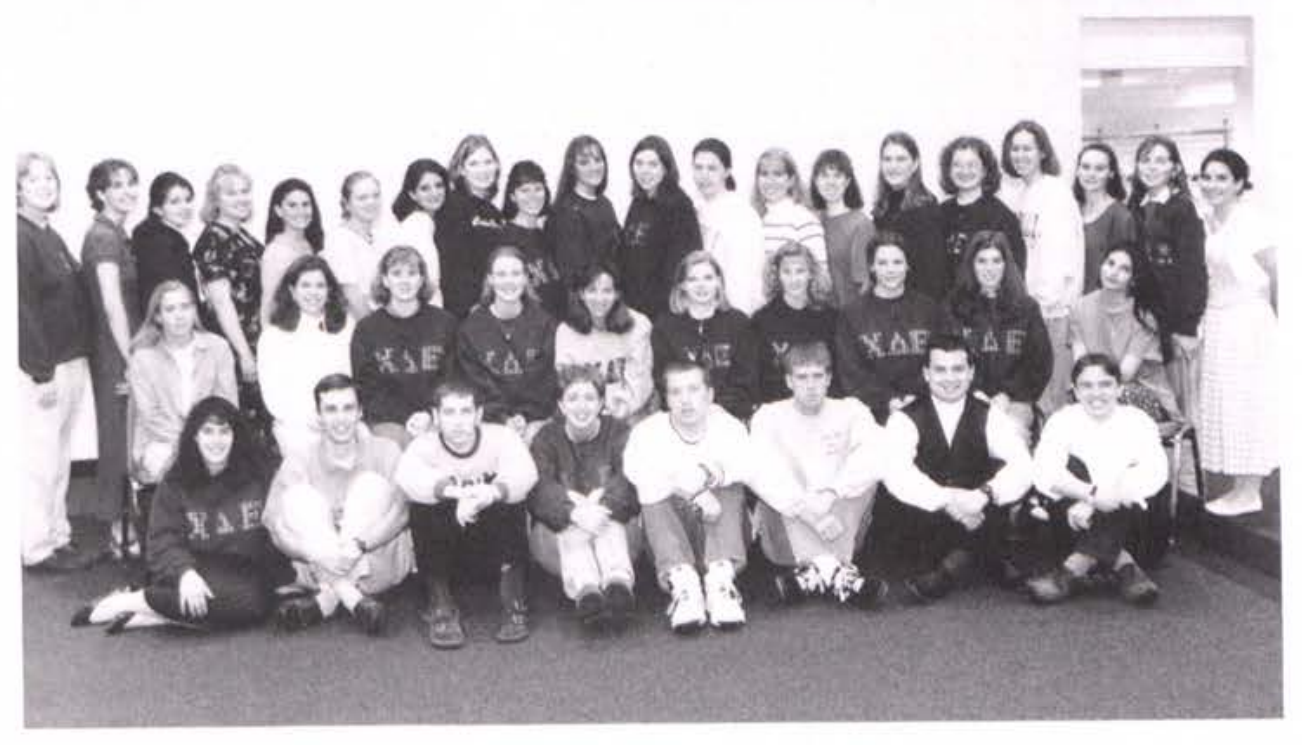

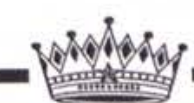

$C h_{i} D_{\varepsilon} l_{t a} \varepsilon_{p s i l o n}$ students strive to increase professional awareness and development, to participate in community outreach and to encourage each other as they prepare for a career in elementary education.

row 1: Amy Nichols, Stephen Kline, Nathan Pohlman, Mandy MacKay, Brian Dye, Josh Bell, Jason O’ Dell, Stephen Slates. row 2: Lisa Mariano, Jessica Meissner, Christa Hill, Danielle Coler, Angela Howe, Valerie Huggler. row 3: Jill Shearer, Angela Justice, Jamie Baker, Michelle Bolt, Jen Alt, Melissa Wabeke, Andrea Dieringer, Haylee Biggs, Susie Dunham, Michelle Clark, Amber Seely, Leanne Heath, Kathryn Johnson, Julie Trout, Emily Davis, Martha Failor, Erin Steelman, Tammy Michaels, Lori Hamilton, Holly Barnett.

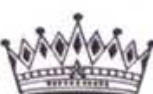

$C h_{i} D_{\varepsilon} l_{t a} \oint_{u}$ is a support group committed to prayer, encouragement, and fellowship for its members.

row 1 : Holly Barnett, Laura Jelinek, Doreen Fuhr, Joey Decker, Kim Miller. row 2: J. Michael Yoder, Tim Hotchkiss, Chad McFadden, Holly Peterson, Shellene Everson, Christine Gerard, Brooke Frazier, Yvette Pizano, Mandy Murray, Dr. Cheryl Fawcett. row 3: Tom Leightenheimer, Justin Colby, Al Anderson, Jeremy Toyer, Andrew Manwiller, James Dewald, Matt Palombo, Bill Stewart, Nate Childers, Bryan Miller, Jason Carrier, Matt Beck, Aaron Cook, B.J. Bechtel, Barry Gorsuch, Bill Workman, Steve Merchant.

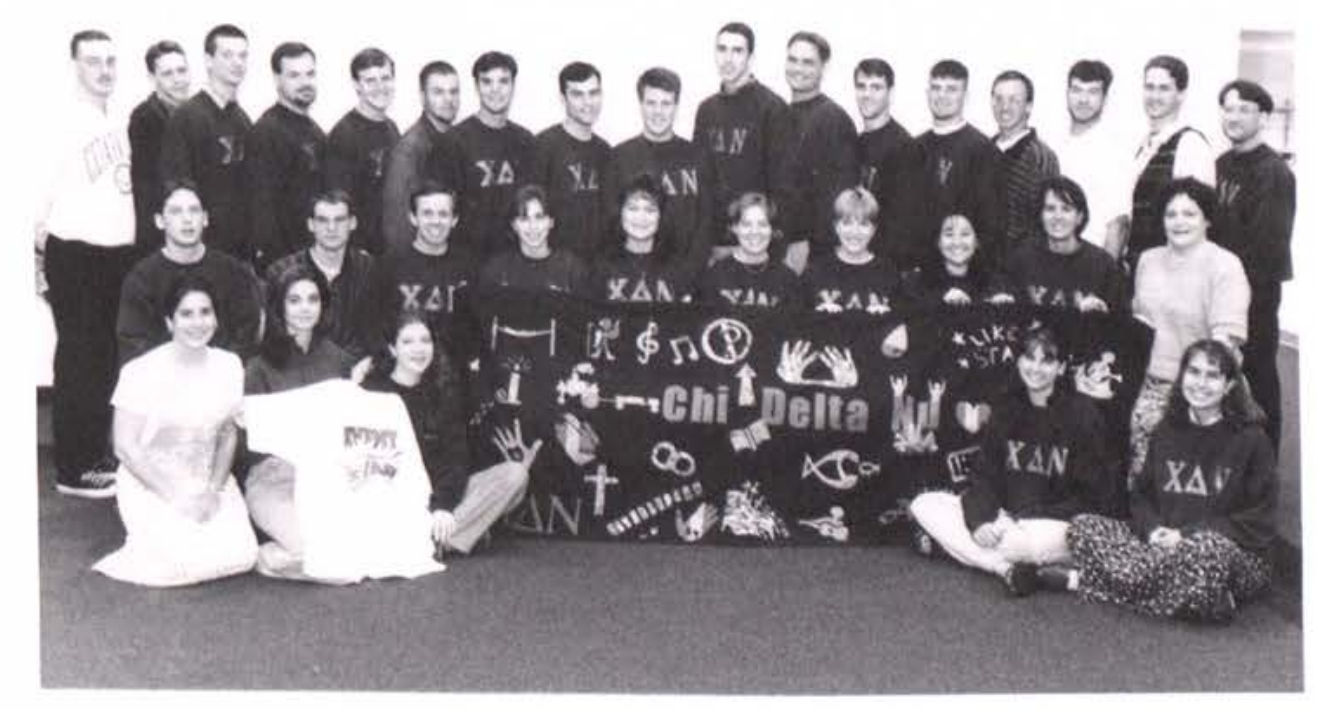




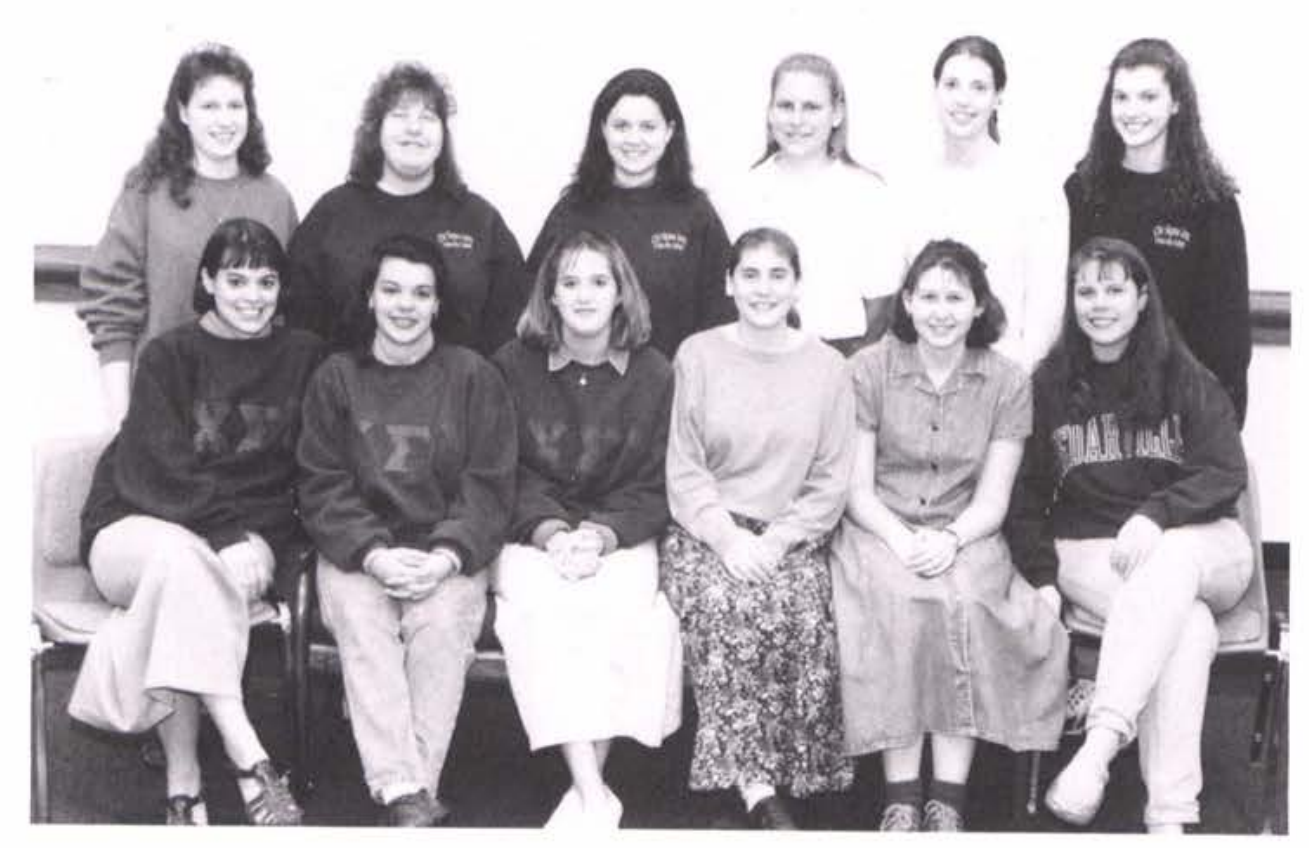

oropidy

Chi Sigma $D_{\text {ota }}$ promotes interest in the office technology profession, provides opportunities for members to observe various business offices in operation, sponsors service projects which benefit the local business community, and promotes fellowship for students with similar interests in related fields.

row 1: Joanna Halsey, Amy Dietz, Julie Witt, Amy Meckley, Sarah Mattke, Kristi Gleason. row 2: Hannah Sherwood, Christine Schmidt, Jessica Griffith, Kelly Reitz, Lindsay Schneider, Kristy Jackson.

\section{ardicy}

$C h_{i}$ Thzta $\mathcal{P}_{i}$ strives to provide opportunities for students in pre-health profession majors to experience various facets of their chosen professions through special activities, speakers, and field trips.

row 1: Vicki Frederickson, Sarah Martin, Naomi Redington, Julie Lamborn, Amanda Mudrey, Caren Furst, Emily Weber. row 2: Michelle Higgins, Jonathan Murphy, Tamara Shrader, Charity Brown, Erin Timco, Alicia Hill, Cara Hines, Erin McLarty. row 3: Joseph Cantor, Melissa Blore, Stephanie Mace, Amy Hurst, Gena Lamoreaux, Amy Thayer, Carrie Gwilt, Kara Doden, Julie Lauritzen, Naomi Kossel, Alicia Baisley, April Johnson, Kim Maynard, Johannah Augustine.

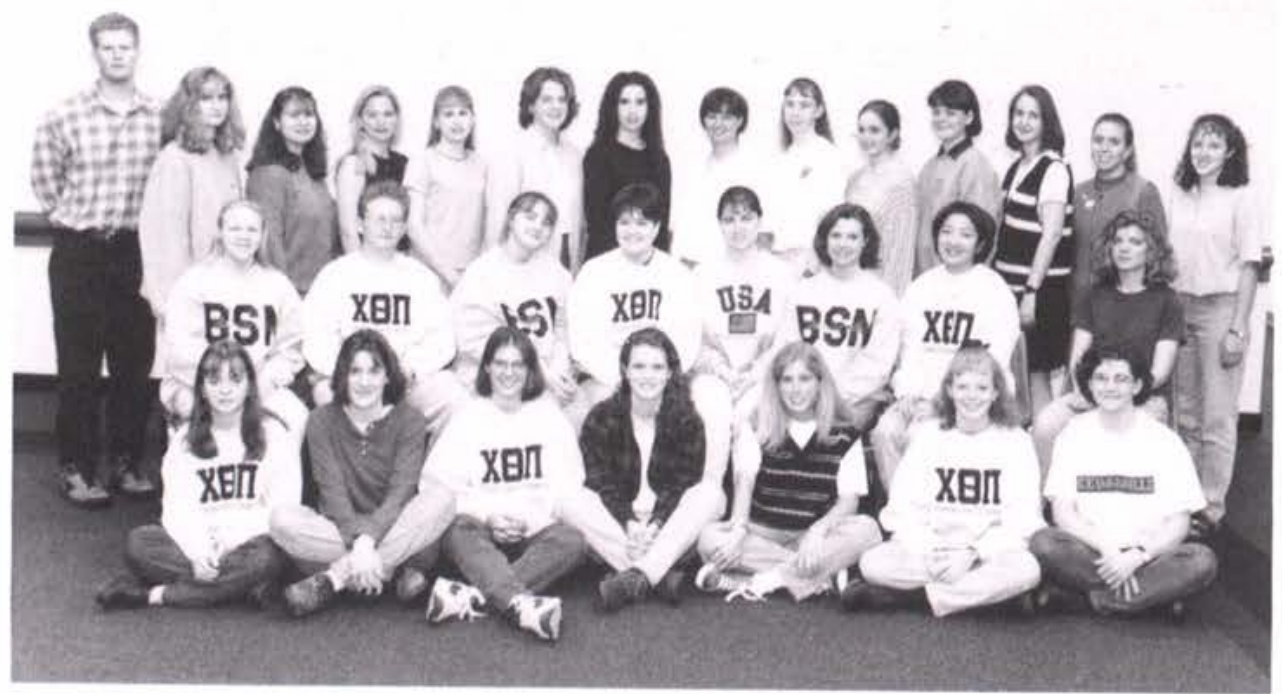




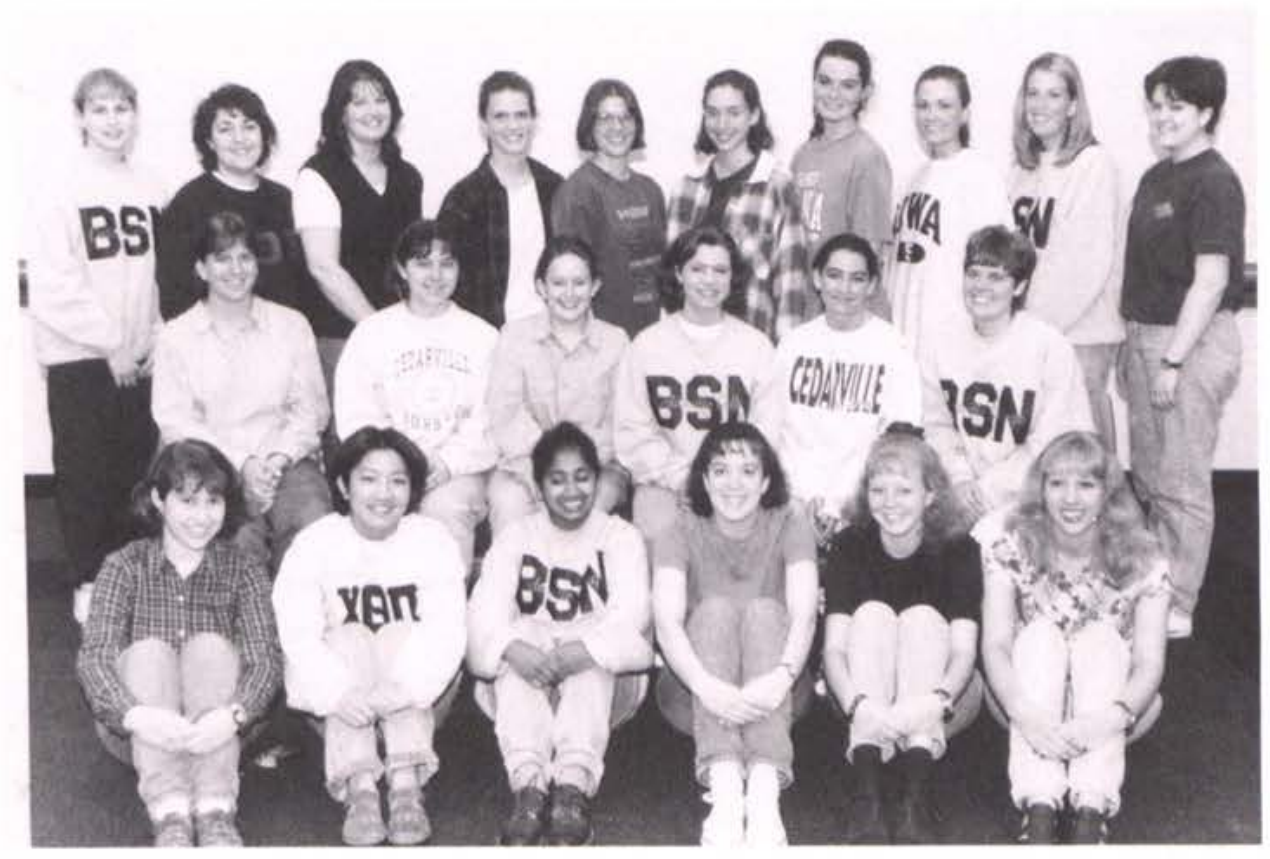

Christian ONurss Afsociation promotes spiritual and professional development for nurses and service for the community.

row 1 : Sarah Rittgers, Cara Hines, Jaya Philip, Jana Glessner, Caren Furst, Melanie Wadlington. row 2: Kristin Marshall, Sarah Svendsen, Becca Ribeiro, Alicia Hill, Dana Scott, Kathy Petersen. row 3: Gena Lamoreaux, Rachel Maki, Tonya Brockman, Julie Lamborn, Naomi Redington, Rachel Ryan, Heather Perrault, Carey Daniels, Kerri Vander Molen, Charity Brown.
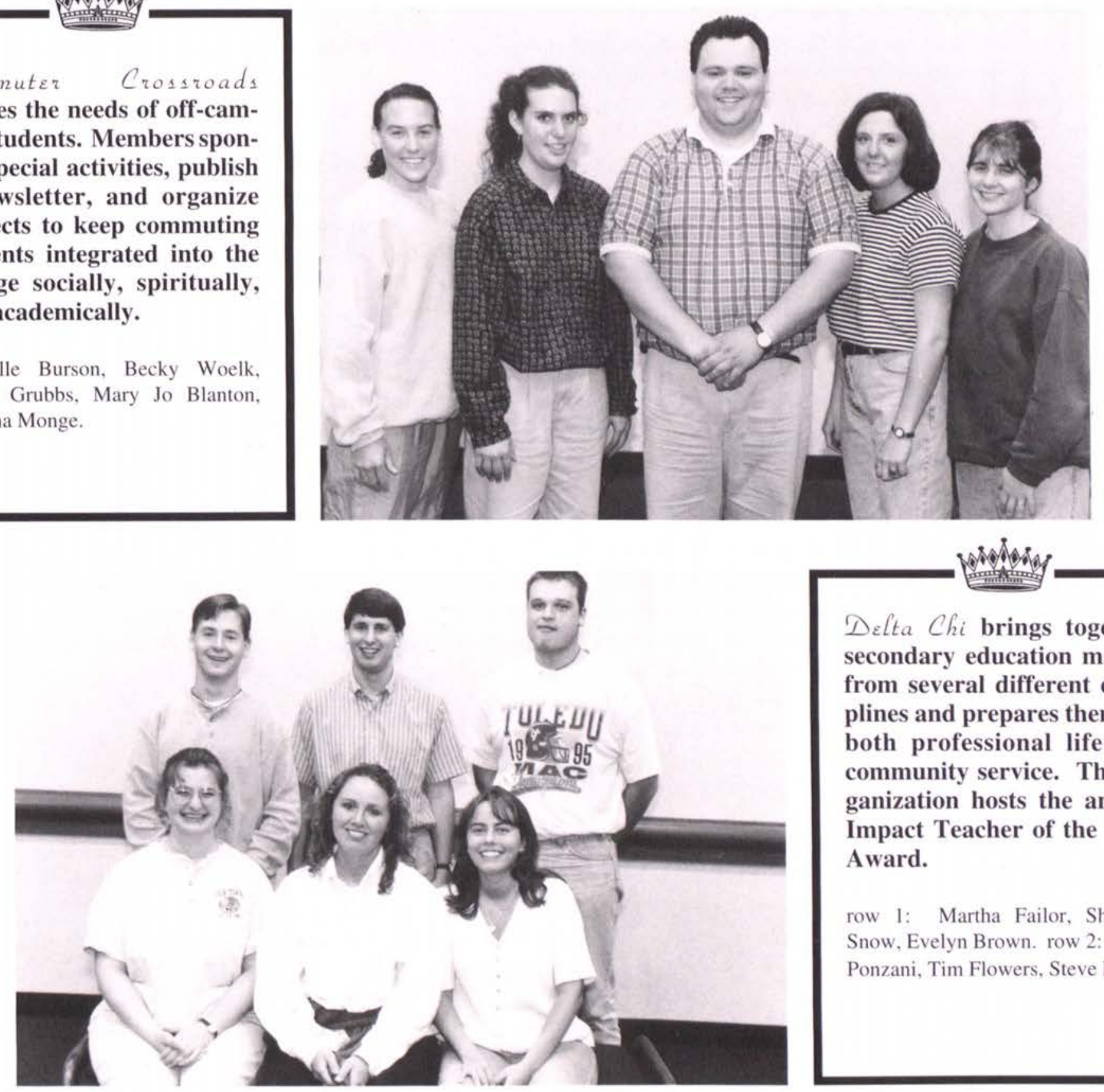

$D_{\varepsilon}$ lta $C h i$ brings together secondary education majors from several different disciplines and prepares them for both professional life and community service. The organization hosts the annual Impact Teacher of the Year Award.

row 1: Martha Failor, Shannon Snow, Evelyn Brown. row 2: Aaron Ponzani, Tim Flowers, Steve Ryan. 
Delta Omega $\varepsilon_{p s i l o n}$ challenges its members to acquire the traits of Discipline, Obedience, and Excellence, and to build those traits into all aspects of life.

row 1: Pete Sutton, Chuck Quarles. row 2: Darin Rosenvold, Paul DiCuirci, Scott Van Loo, Josh Lunney, David Burke, Clinton Rice, Malik Shabazz. row 3: Vance Smith, John Krueger, Ben Haffey, Ted Forrest, Greg Walker, Jason Brown, Chad Deakyne, Scott Kennedy.

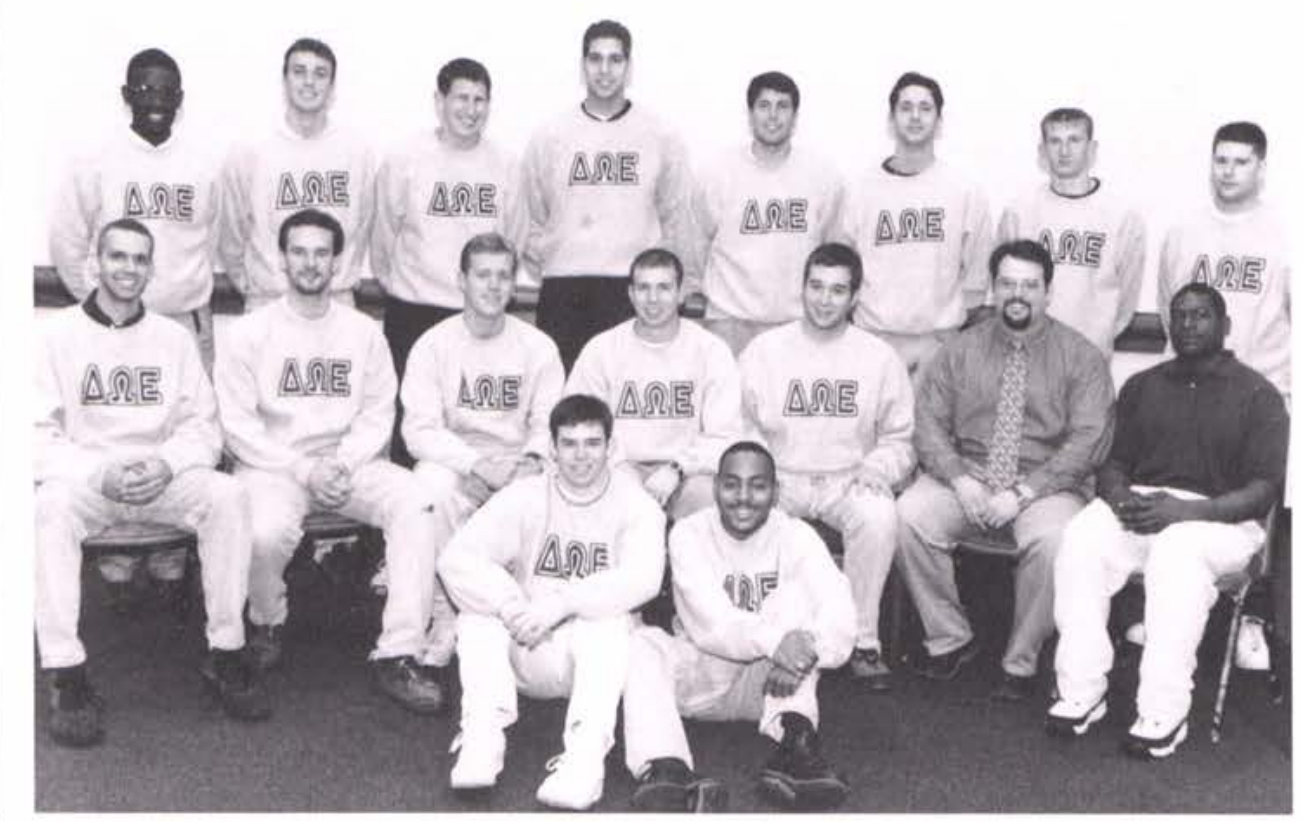

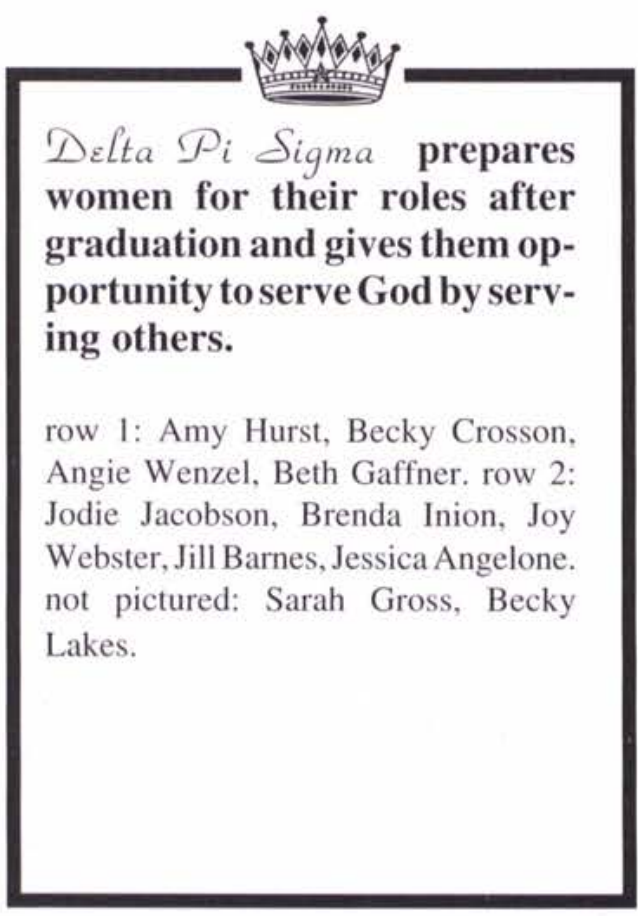

row 1: Amy Hurst, Becky Crosson, Angie Wenzel, Beth Gaffner. row 2: Jodie Jacobson, Brenda Inion, Joy Webster, Jill Barnes, Jessica Angelone. not pictured: Sarah Gross, Becky Lakes.
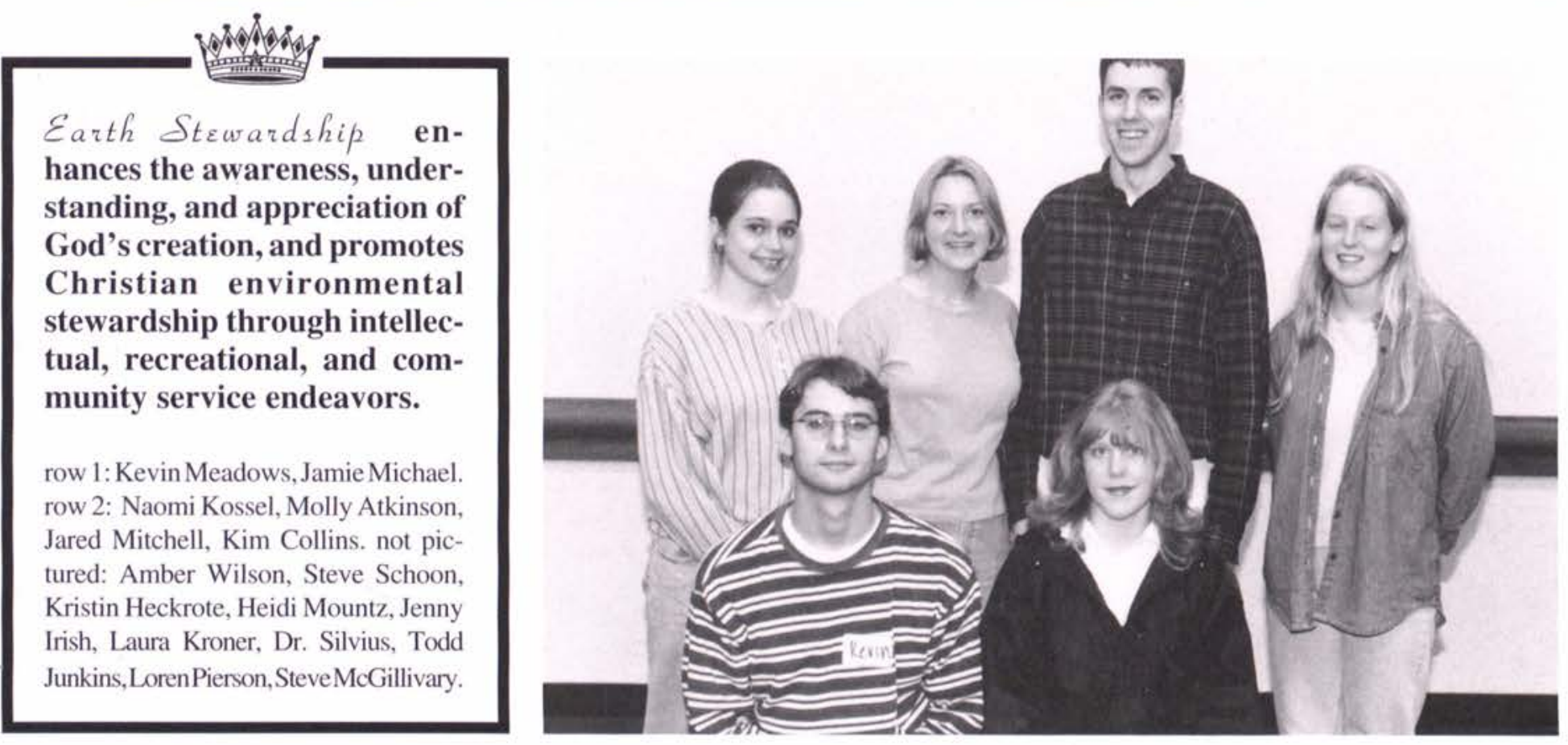

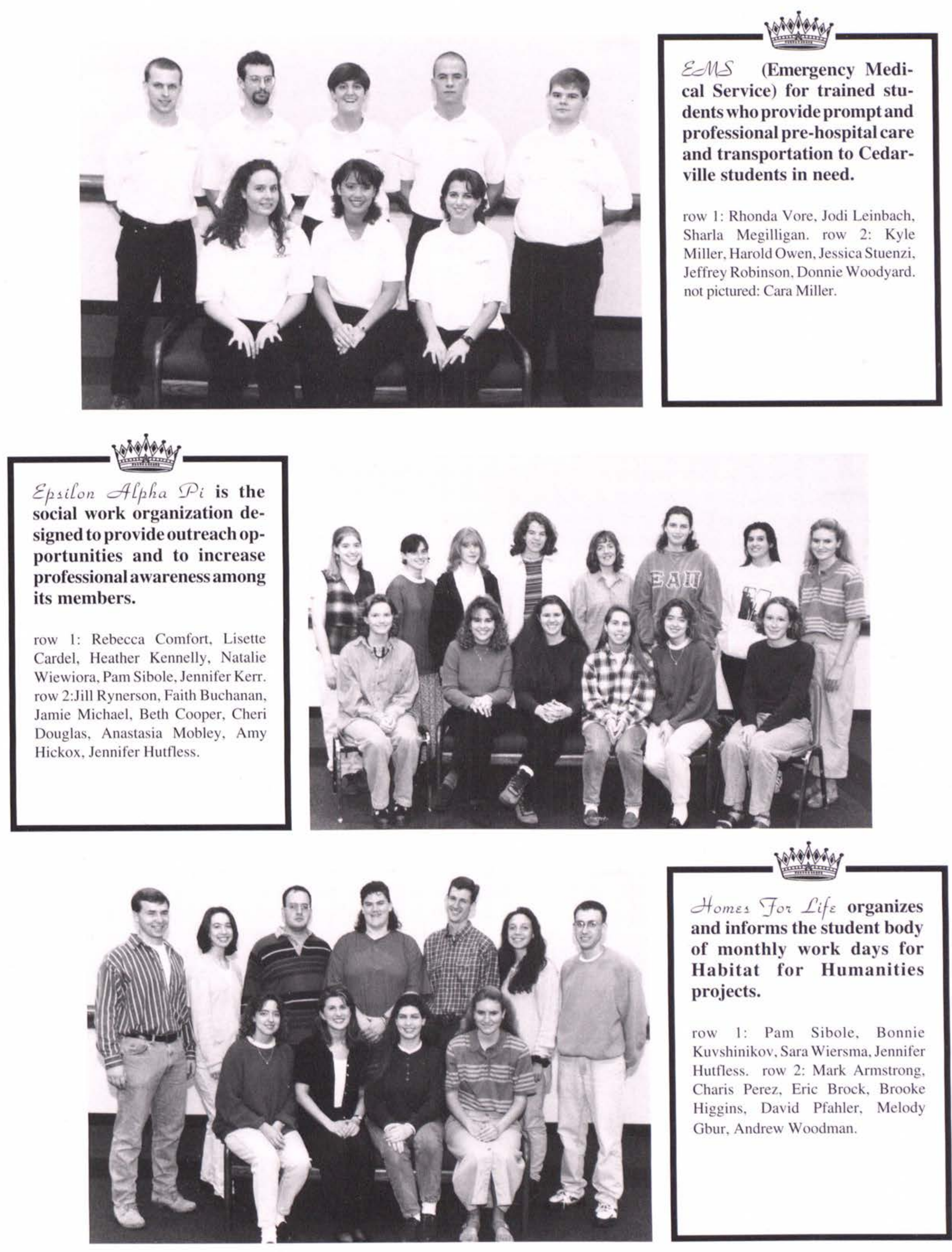


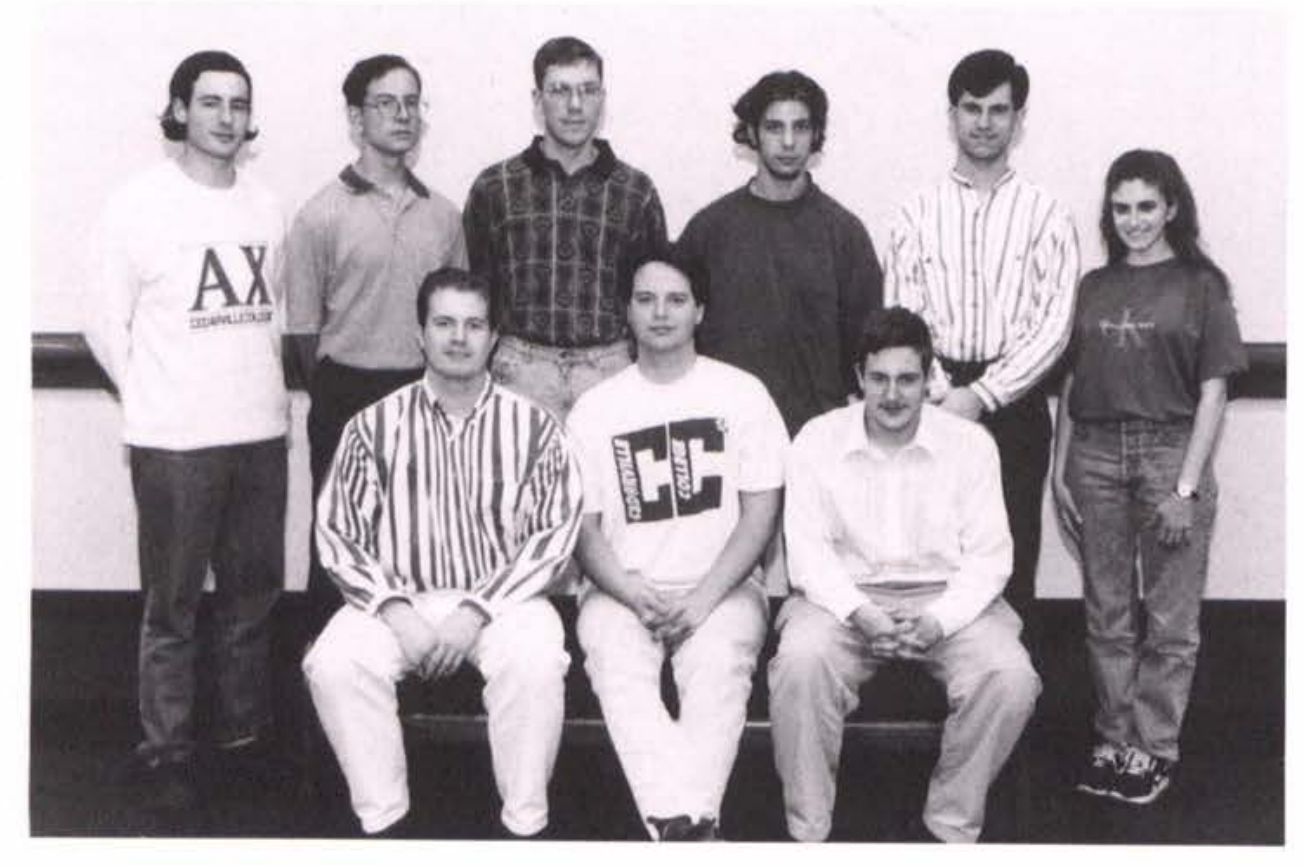

avisos

I.E.E. E. (Institute of Electrical and Electronical Engineers) promotes technical and professional growth among electrical engineering students.

row 1: Jeremy Staley, Charley Walker, Kenneth Witalis. row 2: Tobin Strong, David Carl, Nathan Wright, Matt Freitag, Nathan Wehr, Stacie Bennett.

\section{aropory}

$I_{\text {ota }} C h_{i} \mathcal{P} h_{i}$ (Intercultural Christian Fellowship) has a mission to achieve what God has stamped on the heart of every true believer and which the U.S. has stamped on every coin - "E PLURIBUS UNUM"

- Out of Many, One.

Heather Kunert 

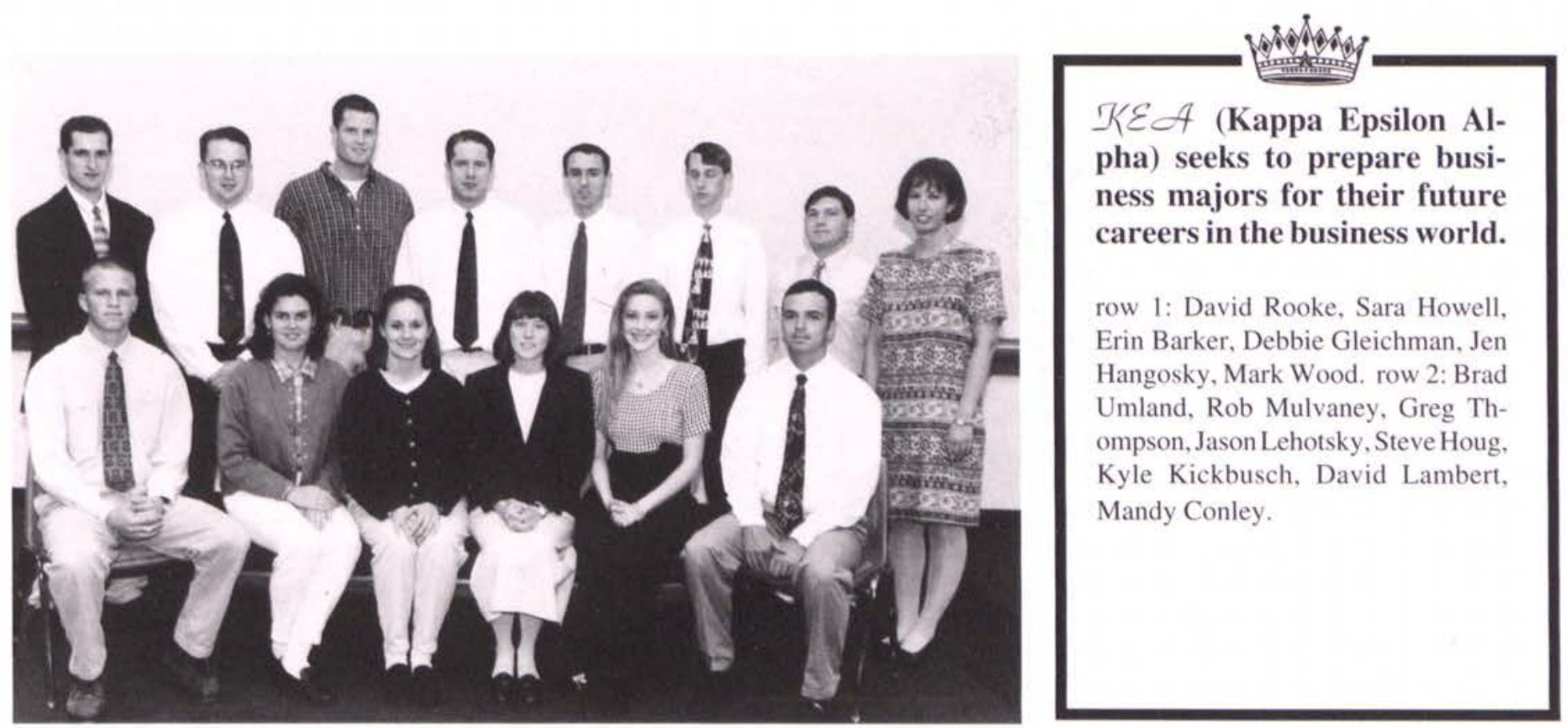

opdosy

ME NC (Music Educators' National Conference) strives to keep music education students informed of what is currently happening in music education.

row 1: Erin Stuenzi, Jenny Armour, Nikki Thomas, Lea Anne Churgovich, Beth Stewart, Eileen McCoskey. row 2: Valerie Smith, Cynthia Lawry, Alisa Whitt, Michael Gregg, Tiffany Zirke, Amy Clutz, Nadine Tomsa.

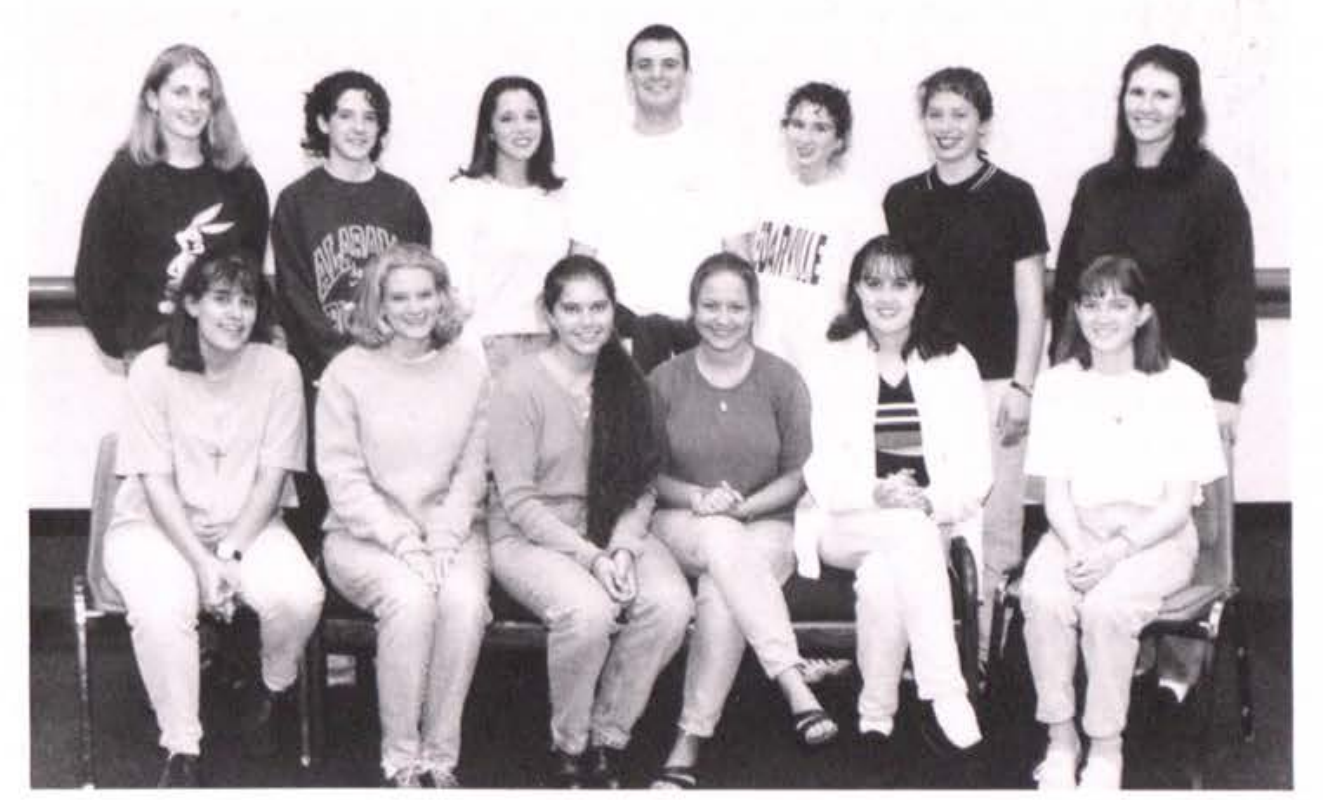

oseresy

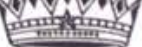

- Mu Z Kappa exists to provide missionary kids and international students with opportunities for fellowship,socialinteraction, and cultural and spiritual growth.

row 1: David Southwell, Stacy Stauffer, Kari Lynn Yoder, Sofie Jorstad, Deborah Woods, Tami Weber, Bart Allen. row 2: Tami See, Kristin Kuhn, Kimberly Ketterer. Tammy Stark, Tiffany Linden, Rob Bouwens, Phil McVey, Kouichi Ikeda. row 3: Janette Baker, Emily Davis, Atsuko Ohtake, Rebecca Border, Jon Shrubsole, Jon Hjembo, Edwin Bett, Steve Kline, Ben Nordaas, Becca Southwell, George Weber, Jeff Schumacher, Karl Hjembo. 

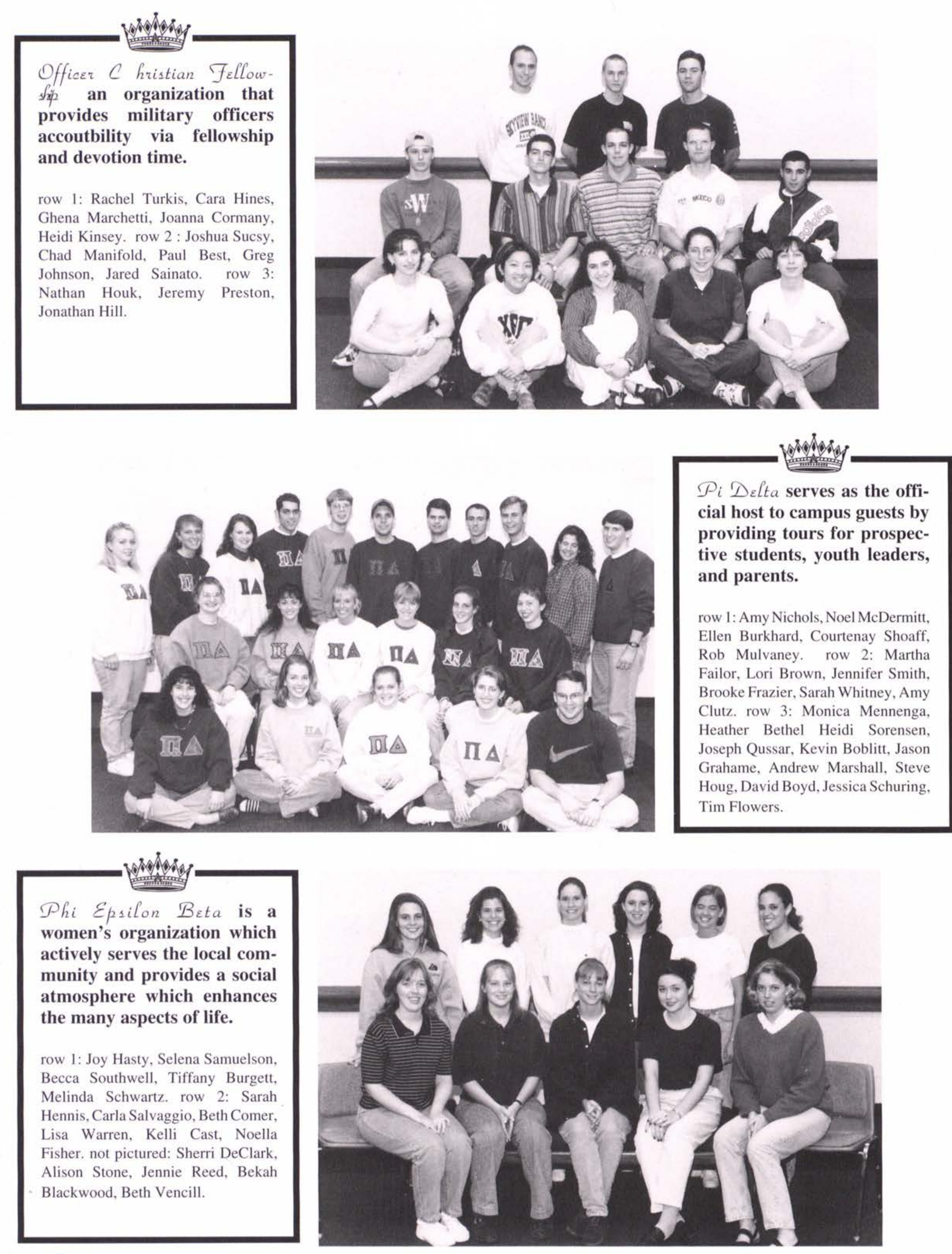


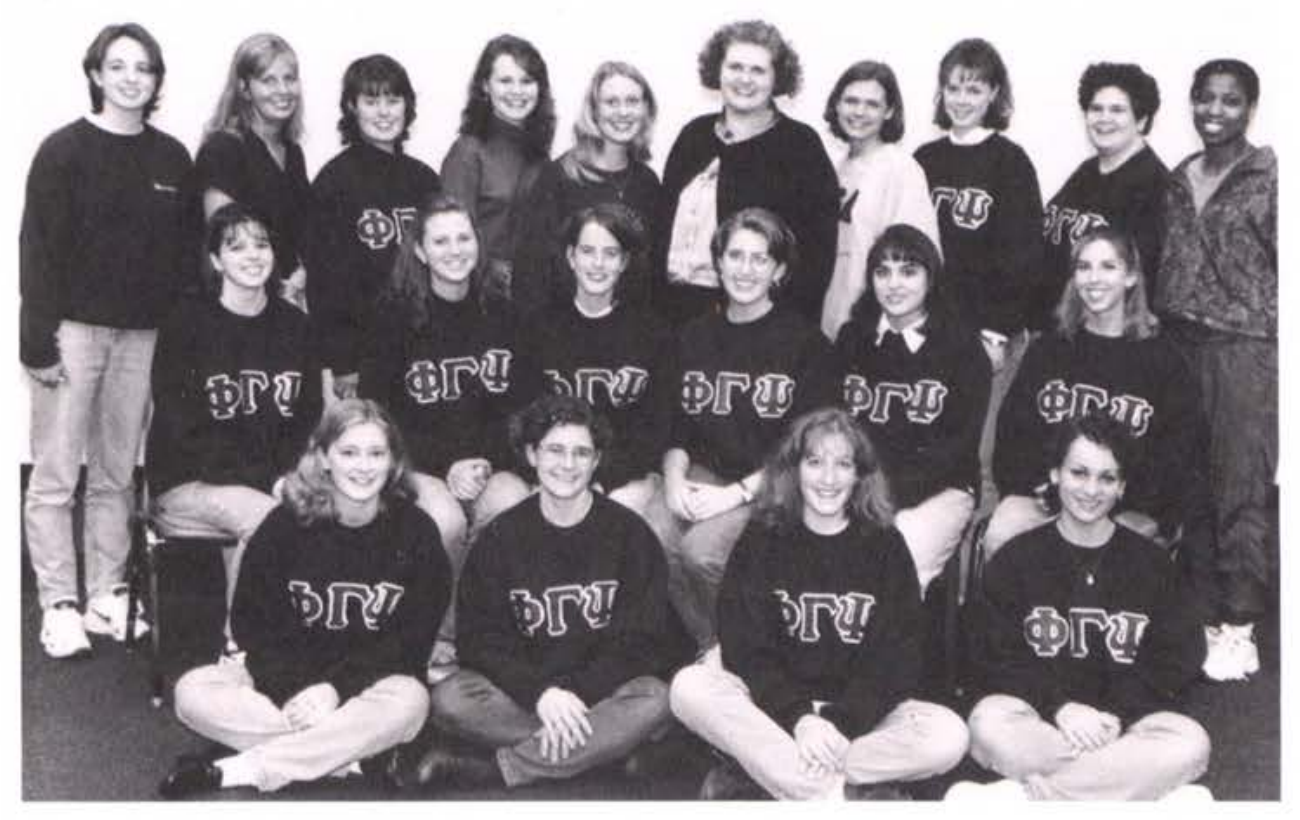

oropisy

$P_{h i} G_{\text {amma }} P_{1 i}$ is a women's social and service organization designed to encourage women through prayer meetings and social activities.

row 1: Melissa Crawford, Emily Weber, Mandy Mullen, Gretchen Dorman. row 2: Julia Horne, Jennifer Ingalls, Jennifer Tegtmeier, Courtenay Shoaff, Heidi Mountz, Jen Vander Bush. row 3: Abby Stroven, Amy Wilder, Cara Harju, Heidi Sorensen, Holly Sorensen, Heather Murdoch, Chrystie Ruba, Heather Brodie, Heather Jackson, Kezia McNeal (advisor).

ardivis

$\mathcal{P}_{i}$ Sigma $\mathcal{N}_{u}$ is a brotherhood that seeks to develop its members into the "whole man" in the areas of body, mind, and spirit.

row 1: Dave Ormsbee, Jim Latourelle, John Pugno, Brent Mullin, Jason Groesser. row 2: Dan Parlin, Ryan Tatum, Steve Rutlinger, Ben Stewart, Jason Davies. not pictured: Damon Derstine.

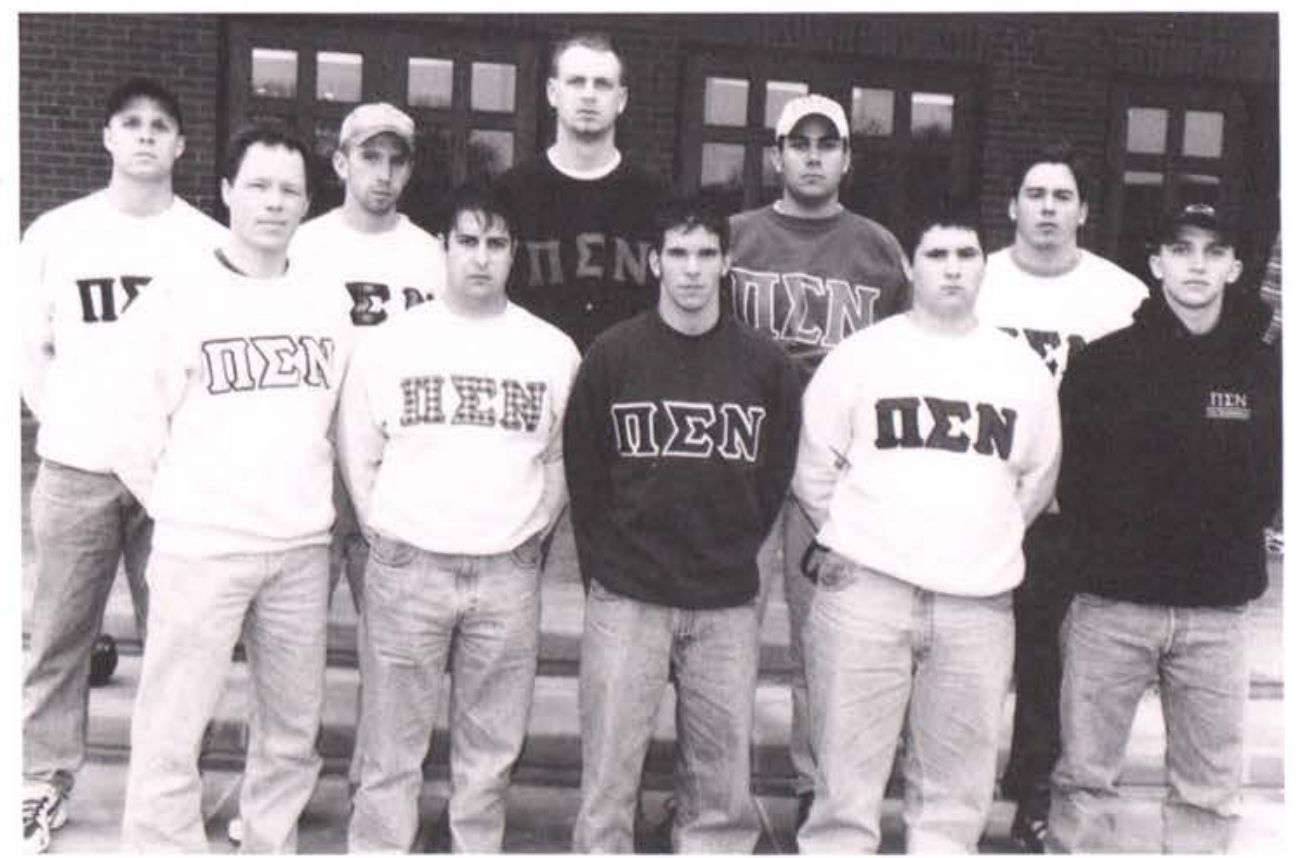




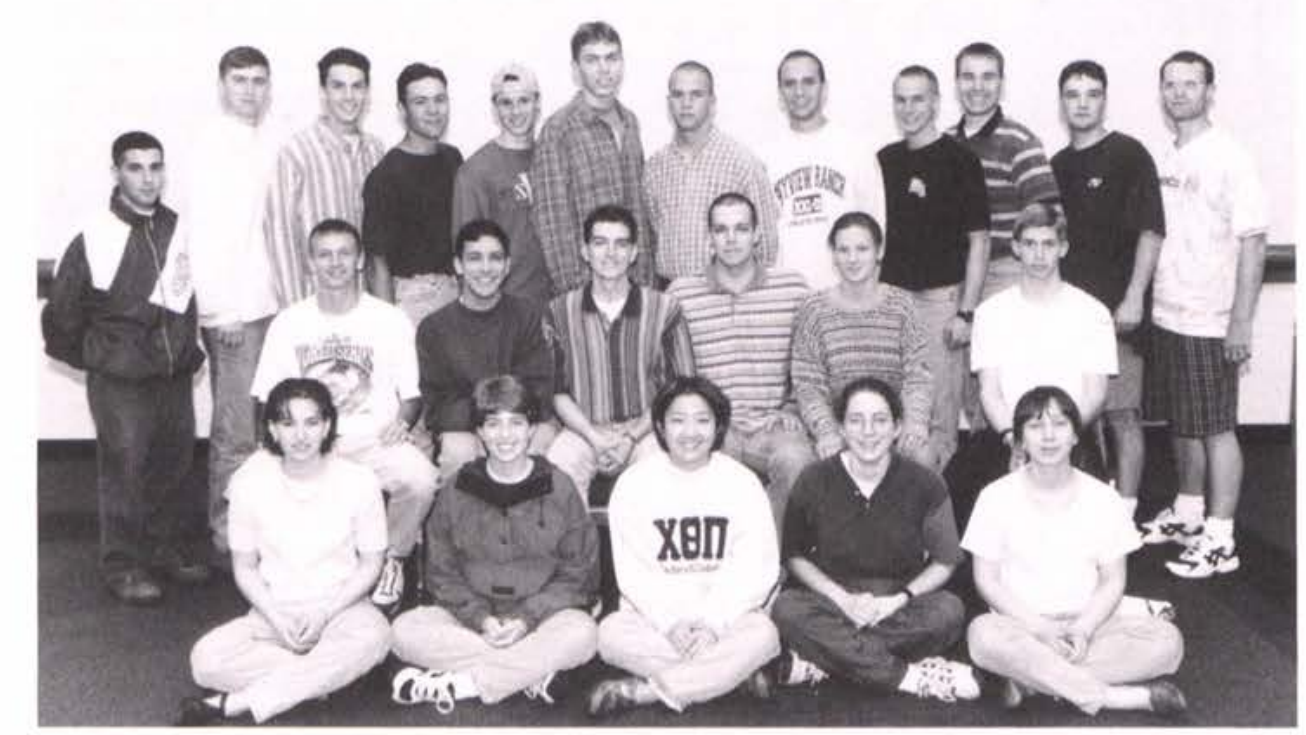

ropios

CPODC (Reserve Officers Training Corp) provides students who will serve in the military with leadership skills.

row 1: Rachel Turkis, Pam Claus, Cara Hines, Joanna Cormany, Heidi Kinsey. row 2: Brett Frey, Jay Mantravadi, Chad Manifold, Paul Best, Sara Laning, Lewis Pulley, row 3: Jared Sainato, Paul Puryear, Adam Rizer, Jonathan Hill, Joshua Sucsy, Andrew Kempe, Jeffrey Robinson, Nathan Houk, Jeremy Preston, Brenon Christofer, Jonathan Gray, Greg Johnson.

orisicy

$\delta \mathcal{A}$ (Society of Automotive Engineers) gives students the chance to learn about their future and talk to other engineers through seminars and projects.

row 1: Debbie Pittman, David Carl, Heidi Kinsey, Amy Carpenter, Scott Koziol, Chris Troupos, row 2: Nathan Houk, Cindy McFadden, Tobin Strong, Scott Husband, Scott Hoadley, Dan Cochrane, Ann Weeks, Erick Anglund, Seth Valentine, Chris Nolt.

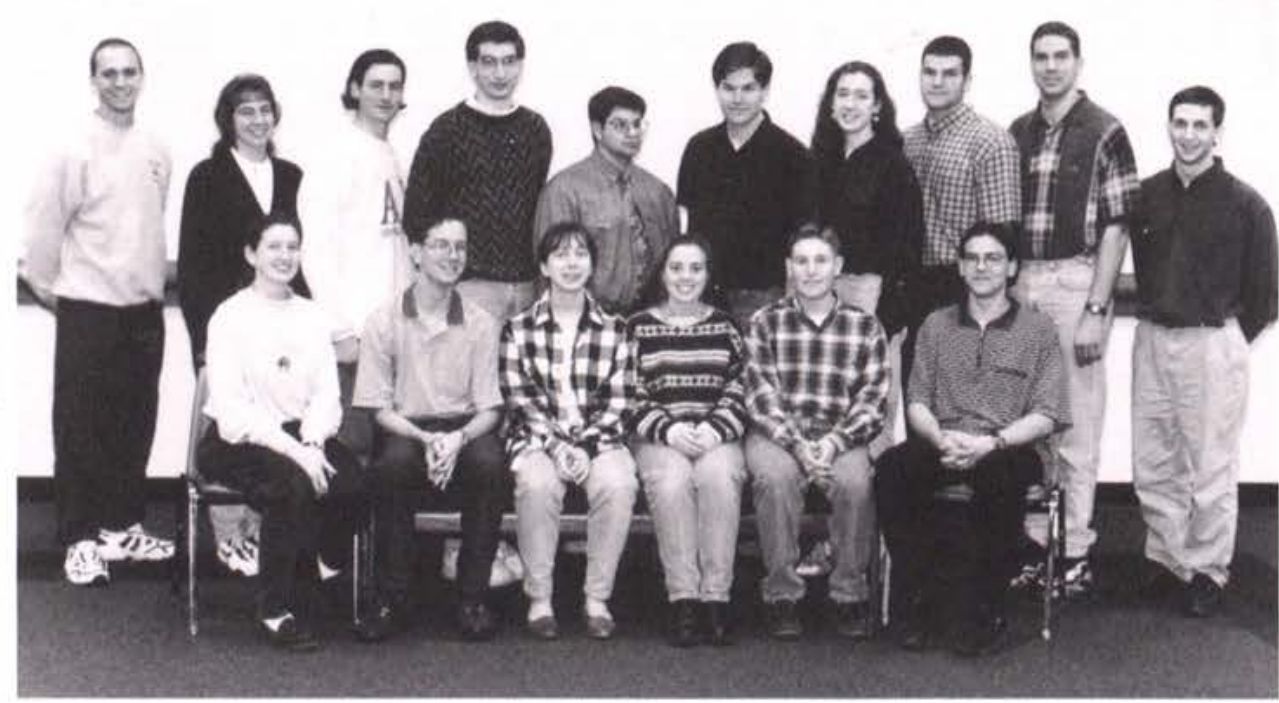




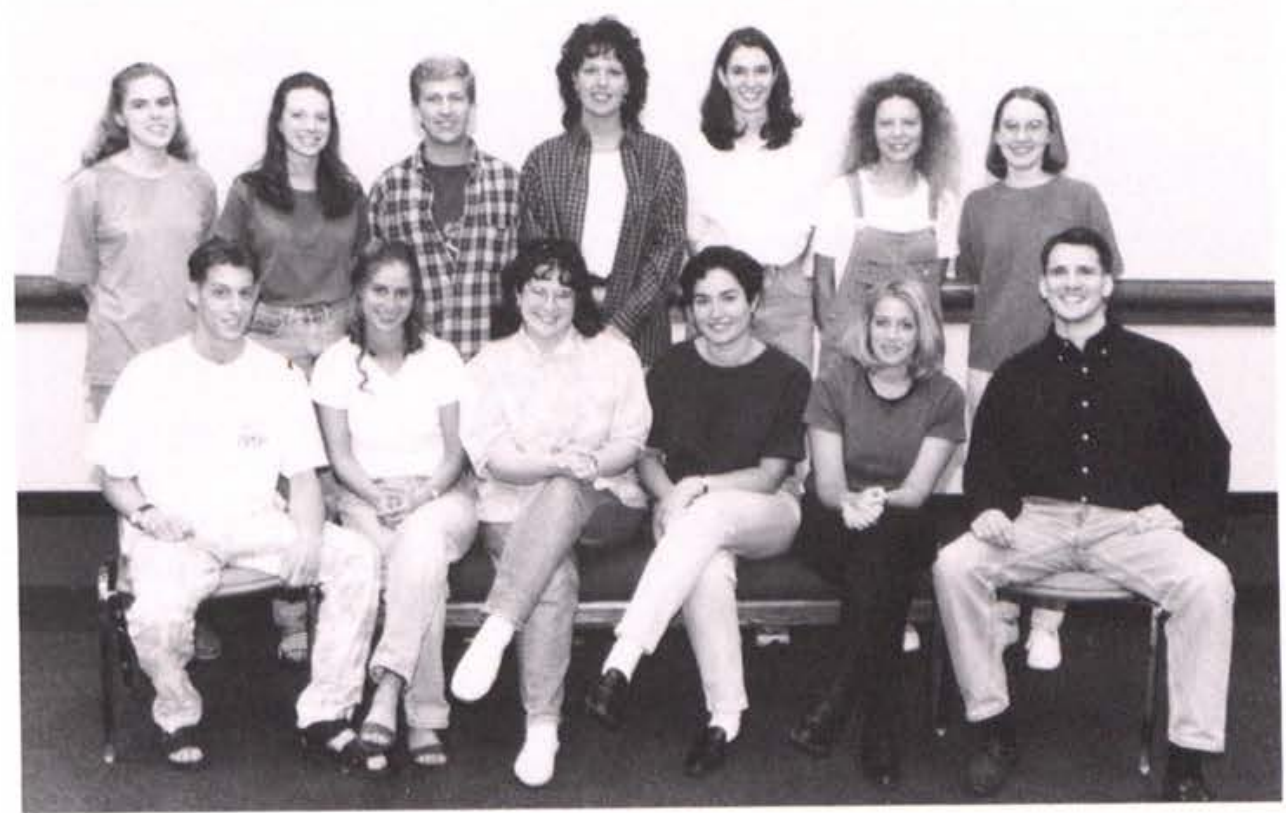

$\delta \square C$ (Society for Technical Communications) gives professional writing students a variety of opportunities to learn about their field, enhance their professional reputation, and find employment.

row 1 : Blake Goulette, Brenda Lynn Tabberer, Christy Cleaver, Joy Wickholm, Angela Lenhart, Chris Ashcraft. row 2: Libby Miner, Samantha Stemad, Brad Bartlett, Angela Pappas, Lisa Van Wormer, Jen Benson, Megan McMacken. Not Pictured: Phil Wittmer, Rachel Ross.

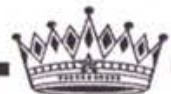

Spanish Cluf offers an opportunity to speak Spanish and have fun in a relaxing atmosphere; members are not required to be fluent.

row 1 : Rachelle Paquin, Nicki Sweet, Elizabeth Rogers. row 2: Michelle Clark, Karen Hayes, Sarah Carr, Adam Rorex, Chris Robertson.
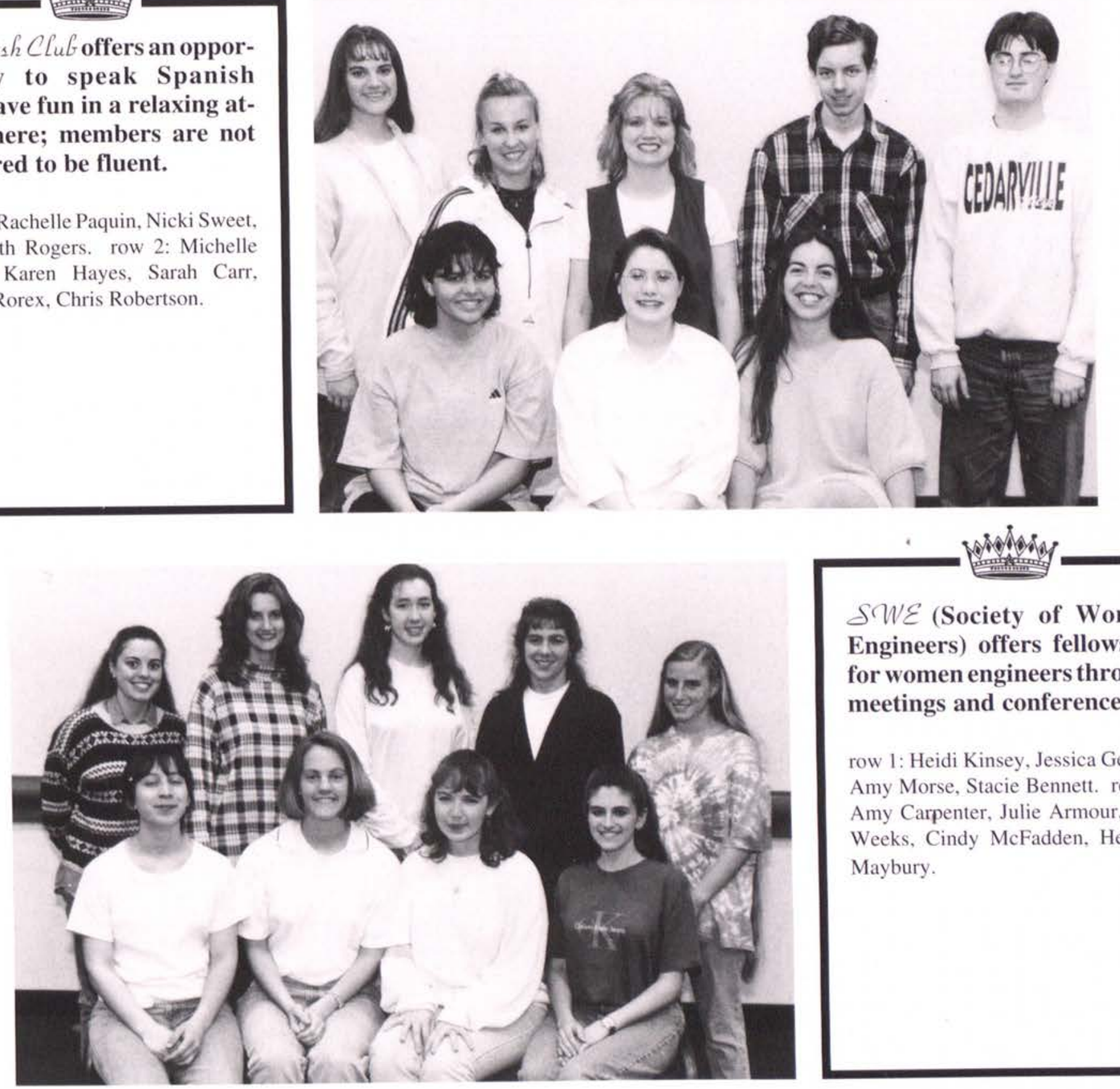

SWE (Society of Women Engineers) offers fellowship for women engineers through meetings and conferences.

row 1: Heidi Kinsey, Jessica Gerber, Amy Morse, Stacie Bennett. row 2: Amy Carpenter, Julie Armour, Ann Weeks, Cindy McFadden, Heather Maybury. 


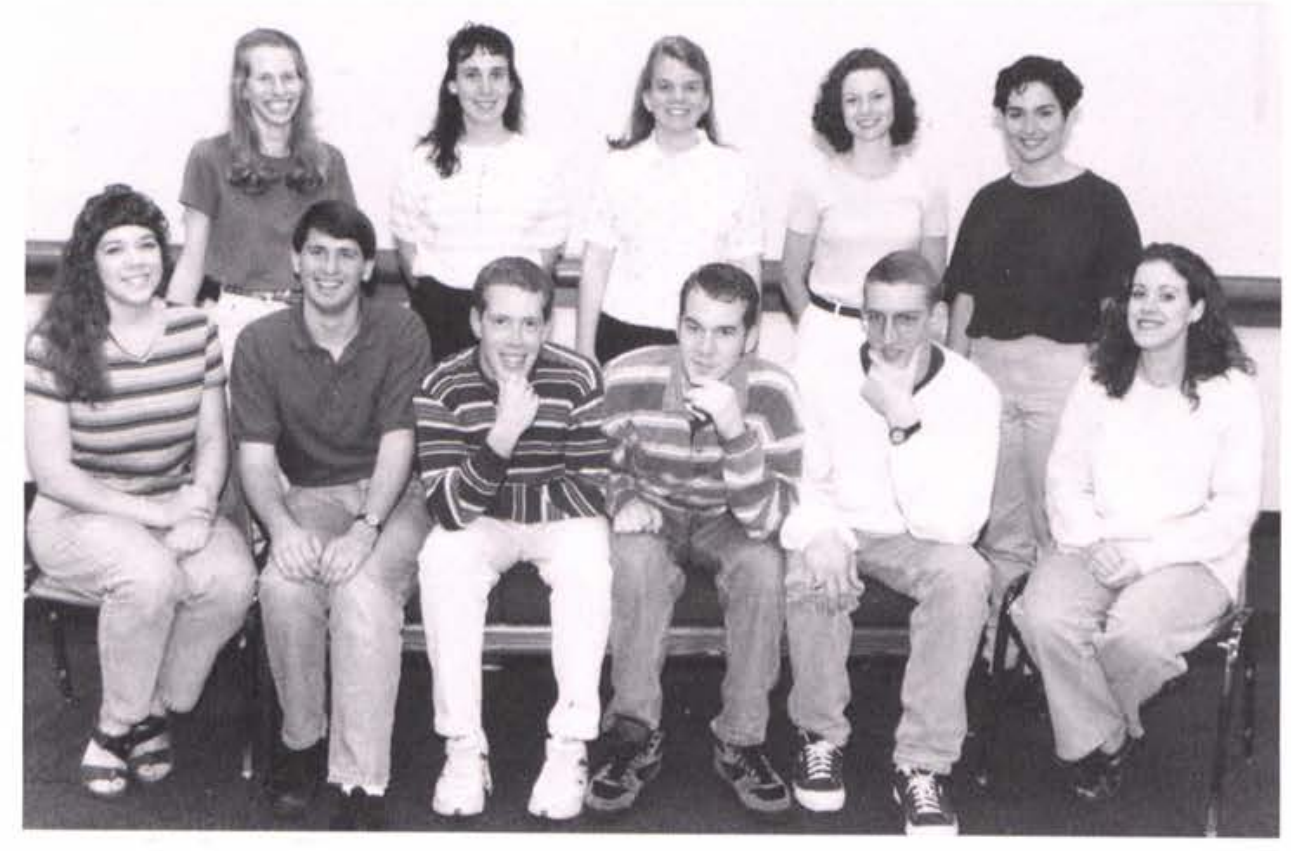

ardes

】au $\searrow_{\varepsilon} l_{t a}$ K Kappa provides an opportunity for fellowship, exchange of ideas, and support for those students enrolled in the Honors Program.

row 1: Summer Schafer, Tim Flowers, Doug Amundson, David Reisenbigler, Jonathan Burson, Lisa Walker. row 2: Christina Baley, Tami See, Erin Tuinstra, Rachel Stewart, Joy Wickholm.

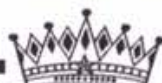

Theta Cho Eksilon is a men's organization dedicated to accountability, building friendships, and having a good time.

row 1: Chris Moles, Jon Woolley, Joshua Ausfahl, Chris Vitarelli, Paul DeKruyter. row 2: Jeff Beste, Jim Amstutz, Ernest Clark, Tim Simon, Shawn Shipp, Mark Price, Jon Wu, Jeff Neuman, Andy Clary.

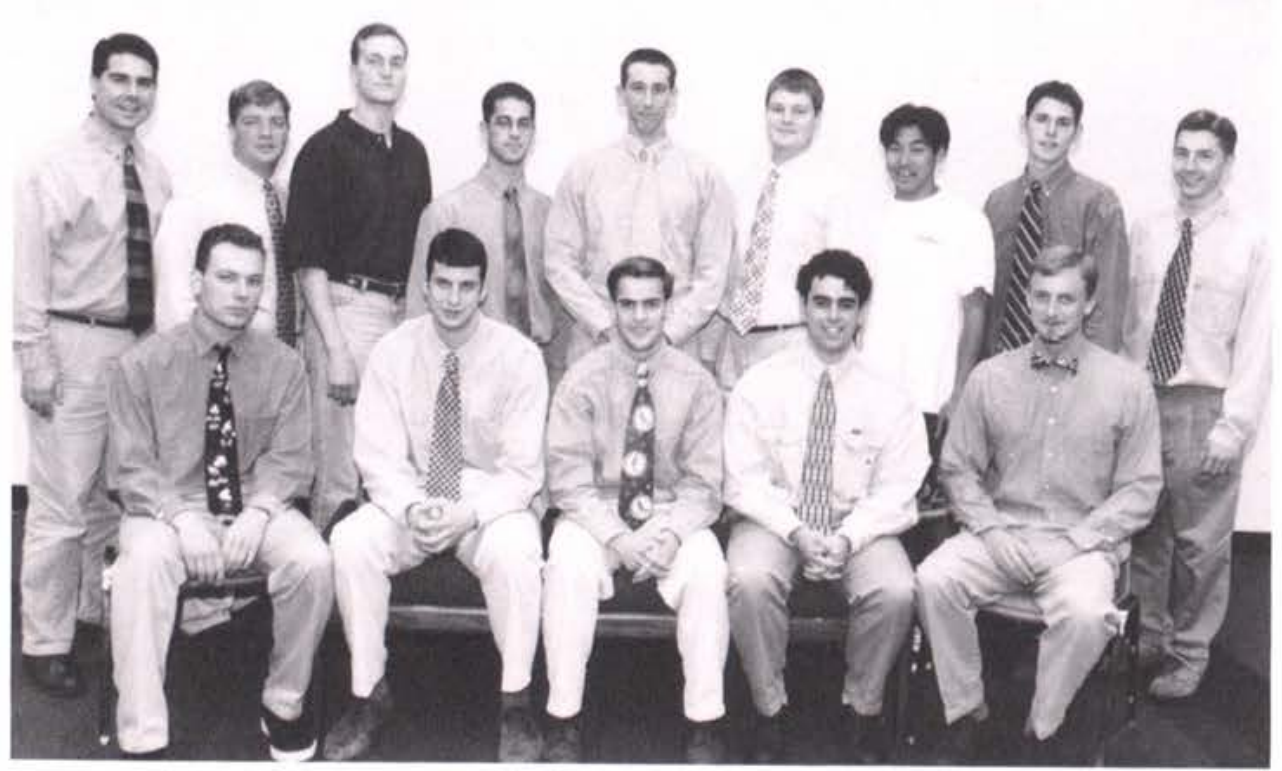




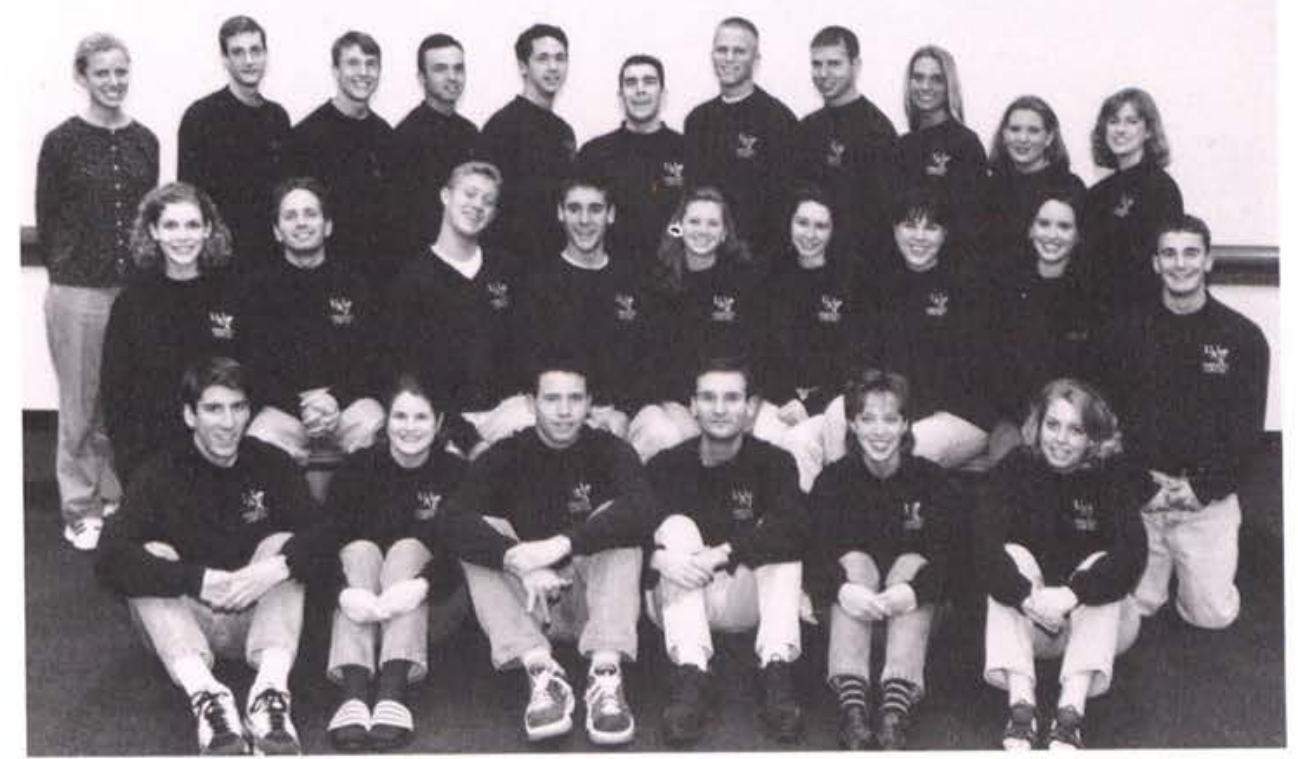

UAA (Undergraduate Alumni Association) assists the CC Alumni Association and office with activities and represents the student body in the Alumni Association.

row 1: Mathew Murphy, Jessica Angelone, Josh Amos, Ryan Anderson, Marianne Palmer, Joy Hasty. row 2: Angie Wenzel, Doug Bayler, Scott Van Loo, Anson Hanbury, Jennifer Ingalls, Elizabeth Price, Sara Gromko, Alissa Whitten, Brad Murray. row 3: Lisa Hunsberger, Aaron Armour, Jason Van Heukelum, Mark Wood, Jason Brown, John Richard, David Rooke, Josh Lunney, Stephanie Newman, Rebecca Patten, Emily Wiljamaa.

Varsity C promotes interac-
tion for varsity athletes, and
runs the concession stand for
basketball games.
row 1: Laura Glessner, Jill Breckenfield,
Lindsey Mitchell, Denver Seely, Travis
Mulanax, Michelle Dick, Megan Stevens.
row 2: Chris Leverette, Josh Bell, Mark
Wood, Josh Lunney, David Rooke, Doug
Amundson, David Swasey, Nathan Houk.
row 3: Jill Bruin, Michelle Burson, Kari
Persons, Kerri Vander Molen, Jodi
duMonceaux, Noella Fisher, Andie Butz,
Erika Miller, Bonnie Schaefer, Becky
Campbell, Becky Woelk,JuliannePletcher,
Andrew Miller.
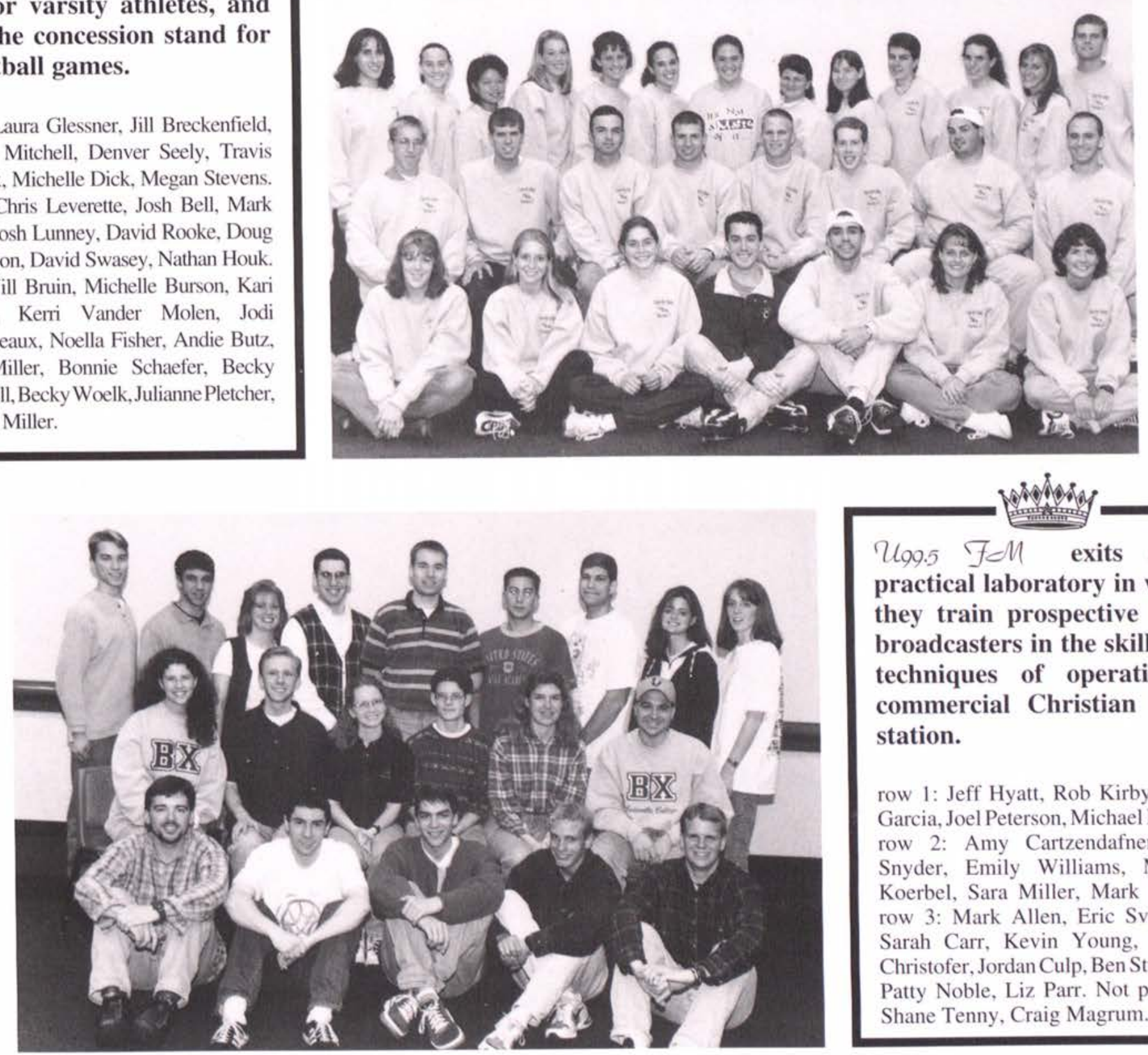

U99.5 $7 \mathrm{CM}$ exits as a practical laboratory in which they train prospective radio broadcasters in the skills and techniques of operating a commercial Christian radio station.

row 1: Jeff Hyatt, Rob Kirby, Steve Garcia, Joel Peterson, Michael Boehm. row 2: Amy Cartzendafner, Josh Snyder, Emily Williams, Michael Koerbel, Sara Miller, Mark Button. row 3: Mark Allen, Eric Svendsen, Sarah Carr, Kevin Young, Brenon Christofer, Jordan Culp, Ben Stutzman, Patty Noble, Liz Parr. Not pictured: Shane Tenny, Craig Magrum. 

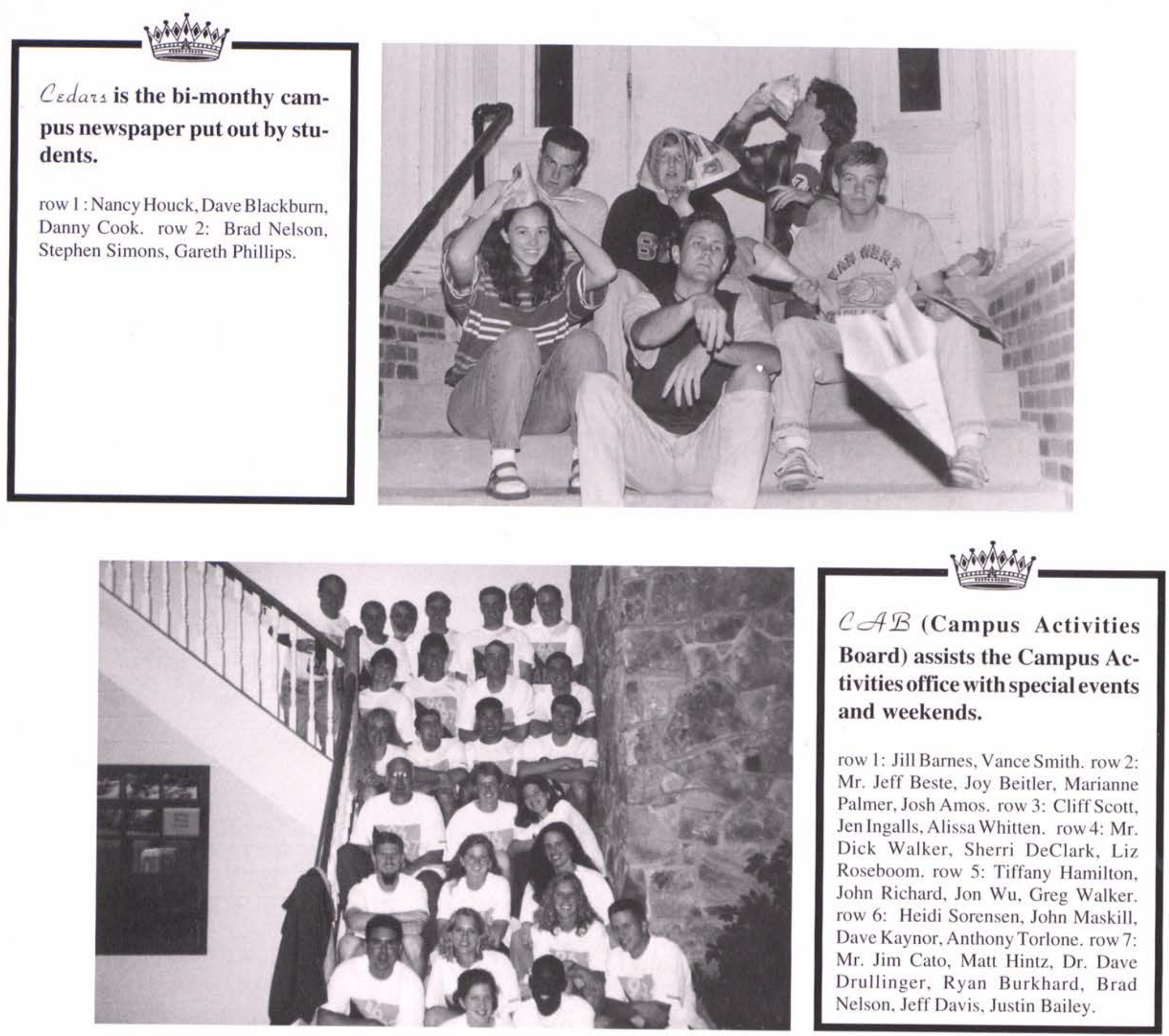
renging

$C Z$ a campus spirit organization that seeks to promote enthusiasm and pride in college traditions and events.

row 1: Dave Kaynor, Sherri DeClark, Brad Nelson, Ellen Burkhard. row 2: We'd have to kill you if we told.

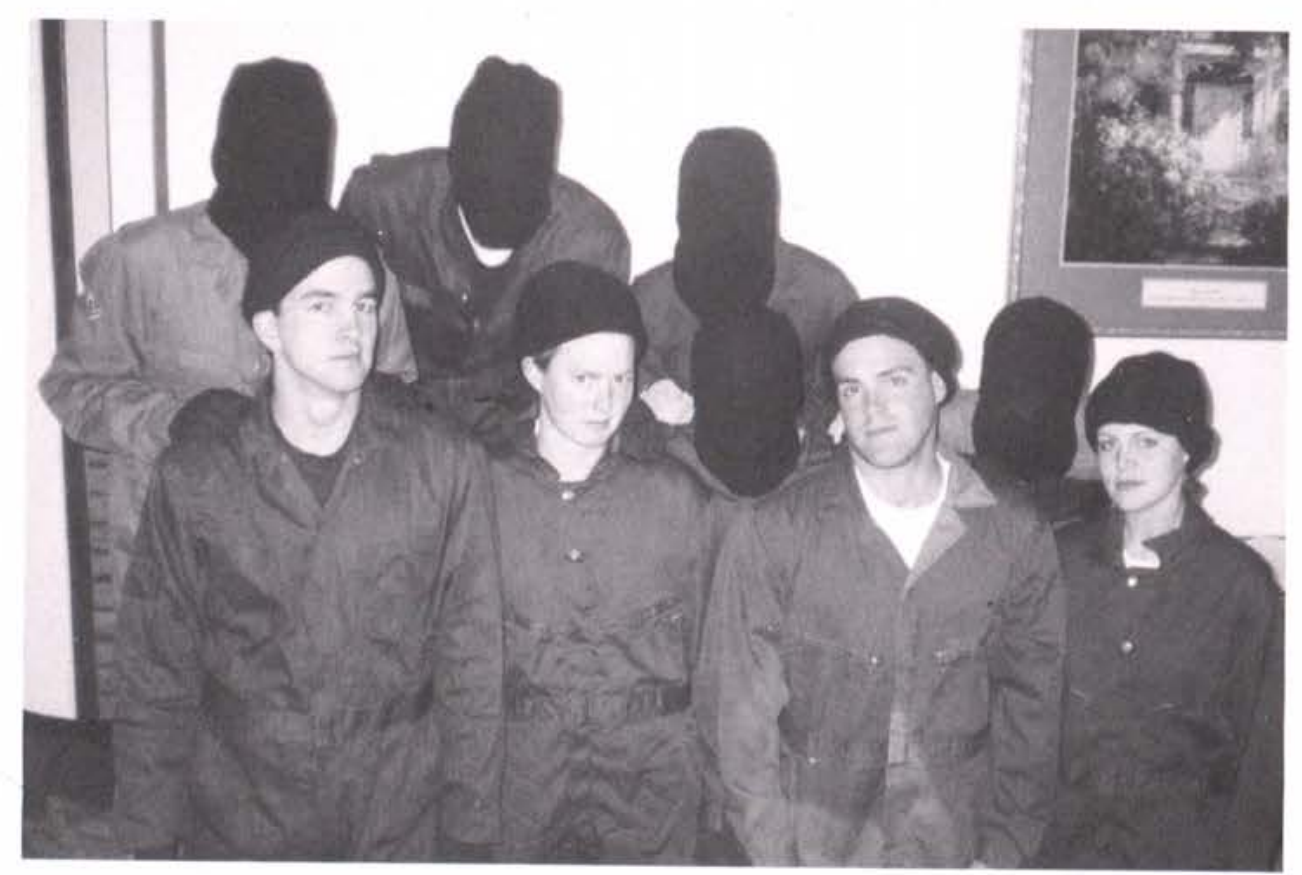




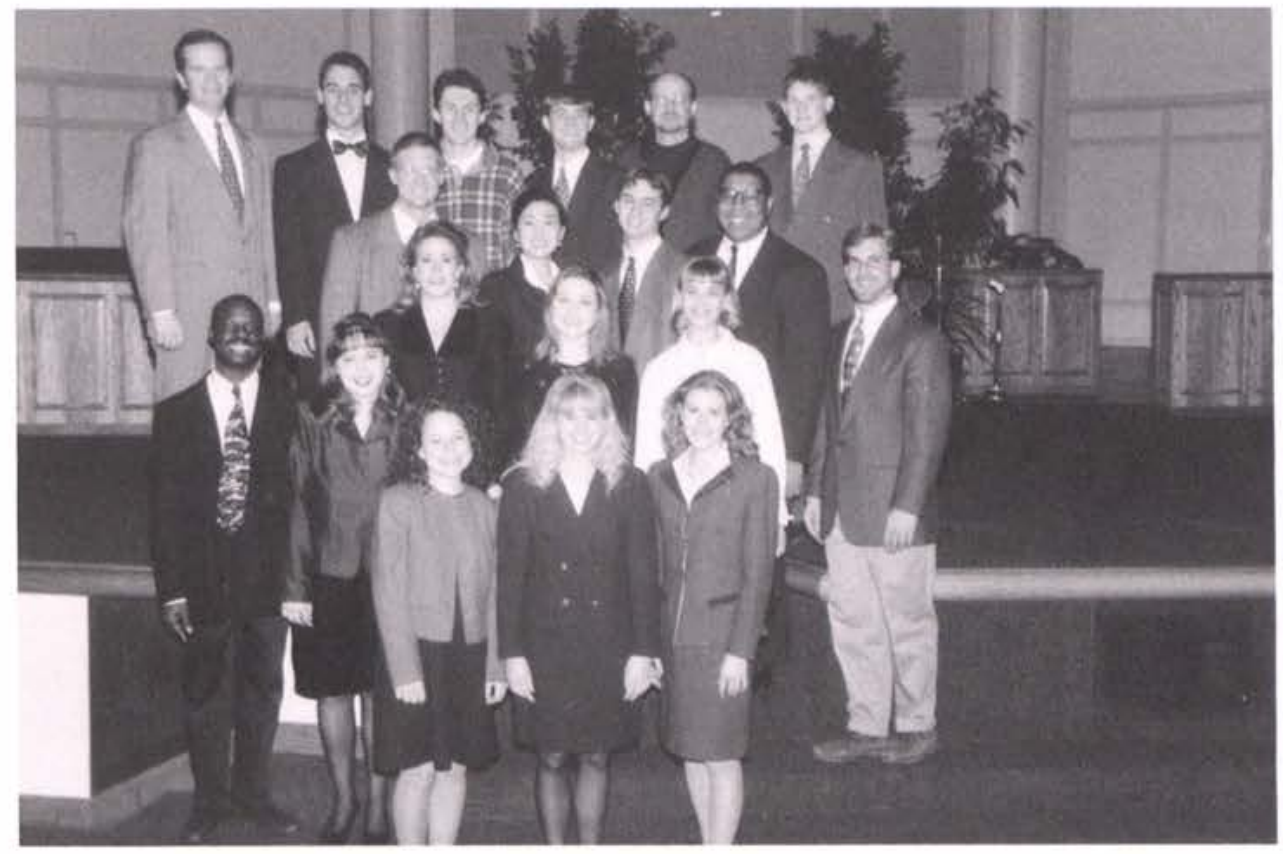

onoso

Forensics students, (involved
in Inter-collegiatespeech com-
petition,) had a very success-
ful year finishing second in
Ohio after The OhioState Uni-
versity.
row 1: Kelsey Perkins, Julie Forstrom,
Becky Crosson. row 2: Derrick
Green, Sara Romang. row 3: Beth
Schloegel, Stacy Saville, Heidi Jo
Dean, Eric Sorensen. row 4: Jamin
Ferner, Ruthanne Pierson, Kevin
Meadows, Michael Dorsey. row 5:
Dr. David Robey, Anson Hanbury,
Matt Rohrer, Daniel Scott, Mr. Gary
Barker, Jeremy Brown.

Defate team members com-
pete as teams and individuals
in debate tournaments
throughout the year.
row 1: Jeff Motter, Jesse DeConto,
Brian White. row 2: Jason Brown,
Heather Smith, Allison Smith, Christa
Jennings, Sara Romang, Jenny Lutz,
Laura Foeldvari, Deborah Haffey-
coach. row 3: Brooke Higgins, Matt
Comrie, Jonathan Hammond. not pic-
tured: Erik Larsen, Joy Wickholm,
Christy Farris, Katie Moon.
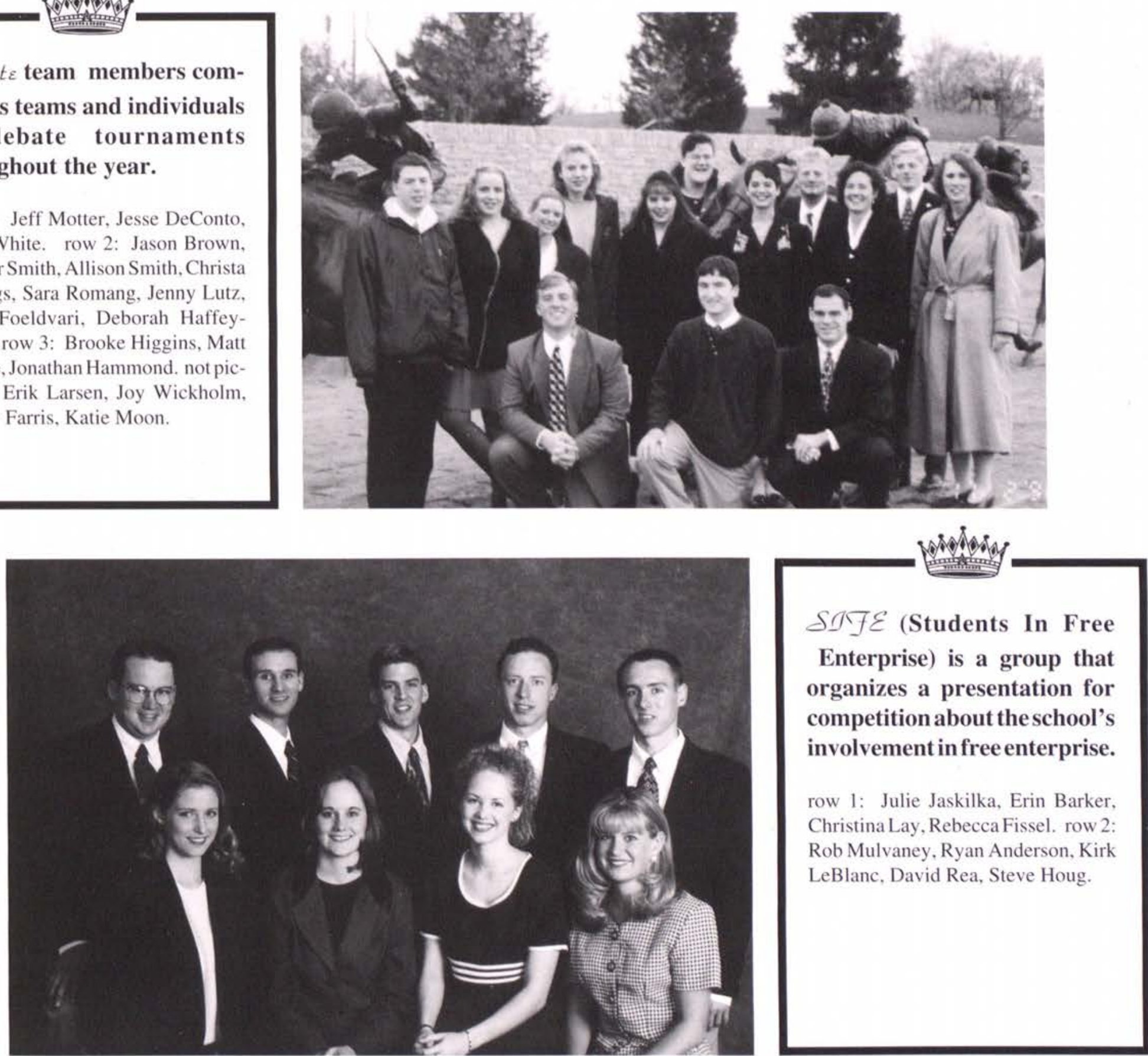

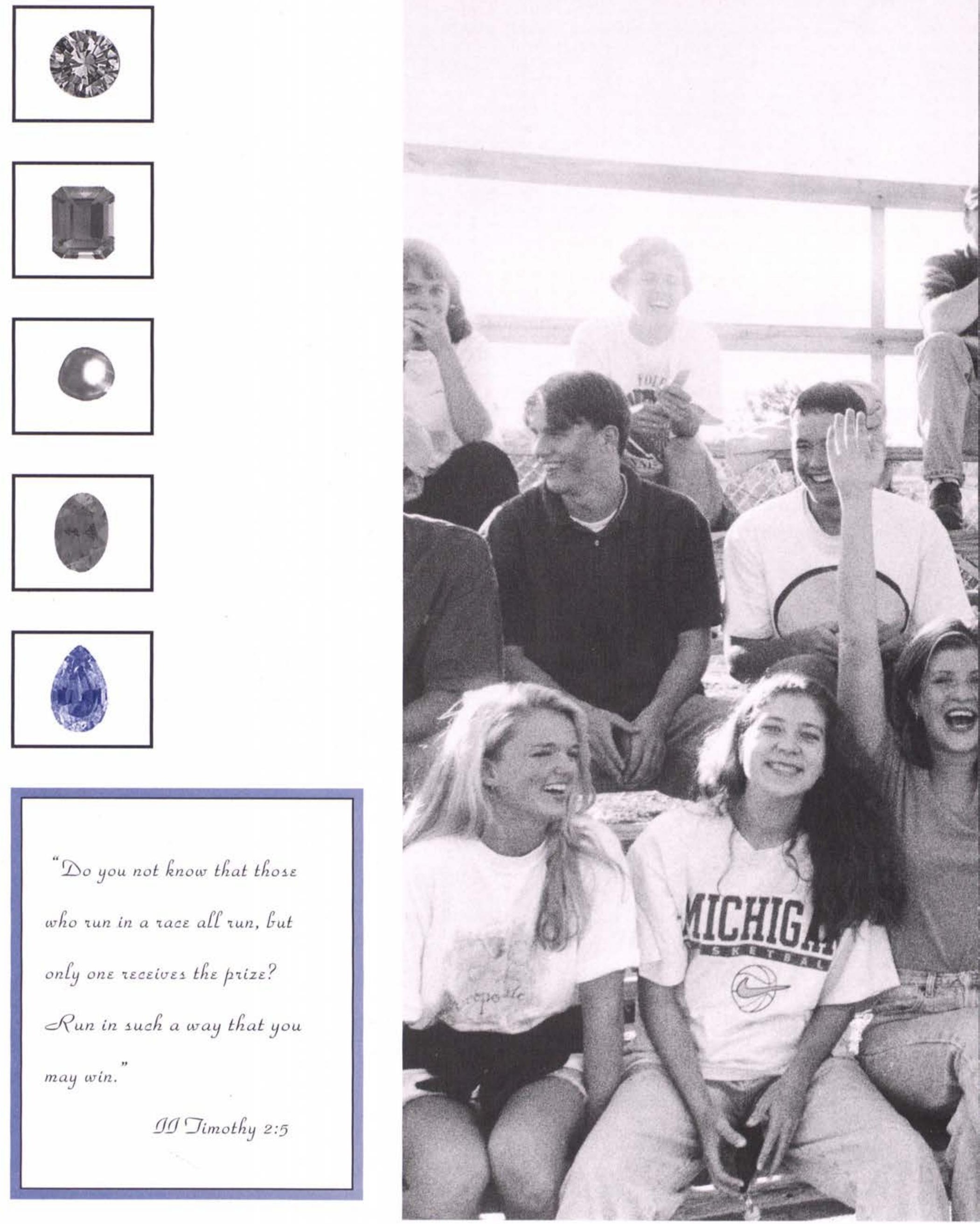

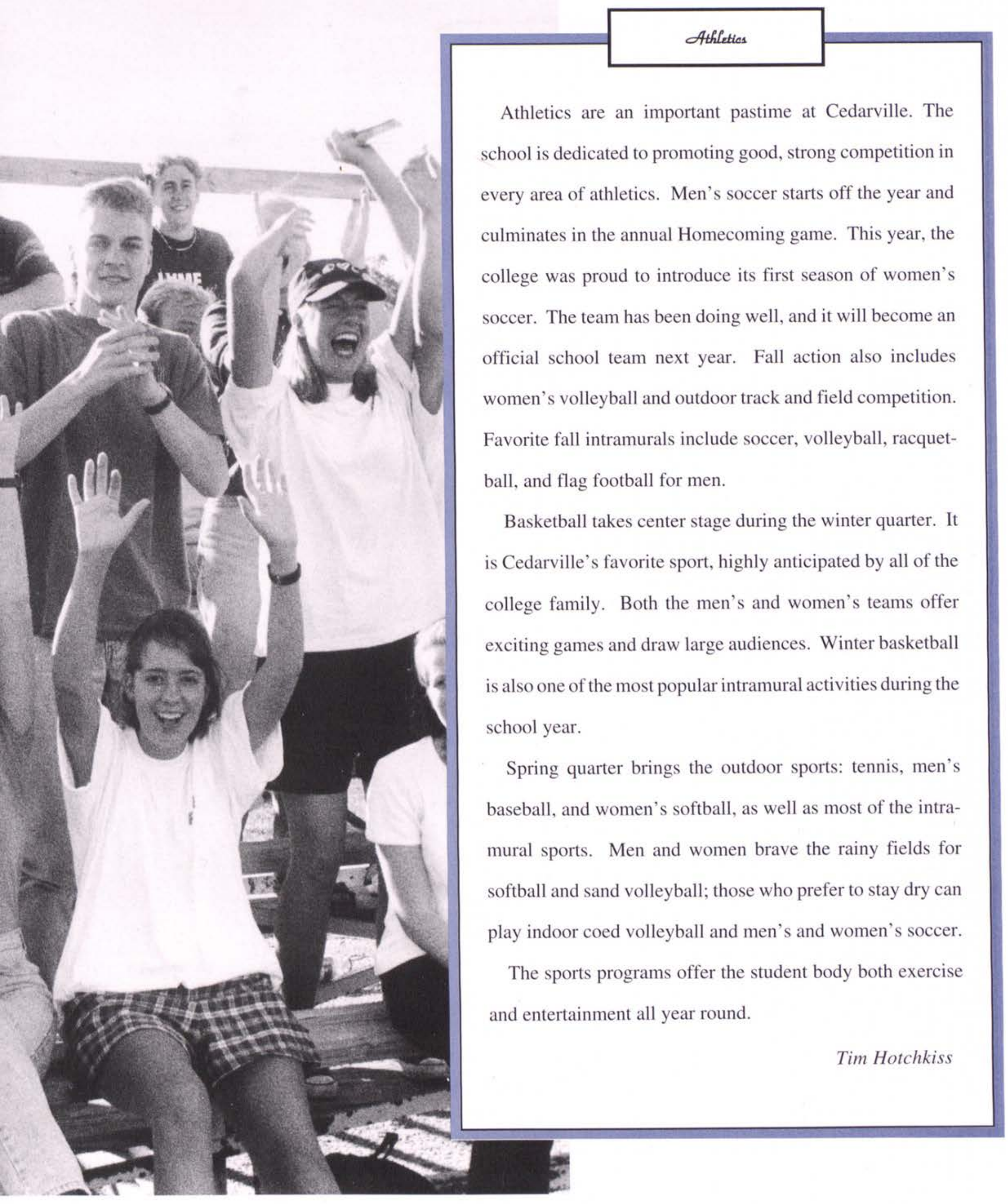

Athletics are an important pastime at Cedarville. The school is dedicated to promoting good, strong competition in every area of athletics. Men's soccer starts off the year and culminates in the annual Homecoming game. This year, the college was proud to introduce its first season of women's soccer. The team has been doing well, and it will become an official school team next year. Fall action also includes women's volleyball and outdoor track and field competition. Favorite fall intramurals include soccer, volleyball, racquetball, and flag football for men.

Basketball takes center stage during the winter quarter. It is Cedarville's favorite sport, highly anticipated by all of the college family. Both the men's and women's teams offer exciting games and draw large audiences. Winter basketball is also one of the most popular intramural activities during the school year.

Spring quarter brings the outdoor sports: tennis, men's baseball, and women's softball, as well as most of the intramural sports. Men and women brave the rainy fields for softball and sand volleyball; those who prefer to stay dry can play indoor coed volleyball and men's and women's soccer.

The sports programs offer the student body both exercise and entertainment all year round. 


\section{Setting the}

The Cedarville College women's cross country team has enjoyed a prosperous and exciting season. The team won the Mid-Ohio Conference meet, which automatically qualified them for the NAIA nationals with an at-large bid. At the MOC meet, seniors Michelle Burson and Julianne Pletcher and junior Becca Jenks were named All-MOC. Sophomores Becky Jordan and Christy Taylor were named Honorable Mention MOC.

After the MOC meet, the team ran at the NCCAA National Meet at John Bryan State Park, where they placed first. Pletcher, Jordan, Burson, Jenks, and senior JillZenner earned All-American honors. Pletcher was the National Cham-

\section{Pace}

\section{Women's Cross Country}

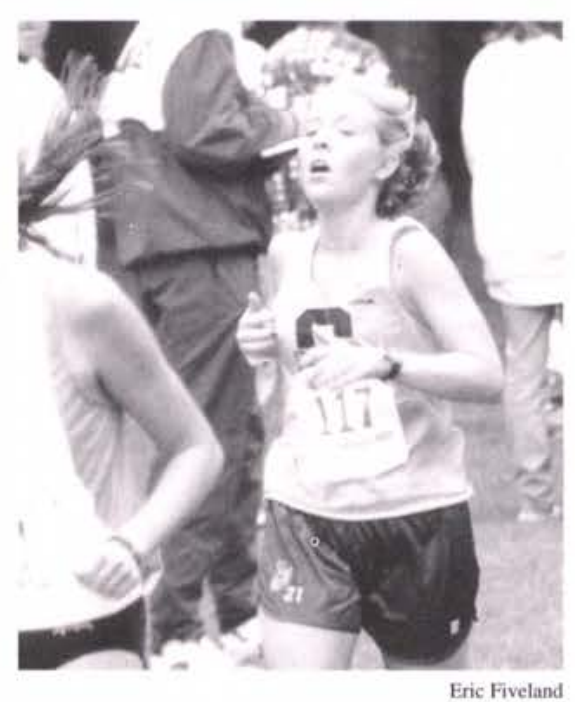

pion. She was also selected as the Wheeler Award winner, which recognized her as the best Christian college runner in the area of academics, Christian witness, and leadership.

Following the NCCAA National Meet, the team traveled to Kenosha, Wisconsin to compete in the NAIA Nationals. There the women finished in fifth place - the highest finish ever for the cross country team and the best placing of any Cedarville athletic team in NAIA competition. Pletcher finished tenth and Jordan twenty-ninth, both earning AllAmerican honors.

Rachel J. Stewart

left: Jill Breckenfeld represents Cedarville at the Friendship Invitational.

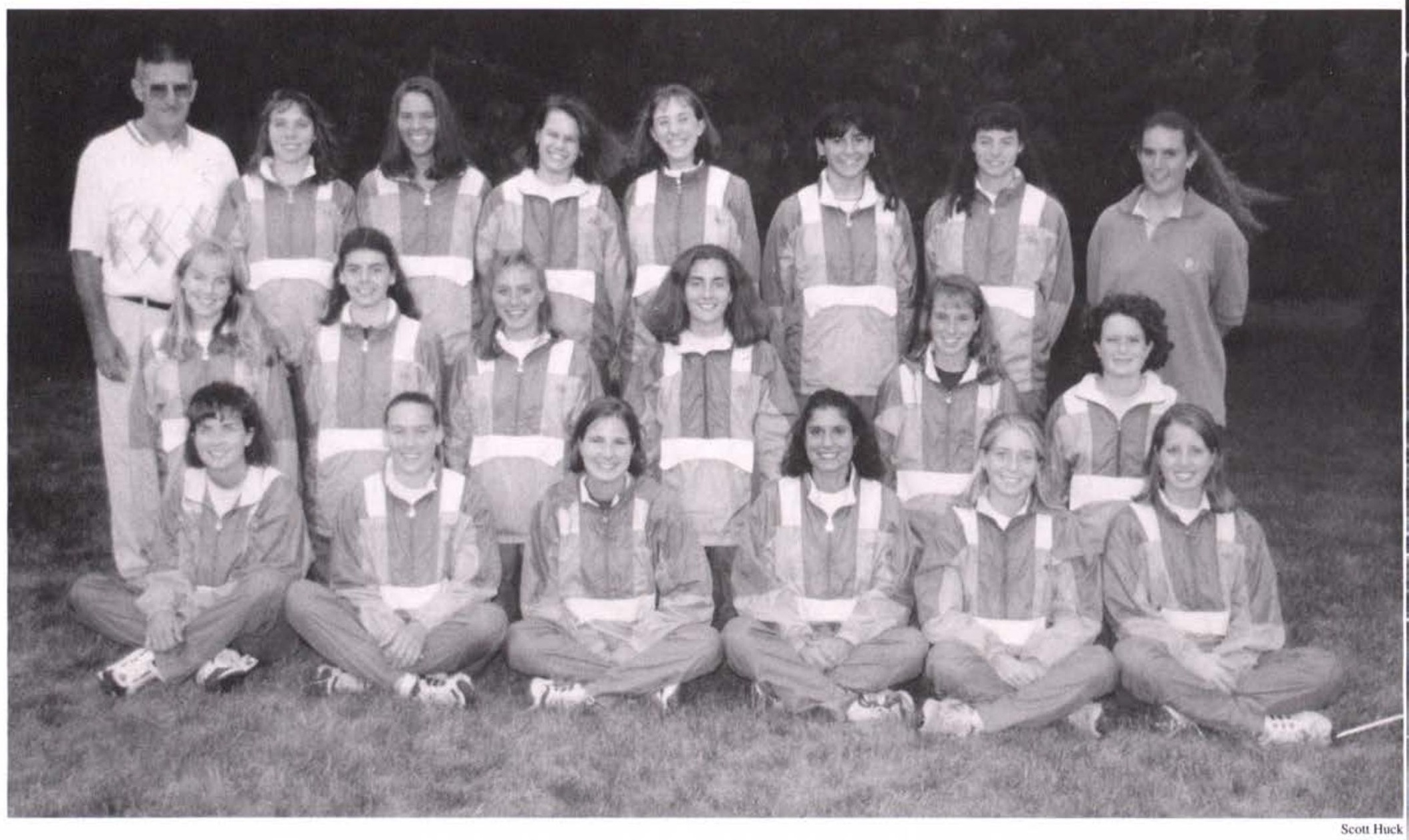

row 1(1-r): Megan Stevens, Michelle Burson, Rachelle Elder, Christy Taylor, Jill Breckenfeld, Jori Forward. row 2: Edith Steele, Rebecca Jenks, Julianne Pletcher, Jessica Chambers, Becky Jordan, Kara Malone, row 3: Head Coach Elvin King, Leah Alley, JeriAnn Goodbar, Nikki Luckmann, Sonya Foster, Jessica Black, Jill Zenner, Student Assistant Becky Woelk. 


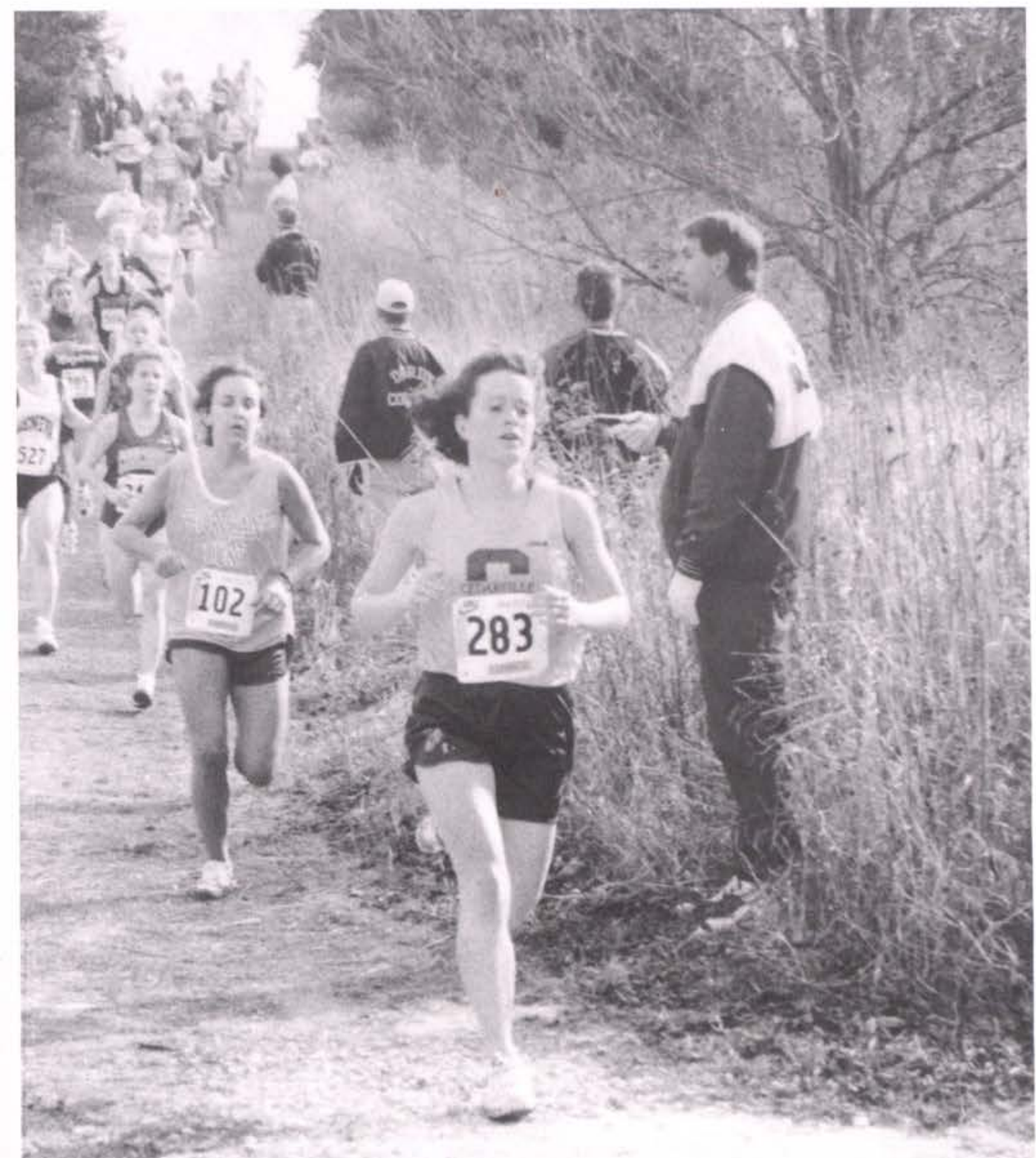

\begin{tabular}{|ll|}
\hline \multicolumn{2}{|c|}{ Statistics } \\
\hline \multicolumn{1}{|c|}{ Meet } & Place \\
\hline Wittenberg Inv. & 1 of 6 \\
Friendship Inv. & 4 of 9 \\
Midwest & 6 of 26 \\
Greensboro Inv. & 1 of 11 \\
Ohio Championships & 5 of 35 \\
Gettysburg Inv. & 1 of 25 \\
Mid-Ohio Conf. & 1 of 6 \\
NCCAA Nat. & 1 of 19 \\
NAIA Nat. & 7 of 31 \\
\hline
\end{tabular}

\section{"This year we gave out wordless book bracelets. It was ex- citing to see four people come to know Christ through this." Rachelle Elder}

left: Kara Malone sets the pace at the NAIA Nationals and pushes herself and the team toward the finish. below (I-r): Jori Forward, Nikki Luckmann, Jessica Black, Jessica Chambers, Christy Taylor, JeriAnn Goodbar, and Sonya Foster take on the Friendship Invitational.

John Ward

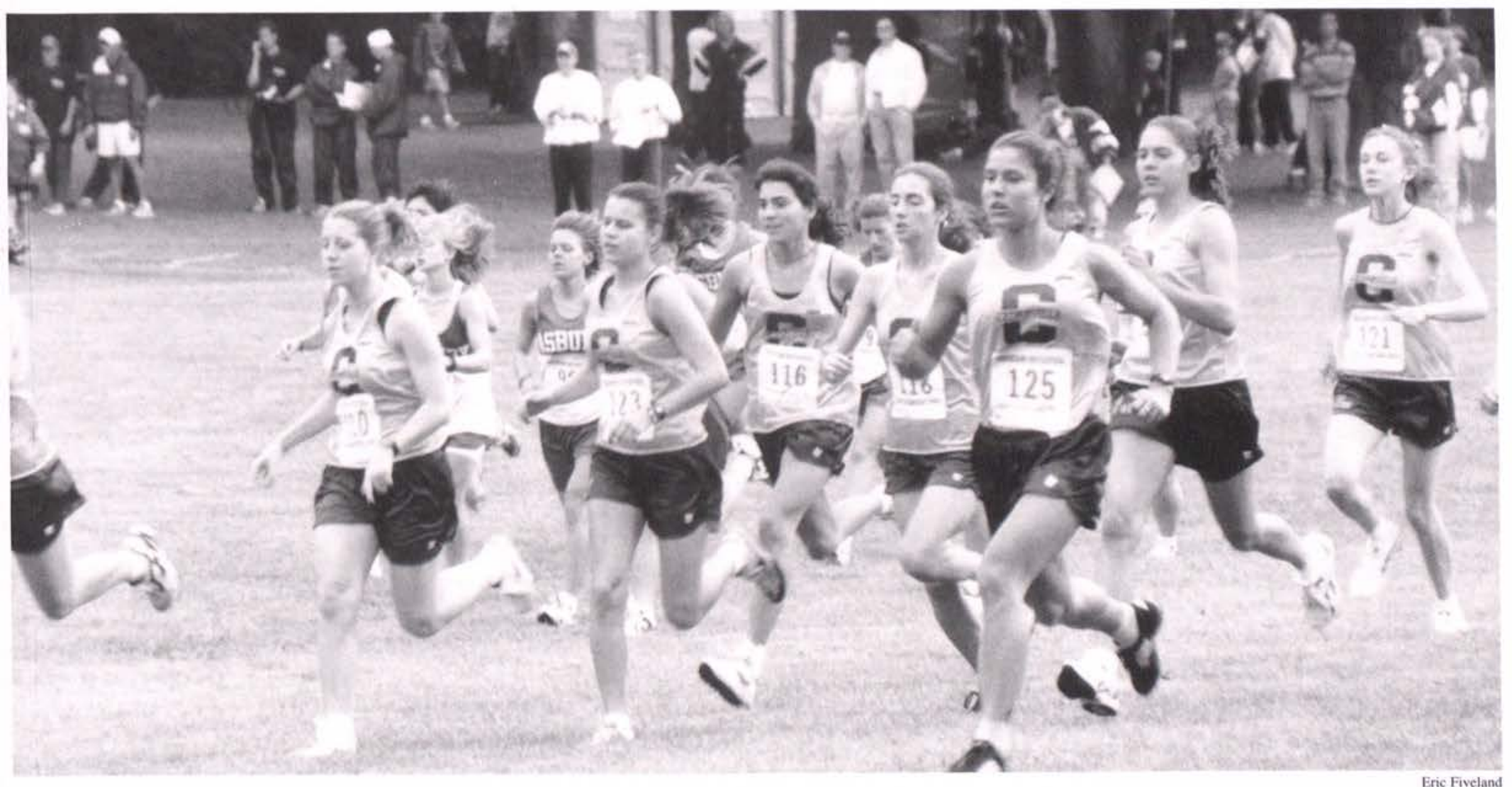




\section{Leading \\ the Pack}

The men's cross country team had a very good 1996-97 season. Members of the team, though faced with both time intensive and physically demanding training regimens, kept up with their academic studies. The team GPA was over 3.0 and one of the athletes maintained a 4.0 throughout the entire season. In addition to academic effort, the athletes found time to continue in personal devotions and invest in ministry opportunities. They took advantage of their weekly meets by sharing the gospel with other athletes competing.

The team consisted of nine members ranging from freshman recruits to junior veterans. Going into the season, Coach Elvin King was confident in the team's abilities and their potential for improvement. "They were even more determined and tenacious than I knew. We deserved to be ranked in the top 25 nationally but the raters wouldn't rank us. We had to beat 16th ranked Walsh in the MOC to have any chance to get to the NAIA nationals and we were not in the top 30 at the time. We beat them, were then ranked seventeenth and did run in the nationals. Each athlete contributed so much to the season that I can't point out one single athlete. They did not lose their confidence even though the raters didn't recognize their accomplishments," he said.
Men's Cross Country
Of the 8 meets in which the team participated during the season, they finished first or second in half of them, and scored highly in the other four. Eric Crawford, a junior, was named NCCAA All-American. Coach King described his team as "consistently improving and determined." In the All-Ohio Championships, the team placed 14th of 36 . The team placed 2 nd of 6 in the Mid-Ohio Conference. After earning a place in the nationals, the team first placed 5 th of 18 in the NCCAA Nationals and then 26th of 34 in the NAIA Nationals.

Voicing his thoughts about the immediate future, Coach King remarked optimistically, "I am excited about next year. We have every athlete back plus a good group of freshman recruits. We have great leadership on the team."

Peter Chevere

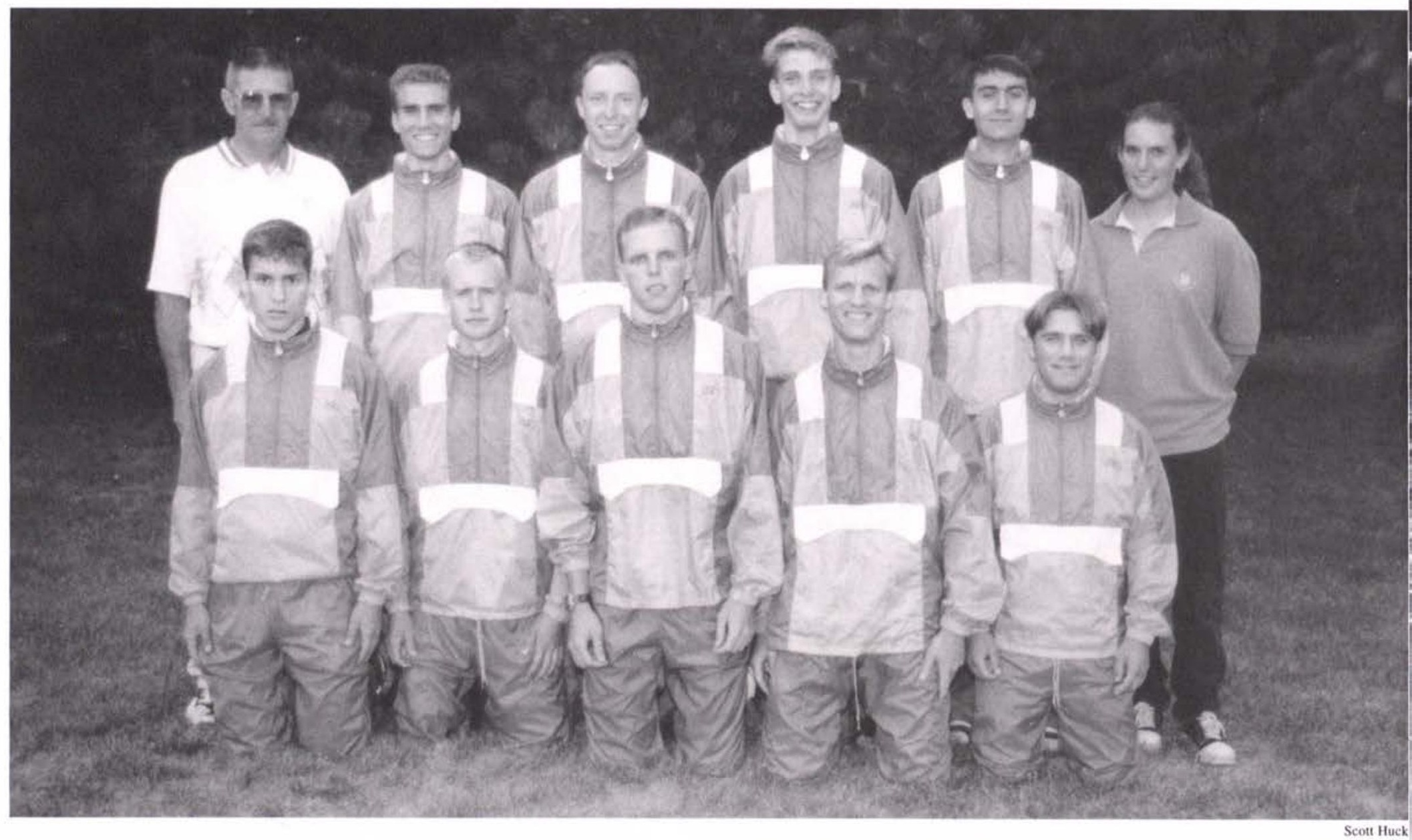

row 1(l-r): Scott Ruhlman, Craig Shank, Chris Merrell, Eric Crawford, Ben Thompson. row 2: Head Coach Elvin King, David Swartzentruber, David Rea, Joel Peterson, Steve McGillivray, Student Assistant Becky Woelk. 


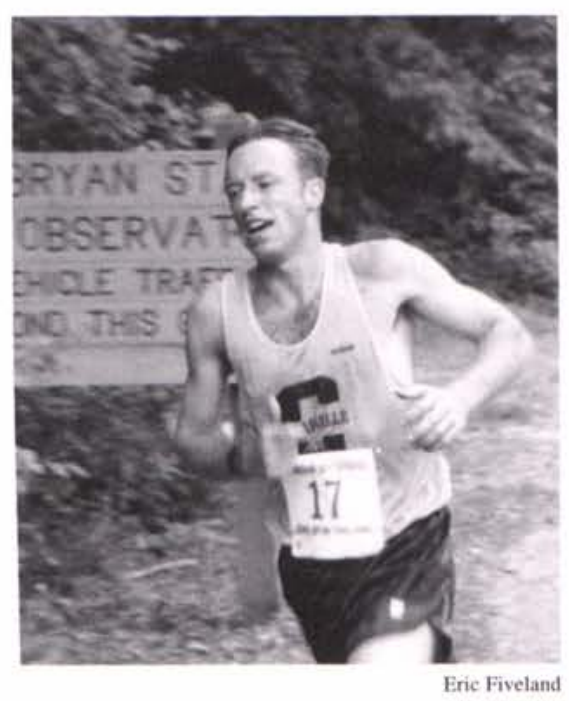

\section{"They were} even more determined and tenacious than I knew."

\section{Coach King}
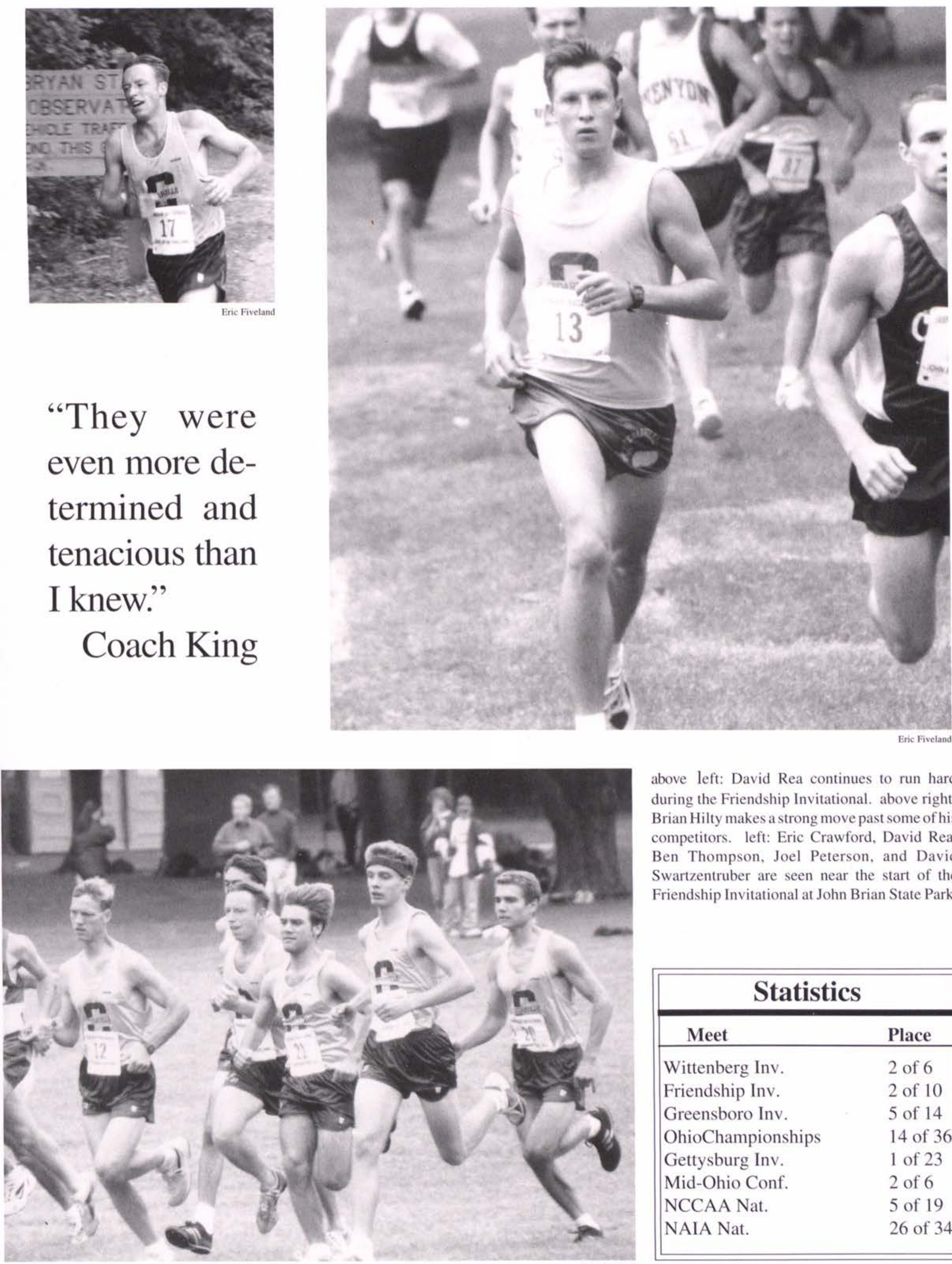

above left: David Rea continues to run hard during the Friendship Invitational. above right: Brian Hilty makes a strong move past some of his competitors. left: Eric Crawford, David Rea, Ben Thompson, Joel Peterson, and David Swartzentruber are seen near the start of the Friendship Invitational at John Brian State Park.

\begin{tabular}{|ll|}
\hline \multicolumn{2}{|c|}{ Statistics } \\
\hline \multicolumn{1}{|c|}{ Meet } & Place \\
\hline Wittenberg Inv. & 2 of 6 \\
Friendship Inv. & 2 of 10 \\
Greensboro Inv. & 5 of 14 \\
OhioChampionships & 14 of 36 \\
Gettysburg Inv. & 1 of 23 \\
Mid-Ohio Conf. & 2 of 6 \\
NCCAA Nat. & 5 of 19 \\
NAIA Nat. & 26 of 34 \\
\hline
\end{tabular}




\section{A New}

Cedarville College added a new athletic team this year by welcoming the women's soccer club to its list of fall activities. The team, founded by senior Ellen Burkhard, has been in the works for over two years, and will reach its ultimate goal next year when, in the fall of 1997 , the women's soccer team will finally become intercollegiate.

Burkhard began her quest for a women's soccer team two years ago when she put together a group of girls to participate in an indoor league in Inglewood, Ohio. The team was open to all women and no tryouts were necessary. Although this was a good experience, Burkhard wanted to pursue other options. She went to John McGillivray, head coach of the men's soccer team, and together the two prepared a proposal for an official women's soccer team at Cedarville. Three

\section{Tradition}

Women's Soccer

weeks before school let out last spring, Burkhard was informed that a club team would be started in the fall of 1996 .

This September, tryouts were held for all women interested in the team. Although over fifty women attended the tryouts, only twenty-five made the final cut. Practices, which ran one hour a day, five days a week, began soon after. The team came under the direction of student coaches Ellen Burkhard and Heather Edwards, as well as the men's team coaches.

In early October, the women competed in their first game against Marion
College of Indianapolis, Indiana. The Lady Yellow Jackets crushed their opponents with an overwhelming 6-0 victory. The team continued to improve over the remainder of the season, and, to keep themselves in shape for next year, the women participated in a winter indoor league.

Burkhard, who began playing soccer on a community team in her hometown several years ago, played midfield and outside for the Yellow Jackets this year, in addition to her coaching duties. Because she has always had an interest in athletics, she is very excited to see her dream of a women's soccer team at Cedarville finally becoming a reality.

Amanda Prusha

Eric Fiveland

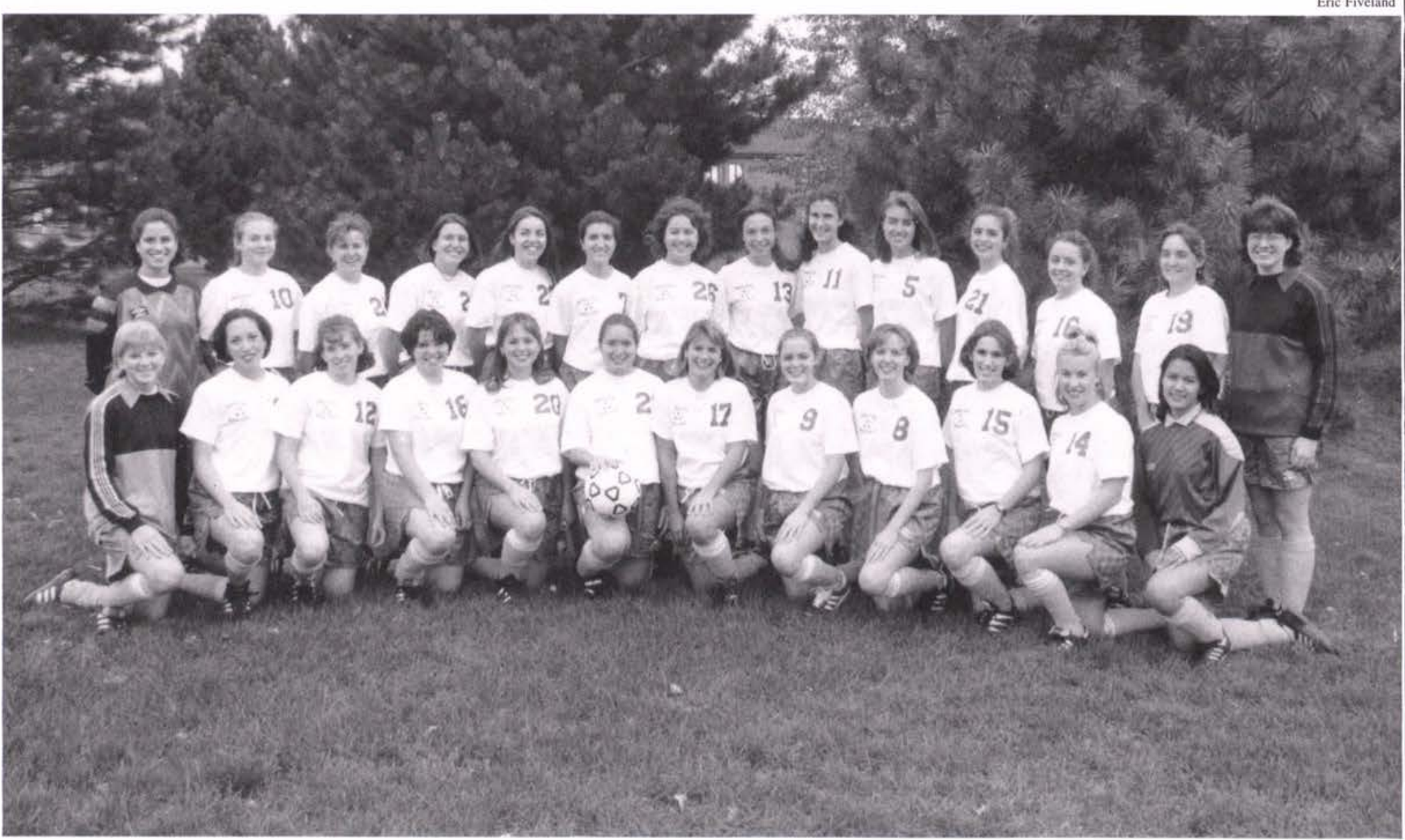

row 1(1-r): Sheryl Buckley, Ehrin Arimura, Heather Edwards, Rachelle Paquin, Rachel English, Ann Reugsegger, Carrie Orme, Ellen Burkhard, Kristine Brown, Kim Howells, Karen Hayes, Anna del Rosario. row 2: Monica Ruth, Andrea Creamer, Rachel Soderstrom, Lisa Thomson, Elizabeth Rogers, Bethany Barch, Rachel Volpe, Melody Gbur, Jessica DeVinney, Meredith Allgrim, Lara Gyurick, Michelle Miller, Jen Wilk, Sarah Hills. 


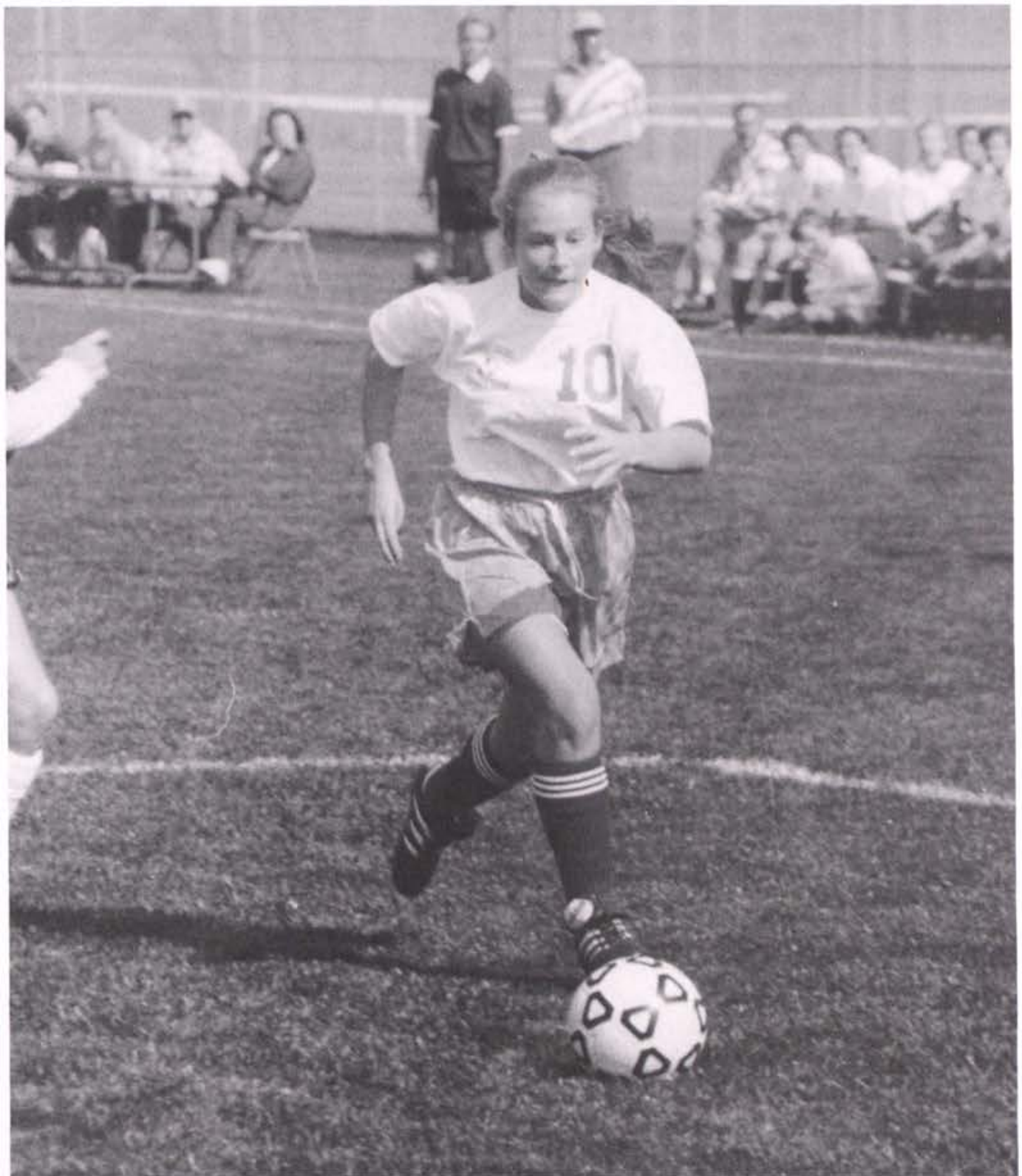

\begin{tabular}{|lc|}
\hline \multicolumn{2}{|c|}{ Statistics } \\
\hline Game & Score \\
\hline Marion College & $5-0$ \\
University of Dayton & $1-2$ \\
Marion College & $9-1$ \\
University of Dayton & $0-6$ \\
\hline
\end{tabular}

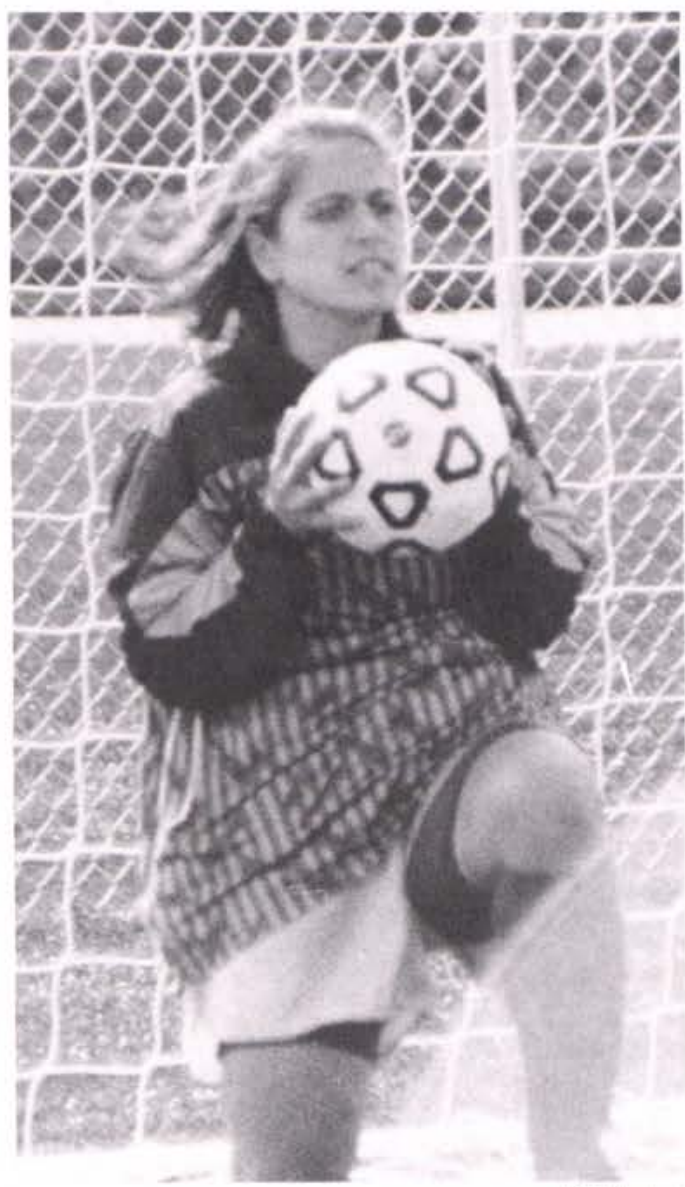

above: Andrea Creamer dribbles the ball down the field, looking for an opportunity to score. above right: Keeper, Monica Ruth makes a save in a game against Marion. right: Melody Gbur moves the ball down the field, warding off her opponent.

"It was so exciting to see the team become a reality. We're thankful for Ellen and her determination in starting the team--I feel her hard work has definately paid off. All of us look forward to becoming a varsity team next year."

Rachel English

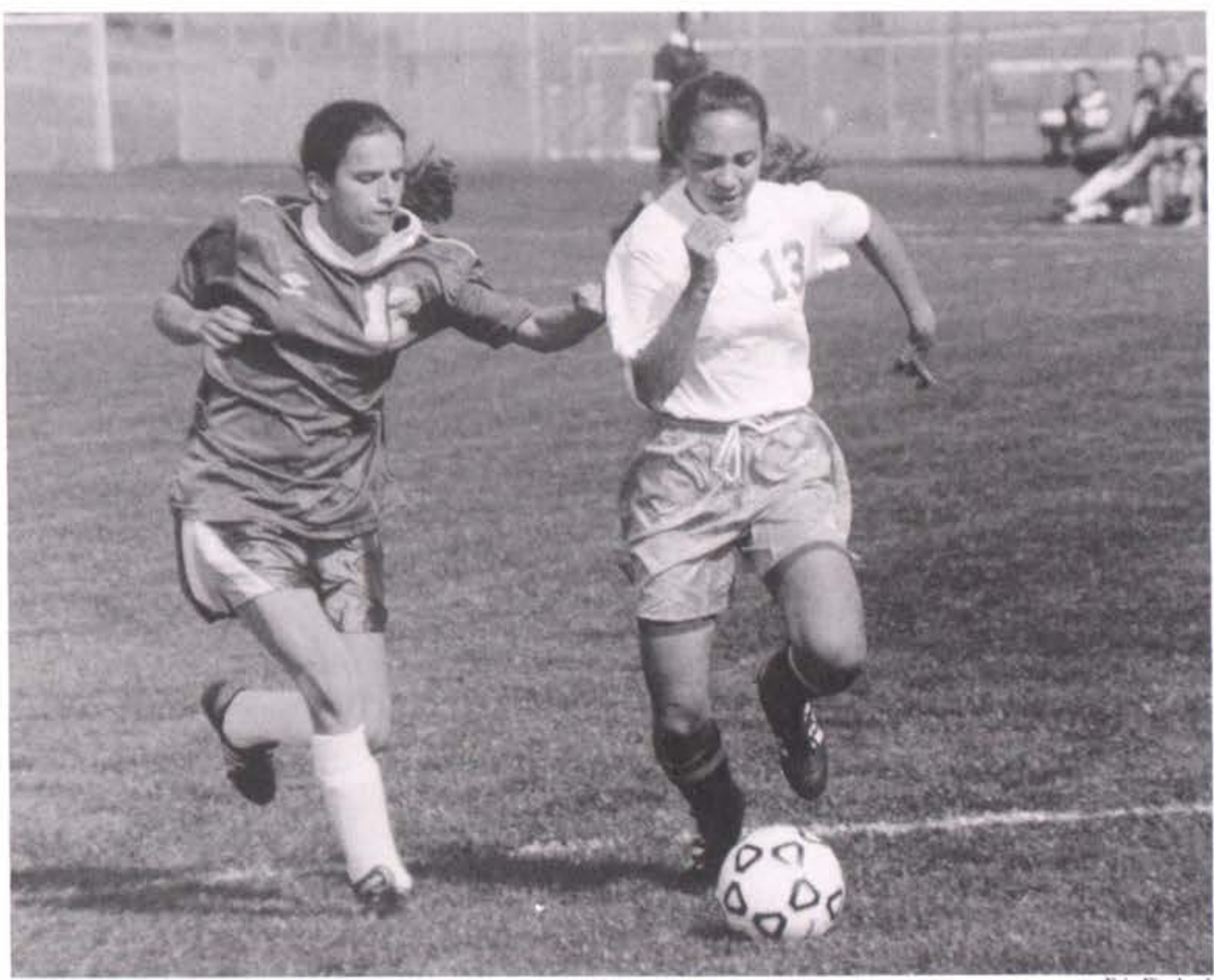


According to Assistant Professor of Physical Education and Coach John McGillivray, this year's men's soccer team included a number of veteran players, and had strong leadership in Senior co-captains Cliff Scott and Matt Towle. The season saw the team get back on track from the year before as they posted a 10-10-2 final win/loss record. The team finished at 3-4-1 in the Mid-Ohio Conference beating Mt. Vernon, Findlay, and Shawnee State. The team faced solid competition early in the season, losing their first two contests at Wittenberg $(0$ 3 ) and Tiffin (0-5). On September 17, the Yellow Jackets played their first home game, winning 1-0 over Mt. Vernon.

The game against Mt. Vernon was not only the team's first victory of the season, but also marked Coach McGillivray's 200th win and his twenty-

\section{Giving it Their Best Shot}

\section{Men's Soccer}

third season as head coach of the Cedarville College men's soccer program. McGillivray said, "The first 100 seemed much easier than the second 100 , but it was great for the guys to win that game and exciting for me." McGillivray has been recognized numerous times for his coaching ability and teams that have demonstrated outstanding sportsmanship.

The team went on from their first win to a streak of five more, but then had several losses. In retrospect the low and high points of the season both came in the last few games. Looking back, McGillivray said, "I think the low point would have to be our close loss to Indiana Wesleyan which, we thought, put us out of the National Tournament. That was what the captains had set before the other guys as our goal and they were obviously very disappointed." Although this was discouraging, news came soon after that Cedarville had received entrance to the tournament by means of an "at large bid" which is based on the scores of the games and the opponents that the team has played. This gave the squad a new burst of energy.

Cedarville lost $0-1$ in the firs round of the NCCAA National Tournament, and then met Indiana Wesleyan for the third time in the season. The teams

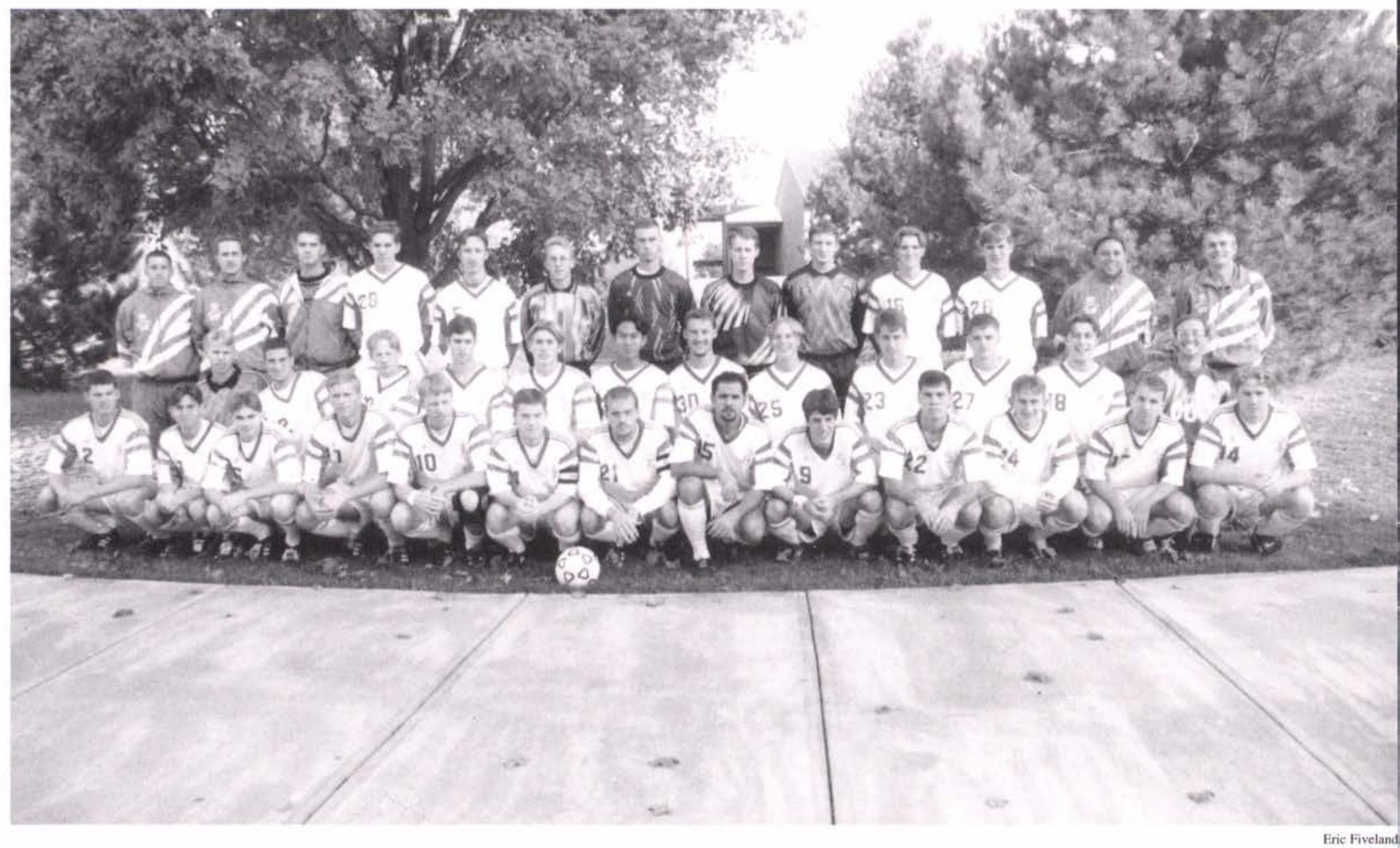

row 1(1-r): Ben Nordaas, Jeff Rockwood, Russ Pound, Dave Rooke, Reade Faulkner, Cliff Scott, Matt Towle, Gabe Sava, Michael Hidalgo, Andy Shaw, Chad Deakyne, Duane Hammond, Dave Rutledge. row 2: Mike Crawford, Jason Tovey, George Weber, Steve Light, Brian Collins, Pete Warinsee, Tim Pfahler, Chris Brock, Derek Chandler, Tim Amstutz, Josh Tackett, B.J. Bechtel. row 3: Roger Swigart (Asst. Coach), Bill Workman (Score Keeper), Fred Ludwig (Trainer), Jed Smith, Jamie Tait, Jeff Barrons, Kevin Yankovich, Brian Gault, Trevor Batt, Dan Bucklew, David Skinner, Kevin Roper (Asst. Coach), John McGillivray (Coach). 

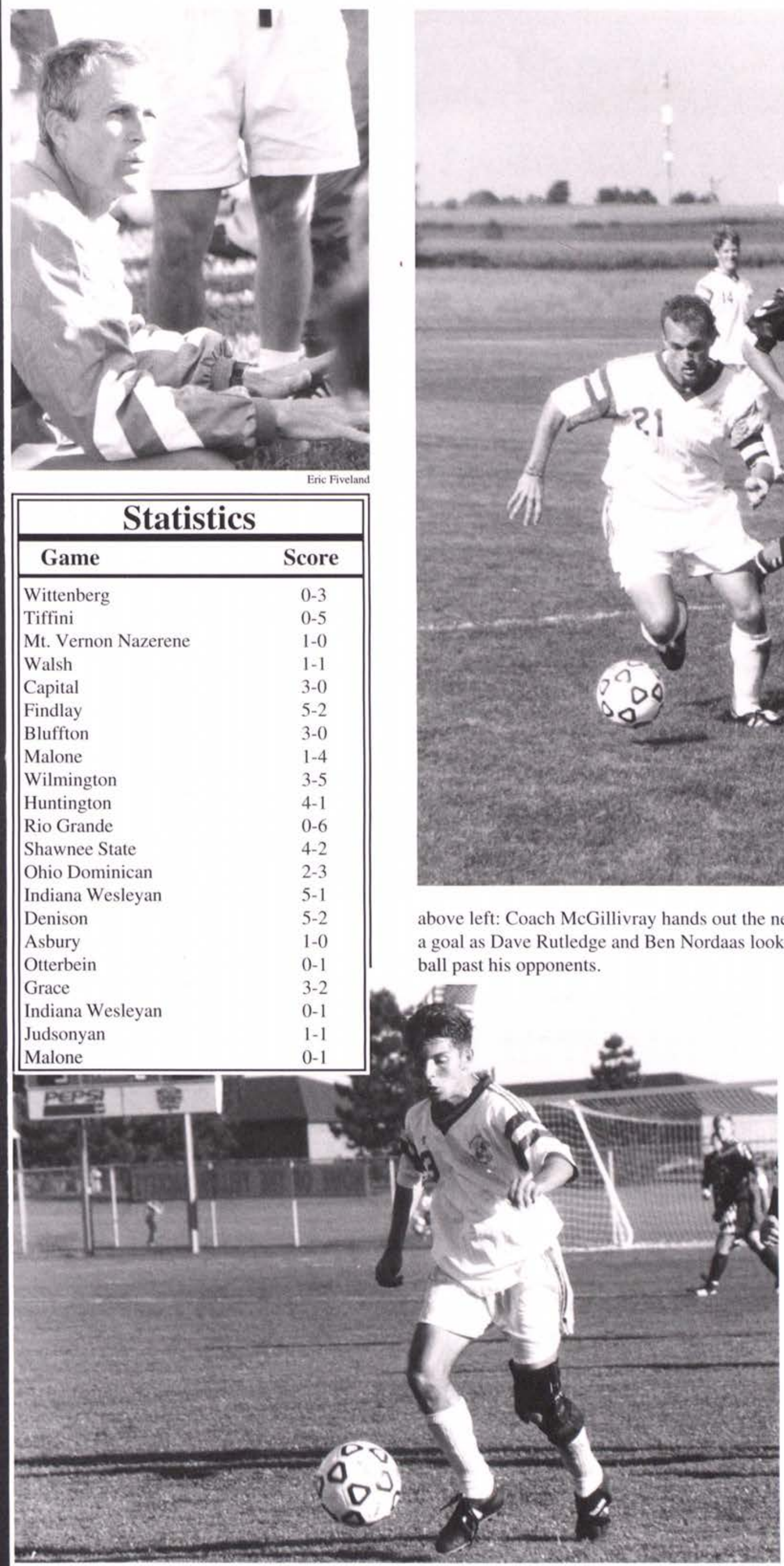

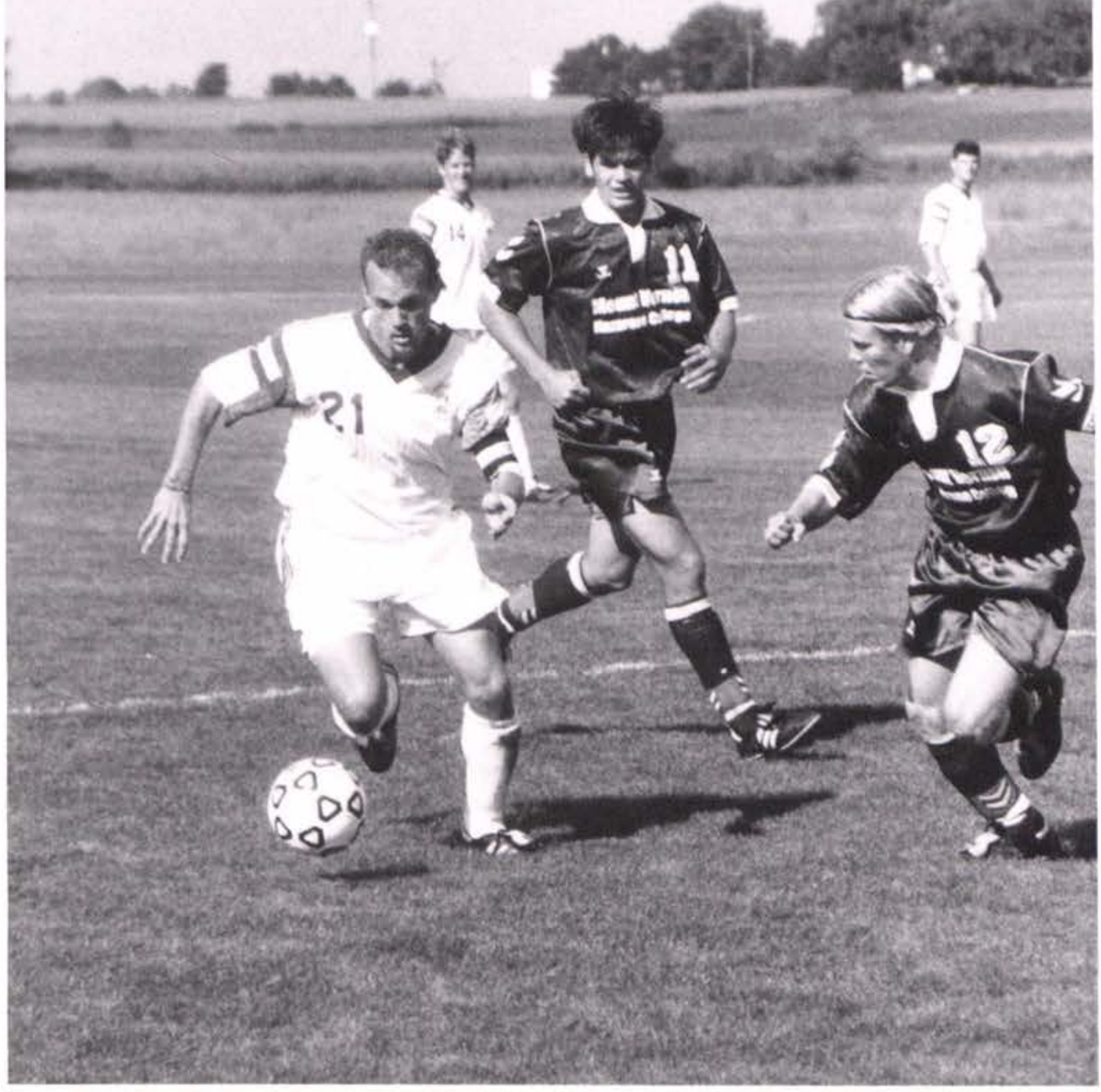

Eric Fiveland ut the next winning strategy. above: Matt Towle goes for goal as Dave Rutledge and Ben Nordaas look on. below: Jeff Rockwood intensely dribbles the struggled to a 1-1 tie, which was decided when Cedarville won the shoot-out 4-2. The Yellow Jackets advanced to meet Malone for fifth place, but lost $0-1$ in a heavy rainstorm. Cedarville ended up receiving the Award for Sportsmanship in the National Tournament.

McGillivray is proud of his team and what they accomplished this year. He gives a great amount of credit to his assistant coach, Roger Swigart and goalkeeper's coach, Kevin Roper, as well as to the team leadership of captains Scott and Towle for motivating their teammates and maintaining positive attitudes throughout the season. McGillivray is optimistic about what he can expect from the team in future.

Peter Bednarek 


\section{Successful}

Under the leadership of new head coach Teresa Clark, the women's volleyball team finished its season with a record of 31-15.

Although this is Clark's first year at Cedarville, she brought with her seven years of coaching experience from Blackhawk Christian School in Fort Wayne.

Once the initial transitional hurdles were overcome, the season had several highlights. For the first time since its introduction nine years ago, the team won the Cedarville College Invitational. Also, in a major victory for the Yellow Jackets, rival Mount St. Joseph was defeated this year on its own home court.

The only home loss was to Walsh, which finished first in the Mid-Ohio

\section{Transition}

Volleyball

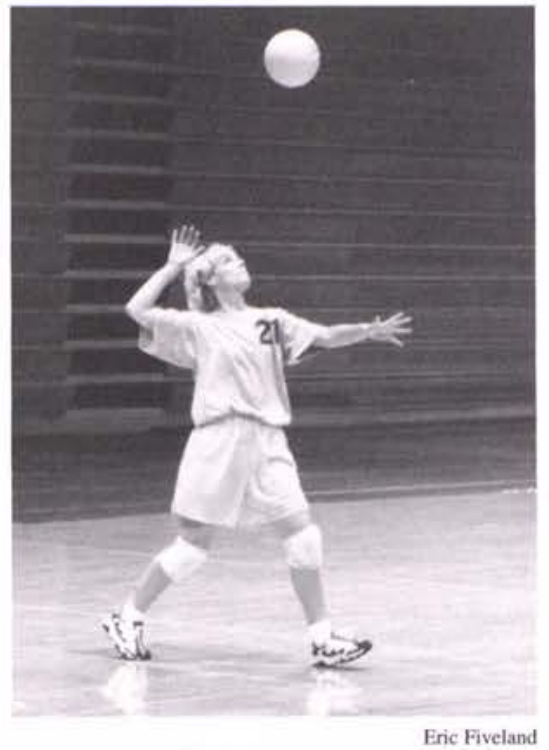

Conference. This year, Cedarville placed third in the MOC, an improvement over last year's fourth place standing.

In the NCCAA, the team was district runner-up to Grace College. The deciding game was played during Grace's Homecoming Weekend, giving Grace the ultimate home court advantage.

An outstanding highlight for the team was to receive the at-large bid for the NCCAA nationals, held at Lee College in Tennessee. Clark said it was interesting that the teams Cedarville lost to were the top three teams, Christian Heritage College, Grace College, and Western Baptist College, respectively. Cedarville held right with them throughout the tournament.

\section{Chris Robertson}

left: Marcie Curry puts the ball into play with a powerful overhand serve.

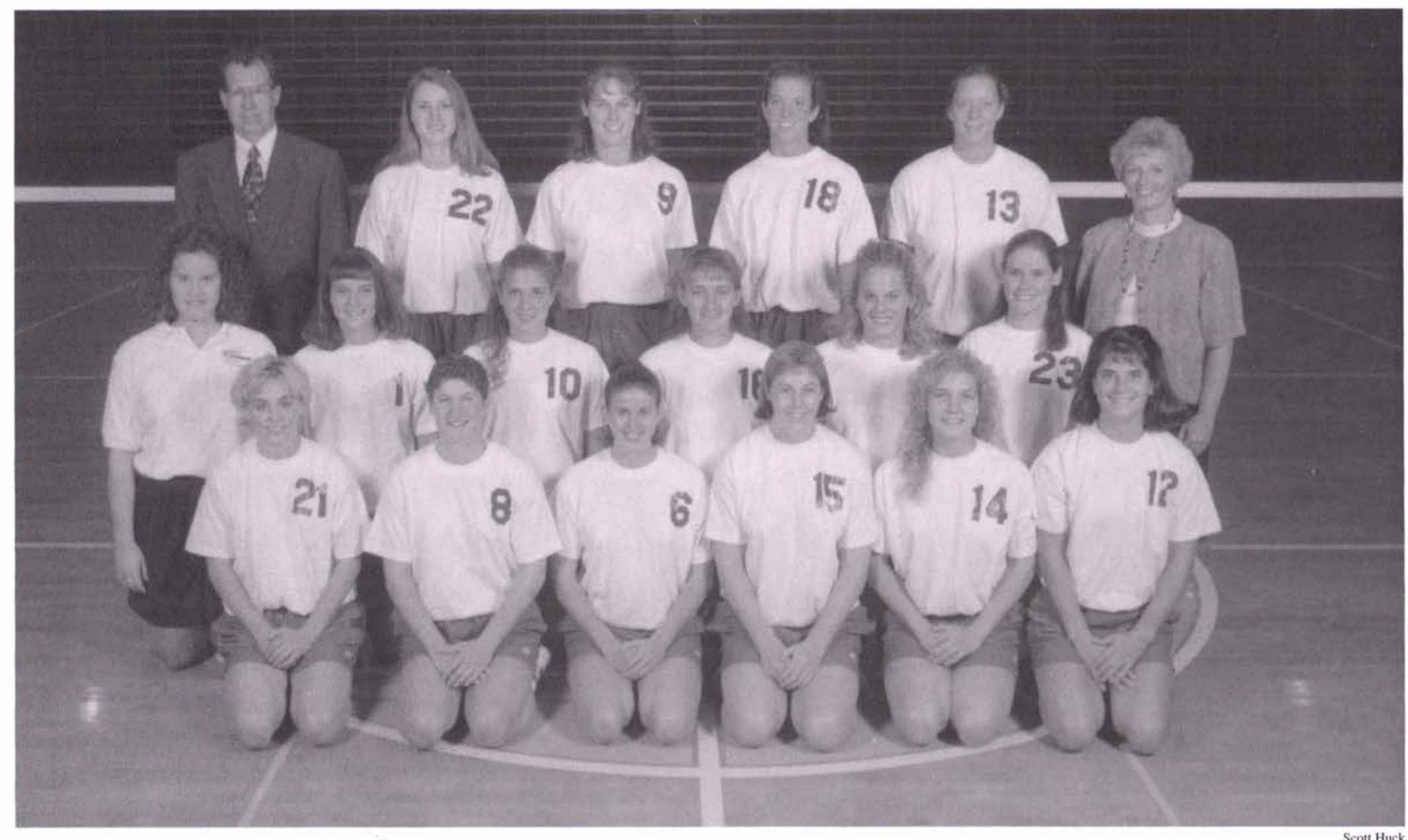

row 1(1-r): Marcie Curry, Rachel Tilton, Amanda Johns, Casey Ruffin, Jody Thompson, Melissa Sprankle. row 2: Student-Trainer Ruthann McAuley, Sarah Jackson, Christine Scheffel, Suzanne Lehman, Julie Barkhaus, Beth Comer. row 3: Assistant Coach Jim Clark, Heather Scheffel, Miranda Woller, Lisa Weirich, Julie Opperman, Head Coach Teresa Clark. 


\section{One Challenging}

The Cedarville women's basketball team faced an extremely challenging 1996-1997 season. The women did not back down, but instead took each game as an opportunity to gain experience and steadily improve.

It is impossible to measure many of the improvements simply by a scale of wins and losses. The Lady Yellow Jackets lacked some of the leadership on the court which they had the previous season and they also suffered a variety of injuries. The women worked hard in spite of adversity. Assistant Professor of Physical Education and Head Coach Kathy Freese spoke highly of the team's work ethic and desire to improve. "The team worked hard. There were tough times, but we have tough times in life and it is during those times that we call upon the Lord so that we can witness the growth he brings about," said Freese. She was always proud of the way her team constantly demonstrated strong Christian character both on and off the court.

\section{Season}

\section{Women's Basketball}

The team picked up three of their four wins early in the season, and all of the wins came at home. Although this year's team was without the help of three seniors who contributed greatly in the previous season, the current seniors provided many solid performances for the team. Co-captains Amy Bathrick and Jill Detwiler were the leading scorers for the Lady Yellow Jackets, and each received special honors for their play in the Conference. Bathrick was named to the first team All Conference (MOC) and was fifteenth in rebounding; Detwiler made the second team. Freese said, "The two captains did an excellent job with the team as far as their leadership off the floor and their contribution on the court."
Junior Summer Bennington brought experience to the team's inside game. The team also had valuable contributions from the sophomore duo of Heather French and Gillian Gombis.

Like the men's basketball team, the women had many new freshmen in the ranks; the total was six. Charity Cole provided a much needed offensive spark at times with her ability to steal the ball, move it quickly, and create openings for her teammates, while Loree Beth Fraley maintained a good outside game. Jodi Quint also developed her game in the post grabbing rebounds through many quality minutes. The addition of these freshmen and Junior transfer Jodee Howard definitely make the outlook for next year bright.

The combination of young players and many injuries would have been enough to make any season seem difficult, but the added difficulty of the Yellow Jacket's schedule increased the challenge. Not only was the Conference competition ex-

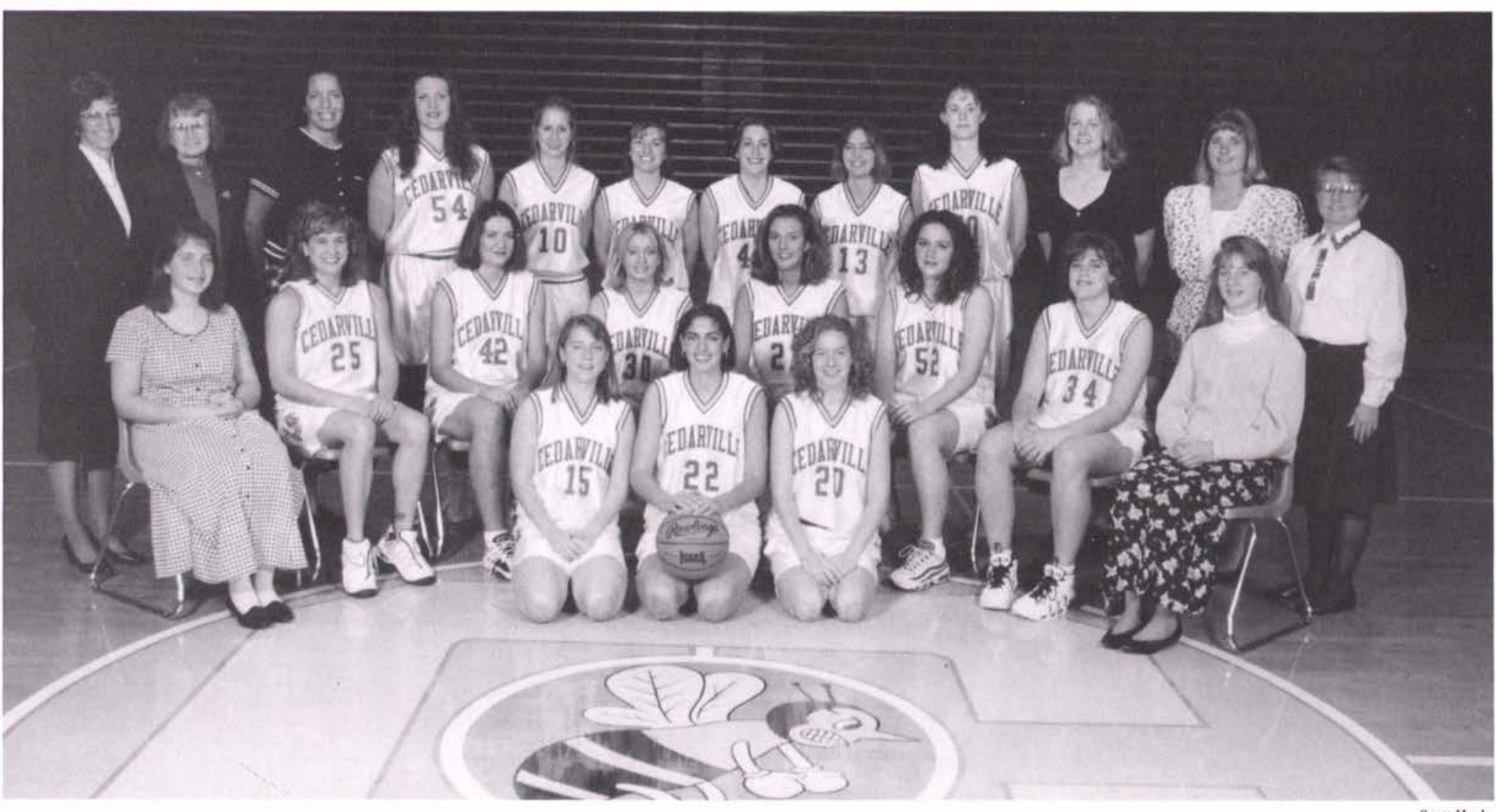

row 1 (1-r): Heather French, Gillian Gombis, Amy Egolf. row 2: Student-Trainer Brenda Johnson, Jodie Howard, Robyn Paulin, Jill Detwiler, Amy Bathrick, Summer Bennington, Elizabeth Hamilton, Student Assistant Tammi Matula. row 3: Head Coach Kathy Freese, Team Chaplain Martha Baldwin, Manager Kelly Ford, Jessica Hooper, Natalie Royer, Loree Beth Fraley, Charity Cole, Tara Swaney, Jodi Quint, Scorekeeper Theresa Olson, Assistant Coach Beth.Callinan, Assistant Coach Joy Williams. 


\begin{tabular}{|lc||}
\hline \multicolumn{2}{|c|}{ Statistics } \\
\hline Game & Score \\
\hline Wilberforce & $74-66$ \\
Georgetown & $58-89$ \\
Olivet Nazarene & $42-68$ \\
Trinity Christian & $58-63$ \\
Taylor & $62-71$ \\
Grace & $78-60$ \\
Central State & $76-88$ \\
Mount St. Joseph & $50-57$ \\
Bluffton & $83-78$ \\
Indiana Wesleyan & $67-89$ \\
Michigan Christian & $72-75$ \\
Rio Grande & $75-87$ \\
Mt. Vernon Nazarene & $67-69$ \\
Wilmington & $73-80$ \\
Urbana & $57-87$ \\
Ohio Dominican & $50-59$ \\
Malone & $67-73$ \\
Findlay & $45-73$ \\
Tiffin & $69-52$ \\
Urbana & $67-84$ \\
Shawnee State & $72-80$ \\
Rio Grande & $80-89$ \\
Mt. Vernon Nazarene & $65-68$ \\
Walsh & $42-61$ \\
Ohio Dominican & $72-80$ \\
Malone & $69-75$ \\
Findlay & $60-86$ \\
Tiffin & $76-83$ \\
Shawnee State & $50-88$ \\
Walsh & $57-67$ \\
\hline
\end{tabular}

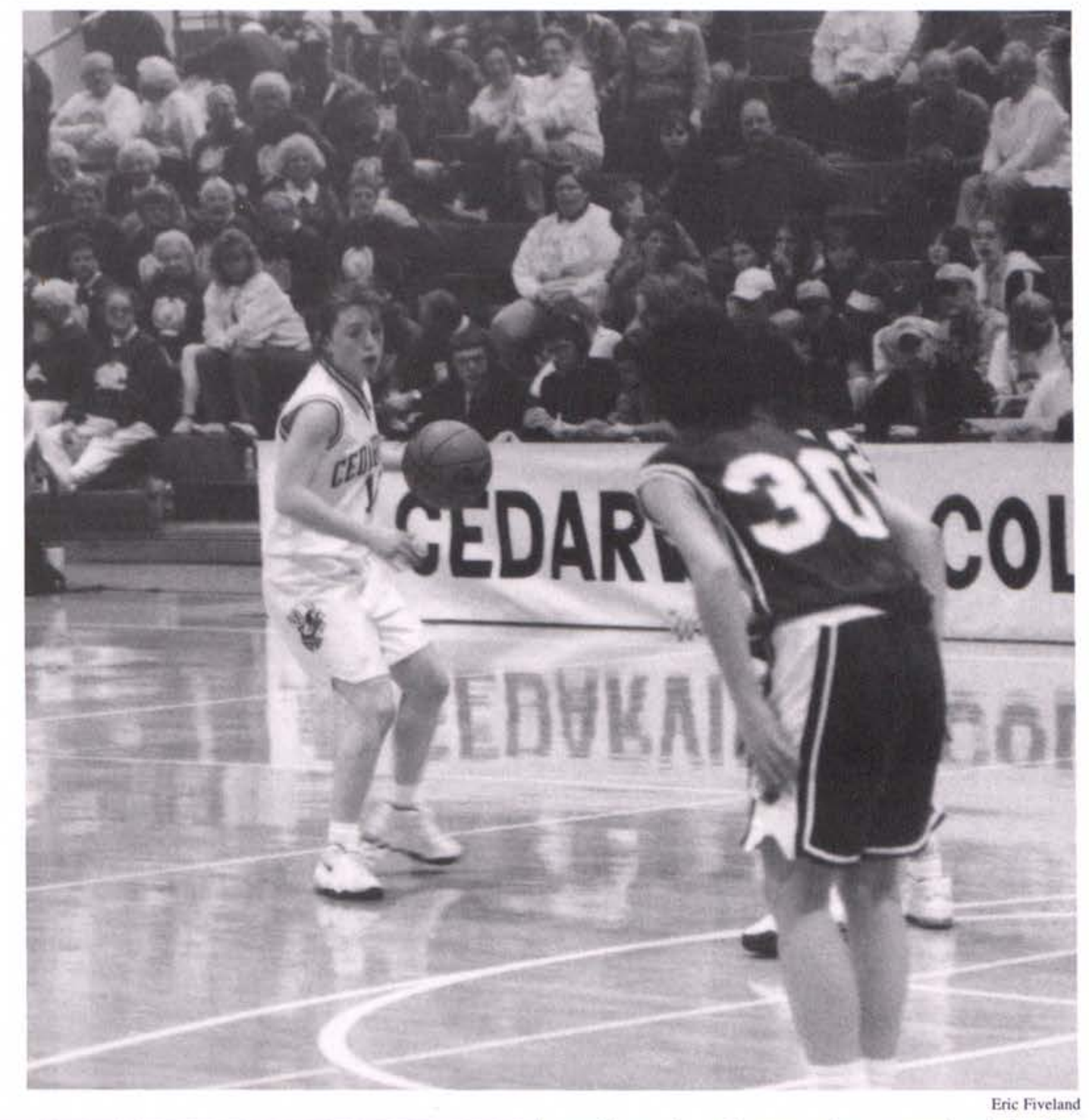

cellent, but the non-league schedule for the women also boasted many tough teams. Shawnee State was the number one ranked team in the NAIA Division II, while Central State was ranked number six in NAIA Division I. Findlay and Rio Grande had solid teams as well.

With the many new players and the experience gained from a competitive schedule, the team looks to build on the improvements they made during the season. "Improvement is the key to success," said Freese, and that improvement was clearly evident in contests like the one away against Shawnee State. The women played one of their best games against the nationally ranked team. It will be the drive of the team and the enthusiasm of its players, as well as strong coaching, which will allow this team to reach for their athletic goals in the year ahead.

\section{Peter Bednarek}

above: Heather French dribbles down the court, looking for a chance to score. below: Natalie Royer shoots for two at the line. 


\section{Finishing}

The Men's basketball team won their first-round game of the NAIA Division I Great Lakes Regional Tournament by a narrow margin against Rio Grande, but the Yellow Jacket's season came to a halt when they faced the University of Findlay (ranked 8th in NAIA Division I) in the second round. Overall the team closed out the season with a win/loss record of 12-21.

The Jackets were down from the previous year, and they did not enjoy the success which generally characterizes the Cedarville program. "This was obviously not a successful season as far as wins and losses are concerned," said Associate Professor of Physical Education and Coach Jeff Reep. Reep said, "There were two things that we knew were going to make things difficult for us going into this season." The first was a roster that included nine freshmen and only one senior. The second was the fact that every team experiences injuries during the course of a season.

What Reep and the team could not know was that the Yellow Jackets would

\section{Strong}

\section{Men's Basketball}

be the most injury-plagued team in the entire MOC. The team's sole senior and co-captain, John Woolley, went down in the middle of the season for nearly six weeks. When a team experiences injuries to the extent that Cedarville did, the effects are far reaching. Not only does the team lack the specific effort and skills that an individual player provides on the court, but also the continuity that he brings to the team in practice. Instead of a group of players becoming accustomed to their roles and understanding each other, they must always adjust to having someone different out on the floor. Reep said, "We had guys out there playing an impact role who in the future will only need to act as role players." This should, however, work to the team's ultimate advantage; the players in this position gained valu- able experience for games to come.

Sophomore John Krueger was often the source of the team's spark this season. Krueger offered solid play throughout the season, and averaged nearly 21 points per game, ranking him second in the Conference in scoring. "He really has an ability to create his own shot, and he can put it on the floor," said Reep. Reep credits Krueger for working hard and, "doing what he could to get the job done."

Freshmen Clint Hayes was ranked fourth in the MOC and was named to the All-MOC Freshmen Team; he averaged 18.4 points per game. Hayes brought great offensive output to the game, but unfortunately missed several games in middle of the season due to a knee injury.

Although Krueger and Hayes combined for an average of almost 40 points, the added scoring of junior co-captain Brent Miller and freshmen Kyle Mraz and Brooks Fry propelled the team to a third-best 3-point field goal percentage in the MOC. Woolley added an average of 11.7 points and ranked third in the Conference for blocked shots.

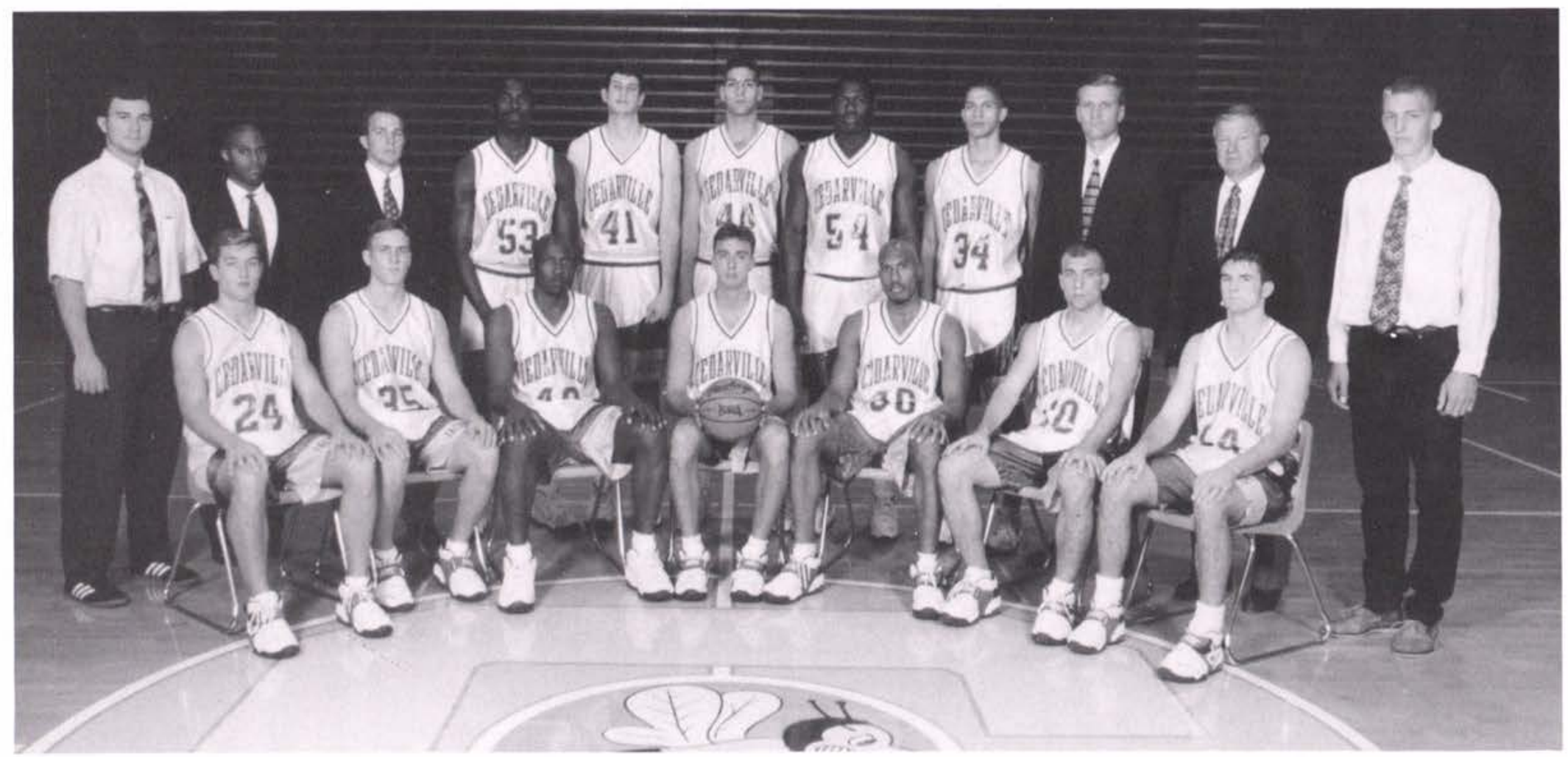

Scott Huck

row 1 (1-r): Kyle Mraz, Brent Miller, Carson Duncan, John Krueger, Dwayne Bryan, Brooks Fry, Clint Hayes. row 2: Assistant Coach Eric Taylor, Assistant Coach Tim Walker, Linton Ellis, Jon Woolley, Ted Forrest, Perry Mattis, Andrew Boyd, Head Coach Jeff Reep, Assistant Coach Pete Reese. 


\begin{tabular}{|c|c|}
\hline \multicolumn{2}{|c|}{ Statistics } \\
\hline Game & Score \\
\hline Wilberforce & $92-80$ \\
\hline Michigan-Dearborn & $105-90$ \\
\hline Cincinnati Bible & $92-50$ \\
\hline Cumberland KY & $59-87$ \\
\hline Fanshawe & $78-50$ \\
\hline Roberts Wesleyan & $84-90$ \\
\hline Ashland & $70-77$ \\
\hline Redeemer & $108-44$ \\
\hline Concord IA & $80-65$ \\
\hline Tiffin & $79-90$ \\
\hline Ohio Dominican & $81-68$ \\
\hline Wilberforce & $96-99$ \\
\hline Sheridan & $100-93$ \\
\hline Walsh & $59-79$ \\
\hline Malone & $77-89$ \\
\hline Rio Grande & 2-0 (forfeit) \\
\hline Shawnee State & $88-75$ \\
\hline Urbana & $57-71$ \\
\hline Michigan-Dearborn & $67-80$ \\
\hline Mt. Vernon Nazarene & $80-107$ \\
\hline Madonna & $85-90$ \\
\hline Urbana & $64-67$ \\
\hline Mt. Vernon Nazarene & $113-119$ \\
\hline Findlay & $67-79$ \\
\hline Tiffin & $61-75$ \\
\hline Ohio Dominican & $73-59$ \\
\hline Walsh & $77-106$ \\
\hline Malone & $69-80$ \\
\hline Rio Grande & $62-77$ \\
\hline Shawnee State & $76-82$ \\
\hline Findlay & $65-79$ \\
\hline Rio Grande & $81-75$ \\
\hline Findlay & $65-83$ \\
\hline
\end{tabular}

Woolley provided much-needed leadership for the young team, both on and off the court. He and Miller organized daily team devotions, and even brought in different speakers to address the team.

In addition to individual's statistics, Reep was proud of the team as a whole. "They all worked hard and they have not quit," he said. The coaching staff will rely on the steady improvement that was evident throughout the season.

The game on the road against Shawnee State University was an example of the character Reep sees in his team. They were down 19 points in the first half but climbed back to within nine late in the second. Reep is proud of this type of effort and hopes that with the changing of just a few factors, wins and losses will begin to take care of themselves. "The note we end every practice on is first in prayer, then with the goal of our team:

\section{'Every day and in every}

\section{way--we will get a little better."'}

\section{Peter Bednarek}

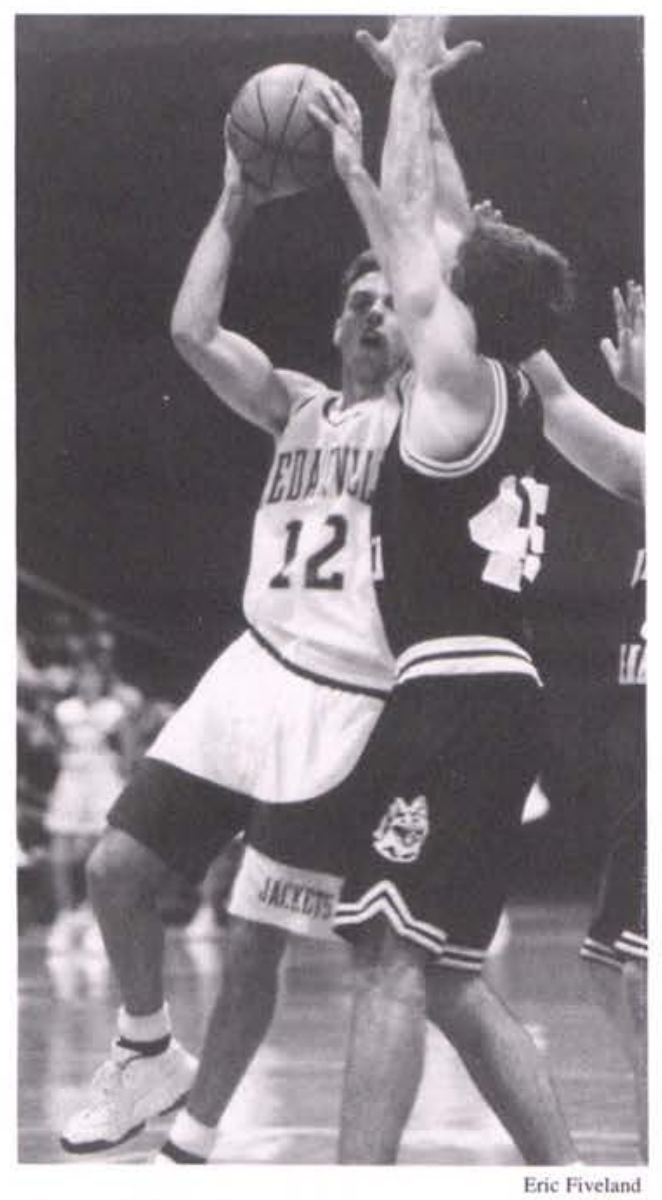

above: John Kreuger puts a shot up past his opponent. below: Dribbling down the court, Kyle Mraz makes his way to the basket.

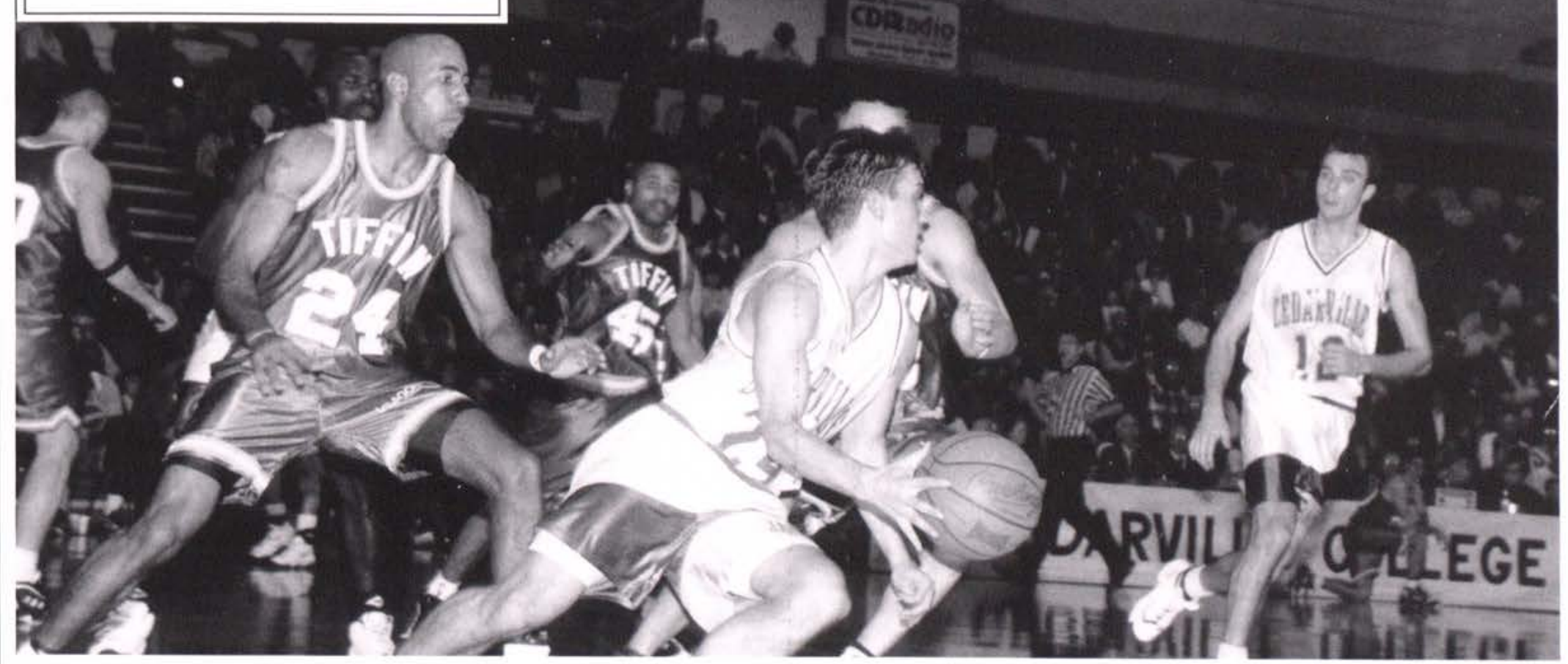




\section{Stack'em}

As basketball players race up and down the court, several voices call out "Cedarville, Cedarville, what do you say?" The crowd replies with a deafening, "We back the Jackets all the way!" The enthusiasm is invigorating. Somersaulting, back-flipping, and performing intricate group stunts, Cedarville's cheerleaders make sure that everybody gets into the game.

Although the crowd only sees the cheerleaders' role during the actual games, each member on the cheerleading team can attest to the hours of commitment surrounding the basketball events. Tryouts begin in September. After the six men and six women learn that they have made it onto the squad, they begin a schedule of rigorous practices, which continue in one form or another until the final game has been played in the middle of March. According to Monica Mennenga, the coach of the team, the group spends three or four evenings together each week; due to their busy indi-

\section{High}

\section{Cheerleading}

vidual schedules, these practices usually occur late at night.

The cheerleaders do not limit themselves to practices and games, however. Throughout the year they hold private cheerleading clinics at several local schools. These events provide a special opportunity for reaching out to the community and encouraging younger cheerleaders to continue to work on their skills and to use their talents. Also, an additional cheerleading squad cheers for all of the freshman teams and the womer's team games.

Participating in cheerleading is appealing for those who love the game of basketball and enjoy athletic challenges. It requires an enormous amount of agility and balance, and excellent tumbling skills. The squad also enjoys traveling to other schools for away games.

Monica's long history of cheerleading testifies to the sense of fulfillment and excitement that comes from being involved on the team. She had her first cheerleading experience at the age of two. She has participated on the team during all four of her years here at Cedarville. In her current capacity of coach, she has the added responsibility of scheduling the practices, arranging the squad's role in the away games, and taking care of the uniforms. She considers this preparation for future jobs, as she finishes her senior year in June with a physical education degree.

Doug Amundson, senior finance major and guys' captain for the team, began cheerleading during his sophomore year at college. He wanted to keep in shape and to do something extracurricular dur-

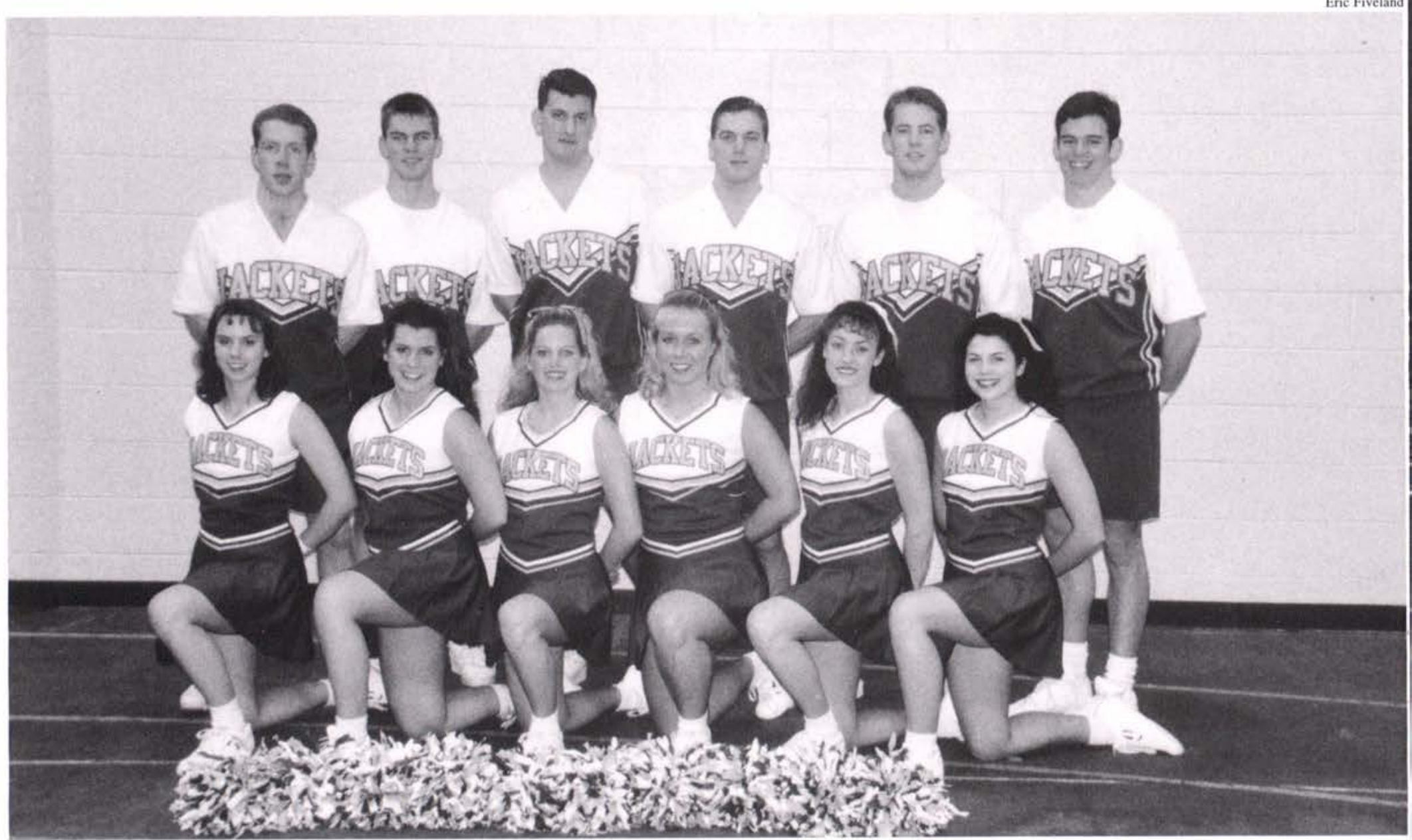

row 1(l-r):Becky Roche, Monique Shoaf, Carmon Huddleson, Monika Mennengae, Lori Brown, Kelly Grady (Sarah Hennis not shown). row 2: Douglas Amundson, Josh Sherwood, Mike Morris, Scott Vandergrift, Tom Sanderson, Pete Sutton. 
ing the winter quarter, the off-season for his other sport: the javelin throw. Doug chose cheerleading because his roommate at the time was on the squad, and enjoyed his own participation. Many special friendships and memories have resulted from the unique trust Doug and his teammates have learned to place in one another.

It is difficult not to catch the cheerleaders' spirit. They make every basketball game a memorable experience. "Fight, Jackets, fight! Victory, that's right!"

\section{Keturah Stork}

right: JV Cheerleaders, row 1 (1-r): Amber Corbin, Cindy Wren. row 2: Lori Wheeler Christa Hill, Rebecca Fissel. Bottom left (t-b,l-r): Monique Shoaf, Karmen Huddleston, Lori Brown, Monica Mennenga, Scott Vandergrift, Tom Sanderson, Douglas Amundson. Bottom right: front row (1r):Monique Shoaf, Monica Mennenga, Karmen Huddleston, back: Kelly Grady.

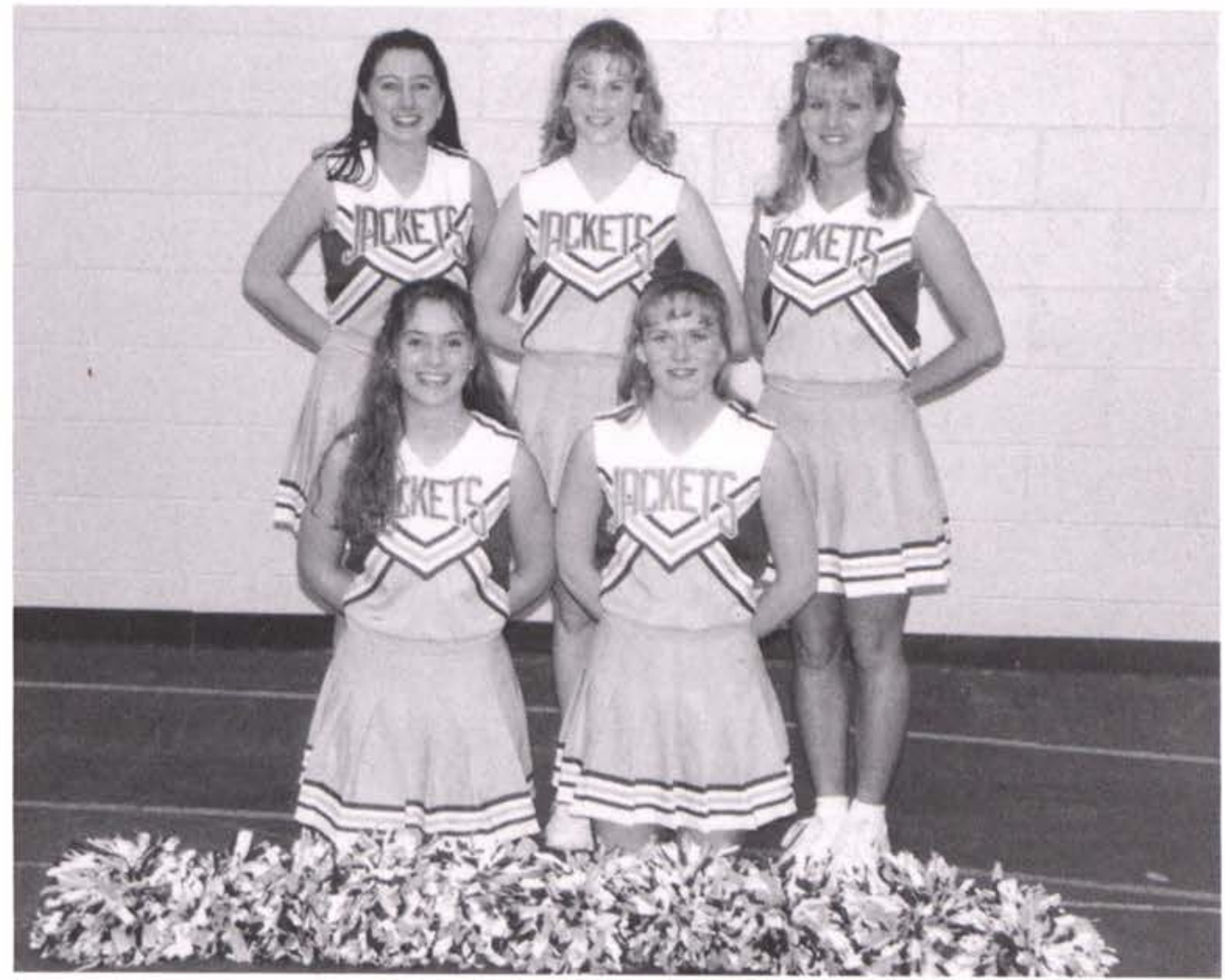

Eric Fiveland
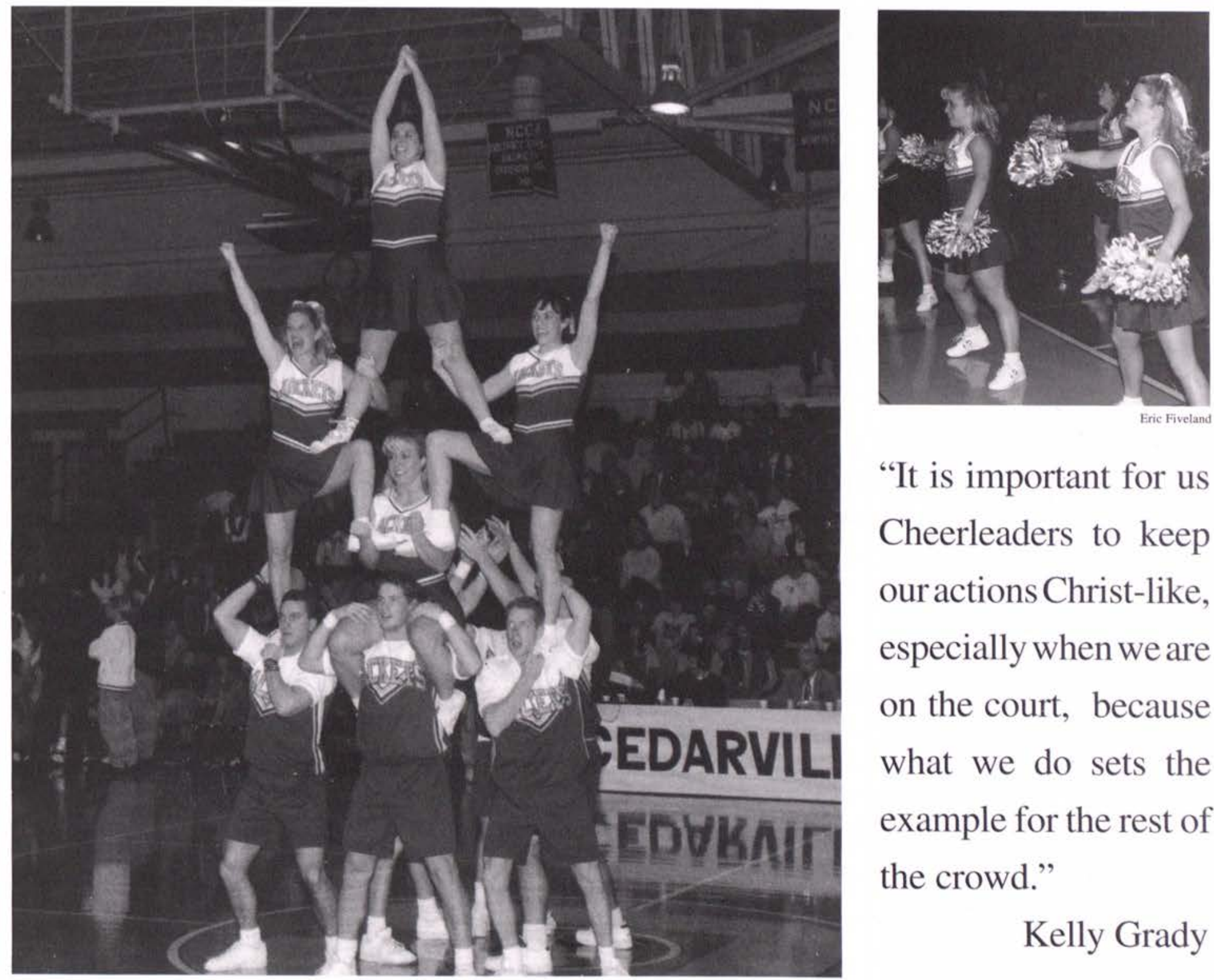

"It is important for us

Cheerleaders to keep our actions Christ-like, especially when we are on the court, because what we do sets the example for the rest of the crowd."

Kelly Grady 


\section{Match}

The 1997 women's tennis players boast one of the most successful seasons of any Cedarville college sports team. The Lady Jackets completed the season with a record of 11-1.

The women's team spent spring break in Florida, training and creating a new line-up. Dr. Pam Johnson, Professor of Physical Education and Director of Academic Assistance, coached the young team. Eleven of the fifteenmembers were sophomoresandfreshmen.Going intothe season, Johnson worried that youth would be a liability. Soon she learned that her fears were unfounded. "It was a wonderful season. They responded very well," Johnson said.

Johnson was assisted by part-time faculty member Dr. Delyte Morris. Eleanor Taylor assisted and led the Bible studies.

Four of the six singles spots were held by freshmen. Casey Ruffin (number two), finished her first season 10-2. At the number three position, Ginger Butler was unde-

\section{Point}

\section{Women's Tennis}

feated during the regular season. Her only loss came during the MOC finals at Walsh. Senior Hasmine Gmuer lead the team, and aftertwoyearsatnumbertwo, Gmuerstepped into the number one singles position. In addition, she picked updoubles with Ruffin. Both made the MOC All-Conference team and took homeCedarville's lone conference title at number two doubles, where they were undefeated. Butler and sophomore Jodie Du'Monceaux comprised the number one doubles team with an 8-3 record.

Highlights for the year included victories over MOC rival Ohio Wesleyan and local nemesis Wittenburg, neither of whom had lost to Cedarville in several years. On April
21, four of the Springfield matches went a grueling three sets, three of which were won by Cedarville. A week earlier, on April 15 . Cedarville scored a comeback victory, 5-4. against Ohio Wesleyan. Singles victories came from Ruffin, Butler, and Wolfe.

Inclement weather was the negative factor at the tennis team's only losses. In the game against Walsh, rain forced them to play on unfamiliar indoor courts where the women fell,4-5.Victors for Cedarville were Gmuer Butler, Gmuer/Ruffin, and sister duo Beth and Mary Wolfe. The same scenariobrought the season to a bittersweet end at the MOC tournament. The Walsh University squad was at home on their indoor courts and repeated as conference champions with 40 points. Cedarville trailed by only six points Johnson said this loss will fuel the younger players to return next year and win.

Rebecca Ritzel

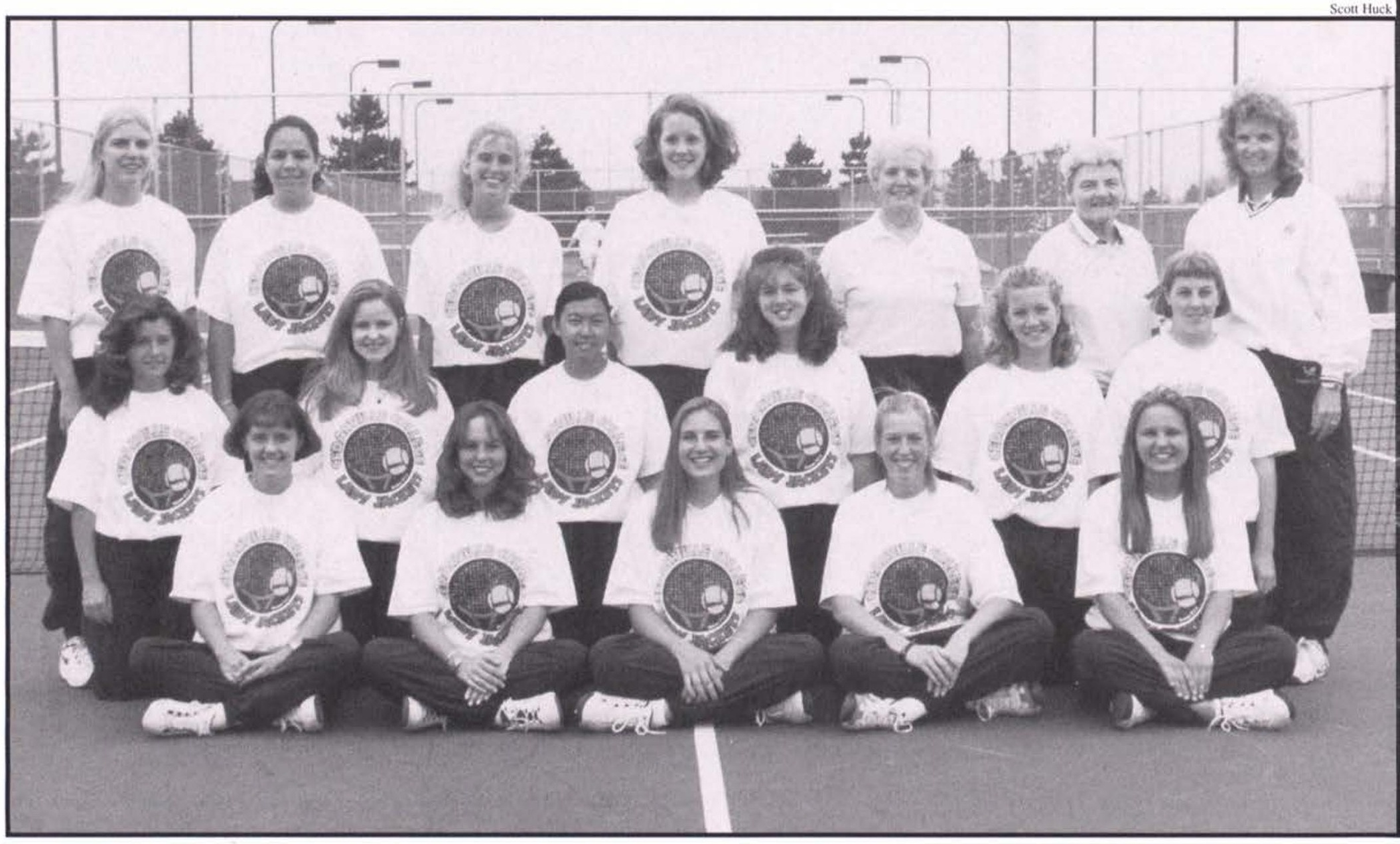

row 1 (1-r): Jodi Du'Monceaux, Rachel English, Christine Wiesert, Kerri VanderMolen, Jennifer Stankiewicz. row 2: Debbie Baker, Darcie Blakemore, Angela Mast, Julie Gregory, Elizabeth Wolfe, Casey Ruffin. row 3: Ginger Butler, Hasmine Gmuer, Amanda Mudrey, Mary Wolfe, Assistant Coach Eleanor Taylor, Assistant Coach Dr. Dee Morris, Head Coach Dr. Pam Johnson. 


\section{A Smash}

The Cedarville Men's Tennis team entered their 1997 season as the underdogs after losing the top three players from their previous year. The Yellow Jackets finished with an overall record of 8-12.

The team opened their season with losses against Transylvania and Merrimack before coming around to defeat Ohio Wesleyan for the first time in four years.

The match against Northern Kentucky found the Jackets down 6-1, but they rebounded against Mount St. Joseph to a 9-0 victory.

Coming from a six-match losing streak, Cedarville strongly defeated MOC rival Malone 8-1.

In their second match against Mount St. Joseph, Cedarville closed them out for a 9-0 win. The Yellow Jackets picked up their third straight win against Tiffin with an 8-1 MOC victory.

\section{Hit}

\section{Men's Tennis}

At the Bluffton Invitational, Cedarville gathered two wins and one loss to place second in this four team tournament, with Brian Wilbur and Tim Haylett picking up the two wins.

At Greenville for NCCAA National play, the Yellow Jackets lost by a narrow 5-4 margin.

The team loses two of its seniors, Tim Haylett and Anthony Torlone, to graduation, and its No. 1 ranked singles player Brian Wilbur, a sophomore, to
Oxford University, though he's promised to come back for his senior year.

"This team was one of the best groups of guys I've ever worked with," Coach Edlund observed. "All the guys pulled together for each other to form a cohesive unit, and they all got along very well."

The team spent their Spring Break in Jacksonville, Florida where they practiced an average of six hours a day.

"The Jacksonville practices were a time for us to get to know each other, with six hour practices and then spending the rest of our time together," Coach Edlund said about their Spring Break.

The Cedarville Yellow Jackets look forward to next year as many of their opponents are losing top-ranked seniors, while the Jackets team is bringing in several top-ranked players to continue building a solid team.

Ben Barnhart

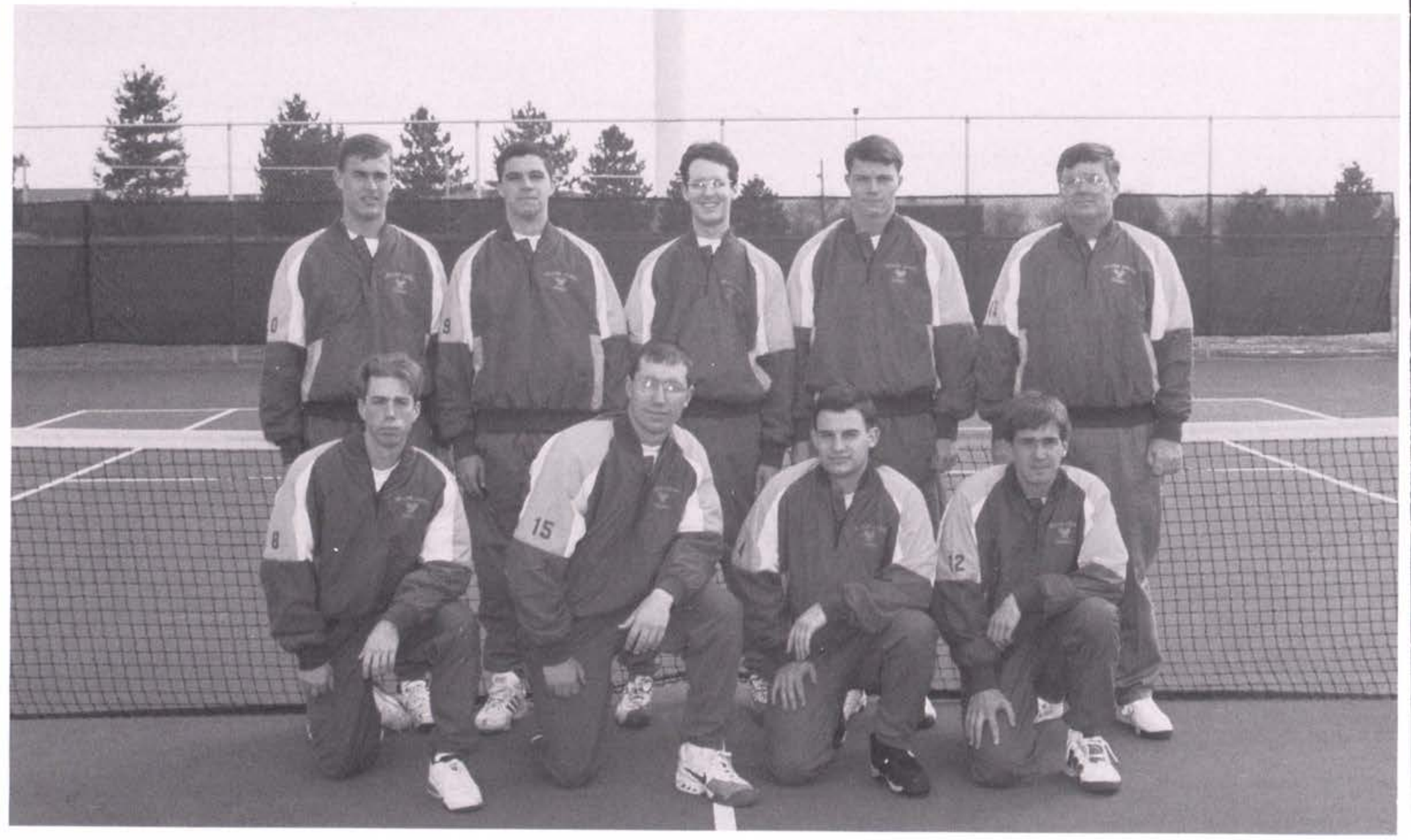

row 1 (1-r): Reuben Duncan, Brett Cooley, Tim Haylett, Matt Taylor. row 2: Carl Weise, Anthony Torlone, Brian Wilbur, Zach Ruffin, Head Coach Alan Edlund. 


\begin{tabular}{|lcc|}
\hline \multicolumn{3}{|c|}{ Statistics } \\
\hline Opponent & W/L & Score \\
\hline Transylvania & L & $2-7$ \\
Merrimack & L & $2-7$ \\
Ohio Wesleyan & W & $4-3$ \\
N. Kentucky & L & $1-6$ \\
Mt. St. Joseph & W & $9-0$ \\
Wittenburg & L & $1-7$ \\
Bluffton & L & $2-7$ \\
Findlay & L & $4-5$ \\
Otterbein & L & $4-5$ \\
Walsh & L & $0-9$ \\
Taylor & L & $1-8$ \\
Malone & W & $8-1$ \\
Mt. St. Joseph & W & $9-0$ \\
Tiffin & W & $8-1$ \\
Bluffton Invitational & 2nd out of 4 \\
Wilmington & W & $9-0$ \\
NAlA Great Lakes Sectional \\
Greenville & L & 3rd out of 6 \\
Lee & L & $0-5$ \\
& & \\
\hline \multicolumn{4}{|c|}{} \\
\hline
\end{tabular}

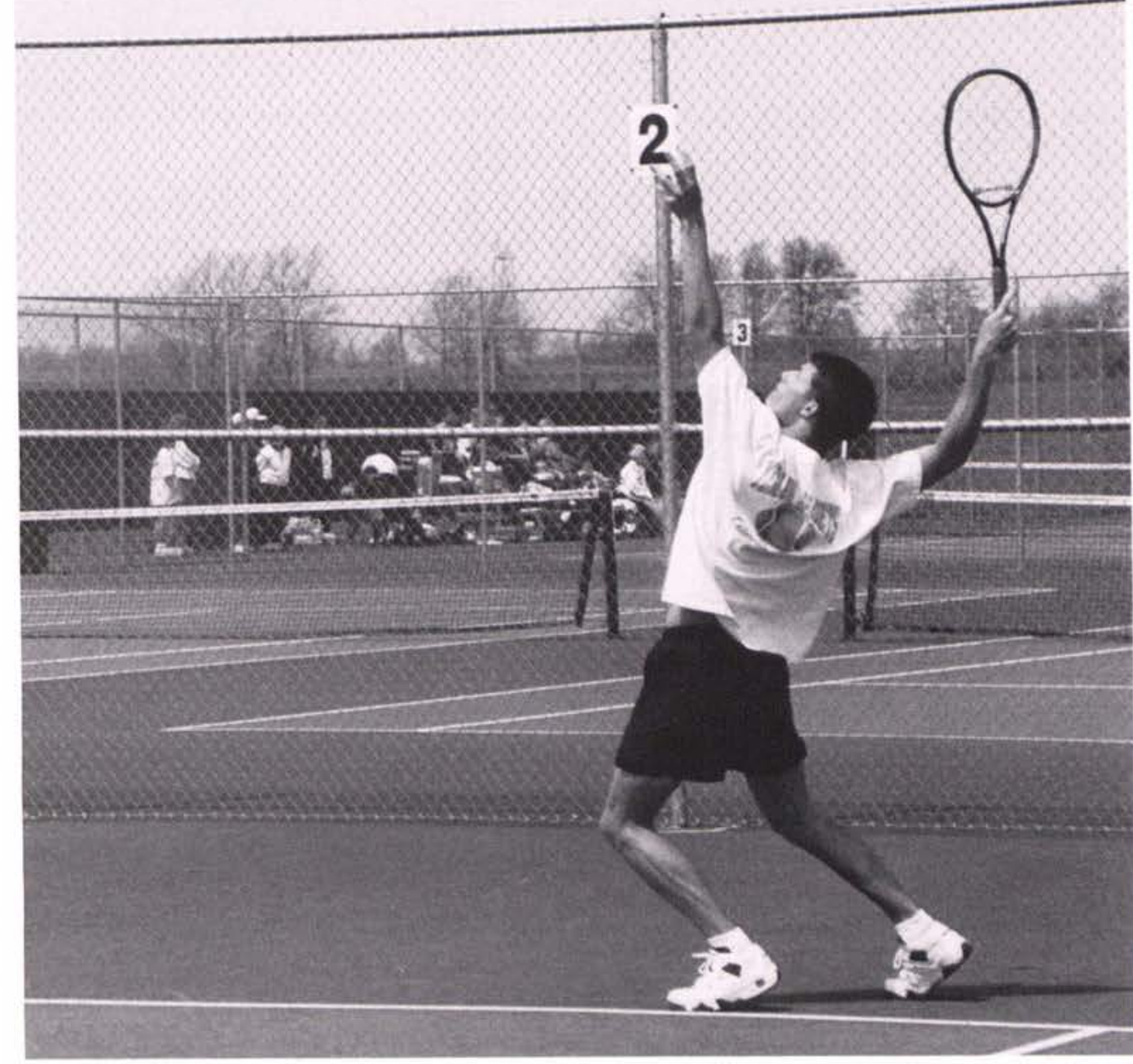

Eric Fiveland

above right: Zach Ruffin shows off his powerful serve. below: Anthony Torlone is ready for his opponent's return.

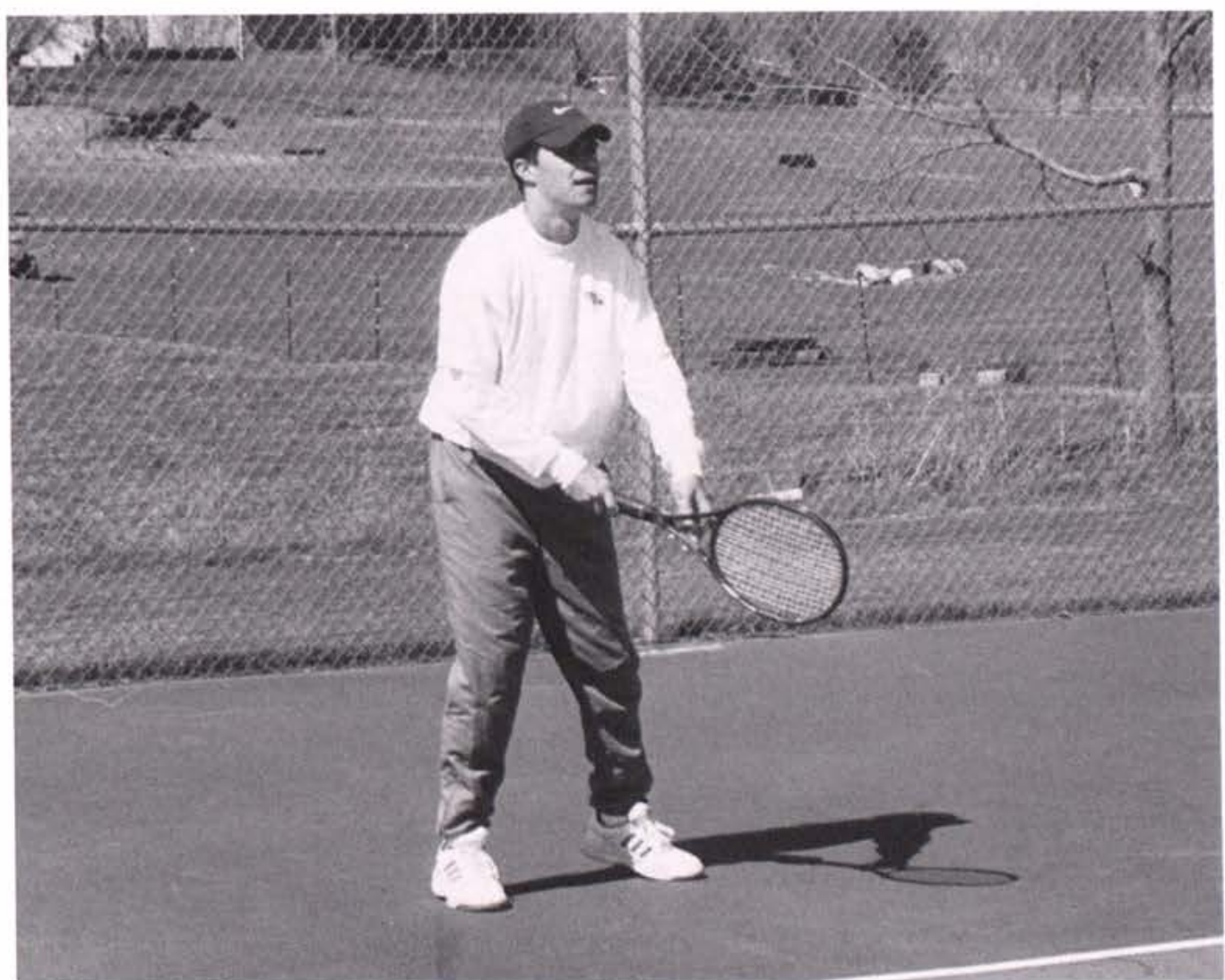

"This team was one of the best groups of guys

I've ever worked with."

--Coach Edlund 


\section{Right on}

This year's golf team was made up of six players. They were led by their two veterans, Steve Burchett and Troy Page. Joining them were Brodie Swanson, David Rooke, Joel Schenk, and Jeremy Birk.

Cedarville's golf team is coached by Dr. Jim Kragel, who is in his third year of coaching the Yellow Jackets. He is an Assistant Professor of Communication Arts and teaches many broadcasting classes. He was a scratch golfer for many years, and he also coached high school golf for three years. Before coming to Cedarville, he lived in Florida, where he played in several mini tour events on the Tommy Armour Tour and the Central Florida Tour. He has even competed in several U.S. Open qualifiers.

In order to get ready for the season, the team took a Spring Break trip to

\section{Course}

\section{Golf}

Myrtle Beach for practice and fellowship. During the season, Cedarville played in ten invitationals in Ohio, Indiana, and Kentucky. The team's best results came in the OSU-Lima tournament, in which they placed 4 th out of 14 teams. They finished a disappointing 7 th in the MOC districts, and in the NCCAA national tournament they finished 8th. Also, in the 16th Annual Cedarville College Invita- tional, Cedarville finished 8th.

Coach Kragel was grateful that the team always had a strong Christian witness wherever they went. "Our entire team loves the Lord and is dedicated to spreading the gospel at every opportunity. Like I told the other coaches we competed against, we might not have the best golfers out there, but we certainly have the nicest players of all the teams that we go up against. It was a pleasure to coach them."

Tim Hotchkiss

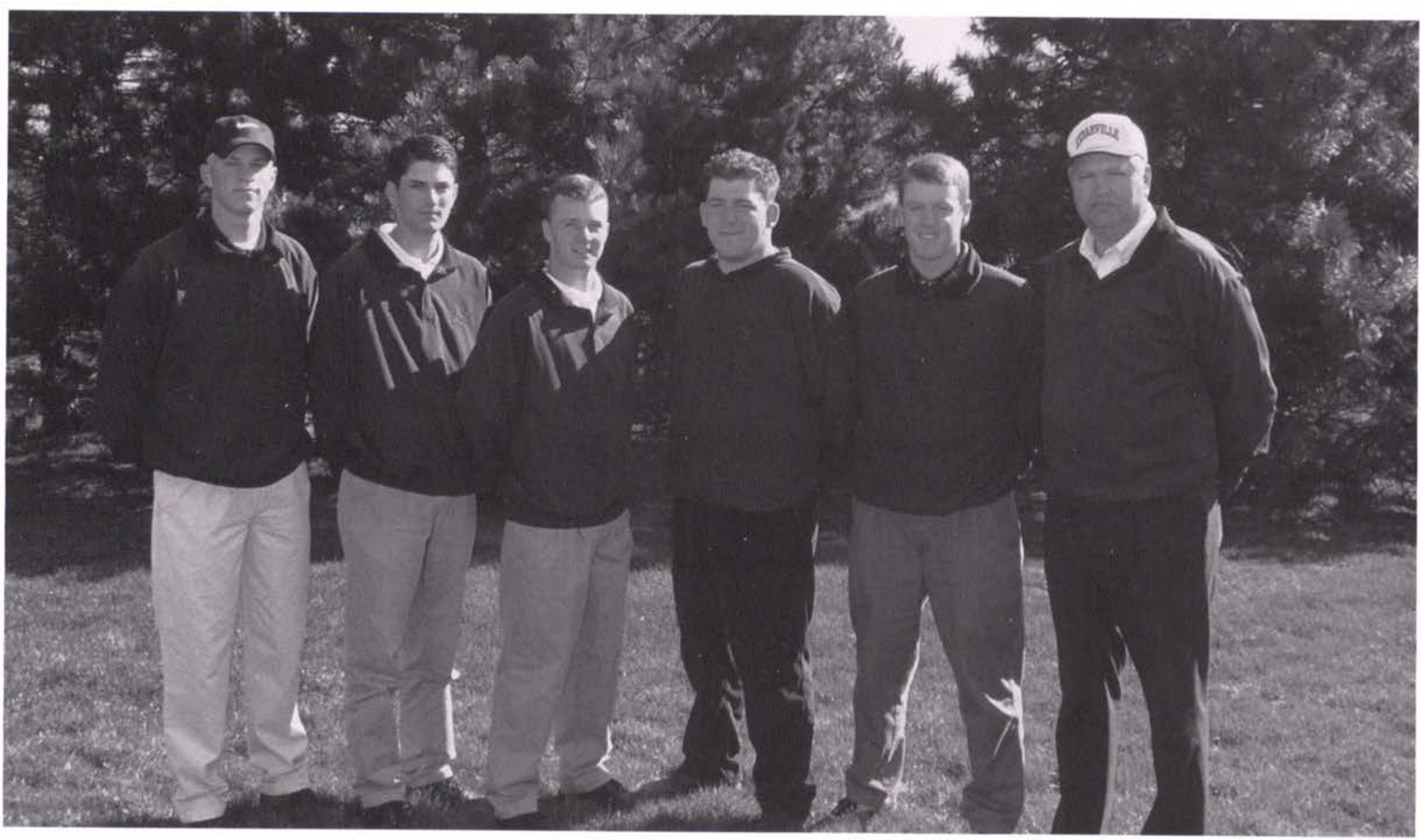

(1-r) Dave Rooke, Jeremy Birk, Troy Page, Joel Schenk, Steve Burchett, Head Coach Jim Kragel. Not Pictured: Brodie Swanson 


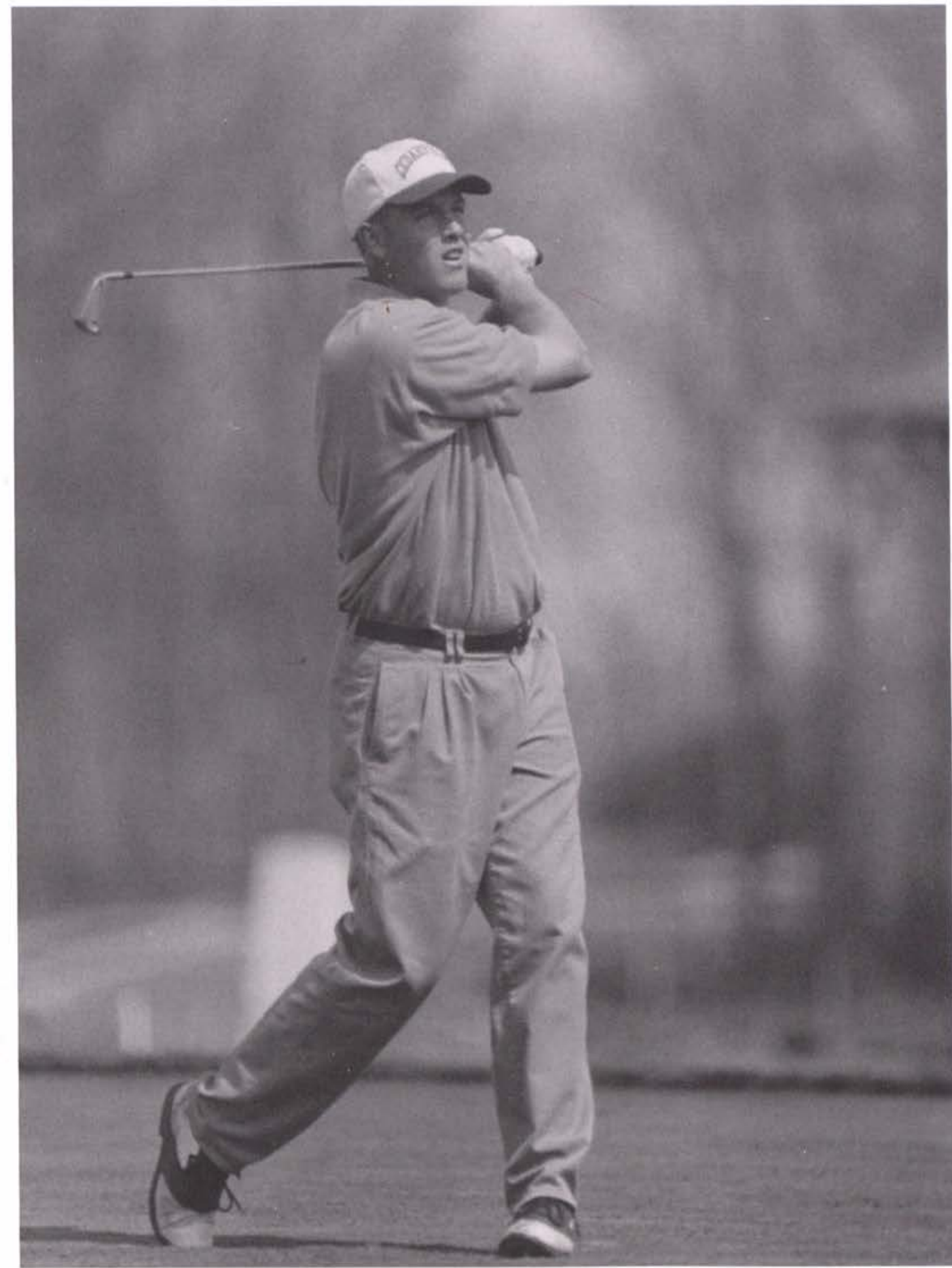

"Our entire team loves the Lord and is dedicated to spreading the gospel at every opportunity. . ."

--Coach Kragel

above: Steve Burchett focuses intensely on the result of his swing. right: Steve Burchett practices his putting skills.

\begin{tabular}{|lr|}
\hline \multicolumn{2}{|c|}{ Statistics } \\
\hline Match & Place \\
\hline Shawnee State Invit. & 11 th \\
Urbana Invit. & 7 th \\
Walsh Invit. & 7 th \\
Tiffin Invit. & 7 th \\
Cedarville Invit. & 7 th \\
Mid-Ohio Conference & 7 th \\
\hline
\end{tabular}

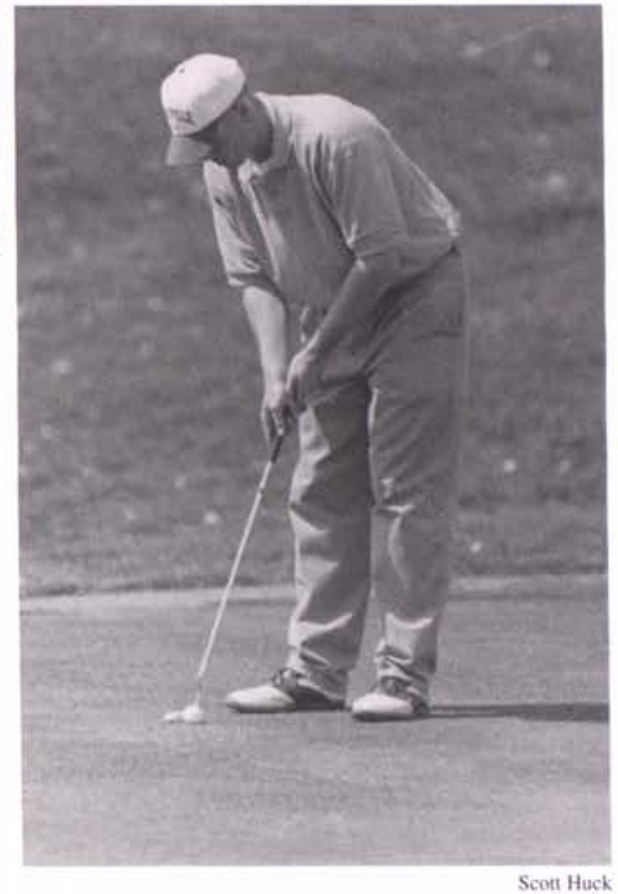




\section{Bringing It Home}

\section{Women's Softball}

the season. Although faced with these obstacles, the team's head coach David Gaffner, Assistant Director for the Academic Assistance Center, described his players as being very competitive. Their competitive spirit showed through in their winning of a double-header against Rio Grande.

Several highlights and awards marked the 1997 season. Gaffner named senior third-baseman Erika Miller as the team's Most Valuable Player. Miller led the team in batting averages with a .441 average. Seniors Melissa Sprankle (outfielder), Michelle Dick (outfielder) and Jennifer Cassidy (first-baseman), made major contributions to the team as well.
Gaffner was careful to stress that not only was the team concerned about their athletic abilities but about spiritual growth as well. The team held devotions and were "Prayer Partners." Each week, the women would pair up with a different partner. They would meet together throughout the week to pray. "We tried to go beyond just softball and make this more than a one-dimensional program," Gaffner said.

Though this season was a bit rough, Gaffner feels that the team has a bright future. "Overall, I think we have a good group coming back, there are a lot of people trying out in the fall, and we have a new pitcher coming in," he said. Sophomore pitcher/outfielder Lara Gyurick, freshman infielder Becky Summers, sophomore outfielder Noella Fisher, sophomore pitcher/second-baseman Bonnie Schaefer, freshman pitcher/out-

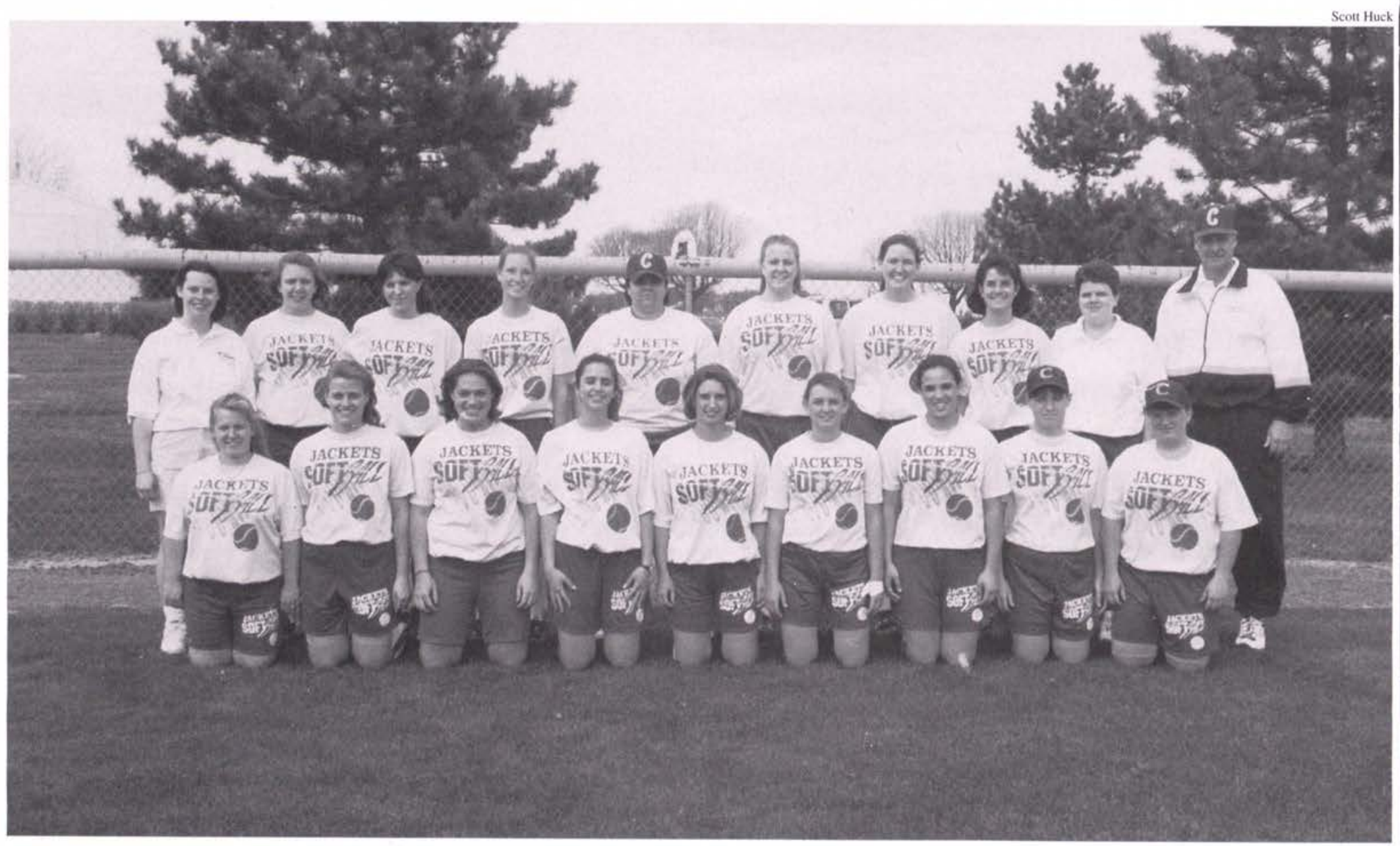

row 1 (1-r): Lana Schwinn, Michelle Dick, Andrea Butz, Lara Gyurik, Christine Olbrich, Maggie Winkels, Noella Fisher, Bonnie Shaefer, Erika Miller. row 2: Student Trainer Gretchen Taylor, Maureen Strang, Becky Summers, Michele Vanlier, Jennifer Cassidy, Theresa Olson, Julie Shaefer, Melissa Sprankle, Student Manager Annie Miller, Head Coach Dave Gaffner. 


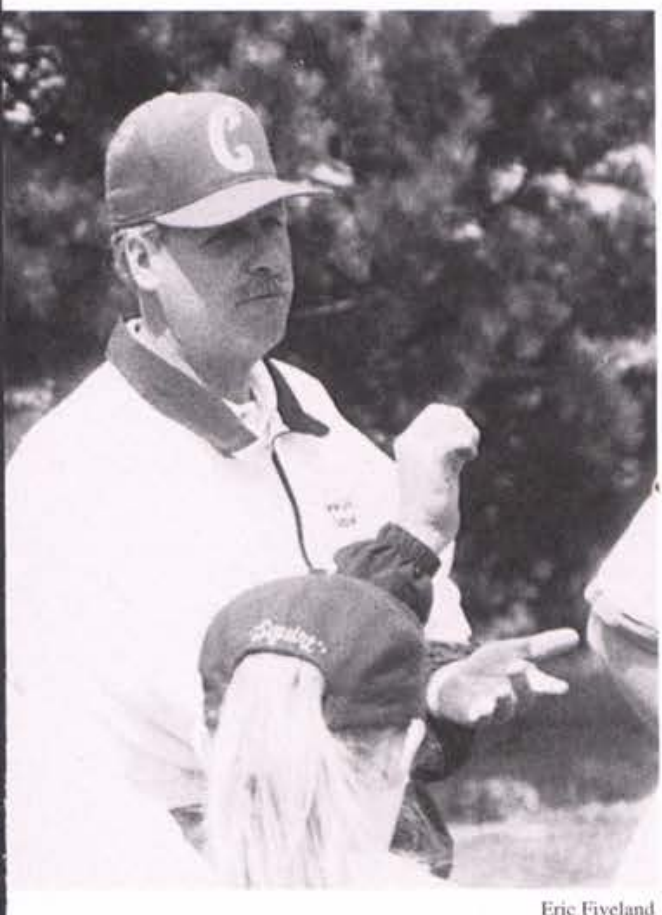

fielder Michele Vanlier and junior firstbaseman Andrea Butz are all returning players who will have a major impact on the team next season. Along with these veterans, a new pitcher, recruited from Kettering Fairmont will help the team to bring their play up to the next level.

The 1997 season record stands at 9-24 overall and 4-14 in the MidOhio Conference. In optimistic perspective, Coach Gaffner added, "We're already on our way up."

Rachel J. Stewart

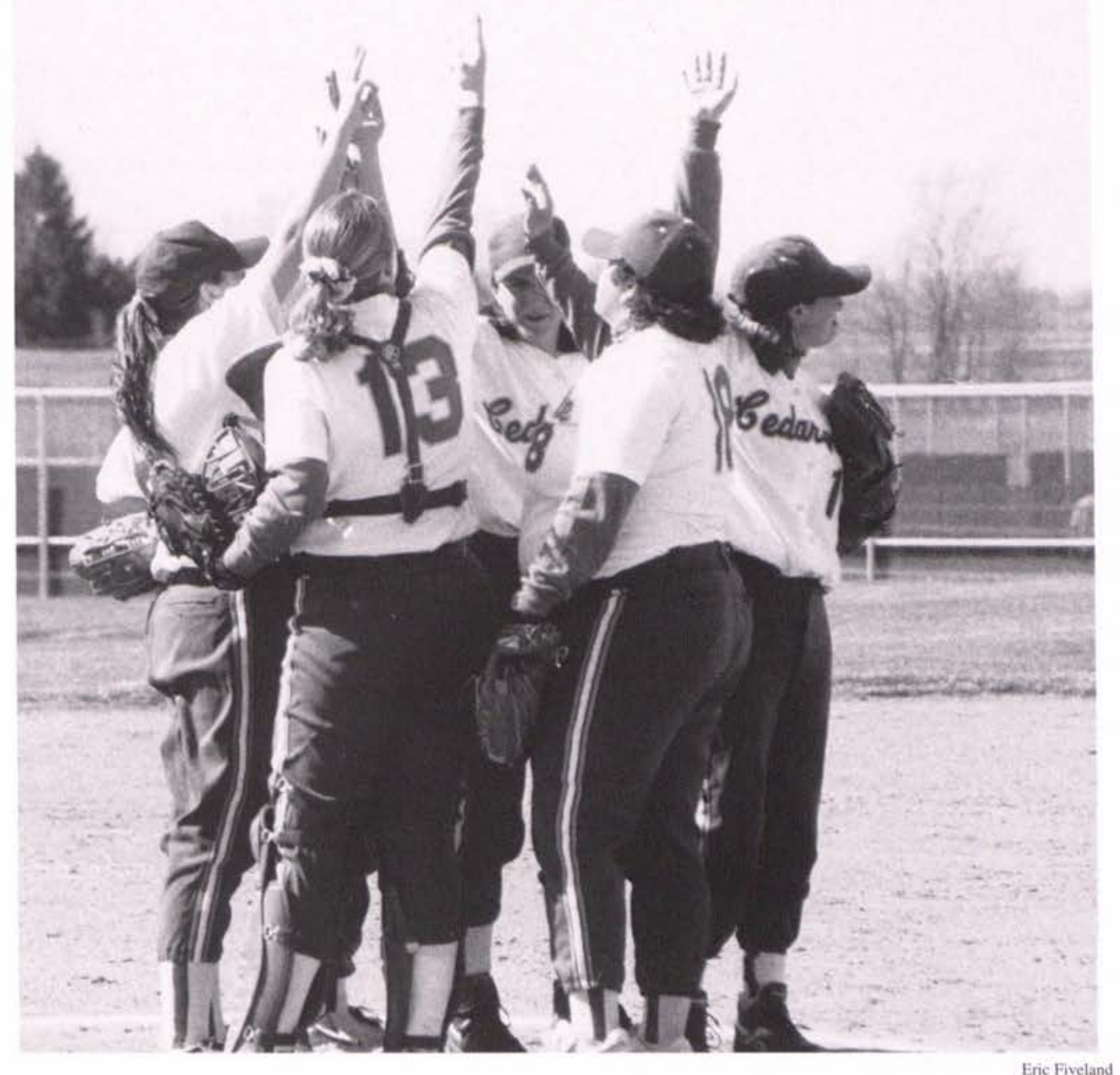

above left: Coach Gaffner gives some last minute instructions. above: Team spirit! below: Lara Gyurik pitches for the Yellow Jackets.

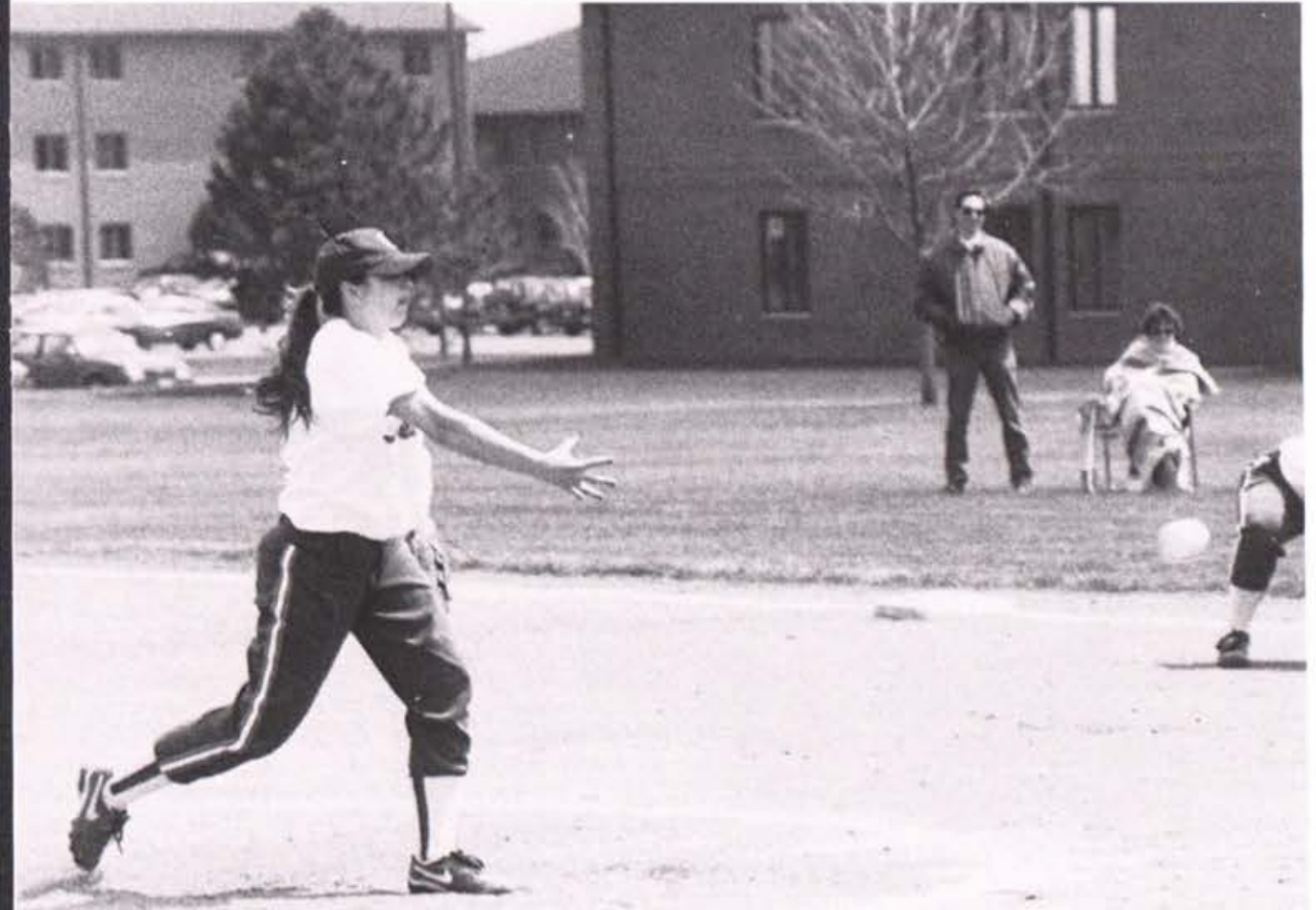

\begin{tabular}{|lcr|}
\hline \multicolumn{3}{|c|}{ Statistics } \\
\hline Opponent & W/L & Score \\
\hline Belhaven & L & $5-7$ \\
Huntington & L & $3-5$ \\
William Carey & L & $2-8$ \\
Belhaven & W & $9-1$ \\
Ohio Dominican & L & $7-8,0-7$ \\
& & (forfeit) \\
Urbana & L & $0-8,2-10$ \\
Mt. Vernon & Split & $2-1,4-6$ \\
Tiffin & L & $0-13,3-5$ \\
Findlay & L & $4-10$ \\
Rio Grande & W & $9-4,8-7$ \\
Urbana & L & $0-2,3-8$ \\
Walsh & L & $0-1,1-7$ \\
Malone & Split & $0-3,4-0$ \\
Findlay & Split & $0-8,3-2$ \\
Wittenburg & L & $0-3,9-11$ \\
Shawnee State & L & $1-4,2-12$ \\
Wilmington & W & $8-6,3-1$ \\
Taylor & Split & $4-3,7-8$ \\
Mt. St. Joseph & L & $6-9,1-7$ \\
\hline
\end{tabular}




\section{Another}

Heading into the 1997 baseball season head coach Norris Smith remarked, "the Mid-Ohio Conference schedule is tough as usual and we'll have some difficult non-league games. We have some players who I believe have the talent to compete so we're looking forward to the challenge." As the difficult schedule and player injuries took their toll, the season did prove to be challenging. "Injuries to the pitching staff killed us," said Smith. Two of the team's top pitchers, senior Sean Moore and sophomore Jay Clark, were both hampered by injuries during the season.

Overall, the team finished with a record of 6-22, 3-15 in the MOC. Coach Smith added that "we were pretty competitive in most ball games." One of those games was on April 8 versus Rio Grande at Yellow Jacket Field. After seven innings, the score was tied 5-5. Rio Grande hit four runs in the top of the eighth to make the score 9-5. Cedarville closed the mar-

\section{Turn at Bat}

Men's Baseball

gin to $9-8$ in the bottom of the eighth.

Cedarville will be losing only two seniors in pitcher/first baseman Sean Moore and co-captain catcher Josh Green. Green, who was voted to his second consecutive NCCAA All District Team, batted .235 with 15 RBIs and .992 fielding percentage over 27 games played. Moore batted .314 with a .381 on-base percentage over 23 games played.

Junior Josh Lunney was voted by his teammates as the Most Valuable Player. $\mathrm{He}$ also received NCCAA All District honors and was an NCCAA All-American Honorable Mention and an MOC Honor- able Mention. He finished with a .302 batting average, and also led the team in runs scored (21), RBIs (16), triples (4) batting percentage (.465), stolen bases (6), and on-base percentage (.408).

Other key contributors were jun ior Mark Wood who led the team with : .318 batting average, sophomore pitcher Matt Neubert (.280 avg., 3-0, 3.14 ERA) and sophomore center fielder Jeremy Howard (.250 avg., 2 Hrs).

Three freshmen made significan contributions: left fielder Mike Reed, second baseman Gabe Schlappi, and pitcher Johnny Freese. Reed batted .286 while Schlappi was .268 at the plate. In five starts and eight appearances, Freese was 1-3 with a 6.44 ERA.

With only two seniors departing, a solid nucleus remaining, and some new recruits arriving, Cedarville expects to have a strong team in 1998.

Mark Allen

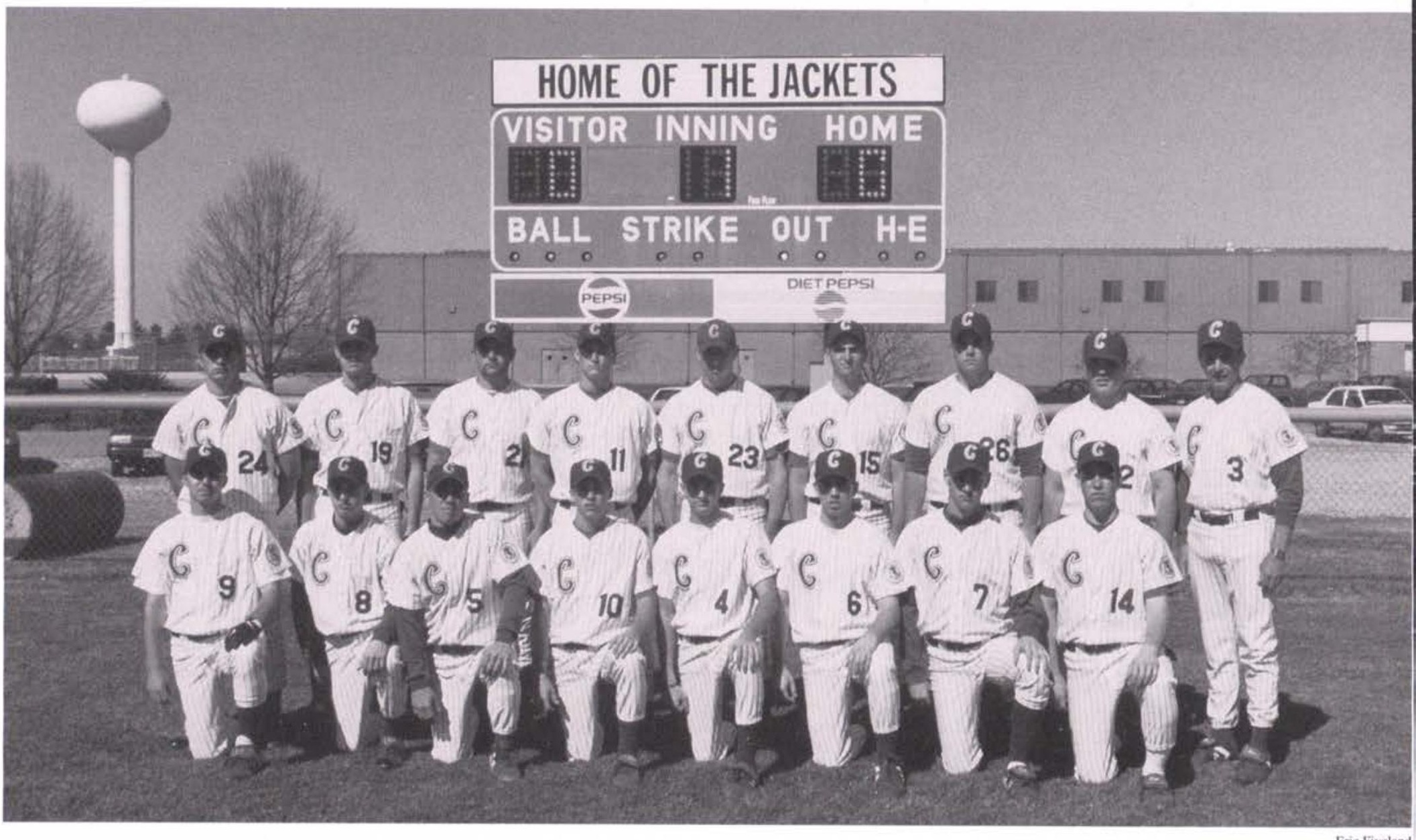

row 1(1-r): Mark Wood, Ryan Urbassik, James Clark, Gabe Schlappi, Mike Reed, Brian Rasey, Matt Neubert, Dustin Crider. row 2: John Freese, Sean Moore, Dustin Klopp, Jeremy Howard, Josh Lunney, Adam White, Steve Strong, Josh Green, Head Coach Norris Smith.

232 athletios 


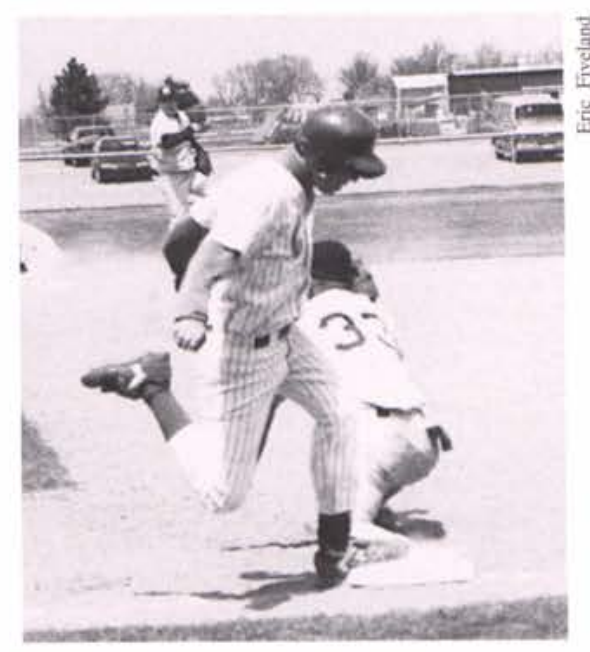

\begin{tabular}{|lcr|}
\hline \multicolumn{3}{|c|}{ Statistics } \\
\hline \multicolumn{1}{|c|}{ Opponent } & W/L & \multicolumn{1}{c|}{ Score } \\
\hline Wilmington & W & $12-2$ \\
Martin Methodist & L & $2-12,7-10$ \\
Warner Southern & L & $1-19$ \\
Tenn. Temple & Split & $3-11,7-4$ \\
Ohio Dominican & L & $0-10,3-9$ \\
Wilmington & W & $7-6$ \\
Mt. Vernon & L & $1-12,7-13$ \\
Findlay & L & $9-11,2-8$ \\
Rio Grande & L & $3-8,8-9$ \\
Tiffin & Split & $7-2,1-10$ \\
Urbana & Split & $0-9,6-3$ \\
Walsh & L & $2-2,0-11$ \\
Malone & Split & $2-9,8-7$ \\
Shawnee State & L & $6-11,5-12$ \\
Mt. Vernon & L & $1-4,0-9$ \\
Wittenburg & L & $4-8$ \\
\hline
\end{tabular}

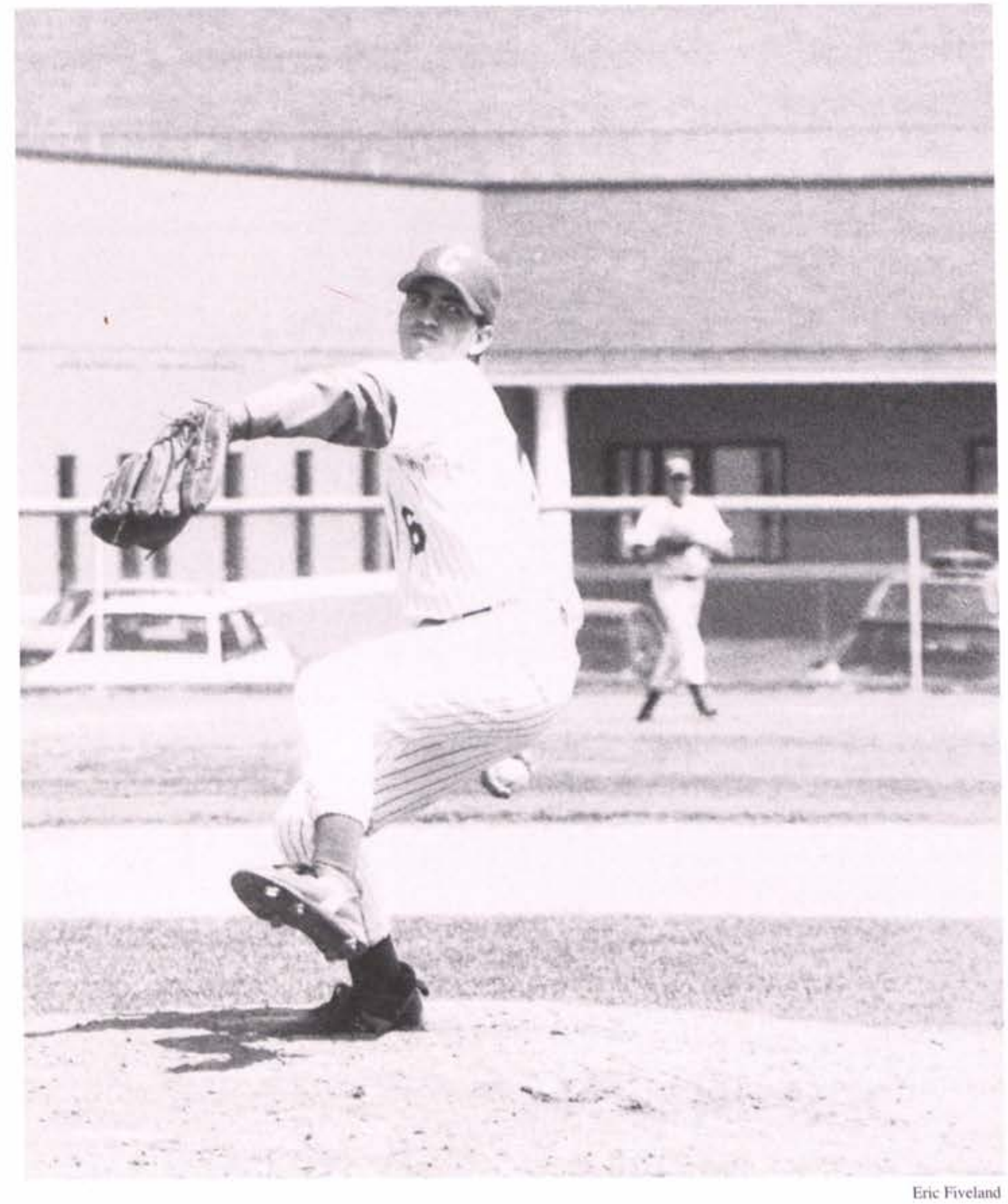

above left: Brian Rasey makes it safely on base. above: John Freese concentrates on throwing a strike. below: Josh Green makes a base hit.

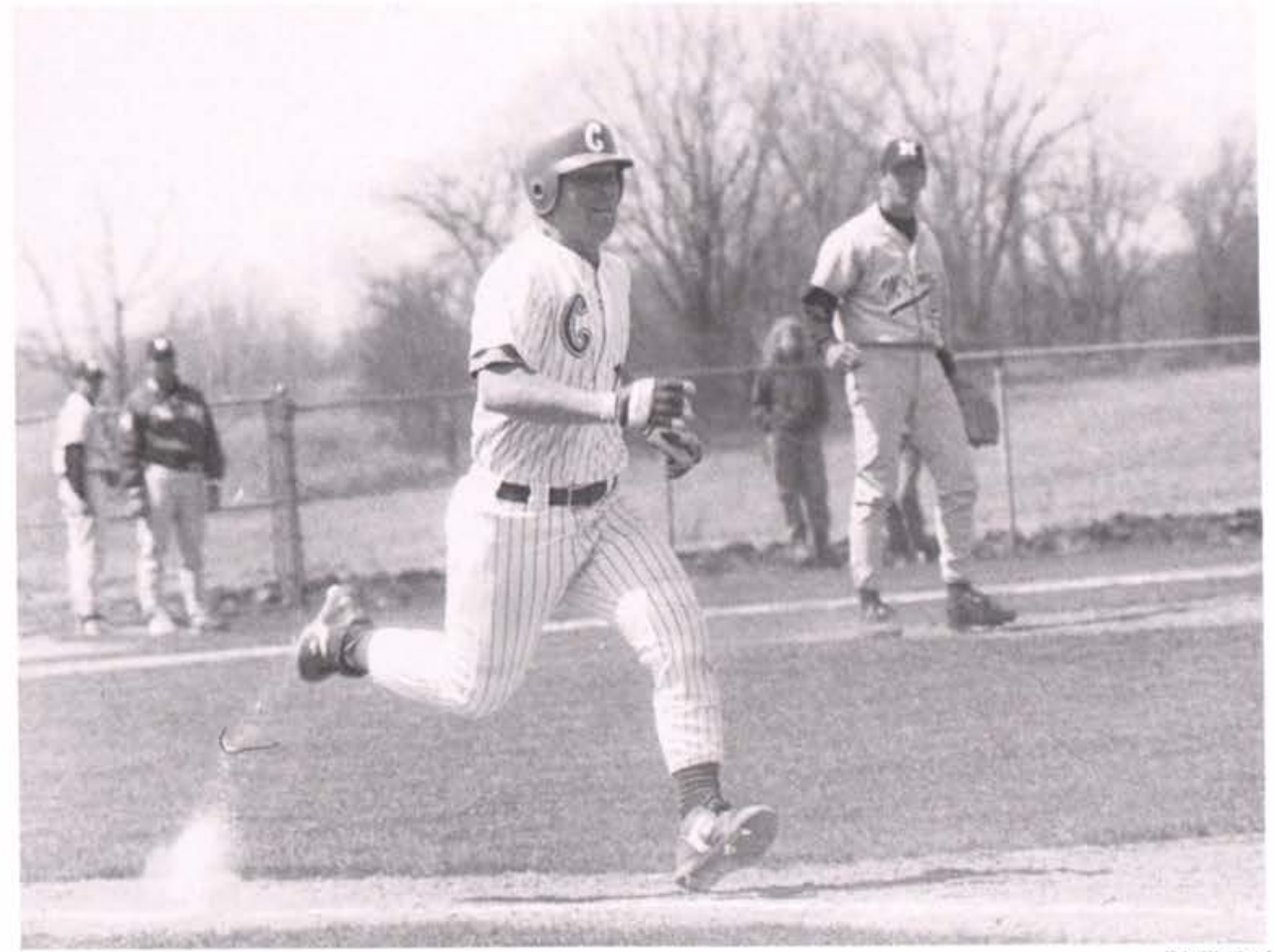

“. . .we'll have some difficult non-league games. We have some players who I believe have the talent to compete, so we're looking forward to the challenge."

--Coach Norris Smith 


\section{Defending}

This year's Cedarville College women's track and field team was marked by numerous individual successes and decisive team victories. The senior members were extremely strong and provided many points for the team. The regular season culminated with the women defending their title for the fourth straight year as National Christian College Meet champions.

At the National Meet, the women's team scored points in all but one event, while taking first in many of them. The Lady Yellow Jackets secured a landslide victory and topped off a successful season. Their point totals were fueled by outstanding senior athletes like Julianne Pletcher and Joy Beitler. Beitler won the 100 meter hurdles, 400 meter hurdles, high jump, and also ran on the winning $4 \times 400$ relay team. Pletcher won the 3,000 meter and the 5,000 meter run. Cedarville women were true to their winning form in the distance events. Sophomore Becky Jordan, Senior Jill Zenner, and Junior Megan Stevens all scored in the 1500 meter. Pletcher, Senior Kara Malone, and Junior Rachelle Elder swept the top

\section{The Top}

\section{Women's Track}

three places in the 5,000 meter, while Junior Becca Jenks, and Senior Michelle Burson were third and fourth in the 10,000 meter. In the field events Cedarville's dominance continued; Sophomore Lindsey Mitchell and Zenner were third and fourth in the high jump behind Beitler's winning jump. Mitchell was fourth in the triple jump and Freshmen Jodi Quint was also fourth in the javelin. Freshmen Sandy Swales and Alison Huizinga took second and fifth in the hammer. In the end, the Lady Yellow Jackets brought home another championship banner and another year of excellent track and field. At the awards ceremony for the meet all junior and senior athletes attaining the scholar athlete awards for high GPA's and athletics combined were honored. Cedarville women represented the vast majority of those receiving the award. Pletcher was named as the outstanding female athlete for the meet. Also receiving "at large" All-American status for the women was Jordan for her 1500 meter race.

A number of the men and women continued on to compete at the NAIA National meet in Atlanta, Georgia on May 21-23. Zenner was named national champion for her victory in the 3,000 meter race walk and set a new national record by 37 seconds. Burson ran an excellent marathon, and Pletcher scored points in the 10,000 meter. Beitler, who tied for second in the high jump and took fourth in the 400 meter hurdles, continued the scoring for the women in the national meet.

Assistant Professor of Physical Education and Coach Paul Orchard is in his fourth season at Cedarville and now sees the first freshmen class that he has coached graduate. Orchard is proud of their achievement and looks forward to the rest of the team benefiting from the leadership this year's seniors provided.

Peter Bednarek

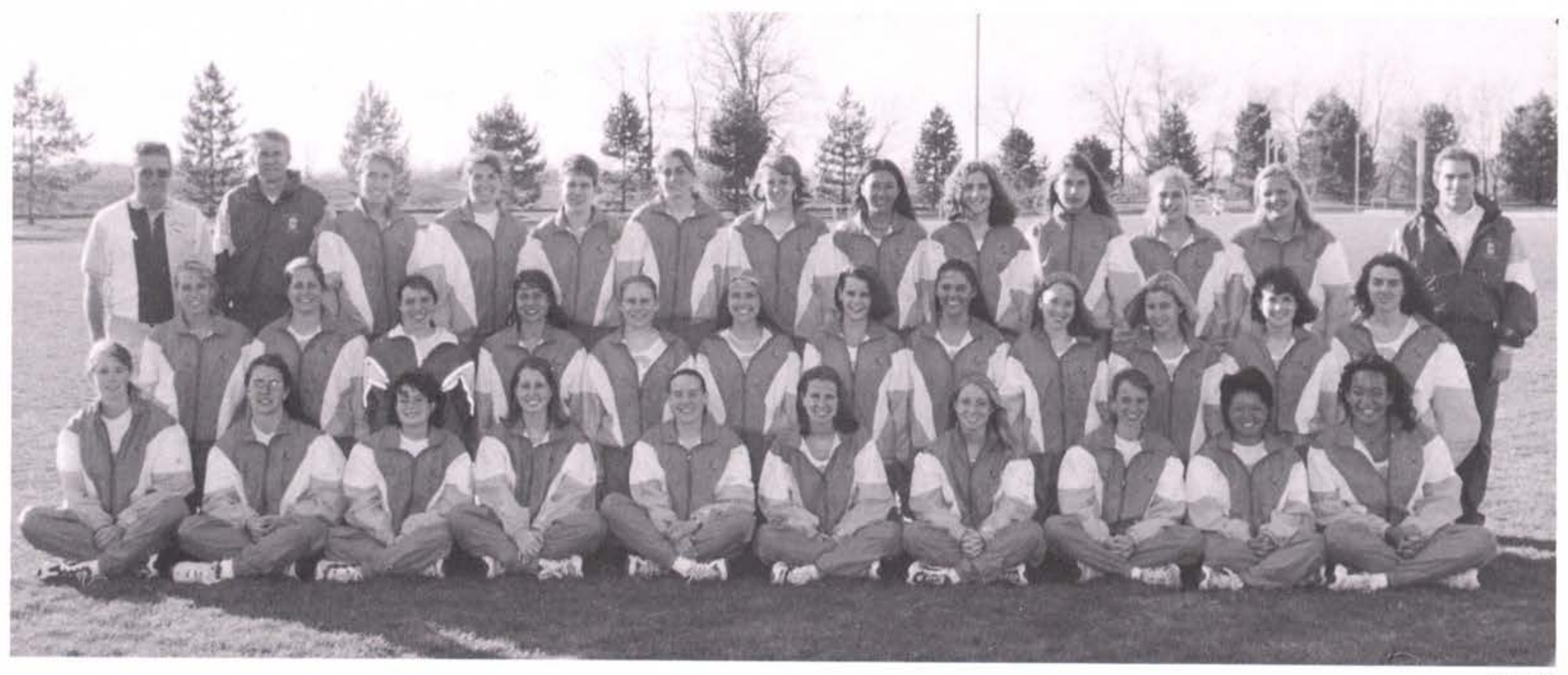

Scott Huck

row 1 (1-r): Jodi Quint, Alison Huizinga, Kara Malone, Jori Forward, Michelle Burson, Rachelle Elder, Jill Breckenfeld, Becky Jordan, Kari Persons, Kelly Ford. row 2: Joy Beitler, Sandra Swales, Leah Alley, Christy Taylor, Kelly Reitz, Loree Beth Fraley, Nikki Luckmann, JeriAnn Goodbar, Edith Steele, Christian Sicard, Megan Stevens, Meredith Allgrim. row 3: Assistant Coach Elvin King, Assistant Coach John McGillivray, Jill Bruin, Kristin Jacoby, Becky Campbell, Lindsey Mitchell, Laura Glessner, Lina Ponder, Jessica Chambers, Debbie Trimble, Jody Thompson, Quincy Kilburn, Head Coach Paul Orchard. 


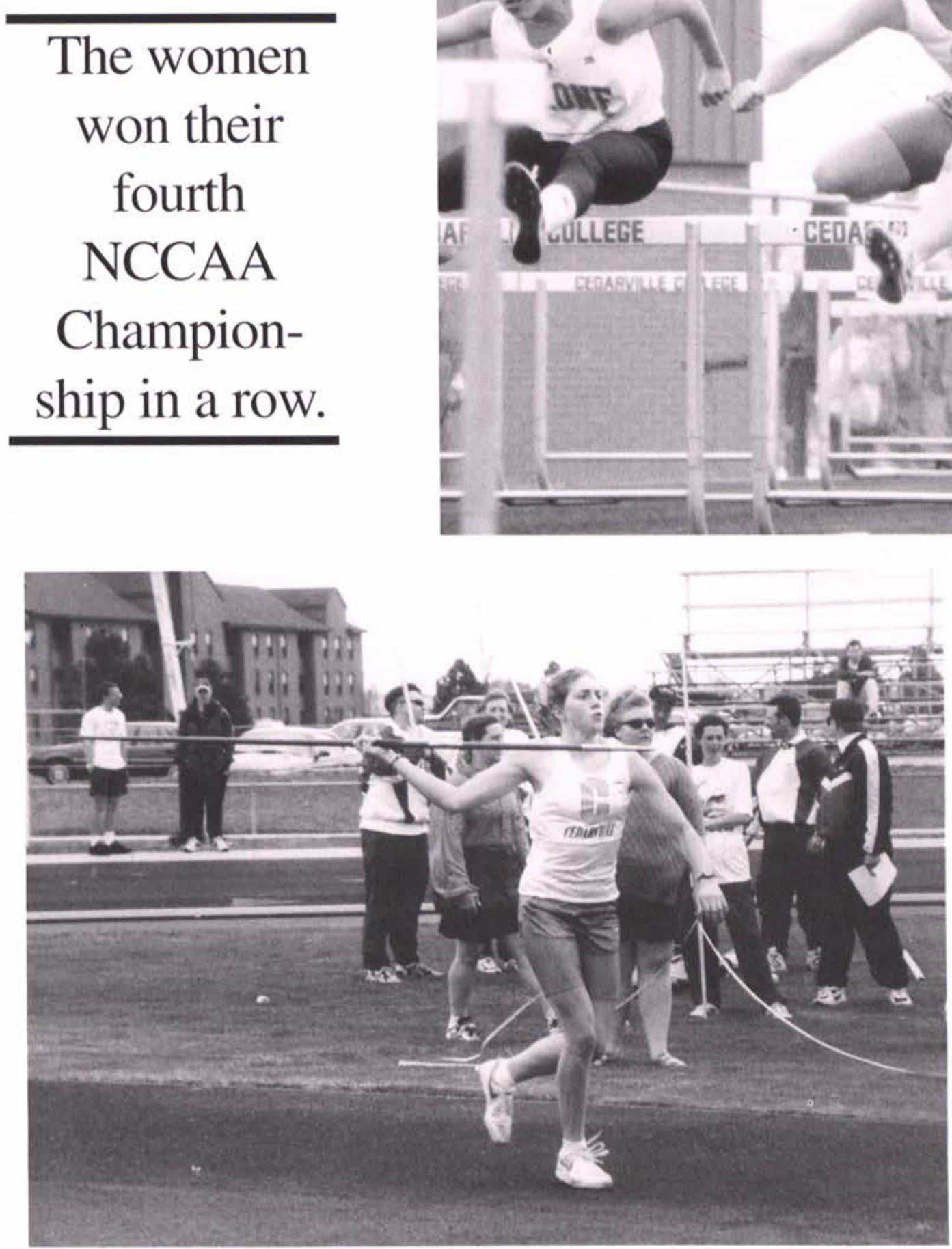

Eric Fiveland

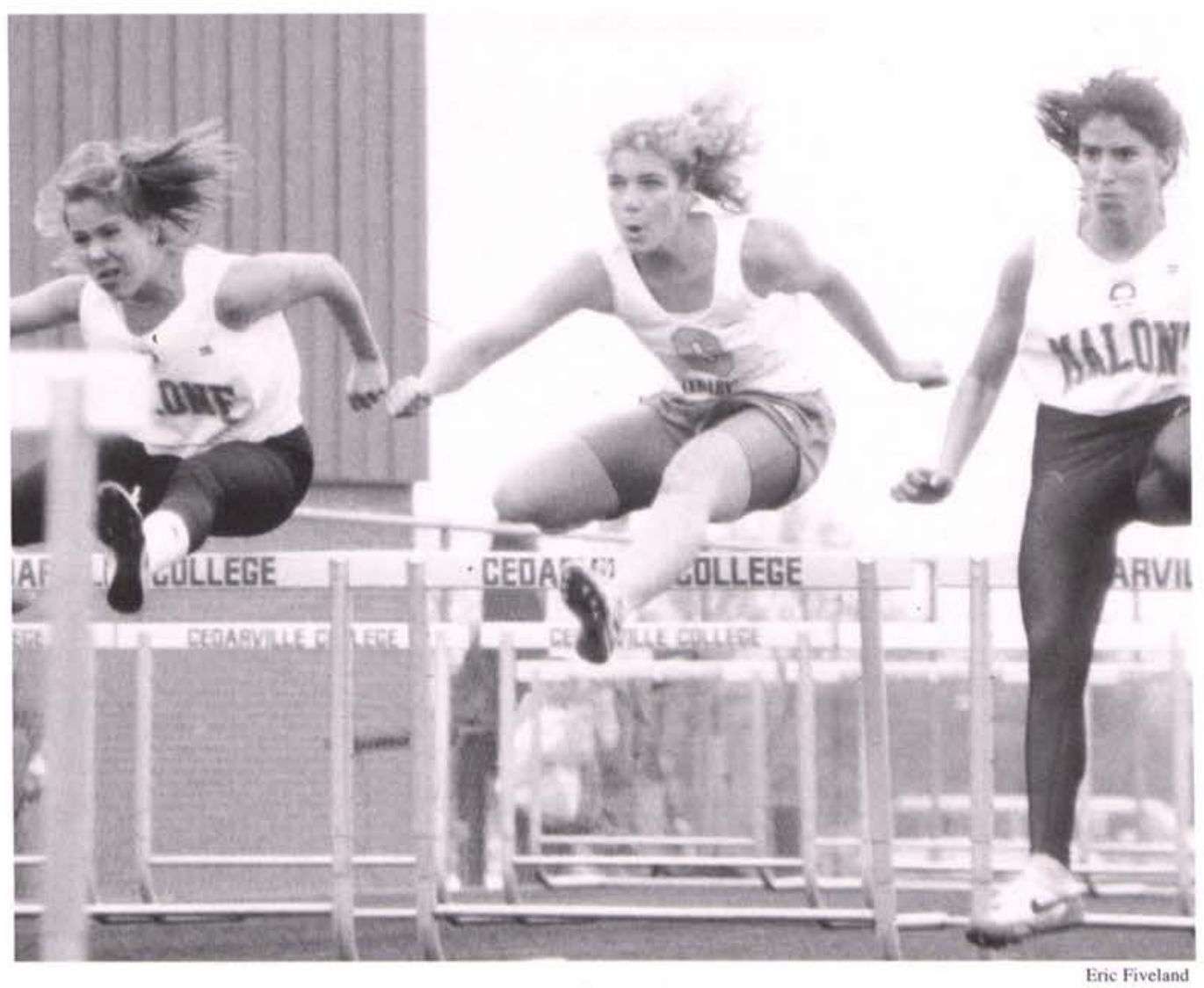

Eric Fiveland

\begin{tabular}{|llr||}
\hline \multicolumn{3}{|c|}{ StatisticS } \\
\hline & \multicolumn{1}{|c|}{ Meet } & Team Place \\
\hline Mar. 21-22 & at Florida State Relays & Non-Scoring \\
Mar. 28 & CEDARVILLE INVITATIONAL & Non-Scoring \\
Apr. 5 & at Wittenberg Invitational & 2nd of 13 \\
Apr. 12 & at Miami Invitational & 8th of 8 \\
Apr. 19 & at Mid-Ohio Conference & 2nd of 5 \\
Apr. 26 & at Baldwin-Wallace Invitational & Non-Scoring \\
May 2-3 & NCCAA NATIONALS & 1st of 15 \\
May 9 & at Indiana Wesleyan Invitational & Non-Scoring \\
May 21-23 & at NAIA Nationals & tie 13th of 47 \\
\hline
\end{tabular}

above: Jody Thompson clears the hurdle. below left: Lindsey Mitchell practices her javelin throwing technique. below right: Julianne Pletcher races for the Yellow Jackets.

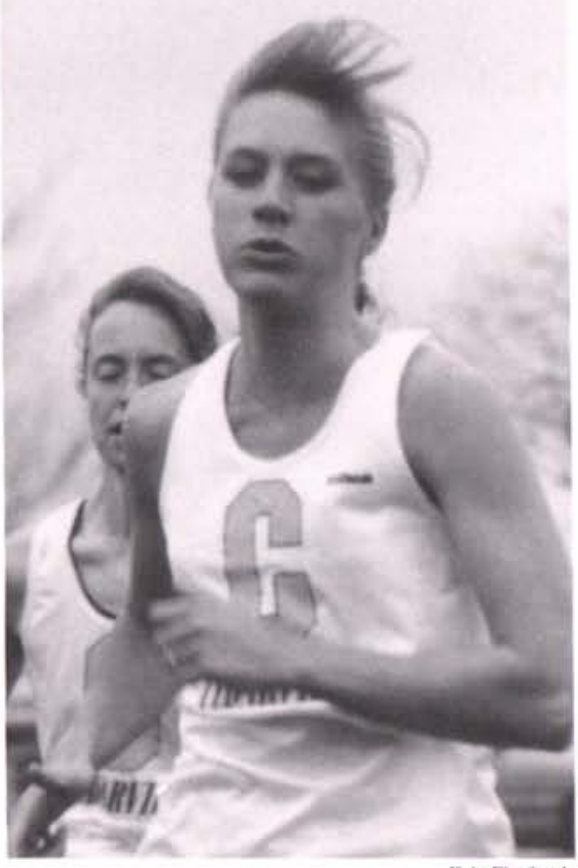

Eric Fiveland 


\section{Making Great}

This year marks the fourth year for Assistant Professor of Physical Education Paul Orchard as head coach for the Cedarville College men's track and field team. The four seniors: Doug Amundson, Josh Bell, Dave Swasey, and James Persenaire are the only members of the team from Orchard's first year.

For most of the Cedarville College track and field team members the May 3 , National Christian College track meet marked the culmination of a strenuous, yet exciting season. The men placed $3 \mathrm{rd}$ out of 15 as a team.

The men were third in the meet behind Taylor and Malone respectively. The only event in which the men took first place was the 100 meter dash. Junior Linton Ellis defended his title for a second straight year in the race. Sophomore Lee Reinhard finished fourth as well. In the 400 meter Senior James Persenaire and Freshmen Nate Jenkins were second and third respectively. Many of the distance events were dominated by strong Taylor and Malone athletes. Taylor University's standout Senior James

\section{Strides}

\section{Men's Track}

Njorage was impressive, winning the 800 meter, 1,500 meter, and 5,000 meter races. Junior Chris Leverette was third in the 400 meter hurdles and also picked up fourth in the 110 high hurdles. Cedarville men finished second in both the $4 \times 100$ meter relay and the $4 \times 400$ meter relay. Sophomore Jon McGinnis finished third in the pole vault and Reinhard was second in the long jump. Junior Dan Hudson threw his way into second place in the discus, third in the javelin, and fifth in the shot put. Senior Dave Swasey took third in the hammer throw. McGinnis was honored as an "at large" NCCAA AllAmerican for his vault of 14'6". In the end, the meet saw many good performances, and the Yellow Jackets had a solid score as a team.
With the conclusion of the regular season competition, a few members of the men's team traveled to Atlanta, GA, to compete at the NAIA National meet. McGinnis was named NAIA All-American for his vault of $15^{\prime} 3^{\prime \prime}$ and Senior Steve McGillivray ran very well in the marathon. Swasey and Hudson represented the throwers in Atlanta, while Leverette and Junior Dave Rea set personal records in the 400 hurdles and 800 meter races respectively.

Looking back on the season for the men, it is evident that this team performed well in spite of youth and many injuries near the end of the season. The majority of the members are freshmen and sophomores. They will return next year after a summer of conditioning to pick up their specific events again and build upon what they have done. The men will lose only four seniors, and definitely expect to return with an even stronger team for next year.

Peter Bednarek

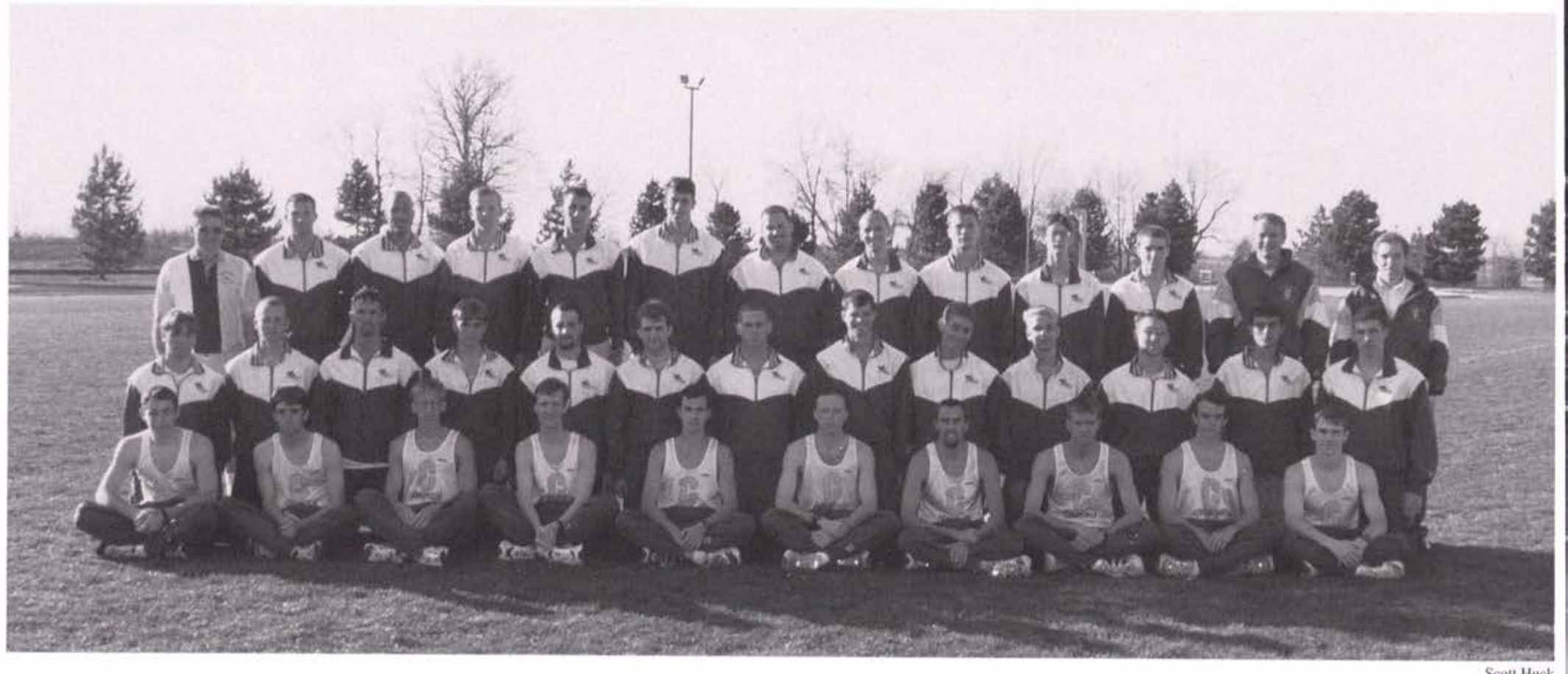

row 1: Mark Hayner, Michael Freeman, Chris Leverette, Eric Crawford, Brian Hilty, David Rea, Greg M. Johnson, Josh Bell, Nate Jenkins, Jon McGinnis. row 2: Ben H. Thompson, Craig Shank, Lee Reinhard, Jeremy Margene, Nathan Arndt, Dan Freeman, Pete Gruhlke, Greg Havens, Scott Ruhlman, Joel Peterson, Ben Stutzman, Steve McGillivray, Pete Bednarek. row 3: Assistant Coach Elvin King, Scott Walker, Dwayne Bryan, Chris Merrell, James Persenaire, Dan Hudson, Dave Swasey, Nathan Houk, Shawn Graves, Lance Harkleroad, Aaron Margene, Assistant Coach John McGillivray, Head Coach Paul Orchard. 


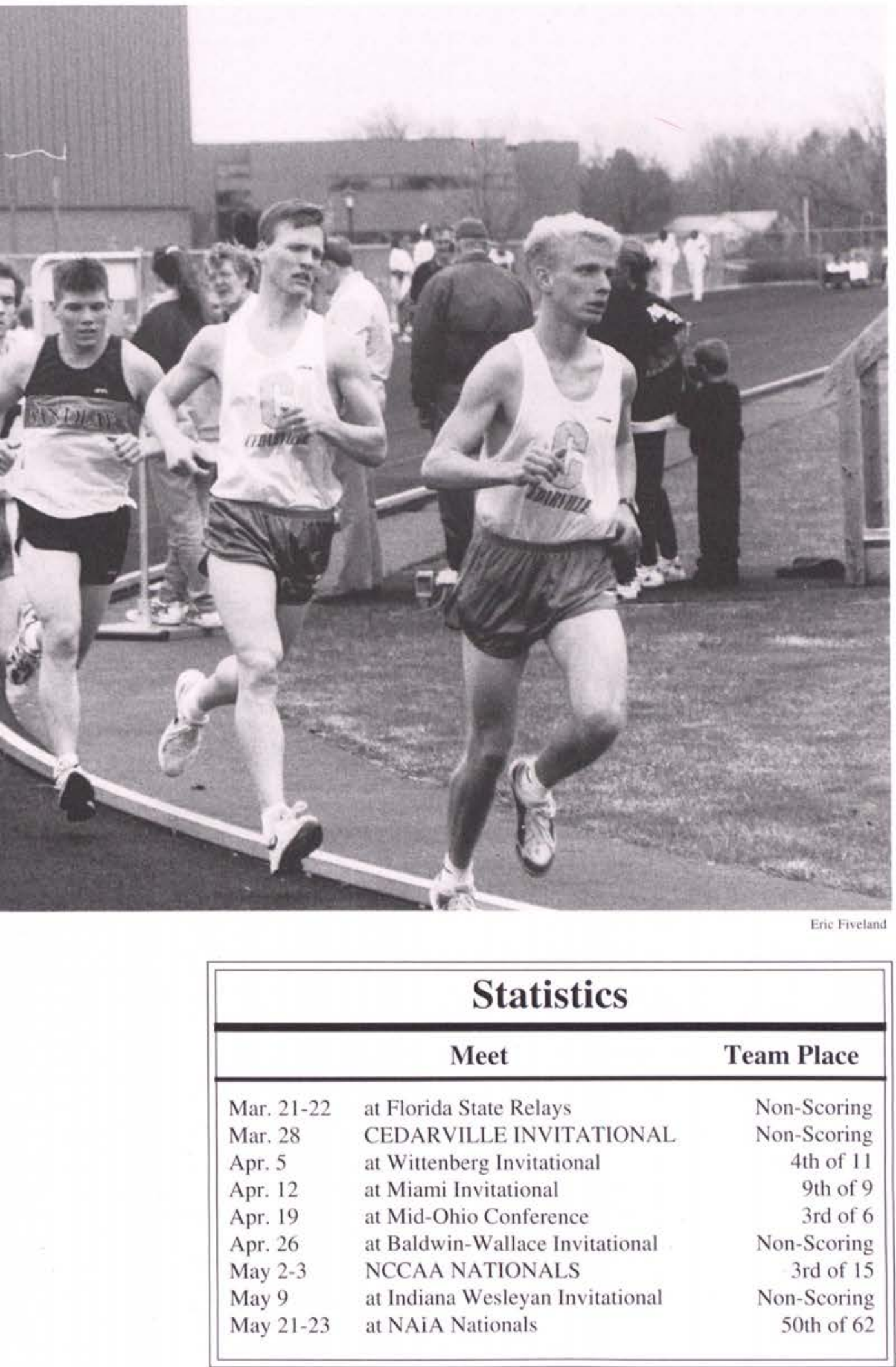

left: Joel Peterson followed by Eric Crawford compete in the 10,000 meter run. right: Jon McGinnis pole vaults for the Yellow Jackets. below: David Rea leads the pack in the 800 meter race.

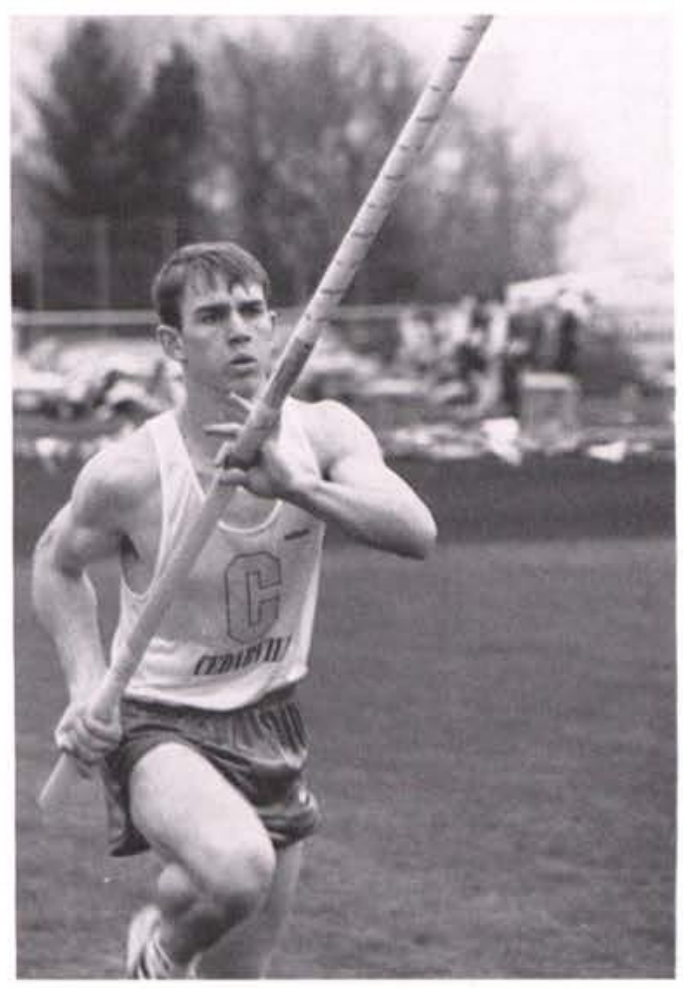

Eric Fiveland

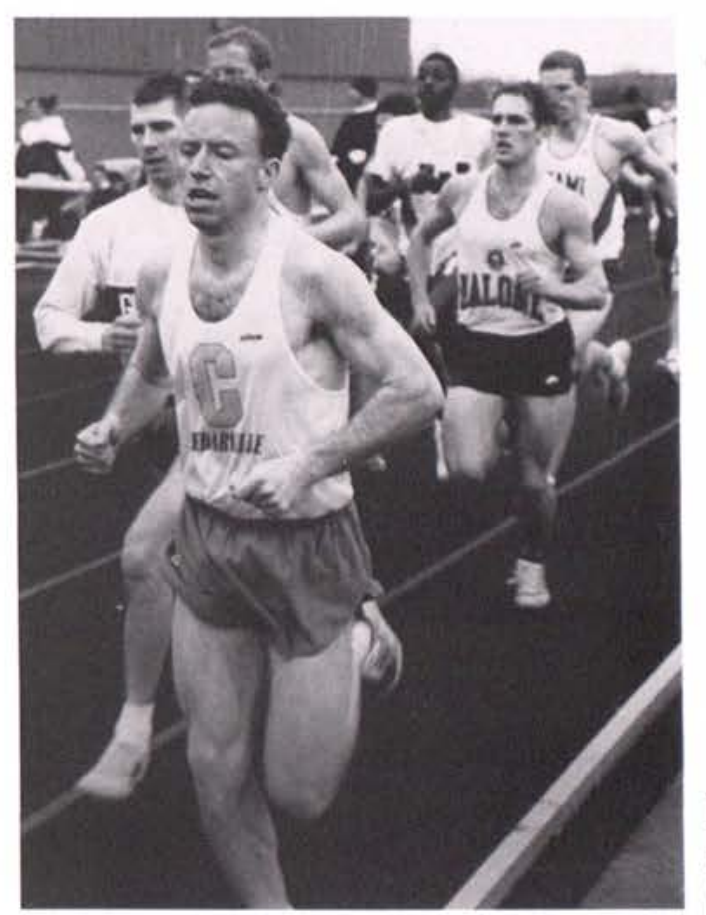




\section{The Spirit of Cedarville}

games is the pep-band. Cedarville's pepband was started in 1979 by Professor MichaelDiCuirci. "Don Callan, then head coach, approached me and asked me to consider organizing and directing a permanent pep-band for men's varsity home games," DiCuirci said. "I was new and enthusiastic and eager. I wanted to do it. I didn't realize that it would catch on so strongly. It is now a Cedarville institution."

The pep band has been present at every home game for 19 years. They play music if there is a pause on the court or inbetween periods. Pep-band also adds life to the game by starting cheers and striking up a beat that fans can stamp or clap to.

\section{Pep Band}

DiCuirci tries to keep the music library current but he also has a few songs that never get old. "Songs like Wipe Out, You Can Call Me Al, and Hang On Sloopy are staples but we add four to five new tunes every year."

Pep-band is a time for college students, faculty, staff, and a few high-school students who enjoy playing instruments to play in a less formal atmosphere. "Pepband is a total fun group that plays tune arrangements and exists for one purpose: to inject life and spirit into the games. We play well, but are not under the same set of rules as the concert ensembles," said DiCuirci.

There are 140 people listed as part of the pep-band, but there are usually only 90 at an average game. Unlike all the other musical instrument groups on campus pep-band does not hold auditions. Anyone interested just needs to show up to the first practices. Ironically the pepband has a larger audience. "We play to more people in a season than all of our concert groups do in five years. Three thousand people a game times 15 home games is quite an audience," said DiCuici.

Stephen Simons

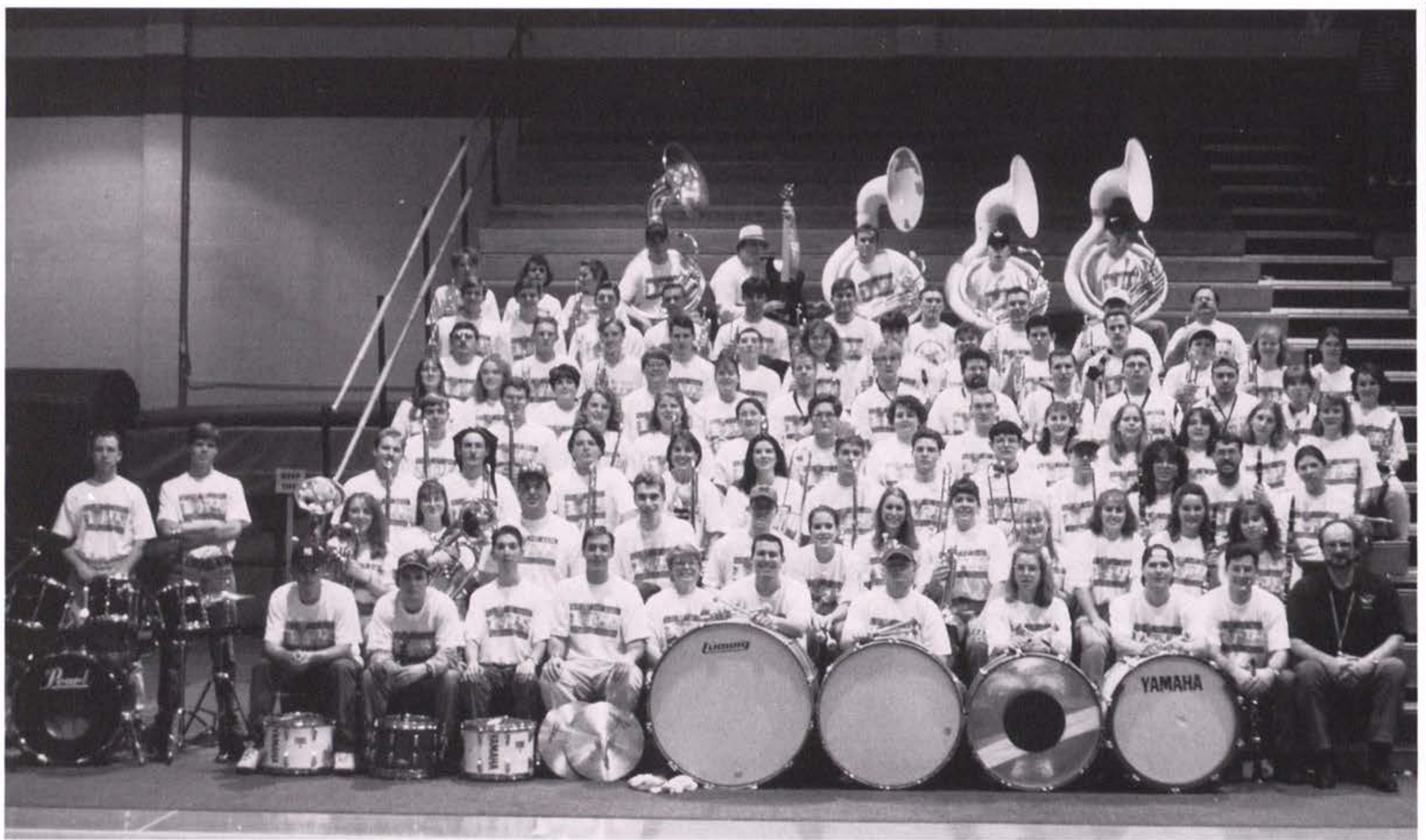

Eric Fiveland 


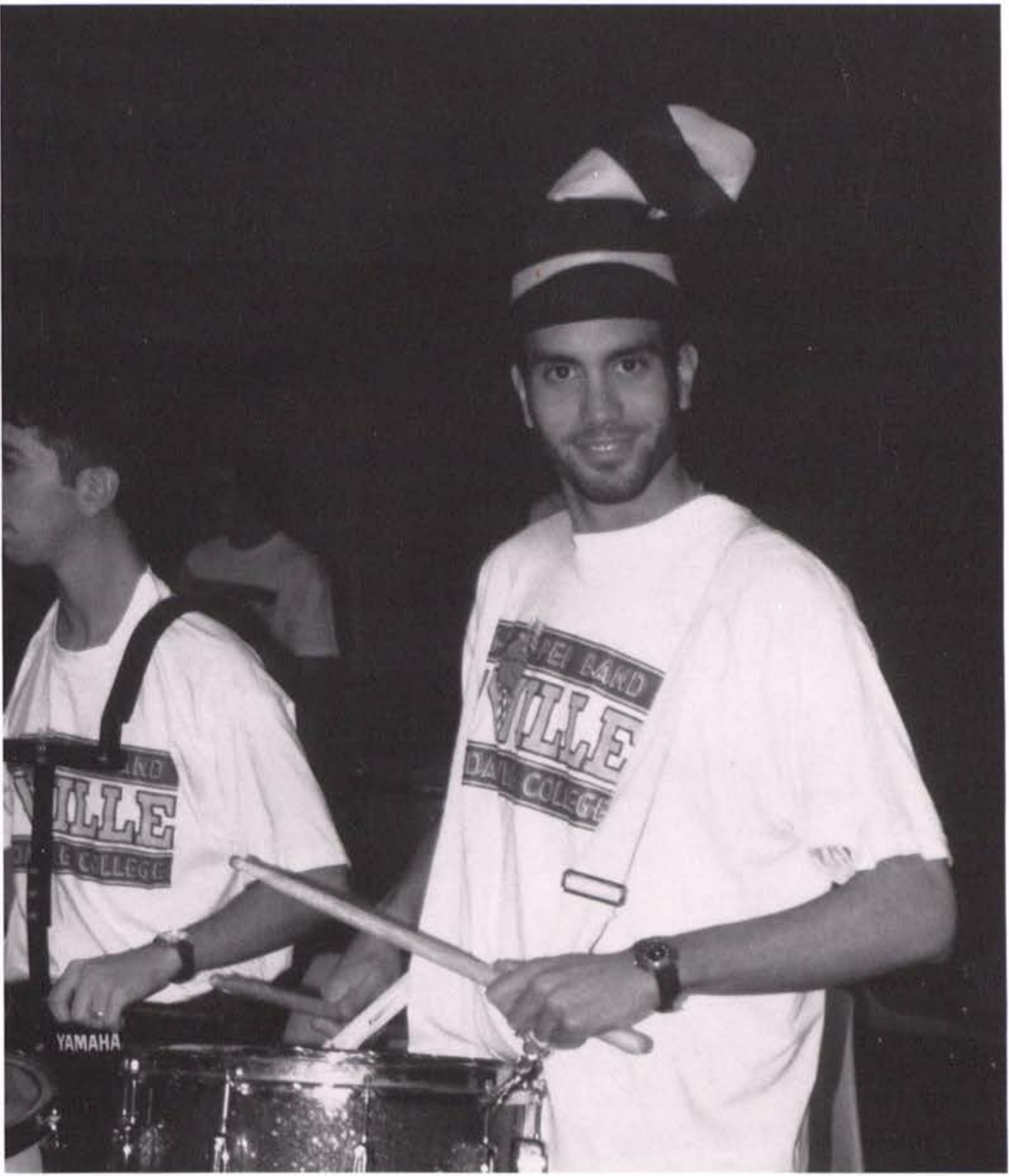

left: Randy Yinger wearing his yellow and blue hat shows his yellow jacket spirit. below: Susan Hunsaker sways to the beat.

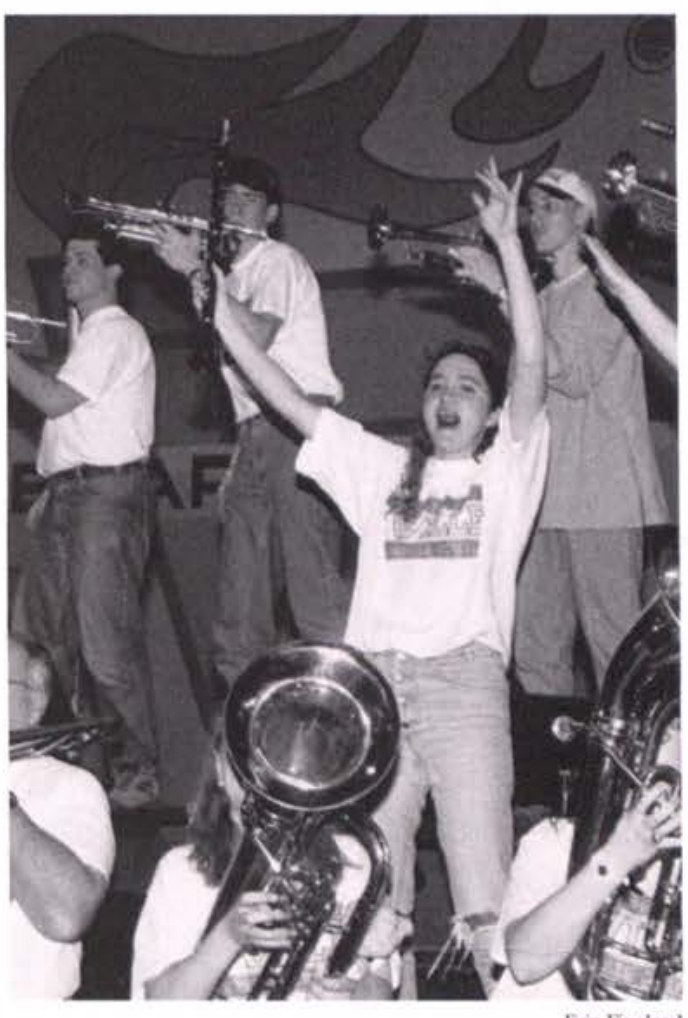

\section{Pep Band}

Baritone

Keith Hamer

Tim Hewitt

Becky Kayser

Bethany Walket

\section{Clarinet}

Matt Baldwin

Suzanna Creps

Angela DeSantis

Cheryl Emmert

Susan Hunsaker

Nathan Lewis

Christel Linerode

Anna Lorenz

Christy Marn

Kim Maynard

Stephanie Mead

Amy Meckley

Sam Polgrady

Lesley Shover

Heather Smith

Flute

Rachael Ayres
Stephanie Buchholtz

Natalie Bunch

Jennifer Cook

Trisha Johnson

Melanie Lehman

Amy Morse

Michaela Murray

Michelle Picuri

Amanda Ratliff

Jennifer Rogers

Stacey Romeyn

Erin Ross

Valerie Smith

Rebekah Sorensen

Renee Tuinstra

Kristy Turner

Rebecca Vanderground

Oboe

Leanne Heath

French Horn

Melody Brickel

Kara Doden

Natalie Keller
Summer Schafer

Amy Stowers

Percussion

Andy Bergman

Kimberly Boesch

Rob Bouwens

Kelly Boyd

Chris J. Brown

Ryan Eby

Michael Graybill

Daniel Hicks

Jim Johnson

Shari Magin

Ethan McQuinn

Joshua Nelson

Cheryl Sims

Josh Smith

Wesley Warriner

Mark Wyse

Randy Yinger

Saxaphone

Brad Barnard

Pam Claus
Gretchen Dorman

Kelly Grindall

Chet Jenkins

Brenda Johnson

Tom Leightenheimer

Brett Pfeiffer

Ted Russell

Ben Schroeder

Paul Sheldon

Kristen Wawro

\section{Trombone}

Kevin Armstrong

Aaron Berning

Joyce Boggs

Shaun Boyle

Brandon Caudill

Steve Hodson

Steven McGillivray

Mandy Murray

Joe Reaper

Scott Ruhlman

Jeremy Staley

Tobin Strong

Amy Thayer
Jill Townsend

Charlie Walker

Andrew Woodman

Trumpet

Isreal Barr

Michelle Boehm

Amy Bohn

Jeff Brown

Josh Childs

James Filson

John Filson

Ben Forshee

Deborah Frank

Caren Furst

Andrew Manwiller

Annie Miller

Andrew Nyveldt

Colin Ovenel

Chris Pagnard

Richard Porter

Naomi Redington

Kimberly Rideout

Dan Ruba

James Scheid
Kevin Sims

Daniel Sines

Ben Stutzman

Ed Supplee

Eric Svendsen

Flynn Tregay

Tim Van Dalen

Becky VanLoon

Justin Work

Bill Workman

Tom Wright

Tuba

Chris M. Brown

Paul DiCuirci

Michael DiCuirci

Steve Fox

Bethany Frank

Chris Grigson

Dan Hacker

Virginia Miller

Dave Nicholas

Josh Rupp 

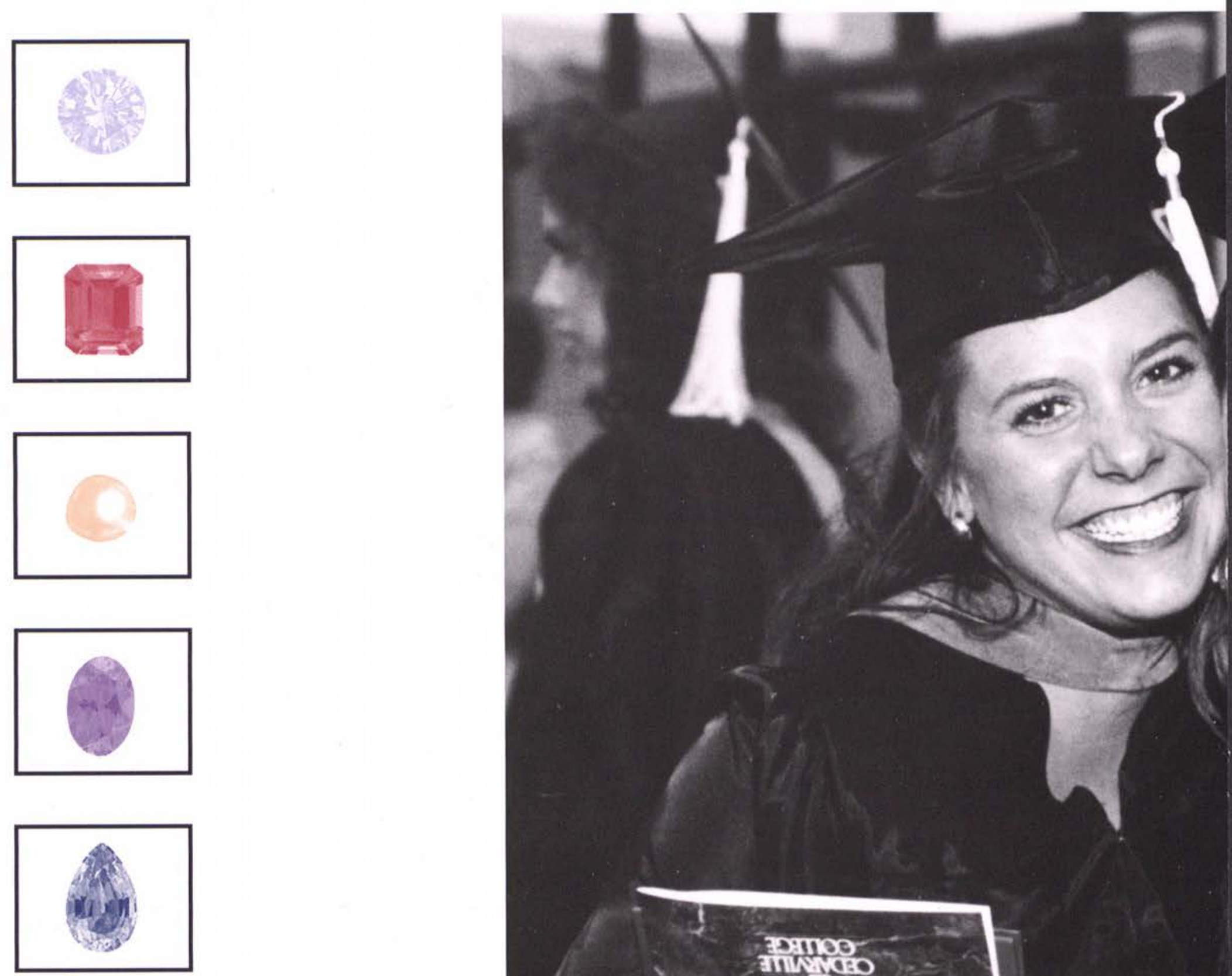

And coming to Him as a living stone, rejected by

men, Gut choice and precious in the sight of God. you also as living stones, are being fuilt up as a spiritual house for a holy priesthood, to offer up spiritual sacrifices acceptable to God through Jesus Christ. 

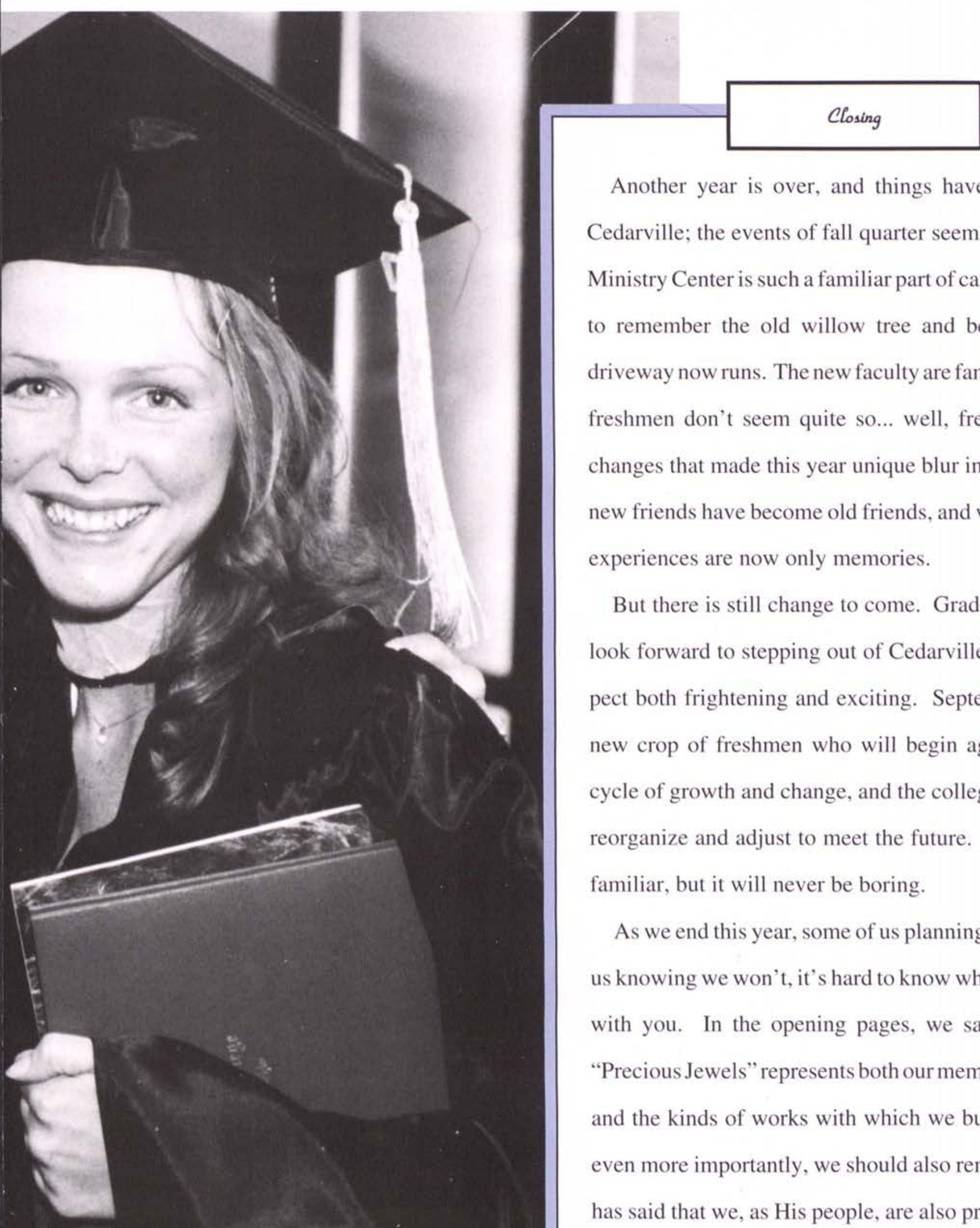

Another year is over, and things have settled down at Cedarville; the events of fall quarter seem so long ago. The Ministry Center is such a familiar part of campus that it's hard to remember the old willow tree and benches where the driveway now runs. The new faculty are familiar faces and the freshmen don't seem quite so... well, freshman-like. The changes that made this year unique blur into previous years: new friends have become old friends, and what began as new experiences are now only memories.

But there is still change to come. Graduating seniors can look forward to stepping out of Cedarville's bubble, a prospect both frightening and exciting. September will bring a new crop of freshmen who will begin again the four-year cycle of growth and change, and the college will continue to reorganize and adjust to meet the future. Life may become familiar, but it will never be boring.

As we end this year, some of us planning to return, some of us knowing we won't, it's hard to know what thought to leave with you. In the opening pages, we said that the theme "Precious Jewels" represents both our memories of Cedarville and the kinds of works with which we build our lives. But even more importantly, we should also remember that Christ has said that we, as His people, are also precious stones. We are the materials out of which $\mathrm{He}$ is building His Church, whether here or out in the wide world.

Matt Sherwood 
The 1997 Miracle staff offers a special than you to all those chuches and parents who through their generous gifts made this Miracle possible. Without their help many special Elements of this yearfooch such as extra pages, photography or desigh elements woul be greatly reduced or Eliminated. Thank you!

Berean Baptist Church

Brunswick, ME

Berean Baptist Church

Springfield, IL

Calvary Baptist Church

Xenia, $\mathrm{OH}$

Emmanuel Baptist Church

Toledo, $\mathrm{OH}$

First Baptist Church

Moweaqua, IL

Grace Bible Church

Newfane, NY

Heritage Baptist Church

Clarks Summit, PA

Patterson Park Church

Dayton, $\mathrm{OH}$

Pike Grace Brethren Church

Johnstown, PA

Sheffield Chapel

Sheffield, MA

Shoaff Park Baptist Church

Ft. Wayne, IN

Larry and Nancy Allen

ABWE Missionaries
James Beckley

Londonberry, NH

Mr. and Mrs. Vernon Berning

Sidney $\mathrm{OH}$

Mr. and Mrs. Rick Bowersox

Dayton, $\mathrm{OH}$

Mr. and Mrs. John W. Boyes, Jr.

Cleveland Heights, $\mathrm{OH}$

Preston and Ruth Butcher

Plantation, FL

Jack and Susan Carnahan

Clarkston, MI

Mr. and Mrs. Dan Crecco

Navarre, $\mathrm{OH}$

John and Marsha Eads

Jackson, MI

Mr. and Mrs. Robert A. Fisher

Northridge, CA

Paul and Kathy Goetz

Westerville, $\mathrm{OH}$

Dr. and Mrs. Leon Gombis

Palos Heights, IL

Mr. and Mrs. Dave Gosman

Madison, IN

Gerald and Linda Grow

Whitehall, MI
David J. and Linda J. Hangosky

Chesterland, $\mathrm{OH}$

Don and Marilyn Harju

Hudsonville, MI

Sharman Hedges

Massillon, $\mathrm{OH}$

Mr. and Mrs. David Hoadley Epsom, NH

Donald and Donna Ireland

West Hampton, NJ

Randy and Vicki Junkins

Downingtown, PA

Mark and Glennie Justice

Indianapolis, IN

Mr. and Mrs. Dennis Kuyper

Creston, IA

Mr. and Mrs. James Latourelle Chatham, MA

Thomas T. Leightenheimer

Cedarville, $\mathrm{OH}$

Mike and Bonnie Luck

Ft. Washington, MD

Dr. and Mrs. William Lyons

Palo Cedro, CA

Clarence and Edna Mast

Tunkhannock, PA 
Veronica P. Mayer

Brookfield, WI

Buryl McFadden

Dayton, $\mathrm{OH}$

Chuck and Toni Miller

Mokena, IL

Tom and Pat Miller

Monroeville, $\mathrm{OH}$

Michael and Sharon Morris

N. Collins, NY

Jeff and Sharon Mraz

Akron, $\mathrm{OH}$

Mark and Diann Murphy

Burdett, NY

Mr. and Mrs. Alan Noble

Westwood, NJ

James and Carole Otten

Xenia, $\mathrm{OH}$

Dr. and Mrs. Kenneth Petersen

Goshen, IN

Mr. and Mrs. Russell D. Pierce

Sinking Spring, PA

Mr. and Mrs. Ronald A. Pierre

Oberlin, $\mathrm{OH}$

Mr. and Mrs. David Reisenbigler

Erie, IL

Mr. and Mrs. Charles Robertson

Bay Village, $\mathrm{OH}$

Lidia Ruba

Philadelphia, PA

Gary and Vicki Ruhl

Derry, NH
Mr. and Mrs. Gary Rutledge

Rochester Hills, MI

Mr. and Mrs. Glenn Saunders

Racine, WI

Mr. and Mrs. Lorne Scharnberg

Des Moines, IA

Icilin Senior

Mahwah, NJ

Mr. and Mrs. Paul R. Snowden

Mason, $\mathrm{OH}$

Young Chil Sohn

Chicago, IL

Joe and Jan Sprankle

Chesapeake, VA

Mr. and Mrs. Richard Staedtler

Langhorne, PA

Robert W. Strong

Chelsea, MI

Keith and Nancy Svendsen

Algonquin, IL

Mr. and Mrs. Michael Thomas

Flint, MI

Mr. and Mrs. Gary Vandemark

Grand Blanc, MI

Leroy, Rosemary, and Scott Vandegrift

North Canton, $\mathrm{OH}$

Tom and Kathy Waardenburg

Nashua, $\mathrm{NH}$

Mr. and Mrs. Chuck Wambold

W. Carrollton, $\mathrm{OH}$

Mr. and Mrs. John Warnken

Factoryville, PA
Allen and Linda Wehr

Mifflinburg, PA

Mr. and Mrs. David L. West

Temperance, MI

Dave and Cheryl Whitten

Romeo, MI

Dr. and Mrs. Ken Wiesert

Indianapolis, IN

Michael and Melanie Wiljamaa

Findlay, $\mathrm{OH}$

Carole Williams

Ozone Park, NY

James and Lynda Wishart

Canton, MI

Mr. and Mrs. James M. Witt

Troy, MI

Paul and Gwendolyn Wright

Tyler, TX

Mr. and Mrs. David C. Wu

Apex, NC

Stan and Leah Yeiter

Warsaw, IN 
Ayres, Rachael .................120. 239

Abas, Jennifer . 120, 150, 151, 176 Abbas, Robert ………………….... 64 Abbeg, Andy ……………….... 132 Abe, Elisabeth ………….......... 132 Abraham, Kristina ..................... 132 Abraham, Laura …………........ 110
Acton, Caleb 132 Acton, Caleb ....................... 132 Adams, Daniel ......................... 120 Adams, Matt ………….......... 110 Ager, Merlin ………............. 62, 181 Ager, Ruth .................................. 65 Albertson, Dawn120, 15I, 154, 162 Alderfer, Andrew ...............68, 176 Aldridge, Jared .................132, 155 Alexander, Heather L.................. 68 Alexander, Jason ............... 120, 172 Allcom, Eric ………………… 132 Allen, Barton ……………....68, 197 Allen, Danette …………............ 132 Allen, Debbie ………....... 110, 169 Allen, Kerry ……..........52, 68, 165 Allen, Luke …....................... 132 Allen, Mark ..... 110, 158, 189, 203 Allen, Titus .............................. 132 Alley, Leah …………….... 208, 234 Allgrim. Meredith .. 110, 155, 212 , 234

Allport, Charles ............................. 63

Alt, Emily …………..... 120, 175 Alt, Jennifer ………... 120, 174, 191 Alyn, Irene _........................... 40,63 Ament, Jennifer ……....... 132, 168 Amos, Elizabeth .............. 9, 68, 151 Amos, Josh 110, 171, 184, 203, 204 Amstutz, Jim $\quad 110,202$ Amstutz, Timothy .... 132, 181, 214 Amundson. Douglas .. 68, 101, 202, 203, 222, 223

Anderson, Al ............ 120, 170, 191

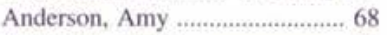
Anderson, Angie ........................... 68 Anderson, Lori 48, 49, 68, 150, 188 Anderson, Lyle ........................... 63 Anderson, Nikki …........... 110, 169 Anderson, Richard …………....1132 Anderson, Ryan …............. 110, 203 Andrews, Adria ………...... 68, 165 Andrews, Fran . Angela, John ............................ 6 Angelone, Jessica ..... 171, 194, 203 Anglea, Marjorie …………….... 65 Anglund, Dana ......... 132, 158, 182 Anglund, Erik _...…… 68, 200 Anna, Laurie ...................... 110, 174 Anthony, Darla .....................68, 190 Anthony, David ........................ 132 Appel, Becci ………….........110 Arimura, Ehrin ………..... 120,212 Armistad, Chris .......................... 69 Armitage, Melissa …………....... 69 Armour, Aaron .. 69, 150, 184, 185 , 203

Armour, Jenny ...... 8, 132, 150, 197 Armour, Julie ........... 110, 150, 20 Armstrong, Kevin 28, 110, 150, 239 Armstrong, Mark ......120, 150, 151 . 170, 195

Arndt, Alyssa 120

Arndt, Nathan

Arnold, Wayne ………....... 28, 120

Arthur, David

Ashcraft, Chris R. .... 110, 173, 188 201

Ashurst, Valerie ........... 47, 69, 190 Assid, Dena ……………... 110, 183 Atkinson, Molly ……....... 175, 194 Atwell, Jason .......37, 110, 150, 15 Augustine, Johannah .. 69, 151, 192 Augustus, Elizabeth …………. 132 Ausfahl, Joshua 110, 155, 176, 202 Ausfahl, Philip ................. 132, 172 Austin, Graigory .......................... 69 Avery, Daniel ………..... 132, 156 Avery, Jill L.

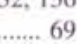

Awbrey, Emilie S

Awbrey, Jeremy K.

\section{B}

Baab, Stephen ………………... 132 Bader, Michal .............................65

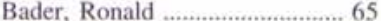
Baer, Allison ………….........110

Bailey, Justin ........ 49, 69, 184, 204 Bailey, Krystal 13, 15, 49, 69, 106 Baise, Barbara

Baise, Louann …… 65 Baisley, Alicia ...........120, 159, 192 Baits, Mark ………………...... 69 Baker, Carolyn ......................... 132 Baker, Deborah ……....... 132, 224
Baker, Glenn Baker, Glenn …………....... 28, 69

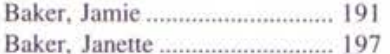

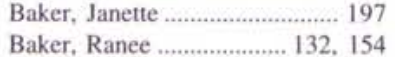
Baker, Rebecca ………………... 24 Baker, Sue …………………........ 62 Baldwin, Allister …………….... 120

Baldwin, Jennifer .

Baldwin, Martha ……….....65, 218 Baldwin, Matthew …….... 132, 239 Baldwin, Richard ………............ 62 Baley, Christina . 49, 69, 106, 179 , 202

Ballard, Stanley

Band, Clarissa

Bandy, Kimberly

Barber, Rebecca

Barch, Bethany ......... 132, 173, 212

Barker, Elizabeth ……………... 120

Barker, Erin ..............110. 171, 197

Barker, Gary ......................... 37, 38

Barkhaus, Julie ……...... 216, 217

Barnard, Bradford ............ 132, 239

Barnard, Jaclene ............ 132
Barnes, Jill _..... 110, 183, 194, 204

Barnett, Holly ........... 110, 160, 191

Barnhart, Ben ……u. I 22

Barr, Israel ................. 150, 151, 239

Barrett, Virginia ……………….... 70

Barrons, Jeff _................ 132, 214
Bartholomew, Raymond .......... 63

Bartholomew, Raymond ............. 63
Bartlett, Brad .................. 110, 20

Basa, Emilio ......................120, 171

Basley, Christie …………….... 188

Basner, Jonathan …………….... 110

Bassett, Philip …………………... 62

Bast, Molly

Batchelder, Rachel ……..... 70, 104 Bath, Lezley ....................120,150 Bathrick, Amy .............. 19, 70, 218 Bathrick, Sara …………......... 110

Batkin, John

Batt, Trevor

169
0.214

Battaglia, Nathan ....................... 109

Baumann, Eddie ……………….... 62

Bayler, Doug ......... 46, 52, 54, 106 ,

203,70

Bayley, Rob ...................... 120, 158 … 70

Beal, Mary Beth .................. 70, 165

Bean, Shannon ........................... 70

Bear, Jennifer …………............ 120

Beary, David ……………........ 132

Bechtel, B.J. ................... 191, 214

Bechtol, Heather ………....132, 168

Beck, Matthew .. 110, 150, 165,191

Becker, Ron ……….............132

Beckley, Matthew …………..... 120

Bedford, Jaime ………......... 110

Bedillion, Eric ..................110, 15

Beecher, Rachel ………….... 70

Beitler, Joy 10, 49, 70, 159, 204, 234

Belding, Karen .......................... 132

Bell, Bryan ……....... 120, 130, 185

Bell, Joshua ….... 70, 191, 203, 236

Bell, Megan .......................170, 176

Bennett, Sandra ................. 132, 169

Bennett, Stacie …..... 132, 196, 201

Bennington, Summer 110, 170, 218

Bensink, Sarah ........................ 132

Benson, Jennifer .... 16, 49, 70, 106 , 188.201 Bahn, Jennie ……………….... 120

Beachy, Susan

Beck, Jamie ................................. 70
Beres, Jennifer.

Bergman, Andrew

Berrus, Josh ................... 120, 150

Berry, Emily ........................... 253

Berry, Sabrina ……….....132, 178

Bertelson, Noelle ………......37, 150

Best, Paul .............. 8, 24, 198, 200

Beste, Jeff ..................65, 202, 204

Bethel, Heather ……... 70, 173, 198

Bett, Edwin ..... 110, 174, 188, 197

Beyer, Joseph ………...... 120, 159

Bielek, Robert …............65

Bielo, Allyson ...........110, 150, 161

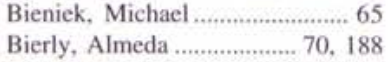

Bigelow, Ryan ………………. 120

Biggs, Haylee ............120, 172, 191

Billing, Stacey .................110, 188

Billock, Jim ..............132, 175, 181

Birk, Jeremy ................... 8, 228
Birmingham, Sandra …….......... 70

Bischoff, Thomas ...................... 132

Bishop, Emily …..............132, 160

Bjerke, Joel ......

Bjornstad, Christine …...... 132, 177

Bjornstad, James

Bjornstad, Karen ……….... 132, 178

Black, Jessica _...........120, 208, 209

Blackburn, Becca ...................... 132

Blackburn, Dave .............. 110, 204

Blackwood, Rebekah .......120, 130

Blair, Brian

Blakemore, Darcic …….... 120, 224

Blanton, Cynthia ………....120, 158

Blanton, Mary Jo _...……71, 193

Bleason, Stephenie ………..... 182

Bledsoe, Erik ………........120, 150

Blodgett, Andrew …..........132, 173
Blore, Melissa ................ 120, 192

120,192
65

Blumenstock. Helen.

Blumenstock, Richard .................62 62

Blythe, Jeremy ……………...... 133

Boblitt, Kevin 120, 150, 151, 175, 188, 198

Boehm, Matt .

Boehm, Michael .... 150

Boehm, Michelle 110,239

Boertje, Julia

Boesch, Kimberly ............. 120, 239

Boggs, Joyce

Boggs, Philip 7....... 239

Bohl, Emily …………....... 133, 158

Bohn. Amy ....... 120, 150, 151, 239

Bollman, Jill .................... 110, 171

Bollmeier, Kristen .............. 133, 154

Bolt, Michelle ............ 120, 151, 178

Bonenberger. Omer ..................... 62

Bonner, Michael ..................... 110

Bonner, Rebecca _................. 8,133

Bontrager, Rachel ........... 133, 181

Bookie, Ben .......................... 188

Boone, Elizabeth Ann ................. 71

Border, Rebecca ....... 120, 151, 197

Bordine, Andrew …………...... 133

Bork, Nichole ................... 133, 154

Borland, Lindsey .................... 133

Borling, Janine ………................. 45
Borling, Scott ................... 45, 71

Borton, Aaron ...... 133

Bosaw, Sara ……….........120,123

Bosworth, Dan

.. 120

Bott, Michelle .......................... 191

Bouwens, Robert 110, 155, 197, 239

Bowersox, Elizabeth ................. 120

Bowersox, James ........................ 65

Boyce, Angela

Boyce, Kelly.

Boyd, Andrew ……........ 133, 220

Boyd, David110, 150, 151, 176, 198 Boyd, Kelly .............. 133, 178, 239

Boyes. Kristen ….......120.151, 158

Boyle, Shaun .............. 133, 151, 239

Boynton, Shannon ...... 29, 120, 189

Bozick, Kelly

Bradds, Connie

Bradley, Scott A.

Brainard, Bradford

Brainard, Matthew

Braithwaite, Edwin

Brame, Michael

Brandfas, Ben.

Branon, Lisa

Brant, Kristin

Brazalovich, Debbic

Brdlik, Laura

Breckenfeld

Breneman, Jeff $\quad 49,71,189$ Brengel, Rachel .. 133, 179

Brennan, Shawn

... 173

rentlinger, Beth

Brewer, Stacy

Melody $110,150,158,239$

150 Briggman, Anthony A.

Brinkley, Chris

Brinkmeier, Derek 
Clark, Jim . .216

Clark, Martin

Clark,

Clark, Teresa ....................... 63, 216

Clary, Andy ............. 121, 161, 202

Claus, Pam ...... 121, 163, 200, 239

Clawson, Nancy ...................... 121

Clawson, Rodney ................ 73, 163

Clayton, Sara. 34, 35, 73, 150, 170

Cleaver, Christy ............... 177. 201

Clevenger, Charles ....................... 63

Click, Paul .........................111, 175

Clutz, Amy121, 150, 151, 167, 197, 198

Coates, Greg

Cobb, Jennifer

121

Cobb, Robert

Coble, Jenny ............ 133, 178, 190

Cochrane, Daniel ................ 111, 200

Cochrell, Kelly ........ 8, 24, 32, 133

Cochren, Constance N. .............. 73

Cohan, Ryan .............................. 133

Colby, Justin .............................. 191

Cole, Charity ........... 133, 170, 218

Cole, Lisa

Coleman, Daniel R. ....................... 73

Coler, Danielle ................. 121, 191

Colinco, Stephen

Collier, Suzanne

Collins, Brian ................... 167, 214

Collins, Kimberly ........ 73, 157, 194

Colman, James

Combs, Sharron Lynn ................. 73

Comer, Beth …........................... 111,216

Comers, Heidi ............................. 150

Comfort, Rebecca .... 111, 171, 195

Compton, Christine .......... 133, 171

Comrie, Matthew James ............. 74

Conant, Johannah ……............... 121

Conley, Mandy ................. 133, 197

Conley, Shawn …....................... 133

Conner, Beth …….................... 198

Connors, Jeff

Conway, Janet

Cook. Aaron

Cook, Danny

Cook, David

Cook, Lisa ....................... 111, 150

Cook, Matthew

Cook, Shauna .................... 134, 150

Cooley, Brett ...................... 134, 226

Cooley, Nicole .................... 74, 150

Coon, Tina ….................... 134, 175

Cooper, Andrew

Cooper, Beth

Cooper, James E. ........................ 121

Cooper, Lonny ......... 121. 154, 189

Cooper, Naomi …................ 121, 161

Cope, Tiffiny ................... 134, 177

Copeland, Aimee ............... 121, 175

Copeland, Deborah ....................... 74

Corbin, Amber .................. 134, 223

Cormany, Joanna ............... 121, 198

Corson, Michael

151

Cory, Mary .....

Cottrill, Erinn

Cottrill, Sean

Couser, Gregory .

Coverdell, Ryan

Covert, Brandi

Cowell, Sallisha 134, 150, 179, 185 Coy, Ronald

Crawford, Eric . 111, 210, 211, 236 237

Crawford, Kim ............................ 74

Crawford, Melissa ............. 121, 199

Crawford, Michael ............ 111, 214

Crawford, Sarah ......................... 12

Creamer, Andrea ...... 134, 212, 213

Crecco, Ryan …................ 121, 168

Creps, Suzanna ................... 121. 239

Creswell, Jim ............................ 111

Crider, Dustin .............................. 232

Croft, Chad ................................... 74

Cromer, Chet ............ 121, 169, 188

Cromie, Matt ….......................... 120
Cronk, Körien ............................ 74

74

nebekah 38, 42, 121, 150,

Crum, Alex ............................... 134

Crunelle, Amy ........................... 134

Culberson, Tom ...

175

Culler, Christa ................... 134, 154

Culp, Jordan ............ 134, 155, 203

Cunningham, Aliel ........... 134, 178

Cunningham, Jeff ........................ 65

Cunningham. Renee ........... 74, 158

Cunningham. William L. ........ 121

Curry, Marcje ….................. 74, 216

Curry. Marie …............................. 74

Curtis, Melody ………………..... 65

111, 171, 188

\section{$D$}

Dabravalskas, Christine 49, 74, 150 Dabravalskas, Shawn 134, 150, 151

Dager, Hannah ........................... 134 Dahms, Amber ............................ 134 Damcott, Kevin ....................... 134

Dangelo, Scott .......................... 134 Daniels, Carey .. 74, 103, 104, 188 , 193

Darr, Aaron

Davids, Cory

121

Davidson. Gina

134

Davies, Jason

74,199

Davis, A'Linda

24,74

Davis, Clay

Davis, Emily ..... 121, 168, 191, 197

Davis, Jeff .... 49. 74, 181, 182, 204

Davis, Jerra A.

Davis, Keith

Davis, Leslie E.

Clark, Sherri ........... 49, 75, 204

De Conto, Jesse …............. 150, 15

De Graw, Bethany ...................... 179

De Hart, Nate …......................... 134

De Koch, Carole ....................... 11

De Kruyter, Paul ........ 75, 155, 202

De Ridder, Nate ................. 134, 182

De Santis, Angela .... 121, 150, 169

239

De Usanio, Toni .......................... 11

De Vera, Antoinette ..................... 75

De Vinney, Jessica ............ 134, 212

Deakyne. Chad ................. 194, 214

Dean, Christopher John ............ 121

Dean. Heidi .................. 37, 38, 121

Dean, Joel 157, 190

Deardorff, Donald …...................... 63
Decker, Jennifer ....................... 111

Decker, Joey ............... 111, 165, 191

Dehnke-Hirschleman, Robert 134

182

Deichert, Kevin

Deister, Andrew

Deister, Jake .

Del Rosario, Anna ....... 111.212

Delaney, Michelle ................. 75, 150

Dellinger, Casey .....

75, 150

Deltaan, Carolyn ……................. 134

Denges, Mark ….................. 75, 163

Denham, Katherine 49, 55. 75, 161

Denlinger, David ………………..... 65

Dennison, Paul ........................... 134

Deranek, Norma

Derstine, Damon

Detwiler, Jill ...

.... 75

Dewald, James ......................... 191

Dewalk, Richard …................... 111

Dewar, Raymond ....... 121, 159, 160

Di Cuirci, Michael63, 150, 151, 239

Di Cuirci, Paul. 121, 150, 151, 194. 239

Diaz, Daphne ............... 29, 121, 166

Dick, Michelle ............ 75, 203, 230

Dickerson, Stephanie ............... 111

Dieringer, Andrea ............ 121, 191

Dietz, Amy ..................... 100, 192

Diggle, John

100,192
134

Dillon, Margaret ........................... 65

Divan, Linda ................................ 65

Dixon, Dr. Paul ....... 52, 54, 61, 117

Dixon, Pat ................................... 52

Dizer, Emily .............................. 122

Doak, Gregory Justin .............. 75
Doden, Kara ..... 135, 173, 192, 239
Dodson, Jamie ……....................... 111

Dina …..................... 122, 172

Doese, Jason ............................ 122

Dolby, Jennifer ........................... 135

Dolph, Laura

75
167

Doran. Richard …....................... 75

Dorman, Gretchen .... 135, 199, 239

Dorsey, Michael ................... 37. 135

Douglas, Cheri .................. 111, 195

Douglas, Daniel ..................75, 163

Douridas, Damon ................ 38, 122

Drager, Kelly ............................ 122

Drullinger, David .................. 62, 204

Drury, Patrick ....

Du Monceaux, Jodi 122, 174, 203, 224, 225

Duhaime, David ............... 111, 170

Dull. Michael

Dumbeck, Erich ............................. 75

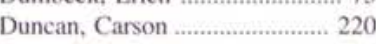

Duncan, Reuben ….................... 226

Dunham, Aaron ........................ 11

Dunham, Susan ........ 122, 183, 191

Durham, Richard ........................ 64

Durham, Traci Lyn ..................... 76

Dyer, Jim ........................ 150, 151

Dyer, Rachel ….................. 122, 160

Dyer, Ryan ...............111, 150,151

\section{$\varepsilon$}

Eaby, Joel .................................. 111

Eads, Adrianne ......................... 135

Eads, Nathan John _................. 76

Eby, Ryan ......................................... 239

Eckstein, Sharon ……………...... 65

Edgington, Kipp ………............ 76

Edlund, Alan ............................. 226

Edlund, Gregory Alan ................ 76

Edwards, David ...........................111

Edwards, Heather ................. 76, 212

Edwards, Melanie ..................... 135

Edwards, Michelle ........... 135, 177

Egolf, Amy ...................... 111, 218

Ehrich, Andrew

Eichom, David ............... 76

Eimers, Leroy

Eimers, Sharon

Elder, Rachelle ....... 111. 208. 234

Eleveld, Sara ……............. 135, 150

Elliott, Jeff .......................... 111, 170

Ellis, Linton

220

Ellison, Debbie

Elmore, Floyd

Elmore, Pamela

Emery, Jonathan ......................... 122, 151

Emment, Cheryl .................. 76, 239

Endicott, Andrea ............... 135, 163

Endsley, Jocelyn …................ 122

England, Roxanne .....32, 122, 173

Engle, Michael L. ...................... 76

English, Rachel122, 166, 180, 212.

Entner, Sandra

Ervin, Kimberly

62

Estep, Stephr.

Estepp, Patrick .............................. 76

Estes, Carol

Estes, Christiana _.............. 135

Estes, Daniel

Estes, Jean ….......24, 111, 170, 176

Etheridge, Rutledge ......... 122, 170

Everson, Shellene …....... 55. 76, 191 7

Fabian, Carrie ……........... 33, 135

Failor, Martha .. 24, 111, 168, 191 .

193. 198

Falk, Brian

Fancher, Angie .......................... 135

Faris, Paula …..... 12, 13, 15, 49, 76

Farkus, Karyn ……........... 135. 168

Farley, Michelle ....................... 135

Farlow, Andrea ........................... 135

Farlow, Jeremy

Farlow, Joyce

arrell. David .............................. 76

Farris, Christ

Faul, Heidi

Faulkner, Reade ................. 10, 214 
Gray, Jonathan ..................2 28, 200 Graybill, Michael .............. 113, 239 Green, Anna Mari .......................... 66
Green, Derrick .................... 47, 78 Green, Harold . 66 Green, Joshua ...... 49, 78, 232, 233 Green, Margaret …...................... 66 Greenwood, Aimee ........... 113, 185 Greenwood, Janice _............. 66 Greer, Kevin ............................. 123 Gregg, Michael 135, 151, 161, 197 Gregory, Julie Ann ........... 135, 224 Gribbin, Mike....................... 28, 78 Griffin, Rachel ......................... 123 Griffith, Jessica _...78, 181, 192 Griffith, Joy ............................... 135 Griffith, Kristy ....................66, 182 Grigson, Chris ......136, 151, 239 Grills, Jason ................................. 78 Grindall, Kelly .................136, 239 Grindall, Lisa Gritsavage, Heidi ....................... 78 Groesser, Jason ................ 136, 199 Gromko, Sara …..................113, 203 Gross, Meredith ................. 136, 177 Gross, Sarah …................ 14, 113 Groves, Heidi ..................... 78, 104 Grow, Lesley .................... 123, 171 Grubbs, Kevin ………..... 78, 193 Grubbs, Michael ........................ 123 Gruet, Jeff .................................. 78 Gruhlke, Pete _........................ 236 Guenther, Stephen ........... 136, 156 Guerette, Carolyn .............. 123, 150 Guikema, Karen ................ 136, 167 Guyn, Kimberly ............................ 79 Gverette, Carolyn .................... 171 Gwilt, Carric .............113, 182, 192 Gwilt, Paul .......................136, 172 Gyurik, Lara .... 123, 212, 230, 231

\section{dt}

Hable, Nathan ……….................. 79 Hacker, Daniel ................. 136, 239 Hacker,

Hacoby, Kristin 79
181

Haffey, Benjamin 113, 194 Hager, Jodie ...................113, 188 Hague, Keith

Haisch, Kevin Hall, James .................................. 136 Hall, Joanna 79

Hallsten, Jonathan ......................... 136

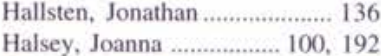
Halsey, Joseph .... 64 Halulko, Josh ....................113, 176 Halverson, Stephanie ................ 66 Halvosky, Sarah ........................ 163 Hamer, Craig ........................ 27, 79 Hamer, Keith ..................... 180, 239 Hamer. Matthew ................ 123, 185 Hamilton, Amy ................. 136, 161 Hamilton, Elizabeth ......... 123, 218 Hamilton, Lori $113,165,176,191$ Hamilton, Ruth ............................. 66 Hamilton, Tiffany .... 113, 179, 204 Hammond, Duane ............ 173, 214 Hammond, Jonathan ................. 163 Hammond, Michael ................. 136 Hamrick, Anna ……… 150 Hanbury, Anson 8, 37, 38, 50, 113 , 150, 203

Hancock, Jillianee …................. 136 Handel, Steve ................... 123, 150 Hangosky, Jennifer ... 45, 113, 173 , 197, 252

Hanks, Josh ......................... 55, 79

Hansell, Timothy ......................... 66

Hardesty, Clinton ...................... 136

Haris, Jody...

Harju, Cara ........................... 113, 171, 199

Harkleroad, Lance ............. 123, 236

Harner, Sandra ............................ 63

Harris, Wade ….................. 8, 185

Harsh. Michael ........................... 113

Hart, Nathan …................. 113, 167

Hart, Rachel L.

123

Hartshorn, Sarah
Hassell, Aaron ............................. 79

Hassenzahl, David 28, 32, 123, 190 Hassenzahl, Kelby …….............. 79 Hastings, Julie .

.. 79

Hasty, Joy ........ 113, 161, 198, 203 Hatfield, Daniel

Havens, Greg ......

Hawkins,

Hayes, Clint ……........................ 220

Hayes, Karen ............ 136, 201, 212

Haylett, Timothy ................ 79, 226

Hayner, Mark .................... 136, 236

Haynes, Jeffrey ........................... 124

Haynie, Kelly

Hazen, Marinus

124

Headings, Andrew 15

Heale-Skillman, Elissa .............. 150

Heath, Leanne 136, 155, 157, 191 239

Heaton, Molly

Heaton, Timothy

137

Hedges, Andy ..... 37, 137, 151, 178

Heffner, Robyn 137

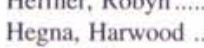

Hegyi, Brian .............

63

Heil. DaciaLea

Hein, Gretchen

Hein, Jessica ........

Heineman, April

Heldreth, Karri

Helfrick, Erika

Hellwig. Evan.....

Helmick, Larry .

Helmuth, Barbara

Helmuth, Dale

Helton, David

Henney, Dawn

Henniger, Jared

Henning, Nelson

Hennis, Sarah ........

Henricks, Rachel .....80, 150, 185

Henry, Chad

.............. 80

Herb, Amy

137

137

Herschberger, Lowell ............ 49, 80

Hess, Aubrey ............................. 137

Hewitt, Dave .... 137, 151, 171, 188

Hewitt, Melissa ….............. 80, 154

Hewitt, Tim ….......... 124, 188, 239

Heyd, Andy ............... 150, 168, 189

Hickox, Amy ...................... 80, 195

Hicks, Daniel ........... 113, 176, 239

Hidalgo, Michael .............. 113, 214

Higgins, Brooke ....... 113, 167, 195

Higgins, Michelle ..... 113, 175, 192

High, Michele ............ 124, 171, 179

High, Sarah .................... 137, 178

Hill, Alicia ............... 113, 192, 193

Hill, Christa ............. 137, 191, 223

Hill,

24, 80

Hill, Dusty

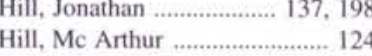

Hill, McArthur

Hille, Anna Ruth

169

Hillman, Geoff

113,178

Hills, Sarah

137,212

Hilty, Brian

Hines, Cara123, 124, 192, 193, 198. 200

Hines, Dan

Hintz, Matthew ..... 49, 80, 173, 204 Hirschelman, Robert ................. 137

Hively, Melissa

Hjembo, Jon ............................. 151, 197

Hjembo, Karl ............. 137, 151, 197

Hlad, Mandy .............................. 137

Hoadley, Scott ......................... 80, 200

Hock, Jeff ................................... 80

Hodel. Emily ................... 49, 52, 80

Hodson, Carolyn ........................ 66

Hodson, Stephen ................ 55, 239

Hoecke, David .......................... 66
Hofert, David .......... 113, 172, 180

Hoff, Jamie L. ......................... 124

Hoff, Ryan ................................. 124

Hoffman, Beth ................................. 80

Hoffman, Jennifer .... 124, 163, 168

Hoffman. Julie ........................... 113
Hofstetter, Kristy ..... 137, 168, 191 Holesovsky, Sarah ........47, 80, 182 Holler, Courtney

Hollins, Marc

Holz, James

Hooper, Jessica

Hoover, Christy

137,218

Hoover, Leah.....

150

Hoovler, Kristin ................. 137, 162

Hoppe, Kelly M.

37.162
$\ldots .174$

Hornback, Mike

... 137

Horne, Julie

Hoskins, David

125. 199

Hotchkiss, Tim

113,191

Houck, Joanna .............37, 137, 150

Houck, Nancy ..........113, 162, 204

Houg. Stephen

Houg, Steve ..................... 197, 198

Houk, Nathan . 175, 198, 200, 203. 236

House, Melissa ........................ 137

House, Shannon ....... 125, 168, 182

Housten, Kathleen .... 113, 156, 165

Hovis, Jody ..... 12, 24, 37, 113,150

Howard, Jen ...................... 137, 183

Howard, Jeremy

.... 232

Howard, Jodie ………………...... 218

Howard, Joel ..................... 125, 163

Howard, Ken ............... 81, 172, 188

Howard, Tim

125
.... 137

Howarth, Sarah

Howder, Scott.

Howe, Abigail ...................113, 176

Howe, Angela ... 125, 151, 169, 191

Howe, Karisa

Howell, Jennifer ……......... 125, 169

Howell, Sara .......... 52, 81, 188, 197

Howells, Kimberly ........... 137, 212

Hsisch, Kevin ........................... 178

Hubbard, Patricia

Huber, Daniel ................ 49, 81, 151

Huber, Patricia …………........ 66

Huck, Abigail .

Huck, Roxy

181

66, 181

Huddleston, Karmen .. 81, 222, 223

Hudson, Dan ….......... 113, 173, 236

Huebner, Casey .......................... 137

Huggler, Valerie ................. 125, 191

Hughes, Brent ................... 113, 177

Huizinga, Alison ............... 125, 234

Hull, Gary

Humphreys, Amy ........................ 81

Hunsaker, Amy ................... 81, 170

Hunsaker, Susan .......... 81, 150, 239

Hunsberger, Lisa ................ 16, 203

Hunt, Barbara _........... 66

Hunt, Leigh

Hunt, Robert

Hunter, Erica

Hunter, Jennic

Hunter, Kristen Lec

Hurley, Jeremy

Hurst, Amy ............... 113, 192, 194

Hurt, Scott

Husband, Scott ................. 113,200

Hutchison, Aaron ……................ 113

Hutchison, Amy ................ 125, 162

Hutchison. Thomas ....................... 62

Hutfless, Jennifer .............. 137, 195

Hyatt, Jeff ............ 24, 81, 113, 203

\section{g}

Ice, Kendra

81

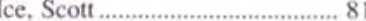

Ikeda, Kouichi .............81, 174, 197

Illian. Bradford

Ingalls, Jennifer.... 49, 50, 81, 199. 203, 204

Ingalls, Margaret ........................ 64 Inion. Brenda ............. 113, 150, 194 Ireland, Drew Irish, Jeanette ……....... 125, 165 Ison, Rebecca …............................ 81 Ivey, Becky

\section{J}

Jackson, Christina .......... 24, 34, 82

Jackson, Dave ......................... 150

Jackson, Heather .......137, 169, 199 
Persons, Kari ..............89, 203, 234 Peter, Leah .......................... 37, 128

Peterman, Jessica ........................ 150 Petersen, Kathy ... 49, 89, 150, 185 Peterson, Amy ........................... 141 Peterson, Ben ............................. 128 Peterson, Holly ......... 116, 172, 191 Peterson, Joel . 203, 210, 211, 236, 237

Peterson, Joy ......................, 89, 162

Peterson, Kathy

193

Peterson, Tiffany ….......... 141, 175

Pfahler, David …....................89, 195

Pfahler, Tim ............................ 214

Pfeiffer, Brett _........... 150, 151, 239

Pfeiffer, Troy ............................. 141

Philip, Jaya ....... 141. 171, 176, 193

Phillips, Douglas .......................... 67

Phillips, Edmond .......................... 67

Phillips, Gareth .................... 89, 204

Phillips, Tim .............................. 128

Phipps, James ............................... 62

Phipps, Sheri …….................. 128

Phipps, Terry ….......................... 64
Pickell. Mindi ................... 116, 150

Picuri, Michele ..... 55, 89, 151, 239

Pierce, Krista ......................... 141

Pierce, Stephen ............................. 89

Pierre, Chris ... 37, 38, 39, 128, 173

Pierson, Loren ................................ 29

Pierson, Michael ...................... 128

Pierson, Ruthanne ...... 37, 116, 150

Pifer, Hilary ....................... 116, 188

Pinkley, Dale ...................... 45, 128

Pitstick, Amy ........................... 141

Pittenturf, Christophan, Debbie 128, 150, 151, 189.

Pittman,
190,200

Pizano, Yvette

128,191

Plaatje, Matthew ................... 8, 141

Platt, Casey …................... 141, 171

Pletcher, Julianne 49, 90, 203, 208, 235

Plummer, Susan ............... 141, 171

Pod, Chris J.

161

Poff, Amanda

.... 128

Pohlman, Nathan ..................... 191

Polgardy, Samantha ......... 116, 239

Poling, Jason

..... 90

Pollard, Amy .....

90,103

Ponder, Lina .............. 141, 166, 234

Ponzani, Aaron 116, 150, 179, 181. 193

Pool, Chris ................................ 141

Porter, Richard 116, 128, 151, 176. 239

Postema, Luke ................. 168, 188

Potter, Cynthia . 116, 150, 168, 181

Potter, Jamie ............................. 150

Pound, Russ .............. 128, 162, 214

Powell, MaryBeth ............ 116, 160

Powell, Melissa ......................... 128

Powell, Sarah ….................. 90, 173

Powers, Edward .......................... 90

Pratt, Sandra .................................... 64

Prediger, Chris ......................... 90
Prentis, Erin ............. 128, 150, 180

Preston, Jeremy ........ 128, 198, 200

Preston, Joseph M. ................... 128

Price, Elizabeth ...............116, 203

Price, Krista _................. 49, 90, 184

Price, Mark …........................... 202
Proper, Marlena $\ldots \ldots \ldots$

Prugh, Ruth

Prusha, Mandy ........... 150, 168, 180

Pryor, Nicole ................................ 90

Puckett, Rachel ......................... 128

Pugh, Kathleen .................. 128, 174

Pugno, John ..................... 141, 199

Pulley, Lewis ...................... 170, 200

Purple, Jonathan …........................ 62
Puryear, P.J. 200

Puterbaugh, Jennifer ................ 128

Quarles, Chuck.. 90, 106, 150, 194 Quint, Jodi ................. 141, 218, 234 Qussar, Joseph.

Qussar, Naseem
Rogers, Jennifer ... 37, 91, 189, 239 Rohm, Emily

67

Radford, Nathan ............... 116, 169

Rafferty, Seth ............................ 116

Ragle, William ........................... 62

Rahilly, Sharon …................... 64

Rainsberger, Dan .............. 150, 151

Rambo, Jacob ........................... 116

Ramsey, Allison ....................... 141

Randall, Jared .......................... 141
Randall, Roger ........ 124, 128, 166

Ranger, Nancy ......................... 67

Rasey, Brian ..................... 232, 233

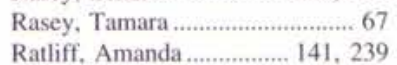

Ratliff. Amanda ................ 141, 239
Rauch, Jonathan .......141, 156, 172

Rea, David 13, 116, 210, 211, 236. 237

Rea, Emily

141. 151

Read, Shari

90,156

Reagan, Erin

141, 160

Reaper, Joe $\ldots \ldots \ldots . . . . . . . . . . .128,239$

Reaper, Joe ..................... 128, 239
Recktenwald, Chris .......... 141, 160

Reda, Jamin .............................. 116

Redington, Naomi ... 141, 161, 192. 193, 239

Reed, Mike

Rohm, Lynn

Rohm, Robert .

Rolliff, Amanda

67

Romaine, Doug ............................ 91, 151

Romang, Sara .. 24, 37, 38, 42, 106

Romeyn, Stacey ................ 141, 239

Romin, Todd …......................... 141

Rooke, David . 116, 171, 197, 203,

214. 228

Roper, Kevin

Rorex, Adam

64,214

Rose, Joel

141. 170

Rose, Matt ................................ 176

Roseboom, Liz 34, 46, 49, 91, 184. 188, 204

Rosencrantz, Rene

Rosenvold, Darin

.. 116

Rosner, Kristi

141,161

Ross, Erin .....

Ross, Julie

128,239

Ross, Rachel

91,175

Rosseau, Brian .......... 141, 156, 176

Rotman, David

141,180

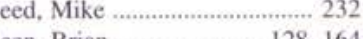

Reep, Jeffrey ...........................63, 63, 220

Reese, Joyce ................................. 67

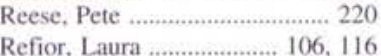

Reibson, Jesse .......................... 116

Reich, Robert ............... 49, 90, 165

Reilly, Jennifer ...................... 56, 90

Reinhard, Lee ………………......... 236

Reis, Janelle .............................. 90
Reisenbigler, David ......... 141, 202

Reitz. Kelly ...... 141. 175, 192, 234

Reno, Rachel ........................... 141

Reugsegger, Ann ..................... 212

Reutlinger, Stephen .................... 90

Rexford, Matt ....................150, 151

Reynolds, Annmarie .......... 128, 158

Rheam, Gregory ............... 128, 170

Ribeiro, Rebecca ................ 116, 193

Rice, Clinton

Rice, Jennifer

67, 194

Rice, Karin $\quad 141,179$

Rice, Patricia .............................. 116

Richard, John ....... 49, 90, 203, 204

Richards, Tom ...................... 90, 184

Rickard, Donald

Riddle, Eric

Rideout, Kimberly ............. 128, 239

Ridley, Brent ...................... 91, 189

Rigg, Amy .

Riggs, Jack

Riggs, Joyce

Riley, Christina

Rotman, Doug

Roumke, Jason $\ldots . . \ldots \ldots \ldots \ldots \ldots \ldots \ldots . . . . .141$
Rowley, Brian .................... 150, 151

Royer, Natalie .......... 141, 218, 219

Ruba, Chrystie .......... 116, 163, 199

Ruba, Dan ………….......... 91, 239

Ruberg. Maranatha ..................... 141

Ruby, Carl

Rudd, Daniel

62
.. .116

Rudolph, Christopher ................. 9]

Ruegsegger, Ann ..................... 116
Ruffin, Casey ........... 141, 216, 224

Ruffin, Casey …....... 141, 216, 224
Ruffin, Zach …......... 91, 226, 227

Ruhl, Kimberly ...........................91

Ruhlman, Scott ........... 210, 236, 239

Rummel, Paula ........................... 116

Rumschlag, Ann ....................... 178

Runnion-Gray, Jason ................. 116

Rupp, Josh .. 34, 116, 150, 151, 239

Russeau, Brian

Russell, Jayne

Russell, Ted.

Ruth, Monic

Rutila, Paul

Rutledge, Dave ........ 166, 214, 215

Rutlinger. Steve ........................ 199

Ryan, Rachel ....................... 142, 193

Ryan, Steve …................. 173, 193

Rynerson, Jill

\section{$\delta$}

Sabella, Mike

Sagraves, Michelle

Sagraves, Thomas

Sainato, Jared ........... 142. 198, 200

Salvaggio, Carla ........ 116, 191, 198

Salyer, Melissa

Salyers, Melinda

Ringler, Amanda …......................... 128

Rising, Chrissy• Luke .. 24, 116,

159

Ritchey, Heather ........................ 91

Rittgers, Sarah .......... 141, 162, 193

Ritzel, Rebecca ................. 141, 179

Ritzer, Jamie .............................. 14

Rives, Hannah ........... 141, 150, 170

Rizer, Adam ..................... 141, 200

Rizer, Charity ............................. 128

Roberts, Geneva .......... 14, 141, 169

Roberts, Jesse …............. 49, 52, 91

Roberts, Micah ................ 116, 140

Roberts, Shawna ...................... 116
Robertson. Chris ............. 158. 201

Robertson, Mark ........................ 128

Robey, Beverly ………................67 67

Robey, David ……............. 37, 62

Robinson, Ben ….............. 150, 151

Robinson, Charles ................... 116
Robinson, Jeffrey ........... 195, 200

Robinson, Mindy ....................... 141

Robyck, Carrie ….................... 116

Roche, Becky ... 141, 155, 222, 223

Rockwood, Jeff ................ 214, 215

Rogers, Elizabeth $143,150,201,212$

Rogers, Gordon .. 57, 91, 173, 188.

190

Schleith, Mark …................ 38, 116

Schlesener. Ivy

Schmidt. Christine ... 142, 178, 192

Schneider, Lindsay .......... 129, 192

Schoon, Stephen ....................... 116

Schradin, Aaron ......................... 92

Schroeder, Ben …............. 142, 239

Schulman, Crystal ............ 142, 168

Schultz, Jon

Schulz, Laura .....

176
$\ldots . . . .116$

Schumacher, Jeff .............. 142, 197

Schumacher, Robert ….............. 64

Schuring, Jessica. 49, 92, 150, 198

Schwartz, Connie .............. 142, 179

Schwartz. Melinda ............ 107, 198

Schweickart, JJ

188

Schwinn, Lana ................116, 230

Scott, Chris ......................129, 168

Scott, Cliff ..... 49, 50, 92, 204, 214

Scott, Dan ...... 37, 38, 39, 150, 151

Scott, Dana ................116, 185, 193

Scott, Dawn

Scott, Kimberly

129
129

Scott, Lisa

142

Scotton, Will

7. 202

Seefried, Andrew 92, 182

Seeley, Jennifer ............................ 142

Seeley, Jessica _..........142, 150, 159

Seely, Amber ... 129, 179, 182, 191

Seely, Denver .................... 92, 203

Selin, Stephanie

..... 142

Sell, Jamie .......................... 129, 174

Sellers, Danny

Sellers, James

Senior, Mandy ..................116, 173

Sepkovich, Melissa ...................... 92

Sevo, Shane ….................. 142, 168

Seyfang. Bethany ...................... 129

Seyfert, Tara ..............129, 156, 174

Shabazz. Malik .......................... 194

Shaefer, Bonnic ........................... 230

Shaefer, Julie ............................. 230 
Stevens, Darin 118, 158 Stevens, Megan 118, 203, 208, 234 Stevens, Ryan …............... 142, 172 Steves, David .............................. 118 Stewart. Amy ........................... 49, 95 Stewart, Ben ............................. 199 Stewart, Beth ... 118, 150, 173, 197 Stewart, Bill ..................... 151, 191 Stewart, Rachel ......................... 202 Stewart, Rachel E. ..................... 142 Stewart, Rachel J............... 142, 179 Stewart, William ....................... 142 Stock, Shelley ........................... 180 Stone, Alison .....................118, 183 Stone, David ............................ 142 Stone, Deana ….......................... 67

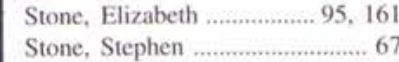
Stork, Keturah ............................ 130 Stout, Aimee .............118, 167, 185 Stover, Jennifer …....................... 130 Stowell, Joseph ......................... 139 Stowers, Amy .. 142, 163, 176, 239 Strang, Maureen ................ 142, 230 Stratton, Jeff …………… Street, Joan ................................... 67 Streetman, Stephanie …....130, 166 Streit, Nikki ...................... 118, 150 Stribling, Diane ……..................... 64 Strong, Daniel ……...................... 8 Strong, Randall ........................ 118
Strong, Steve 230,232 Strong. Steve ................. 130, 232
Strong. Tobin ... 95, 188, 196, 200, 239

Stroven, Abby ........... 118, 161, 199 Strunk, Jennifer ……................... 142 Strychalski, Christopher ........... 118 Strychalski, Joshua ..............24, 130 Stryker, Jenny ……............142, 155 Stuenzi, Erin ..... 130, 164, 176, 197 Stuenzi, Jessica ........ I18, 176, 195 Stumbo, Melissa ................ 130, 155 Stupnicki, Dale ........................ 142 Stutes, Patty ……................. 67 Stutzman, Ben .........203, 236, 239 Stutzman, Ben H..................... 163
Stutzman. Benjamin ................ 142 Stutzman, Bobby ….................... 118 Stutzman, Duane ........................... 95 Stutzman, Monica ..................... 130 Sucsy, Alisha ...................... 142, 151 Sucsy, Joshua ............ 142, 198, 200 Suhayda, Juliana .......................... 95

Sullivan, Michael ....................... 142 Summer, Shauna ........................ 188 Summers, Rebecca ........... 142, 230 Supplee, Ed ................................ 239 Sutton, Pete ......... 49, 95, 194, 222 Sutton, Will ................................ 170 Svendsen, Eric .........142, 203, 239 Svendsen, Sarah ............... 118, 193 Swales, Sandy ….......142, 171, 234 Swaney, Tara .............142, 170, 218 Swank, Sharon ......................... 118 Swanson, Brodie …….................. 95 Swanson, Justin ......................... 142 Swanson, Kevin …..................... 95 Swartz, Janelle ................. 142, 150 Swartzentruber, David .....2 210, 211 Swartzentruber, Earl ................... 130 Swasey, David ............95, 203, 236 Sweeney, Deborah …………....... 95 Sweet. Nicki ...................................... 201 Sweetser, Wesley .........................62 62 Swenson, Paul ........................... 142 Swigart, Roger .......................... 214 Swiger, Tara …….................95, 103 Swiger, Tonya ............................. 143

\section{כ}

Tabberer, Brenda .....118, 156, 201 Tackett, Josh ................... 130, 214 Tait, Douglas ................................. 143 Tait, Jamie ….............................. 214 Tatum, Ryan …….................... 95, 199 Tatum. Scott .............................. 143

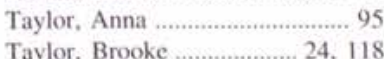
Taylor. Brooke ............... 24, 208 209, 234
Taylor, Eleanor

224

aylor, Eric ........................95, 220 Taylor, Gretchen ......130, 166, 230 Taylor, Matt …..................2226, 227 Taylor, Stacy ............................ 143 Tegtmeier, Jamie .......................... 95 Tegtmeier, Jennifer ... 14, 130, 173 . 199

Temmesfeld, Daniel ................ 118 Tenny, Shane …............. 49, 96, 180 Terkelsen, Susan .......................... 67 Terpstra, Sarah ............ 52, 96, 103 Tessmann, Elizabeth ........... 24, 143 Tharngan, Rebecca ... 143, 170, 174 Tharyer, May ............................. 192 Thayer. Amy ….........118, 163. 239 Thayer, Katrina …..................... 170 Thirey, Micah ................... 143, 172 Thomas, Nikki .......... 143, 163, 197 Thomas, Robert ........................... 96 Thomas, Sarah .................... 143, 182 Thomas, Scott ........................ 182 Thompson, Ben 143, 210,211, 236 Thompson, Debra ....... 96, 164, 183 Thompson, Greg ....... 118, 171, 197 Thompson, Jody 143, 216, 234, 235 Thompson. Matthew

Thompson. Patricia ................... 143 Thompson, Thomas ..................... 63 Thomson, Brian ................ 143, 177 Thomson, Lisa ................... 130. 212 Thomson, Scott ..................... 10,96 Thure, Michael ........................... 143 Tiel, Jennifer ............ 130, 157, 190 Tilton, Rachel .................. 143, 216 Timco, Erin ….................. 130, 192 Titland, Ellie ................................ 143 Titus, Damon ..................... I50, 151 Tocknell, John …..........................67 67

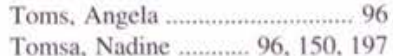
Tomsa, Stephanie ............. 130, 165 Torlone, Anthony 49, 96, 204, 226. 227

Tovey, Jason

183,214

Towle, Matt $96,214,215$ Townsend, Jill ................... 130, 239 Townsend, Ray ...................96, 162 Townsend, Sarah ……................ 118 Toyer, Jeremy ..................... 118, 191 Toyer, LeeAnne ……... 8, 143, 157 Tracey, Heather ........................ 143
Treadwell, Mark _............ 143, 158 Tregay, Flynn 150,151, 239 Trimble, Deborah ...............96, 96, 234 Trimble, James .......................... 143 Trimble, Melany …...................... 96 Tromp. Joshua ............................ 143 Trost, Trisha .................... 118, 161 Troupos, Chris _.......... 130, 200 Trout, Julie ....... 143, 159, 169, 191 Trylick, Aaron ......................... 143 Tuinstra, Erin …........ 143, 151, 202 Tuinstra, Renee .. 96, 174, 191, 239 Turkis, Rachel .......... 143, 198, 200 Turner, Jonathan ......................... 143 Turner, Kristy .................. 143, 239 Twigg, Amy .................... 143, 159 Tyson, Angie ...................... 144, 174 Tyson, Heather

$u$

Umland, Brad ............................ 197 Upham, Rebecca ............... 130, 150 Urbassik, Ryan .................. 144, 232

\section{$v$}

Vaduva, Virgil

Valade, Megan

144

Valentine, Seth ................. 130, 200

Valiknac, Traci ....................... 144

Van Dalen. Tim ................. 144, 239

Van Gorp, Heather ........... 144, 174 Van Heukelum. Jason ...... 184, 203 Van Loo, Scott ..........118, 194, 203 Van Loon, Rebecca .......... 130, 239 Van Wormer, Lisa 56, 96, 174, 201 Vandegrift, Scott106, 118, 222, 223 Vandemark, Natalic ................... 96 ander Bush, Jennifer ..... 118, 199 ander Molen, Kerri .97, 193, 203. 224

Vanderground, Rebecca .. 130, 239 Vanlier, Michele ............... 144, 230 Vannatta, Lisa ....................97. 103

Vare, Rhonda …...................... 195

Vasquez, Jason ........................... 150

Vaught, Bethany ..............130, 179

Vawter, Ben $\quad 118,150$

Vencill, Beth ................... 130, 165

Vitarelli, Chris24, 37, 42, 119, 159 ,

202

Voigt, Becky ................... 119, 150

Voigt, Jared 144

Voldness, Micah 144 Volpe, Rachel ................... 144, 212 Vore, Rhonda …........................ 119

Vorlicky, John .......................... 144

Voumard. Bradley ...................... 130

\section{w}

Waardenburg, Ty 144

Wabeke, Angela

Vabeke, Melissa 130, 172, 191 Waddell, Laura .................. 130, 160 Wade, Michael ................. 145, 161 Wadlington, Melanic 145, 150, 193 Waechter, Holly .......119, 150, 177 Wagner, Jessica ................ 145, 157

Wagner, Lindsay ........................ 145 Wagner, Trudee ........................... 67 Waibel. Keely ....................97. 97. 188 Wainwright, Kathleen ................. 97 Walbright, Kay ........................... 97 Walcott, Catherine ........... 130, 176 Walder, Charley ........................ 196 Walker, Amy ............................... 97 Walker. Bethany ......130, 154, 239 Walker, Charlie ......................... 239 Walker, Dick ....................... 67, 204 Walker, Greg. 12, 49, 97, 194, 204 Walker, Lisa ...... 37, 130, 150, 202 Walker, Ronald .......................... 62 Walker, Scott ............ 145, 182, 236 Walker, Tim ….......................... 220 Walker, William ................................ 97 Wallis, Philip ..........119, 181, 189 Walter, Dana …………............. 119 Walworth, Justin ......................... 8, 145 Wambold, Eric ............................. 97 Wannemacher, Amy .................. 97 Ward, Christy ............................ 130 Ward, John …............................ 131 Ware, Charles .............................. 97 Ware, Paul. Warinsee, Pete 145,214 Warner, Melissa ............... 145, 171 Warnken, Adam .......................... 145 Warren, David …………........... 62 Warren, Lisa ............. 119, 156, 198 Warren, Mark ..................... 119, 150 Warriner, Aaron .......................... 145 Warriner, Kelly .......................... 119 Warriner. Wesley ............... 145, 239 Watson, Ben …. 119, 158 Wawro, Kristen ............49, 97, 239 Wawro, Megan .................. 145, 169 Wear, Elizabeth ….......................... 97 Weaver. Celeste .......................... 97 Weaver, Darren …...................... 131 Weaver, Paul ............................... 98 Weaver, Woody .......................... 188 Webb, Lee ……........................... 67 Weber, Emily .. 131, 179, 192, 199 Weber, George ...........98, 197, 214 Weber, Jonathan . 49, 98, 150, 179 Weber, Laurie .............................. 119 Weber, Rhonda .................... 119, 184 Weber, Steven ........... 119, 150, 161 Weber, Tami ....................... 131, 197 Weber, Tracy ..................... 171, 183 Webster, Heather .........38, 119, 155 Webster. Joy ……........ 50, 119, 194 Webster, Todd ….......................... 98 Weeks, Ann ..... 131, 189, 200, 201 Wehr, Nathan ….................98, 196 Weirich, Lisa ..................... 216, 217 Weise, Carl ......................... 131, 226 Weiss, Jamie …....................100, 150

eldy, Stephanie 98 Weller, Natalie .......................... 164 Welner, Rob 131
119

Wenger, Michael ...... 131, 172, 182 Wenzel, Angela8, 38, 119, 194, 203 West, Jenni

West, Linda

West, Nellie.....

.145

West, Susan ..... .... 67 98,150 Wetzel, Daniel ...............40,64, 149 Wetzel, Phyllis 67 Whaley, Jeremy …............. 131, 15 Whaley, Laurie ..............................98 98 Whaley, Vernon ....................64, 127 Wheeler, Lori .................... 145, 223 


\section{Contributing}

Photographers

Shannon Boynton

Eric Boothe

Scott Huck
Laura Milligan

John Ward

Mike Apice

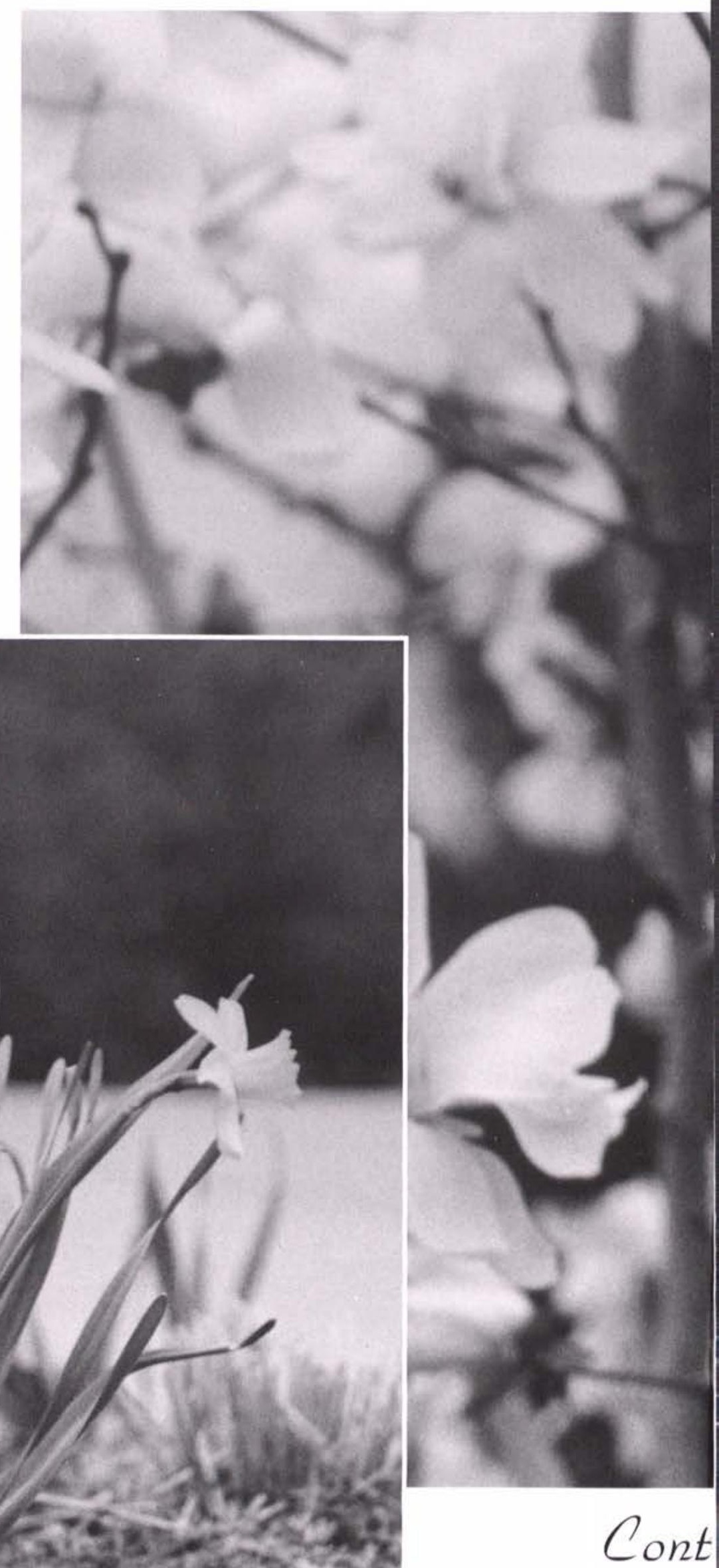

Mark Allen

Chris Ashcraft Jennifer Baer Ben Barnhart Peter Bednarek Jennifer Benson Ben Bookie 
Mr. Jack Simons

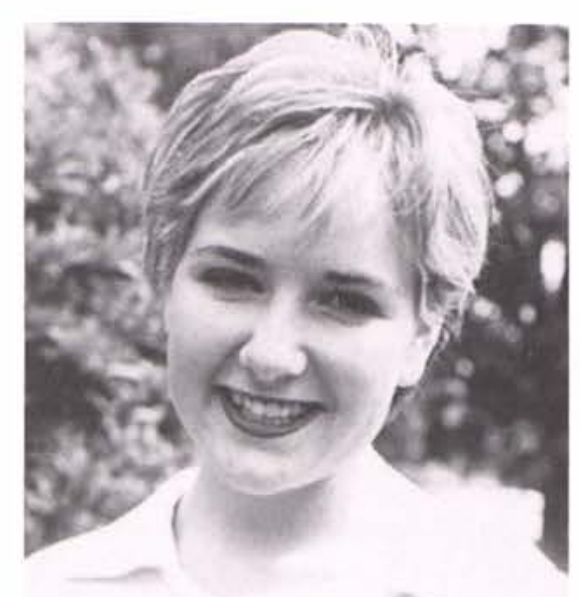

Editor-in-Chirf

Sarah Simons

Sarah Simons

Layout Editor

Tiffany Zimmerman

Busines1 Manager/Layout Arsistant

Jennifer Hangosky

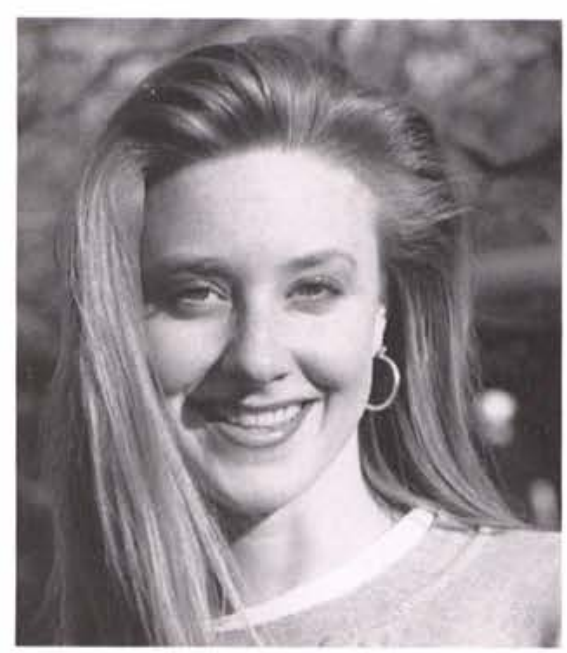

Innifer Hangosky

Photography Editor

Eric Fiveland

Copy Editor

Matt Sherwood

Secretary

Emily Berry

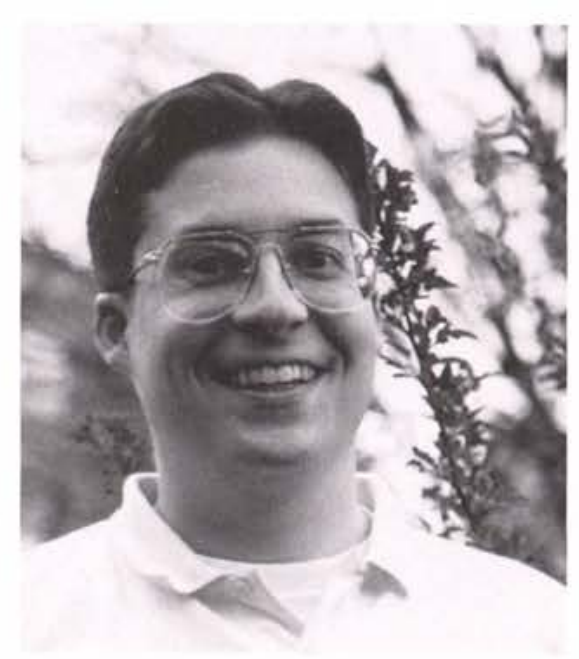

Matt Sherwood 


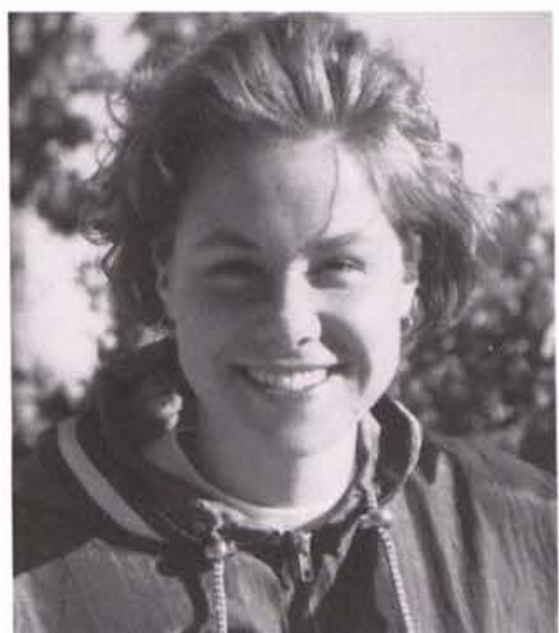

Tiffany Zimmerman

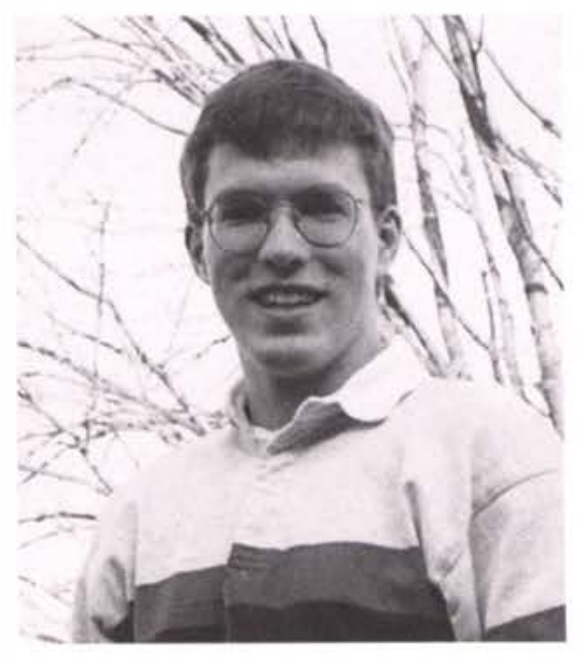

Evic Fiveland

\section{Layout Staff}

Chris Ashcraft

Emily Bohl

RachelEnglish

MattFjare

Lance Harkleroad

Sarah Holesovsky

Kristin Marshall

Dale McCrory

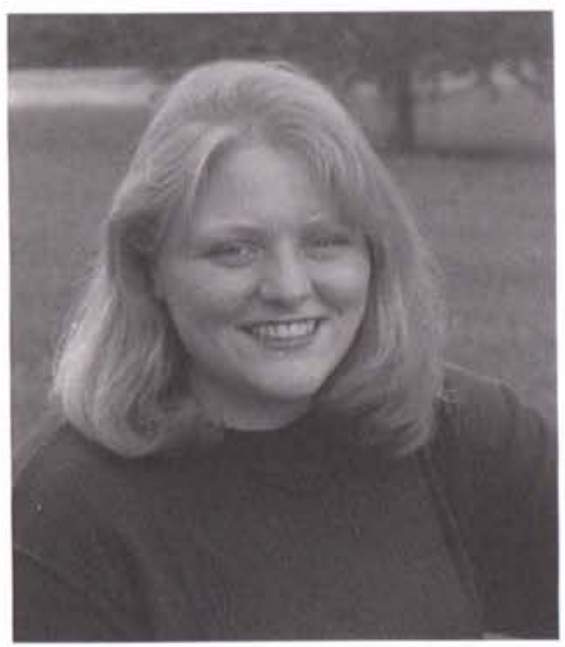

$E_{\text {mily }} \mathcal{B}_{\text {erty }}$ 
"It has been said,

\section{'...This will be the}

crowning jewel of the

\section{Cedarville College}

campus." "

Dr. Paul Dixon 


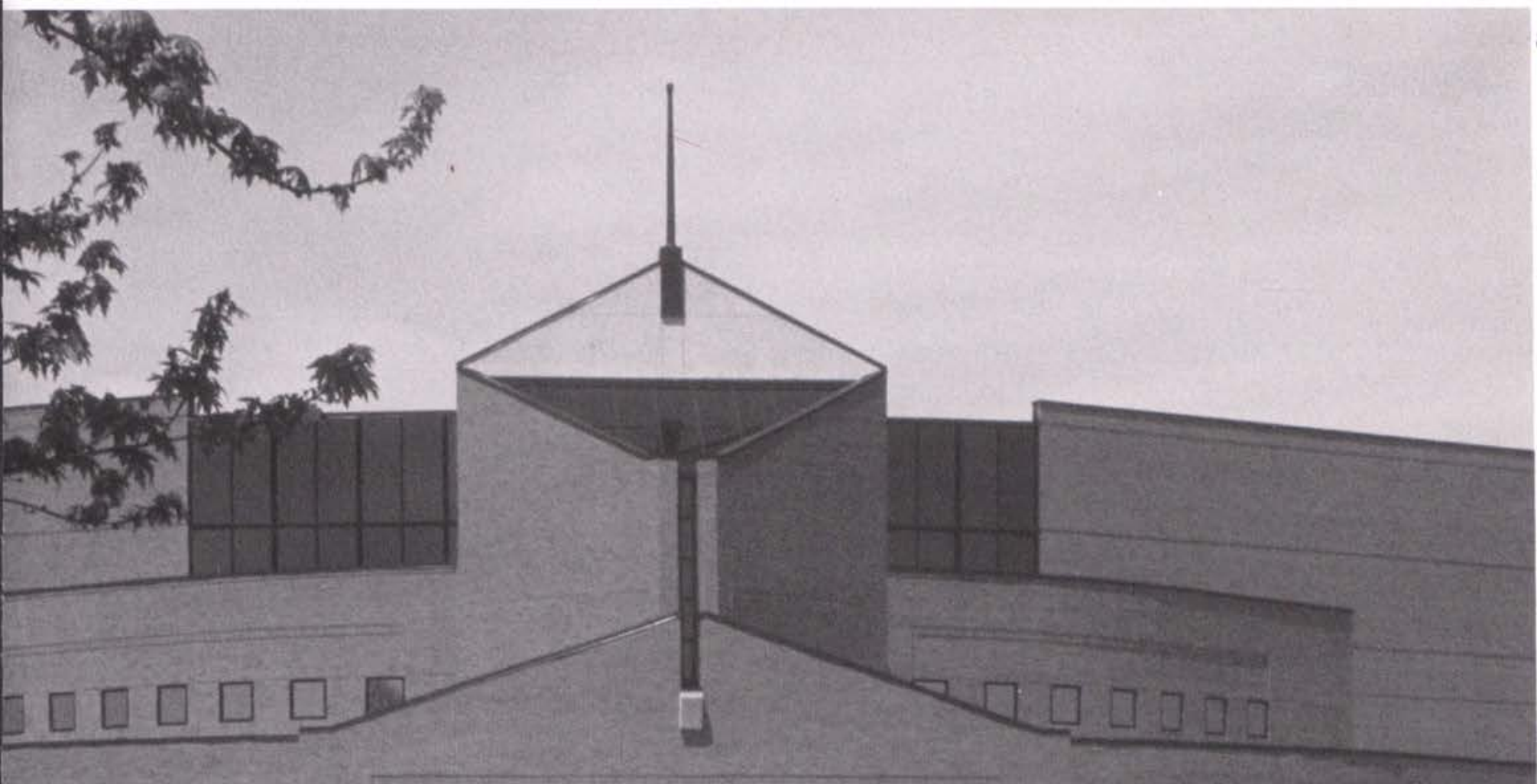

Dixon Minisfay cuiru

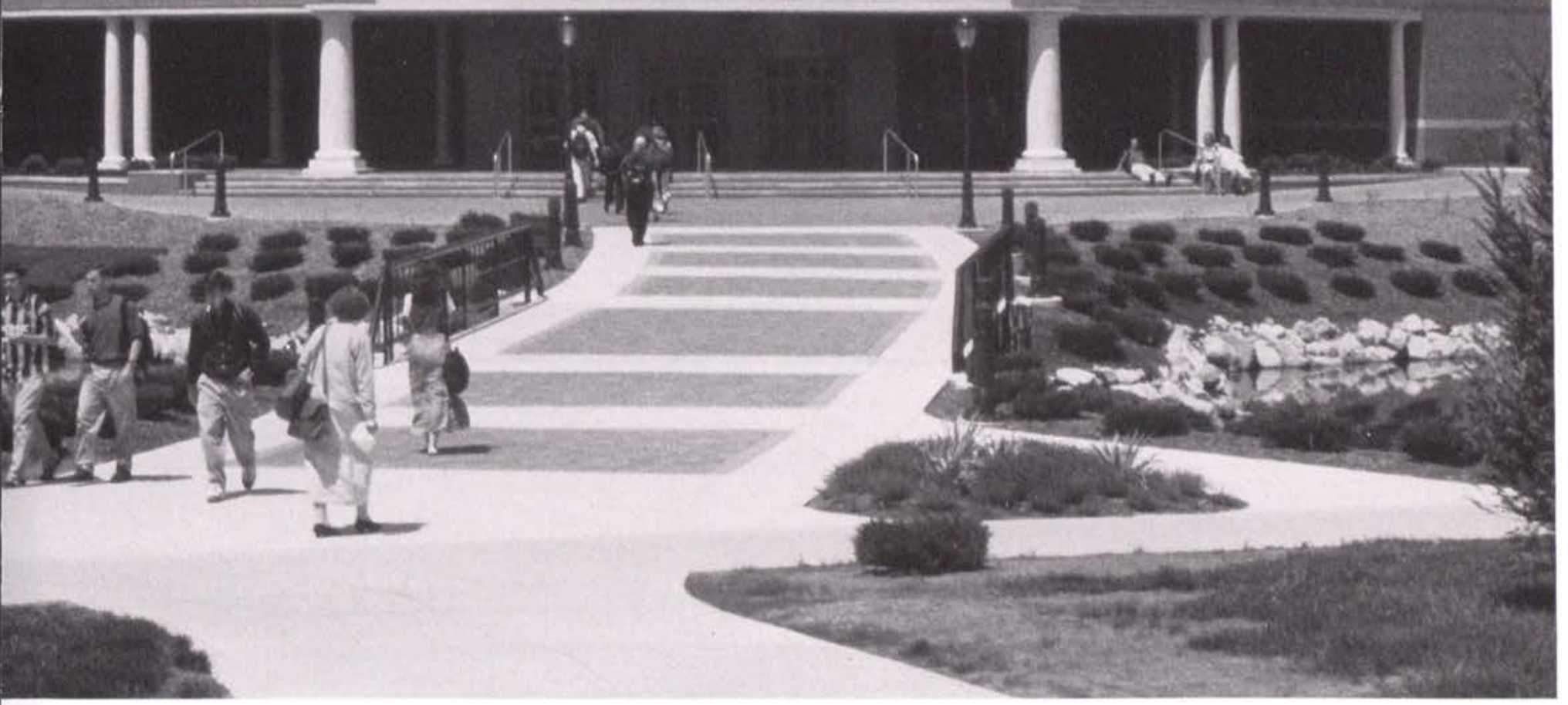

Scott Huck

The Dixon Ministry Center 
When $H_{\varepsilon}$ cometh, when $H_{\varepsilon}$ cometh, to make up His jewrls, all His jewrls, precious jewrls, His loord and His own. He will gather, Ote will gather the gems for His kingdom, all the purr ones, all the Gright ones, His Lourd and His own. Little children, Little children, who Love their CRedermer, are the jewrels, precious jewrels, His lourd and His own. Like the stars of the morning, His bright crown adorning, thry shall shine in their Grauty, Gright gems for His crown. 




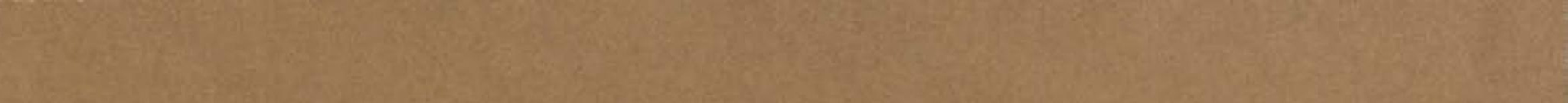

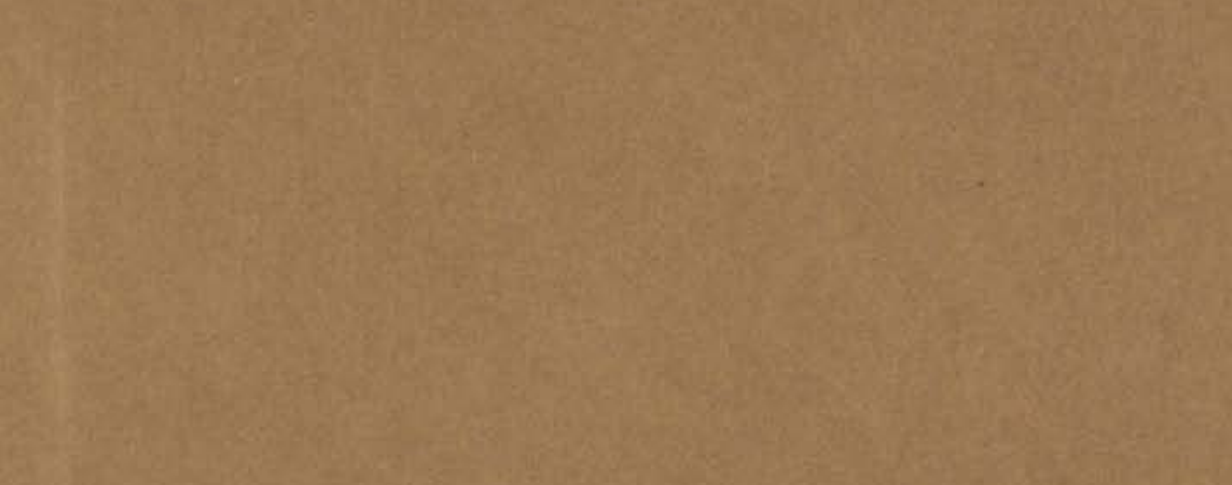

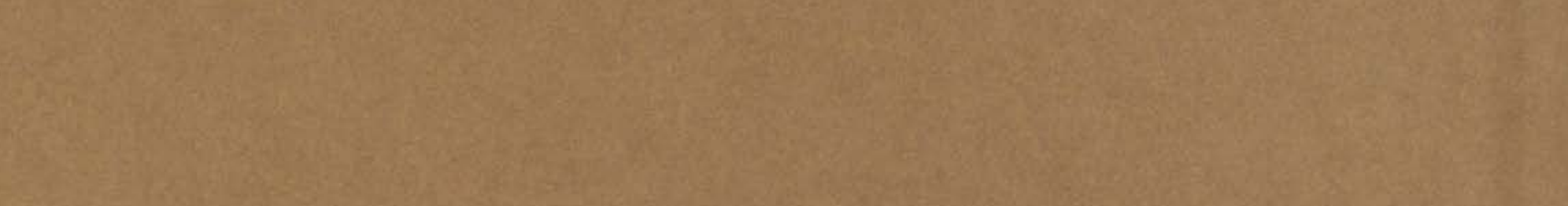

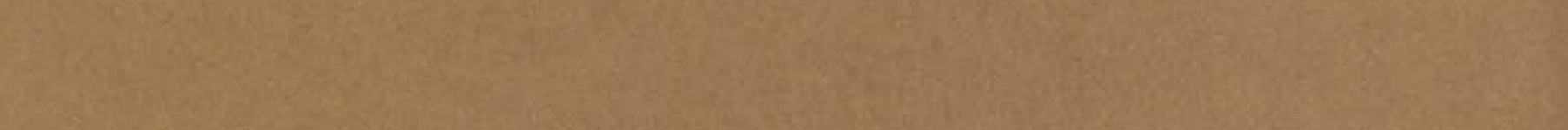

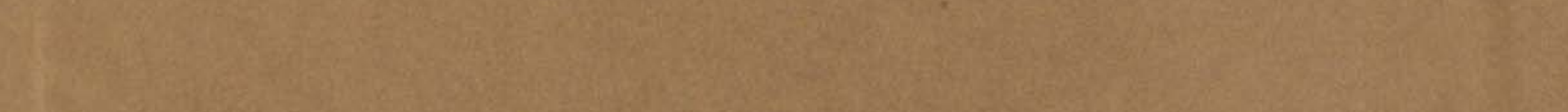

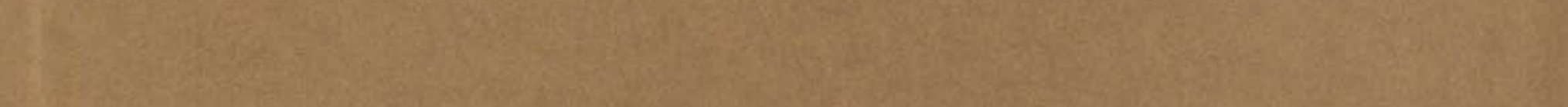

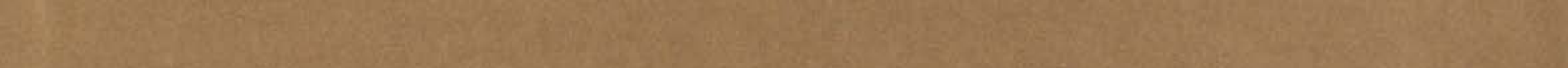
Ly

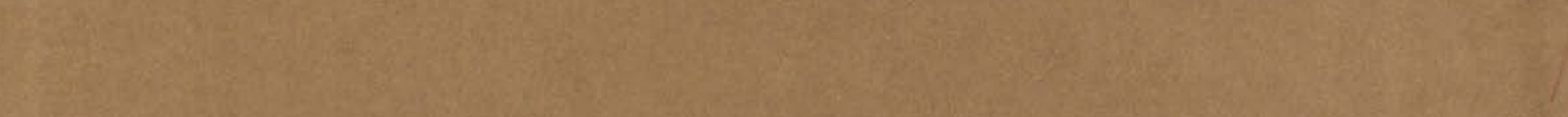
Sy

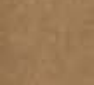

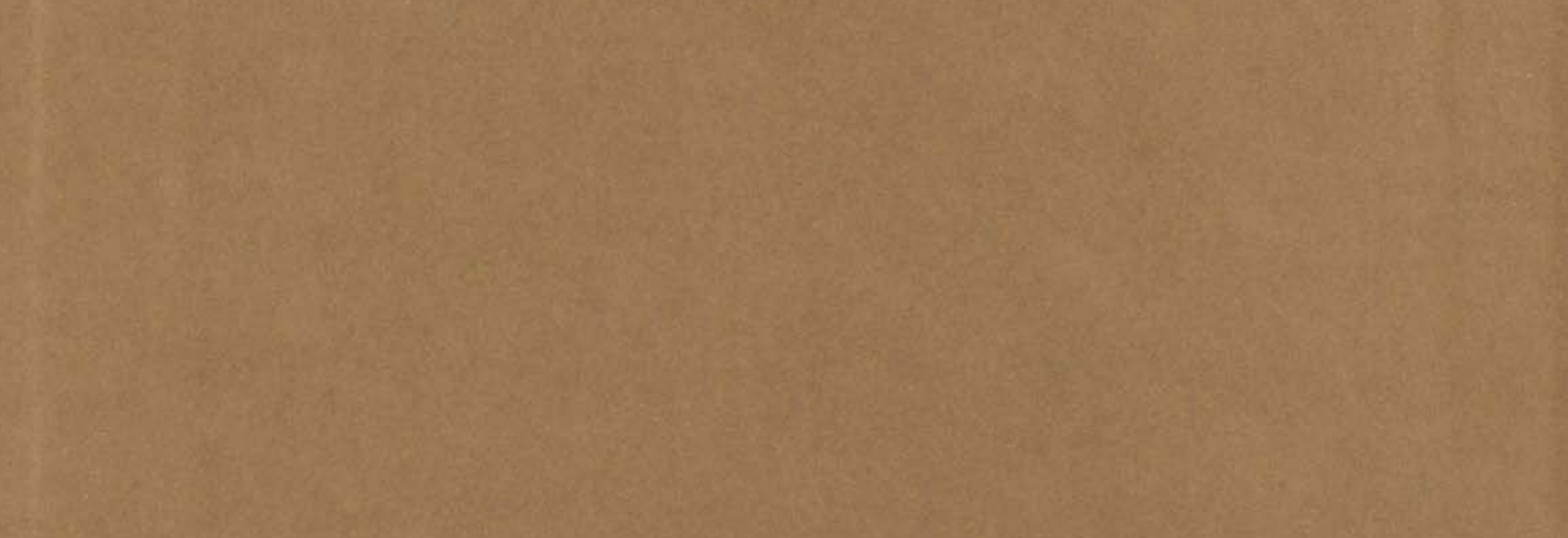

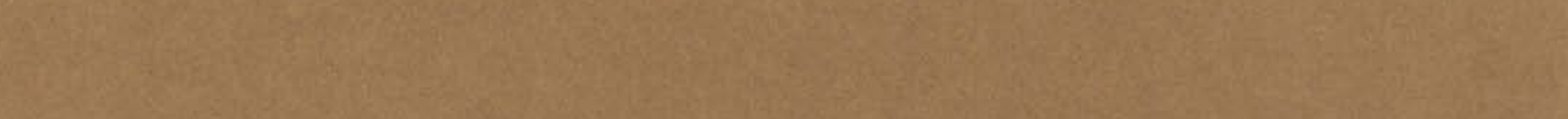

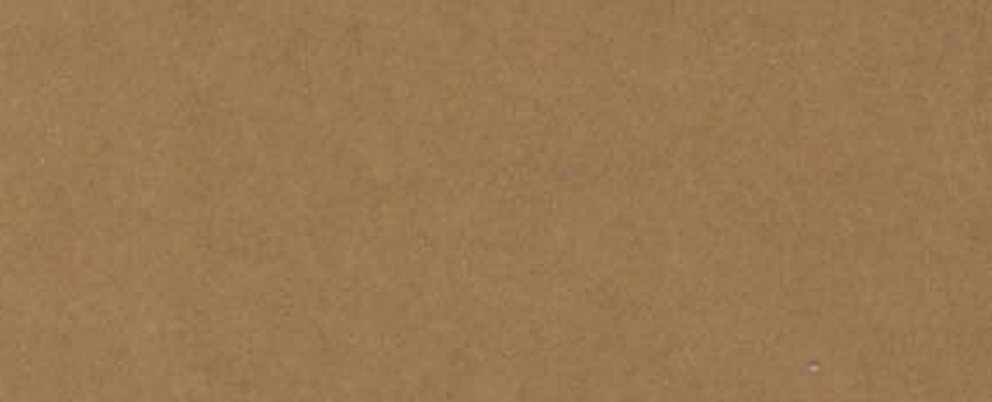
- Sid

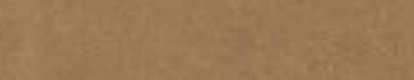

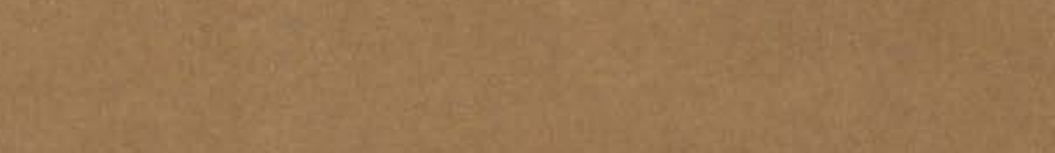

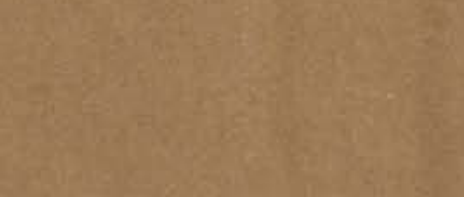

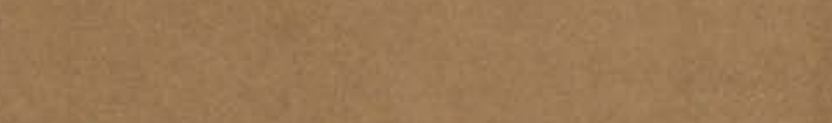
- 



$$
\because 2-2=
$$

